\title{
SYSTEMATIC COMPARISON \\ OF THE MINOS \\ NEAR \& FAR DETECTOR \\ READOUT SYSTEMS
}

Anatael Enrique Cabrera Serra

University of Oxford

The Queen's College

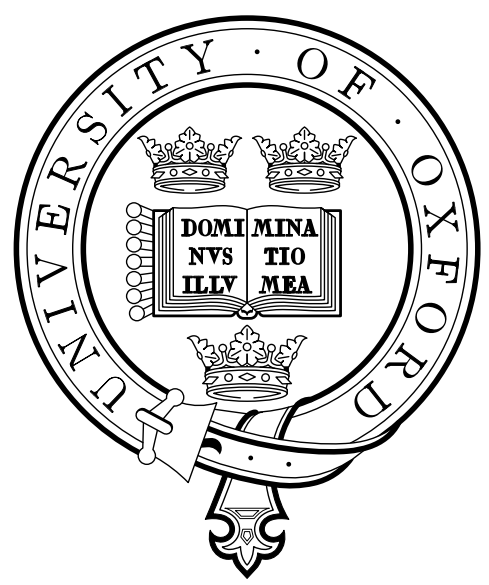

Submitted in partial fulfillment of the requirements for the degree of Doctor of Philosophy

Trinity Term - 2005 
This thesis was supervised by:

Dr Alfons Weber - Oxford University / Rutherford Laboratory (UK)

and examined on the $22^{\text {nd }}$ of June 2005 by:

Dr Steve Biller - University of Oxford (UK)
and
Dr Kai Zuber - University of Sussex (UK)

for approval by the University of Oxford. 


\title{
Systematic Comparison of the MINOS Near \& Far Detector Readout Systems
}

\author{
Anatael Enrique Cabrera Serra \\ The Queen's College - University of Oxford \\ Submitted in partial fulfilment of the requirements for \\ the degree of Doctor of Philosophy \\ Trinity Term - 2005
}

The MINOS experiment is a neutrino oscillation baseline experiment intending to use high resolution L/E neutrinos to measure the atmospheric neutrino oscillations parameters to unprecedented precision. Two detectors have been built to realise the measurements, a Near detector, located about $1 \mathrm{~km}$ downstream from the beam target at the Fermi Laboratory, and a Far detector, located at $736 \mathrm{~km}$, at the Soudan Laboratory. The technique relies on the Near detector to measure the un-oscillated neutrino spectrum, while the Far detector measures the neutrino spectrum once oscillated. The comparison between the two measurements is expected to allow MINOS to measure $\Delta m^{2}$ beyond $10 \%$ precision level.

The Near and Far detectors have been built similarly to minimise possible systematic effects. Both detectors have been endowed with different readout systems, as the beam event rates are very different. The MINOS calibration detector (CalDet), installed at CERN, was instrumented with both readout systems such that they can simultaneously measure and characterise the energy deposition (response and event topology) of incident known particle from test-beams.

This thesis presents the investigations to quantify the impact of the performance of both readout systems on the MINOS results using the measurements obtained with CalDet. The relative comparison of the responses of both readout systems have been measured to be consistent with being identical within a systematic uncertainty of $0.6 \%$. The event topologies have been found to be negligibly affected. In addition, the performance of the detector simulations have been thoroughly investigated and validated to be in agreement with data within similar level of uncertainties. 


\section{Dedicatoria}

"A mi Mamá y a mi Papá,

por ser las personas que maś me han influenciado en mi vida,

y por haberme ayudado a ser casi un 'Hombre'...

a Eva,

con quien empezó éste viaje que Hoy termina,

porque sin su apoyo de entonces Hoy tan sólo sería el sueño que ayer fue...

a Erhan,

por todo ese cariño generoso que me ha siempre brindado

y por haberle dado generosa forma a ese esbozo de 'Hombre' que conoció y ahora soy...

y a Annalisa,

per fare cosí felice questo 'Hombre'...

e sopratutto per darmi tanta voglia e senso per andare avanti!" 


\section{Acknowledgement}

Firstly, I would like to start by thanking my supervisor: Dr Alfons Weber. His impact, during my time in Oxford, ranges from my preparation as a better physicist to becoming a better person. He changed my appreciation for experimental physics dramatically... and he was right about it! He also showed me the way to enjoy research while keeping a high level of performance on the subject investigated. In addition, I deeply appreciate his effort to comprehend my perspective, even in the (many) difficult moments. The only thing I can say I regret is not have learnt more physics from him and, maybe also, not to have managed to engage him a bit more on my work, at least, as much as I would have liked. For all that, and for his friendship, I would like to deeply thank him, as well as Jutta Weber, who I felt all the time present supporting both of us during all these years.

Secondly, I would like to stress the joy of sharing my "graduate path" with so many nice and talented people I met in Oxford and within the MINOS collaboration. Unfortunately, I cannot name them all while I hope I have made them all aware of my gratitude. Nevertheless, I make special mention to those who very especially had a critical impact in my life and studies over the last years. In such a list, I have to mention Dr Nathaniel Tagg (he generously taught me a lot -from the first to the last day- even on how to get stronger), Dr Chris Smith (a friend and an excellent physicist... thanks also for very carefully reading and commenting the manuscript of this thesis) and Dr Costa Andreopoulos (a friend and a source of ideas and plans for today and tomorrow). Last and definitely not the least, of course: Jeff (now Dr Jeff Hartnell)... with whom I have shared planes, buses, beams, detectors, barracks, hotels, whiskies (and other cocktails), a jacuzzi and lots of pain \& pleasure during our DPhil's. It has been a great honour and pleasure to have shared my path with Jeff during all these years, while witnessing how he has become an excellent physicist.

In Oxford, I had the pleasure to meet other excellent physicist whose contribution to my education and motivation have been important such as Dr Giles Barr, 
Dr John Cobb, Dr Antonella de Santo, Dr Katarzyna Grzelak, Dr Mathew Barker and Dr Paul Miyagawa (great C++ "tutor"). In the office, during the painful last months of the DPhil, I had the joy and great pleasure to share the office with Tobi Raufer, Phil Litchfield (thanks to whom submitting was always less remote) and Justin Evans... thanks to them for their friendship and for coping with my stress over the last days while reminding me that the office can be a place for joy! In MINOS, I met some superb physicists with many backgrounds and styles, they were the basis of an enriching and "professionally luxurious" environment for my education. In particular, I have met one of the best physicist I have ever met: Dr Doug Michael, from whom I wish I could have learnt more from his very inspiring character and talent.

At CalDet, I had the pleasure to help to run a real detector in a real beam at CERN. This was a task, as wonderful as painful, from which I have learnt the most. I would like to thank all the CalDet crew for their presence, contributions and lessons kindly given all the way through. In particular, I would like to express my respect for Prof. Jenny Thomas, for having the character and talent to lead the whole project to success while also being able to spend time with each member of the team always opened to teach or learn. I think, I have learnt much from her too.

Despite not being the best time of my life, my time at the MINOS Far Detector did not lack an excellent company (all the mine crew and MINOS physicist on shift) but also granted me with two unexpected surprises: two good friends (Rodolphe Piteira and Simona Murgia) and meeting two wonderful persons: Andy Blake and Bill Miller.

Thirdly, I would like to thank some of my mentors who encouraged and significantly helped me during my path in Physics (chronologically ordered): Dr Ashley Clarke (my tutor at Leeds), Dr D. Adolf (Leeds), Dr Joachim Rose (Leeds) and Dr Olav Ullaland (CERN). I own them much of my strong motivation, enthusiasm and preparation in physics.

Fourthly, I also would like to thank all funding and supporting bodies that helped my DPhil be such a fruitful experience offering opportunities to improve my preparation as a better (neutrino) physicist. In this list, I have to start by thanking the Department of Particle Physics at the University of Oxford (very especially to Prof. Susan Cooper) which granted me with full funding to carry out my studies as well as my college: the Queen's College; which kindly hosted me during those (never too many) days at Oxford. I would like to thank the 
organisation of the Neutrino 2002 conference at Munich, Neutrino Interaction 2004 (to Dr Flavio Cavana) at Gran Sasso Laboratory, Neutrino 2004 conference at Paris and XVI Indian School of Physics (Neutrino Physics) at Prague for the funding provided for my participation. I also thank the ECT*/Marie-Curie-Training (Prof. George Ripka) for having granted me with training-fellowship over the summer 2004

Fifthly, I would to thank my examiners Dr Steve Biller and Dr Kai Zuber for having so kindly accepted to assess my thesis (despite its intimidating thickness) and for having thoroughly read my thesis adding copiously comments on the physics and the editing. Thanks very much to both of them for their effort.

Finally, I would like to thank my Friends - all of them wherever they may be now! - for having flavoured so sweetly the path of life and for having allowed me to hide myself behind the silence I needed to culminate this thesis. Thanks to you all (hoping not to miss any!): Giovanni, Erik, Gianluca, Olga, Joao, Franco, Miguelon, Vianita, Pablito, "Stefano Chiara \& Leonardo", Zora*, Rodrigo (\& his family), Carolina (\& her new family), Lucia, Ruben, Gautam, Paul, Rob, Pete (\& family), Maria, Susan \& Peter (\& family), Nancy, Cesar \& Ade, Gerrit, those already named above and maybe one more that I am sure I forget!

And most especially thanks to all my Families: the one in Tenerife (Mama, Papa, Maria y Gabriela), the one in Turkey (Erhan and all at members of "Ev"), the one at Conversano and the one Here with me: Annalisa... without whom most of this would have been simply impossible and not even worth it!

Allow me to thank you (the "reader" of this thesis) too for reading this document and let me know of any possible comments you might have. I hope you can find the information you want easily and forgive me, in advance, for not being a good writer (but a mere physicist forced to write), while I would sympathise you for being my dear reader.

Anatael Cabrera. Paris, October 2005. 


\section{Contents}

List of Figures vi vi vis

List of Tables vii

1 Introduction 1

2 Neutrino Oscillation Physics 5

2.1 The Standard Model in Brief . . . . . . . . . . . . . . 5

2.1.1 Fundamental Building Blocks . . . . . . . . . . . . 6

2.2 Neutrinos So Far . . . . . . . . . . . . . . . . . . . . . . . . 9

2.3 The Neutrino Anomalies . . . . . . . . . . . . . . . . . . 12

2.3.1 The Solar Neutrino Anomaly . . . . . . . . . . . . . 12

2.3.2 The Atmospheric Neutrino Anomaly . . . . . . . . . . . 18

2.4 Need for a Non-Diagonal Free Hamiltonian . . . . . . . . . . . . . . 20

2.5 Towards Neutrino Oscillations . . . . . . . . . . . . . . . 25

2.6 The Neutrino Community "To-Do-List" . . . . . . . . . . . . . . . . 31

3 The MINOS-NuMI Project 33

3.1 The NuMI Beam . . . . . . . . . . . . . . . . . . 33

3.2 The MINOS Experiment . . . . . . . . . . . . . . . . 37

3.2.1 The MINOS Detector Technology . . . . . . . . . . 37

3.2 .2 The Far Detector . . . . . . . . . . . . . . . . . . . . . . . 44

3.2 .3 The Near Detector . . . . . . . . . . . . . . . . . . . . 49

3.3 Physics Capabilities of MINOS . . . . . . . . . . . . . . . 54

3.3.1 Neutrino Oscillation Physics . . . . . . . . . . . 55

3.3.2 Non-Oscillation Physics . . . . . . . . . . . . 61 
4 The Calibration Detector $\quad 65$

4.1 Objectives of CalDet . . . . . . . . . . . . . . . 65

4.2 Description of CalDet . . . . . . . . . . . . . . 66

4.3 The CalDet within the PS Beam Complex at CERN . . . . . . . . 70

4.4 PID and Triggering Instrumentation . . . . . . . . . . . . . 71

4.4.1 The Time of Flight System . . . . . . . . . . . . . . . . . . 72

4.4 .2 The Beam Trigger . . . . . . . . . . . . . . . . . . . 74

4.4.3 The Cherenkov Counters . . . . . . . . . . . . . . 75

4.5 Physics Runs at CalDet . . . . . . . . . . . . . . 76

5 The MINOS Calibration Chain $\quad 78$

5.1 MINOS Calibration Chain Overview . . . . . . . . . . . . . . 79

5.2 Dedicated Calibration Runs at CalDet . . . . . . . . . . . . 80

5.3 FEE Calibration . . . . . . . . . . . . . . . . . . . . 82

5.3 .1 VA-chips Pedestal . . . . . . . . . . . . . . 82

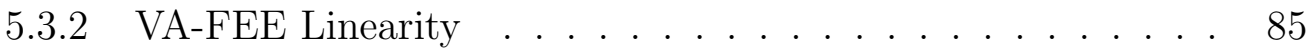

5.3 .3 QIE-chip Calibration .............. 86

5.3 .4 QIE-FEE Linearity . . . . . . . . . . . . . . . . 86

5.3.5 Sparsification Thresholds . . . . . . . . . . . . . 89

5.4 Light Injection Calibration . . . . . . . . . . . . . . . . . . . . . . 91

5.4.1 Gain \& Drift-Point Calibration . . . . . . . . . . . . . . . 92

5.4 .2 Linearity of Readout Systems . . . . . . . . . . . . . . . 96

5.4.3 ND and FD Non-Linearity Parametrisation . . . . . . . . . 97

5.5 Muon Calibration . . . . . . . . . . . . . . . . . . . . 99

5.5.1 Intra-Detector Calibration: "Strip-to-Strip" . . . . . . . . . 99

5.5.2 Inter-Detector Calibration: "MIP" . . . . . . . . . . . . . 100

5.5.3 Attenuation Calibration at CalDet . . . . . . . . . . . . 102

5.6 Absolute Energy Calibration . . . . . . . . . . . . . . . . . 109

5.6.1 Energy Calibration at CalDet . . . . . . . . . . . . . 109

5.6.2 Neutrino Energy Calibration . . . . . . . . . . . . . . . . 110

6 Selection of Events $\quad 112$

6.1 Event Selection Cuts . . . . . . . . . . . . . . . . . . . . . 113

6.1.1 Quality Cuts .................. 113

6.1 .2 Comparison Cuts . . . . . . . . . . . . 115

6.1 .3 PID Cuts . . . . . . . . . . . . . . . . 121

6.1 .4 Topology Cuts . . . . . . . . . . . . . . . . . . . 124 
6.2 Particle Candidates Samples . . . . . . . . . . . . . . . . . . . 131

6.2.1 Electron Candidates Sample . . . . . . . . . . . . . . . . . 132

6.2.2 Muon Candidates Sample . . . . . . . . . . . . . . . . 136

6.2.3 Proton Candidates Sample . . . . . . . . . . . . . . . . . . 139

7 The Near/Far Readout Systems Comparison 142

7.1 Analysis Objectives . . . . . . . . . . . . . . . . . . . . . . . . . 142

7.2 Analysis Strategy . . . . . . . . . . . . . . . . . . . 143

7.2.1 Analysis Probes . . . . . . . . . . . . . . . . . . . 144

7.2 .2 MC Generated Events . . . . . . . . . . . . . . . . 146

7.2 .3 Data Status . . . . . . . . . . . . . . . . . . . . . 147

7.3 Energy Comparison Framework . . . . . . . . . . . . . . . . . . . 148

7.3.1 The Energy Asymmetry Definition: $A_{N / F} \ldots \ldots$. . . . . 148

7.4 Strip-wise Energy Comparison . . . . . . . . . . . . . . . . . 150

7.4.1 Double-Ended Strip Relative Comparison . . . . . . . . . . . 150

7.4.2 Single-End Strip Relative Comparison . . . . . . . . . . 157

7.5 Event-wise Energy Comparison . . . . . . . . . . . . . . . . 159

7.5.1 Analytical Calculations of $A_{N / F} \ldots \ldots \ldots$. . . . . 160

7.5.2 Measurements of $A_{N / F} \ldots \ldots \ldots$. . . . . . . . . 162

7.5.3 $A_{N / F}$ Evolution with Energy . . . . . . . . . . 168

7.5.4 Calibration Repercussions on $A_{N / F} \ldots \ldots$. . . . . . 172

7.5.5 $A_{N / F}$ Width Studies . . . . . . . . . . . . . . . . 180

7.5.6 Systematic Uncertainties . . . . . . . . . . . . . . . . 181

7.6 Topology Comparison . . . . . . . . . . . . . . . . . 186

7.6.1 Basic Topology Comparison . . . . . . . . . . . . . . 187

7.6.2 Longitudinal Energy Profile . . . . . . . . . . . . . . . . . . 194

7.6.3 Transverse Energy Profile . . . . . . . . . . . . . . . 196

7.7 Time Comparison of the ND Readout System . . . . . . . . . . . . 198

7.7.1 Time Structure of Pulses into the Readout System . . . . . 198

7.7.2 Time Spread over Readout Buckets . . . . . . . . . . . . . . 200

7.7.3 Definition of the QIE-FEE Readout Window . . . . . . . . 200

7.8 Specific Instrumental Effects . . . . . . . . . . . . . . . . . . 201

7.8.1 Bucket Sparsification on Muons . . . . . . . . . . . . . . 202

7.8.2 Crosstalk Measurements . . . . . . . . . . . . . . . . 203 
8 Conclusions $\quad 207$

8.1 Readout Systems Comparison at CalDet . . . . . . . . . . . . . 207

8.2 Readout Systems Comparison in MINOS . . . . . . . . . . . . . . . 209

8.3 Repercussions on the Physics Capabilities of MINOS . . . . . . . 211

A Cross-Talk Studies of the Hamamatsu M64 i

A.1 PMT Crosstalk . . . . . . . . . . . . . . . . . . . i i

A.2 Experimental Setup: the Oxford PMT Test-Stand . . . . . . . . . ii

A.3 PMT Crosstalk Separation . . . . . . . . . . . . . ii

A.3.1 Implementation of the 2D Cut . . . . . . . . . iv

A.4 Characterisation of PMT Electrical Crosstalk . . . . . . . . . v v

A.4.1 Readout Electronics Crosstalk Studies . . . . . . . . . . vii

A.5 Characterisation of PMT Optical Crosstalk . . . . . . . . . vii

A.6 Summary of Analysis . . . . . . . . . . . . . . . . . . . xii

B Near/Far Single Noise Measurements xiii

C Slicer Implementation $\quad$ xxii

D The Swimmer Algorithm xxiv

D.1 Swimmer Algorithm Criteria . . . . . . . . . . . . xxv

E Analysis Software Developed for MINOS xxx

E.1 "SexyPedestal" . . . . . . . . . . . . . . . . . . xxx

E.2 "PedStability" . . . . . . . . . . . . . . . . . . . xxxi

E.3 "LookAtLUT" . . . . . . . . . . . . . . . . . . . . xxxi

E.3.1 "TreeMyLUT" . . . . . . . . . . . . . . . . . xxxi

E.3.2 "LookAtLUT" . . . . . . . . . . . . . . . . . . xxxii

E.4 "LIGainCurve" . . . . . . . . . . . . . . . . . . . . . . xxxxii

E.5 "NFComp" \& "PIDSurveyor" . . . . . . . . . . . . . . . . . xxxii

E.5.1 "NFCompReco" . . . . . . . . . . . . . . . . xxxiii

E.5.2 "PIDSurveyor" ................. . . xxxiii

E.5.3 "NFCompAna" . . . . . . . . . . . . . . xxxiv

E.5.4 "NFCompMvsD" and "NFCompVsE" . . . . . . . . . . xxxv

Bibliography xxxvi 


\section{List OF Figures}

2.1 Diagram of an Atom as Understood Today . . . . . . . . . . . . 6

2.2 Solar Neutrino Fluxes as Predicted by SSM . . . . . . . . . . . . . 13

2.3 Solar Neutrino Results as Today . . . . . . . . . . . . . . . . . . . . 14

2.4 SNO Solar Neutrino Fluxes Measurements . . . . . . . . . . . . . . 16

2.5 Atmospheric Neutrinos Generation Diagram . . . . . . . . . . . . . 19

$2.6 \mathcal{R}^{\prime}$ as Today . . . . . . . . . . . . . . . . . . . 20

2.7 SK Atmospheric Azimuthal Angular Distribution . . . . . . . . . . 21

2.8 The Neutrino Oscillations Unique Signature . . . . . . . . . . . . . 25

2.9 KamLAND Evidence towards Solar Neutrino Oscillations . . . . . . 26

2.10 The K2K Evidence for Neutrino Oscillations . . . . . . . . . . . . . 27

2.11 Experimental Evidence for Neutrino Oscillation . . . . . . . . . . . 28

2.12 L/E Modulation of the Survival Probability . . . . . . . . . . . . . 29

2.13 The Neutrino Oscillation Parameter Space Today . . . . . . . . . . 30

3.1 MINOS Experiment on a Map . . . . . . . . . . . . . . . . 34

3.2 NuMI Beam Production Diagram . . . . . . . . . . . . . . . . . 35

3.3 NuMI Beam Spectra and Configuration . . . . . . . . . . . . . 36

3.4 The MINOS Readout System . . . . . . . . . . . . . . . . . 38

3.5 A MINOS Detector Plane . . . . . . . . . . . . . . . . . 39

3.6 Scintillator Strip Diagram and Photo . . . . . . . . . . . . . . . . . 40

3.7 The MINOS Multi-Anode PMT . . . . . . . . . . . . . . . . . 41

3.8 Dynode Trigger Threshold Setting at CalDet . . . . . . . . . . . . . 41

3.9 MINOS Far Detector: Site and Photo . . . . . . . . . . . . . . . . . 44

3.10 Overall Structure of MINOS Far Detector . . . . . . . . . . . . 45

3.11 VA-chip Digitisation . . . . . . . . . . . . . . 46

3.12 Diagram of the VA-chip Electronics . . . . . . . . . . . . . 47 
3.13 MINOS Near Detector: Site and Photo . . . . . . . . . . . . . . . . 49

3.14 MINOS Near Detector Plane: NuMI Beam Spot . . . . . . . . . . . 50

3.15 Overall Structure of MINOS Near Detector . . . . . . . . . . . . . . 51

3.16 QIE Electronics: Logic Diagram and Calibrated Response . . . . . 53

3.17 Types of Event Topology Diagram . . . . . . . . . . . . . . . 55

$3.18 \mathrm{CC} \nu \mathrm{N}$ Cross-Sections: Data and MC . . . . . . . . . . . . . 56

3.19 MINOS Experiment on the Earth . . . . . . . . . . . . . . 56

$3.20 \nu_{\mu}$ CC Spectral Distortion . . . . . . . . . . . . . . 57

$3.21 \nu_{\mu}$ CC Disappearance: Neutrino Oscillation Parameter Fit Plot . . . 59

$3.22 \nu_{e}$ CC Appearance Oscillation Parameter Sensitivity Plot . . . . . . 60

3.23 Sterile Neutrino Search Oscillation Parameter Extraction Plot . . . 62

4.1 The CalDet at T7 in Near/Far Comparison Configuration . . . . . . 68

4.2 The CalDet Planes and their Readout Fibres Configuration . . . . . 69

4.3 Average Relative Particle Composition of the T7 Beam . . . . . . . 70

4.4 Diagram of the T7 Beam Line within the PS Beam Complex . . . . 71

4.5 Diagram of the CalDet External PID Instrumentation . . . . . . . . 72

4.6 ToF System Time Distribution at CalDet . . . . . . . . . . . . . . . 73

4.7 Cherenkov Counters: Pressure and Efficiency . . . . . . . . . . . . . 76

5.1 The MINOS Calibration in a Nut-shell . . . . . . . . . . . . . . . . 79

5.2 Pedestal Distribution . . . . . . . . . . . . . . . . 82

5.3 Effect of Pedestal Truncation _. . . . . . . . . . . . . . . 83

5.4 Pedestal Stability Studies . . . . . . . . . . . . . . . . . 85

5.5 VA-FEE Non-linearity Curve . . . . . . . . . . . . . . . 86

5.6 QIE-FEE Non-linearity Curve . . . . . . . . . . . . . . 87

5.7 QIE-FEE Calibration Quality Studies . . . . . . . . . . . . 88

5.8 Sparsification Threshold Definition Criteria . . . . . . . . . . . . . . 89

5.9 Sparsification Thresholds Per Readout Type . . . . . . . . . . . . . 90

5.10 Light Injection System Diagram at CalDet . . . . . . . . . . . . . . 91

5.11 Drift-Point Calibration Capability . . . . . . . . . . . . . . . 93

5.12 Long Term PMT Gain Variation at FD . . . . . . . . . . . . . . . . 95

5.13 Readout System Non-linearity Measurement . . . . . . . . . . . . . 96

5.14 ND and FD Readout Systems Non-Linearities . . . . . . . . . . . . 98

5.15 MIP Calibration Definition: MIP Window . . . . . . . . . . . . . . 101

5.16 Attenuation Measured with Cosmic Muons . . . . . . . . . . . . . . 102

5.17 Integrated Attenuation Curve at CalDet . . . . . . . . . . . . . . . 104 
5.18 Beam Spot Variation at CalDet . . . . . . . . . . . . . . . 105

$5.194 \times$ MIP Calibration Definition . . . . . . . . . . . . . . 108

5.20 Absolute Energy Calibration of Electrons . . . . . . . . . . . . . . 110

6.1 Readout Systems Time Correlation . . . . . . . . . . . . . . . 116

6.2 Time Fiducial Cut Definition . . . . . . . . . . . . . . . . . 117

6.3 Example of a Multiple Particle Trigger Tagged by the Slicer . . . 119

6.4 Efficiency of Comparison Cuts . . . . . . . . . . . . . . . . 120

6.5 Overall Multiple Events Rejection Performance . . . . . . . . . . . 121

6.6 ToF Time Difference Distribution Sample . . . . . . . . . . . . . . . 122

6.7 Cherenkov Counters Charge Distribution Sample . . . . . . . . . . 123

6.8 Beam-Trigger Tag to Cherenkov Counters Difference Distribution Sample . . . . . . . . . . . . . . . . . . 125

6.9 Range Distribution Sample for Electrons Candidates . . . . . . . . 126

6.10 Range Distribution for Pions Candidates . . . . . . . . . . . . . . 127

6.11 Range Distribution of Muons after Tracking . . . . . . . . . . . . . 129

6.12 Track-End Location for Calibration Quality Muons . . . . . . . . . 130

6.13 Efficiency of Range Cut for Muons . . . . . . . . . . . . . . . . . . 131

6.14 Electrons 3D Hit Map . . . . . . . . . . . . . . . . . . . . . . . . . 133

6.15 Electron Candidate Sample Selection Cuts (1) . . . . . . . . . . . . 134

6.16 Electron Candidate Sample Selection Cuts (2) . . . . . . . . . . . . 135

6.17 Muons 3D Hit Map . . . . . . . . . . . . . . . . . . . . . . . . . . 137

6.18 A Well-defined Stopping Muon . . . . . . . . . . . . . . . . 137

6.19 Muon Candidate Sample Selection Cuts . . . . . . . . . . . . . . . 138

6.20 Protons 3D Hit Map . . . . . . . . . . . . . . . . . . . . . . . . . . 139

6.21 Proton Candidate Sample Selection Cuts . . . . . . . . . . . . . . 140

7.1 Correlation of Responses Strip-by-Strip . . . . . . . . . . . . . . . . 149

7.2 Strip-by-Strip $A_{N / F}$ Evolution . . . . . . . . . . . . . . . . . . 152

7.3 Relative Non-Linearities as Measured by LI . . . . . . . . . . . . . 153

7.4 Bucket Sparsification Effect . . . . . . . . . . . . . . . . . . . . . 154

7.5 Double/Single Ended Hits Contributions . . . . . . . . . . . . . . . 158

$7.6 \quad A_{N / F}^{4 \times \mathrm{MIP}}$ for Electrons: Data versus MC predictions . . . . . . . . . . 164

$7.7 A_{N / F}^{\text {Beam-Spot }}$ for Electrons: Data versus MC predictions . . . . . . . . 166

$7.8 A_{N / F}^{S 2 S}$ for Electrons: Data versus MC predictions . . . . . . . . . . 167

7.9 Event-by-Event $A_{N / F}^{S 2 S}$ Evolution for Electrons . . . . . . . . . . 168

7.10 Event-by-Event $A_{N / F}^{S 2 S}$ Evolution for Muons . . . . . . . . . . . 169 
7.11 Event-by-Event $A_{N / F}^{S 2 S}$ Evolution for Protons . . . . . . . . . . 170

7.12 Event-by-Event $A_{N / F}^{4 \times \text { MIP }}$ Evolution . . . . . . . . . . . . . . . 171

7.13 Demonstration of the Attenuation Correction . . . . . . . . . . . . 172

7.14 Remnant Temperature Dependence of $A_{N / F} \ldots \ldots$. . . . . . . . . 174

7.15 Systematic Uncertainty on $A_{N / F}$ due to the LI-drift Calibration . . 175

7.16 Dependence of $A_{N / F}$ on the Trigger Rate . . . . . . . . . . . . 176

7.17 Calibration Muon Diagram . . . . . . . . . . . . . . . . . . . 177

7.18 Calibration Muon Results . . . . . . . . . . . . . . . . . . 178

7.19 Event-by-Event $4 \times \mathrm{MIP} A_{N / F}$ Evolution for Muons . . . . . . . . 179

7.20 Near/Far Energy Asymmetry Width . . . . . . . . . . . . . . . . . 181

7.21 Near/Far Energy Asymmetry Width Evolution . . . . . . . . . . . . 182

7.22 Basic Topology Features . . . . . . . . . . . . . . . . . . . . . . . 188

7.23 Topology Characterisation Evolution for Electrons . . . . . . . . . . 191

7.24 Topology Characterisation Evolution for Muons . . . . . . . . . . . 192

7.25 "Shower-Max" Plane Location . . . . . . . . . . . . . . . . . . . . . 193

7.26 "Shower-Max" Strip Location . . . . . . . . . . . . . . . . . . . . . 194

7.27 Muon Simulation Accuracy in GEANT3 . . . . . . . . . . . . . . . 195

7.28 Longitudinal Energy Profiles of Electrons . . . . . . . . . . . . . . . 196

7.29 Transverse Energy Profiles of Electrons . . . . . . . . . . . . . . . 197

7.30 Time Pulse Shape . . . . . . . . . . . . . . . . . . . . . . . . . . . 198

7.31 Time Spread . . . . . . . . . . . . . . . . . . . . . . 199

7.32 Number of Buckets Hit . . . . . . . . . . . . . . . . . . . 200

7.33 ND Bucket Integration: MC/Data Comparison . . . . . . . . . . . . 201

7.34 Strip-by-Strip $A_{N / F}$ Evolution for Muons . . . . . . . . . . . . . . 202

7.35 Unread-Crosstalk Measurements . . . . . . . . . . . . . . . . . . . . 204

7.36 Unread-Crosstalk for Muons and Electrons . . . . . . . . . . . . . . 205

7.37 PMT Crosstalk from Muons . . . . . . . . . . . . . . . . . 206

$8.1 A_{N / F}^{\text {Total }}$ versus Event Energy . . . . . . . . . . . . . . . . . . . . 210

8.2 Fractional Error Induced in $\Delta m^{2} \ldots \ldots \ldots$. . . . . . . . . 211

A.1 Charge Spectrum of a Cross-Talked Pixel . . . . . . . . . . . iii

A.2 Correlation Between Injected Pixel versus Cross-Talked Pixel . . . . iv

A.3 Diagram of Crosstalk in the M64 . . . . . . . . . . . . . v v

A.4 EXT versus Inter-Pixel Distance . . . . . . . . . . . . . . vi

A.5 Broadening of the EXT Distribution . . . . . . . . . . . vii

A.6 Readout Electronics Crosstalk . . . . . . . . . . . . . . . . viii 
A.7 Characterisation Model for OXT . . . . . . . . . . . ix

A.8 Evidence for Pre-First-Dynode Nature of OXT . . . . . . . . . . $\mathrm{x}$

A.9 Evidence for OXT Origin Pattern . . . . . . . . . . . . . . . xi

B.1 Single-Noise Occupancy during Beam Running . . . . . . . . . . xiv

B.2 Charge \& Time Distribution of Single . . . . . . . . . . . . . . . xv

B.3 Number of Hits per Strip-end . . . . . . . . . . . . . . . . . xvi

B.4 Beam Single versus Beam Momentum . . . . . . . . . . . . . . . xvii

B.5 Selection of Singles During Cosmic Runs . . . . . . . . . . . . . . . xix

B.6 Pattern of Single During Cosmic Runs . . . . . . . . . . . . . . . . xix

B.7 Charge and Time Distribution of Singles . . . . . . . . . . . . xx

C.1 Time Distribution of Energy Deposition . . . . . . . . . . . . xxiii

D.1 Swimmer Diagram . . . . . . . . . . . . . . . xxiv

D.2 Swimmer Weight Diagram . . . . . . . . . . . . . . xxvi

D.3 Swimmer Step-1 . . . . . . . . . . . . . . . . . xxvii

D.4 Swimmer Step-2 . . . . . . . . . . . . . . . . xxvii

D.5 Swimmer Step-3 . . . . . . . . . . . . . . . . . . xxvii

D.6 Swimmer Examples . . . . . . . . . . . . . . . . . . . . xxix

E.1 NFComp: Data Flow Diagram . . . . . . . . . . . . . . . . xxxiv

E.2 NFComp: Execution Structure . . . . . . . . . . . . . . xxxx 


\section{LIST OF TABLES}

2.1 Properties and Quantum Numbers of Fermions. . . . . . . . . . . 7

2.2 Properties and Quantum Numbers of Bosons . . . . . . . . . . . . 8

2.3 Solar Neutrino Production Reactions . . . . . . . . . . . . . . . . . 13

2.4 Results of Radio-Chemical Experiments . . . . . . . . . . . . . . . . 15

2.5 Results of Kamiokande Experiments . . . . . . . . . . . . . . . . 17

4.1 List of CalDet Configurations . . . . . . . . . . . . . . 67

5.1 Pedestal Stability: Studied Time Scale Variations . . . . . . . . . . 84

5.2 MIP Calibration Constant for CalDet Near/Far 2003 . . . . . . . . 101

5.3 Integrated Attenuation Computation Measurements . . . . . . . . . 104

$5.44 \times$ MIP Calibration for Near/Far Running . . . . . . . . . . . . . . 107

5.5 Absolute Calibration of Electrons . . . . . . . . . . . . . . . . 109

6.1 Range Cut for $\mu / \pi$ Separation . . . . . . . . . . . . . . . . . . 129

6.2 Sequence of Cuts for Electron Selection . . . . . . . . . . . . . . 132

6.3 Sequence of Cuts for Muon Selection . . . . . . . . . . . . . 136

6.4 Sequence of Cuts for Proton Selection . . . . . . . . . . . . . . . . . 139

7.1 MC Generation Configuration: Detector Simulation . . . . . . . . . 146

7.2 MC Generation Configuration: Calibration . . . . . . . . . . . . . . 146

7.3 MC Generation Configuration: Event Location . . . . . . . . . . . . 147

7.4 Data Calibration . . . . . . . . . . . . . . . . . 147

7.5 List of Rejected Strip-Ends . . . . . . . . . . . . . . . . . . . . . . . 148

$7.6 A_{N / F}$ Uncertainty Budget . . . . . . . . . . . . . . . . 183

7.7 Final $A_{N / F}$ Measurements . . . . . . . . . . . . . . . . . . . . . 183

7.8 Final $A_{N / F}^{T o t a l}$ Measurements . . . . . . . . . . . . . . . 186 


\section{Introduction}

Neutrinos have been in focus of fundamental research since the moment that Pauli proposed their existence up to today. Moreover, learning about the nature of neutrinos has been rewarded with the fruitful improvement of our understanding of the nature of the weak force. With neutrinos, critical contributions have been made to different aspects of our model for the electroweak sector: the so-called "Standard Model" of Particle Physics $(\mathcal{S M})$.

Due to lack of experimental evidence or theoretical notion, neutrinos were thought to be massless from very early stages. In fact, the $\mathcal{S M}$ was built in the beginnings adopting such an assumption. However, massive neutrinos can readily fit into the framework of the $\mathcal{S M}$. The evidence for massive neutrinos comes from the possible solution to two long standing and intriguing neutrino problems, characterised by neutrino fluxes having been measured to be lower than expected. These two problems were the so-called "Solar Neutrino \& Atmospheric Anomalies". The solution to these two problems is currently believed to be due to so-called "Neutrino Oscillations".

Neutrino oscillations come about within a similar theoretical framework, already familiar to the $\mathcal{S M}$, needed to explain some of the phenomenology observed in the quark sector. This framework, called "Flavour Mixing", relies on the fact that weak eigenstates (states manifested during weak interaction) can be regarded as superpositions of mass eigenstates (freely propagated states in time-space), and vice versa. In the case of quarks, the mixing happens between strong and weak eigenstates. This superposition of states can be mathematically formulated through a mixing matrix, whose parametrisation has to be measured as the $\mathcal{S M}$ makes no 
prediction about it. This framework was developed when studying the weak decay on kaons in the 60's and later gave rise to the discovery that the weak interaction violated the CP symmetry by a small amount. CP violation is now known to be intrinsically built in to the framework of flavour mixing, due to the unitarity and dimensional constraints of the flavour mixing matrix, to the extent the unitary triangle is closed. Neutrino oscillations are simply the macroscopic manifestation of flavour mixing into the leptonic sector, which had been neglected from the original $\mathcal{S M}$.

Like in the quark sector, the flavour mixing insight has been and still is dominated by the experimental developments. Many experiments have been devoted to measure the solar and atmospheric fluxes to better accuracy and precision using different techniques to build up a coherent picture within which neutrino oscillations are the most favoured hypothesis compatible with all data available as today. Much effort has been dedicated to cross-checking results by conceiving experiments with different systematics uncertainties and far reaching capabilities. Despite the fact that "global analyses" are strongly consistent with neutrino oscillations, there are still a few non-standard models allowed, which could yield similar experimental results, such as neutrino decay and decoherence.

The MINOS experiment (Main Injector Neutrino Oscillation Search) has been designed under the hypothesis of neutrino oscillation to further demonstrate it and to perform high precision measurements, as explain in Chapter 3. MINOS will measure to an unprecedented precision the unique signature of neutrino oscillations: the dependence of the disappearance probability upon $\mathrm{L} / \mathrm{E}$ (the ratio of the the distance travelled to the neutrino energy), as will be discussed in Chapter 2. Measuring such a L/E modulation of the disappearance for the solar and atmospheric neutrino oscillations cases has been shown by KamLAND and SuperKamiokande$\mathrm{K} 2 \mathrm{~K}$ experiments, respectively. MINOS is a long base-line experiment that relies on neutrinos generated by a beam, whose can be measured and the distance between production and detection points is also accurately known. Therefore, only few hundreds high resolution L/E events are needed to show the L/E dependence caused by neutrino oscillation, as the $\mathrm{K} 2 \mathrm{~K}$ experiment has already proved using the same approach. Indeed, $\mathrm{K} 2 \mathrm{~K}$ has, this way, proved the neutrino oscillation hypothesis to $\sim 4 \sigma$ (statistically limited: less 200 events) as the explanation for their observations: rate and spectral distortion measurements. Long base-line experiments need to intelligently choose their L/E such that the effect of oscillation is maximal rendering their sensitivity to oscillation also maximal. 
MINOS will produce a few thousand high resolution L/E events allowing high precision measurements of the neutrino oscillation parameters. In particular, MINOS aims to measure the atmospheric $\Delta m^{2}$ with a precision $<10 \%$. To this end, MINOS intends to map the disappearance probability by measuring the unoscillated spectrum of beam muon-neutrinos with a the Near Detector (ND), located at $\sim 1 \mathrm{~km}$ away from the source, and the oscillated spectrum at the Far Detector (FD) located at the Soudan National Laboratory ( $730 \mathrm{~km}$ away). In order to map the oscillation disappearance probability MINOS has to measure the energy of the neutrinos in both detector precisely, nominally $\sim 5 \%$. Therefore, calibration is a critical concern in MINOS. Particularly important is the capability of comparing results across the ND and FD for which a precise inter-detector calibration is needed (nominally $\sim 2 \%$ ).

Since the ND and FD are located at two different distances with respect to the neutrino beam source, the rate of neutrinos per beam spill is very different. This fact led the MINOS collaboration to opt for instrumenting the two detectors with different types of readout systems: a combination of Photo-Multiplier Tubes (PMT) and Front End Electronics (FEE). The design is such that ND readout system can digitise charge pulses efficiently at high rates (up to 20 events within $10 \mu \mathrm{s}$ ), while the FD is a fast time-stamping electronics, which goes dead for a few $\mu \mathrm{s}$ upon triggering, therefore it is designed for very low event rates.

The design of MINOS has assumed that, after calibration, the energy reconstruction and event topology are identical for both readout systems. This thesis is devoted to the investigation and quantification of this assumption. To this end the third MINOS detector (the MINOS Calibration Detector) was instrumented with both readout systems (one on each side), such that the responses can be simultaneously calibrated, measured and compared in a hit-by-hit basis upon incidence of known particles with known energies from the CERN PS T7 test-beam. Chapter 4 explains the experimental setup, i.e. the ND and the FD readout systems at CalDet, as well as all specific features needed to run the CalDet efficiently in a beam environment. Chapter 5 is devoted to the calibration sequence used for for the CalDet "Near/Far" running mode. A few calibration stages described do not belong to the standard MINOS calibration chain, but had to be developed by the author or through collaboration with MINOS colleagues for the specific purposes of the analysis. Chapter 6 describes the critical stage of event selection. During this stage, measures have been taking to ensure that both differently functioning readout systems have equivalent acceptances for the events to be used in the read- 
out system performance comparison. The contents of Chapters 5 and 6 are central to the results shown during the Near/Far comparison.

Finally, Chapter 7 is devoted to the Near/Far readout system comparison per se. The main goal of this analysis is to characterise the ability of MINOS to calibrate the inter-detector normalisation at the level of the readout systems responses, while the readout systems are physically located in the same detector and exposed to the same energy depositions. This analysis also generally provides a sensitive investigation and test-bed of the MINOS calibration and detector simulation frontends.

Much of the discussion in Chapter 7 will involve familiarisation with the concept of PMT crosstalk. Appendix A summarises the results, accomplished by the author, on PMT crosstalk at the Oxford PMT test-stand, which can serve the reader as a background to understand better the basis of PMT crosstalk. 


\section{CHAPTER 2}

\section{Neutrino Oscillation Physics}

This chapter will be dedicated to give the short introduction into the Standard Model and the current status of neutrino physics to familiarise the reader with the developments in the field leading to the neutrinos oscillation hypothesis. The first section will fully devoted to introducing the Standard Model framework. The second section will be devoted to a short historical account related to the advances led by neutrino physics to our elaboration of the Standard Model. The following three sections will be focused on illustrating the experimental evidence justifying the need for a neutrino flavour changing mechanisms and a short discussion about the theoretical framework provided by neutrino oscillations to account for the observations. The final section will aim to summarise some of the most crucial open questions still waiting to be answered about the fascinating nature of neutrinos.

\subsection{The Standard Model in Brief}

The Standard Model of Particle Physics $(\mathcal{S M})[1]$ is describes how nature works at the level of fundamental particles, explaining most (while not all) of the so far accessible phenomena. Mathematically speaking, the $\mathcal{S M}$ is a renormalisable gauge field theory describing how fundamental particles interact with each other. Within its framework fermions interact with each other by the exchange of gauge boson particles. The types of interactions accounted are electromagnetism, weak and strong interactions, although strong interaction is often not regarded as part of the originally proposed $\mathcal{S} \mathcal{M}$. Particles having electromagnetic charge interact electromagnetically while those endowed with weak isospin (weak-charge-like) and colour 
(strong-charge-like) couple through weak and strong forces respectively. Gravitational interactions, sensed by massive particles, are not described by the $\mathcal{S M}$, as the regime over which the model is believed to be valid is such that gravitational interactions are negligible.

At low energies, the atom (see Figure 2.1) can be understood as consisting of electrons "revolving" around the atomic nucleus due to electromagnetic interaction. The nucleus is made of protons (p) and neutrons (n) which themselves can be thought of consisting of combinations of three of the two lightest quarks: up $(u)$ and down (d) quarks. Quarks, unlike electrons, feel the strong force. Therefore, a proton and a neutrons are not fundamental particle but $\mathrm{u}-\mathrm{u}-\mathrm{d}$ and $\mathrm{u}-\mathrm{d}-\mathrm{d}$ quark combinations. The atomic nucleus is held together due to the strong force. The weak force is responsible, for example, for the so-called $\beta$-decay of the atomic nuclei.

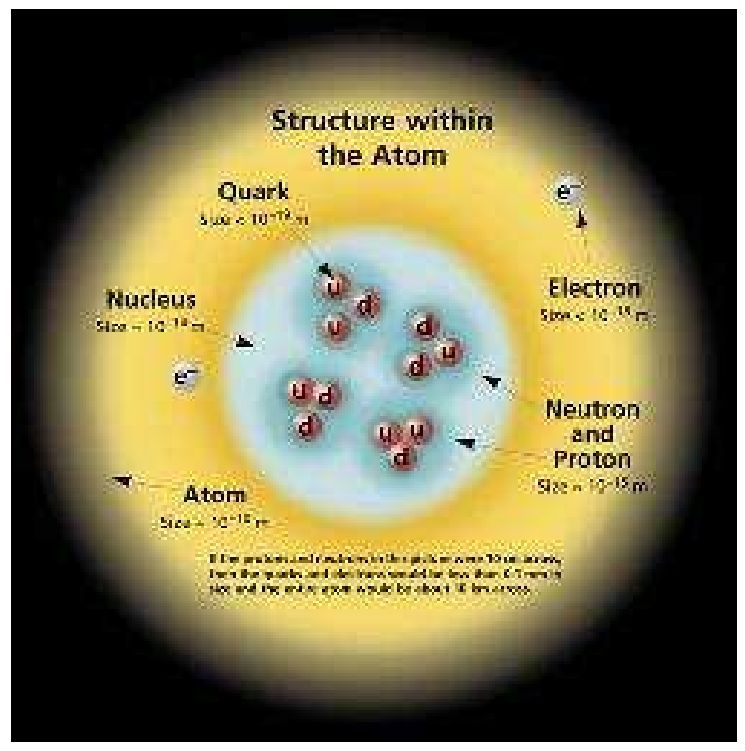

Figure 2.1: Diagram of an Atom as Understood Today. Plot from [2].

Electrons, proton and neutrons are the lightest and most stable known particles therefore making up most known matter at the chemical level, i.e. the atom.

\subsubsection{Fundamental Building Blocks}

\section{Fermions}

Electrons and $\mathrm{u}, \mathrm{d}$ quarks are fermions, as they are spin $\pm 1 / 2$ particles and therefore they must obey the Pauli's Principle of Exclusion or the so-called Fermi-Dirac statistics. Electrons and u,d quarks are however very different as they are sensitive 
to different interactions. This difference is further classified by grouping electrons within the family of the so-called leptons and the u,d quarks are grouped within already introduced family of the quark. Leptons are characterised by not interacting strongly, they are colour singlets. Quarks are, instead, characterised by interacting strongly. Another member of the lepton family are the neutrinos, which do not form part of the atom but which can be found copiously in nature as they are also stable.

At high energies, or during short enough time scales, more massive leptons and quarks can be generated, typically occurring in cosmic ray interactions and particle accelerators. All leptons and quarks so far known are listed in Table 2.1.

\begin{tabular}{||c|c|c|c|c||}
\hline Gen. & Flavour & $\mathrm{Q}$ & $\left(\mathrm{T}_{3}\right)_{L}$ & Mass $(\mathrm{MeV})$ \\
\hline \hline \multirow{2}{*}{1} & $\mathrm{e}$ & -1 & $-\frac{1}{2}$ & 0.51 \\
\cline { 2 - 5 } & $\nu_{e}$ & 0 & $+\frac{1}{2}$ & $<3 \times 10^{-6}$ \\
\hline \multirow{2}{*}{2} & $\mu$ & -1 & $-\frac{1}{2}$ & 105.7 \\
\cline { 2 - 5 } & $\nu_{\mu}$ & 0 & $+\frac{1}{2}$ & $<0.19$ \\
\hline \multirow{2}{*}{3} & $\tau$ & -1 & $-\frac{1}{2}$ & 1777 \\
\cline { 2 - 5 } & $\nu_{\tau}$ & 0 & $+\frac{1}{2}$ & $<18.2$ \\
\hline \hline \multirow{2}{*}{1} & $\mathrm{u}$ & $+\frac{2}{3}$ & $+\frac{1}{2}$ & $1.5-5$ \\
\cline { 2 - 5 } & $\mathrm{d}$ & $-\frac{1}{3}$ & $-\frac{1}{2}$ & $17-27$ \\
\hline \multirow{2}{*}{2} & $\mathrm{c}$ & $+\frac{2}{3}$ & $+\frac{1}{2}$ & $1100-1400$ \\
\cline { 2 - 5 } & $\mathrm{s}$ & $-\frac{1}{3}$ & $-\frac{1}{2}$ & $60-170$ \\
\hline \multirow{2}{*}{3} & $\mathrm{t}$ & $+\frac{2}{3}$ & $+\frac{1}{2}$ & 173800 \\
\cline { 2 - 5 } & $\mathrm{b}$ & $-\frac{1}{3}$ & $-\frac{1}{2}$ & $4100-4400$ \\
\hline \hline
\end{tabular}

Table 2.1: Properties and Quantum Numbers of Fermions Q is the electromagnetic charge of the particle in units of $\mathrm{e} ; \mathrm{T}_{3}$ is the weak isospin of the particle, the quantum number for the weak force. The subscript $\mathrm{L}$ indicates that only left handed chiral particles and right handed anti-particles feel the weak force. The quantum numbers for the anti-particles are obtained by multiplying those shown by -1 . Quarks also posses colour (red, blue and green), which is the quantum number for the strong force.

There are three generations of leptons and quarks, therefore the $\mathcal{S M}$ is symmetric in terms of the number of leptons and quarks. $\mu$ and $\tau$ are like heavy electrons, which are therefore unstable decaying into lighter particles. While $e, \mu$ and $\tau$ are electrically charged, massive, neutrinos were modelled to be neutral and massless, therefore allowed to interact through weak interaction only. The charm (c) and top (t) quarks are heavier versions of the $\mathrm{u}$ quark, while the strange (s) and bottom (b) quarks are the corresponding heavier versions of the d quark. Quarks are sensitive to all three interactions (strong, weak and electromagnetic) endowing them with a 
rich phenomenology.

Neutrinos $(\nu)$ are associated by flavour to $e, \mu$ and $\tau$ (left-handed isospin doublets for particles) during their interactions: $\nu_{e}, \nu_{\mu}$ and $\nu_{\tau}$. u-like and d-like quarks are also paired during weak interactions. The weak interactions are characterised $\mathrm{V}$-A theories for which parity is violated maximally and the fact that neutrinos have a fixed helicity (always left-handed). The weak interaction violates CP (chargeparity) symmetry in a very small fraction in the quark sector. This observation has been proved to arise from the non-diagonal mixture of weak and strong eigenstates. Currently, there is compelling evidence for neutrino mixing (to be explained later). Such an observation is beyond the $\mathcal{S M}$ is as far as requiring neutrinos to be massive.

\section{Bosons}

As anticipated, interactions are mediated by bosons which obey the Boson-Einstein statistics due to their integral spin $( \pm n)$. For example, the electromagnetic interaction is mediated by photons $(\gamma)$. Similarly, the weak and the strong interactions are mediated by the $\mathrm{W}^{ \pm}$and $\mathrm{Z}^{\circ}$, and gluons (eight gluons) respectively. A list of all known bosons is shown in Table 2.2 .

\begin{tabular}{|c|c|c|c||}
\hline \hline Boson & Q & Spin & Mass (GeV) \\
\hline \hline$\gamma$ & 0 & 1 & 0 \\
\hline \hline $\mathrm{W}^{ \pm}$ & \pm 1 & 1 & 80 \\
\hline $\mathrm{Z}^{o}$ & 0 & 1 & 91 \\
\hline \hline $\mathrm{g}$ & 0 & 1 & 0 \\
\hline \hline $\mathrm{H}$ & 0 & 0 & $>115(\mathrm{LEP})$ \\
\hline \hline
\end{tabular}

Table 2.2: Properties and Quantum Numbers of Bosons Q is the electromagnetic charge of the particle in units of e. The bosons in question have also an associated spin. Gluons, also posses colour (quantum number of strong interaction).

Part of greatest achievements of the $\mathcal{S M}$ is to have realised the fact that the weak and the electromagnetic interactions can be regarded as deriving from common type of interaction: "Electroweak". At high enough energies (when the masses of the corresponding bosons are negligible), the strengths of both interactions are similar. At low energies, however, the weak interaction appears weaker $\left(\mathcal{O}\left(10^{5}\right)\right)$ than the electromagnetism because the weak gauge bosons are very massive (about $90 \mathrm{GeV}$ ) while photons are massless. Therefore, the apparent difference in strength 
at low energies is not due to the gauge coupling of the interactions but to the contribution of the boson masses through their propagators.

Gluons despite being massless (infinite range), they give rise to a short-range type of interactions which is due to the fact that the gluons themselves are colourcharged. This allows gluons to self-couple causing the force to be about constant within very short distances (comparable to the diameter of nuclear radius). This same characteristic gives rise to the so-called "asymptotic freedom" of the strong interaction, whereby no "colourful" quark combination can be seen in isolation. Therefore, quark composites can be seen only in colourless spin- $\pm(2 n-1) / 2$ triples (hadron, such as $\mathrm{n}$ and $\mathrm{p}$ ) or in colourless spin- $n$ doubles (mesons, such as pions).

Finally, it is worthwhile emphasising that the $\mathcal{S M}$ does not provide any explanation for the origin of the quark and leptons observed spectra and the mixing pattern. These, as well as others, remain as open questions for physics beyond the $\mathcal{S M}$ to answer.

\subsection{Neutrinos So Far}

In order to study neutrinos, they need to be to produced and detected. Neutrinos used for experiments are produced in six basic scenarios. First, the Sun, like any other star, carries out thermonuclear fusion in its core generating large amount of energy and copious amounts of electron neutrinos. Second, stars also emit neutrinos during the gravitational collapse and colling phase of Super-Novae. Third, nuclear fission reactors produce $\bar{\nu}_{e}$ from the radioactive debris of the fission of heavy nuclei. Fourth, cosmic rays also give rise to all kinds of neutrinos and anti-neutrinos (but mostly electron and muon neutrinos) from the decay of unstable particles (like $\pi)$ generated from the interaction between atmospheric nuclei and the energetic cosmic rays. Fifth are neutrino beams, which generally, but not uniquely, use the decay of pions to generate rather pure beams of $\nu_{\mu}$ and $\bar{\nu}_{\mu}$ (with some electron neutrino contamination). Sixth, ultra-high energy neutrinos may be generated in astrophysical exotic scenarios, such as those where cosmic rays may be accelerated for example.

The detection of neutrinos also has characteristic features which affect the technology adopted. Neutrino detectors tend to be large (kilo-tonnes), to compensate for the tiny cross-sections and, so, they tend to be made using a simple technology to keep costs reasonable. Moreover, neutrino detectors (not all) are usually placed at $\mathcal{O}(100)$ meters underground to reduce cosmic ray muons background by orders 
of magnitude.

These characteristics of production/detection will be present throughout the entire history of neutrino physics, which starts here:

- Neutrino is hypothesised by W. Pauli (1930) W. Pauli's hypothesis explains the continuous spectrum and angular momentum conservation during in $\beta$-decays (discovered by J. Chadwick [3]) as both consequences of 3-body decay process in which one particle, the "neutrino", of spin- $\frac{1}{2}$ eluded detection. The particle in question has to be electromagnetically neutral (because of conservation of charge) with a tiny mass, if anything at all, to prevent detection.

Due to the smallness of the mass and the neutral character the particle in question, they were baptised as "neutrino" by E. Fermi.

- First weak theory by E. Fermi (1934) Further developments in the understanding of weak decays culminated with the first theory of weak decay[4]. This theory provides the first steps towards predictability in weak interactions for processes whose $\mathrm{Q}^{2} \ll m_{W}^{2}$.

- Neutrino is first discovered by Cowan \& Reines (1956) The first detection of the neutrino was based on inverse $\beta^{+}$decay $\left(\bar{\nu}_{e}+p \rightarrow e^{+}+\right.$ $n$ ) by measuring the coincidence of the $0.511 \mathrm{MeV} \gamma$ from the annihilation of the $e^{+}$(prompt) and the delayed capture of the $n$ a few $\mu$ s later using a liquid scintillator detector and neutrinos from a nuclear reactor [5][6][7]. They estimated, for the first time, stunningly small average cross-section $\mathcal{O}\left(10^{-42}\right) \mathrm{cm}^{2}$, which explains the elusive character of neutrinos.

- Parity violation in weak interaction Lee, Yang and Wu (1956-1957) Lee and Yang [8] suggested that parity violation in the weak sector could be the explanation for some unexplained observations in some kaon decay channels. The experiment devised by $\mathrm{Wu}[9]$ tested the parity violation hypothesis, finding that parity is violated maximally in weak interactions.

- Helicity of neutrinos is measured by Goldhaber (1958) Neutrinos are determined to be right-handed (helicity: $\mathcal{H}(\nu)=-1$ ), while anti-neutrino are left-handed (helicity: $\mathcal{H}(\bar{\nu})=+1$ ) [10]. This evidence sets a crucial step toward the $\mathrm{V}$-A theory for weak interactions. 
The weak interaction has also been found to violate CP symmetry in hadronic systems, by J. Cronin and V. Fitch in 1964 [11].

- Muon neutrino is discovered by L. Lederman, M. Schwartz \& J. Steinberger (1962) Up to then, it was not clear whether the neutrino emitted in the pion decay was the same as the one emitted in $\beta$ decays. This was tested by directing a beam of neutrinos produced from the decay of pions into a detector behind many meters of shielding. The detector found 29 muons and 6 electrons out the neutrino interactions, which proved that $\nu_{e}$ is different from a $\nu_{\mu}[12]$. The 6 electrons are due to $\nu_{e}$ from the kaon decay. This experiment proved that there are at least two generations of leptons: $\left(\mathrm{e}, \nu_{e}\right)$ and $\left(\mu, \nu_{\mu}\right)$ and their production and detection occurs in pairs: charge current interactions (CC).

- Discovery of weak neutral currents: towards the $\mathcal{S M}$ (1973-1974) First observation of neutral currents (NC) at CERN [13][14] in the Gargamelle bubble chamber confirmed the prediction made by Glashow, Weinberg and Salam about their existence [1].

Later on, the decay of on-shell $\mathrm{W}$ and $\mathrm{Z}$ were observed in UA1 and UA2 at CERN [15]. The neutrino channel was used for the discovery of the $\mathrm{W}$, based on the observation of a high energy electron/muon associated with missing momentum, reflecting the lack of detection of the neutrino.

- Number of neutrinos coupling to the Z boson at LEP (2001) The number of neutrinos coupling to the $\mathrm{Z}$ resonance was measured at LEP to be $2.984 \pm 0.008$ [16]. Note that this does not exclude the existence of heavier $\left(\mathrm{m}>m(Z) / 2 \sim 40.5 \mathrm{GeV} / \mathrm{c}^{2}\right)$, which could not be produced from the decay of a real Z; or light "sterile" neutrinos which might not couple to the W or Z bosons.

- The expected tau neutrino is finally seen by the DONUT experiment (2000) The observation of $\nu_{\tau}$ [17] was expected since the discovery of the $\tau$ (1976) when a third generation of leptons was realised. The observation of the $\nu_{\tau}$ took a long time due to the technical difficulties encountered to unambiguously identifying the decaying $\tau$ (a very short track before decay with a mean life of about $3 \times 10^{-12}$, hence $\sim 0.1 \mathrm{~mm}$ ) produced in a CC interaction. Emulsions techniques were needed to provide the spacial resolution needed for identification. 


\subsection{The Neutrino Anomalies}

When Raymond Davis measured the solar neutrino flux for the first time and the Kamiokande experiment studied their background to proton-decay: atmospheric neutrinos, they found that their measurements did not agree with their expectations. These two problems are referred to as the "solar and atmospheric neutrino anomalies". Increasing evidence points to an elegant common solution to both problems: neutrino oscillations, which was suggested by Pontecorvo at very early stages to explain the solar neutrino deficit.

In this section, we shall explain what is meant by these anomalies and go through the most relevant evidence that characterise them and, therefore, characterise the signature for neutrino oscillations. The theoretical background behind neutrino oscillations will be postponed until next section, after their need is motivated by the experimental evidence.

\subsubsection{The Solar Neutrino Anomaly}

The fact that experiments measuring different fractions of the solar neutrino spectra observed a deficit on the solar neutrino flux relative to the calculations was called the "solar neutrino anomaly". This was first identified by R. Davis [18] in 1968. Roughly speaking, the measurements obtained are about a third of the expected flux (see later Figure 2.3 for details).

Chronologically, much of the effort was devoted to both understanding and validating the accuracy of both the experimental results (by using experiments with different techniques) and the solar models used to derive the expected neutrino fluxes.

\section{Standard Solar Model}

The so-called "Standard Solar Model" (SSM) is the most accurate model for describing the physical processes taking place in the Sun. The SSM simulates all the known effects associated with the "burning" of hydrogen into helium (dominating the current era of the Sun) mainly via the reaction indicated in Equation 2.1 and other sub-dominant reactions.

$$
4 p+2 e \rightarrow{ }^{4} \mathrm{He}+2 \nu_{e}+26.73 \mathrm{MeV}
$$

The validation of the model is based on the comparison between predictions 


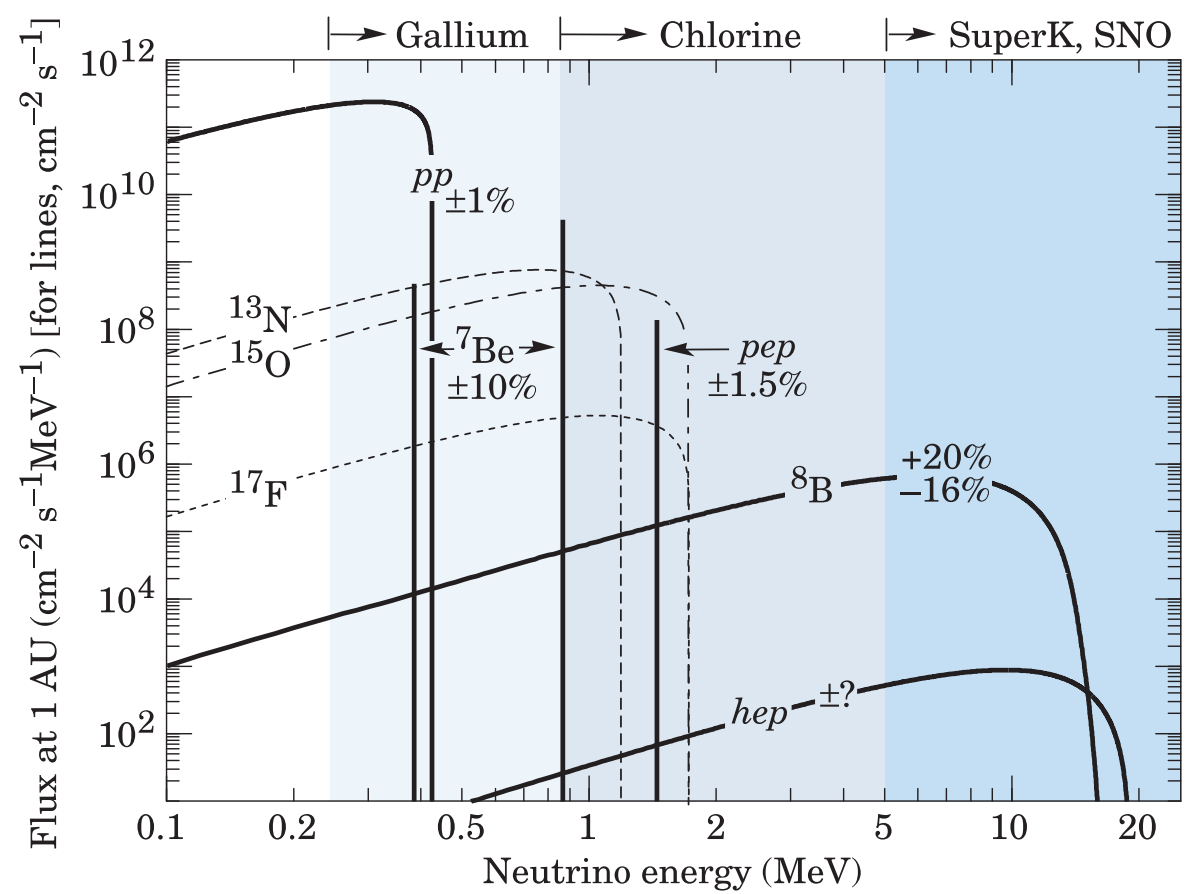

Figure 2.2: Solar Neutrino Fluxes as Predicted by SSM. Plot obtained from [19].

and measurements of the Sun's radius, luminosity, mass and the $\mathrm{He} / \mathrm{H}$ ratio. An additional test-bed for the accuracy of the model is provided by the most recent helio-seismologic data [20], collected by dedicated satellites. Helio-seismology together with the solar neutrino fluxes are, from the astrophysical point of view, among the most direct avenues to extract information about the internal activity of the sun.

\begin{tabular}{l|c}
\hline Reaction & Energy of $\nu(\mathrm{MeV})$ \\
\hline$p+p \rightarrow{ }^{2} \mathrm{H}+e^{+}+\nu_{e}$ & $\leq 0.42$ \\
$p+e+p \rightarrow{ }^{2} \mathrm{H}+\nu_{e}$ & 1.44 \\
${ }^{7} \mathrm{Be}+e \rightarrow{ }^{7} \mathrm{Li}+\nu_{e}$ & $(90 \%) 0.86$ \\
& $(10 \%) 0.38$ \\
& $\sim 15$ \\
${ }^{8} \mathrm{~B} \rightarrow{ }^{8} \mathrm{Be} *+e^{+}+\nu_{e}$ & $\leq 18.77$ \\
${ }^{3} \mathrm{He}+p \rightarrow{ }^{4} \mathrm{He}+e^{+}+\nu_{e}$ & $\leq 1.2$ \\
${ }^{13} \mathrm{~N} \rightarrow{ }^{13} \mathrm{C}+e^{+}+\nu_{e}$ & $\leq 1.7$ \\
${ }^{15} \mathrm{O} \rightarrow{ }^{15} \mathrm{~N}+e^{+}+\nu_{e}$ & \\
\hline
\end{tabular}

Table 2.3: Solar Neutrino Production Reactions. Plot obtained from [21].

The emission of neutrinos is intrinsic to the main solar fusion process. Moreover, 
neutrino emission is also associated with other reactions taking place, explicitly listed in Table 2.3. Figure 2.2 shows the decomposed solar flux predictions of the SSM in its different reaction contributions.

\section{Experimental Evidence}

Seven experiments have so far measured the solar neutrino flux and all of them have measured a deficit with respect to predictions from the SSM, as summarised in Figure 2.3. The experiments in questions can be roughly sub-divided into two categories: radiochemical and water-Cherenkov. The most important differences between the two techniques are:

- The ability to measure the energy of the incident neutrinos

- The ability to measure arrival time and direction of the incident neutrino

- The energy threshold. The lower the energy threshold the higher the statistics, as the integrated flux measured is larger (see Figure 2.2). The energy threshold is determined by the detection reaction used, in combination with detector/analysis cuts.

Total Rates: Standard Model vs. Experiment Bahcall-Pinsonneault 2000

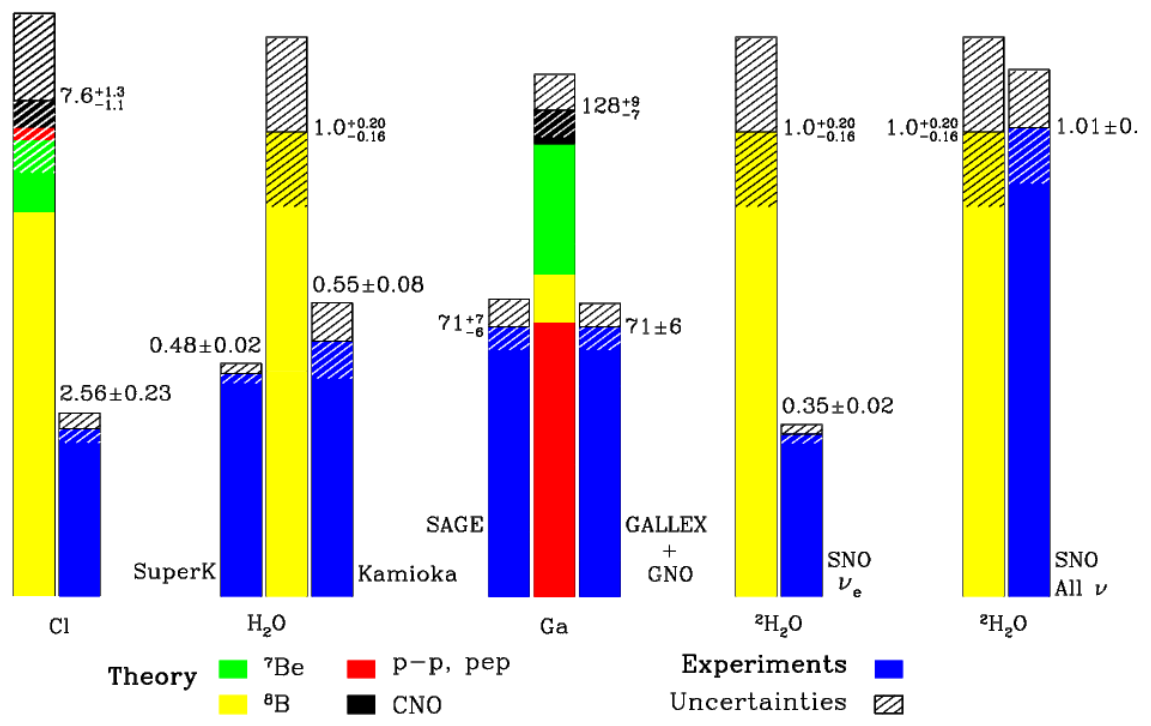

Figure 2.3: Solar Neutrino Results as Today. 


\begin{tabular}{lccc}
\hline Experiment & Measured & BP2000 & Measured/BP2000 \\
\hline Homestake & $2.56 \pm 0.23$ & $7.6_{-1.1}^{+1.3}$ & $0.34 \pm 0.06$ \\
GALLEX + GNO & $74.1_{-7.8}^{+6.7}$ & $128_{-7}^{+9}$ & $0.58 \pm 0.07$ \\
SAGE & $74.1_{-7.4}^{+7.8}$ & $128_{-7}^{+9}$ & $0.59 \pm 0.07$ \\
\hline
\end{tabular}

Table 2.4: Results of Radio-Chemical Experiments. The results are expressed in Solar Neutrino Units $\left(1 \mathrm{SNU}=10^{-36}\right.$ events atoms $\left.{ }^{-1} \mathrm{~S}^{-1}\right)$.

Radio-Chemical Experiments The radio-chemical experiments are divided into two types: "Cl-experiments", such as Homestake [18][22]; and "Ga-experiments", such as SAGE [23], GALLEX [24] and GNO [25]. They differ in the target material used and therefore on the neutrino detection reaction. The reactions are indicated in Equation 2.2 and 2.3 for the Cl-experiment and Ga-experiments respectively.

$$
\begin{aligned}
\nu_{e}+{ }^{37} \mathrm{Cl} & \rightarrow e+{ }^{37} \mathrm{Ar} & & \text { Threshold: } 0.8 \mathrm{MeV} \\
\nu_{e}+{ }^{71} \mathrm{Ga} & \rightarrow e+{ }^{71} \mathrm{Ge} & & \text { Threshold: } 0.2 \mathrm{MeV}
\end{aligned}
$$

Both types of experiments relied on having a large active volume holding a solution containing the $\mathrm{Cl}$ or $\mathrm{Ga}$. The incoming neutrinos then interact via $\mathrm{CC}$ producing radioactive isotopes of $\mathrm{Ar}$ or Ge, respectively. The radioactive isotopes were extracted with periodicity of about $20-30$ days, which is comparable to the half-life of the radioactive isotopes produced, so that they can be identified (by their decays) and counted. The number of isotopes found is proportional to the number of neutrino interactions occurring in the volume over the exposed period. Radio-chemical experiments are characterised by having low energy thresholds, however are unable to tell anything about the specific energy, arrival time or direction of individual neutrinos. They are able provide the solar neutrino flux measurement integrated over the spectra they are sensitive to on a timescale of about one month.

The main results of radiochemical experiments are summarised in Figure 2.3 and Table 2.4. All presented radiochemical experiments has reached their end, as the uncertainties have become dominated by the systematic errors intrinsic to the technique [26] and the information they offer is limited. However, some of the new proposal for solar neutrino experiments to measure the 
pp-flux rely on similar approaches [21].

Water Cherenkov Experiments Water Cherenkov experiments include Kamiokande [27], SNO [28] and Super-Kamiokande [29] (SK) experiments. All three detectors are sensitive to solar neutrino through electron-neutrino elastic-scattering (ES), indicated in Equation 2.4, whose cross-section is calculated from the interference diagram between $\mathrm{CC}$ and NC. Therefore, ES is sensitive to all types of neutrinos, but approximate $6.5 \times$ more to $\nu_{e}$ (at this energy regime) due to the dominant contribution of the CC. ES is very useful for the determination of the direction of the incident neutrino, which has been measured unambiguously to be correlated with the location of the Sun.

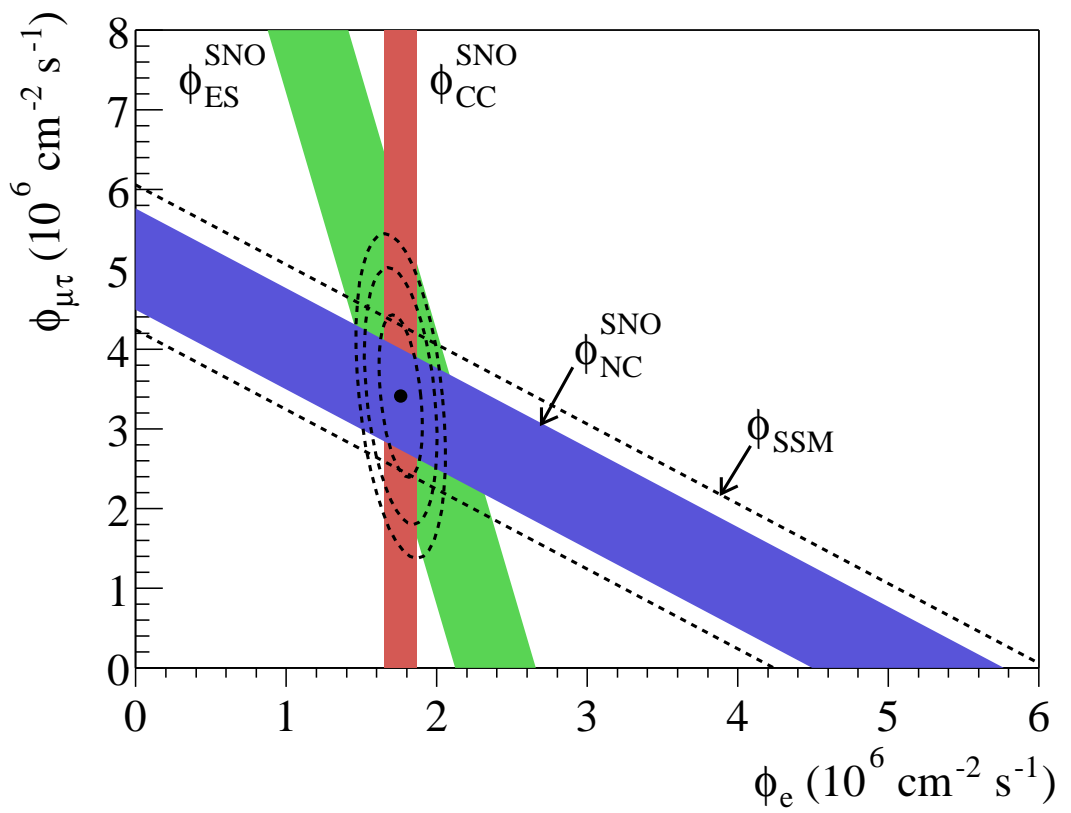

Figure 2.4: SNO Solar Neutrino Fluxes Measurements. This plot [30] shows the $B^{8}$ flux of $\nu_{\mu}-\nu_{\tau}$ versus $\nu_{e}$ as measured with different interactions (ES, CC and NC) by the SNO detector. The broken lines illustrate the flux as predicted by the SSM, which matches the measured flux by NC events. The width of all bands are restricted to the $\pm 1 \sigma$ uncertainty level. The best fitted values of the fluxes measured by SNO are illustrated by the oval contours, showing up to the $3 \sigma$ level.

$$
\begin{array}{llll}
\mathrm{ES} & \nu_{x}+e \rightarrow \nu_{x}+e & & \text { Threshold: } 5.0 \mathrm{MeV} \\
\mathrm{CC} & \nu_{e}+d \rightarrow p+p+e & & \text { Threshold: } 1.4 \mathrm{MeV} \\
\mathrm{NC} & \nu_{x}+d \rightarrow \nu_{x}+p+n & & \text { Threshold: } 2.2 \mathrm{MeV}
\end{array}
$$


SNO, however, uses heavy water $\left(\mathrm{D}_{2} \mathrm{O}\right)$ (1kt), which allow neutrinos to additionally interact with the deuterium through $\mathrm{CC}$ (Equation 3.2) and NC (Equation 3.3) reactions. Indeed, the power of SNO is granted by their ability to measure $\mathrm{CC}$ and $\mathrm{NC}$ interactions of solar neutrinos in the same detector. Since the rate of $\mathrm{NC}$ is immune to the flavour changing mechanism causing the solar neutrino deficit, the NC flux should be proportional to the total rate of solar neutrinos generated by the sun, which should, therefore, agree with the SSM expectations, as shown in Figure 2.4.

The energy threshold of SNO and both Kamiokande detectors limits their sensitivity to the ${ }^{8} B$ neutrinos. However, the three detectors enjoy instantaneous energy, time and direction information about the incident neutrinos. The results from the Kamiokande experiments are summarised in Table 2.5 $[31]$.

\begin{tabular}{lccc}
\hline Experiment & Measured Flux & BP2000 Flux & Measured/BP2000 \\
\hline Kamiokande & $2.80 \times(1.00 \pm 0.14)$ & $5.05 \times\left(1.00_{-0.16}^{+0.20}\right)$ & $0.55 \pm 0.13$ \\
Super-Kamiokande & $2.40 \times\left(1.00_{-0.03}^{+0.04}\right)$ & $5.05 \times\left(1.00_{-0.16}^{+0.20}\right)$ & $0.48 \pm 0.09$ \\
\hline
\end{tabular}

Table 2.5: Results of Kamiokande Experiments. The results are expressed in terms of $10^{6} \mathrm{~cm}^{2} \mathrm{~s}^{-1}$ units.

$$
\begin{aligned}
& \phi_{\mathrm{CC}}^{\mathrm{SNO}}=1.76_{-0.05}^{+0.06} \text { (stat) }{ }_{-0.09}^{+0.09} \text { (syst) } \\
& \phi_{\mathrm{ES}}^{\mathrm{SNO}}=2.39_{-0.23}^{+0.24} \text { (stat) }{ }_{-0.12}^{+0.12} \text { (syst) } \\
& \phi_{\mathrm{NC}}^{\mathrm{SNO}}=5.09_{-0.43}^{+0.44} \text { (stat) }{ }_{-0.43}^{+0.46} \text { (syst) }
\end{aligned}
$$

The SNO fluxes measured [30] are shown in Figure 2.4 and summarised in Equation 2.8. Such fluxes can also be converted into flavour fluxes, as indicated in Equation 2.8 .

$$
\begin{aligned}
\phi_{e} & =1.76_{-0.05}^{+0.05}(\text { stat })_{-0.09}^{+0.09} \text { (syst) } \\
\phi_{\mu \tau} & =3.41_{-0.45}^{+0.45} \text { (stat) }{ }_{-0.45}^{+0.48} \text { (syst) }
\end{aligned}
$$

The contribution of SNO resolved many of the ambiguities of the solar neutrino anomaly. SNO also settled the flavour changing nature of the solution 
of the solar neutrino puzzle. Details can be found in [32]. In this thesis, the SNO impact will be explicitly discussed in forthcoming sections of this chapter.

\subsubsection{The Atmospheric Neutrino Anomaly}

The atmospheric neutrino anomaly was discovered by Kamiokande [27] and IMB [33] experiments, while they studied one of their backgrounds: atmospheric neutrinos. Both experiment were built to search for the still unobserved proton decay.

$$
p+N \rightarrow \pi^{ \pm}+X
$$

Atmospheric neutrinos are copiously produced from cosmic ray interactions at the Earth's atmosphere. Cosmic rays, therefore, upon interacting with atmospheric atoms (mainly nitrogen: $N$ ), generate many short-lived particles (mainly $\pi \mathrm{s}$, but also some kaons), as indicated by Equation 2.9. Pions and a fraction of the kaons undergo weak decays, as illustrated in Figure 2.5, during their flight producing neutrinos along the line towards the Earth.

$$
\begin{aligned}
& \pi^{ \pm} \rightarrow \mu^{ \pm}+\nu_{\mu}\left(\overline{\nu_{\mu}}\right) \\
& \mu^{ \pm} \rightarrow e^{ \pm}+\nu_{e}\left(\overline{\nu_{e}}\right)+\overline{\nu_{\mu}}\left(\nu_{\mu}\right)
\end{aligned}
$$

The atmospheric neutrino production through pions is explicitly indicated in Equation 2.11, from which it can be inferred that the expected ratio of $\nu_{\mu}$ to $\nu_{e}$ neutrinos is $\sim 2$ (ignoring energy dependences and Earth magnetic field effects).

It is useful to define the $\mathcal{R}$ parameter, as indicated in Equation 2.13. The purpose of defining $\mathcal{R}$ is to characterise the rate of different atmospheric neutrino flavours without suffering from the up to $\sim 30 \%$ uncertainties present in the absolute flux calculations [34]. The uncertainty associate with $\mathcal{R}$ is about $\sim 5 \%$, as all the common dependencies with large uncertainties cancel in the ratio.

$$
\begin{aligned}
\mathcal{R} & =\frac{\nu_{\mu}+\overline{\nu_{\mu}}}{\nu_{e}+\overline{\nu_{e}}} \\
\mathcal{R}^{\prime} & =\frac{\mathcal{R}_{D A T A}}{\mathcal{R}_{M C}}
\end{aligned}
$$




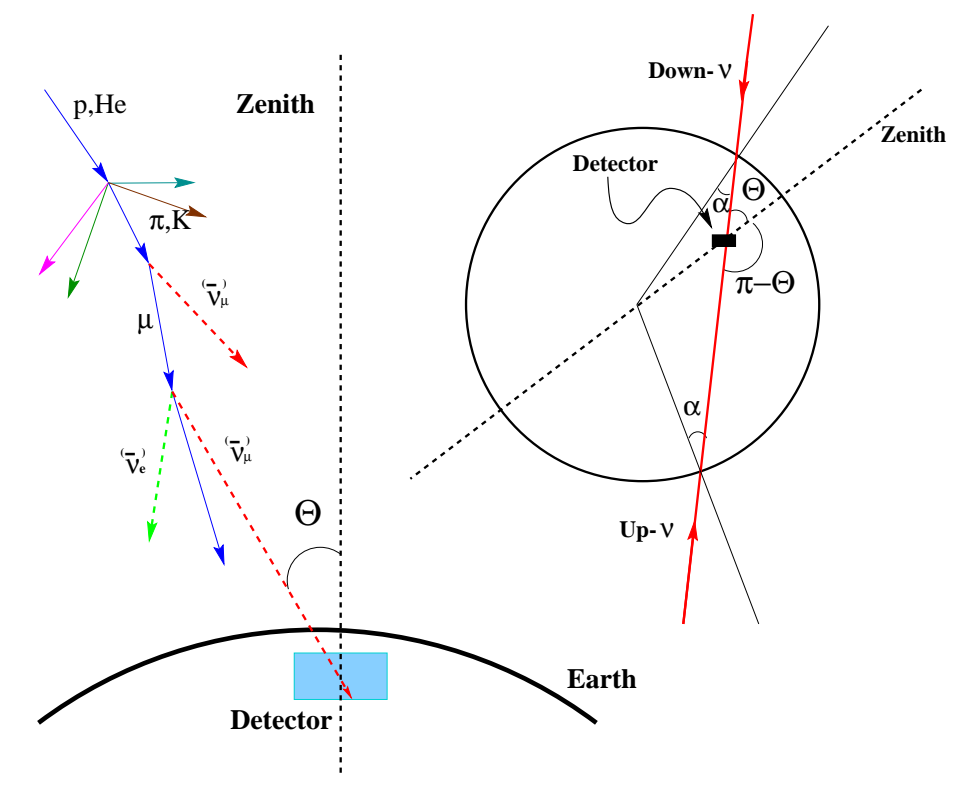

Figure 2.5: Atmospheric Neutrinos Generation Diagram.

$\mathcal{R}_{M C}$ is the expectation assuming no neutrino flavour changing mechanisms. $\mathcal{R}^{\prime}$ can therefore be used to compare measurements and expectations from different experiments. Therefore, $\mathcal{R}^{\prime}<1$ means that there is some mechanism causing the expected muon atmospheric neutrinos to disappear. Indeed, that is essentially the so-called "atmospheric neutrino anomaly", whereby $\mathcal{R}^{\prime}$ is measured to be $\sim 0.6$ on average.

\section{Experimental Evidence}

Many experiments have measured the $\mathcal{R}^{\prime}$ parameter, as summarised in Figure 2.6. However, we shall concentrate on the results provided by Super-Kamiokande [29] as the for neutrino flavour changing is particularly illustrative and compelling.

Super-Kamiokande has measured the $\mathcal{R}^{\prime}$ to be $0.649 \pm 0.016 \pm 0.051$ and $0.675 \pm$ $0.031 \pm 0.083$ for the sub-GeV $(<1.33 \mathrm{GeV})$ and multi-GeV $(>1.33 \mathrm{GeV})$ samples [35] respectively. The most illustrative evidence for flavour changing signature in atmospheric neutrino is found in the azimuthal angular distributions of SK, shown in Figure 2.7. The evidence is shown as histograms of the number events measured versus $\cos \theta$, where $\theta$ is the azimuthal angle, whose meaning is explained in Equation 2.14 . 


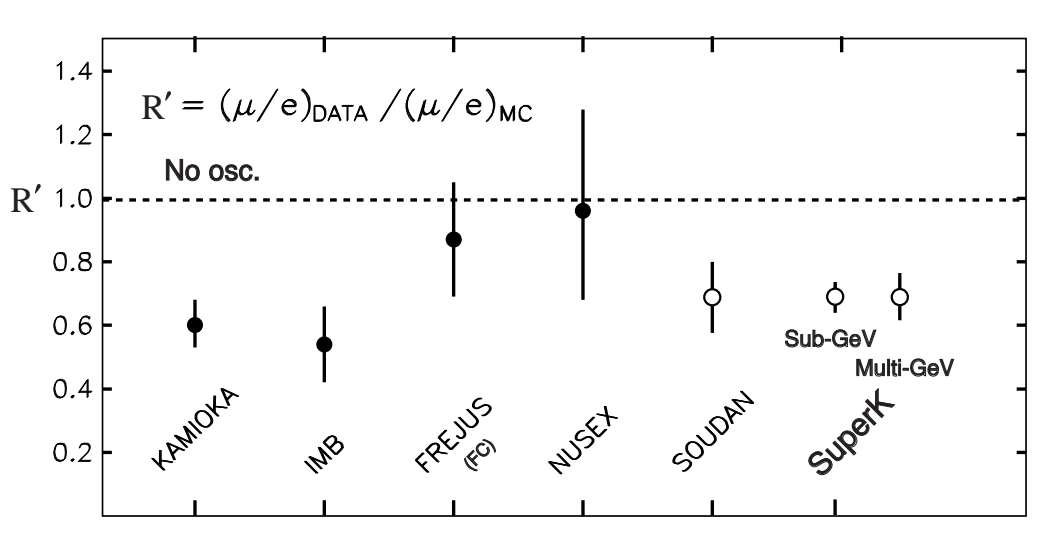

Figure 2.6: $\mathcal{R}^{\prime}$ as Today.

$$
\cos (\theta): \begin{cases}\cos (\theta)=1 & \Longrightarrow \nu \text { come from above } \\ \cos (\theta)=0 & \Longrightarrow \nu \text { come from horizon } \\ \cos (\theta)=-1 & \Longrightarrow \nu \text { come from below }\end{cases}
$$

In Figure 2.7 there are four distributions. The $S$ ub-GeV sample and $M$ ulti-GeV sample for fully contained events correspond to the distributions locate at top and bottom, respectively. The distributions on the left side correspond to electrons and while those on right side correspond to muons. The $\nu_{\tau}$ cannot be identified on an event-by-event basis in SK. The red distributions correspond to the MC expectations with no neutrino oscillations. Data is depicted in black. A clear discrepancy can be seen between the data and un-oscillated MC for muons while electrons agree well with the un-oscillated hypothesis. The green distribution corresponds to the best neutrino oscillation fit. Note that the fit matches the data very well, indicating that the data is consistent with the neutrino oscillation hypothesis. These plots suggest that neutrinos are must massive, therefore undergoing $\nu_{\mu} \rightarrow \nu_{\tau}$ or $\nu_{\mu} \rightarrow \nu_{\text {sterile }}$ transitions and/or decaying.

\subsection{Need for a Non-Diagonal Free Hamiltonian}

The results of all experiments presented so far has made evident the need for a theoretical framework that is able to account for neutrino flavour transitions as neutrinos propagate from the source to the detection point. This condition is identical to requiring that the Hamiltonian operator dictating the dynamics of freely propagating neutrinos (mass eigenstates) is non diagonal for neutrino weak 

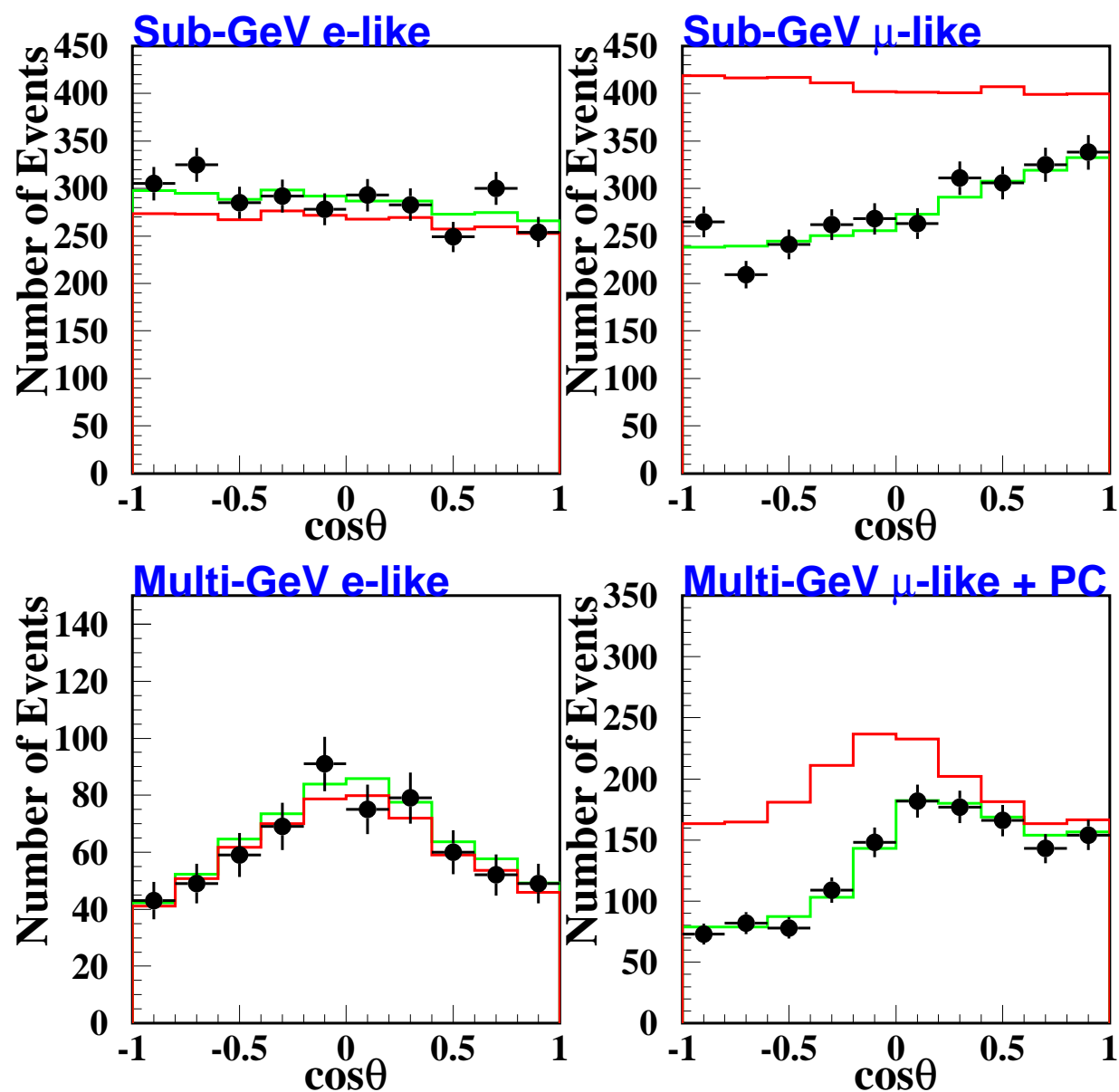

Figure 2.7: SK Atmospheric Azimuthal Angular Distribution.

eigenstates leading to inter-flavour transitions during the propagation of the mass eigenstates in vacuum. The most favoured mechanism providing such a framework is neutrino oscillations.

The basic idea behind neutrino oscillations is that mass-eigenstates can be regarded as a mixture of weak-eigenstates, and vice versa. The formalism for such a "mixing" of states is already present in the $\mathcal{S M}$ [1] characterised by a mixing matrix called "PMNS"1, which plays similar role to the "CKM" ${ }^{2}$ matrix in the quark sector (between weak and strong eigenstates).

The phenomenology of neutrino oscillations can be easily obtained by merely letting a weak-eigenstate $\alpha$ propagate in space-time. Each weak-eigenstate $\alpha$ is

\footnotetext{
1 "Pontecorvo-Maki-Nakagawa-Sakata" Mixing Matrix.

2 "Cabibbo-Kobayashi-Maskawa" Mixing Matrix.
} 
mathematically represented by

$$
\left|\nu_{\alpha}\right\rangle=\sum_{i} U_{\alpha i}^{*}\left|\nu_{i}\right\rangle \quad(\alpha=e, \mu, \tau)
$$

a superposition of all mass-eigenstates. $U_{\alpha i}$ corresponds to the mixing matrix element for the neutrino weak-eigenstate $\alpha$ and neutrino mass-eigenstate $i$.

Note that neutrinos interact, essentially only through the weak force, therefore they are produced (at the source) and detected (at the detector) as weakeigenstates. However, during propagation between source and detection point, their dynamics are dictated by the free-particle Hamiltonian affecting the masseigenstates according to the Schrödinger Equation:

$$
\left|\nu_{\alpha}(t>0)\right\rangle=\sum_{i} e^{-i E_{i} t} U_{\alpha i}^{*}\left|\nu_{i}\right\rangle
$$

Neutrinos with different masses, will have different energies causing a change in the relative phase between the propagated mass-eigenstates at $x>0$, with respect to the relative phase at $x=0$ (production point). Therefore a different weak eigenstate $\beta$ could be detected at $x>0$. The probability for this transition to happen is given by:

$$
\mathrm{P}\left(\nu_{\alpha} \rightarrow \nu_{\beta}\right)=\left|\left\langle\nu_{\beta} \mid \nu_{\alpha}(t)\right\rangle\right|^{2}=\left|\sum_{i} U_{\beta i} e^{-i E_{i} t} U_{\alpha i}^{*}\right|^{2}
$$

Using the approximations that first, neutrinos are always ultra-relativistic $(v \approx$ c), as their masses are very small, hence $E_{i} \simeq p+m_{i}^{2} / 2 E$; and second, $L \equiv x$; we can express the transition probability in terms of experimental variable ( $\mathrm{E}$ and $\mathrm{L})$ :

$$
\mathrm{P}\left(\nu_{\alpha} \rightarrow \nu_{\beta}\right)=\sum_{i}\left|U_{\beta i}\right|^{2}\left|U_{\alpha i}\right|^{2}+2 \operatorname{Re} \sum_{j>i} U_{\beta i} U_{\beta j}^{*} U_{\alpha i}^{*} U_{\alpha j} \exp \left(-i \frac{\Delta m_{i j}^{2} L}{2 E}\right)
$$

where $\Delta m_{i j}^{2}=m_{i}^{2}-m_{j}^{2}$, such that $\sum \Delta m_{i j}^{2}=0$. Therefore, neutrino oscillations requires that the neutrinos mass spectra is non-degenerate. Note that neutrino oscillations are nothing but a manifestation of quantum phase interference, whose macroscopically magnitude is directly related to the smallness of $\Delta m_{i j}^{2}$ involved.

The structure of the PMNS mixing matrix can be parametrised in the following way: 


$$
U_{P M N S}=\left(\begin{array}{ccc}
c_{12} c_{13} & s_{12} c_{13} & s_{13} e^{-i \delta} \\
-s_{12} c_{23}-c_{12} s_{23} s_{13} e^{i \delta} & c_{12} c_{23}-s_{12} s_{23} s_{13} e^{i \delta} & s_{23} c_{13} \\
s_{12} s_{23}-c_{12} c_{23} s_{13} e^{i \delta} & -c_{12} s_{23}-s_{12} c_{23} s_{13} e^{i \delta} & c_{23} c_{13}
\end{array}\right)
$$

where $c_{i j}=\cos \theta_{i j}$ and $s_{i j}=\sin \theta_{i j}(i, j=1,2,3)$ and three mixing angles $\theta_{12}$, $\theta_{13}$ and $\theta_{23}$ represent ad-mixture between the weak/mass eigenstates.

The $\delta$ parameter represents a complex CP-violating phase if one assumes the most general form of the $3 \times 3$ dimension of the unitarity mixing matrix. So, there is a interesting possibility allowed through the neutrino oscillations framework: CPviolation in the leptonic sector, much like the $\mathrm{CP}$-violation present in the quark sector. This phase is often referred as the "Dirac-phase", as opposed to the CPviolating "Majorana-phases" that should be added to the formalism, if neutrinos were Majorana particles. The Majorana-phases cannot be observed in neutrino oscillations as their effect cancels when obtaining transition probabilities.

In addition, the PMNS matrix can be factorised as the product of three matrices:

$$
U_{P M N S}=\left(\begin{array}{ccc}
1 & & \\
& c_{23} & s_{23} \\
& -s_{23} & c_{23}
\end{array}\right)\left(\begin{array}{ccc}
c_{13} & & s_{13} e^{i \delta} \\
& 1 & \\
-s_{13} e^{i \delta} & & c_{13}
\end{array}\right)\left(\begin{array}{ccc}
c_{12} & s_{12} & \\
-s_{12} & c_{12} & \\
& & 1
\end{array}\right)
$$

hence $U=M_{23} \times M_{13} \times M_{12}$.

Note that $M_{13}$ is parametrised in terms of $\theta_{13}$, which is the mixing angle driving the yet unobserved $\nu_{\mu} \rightarrow \nu_{e}$ transitions. $\theta_{13}$ is small: $\sin ^{2}\left(2 \theta_{13}\right)<0.11$, as limited by the Chooz experiment [36]. Indeed, it is the smallness of $\theta_{13}$ that grants the effective de-coupling exhibited between the atmospheric and solar problems. Correlation terms between the two problems are proportional $\mathcal{O}\left(\sin ^{2}\left(2 \theta_{13}\right)\right)$ at least. Note also that terms $s_{13} e^{i \delta}$ always appear together, which means that any chance to measure the leptonic CP-violating Dirac-phase is subjected to a non-vanishing value of $s_{13}$.

On the other hand, $M_{23}$ corresponds to the matrix dominating the neutrino oscillations in the atmospheric case $\left(\theta_{23} \sim \theta_{\text {atm }}\right)$ with a $\Delta m_{a t m}^{2} \sim 2 \times 10^{-3} \mathrm{eV}^{2}$, which are dominated by $\nu_{\mu} \rightarrow \nu_{\tau}$ transitions, as explicitly shown by the SK azimuthal distributions. $M_{12}$ corresponds to the solar neutrino oscillations $\left(\theta_{12} \sim \theta_{\text {solar }}\right)$ with a $\Delta m_{\text {solar }}^{2} \sim 8 \times 10^{-5} \mathrm{eV}^{2}$, which are dominated by $\nu_{e} \rightarrow \nu_{\mu / \tau}$ transitions. 
$M_{23}$ and $M_{12}$ can be therefore simplified as $2 \times 2$ matrices (a la Cabbibo matrix) of the form:

$$
U=\left(\begin{array}{rr}
\cos \theta & \sin \theta \\
-\sin \theta & \cos \theta
\end{array}\right)
$$

for which the transition probability can be expressed in terms of the relevant oscillation parameters for each problem, i.e. atmospheric $\left(\Delta m_{23}^{2}\right.$ and $\left.\theta_{23}\right)$ or solar $\left(\Delta m_{12}^{2}\right.$ and $\left.\theta_{12}\right)$, as

$$
\mathrm{P}\left(\nu_{\alpha} \rightarrow \nu_{\beta}\right)=\sin ^{2} 2 \theta \sin ^{2}\left(\frac{1.27 \Delta m^{2} L}{E}\right)
$$

Equation 2.22 represents the "appearance" probability if $\alpha \neq \beta$ or the "disappearance" (or survival) probability if $\alpha=\beta$. This equation will be used for the rest of this thesis, as current atmospheric, solar and long-baseline disappearance experiments only need a 2-neutrino oscillation framework. Future higher precision neutrino oscillation programmes will require the 3-neutrino oscillation framework for the interpretation of the results.

Although the original $\mathcal{S M}$ did not include massive neutrinos (as there was no evidence for it), there is no symmetry/argument for the $\mathcal{S M}$ not to accommodate the corresponding Yukawa terms for neutrino masses, as described in [37]. The values of the masses and the PMNS and CKM matrices are already beyond the $\mathcal{S M}$ itself, they are not predicted but introduced as experiments measure them. An interesting consequence is that massive neutrinos allow for other types of interactions, such as EDM [38] and gravitational interactions, enriching the neutrino phenomenology and their possible role in the universe.

Neutrino oscillations, however, are far from being the only mechanism providing flavour transitions. There are two alternatives mechanisms which, while already disfavoured, are still somewhat compatible with the experimental results: neutrino decay [39] and decoherence [40]. An illustration of the analytical difference in the shape of the survival probability between those two mechanisms (red is decoherence and green is decay) and neutrino oscillations is shown in Figure 2.8.

Note that neutrino oscillations is very distinguishable with respect to the other two hypotheses:

- Only neutrino oscillation should exhibit a deep "dip", depending on the L/E resolution of the experiment.

- Only neutrino oscillation have a subsequence rise, just after the dip. 


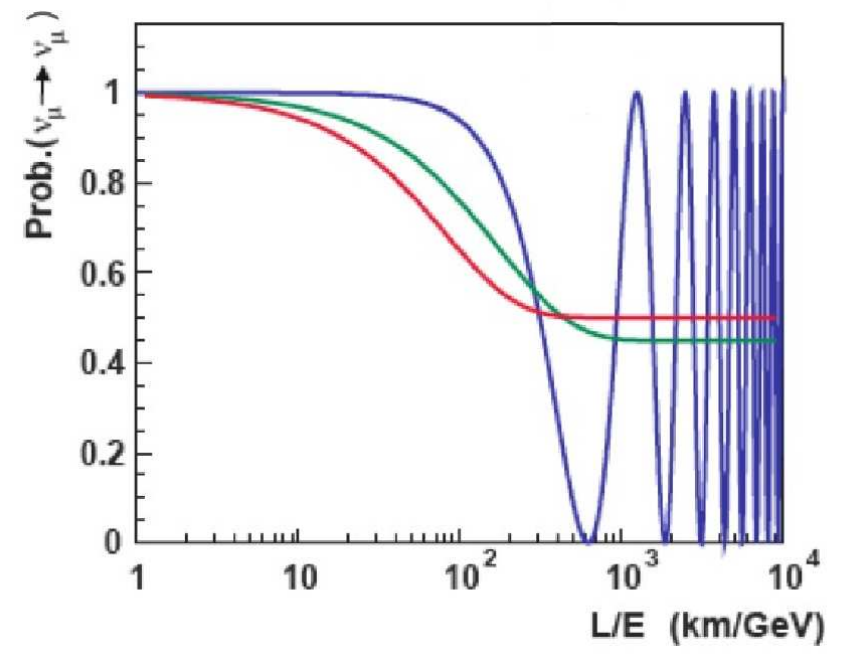

Figure 2.8: The Neutrino Oscillations Unique Signature.

- The falling slope of the three models is different.

\subsection{Towards Neutrino Oscillations}

To the experimental evidence shown so far, there are essentially two more experiments whose conception and results significantly support the neutrino oscillation hypothesis. Experiment aiming to characterise neutrino oscillations are designed such that the events obtained have a high L/E precision, as neutrino oscillation, unlike any other hypothesis, modulates with L/E.

The KamLAND Experiment The KamLAND experiment [41] is a fascinating experiment that uses $\overline{\nu_{e}}$ from about 16 nuclear reactors with an average energy of $4 \mathrm{MeV}$ and an average base-line of about $180 \mathrm{~km}$. Therefore KamLAND's sensitivity covers the neutrino oscillation parameter space favoured by solar neutrino experiments $\left(\Delta m^{2} \sim 5 \times 10^{5} \mathrm{eV}^{2}\right)$ but, for the first time, using neutrinos produced on Earth. Note, however, that solar neutrinos are $\nu_{e}$ rather than $\bar{\nu}_{e}$. Therefore the direct comparison of KamLAND results with solar neutrino results assumes CPT invariance ${ }^{3}$.

KamLAND measured the fraction of the nuclear reactor neutrinos to be $0.582 \pm 0.069 \pm 0.039$, proving disappearance of $\bar{\nu}_{e}$. The spectral distortion

\footnotetext{
${ }^{3}$ If there was no CPT invariance, the $\Delta m^{2}$ exhibited by neutrinos could be different from that of anti-neutrinos.
} 


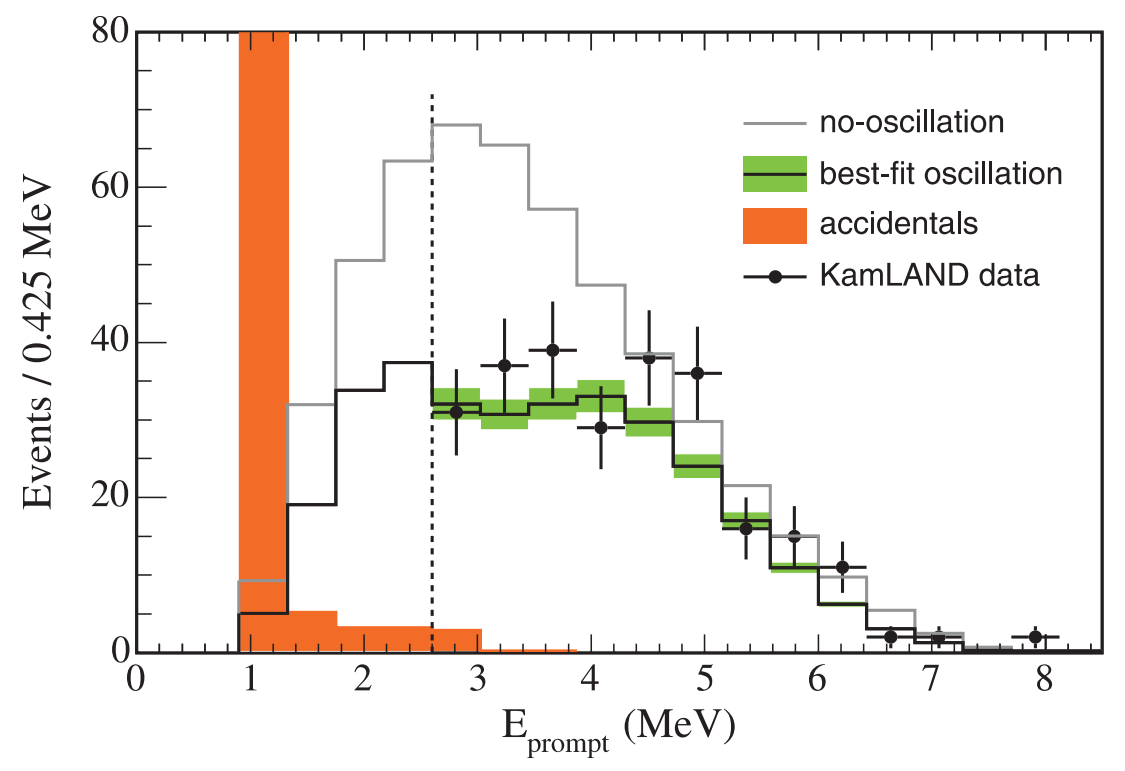

Figure 2.9: KamLAND Evidence towards Solar Neutrino Oscillations. Plot obtained from [41].

observed at KamLAND is consistent with the neutrino oscillations hypothesis see Figure 2.9. But the largest power for extraction neutrino oscillation parameters is obtained by including the KamLAND results in a global fit with the entire solar neutrino data, as will be shown shortly.

The K2K Experiment The K2K [42] is the first long-baseline neutrino experiment. K2K uses neutrinos $\left(\nu_{\mu}\right)$ produced at the KEK laboratory. The experiment consist of a wide-band neutrino beam whose mean energy is about $\sim 1.3 \mathrm{GeV}$ and two detectors. The near detector is located within the same laboratory at about $300 \mathrm{~m}$ and the far detector is the SK detector located about $\sim 250 \mathrm{~km}$ away from the source. The mean energy of the beam and the baseline were chosen to allow $\mathrm{K} 2 \mathrm{~K}$ to be sensitive to atmospheric neutrino oscillation dictated by $\Delta m_{a t m}^{2}$.

The aim of the experiment was to provide an experimental setup that would improve the L/E resolution of each event such that K2K can use the distortion of the oscillated spectrum to measure to test the neutrino oscillations hypothesis. Figure 2.10 shows the measured spectral distortion [43]. Data is shown in black while the un-oscillated spectrum is depicted in red and the best fit to the oscillated data is shown in blue. From rate and spectral distortion measurements, $\mathrm{K} 2 \mathrm{~K}$ has been able to confirm the neutrino oscil- 


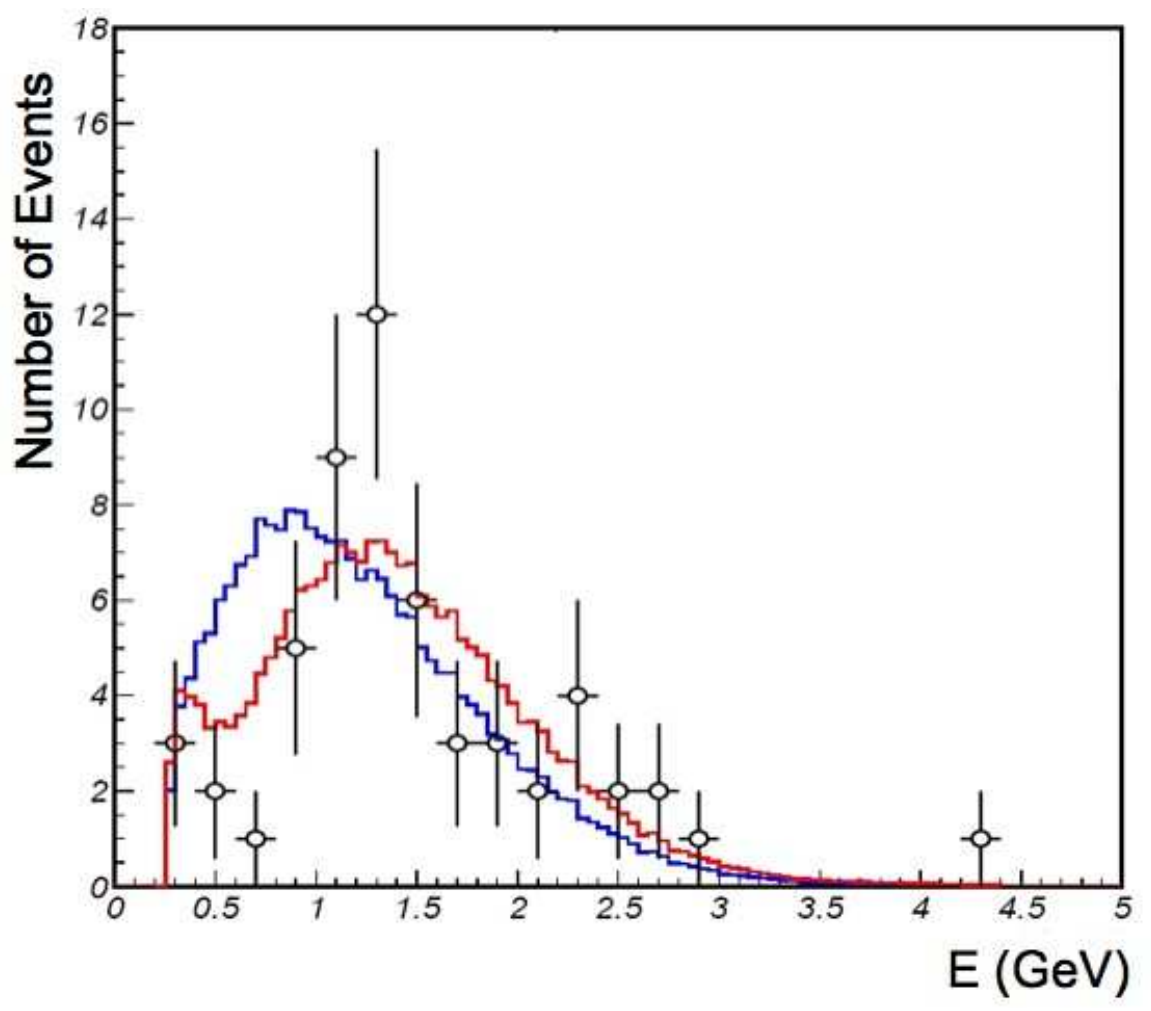

Figure 2.10: The K2K Evidence for Neutrino Oscillations. Plot obtained from Jeremy Argyriades - K2K Collaboration.

lation hypothesis to the $3.9 \sigma$ level, a result essentially limited by statistical sensitivity.

There are essentially two ways to prove neutrino oscillations.

- The "indirect" way to prove neutrino oscillation is by testing the neutrino oscillations hypothesis against all data available (shown in Figure 2.11), through "global analyses". In this particular scenario, the data from all experiments have a varying impact in our confidence about the truth of the hypothesis. Currently, all experiments are very consistent with the neutrino oscillations and alternative solutions (decay and decoherence) are already disfavoured by up to about $4.0 \sigma$ levels.

- The "direct" way is by proving that the neutrino appearance/disappearance probability modulates with L/E (see Figure 2.8). Some evidence for L/E modulation of the survival probability has been provided by SK (Figure 2.12), KamLAND (Figure 2.12) and K2K (implicit in their spectral distortion shown in Figure 2.10). 


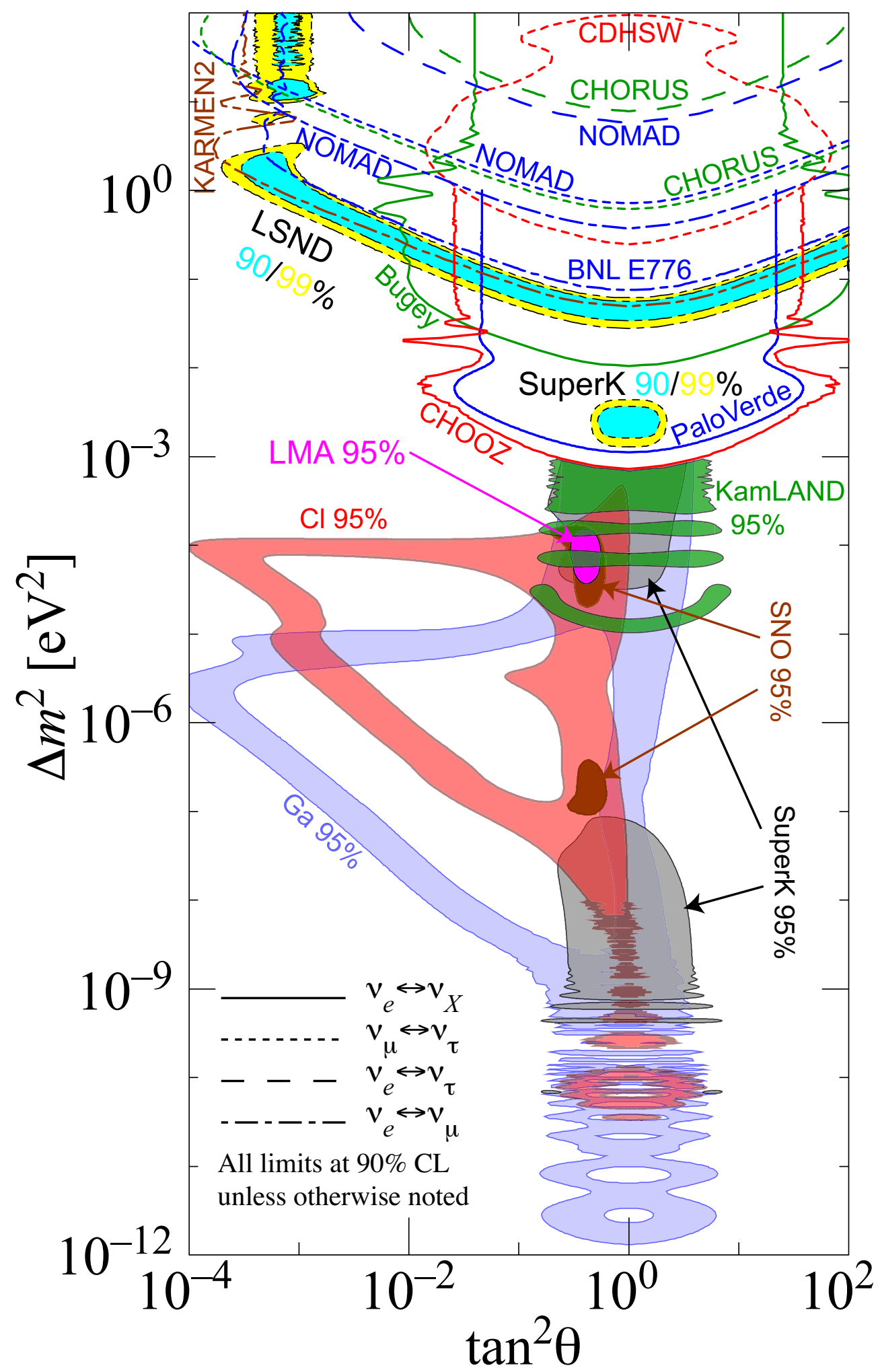

Figure 2.11: Experimental Evidence for Neutrino Oscillation. Plot obtained from [16]. 

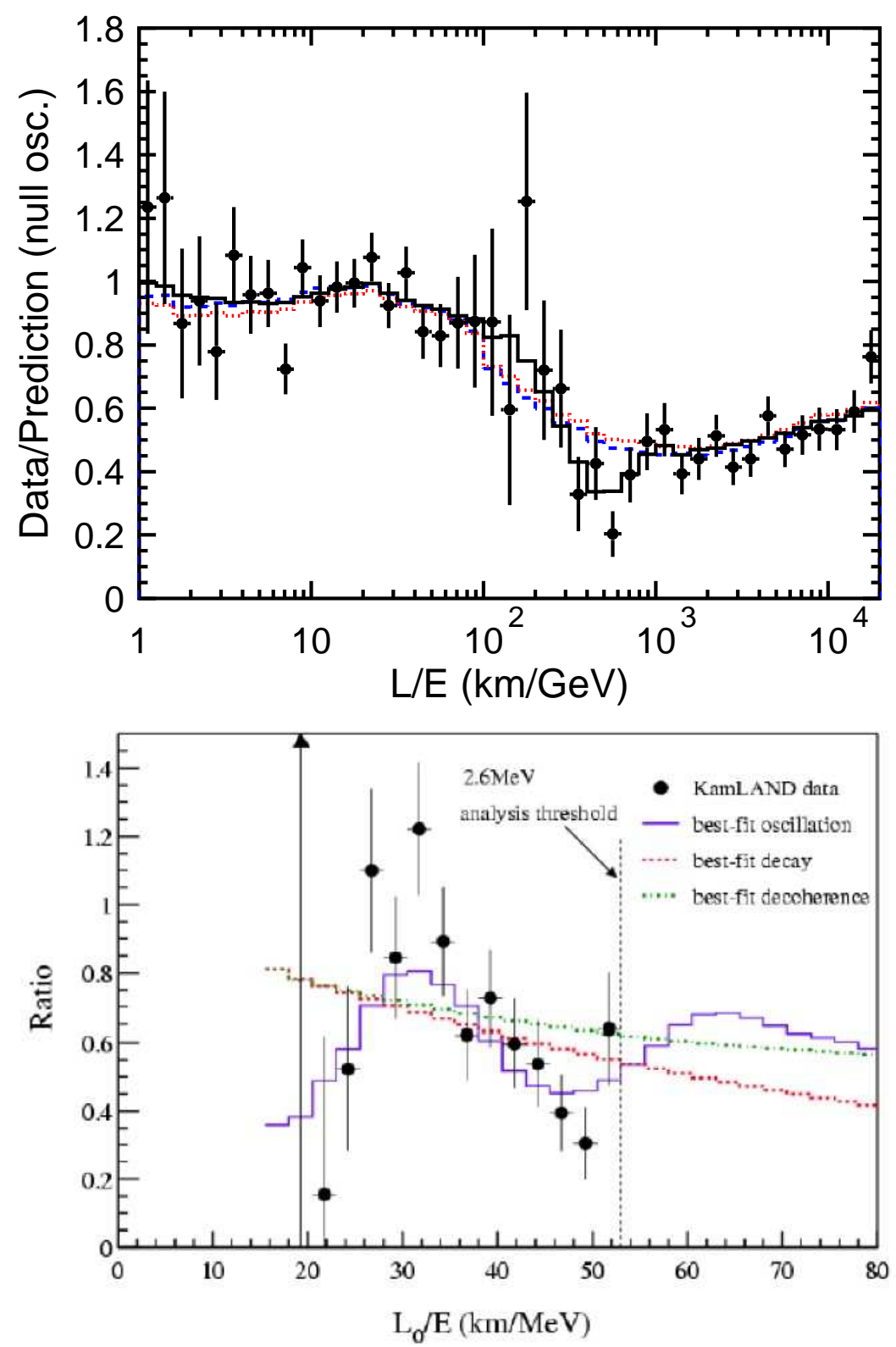

Figure 2.12: L/E Modulation of the Survival Probability. The top plot [44] shows the L/E analysis of the SK experiment. The neutrino oscillation fit to the data black is contrasted with the decoherence red and decay blue hypothesis. With this analysis only, decoherence and decay are disfavoured to $3.8 \sigma$ and $3.4 \sigma$ respectively. The bottom plot [45] shows the L/E analysis of the KamLAND experiment. The neutrino oscillation fit to the data blue is contrasted with the decoherence green and decay red hypotheses. In this analysis, decoherence and decay become disfavoured to 94\%CL and 95\% CL respectively. Note that in none of the cases the signature for neutrino oscillation ("dip") has not yet been resolved with high precision while some discrimination among hypotheses is possible. 
The sensitivity of SK to L/E arises from the correlation between $\cos \theta$ and the distance travelled by the neutrinos from the production point to the detector. So, a neutrino coming from the zenith will have travelled on average distance of $\sim 20 \mathrm{~km}$ before it reaches the detector, while those coming from its antipode may have travelled $\sim 12000 \mathrm{~km}$. Extracting the L/E dependence of the disappearance probability with atmospheric neutrinos is, however, difficult as the estimation of $\mathrm{L}$, relies on the input from simulations limiting the ability to resolve the smallest L/E features of the survival probability.

The advantage of proving neutrino oscillation directly is that the possibility of an unknown contribution to the observations is more limited than through the "indirect" observations. Very precise (i.e. beyond $5 \sigma$ ) L/E modulation of the survival probability is expected to be first obtained by the MINOS experiment within $3 / 4$ years from now.
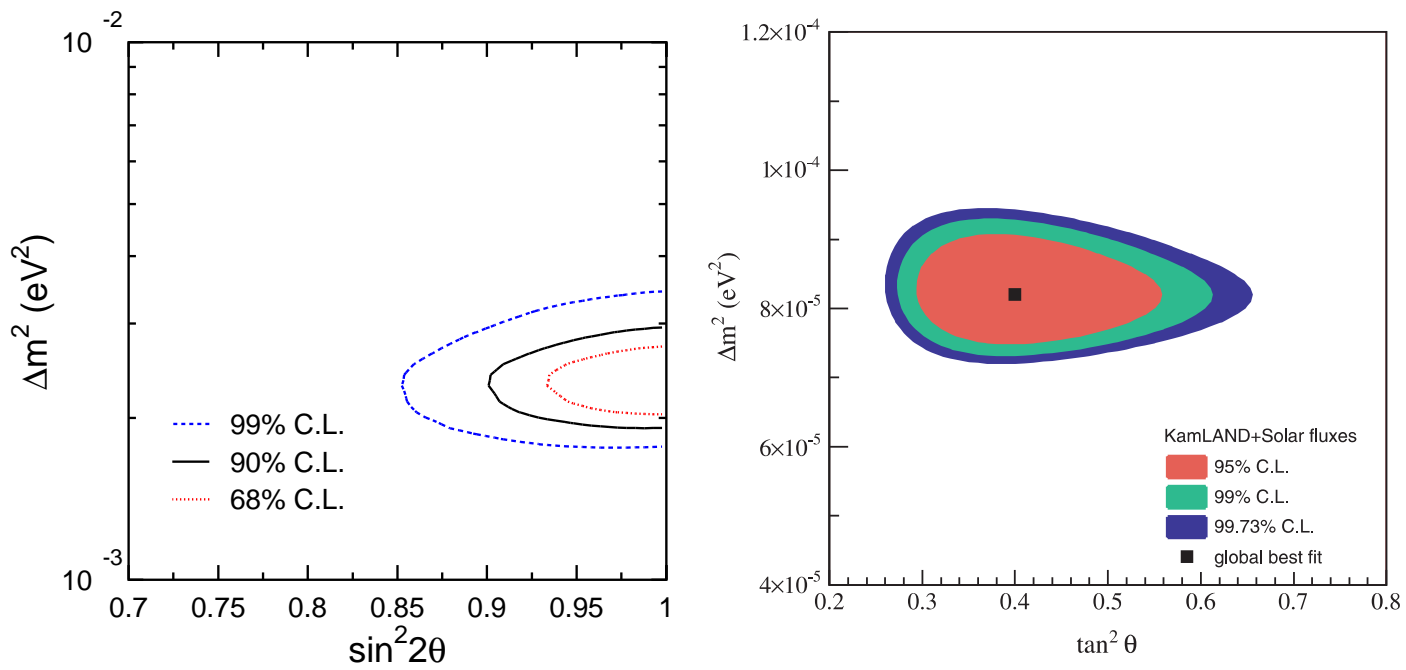

Figure 2.13: The Neutrino Oscillation Parameter Space Today. The left plot [44] shows the limits corresponding to the atmospheric neutrino oscillations parameter space, as measured by the SK experiment. The right plot [45] shows the the limit in solar neutrino oscillations parameter space obtained from the combination of all solar data in combination with KamLAND results. KamLAND significantly constrains the value of the solar $\Delta m^{2}$, as the $\mathrm{L} / \mathrm{E}$ resolution of events used help to obtain a precise determination of $\Delta m^{2}=8.3 \times 10-5 \pm 5 \%$. The solar neutrino oscillation parameter space has since been reduced significantly, rejecting many possible solutions to the solar neutrino anomaly prior to KamLAND data.

Our current best knowledge about neutrino oscillations is summarised in Figure 2.13 and in the following tables: 


$$
\begin{gathered}
\Delta m_{12}^{2} \sim+8.2 \times 10^{-5} \pm 5 \% \mathrm{eV}^{2} \quad(\text { KamLAND+solar }) \\
\Delta m_{23}^{2} \sim \pm 2.4 \times 10^{-3} \pm 30 \% \mathrm{eV}^{2} \quad(\mathrm{SK}-\mathrm{L} / \mathrm{E}+\mathrm{SK}-\text { zenith }+\mathrm{K} 2 \mathrm{~K}) \\
\tan ^{2}\left(\theta_{12}\right) \sim 0.4 \pm 30 \% \\
\sin ^{2}\left(2 \theta_{23}\right)>0.94 \\
\sin ^{2}\left(2 \theta_{13}\right)<0.11 \quad(\mathrm{Chooz})
\end{gathered}
$$

The only exception to the global harmony across the data available are the results from the LSND experiment [46], for which the existence of a "sterile" 4 neutrino is hypothesised in order to reconcile, while not fully, some agreement with the observations. There is however an experiment currently taking data to verify/falsify the LSND results. The experiment is called MiniBooNE [47].

The current effort in neutrino oscillations is generally characterised by experiments assuming neutrino oscillations from their designs to perform high precision measurements or, in some cases still, to further confirm the neutrino oscillation hypothesis. Examples of such experiments about to start taking data are MINOS (Chapter 3), OPERA [48] and ICARUS [49].

\subsection{The Neutrino Community "To-Do-List"}

The most important unknowns that still need to be understood are the following:

1. PMNS Precision Era (assuming neutrino oscillations) PMNS parameters should be measured to high precision, especially $\theta_{13}[50]$ and leptonic CPviolating Dirac-phase. Determining the extract values of the PMNS matrix may elucidate the physics dictating its structure, for example by testing its unitarity.

Examples of experiments endeavoured in such enterprises to come online within the following 10 years are Double-Chooz [51], Braidwood [52], T2K [53] and NOVA [54].

2. Sign $\Delta \mathbf{m}_{\mathbf{2} 3}^{\mathbf{2}}$ The sign of $\Delta m_{12}^{2}$ has been measured through solar neutrino oscillations, as these oscillations are affected by matter effects [55] from which one becomes sensitive to the sign of $\Delta m^{2}$. Future long-baseline neutrino

\footnotetext{
${ }^{4} \mathrm{~A}$ sterile light neutrino, whose mass is $\mathcal{O}(1) \mathrm{eV}$ and does not couple to the $\mathrm{Z}$ and $\mathrm{W}$ bosons. A good dark-matter candidate.
} 
oscillation experiments aiming to measure the appearance of $\nu_{e}$ from a $\nu_{\mu}$ could also allow that.

3. Absolute Mass Current knowledge about the absolute mass scale of neutrinos comes from kinematic limits $\left(\nu_{e}\right)[56]\left(\nu_{\mu}\right)[57]\left(\nu_{\tau}\right)[58]$, from neutrino-less double-beta decay [59] and by astrophysical/cosmological considerations [60].

4. Majorana or Dirac? The answer to this question is only possible through the observation neutrino-less double-beta decay [59]. Evidence for a possible observation of neutrino-less double-beta decay has been presented by a few members of the Heidelberg-Moscow experiment [61] at the Gran Sasso laboratory (Italy). However, this observation has been received sceptically by the experts in the neutrino community and other members of the same collaboration. Nevertheless, even if the observation was correct, a confirmation is needed, ideally with a different isotopes and technique, as the level of sensitivity achieved by such an experiment is unprecedented and the possibility of finding unknown backgrounds cannot be unambiguously ruled out by the same experiment.

If the above observation was confirmed, Majorana neutrinos would provide the avenue for rich phenomenology [16][21]: "See-Saw" mechanism and possible "Lepto-genesis" scenarios, which may give rise to the "Baryon Asymmetry" observed.

All the posed questions have something in common: they remain unsolved unless more precise data is collected. Therefore, the field is still dominated by experimental developments. This is a well known fact and, consequently, vast experimental neutrinos programmes are underway. 


\section{Chapter 3}

\section{The MINOS-NuMI Project}

The MINOS (Main Injector Neutrino Oscillation Search) experiment and the NuMI (Neutrinos at the Main Injector) beam project constitute a dedicated long baseline neutrino oscillation experiment. The main goal of the MINOS/NuMI ${ }^{1}$ neutrino oscillation physics program is to observe the unambiguous neutrino oscillation signature. MINOS is designed to measure the L/E dependence of the neutrino survival probability of neutrinos as they propagate away from a source. This L/E pattern serves to perform precise measurements of neutrino oscillations parameters associated to the "Atmospheric Neutrino Anomaly". The oscillation signature offers the framework for the measurements of the associated oscillation observables: $\Delta m_{23}^{2}$ and $\sin ^{2}\left(2 \theta_{23}\right)$. The main contribution of MINOS is the precision measurement of $\Delta m_{23}^{2}$.

This chapter is dedicated to describing the components of the MINOS experiment in some degree of detail: the NuMI beam in Section 3.1 and the MINOS detectors in Section 3.2. The chapter will culminate with a summary of the envisaged MINOS physics programme in Section 3.3.

\subsection{The NuMI Beam}

The NuMI beam [63] is a wide-band beam (WBB) [21] of mainly $\nu_{\mu}$. The neutrinos are produced with energies in the range $1 \mathrm{GeV}$ to about $40 \mathrm{GeV}$. The NuMI facilities are located adjacent to the FNAL Main Injection (MI) beam (see Figure 3.1), from which a fraction of protons is extracted for MINOS. The production of the

\footnotetext{
${ }^{1}$ Will be referred to as "MINOS" for brevity.
} 


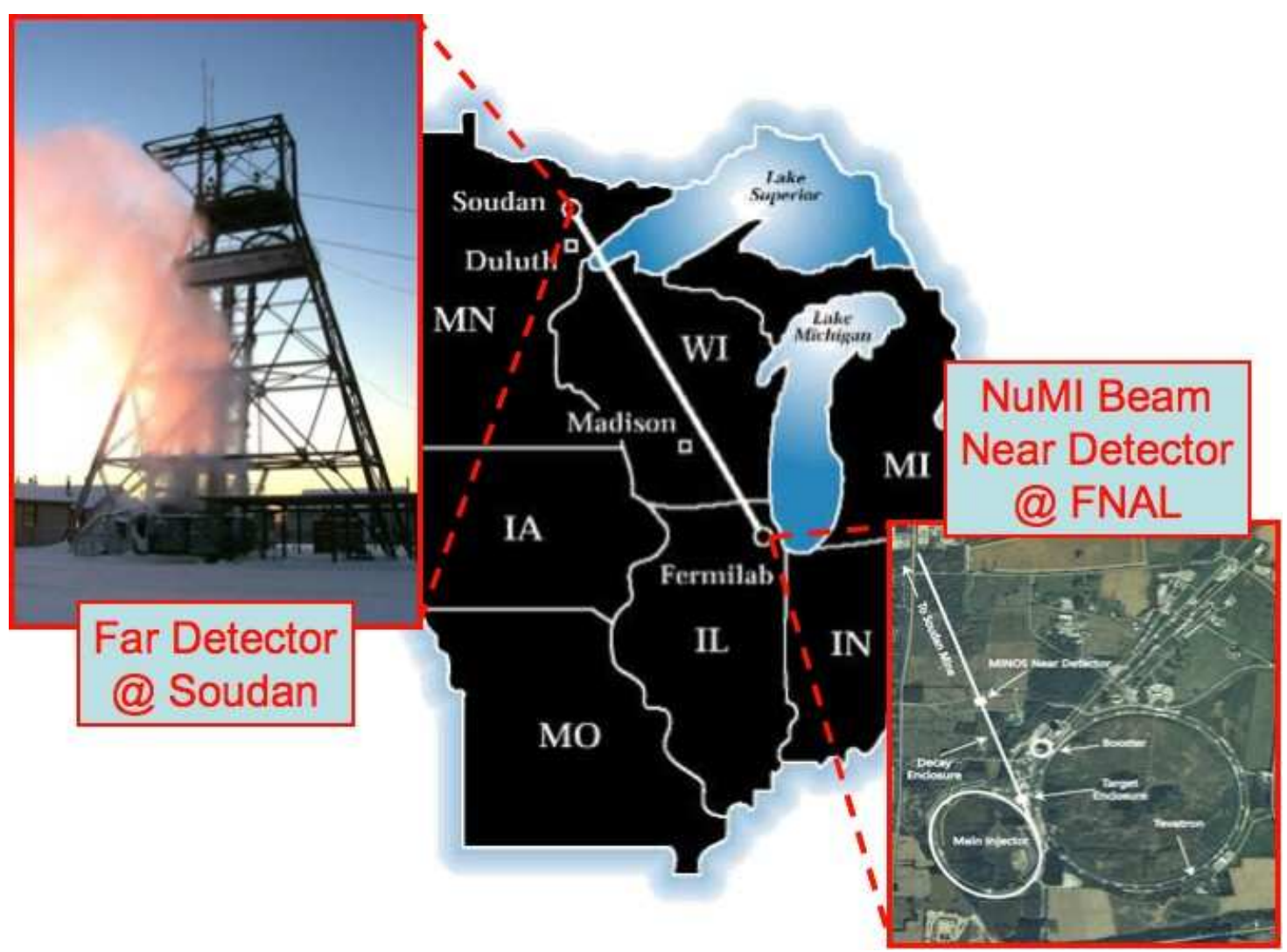

Figure 3.1: MINOS Experiment on a Map. Journey of neutrinos produced at FNAL (IL) and detected at the MINOS far detector in the Soudan National Laboratory (MN).

NuMI beam starts by directing MI protons at $120 \mathrm{GeV}$ onto a slim carbon target. Secondary mesons (mainly $\pi \mathrm{s}$ and $K \mathrm{~s}$ ) are produced from the target due to the strong interactions of the protons. Two magnetic horns located close to the target serve to maximise the collection of secondary mesons. $\nu_{\mu}$ are obtained from the leptonic decay of the mesons in flight along the evacuated decay pipe. Most of the $\nu_{\mu}$ are from the decay of $\pi^{ \pm}$, and correspondingly the length of the decay pipe $(675 \mathrm{~m})$ is about one decay length of a $12 \mathrm{GeV} \pi^{ \pm}$. Some minor contribution is expected from the (semi-)leptonic decay of $K^{ \pm} \mathrm{S}$. A hadron absorber and $250 \mathrm{~m}$ of rock located downstream ensure that only neutrinos reach the MINOS near detector. Figure 3.2 illustrates the whole NuMI production process.

The horns are among the most important components of the NuMI beam. A horn produces a strong pulsed magnetic field in synchronisation with the arrival of the MI protons. The target is located very close to or even inside the horn. The magnetic field inside the horn focuses charged mesons of one polarity along the beam-line and de-focuses those of the opposite polarity. The horns collect a large 


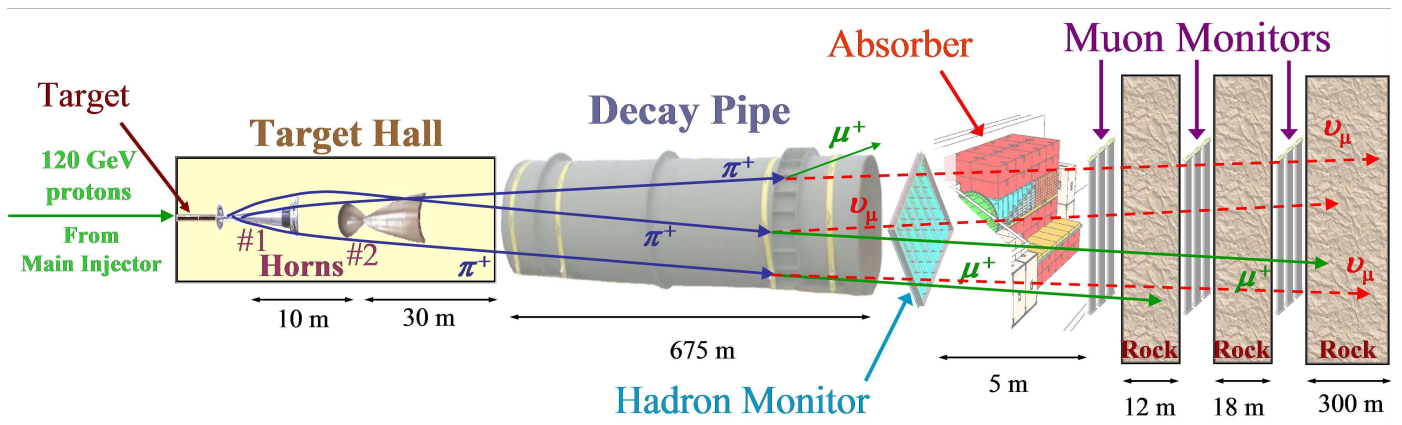

Figure 3.2: NuMI Beam Production Diagram. (Courtesy of Robert Zwaska).

fraction of mesons that would have otherwise been too divergent to have contributed to the neutrino beam. The flux and the spectrum of the beam are therefore largely determined by the horns, as they maximise the collection of secondary mesons, thus enhancing the overall luminosity. The neutrino beam spectrum is determined by the kinematics of the accepted mesons. Altering the relative position of the horns and the target is possible and different configurations will lead to different beam energy spectra since this also changes the kinematics of the mesons accepted. The spectral mean can be varied from $3 \mathrm{GeV}$ to about $15 \mathrm{GeV}$ in this way. Figure 3.3 shows a diagram of the different configurations of the NuMI beam line.

The maximum L/E sensitivity of MINOS is obtained with the "Low Energy" beam configuration: $\Delta m^{2}=\pi E / 2.53 L \approx 5 \times 10^{-3} \mathrm{eV}^{2}$. The focusing/defocusing capability of the horns is used to obtain fairly pure samples of either $\nu$ or $\bar{\nu}$. This is a direct consequence of the purer selection of decaying $\pi^{+} / \pi^{-}$by the horns. Independent $\nu / \bar{\nu}$ running modes is an extra capability that could be used to compensate for cross-sections difference. With "Low Energy" beam, the relative contributions are expected to be $\sim 98.6 \%: \sim 0.9 \%: \sim 0.5 \%: \sim 0.02 \%$ for $\nu_{\mu}: \overline{\nu_{\mu}}: \nu_{e}: \overline{\nu_{e}}$, respectively.

The NuMI beam is intrinsically divergent, as it arises from the decay of highly relativistic particles. The beam spot is expected to be $\sim 0.5 \mathrm{~m}$ and $\sim 10 \mathrm{~km}$ wide at the Near Detector (ND) and Far Detector (FD) sites respectively. Therefore, although the ND spectrum gives a reference of what the neutrino spectrum would be like with no oscillations, the spectrum of the neutrinos measured at the FD is intrinsically different. As a consequence, a well tuned beam MC has to be used to infer the un-oscillated spectra at FD from the ND spectra - and this is one of the most critical steps in the procedure to allow high precision oscillation parameter measurements. The beam MC is used to disentangle the fraction of neutrinos measured in the ND contributing to the FD neutrino spectrum. The beam MC 

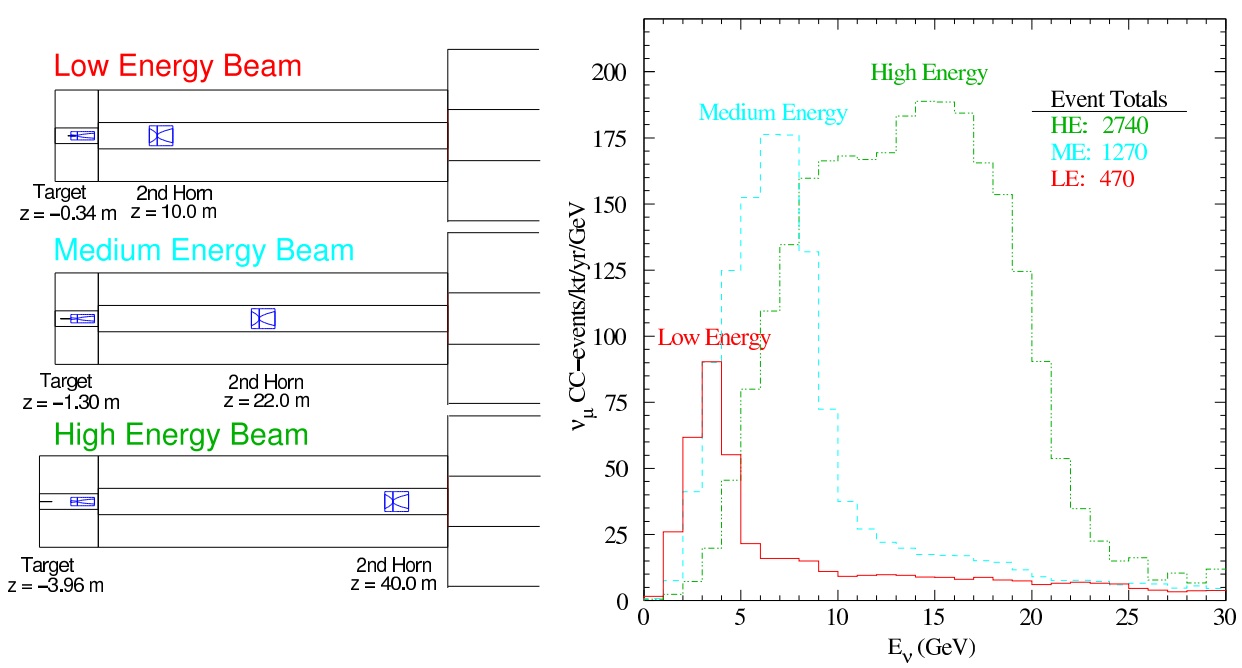

Figure 3.3: NuMI Beam Spectra and Configuration. The diagram on the left shows the different configuration of the NuMI beam horns-target to attain three nominal different neutrino energy spectra. The spectra shown on the right correspond to the $\nu_{\mu} \mathrm{CC}$ to be detected at the FD if no oscillations were to occur, for an equal relative exposure. Note that the corresponding rates differences are essentially dominated by the increase in cross-section with average energy. The mean energy of the "Low-Energy" (LE), the "Medium-Energy" (ME) and the "High-Energy" (HE) beam configurations are $\sim 3 G e V$, $\sim 6 \mathrm{GeV}$ and $\sim 15 \mathrm{GeV}$ respectively. The original MINOS proposal called for the three different beam configurations in order to detect oscillated $\nu_{\tau}$ s from the higher energy beam.

relies on the accurate knowledge of hadronic-yields off the proton-target for this type of calculations to be precise. The MIPP (Main Injector Particle Production experiment) [64] is a dedicated experiment at FNAL whose goal is to measure hadronic-yields. As part of their programme, a NuMI target replica has been be struck by MI protons in order to measure the specific hadronic-yields for MINOS. The aim is therefore to reduce the systematic errors in the extrapolation from the ND to the FD to a nominal $2 \%$ level.

The NuMI beam is expected to provide about $\sim 1.5-2.4 \times 10^{13}$ protons-ontarget $(p o t)$ per spill. Each spill lasts about $10 \mu$ s with a period of about 1.9s. The NuMI beam is expected to deliver around $\sim 2.5 \times 10^{20}$ pot per year $(0.25 \mathrm{MW})$ which corresponds to about $2000 \nu_{\mu}$ CC events per year in the FD with the "Low Energy" beam, if no oscillations occurred. The MINOS experiment is planned to run for 5 years to achieve the statistical sensitivity needed. Further statistics may however be beneficial for some of the measurements that are within the reach of MINOS (see Section 3.3 for details). 


\subsection{The MINOS Experiment}

The MINOS experiment consists of three detectors and a collaboration of about 150 physicists. The two large detectors are dedicated to the beam neutrino oscillation measurements: the ND and FD. This section will be broken down into three logical sub-sections. First, the common technology used on all MINOS detectors will be described. Subsequently, the most relevant features of both FD and ND will be discussed in some degree of detail. The original MINOS design can be found in [65].

The third of the MINOS detectors, designed for calorimetric energy calibration, is called the Calibration Detector or "CalDet". The CalDet will be the subject of Chapter 4. In the description of the CalDet, the emphasis will be restricted to only those aspects of the detector that deviate from the common MINOS technology.

\subsubsection{The MINOS Detector Technology}

The MINOS detectors are magnetised ${ }^{2}$, tracking, sampling, uncompensated calorimeters. This approach is inspired by the original CDHS [66] neutrino detector developed at CERN in 1978, as well as later versions developed by the CCFR [67] and $\mathrm{NuTeV}$ [68] collaborations. Such a design was chosen by the MINOS collaboration to achieve the high precision envisaged on the measurement of $\Delta m_{23}^{2}$. Such a precision can only be attained by accurately estimating the energy of CC interacting $\nu_{\mu}$ s over the energy regime in question. The principle is to measure the "visible", induced hadronic activity by calorimetry (as a sampling-calorimeter), while estimating the energy of the accompanying muon by spectrometry (as a magnetised-tracker). The visible energy of the interacting neutrino is then inferred by summing the visible hadronic energy and the energy of the muon.

\section{Detectors Concept}

All the MINOS detectors were designed to be as identical as possible in order to readily translate responses and event topologies across the detectors, thus minimising potential systematic errors that may arise. The granulating and sampling of the detectors was chosen to be rather coarse as a trade-off between the following considerations:

\footnotetext{
${ }^{2}$ Except CalDet
} 
- Need to achieve high enough hadronic-shower energy resolution to measure the visible energy of neutrinos precisely.

- Need to be able to efficiently separate $\nu_{\mu} \mathrm{CC}$ events from NC events based on the charge deposition topology down to $\sim 1 \mathrm{GeV}$,

- Essential to construct inexpensive detectors whose fiducial volume is large enough to attain reasonable statistical sensitivity

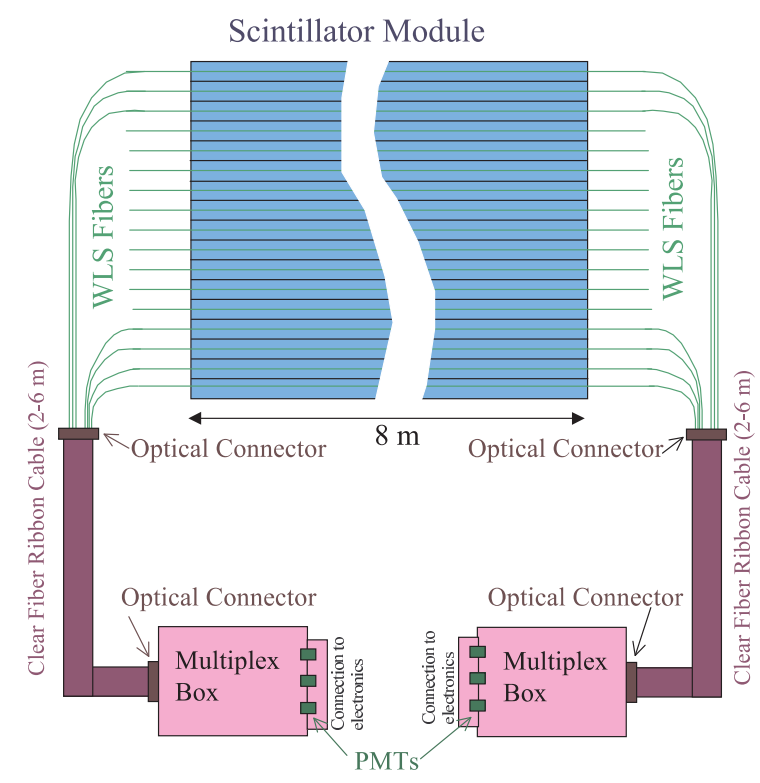

Figure 3.4: The MINOS Readout System.

The general principle is that charge particles going through the detector would cause the emission of scintillation light in the active instrumented planes. The scintillation light is then conducted by optical fibres to PMTs connected to the readout electronics that digitises and time-stamps the hits (or "digits"). The optical readout system, starting from scintillator strips and ending at the PMT face, is illustrated in Figure 3.4.

\section{Detectors Structure}

The ND and the FD are endowed with a magnetic field. The field is induced electro-magnetically in the steel by current running through a coil at the centre of the detector. The magnetic field is used to infer the momentum and polarity of muons, just like in a conventional spectrometer. The muons in question are, 
for example, those emitted in a $\nu_{\mu} \mathrm{CC}$ interaction or through going cosmic muons. In fully contained $\nu_{\mu} \mathrm{CC}$ events, the momentum of the muon can be additionally measured from range (or even calorimetry) measurements. Range estimations have the best resolution at low energies $(\sim 6 \%$ at $10 \mathrm{GeV} / \mathrm{c})$, since the random-scattering of muons degrades the precision of a curvature measurement $(\sim 14 \%$ precision at $10 \mathrm{GeV} / \mathrm{c}$ ). The momentum of partially contained interacting muons from neutrinos within the detector can only be measured from curvature.

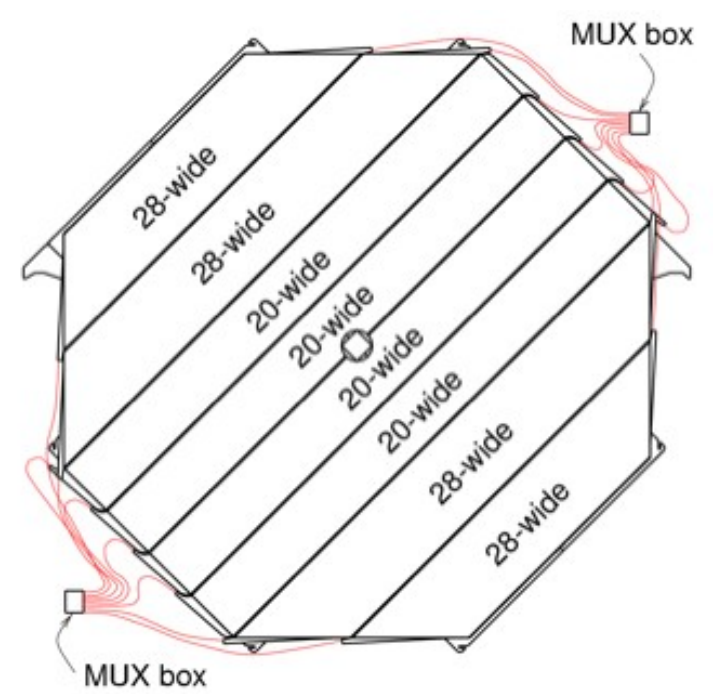

Figure 3.5: A MINOS Detector Plane.

The sampling was achieved by interleaving active planes of plastic scintillator and passive planes of steel. In terms of radiation lengths, the sampling was 0.024 and 1.44 radiation lengths corresponding to $1.0 \mathrm{~cm}$ of scintillator and $2.54 \mathrm{~cm}$ of steel, respectively. A gap of air $(2.5 \mathrm{~cm}$ thick) was left in between every scintillatorsteel pair of planes. Each scintillator plane was made up of parallel scintillator strips $4.1 \mathrm{~cm}$ wide, as illustrated in Figure 3.6. The length of the scintillator strips depends upon their location in the detector, which can be up to $8 \mathrm{~m}$ long in the FD, as illustrated in Figure 3.5. Tracking of particles through the detector is made possible by having alternate scintillator planes with their strips in orthogonal orientations, as shown in Figure 3.10 for the FD.

\section{Detectors Optical System}

The scintillator strips are made of polystyrene doped with fluor PPO (1\%) and POPOP (0.03\%). The fluor PPO helps to convert the scintillation UV-photons 
into longer wavelength photons, where the polystyrene is more transparent. The POPOP is yet another wavelength-shifting material that helps to effectively lowers the attenuation of the scintillation light produced by shifting the scintillation light to a lower wavelength where the scintillator itself is less opaque.

The scintillator strips emit blue/UV light. The scintillation photons are collected by green wavelength-shifting-fibres (WLSF) which then re-emit a proportional number of green photons. The WLSF (Kuraray Y11 of $1.2 \mathrm{~mm}$ of diameter) is located in a groove that runs along the middle of every scintillator strip, as shown in Figure 3.6. Every scintillator strip is painted by an internally reflecting coat of polystyrene with $\mathrm{TiO}_{2}$.

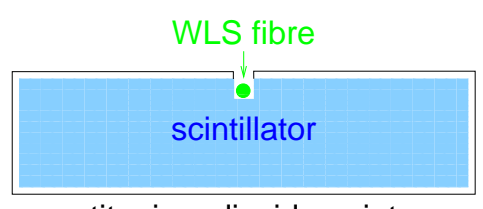

titanium dioxide paint

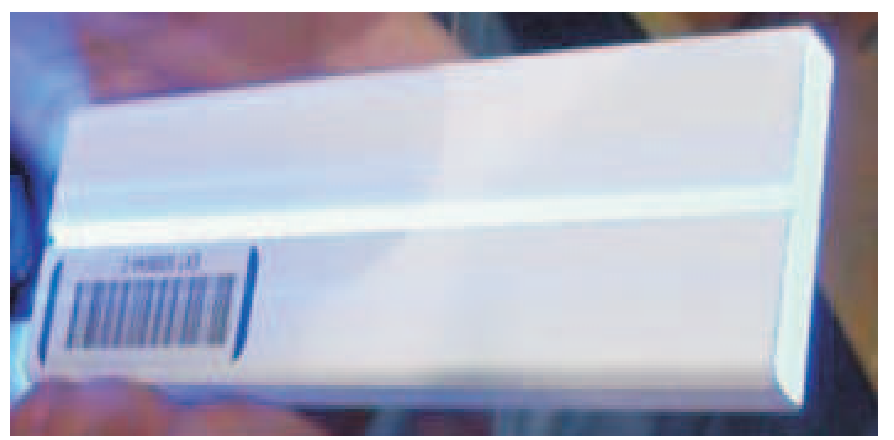

Figure 3.6: Scintillator Strip Diagram and Photo.

The reflective coat enhances the collection efficiency of scintillation light by the WLSF. WLSF green photons are then conducted by clear fibres from each stripend to the readout PMTs. Clear fibres (made of polystyrene by Kuraray) are used because of their longer attenuation length $\left(\lambda^{\text {clear }} \sim 13 \mathrm{~m}\right)$ with respect to that of the WLSFs. The attenuation of light in WLSF is characterised by the sum of two double exponential with attenuation indexes: $\lambda_{1}^{W L S F}=0.7 \mathrm{~m}$ and $\lambda_{2}^{W L S F}=3.9 \mathrm{~m}$.

\section{Detectors Readout System}

The MINOS PMTs are all Hamamatsu multi-anode PMTs [69] [70] [71]. An example of a MINOS PMT can be found in Figure 3.7. Each clear fibre end is coupled to a pixel of a PMT. The light pulses are converted into current pulses by the PMTs and are subsequently integrated, digitised and time-stamped by the FrontEnd-Electronics (FEE). The gains of the PMTs were chosen to output a signal of $\sim 10^{6}$ electrons $(\sim 160 \mathrm{fC})$ per PE (Photo-Electrons).

The multi-anode nature of the MINOS PMTs introduce special features which may change the event topology and, consequently, the performance of MINOS. 


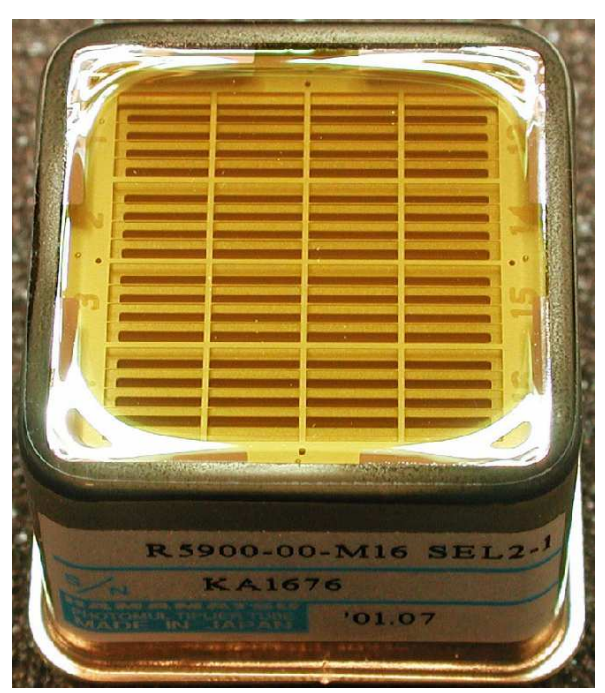

Figure 3.7: The MINOS Multi-Anode PMT.

These features were thoroughly investigated, in particular, the effective uniformity of gain across photo-cathodes and crosstalk. Appendix A summarises the PMTcrosstalk studies performed on some of the MINOS PMTs.

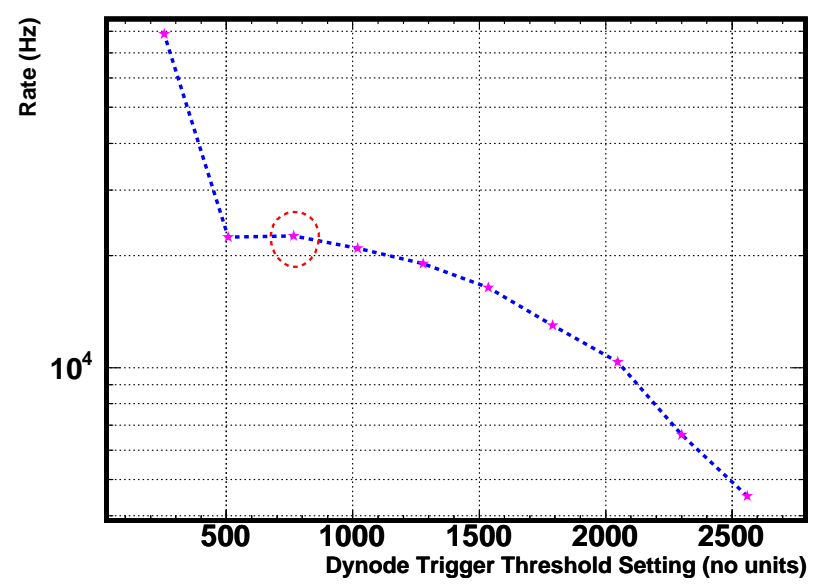

Figure 3.8: Dynode Trigger Threshold Setting at CalDet. This plot exemplifies the empirical method used to tune the dynode thresholds as low as possible during CalDet 2003. The plots shown the average rate of detector versus dynode threshold setting. The point circled (in red) shows the optimal dynode threshold, for which the FEE is not triggering on the pedestal (lower settings) or the setting is unnecessarily low reducing the efficiency on the 1PE peak.

The most basic trigger criterion in all MINOS detectors uses the signal from the last dynode on each PMT. The dynode trigger uses the analogue sum of the 
charge at the last dynode of all pixels: "last-dynode" signal. The readout of the full PMT is triggered when the dynode signal exceeds some threshold, nominally set to 1/3PE. However, the technique used for setting the dynode thresholds, exemplified in 3.8, brings the dynode threshold closer to the level of the sparsification thresholds: $\sim 1 / 5 \mathrm{PE}$. Since the detectors were designed to deliver about 4 to 5PEs per minimum ionising particle ${ }^{3}$ per strip-end, the probability of missing a minimum ionising particle due to photon statistics fluctuations ${ }^{4}$ with this dynode threshold less than 1.4\% (assuming that, on average, only one pixel is hit per PMT per minimum ionising particle). The dead-time is related to the full triggering scheme, which is detector dependent and, so, they will be discussed in the forthcoming relevant sections.

\section{Detectors DAQ}

The MINOS detectors are large detectors located underground in order to be shielded from most cosmic rays. Most of the time that a trigger occurs in the detector, only a few strips exhibit any charge deposition. The remaining channels show pedestal distributions and some 1PE noise from the optical system. Therefore the FEE electronics were endowed with online zero-suppression, also called sparsification, capabilities. The online zero-suppression reduces the instantaneous DAQ rates by removing all read out channels whose charge deposition is below sparsification threshold. Studies showed that tuning the sparsification threshold to the pedestal width is sufficient to reduce DAQ rates, while keeping the $1 \mathrm{PE}$ sensitivity above $90 \%$.

All the MINOS FEE are mounted within VME crates controlled by dedicated VME computers. VME computers also referee the DAQ traffic from the FEE crates to the DAQ computers. The overall synchronisation of all VME computers and DAQ computers is supervised by an external master computer. The DAQ computers are dedicated to collecting all the digits from all FEE channels. Once collected, digits are time-sorted within "time-frames" (a second's worth of data). The identification of digit clusters in time is used as the most basic event construction criterion. Such clusters are called snarls in MINOS. Snarls are not necessarily single events. Offline reconstruction software is required for event formation. This is particularly critical in the ND where a snarl corresponds to a full spill, which

\footnotetext{
${ }^{3}$ Actually refers to a minimum ionising particle crossing each strip at its centre orthogonally to its length and to its width

${ }^{4}$ Assuming a Poissonian distribution
} 
can contain several neutrino interactions. The isolation of snarls relies on finding time "gaps" (typically larger than $\sim 400 \mathrm{~ns}$ ) between clusters of hits.

Once snarls have been formed, the triggering processors can apply different online-trigger criteria to reject/accept each snarl. There are, at the moment, two main schemes of online-trigger depending on whether the system is on-spill or offspill. During a beam spill, all events triggered by the detector FEE are collected since beam neutrino events are expected. Outside of the beam-spill, the collection of data depends on whether a "plane-trigger" criterion OR an "energy-trigger" criteria are met. The "plane-trigger" requires that $\mathrm{M}$ planes are hit out of $\mathrm{N}$ consecutive planes - typically a $4 / 5$ trigger is used for the collection of cosmic muons. On the other hand, the "energy-trigger" puts a minimum requirement on the amount of energy deposited within a certain number of planes for read out. Therefore, the online trigger is most sensitive during spill, maximising the collection of $\mathrm{NC}$ events, which would have been otherwise rejected by both plane and energy criteria. Once snarls meet the on-line trigger condition, they are written out by the DAQ and stored as ROOT [72] files. Further details about the MINOS DAQ system can be found in [73].

\section{Detectors Performance}

The overall energy resolution of MINOS detectors to electromagnetic and hadronic showers was measured at CalDet to be

$$
\begin{array}{cccc}
\frac{(21.42 \pm 0.06) \%}{\sqrt{E}(G e V)} \oplus & (4.1 \pm 0.2) \% & \text { (electrons) } \\
\frac{(56.6 \pm 0.6) \%}{\sqrt{E}(G e V)} \oplus & (4.2 \pm 1.4) \% & \text { (protons) } \\
\frac{(56.1 \pm 0.3) \%}{\sqrt{E}(G e V)} \oplus & (2.1 \pm 1.5) \% & \text { (pions) }
\end{array}
$$

as reported in [74] [75]. The $e / \pi$ ratio was also measured at CalDet to be $\sim 80 \%$. Note that the energy resolution measured at CalDet should be slightly better because the CalDet steel planes were $\sim 2 \%$ thinner than nominal, while the thickness of the scintillator planes was nominal. 


\subsubsection{The Far Detector}

The Far Detector (FD) is located in the Soudan Underground Laboratory in Minnesota (showed in Figure 3.1). SUL is $\sim 710 \mathrm{~m}$ deep underground $(\sim 2000$ water equivalent meters). At such a depth, the rate of cosmic muons is about $\sim 1 \mathrm{~Hz}$. About 4 beam neutrinos per day are expected to interact in the FD during beam spill at nominal luminosity.

There are two main reasons for the location of the FD. First, the tuning of the $\mathrm{L} / \mathrm{E}$ ratio to allow maximal sensitivity to the atmospheric neutrino oscillations: $L / E \times \Delta m_{23}^{2} \sim 1$. The desired L/E ratio can be attained with the baseline of $735 \mathrm{~km}$ (distance between SUL and FNAL) by configuring the NuMI beam to produce neutrino energy spectra peaking at a few GeV, i.e. the "Low Energy" beam. Second, the underground location provides natural shielding from cosmic muons, granting an overwhelming background and rate reduction (for atmospheric neutrino analyses). Such low rates give MINOS the chance to detect beam neutrinos with a virtually trigger-less detector during spill.
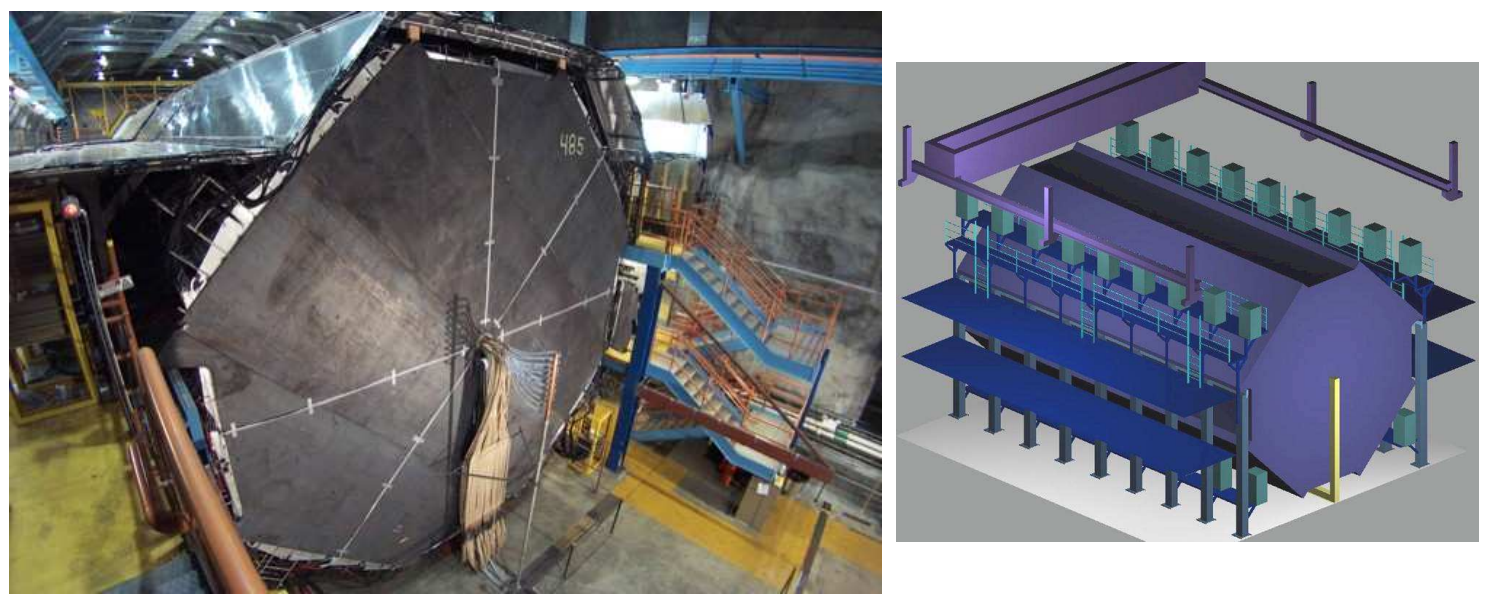

Figure 3.9: MINOS Far Detector: Site and Photo.

Due to the modular design of the detector, diagnosis and calibration data was taken as the detector was constructed. Atmospheric neutrino data has been taken since the first super-module (out of the two making up the whole FD) was fully constructed, magnetised and debugged by summer 2003. The FD was finished during early summer 2003. 


\section{The Far Detector Structure}

The FD is the largest of the MINOS detectors and is shown in Figure 3.9. FD has a mass of $5.4 \mathrm{kT}$ made up of 498 instrumented planes, with 192 scintillator strips each. Each strip is read out on both ends to increase the light collected per event. Each plane is octagonal in shape with a diameter of $8 \mathrm{~m}$. The strips are oriented at $\pm 45^{\circ}$ with respect to the zenith in alternative planes, as shown in Figure 3.10. Such a configuration ensures that all planes exhibit similar occupancy for down-coming atmospheric muons, used for calibration. The magnitude of the field ranges from $1 \mathrm{~T}$ to $1.5 \mathrm{~T}$ within the central volume of the detector.

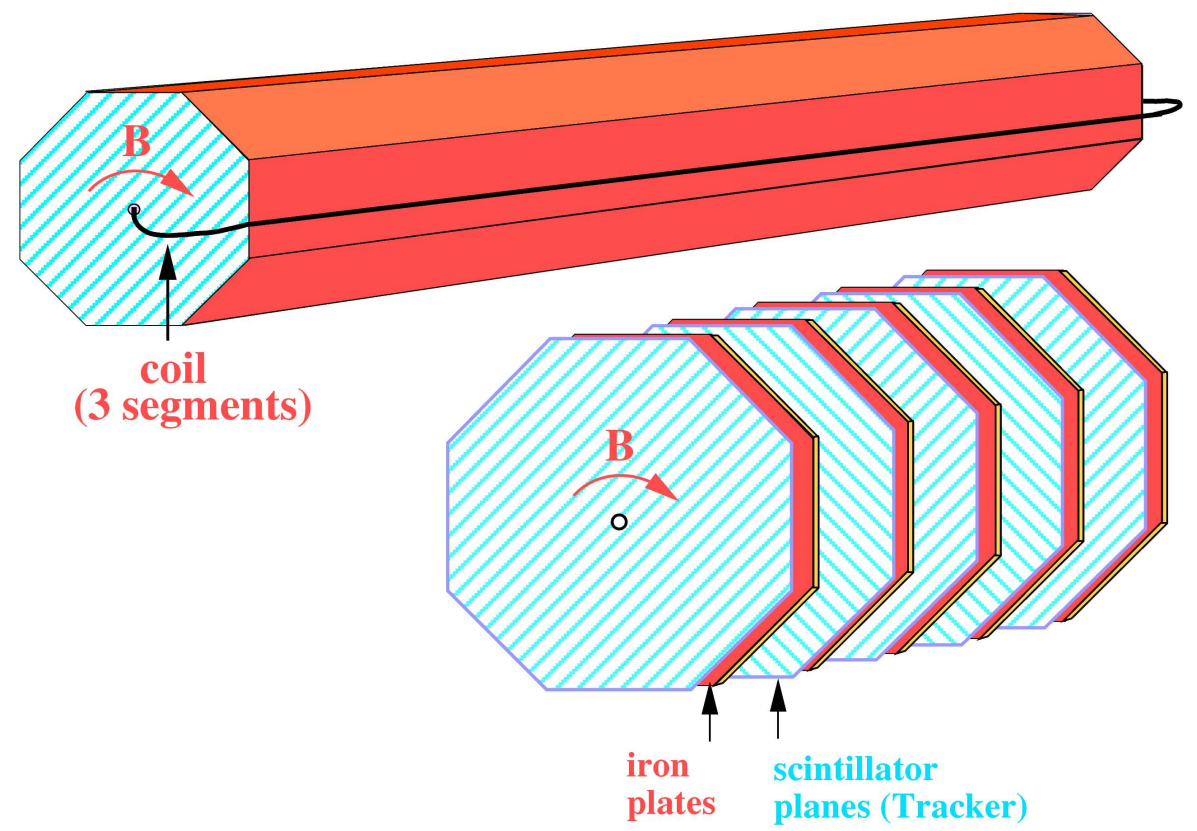

Figure 3.10: Overall Structure of MINOS Far Detector.

\section{The FD Readout System}

The PMTs selected for the FD are the 16-pixels Hamamatsu R5900-00-M16, called M16. The large pixels allow an 8-fold optical sum, meaning that each PMT pixel is simultaneously coupled to 8 strips from the same plane. Each pixel however, has a one-to-one relationship to a FEE channel. The deconvolution of the optical sum in the events is carried out by offline algorithms (as part of the reconstruction), that utilise, as the principle, that the solution is not the same on both side of the detector. Optical sum was considered viable at the FD due to the very low event rates. Of course, this measure caused the required number of PMTs needed 
to be consequently reduced by a factor of eight and the consequent saving in the cost of the FD construction. The major disadvantage of this scheme is that acceptance to the most vertical incoming events is sacrificed, for which no unambiguous reconstructed solution can generally be obtained.

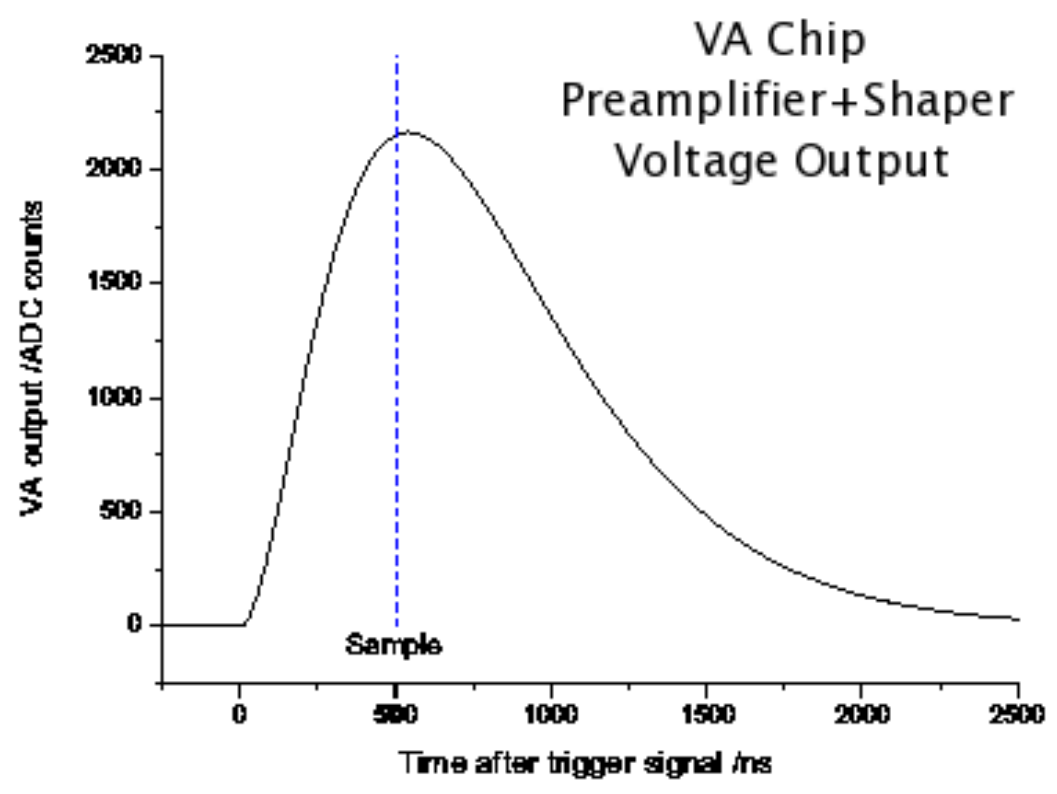

Figure 3.11: VA-chip Digitisation. The main features of the digitisation process carried out by the VA-chip are illustrated in this plot. Upon arrival of a hit, the charge sensitive preamplifier and shaping $(\mathrm{CSP}+\mathrm{S})$ circuitry potential grows monotonically to be stored after about 500ns (blue line) by the VA-chip. The $\mathrm{CSP}+\mathrm{S}$ voltage rises proportionally to the incoming integrated charge granting a linear relation between the incoming charge injected and the CSP voltage as sampled by the VA-chip. (Original plot from Phil Litchfield).

The FEE used at the FD is based on the IDE AS Viking chip (VA32 HDR11), known in MINOS as VA-chip [76]. Each VA-chip reads out one full M16, although the VA-chip has 32 channels (some of the excess channels are used for calibration tasks). An incoming PMT pulse is first fed into a Charge Sensitive Preamplifier and Shaper $(\mathrm{CSP}+\mathrm{S})$ circuitry in each VA channel. The output of the $\mathrm{CSP}+\mathrm{S}$ is then passed to the sample-and-hold circuitry also on the VA-chip. The VA-chip stores the analogue output of the $\mathrm{CSP}+\mathrm{S}$ about 500ns after the dynode triggering was accepted. The $\mathrm{CSP}+\mathrm{S}$ works continuously, independent of whether there is a trigger. Figure 3.11 shows a diagram of how the digitisation is carried out by the VA-chip. The effective response of the VA-chip circuitry is that the voltage held 
by the VA-chip is linearly proportional to the integrated charge of the incoming PMT pulse. The time-stamp associated with each hit corresponds to the arrival of the dynode-trigger for the chip; i.e. the time-stamp per trigger is VA-chip-wise.

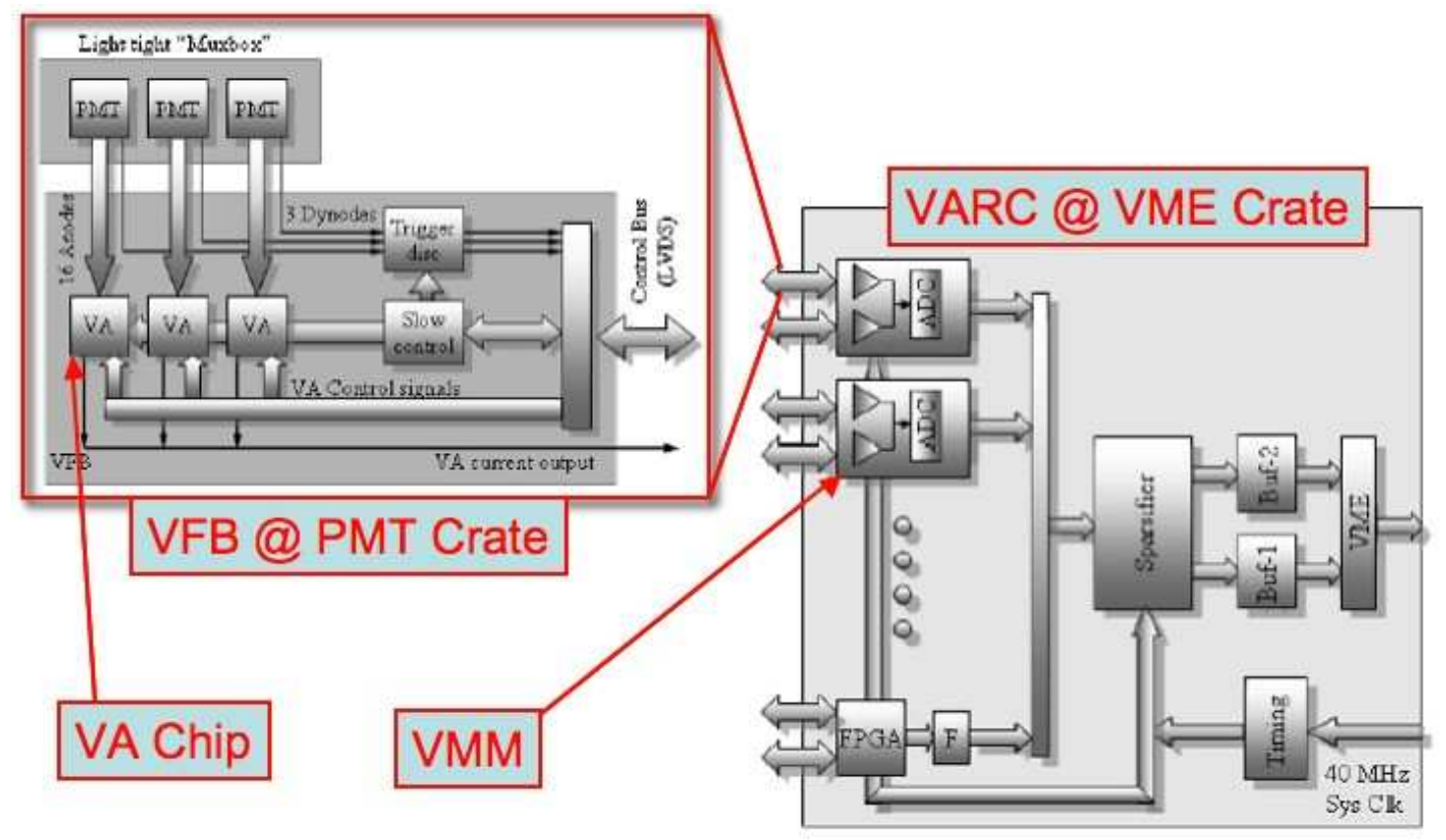

Figure 3.12: Diagram of the VA-chip Electronics. Logic diagram of the breakdown of the components of the electronics based on VA-chips of the MINOS far detector.

Oxford and Harvard universities designed the remaining FEE based on the VAchip, which we shall refer as VA-FEE for short [77]. The building blocks of the VA-FEE are: the VFB (VA Front-end Board), the VMM (VA Mezzanine-Module) and the VARC (VA Readout Card). The VFB is the mother board for the VA-chip. VFBs allow the VA chips to be as close as possible to the PMT anodes, reducing noise from capacitive coupling. The VFB endows the VA-chip with interface to the rest of the VA-FEE to allow data transfer and control. Furthermore, the VFB holds the dynode trigger circuitry for each VA-chip. There are three VA-chips per VFB, while two VFBs (or six VA-chips) are readout by each VMM. A VMM is a card plugged into the VARC (where six VMMs are held) where the 14-bit ADC is located. The VA-FEE sensitivity is $\sim 2.0 \mathrm{fC} / \mathrm{ADC}$, which leads the PMT gain to be $\sim 70 \mathrm{ADC} / \mathrm{PE}$. The VA-FEE uses an effective $640 \mathrm{MHz}$ clock for time-stamping the digitised hits (circuitry on the VARC), allowing a $\sim 1.5 \mathrm{~ns}$ time resolution hitto-hit. This is critical for the atmospheric neutrino physics at the FD, for which 
time allows the separation between up/down going events ${ }^{5}$. Finally, the VARC is the "brain" of the VA-FEE providing control and VME interface to the rest of the DAQ. The VARC carries out some data processing such as sparsification, common mode correction (see Section 5.3.1) and pedestal subtraction. One VARC serves 36 VA-chips (or 36 PMTs) in total. Every VME crate holds three VARCs.

The dead-time in the VA-FEE is VMM-wise since the digitisation stage, which occurs in the VMM, leads the dead-time. The dead-time per triggered VA-chip was configured to be $\sim 5 \mu \mathrm{s}$ in MINOS. Therefore, if the six chips served by the same VMM were hit, a maximal dead-time of about $6 \times 5 \mu \mathrm{s}=30 \mu \mathrm{s}$ is expected.

The VA-chip is known to have non-linear behaviour at very high charges and variation of its gain with temperature. The mechanism causing the VA-chip response (or gain) to vary with temperature arises from a slower response of the $\mathrm{CSP}+\mathrm{S}$ relative to the sampling time as temperature increases (Figure 3.12). The mechanism inducing the VA-chip non-linearity is, however, far more complicated. Both effects yield an effective lower response relative to the incoming pulse and are tackled by the MINOS calibration chain.

\section{The Veto Shield}

A veto shield was constructed for the FD, which can be seen in Figure 3.9. The veto was designed to improve the capability for MINOS to perform competitive atmospheric neutrino physics. The veto shield provides unambiguous tagging of cosmic muon events (veto hit) with respect to neutrino induced events (no veto hit). The need for the veto is due in great part to the beam-oriented geometry of MINOS, i.e. vertical planes. Neutrino beam induced events are expected to be horizontal, thus vertical planes are ideal for their tracking. If there was no veto shield, the vertical incident cosmic muons could sneak through the passive elements of the detector: air gaps and steel. These muons would constitute an indistinguishable background, since the muon would first interact towards the centre of the detector (within the fiducial volume), thereby mimicking the pattern of an atmospheric neutrino. The veto shield was constructed using the same kind of scintillator strips, PMTs and FEE as the rest of the FD.

\footnotetext{
${ }^{5}$ As long as, events produce a trail, at least, $\sim 1 \mathrm{~m}$ long, which is $\sim 2 \times 1.5 \mathrm{~ns} \times \mathrm{c}$.
} 


\subsubsection{The Near Detector}

The Near Detector (ND) is a smaller detector: 980 tons. The ND is located at $100 \mathrm{~m}$ underground ( 300 water equivalent meters) and about $1 \mathrm{~km}$ downstream from the NuMI beam target. Due to its shallower depth, the rate of cosmic muons in the ND is about $20 \mathrm{~Hz}(20 \times$ higher than at FD). Figure 3.13 shows a diagram of the ND site and a photo of the ND once completed.
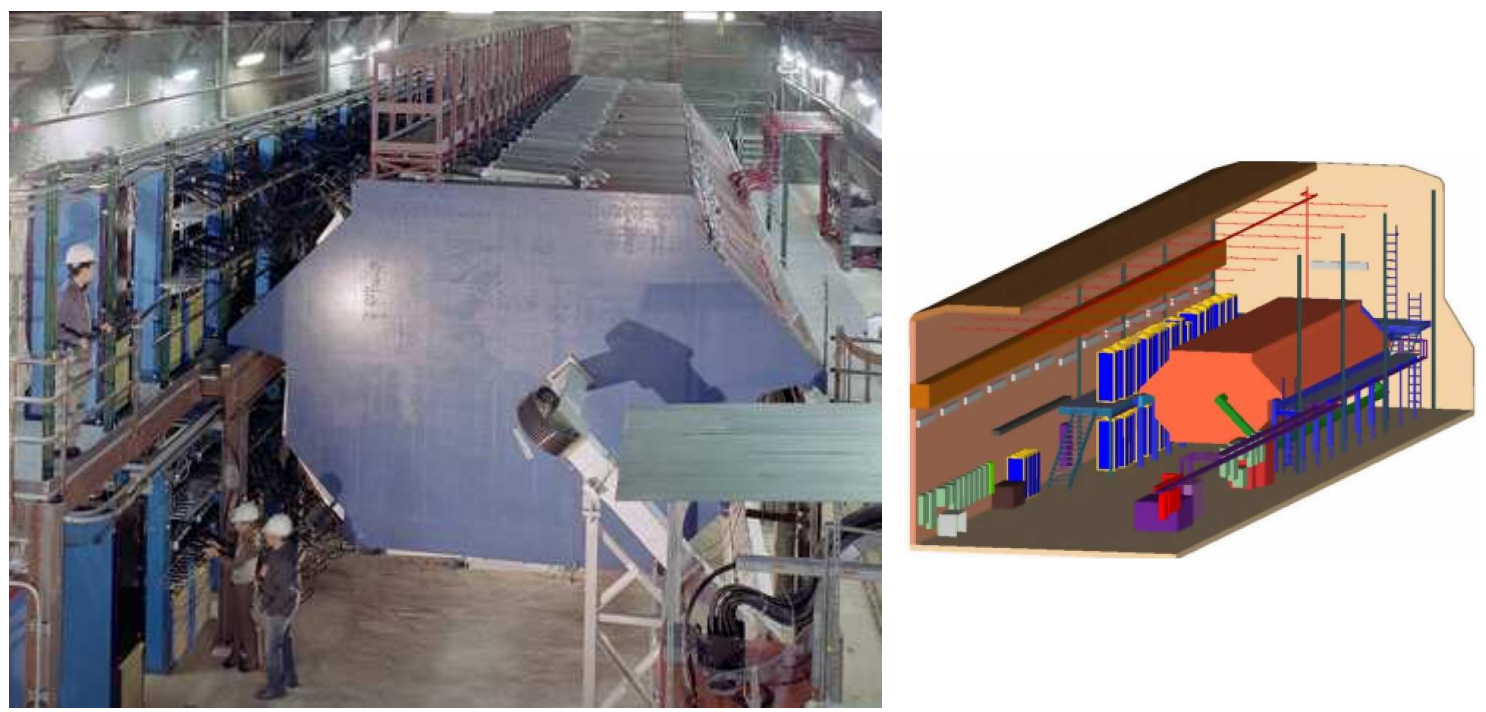

Figure 3.13: MINOS Near Detector: Site and Photo.

\section{The Near Detector Structure}

The planes have only single-sided read out, so fewer readout electronic channels are needed. A mirrored surface was placed at the opposite strip-end (end of the WLSF) to increase the light yield per event. The ND is made up of 282 planes arranged in four different regions (from forward to backward): veto, target, hadron and spectrometer regions as shown in Figure 3.15.

The Veto Region (20 planes) is used to eliminate background from potential incident neutrons created by beam interaction with the rock upstream. Events in this region will not be used for oscillation analyses.

The Target Region (40 planes) is where any useful neutrino interaction should have its vertex.

The Hadron Region (60 planes) is used to contain the hadronic showers present in most neutrino interactions. 
The Spectrometer Region (160 planes) is used to measure muons in $\nu_{\mu} \mathrm{CC}$ interactions and extract their momentum based on curvature in the magnetic field.

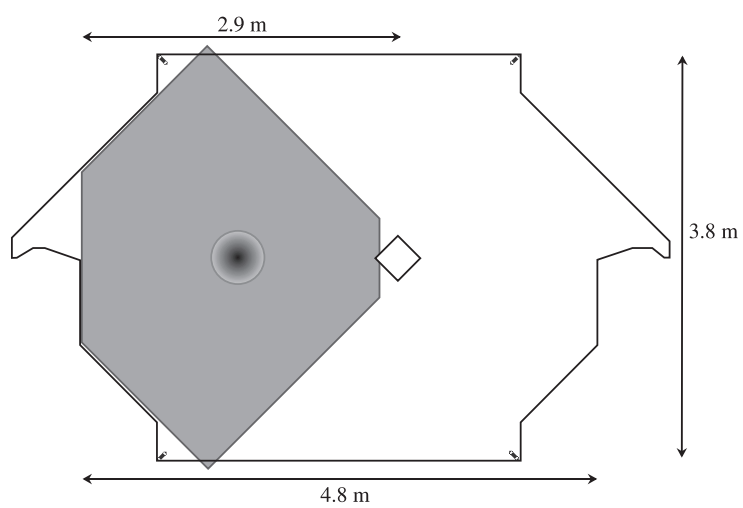

Figure 3.14: MINOS Near Detector Plane: NuMI Beam Spot.

The ND is so close to the beam target that the cross-section of the beam is still narrow: between $0.5 \mathrm{~m}$ and $1 \mathrm{~m}$. Since most of the beam neutrinos pass through the detectors very high rates are expected, up to about 10 events per spill can happen at nominal luminosity with the "Low Energy" beam configuration. The geometry of the ND was therefore optimised to attain some savings on the cost of the ND. First, most of the planes were only partially equipped with readout (see Figure 3.14) while still fully covering the beam spot, and the arising interaction debris. Second, the ND was designed as a "squeezed" octagon of $3.8 \mathrm{~m}$ of height and $4.8 \mathrm{~m}$ of width. The hole for the magnetic field coil and the centre of the beam spot are offset by about $50 \mathrm{~cm}$ on opposite sides of the geometrical centre of the detector. This way events interact within the active volume of the detector with a magnetic field of similar magnitude to that of the FD. Third, only 1 out of every 5 planes is equipped completely in the veto, target and hadron regions (Figure 3.15). This was intended to provide a handle on escaping muons from the fully instrumented detector core. Studies showed that by instrumenting the spectrometer region with 1 out 5 planes would suffice to achieve the precision required in the momentum measurements based on curvature.

\section{The ND Readout System}

The FD readout system, as described in Section 3.2.2, was not designed to cope with the beam rates that the ND will be exposed to during a spill. Therefore a 


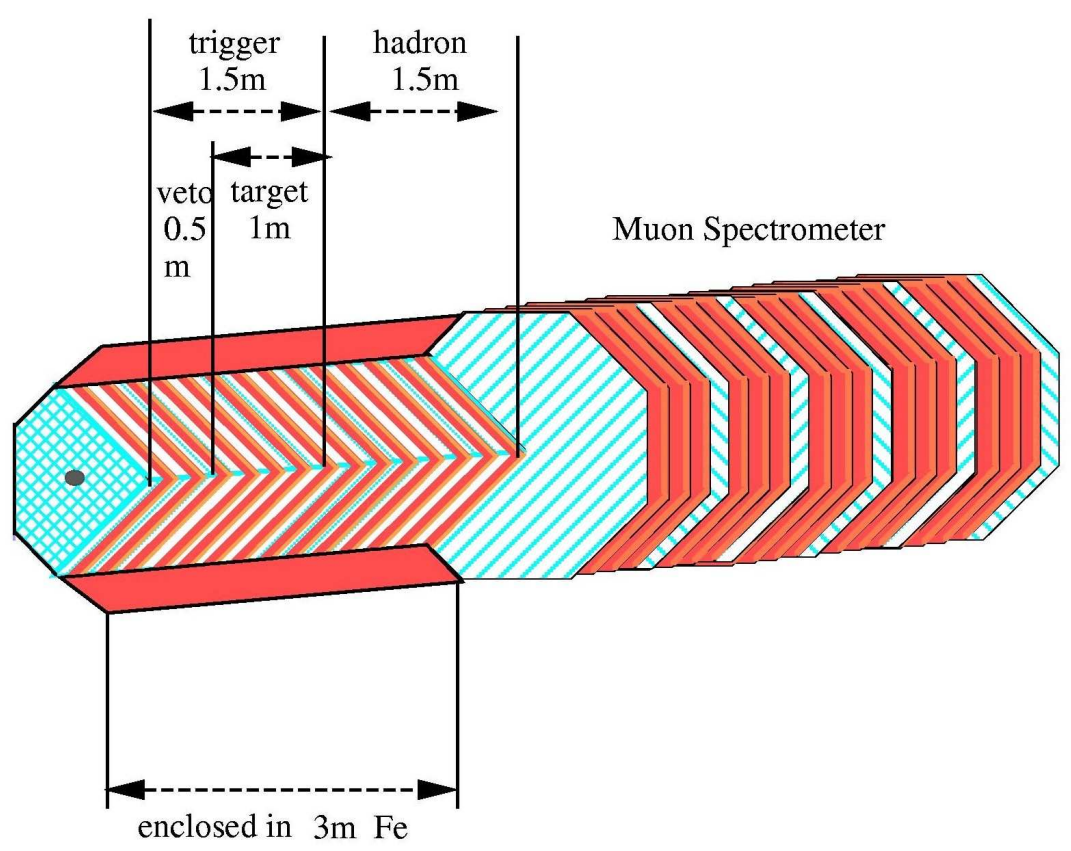

Figure 3.15: Overall Structure of MINOS Near Detector.

different readout system had to be chosen for the ND. A robust FEE at high rates was designed by Argonne National Laboratory based on the QIE-chip developed at FNAL [78]. The ND FEE also has been designed to be dead-timeless during the spills, i.e. about $10 \mu$ s worth of data. We shall call the ND electronics collectively QIE-FEE.

The 64-pixel Hamamatsu R5900-00-M64 PMT (called M64 within MINOS) was a more effective cost/performance choice for the ND. Each strip is coupled to only one PMT pixel, since the optical sum employed at the FD, would not be possible due to the expected high rates/occupancy per spill. The anode of each pixel is read out by one FEE channel in all regions but the spectrometer region. In the spectrometer region, four anodes - four strips - are electrically coupled into one FEE channel. The electrical sum relies on the low occupancy of muons tracks across the spectrometer region per spill. Offline reconstruction is used to achieve the corresponding reconstruction over the spectrometer region.

Ideally, we would have liked to have made the ND and FD identical for neutrino oscillation physics in order to reduce systematic errors on the inter-detector comparisons. However, endowing the FD with the ND readout system would have not been a financially affordable alternative. Therefore, MINOS engaged to study 
in detail the impact of the relative performance of both readout systems into its physics capabilities. The results of such studies are the main topic of this thesis: Chapter 7.

The ND electronics digitises incoming PMT current pulses using capacitive integrating over an pseudo-16-bit dynamic range. The integrated-sampling rate is 53MHz. Therefore, the total charge of an incoming PMT pulse is divided into $\sim 19$ ns integrated "snap-shots" called buckets. The integration over the buckets is carried out offline. During the digitisation process the electronics is dead-timeless: the FEE digitises and stores locally data until a readout command its received or the buffer is full. The data buffer is local FIFO for each channel, whose depth is equivalent to $\sim 20 \mu \mathrm{s}$ ' worth of data, capable of storing a full spill comfortably. When the FEE is readout, the electronics becomes dead to any new triggers. Such a subsequent dead-time, however, depends on how many buckets are read out from the FIFO - even if they were sparsified. As a rule of thumb, the dead-time is $\sim 600$ ns per bucket (or $32 \times$ the buckets readout). During cosmic runs, 8 buckets are read out, therefore the dead-time is about $4.8 \mu \mathrm{s}$.

The effective dynamic range is due to the combined action of an 8-bit-ADC and the QIE-chip. The task of the QIE-chip is to split the incoming signal into eight binary-weighted ranges (identified by the 3-bit RANGE word) and then integrate the resulting fractional currents on a corresponding capacitors per range. Then, a sample-and-hold circuit per range stores the integrated charge of each capacitor. The QIE then selects the first non-saturated range and passes it to the 8-bits for digitisation. Therefore, the QIE-chip $\oplus 8$-bit-ADC is better regarded like $8+8$-bits $\mathrm{ADC}$ covering the same range as a 16-bit ADC with an approximately constant fractional error on the digitisation across the dynamic range.

The described behaviour of the circuit does not provide a linear response by itself. Each different range has different offset and gain according to the range and channel in question, as shown in Figure 3.16 (left). Calibration of the QIEFEE response is therefore needed to linearise its response. The calibration relies on the injection of charge linearly across the dynamic range by an in-built DC charge injector. The calibration data is then analysed online, by the VME computer, for the results to be written out in a Look-Up-Tables (LUT) per MASTER. The LUT stores the relation between the calibrated linear value for every single QIE-chip $\oplus 8$-bit-ADC data word for each electronics channel associated to that MASTER. Therefore, once the LUT is populated, the QIE is said to be calibrated and the output of the electronics is linearised online by each MASTER upon ref- 
erence to its LUT.
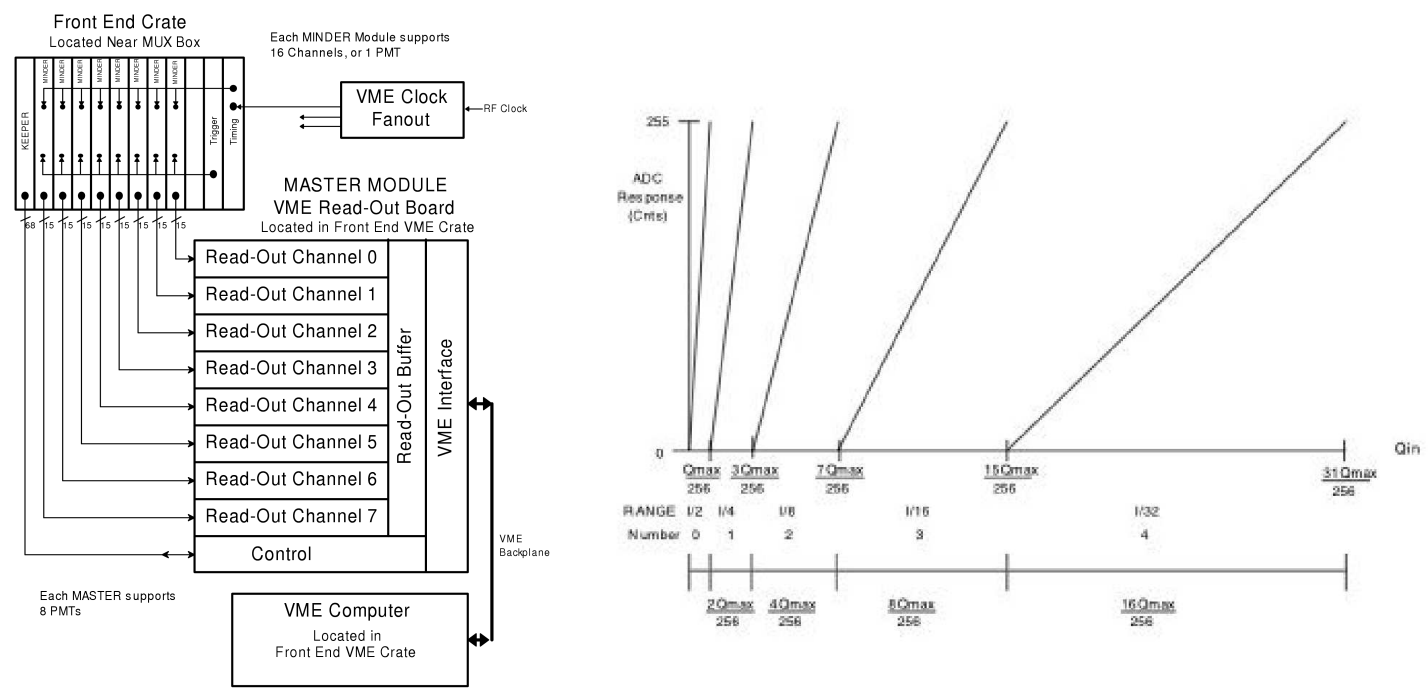

Figure 3.16: QIE Electronics: Logic Diagram and Calibrated Response. On the left, a QIE electronics diagram is shown where all components and their logical dependencies are suggested. While on the right, the expected response of the QIE electronics is shown as it would look after calibration to render the overall response of the QIE electronics linear.

The different components of the QIE-FEE [78] are: MENU, MINDER, KEEPER and MASTER, as shown in Figure 3.16 (right). The MENU is where the ADC and QIE chips are located together with a FIFO with enough memory to store hits for the full spill worth of data. One MENU reads out the anode of one M64 pixel. The MINDER is the mother-board to which 16 MENUs - 1/4 of an M64 - are connected. The time-stamping and data funnelling control for the MENUs are the main tasks of the MINDER. Eight MINDERs together with a KEEPER are seated in each front-end crate located very close to the PMTs and the detector. All components of each front-end crate are read out and controlled by one MASTER, remotely located in a VME crate. The role of the KEEPER is to serve the MASTER to control the FEE crates, i.e. the MINDERs. Each KEEPER also holds the charge injection calibration circuitry and the FEE triggering capabilities. The MASTER does the sparsification and provides the FEE crates with VME interface to the rest of the DAQ.

The sparsification in the QIE-FEE is performed on each bucket, which differs from the method applied on the VA-FEE. The difference in performance of both readout systems arising from their different sparsification methods will be thoroughly studied in Chapter 7. 


\subsection{Physics Capabilities of MINOS}

MINOS relies on a very efficient event-by-event neutrino interaction identification: Charge Current (CC) and Neutral Current (NC) interactions. Equations 3.2 and 3.3 represent respectively the types of transitions caused by $\mathrm{CC}$ and $\mathrm{NC}$ neutrino interactions with the detector target $^{6}$.

$$
\begin{gathered}
\nu_{\lambda}+N \stackrel{W^{ \pm}}{\longrightarrow} \lambda+X: C C \text { Interaction } \\
\nu_{x}+N \stackrel{Z^{\circ}}{\longrightarrow} \nu_{x}+X: N C \text { Interaction }
\end{gathered}
$$

According to the momentum transferred $\left(Q^{2}\right)$, the interactions can be classified (see Figure 3.18) by the kinematics into [79]:

Quasi-Elastic: $N$ and $X$ represent nucleons: $Q^{2}<\sim 1 \mathrm{GeV}^{2}$.

Resonant: $N$ is a nucleon and $X$ represents a resonant excitation of the proton, say a $\Delta^{+}(1.4 \mathrm{GeV}): \sim 1 \mathrm{GeV}^{2}<Q^{2}<\sim 1.9 \mathrm{GeV}^{2}$.

Deep Inelastic Scattering(DIS): $N$ is a quark and $X$ represents outgoing mesons: $Q^{2}>\sim 1.9 \mathrm{GeV}^{2}$.

$\mathrm{CC}$ interactions are identified by tagging the outgoing lepton $(\lambda: e, \mu, \tau)$. Measuring the polarity of the lepton provides discrimination between neutrinos and anti-neutrinos interactions. In MINOS, the recognition of the lepton polarity is only possible for muons. In addition to the outgoing lepton, CC events also present some hadronic activity. The NC interactions are characterised by transferring energy only to the hadronic system. In NC events, the outgoing neutrino $\left(\nu_{x}\right)$ cannot be feasibly detected or identified. Therefore, NC event rates are flavour independent making the NC events neutrino oscillation independent - unless there is some degree of admixture to sterile neutrinos, as discussed below.

Clearly, CC interactions provide the largest amount of information and are neutrino oscillation sensitive. The success of the CC ID resides in the efficiency of the detector to identify the produced leptons. MINOS was optimised for $\nu_{\mu} \mathrm{CC}$ detection, therefore most emphasis was given to the ID of muons. Electrons can be efficiently detected, however, distinguishing electrons from $\pi^{\circ} \mathrm{s}$ - present in $\mathrm{NC}$

\footnotetext{
${ }^{6}$ Since the cross-section scales with the mass of the target particle, interaction of neutrinos with nuclei constituents $(>A)$ should dominate over interactions with atomic electrons $(Z)$ of the target.
} 


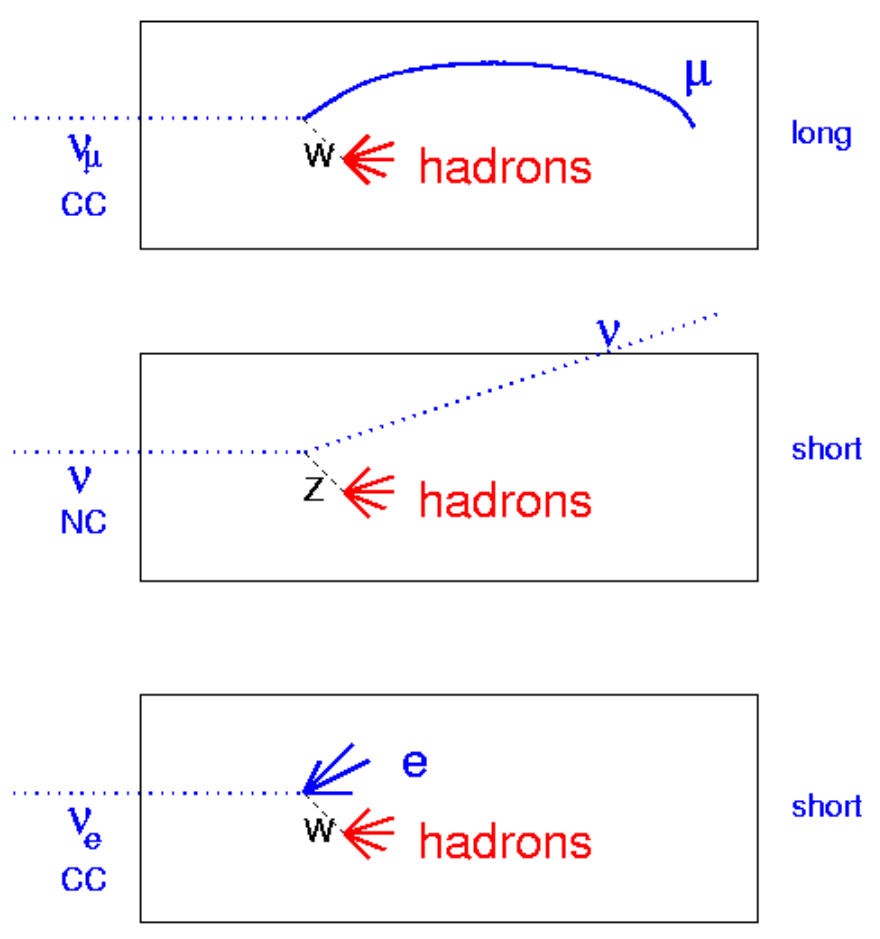

Figure 3.17: Types of Event Topology Diagram. Note that the propagation of the $\mathrm{W} / \mathrm{Z}$ bosons cannot be appreciated, this is just an exagaration of the diagram for the sake of the illustration of the event topologies and particle contents involved.

events - will be very hard in MINOS. $\nu_{\tau}$ CC interactions are not expected to be identified on an event-by-event basis.

To first order, the $\mathrm{CC} / \mathrm{NC}$ interaction identification is based on event shape topology. Energy deposition profiles will also be used for shower events, for example for the discrimination between $\nu_{e} \mathrm{CC}$ and $\mathrm{NC}$ events. Event topology discrimination will be a challenge for MINOS when it comes to separate $\nu_{\mu} \mathrm{CC}$ and $\mathrm{NC}$ events at visible energies below $1.5 \mathrm{GeV}$ - range particularly relevant for the $\mathrm{NC}$ event analysis.

In this chapter we shall subdivide the physics scope of MINOS into two logical sections: the neutrino beam oscillation physics (done with both detectors simultaneously) and those physics programmes that require either the FD or the ND alone.

\subsubsection{Neutrino Oscillation Physics}

As mentioned previously, the main goal of MINOS is to observe the L/E dependence of the survival probability of $\mathrm{CC}$ interacting $\nu_{\mu}$ as they propagate from FNAL 


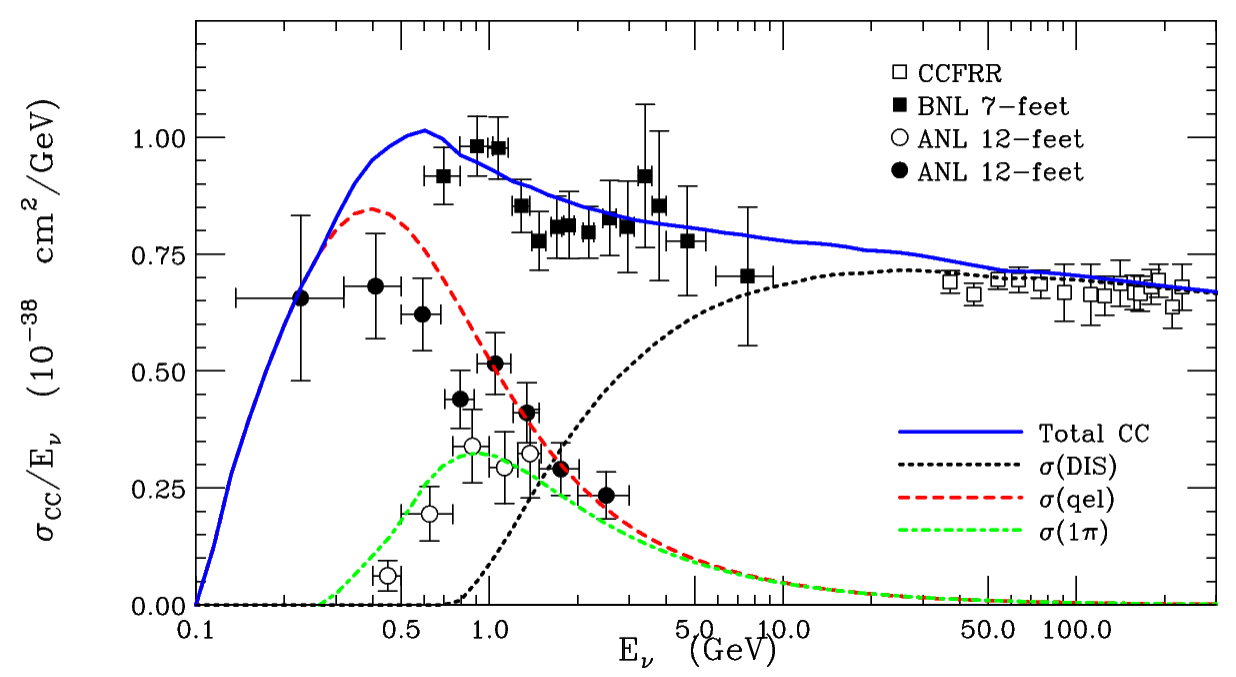

Figure 3.18: $C C \nu N$ Cross-Sections: Data and $M C$ Different interaction regimes have different contributions to the total $\mathrm{CC} \nu \mathrm{N}$ cross-section. The modulation of the different contributions is mainly dominated by threshold effects due to the physics involved.

to Soudan. The L/E pattern provides strong discriminating power for the rejection of alternative models still not fully ruled out such as neutrino decay [39] and de-coherence [40] while confirming that neutrino oscillations are the responsible mechanism. The same pattern provides the framework for high precision measurements of the neutrino oscillation parameters attainable by MINOS: $\Delta m_{23}^{2}$ and $\sin ^{2}\left(2 \theta_{23}\right)$. MINOS has additional sensitivity on the admixture of sterile neutrinos and marginally on the value of $\theta_{13}$. In this section, the four main neutrino oscillation channels that MINOS can address will be briefly described.

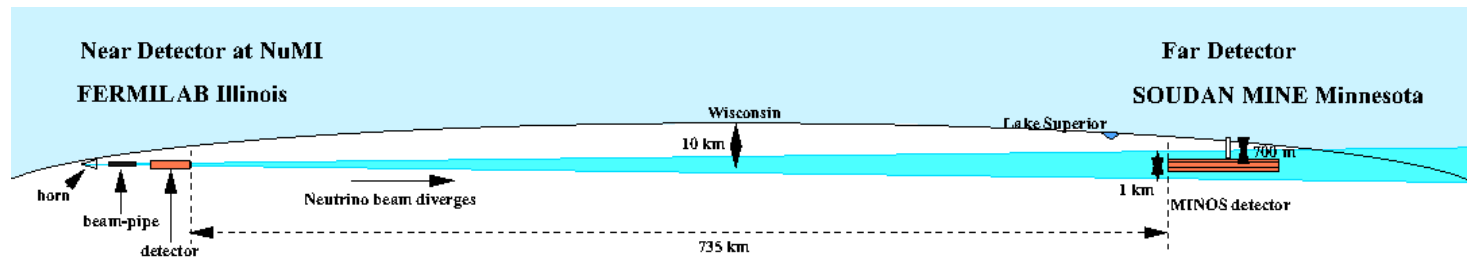

Figure 3.19: MINOS Experiment on the Earth.

The $\nu_{\mu}$ CC Disappearance is the principal physics channel in MINOS: a precision up to about $4 \%$ is envisaged on the measurement of $\Delta m_{23}^{2}$. The quoted level of precision is expected to be limited by systematic uncertainties. The MINOS ND and FD detectors will measure the neutrino beam spectra and 
rates at different distances from the source in order to map the disappearance of $\mathrm{CC} \nu_{\mu} \mathrm{s}$. Note that neutrino oscillations intrinsically change the flux of neutrinos of one type along its propagation which, consequently, induces a change in the energy spectrum (flux $\times$ cross-section). A precise measurement of the neutrino spectra at both sites is required, for which calorimetry technology and accurate calibration systems have been designed to precisely measure the energy of the interacting neutrinos.
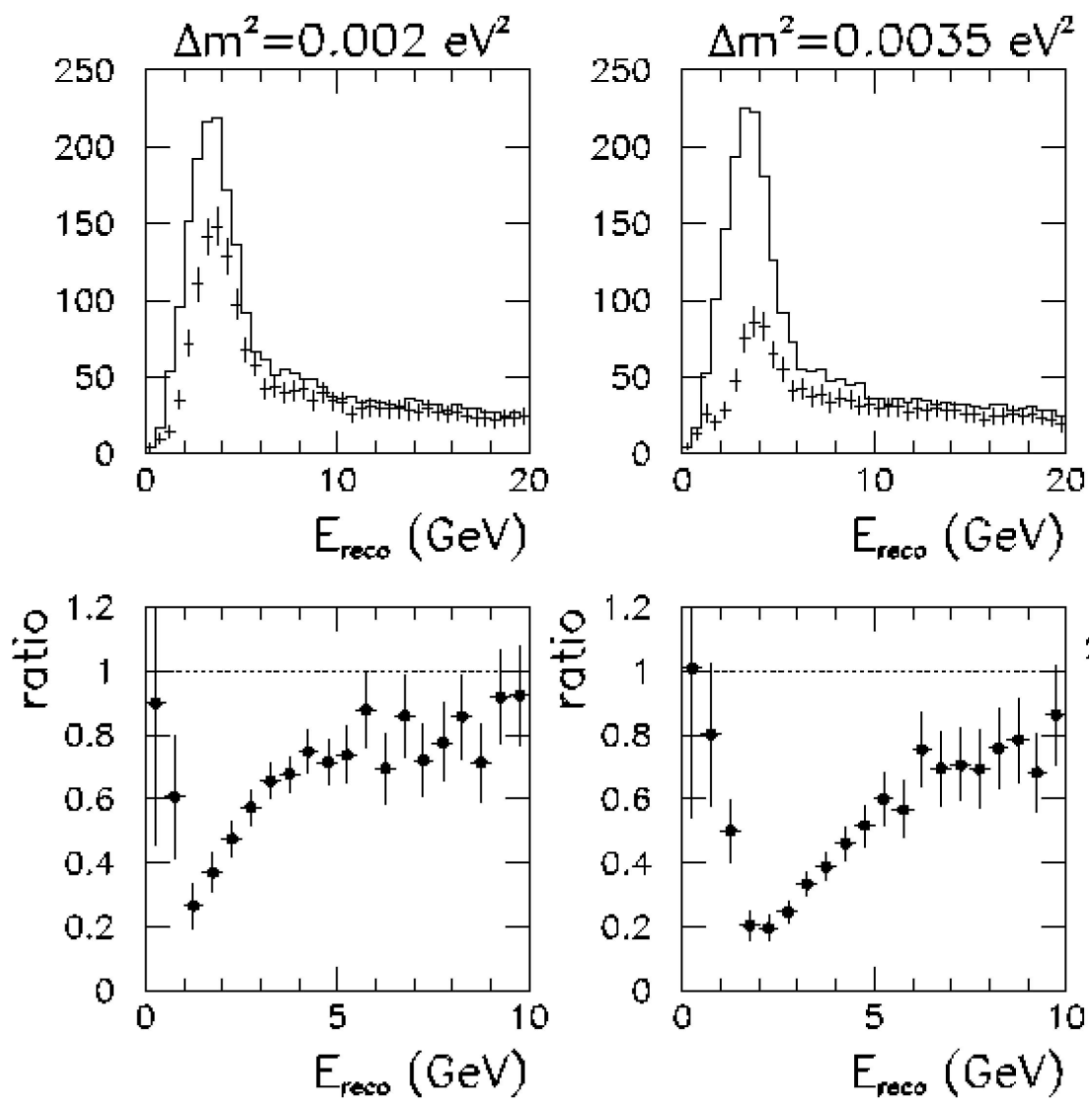

Figure 3.20: $\nu_{\mu} C C$ Spectral Distortion. At the top, simulated oscillated and unoscillated spectra are shown for different $\Delta m_{23}^{2}$ s. Note that, based on the measured rates (integral over spectra), disappearance is already proved. However, the ratio of the two spectra, shown at bottom, provides far more information as the rate of disappearance of neutrinos strongly depends on the energy of neutrinos in question. These plots have been produced under an assumed exposure to $5 \times 10^{20}$ pot with the "Low Energy" NuMI beam configuration at nominal luminosity and $\sin ^{2}(2 \theta)=0.9$.

Neutrinos detected at the ND are unaffected by neutrino oscillations because of the proximity to the source. Therefore, the $\nu_{\mu} \mathrm{CC}$ spectrum measured at the ND provides a handle on both the spectral shape and the normalisation 
of the non-oscillated NuMI neutrinos. Obtaining the neutrino spectrum that would be observed at the FD in the absence of neutrino oscillations ("unoscillated" spectrum) from the information gathered at the ND is far from trivial. The beam MC has to provide a flux correction-function which can be applied to the spectrum measured at the ND in order to predict the FD spectrum. This will need the input from MIPP. The flux correction-function should quantify the fraction of the NuMI neutrinos (and their energies) that would be likely to interact in both detectors relative to those interacting in the ND. Further complications arise from the poor knowledge of the neutrino cross-sections. The cross-sections uncertainties can be as high as $\sim 25 \%$ [80], as illustrated in Figure 3.18. The impact of the cross-section uncertainties to the shape of the measured visible energy makes the de-convolution of the cross-section from spectra measurements difficult. The spectrum measured at the FD and the inferred un-oscillated spectrum from the ND, corrected with the beam MC, are shown in Figure 3.20 (top).

Most of the power for the extraction of oscillation parameters comes from the spectral distortion, which is illustrated best through the ratio of the oscillated to the un-oscillated spectrum. The ratio emphasises the spectral distortion in the form of the dip shown in Figure 3.20 (bottom) caused by neutrino oscillations. The ratio has the additional advantage that some (but not all) unknown systematic effects that could be affecting both spectra in an equal fashion can cancel out.

The ratio is actually nothing more than the $\nu_{\mu}$ CC survival probability dependence on energy (E), for which the distance (L) is fixed. Note that the dip has a "fall" (at low energies) and "rise" (at high energies). Alternative mechanisms causing flavour transitions do not exhibit this characteristic shape. Therefore, the dip, like the one shown previously, is the unambiguous proof and manifestation of the mass-eigenstate composition of each weakeigenstate neutrino and the consequent inference that neutrinos are massive and non-degenerate. The shape of the ratio provides rejection power against alternative models: $>5 \sigma$ by the end of data running.

The accurate characterisation of the dip directly gives information about the neutrino oscillation parameters. The position of the dip corresponds to the energy at which the oscillation probability is largest, where $\sin \left(1.27 \Delta m_{23}^{2} L / E\right) \sim$ 1 and so 

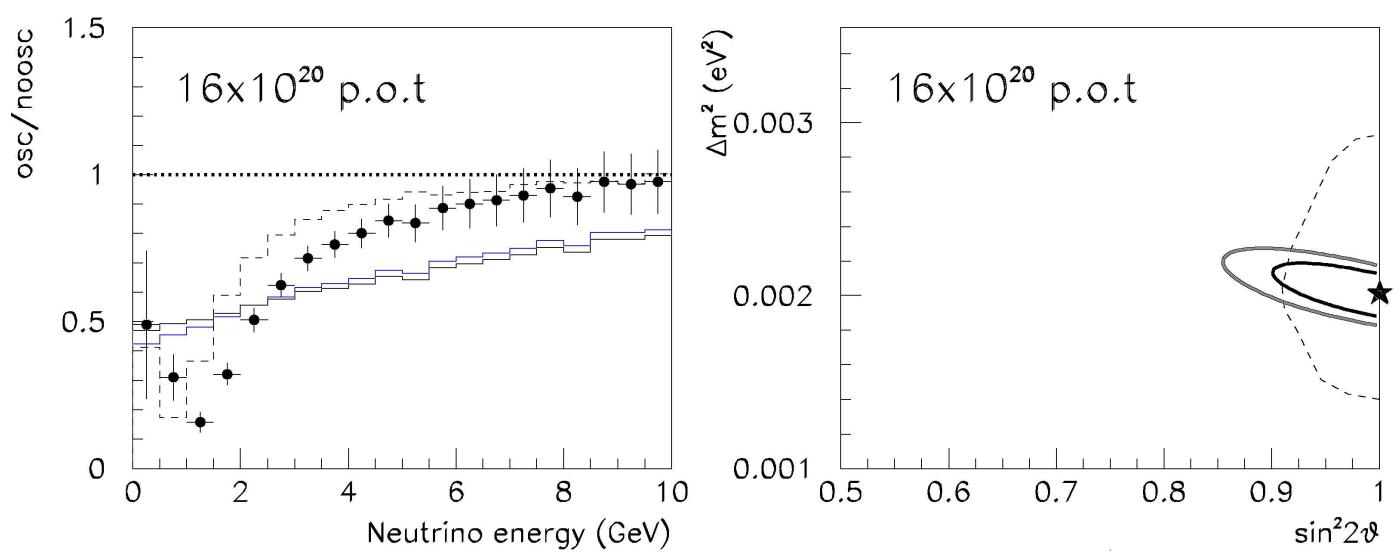

Figure 3.21: $\nu_{\mu}$ CC Disappearance: Neutrino Oscillation Parameter Fit Plot. Note that the $d$ ip is unique to neutrino oscillation and de-coherence (grey) and (blue) decay can be strongly disfavoured by MINOS (left). Our current knowledge on $\Delta m_{23}^{2}$ is most precisely provided by the SK and $\mathrm{K} 2 \mathrm{~K}$ experiments. Their results are best quoted as an range: $\Delta m_{23}^{2} \in[1.5,3.4] \mathrm{eV}^{2} 90 \% \mathrm{CL}[35]$. The results are also shown in parameter space (right), where the bold-lines correspond to MINOS 90\% and 99\% CL limits. The SK limit is depicted by a dashed line. These plots have been produced assuming a 5 year exposure of MINOS to $16 \times 10^{20}$ pot and $\Delta m_{23}^{2}=0.002$.

- $\sin ^{2}\left(2 \theta_{23}\right)$ is the minimum probability measured (maximal depth in yaxis), since, trivially, $P\left(\nu_{\mu} \rightarrow \nu_{\mu}\right)=1-\sin ^{2}\left(2 \theta_{23}\right)$.

and

- $\Delta m_{23}^{2}$ is proportional to the energy (x-axis), for which the probability is minimal (or the dip is maximal): $1.27 \Delta m_{23}^{2} L / E=\pi / 2$ (at first maxima).

Therefore, measuring the dip very precisely grants a highly constrained fit, which yields an precise estimation of the oscillation parameters. This is reflected in the shrinking of the allowed region in parameter space relative to SK results, as shown in Figure 3.21. The measurement of $\Delta m_{23}^{2}$ is expected to be dominated by systematic uncertainties, while the uncertainty on $\sin ^{2}\left(2 \theta_{23}\right)$ will be limited by the statistical sensitivity. With higher than nominal statistics MINOS would be able to explore whether or not $\sin ^{2}\left(2 \theta_{23}\right)$ is maximal. A non-maximal $\sin ^{2}\left(2 \theta_{23}\right)$ would help theorist to pin down the structure of the PMNS matrix and may even help to shed light on the new physics behind such a matrix symmetry. 
The $\nu_{e}$ CC Appearance Current knowledge implies that the atmospheric neutrinos phenomenology is dominated by $\nu_{\mu} \rightarrow \nu_{\tau}$ oscillations. The yet unobserved oscillations $\nu_{\mu} \rightarrow \nu_{e}$ are expected to occur via sub-dominant processes within the $3 \nu$ oscillation framework for long baseline experiments. The process is modulated by $\sin ^{2}\left(2 \theta_{13}\right)$. A handful of oscillated $\nu_{e}$ could appear in MINOS depending on the value of $\sin ^{2}\left(2 \theta_{13}\right)$, which is known to be $<0.11$ [81]. The MINOS sensitivity to the value of $\theta_{13}$ beyond the Chooz limit [36] is shown in Figure 3.22.

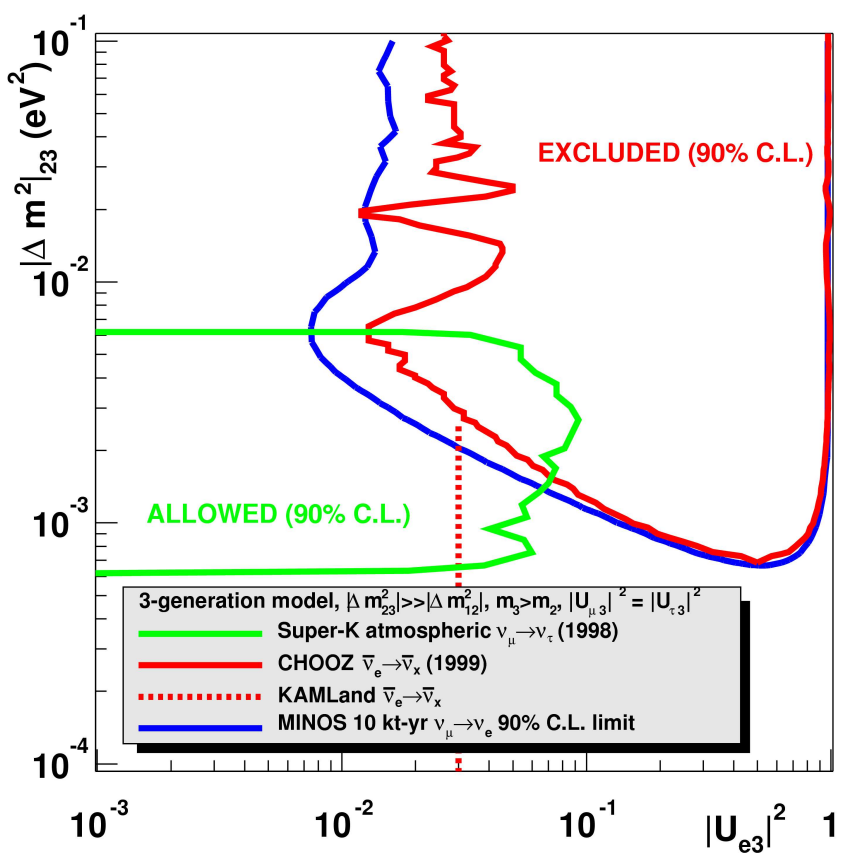

Figure 3.22: $\nu_{e} C C$ Appearance Oscillation Parameter Sensitivity Plot. This plot shows the expected sensitive of MINOS to $\theta_{13}$ (blue), where the regions already explored by Chooz (red) and SK (green) are also indicated.

However, the degree of $\nu_{e} \mathrm{CC}$ excess has to be statistically significant beyond a background, which has to be well understood. The major contributors to the background are an irreducible contribution of beam $\nu_{e}$ contamination for kaon decay and NC events with a leading $\pi^{o}$ events being produced. $\pi^{o}$ are believed to be generally indistinguishable from $\nu_{e}$ in the MINOS, due to the coarse graining. In addition, the expected number of the background events is not very certain, since the cross-sections for their production are not well known [80]. The contents of the beam will need to be very well understood and quantified to accurately account for expected beam $\nu_{e}$, a task for which 
the ND will be crucial. Furthermore, some $\nu_{\mu} \mathrm{CC} / \mathrm{NC}$ events at very low energies can mimic similar energy profiles to electromagnetic showers. These events can also be mistakenly selected and will contaminate the sample.

Measuring $\theta_{13}$ in a long baseline experiment is not trivial. A possible measurement is linked to the intrinsic uncertainties of the physics manifested within a $3 \nu$ oscillation framework due to the degeneracies and the correlations. For example, the complete ignorance about the value of the $\mathrm{CP}$-violating phase accounts as part of those uncertainties. Furthermore, the presence of matter effects can no longer be ignored since it affects the oscillation probability for of $\nu_{e}$ [82]. However, if $\theta_{13}$ was first measured by MINOS, a forthcoming reactor neutrino experiment, like DoubleChooz [51] [50] would also measure $\theta_{13}$ helping to resolve some of the uncertainties and providing MINOS with a slighter deeper scope into the problem.

NC Disappearance NC event rates are, in principle, immune to neutrino oscillations, since they are flavour independent and there is no lepton mass production threshold. However, the disappearance of $\mathrm{NC}$ events would imply that, as well as the 3 active neutrinos (proved to couple to the $\mathrm{Z}$ resonance [16]), there must also be at least a sterile neutrino. MINOS is believed to have a competitive sensitivity to that of SK on the value of $f_{\text {sterile }}$ (admixture fraction of sterile neutrinos) as shown in Figure 3.23. These studies are expected to be limited by the statistics available at MINOS as well as by the value of $\Delta m^{2}$.

The high energy NC events can be safely used for flux measurements across the MINOS detectors, as an useful normalisation check for the experiment. Further details on the sensitivity of MINOS using NC events can be found in [83].

\subsubsection{Non-Oscillation Physics}

\section{Physics at the Near Detector}

The main advantage of the ND is the very high rates of beam neutrinos available. Therefore, neutrino matter interaction physics is the main focus of the physics programs at the ND. A rich program of measurements is envisaged:

- $\nu$-N cross-sections 


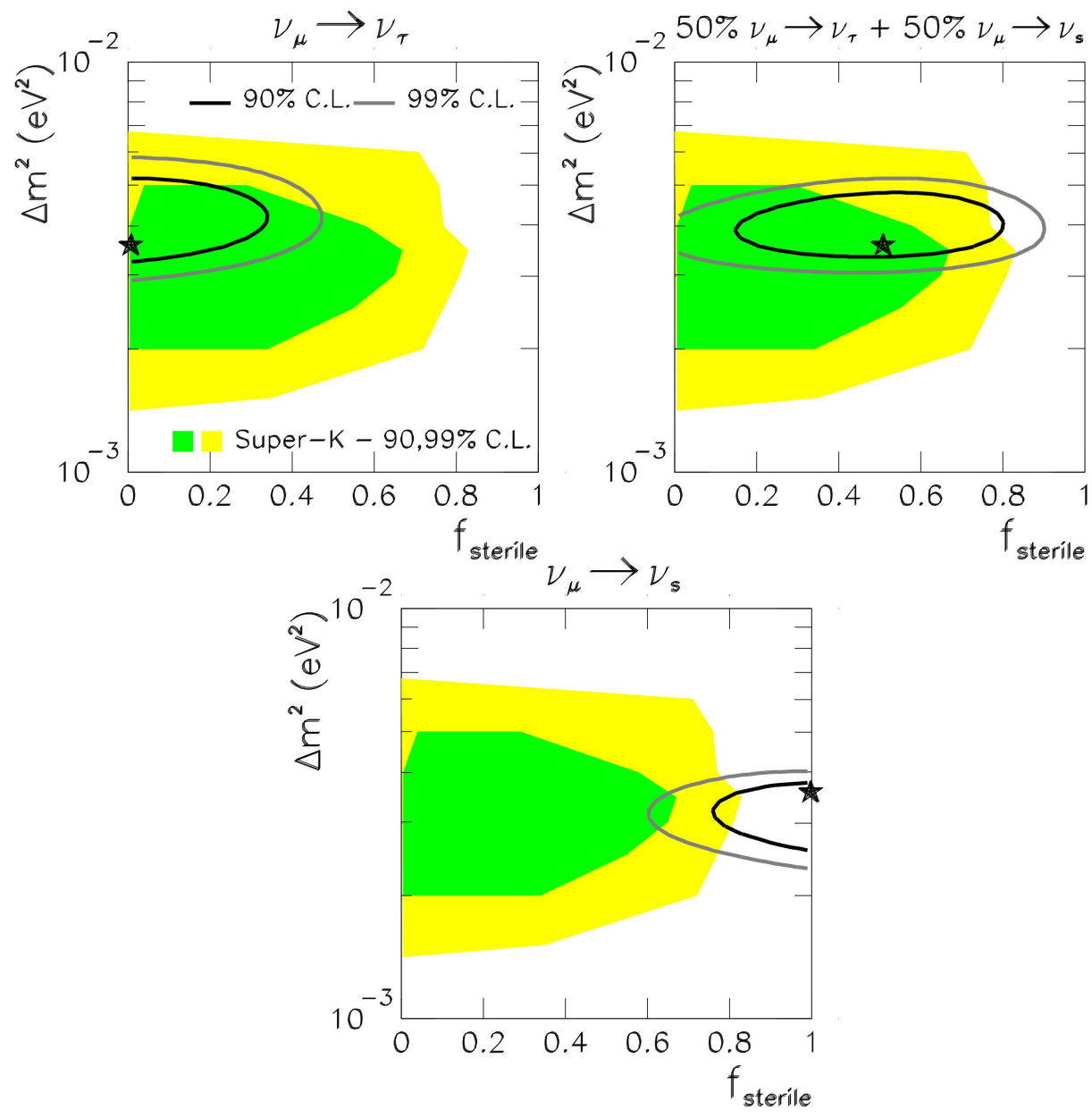

Figure 3.23: Sterile Neutrino Search Oscillation Parameter Extraction Plot. The plots have been produced assuming the nominal "Low Energy" beam with an exposure of $5 \times 10^{20}$ pot and $\Delta m_{23}^{2}=0.0035$.

- study of nuclear effects affecting the neutrino-matter interaction

- parton distribution functions parametrisation

within the kinematic regime available $\log _{10}\left(Q^{2}\right) \in[-2,1.5]$ and $\log _{10}(x) \in[-1.5,0.5]$.

As stressed above, the experimental community is struggling due to the generally poor knowledge of neutrino-matter interactions over certain energy regimes [84]. The NuMI beam events at the ND site provide an ideal opportunity to measure some of the unknowns.

Furthermore, the MINER $\nu \mathrm{A}$ experiment [85] may be built and located in the cavity of the ND. The MINER $\nu \mathrm{A}$ experiments relies on a finer grained detector 
to significantly improve the $\pi^{o} / e$ separation for cross-section measurements. This experiment may eventually help MINOS to better understand the contribution of $\nu_{e}$ in the NuMI beam.

\section{Physics at the Far Detector}

The physics capability of the FD relies mainly on its magnetic field, large fiducial volume and low cosmic background. For some purposes, the veto-shield is also important for the FD physics programme. Atmospheric neutrinos and cosmic ray physics are the main objectives of the FD physics programme. The magnetic field provides charge separation and momentum measurements, making it unique for certain physics channels [86] [87]. However, the statistics accumulated in the FD will never be comparable with that of SK: $\sim 10 \times$ smaller per year.

Cosmic Ray Physics The unprecedented ability to measure the sign and momenta of cosmic muons can be exploited. Mapping the positive/negative muon ratio versus energy of incoming muons can be used for tuning cosmic ray $\mathrm{MC}$ simulations to enhance their accuracy. It may also be possible to provide input to the MC hadronic modelling and models describing the sign composition of primary cosmic rays.

Atmospheric Neutrinos: MINOS can for the first time measure the atmospheric up/down-neutrino/antineutrino flux with energy. Besides being an interesting opportunity to test the oscillation probability of atmospheric neutrinos and anti-neutrinos, a deviation from expectations may also show up. This capability may also become an important benchmark for MC simulation packages available in the community.

Furthermore, MINOS has some sensitivity to the oscillation of atmospheric neutrinos by mapping the disappearance with respect to the azimuthal angle, in the same way as SK [29], Soudan2 [88] and MACRO [89] for $\nu / \bar{\nu}$ separately (when possible), which could test CPT conservation. The MINOS sensitivity is expected to be comparable to that of Soudan2 and MACRO.

Magnetic Monopole: Another interesting channel that becomes available due to the large magnetised volume of MINOS is the search for magnetic monopoles. A magnetic monopole, unlike electrical charged particles, will exhibit a unique pattern in MINOS: they will move along the magnetic field lines. Therefore, if a monopole strikes the FD, it is expected to orbit around the current coil. The 
motion will spiral down to the centre of the detector as the monopole loses energy through ionisation. The sensitivity of MINOS to magnetic monopoles as compared to previous especialised experiments in the matter such as MACRO has not been studied. 


\section{The Calibration Detector}

The Calibration Detector (CalDet) is the third of the MINOS detectors. The CalDet was envisaged to accomplish some of the steps in the MINOS calibration chain that no other MINOS detector could. The goal was to set up the CalDet to be exposed to dedicated calibration beams, which were used to characterise the responses of a MINOS-like detector to the single particles. The particles studied were those to be encountered in other MINOS detectors as produced in neutrino interactions ${ }^{1}: e, \mu, \pi$ and $p$. Therefore, CalDet was designed to be a "portable" detector and was located at the CERN test-beam complex.

One of the major contributions that CalDet provides is the link to the absolute energy scale for MINOS. Further information from the measurements taken at CalDet will be used in MINOS in multiple ways, some of which are the studies shown in this thesis.

\subsection{Objectives of CalDet}

The physics programme of the CalDet is very important to MINOS. A breakdown of the main objectives of the CalDet are as follows:

- Validate the full MINOS calibration chain, including the definition of the absolute energy scale. Demonstrate that the expected calibration accuracy

\footnotetext{
${ }^{1}$ Note that the multiplicity of single particle in a neutrino interaction in the MINOS energy regime is not always one. CalDet measurements instead will be used to tune the MC simulation description and corrections for higher multiplicity events will come from the MC.
} 
goals can be achieved: absolute calibration and relative calibration can be understood to $\sim 5 \%$ and $\sim 2 \%$ precision levels respectively.

- Demonstrate that, having different readout systems in the Near Detector and Far Detector does not introduce significant systematic errors that can affect the physics capabilities of MINOS. This objective is the main topic of this thesis: Chapter 7.

- Parameterise the responses of the MINOS detectors to known particles at various energies. Measure the detector energy resolution for electromagnetic and hadronic showers. These studies can be found in [74] [75].

- Tune and validate the MINOS simulation packages: physics MC and detector simulation. The major efforts on studying the quality of physics MC for CalDet (and MINOS) can be found in [75] [74]. Studies on the performance of the detector simulation packages to characterise the MINOS detectors can be found in [75] [90] and in Chapter 7.

In addition, CalDet was very important preparation for MINOS. Much of the experience gathered on installing and running the MINOS technology during CalDet proved crucial for commissioning, understanding and optimising the remaining MINOS detectors and subsystems. The full calibration chain was first developed, exercised and validated to a large extent at CalDet.

Throughout the CalDet programme, different configurations were required to meet all the goals. The complete set of configurations are listed in Table 4.1.

\subsection{Description of CalDet}

In the following description of the CalDet, only those aspects that are different from the standard MINOS detector design are highlighted. The full description of the MINOS technology was provided in Section 3.2.1 and the specification and functioning of the different readout systems can be found in Sections 3.2.2 and 3.2.3. In this section, there will be reference only to the configuration adopted for the Near/Far Comparison ${ }^{2}$, which is shown in Figure 4.1, while the CalDet was situated in the beam-line at CERN.

\footnotetext{
${ }^{2}$ Note that so far ND and FD were referred to the actual near and far detectors. From this point onwards, unless otherwise specified, ND and FD means the the readout systems of the ND and FD installed at CalDet.
} 


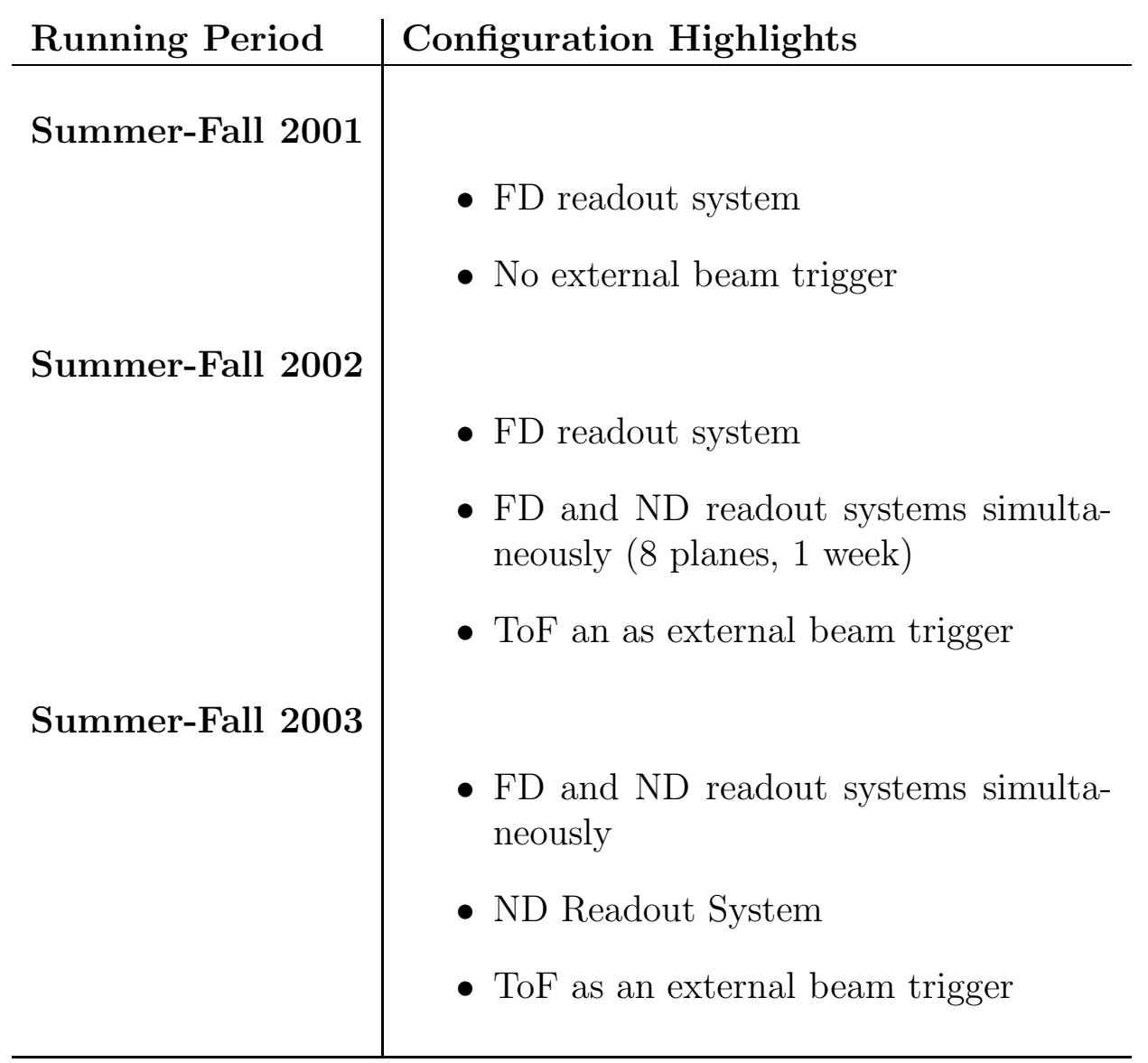

Table 4.1: List of CalDet Configurations.

The CalDet was designed to be a smaller detector (12 tonnes), while still having the same technology principle as the other MINOS detectors. The size was constrained by the mobility needed, since locating the CalDet in different beam lines across the PS complex was a frequent practise during data-taking campaigns. The CalDet planes were square $(1 m \times 1 m)$ with an XY-orientation. An example can be seen in Figure 4.2. The orientation of the strips changes in alternative planes to allow tracking. Each plane was made up of 24 strips. The total length of the detector was about $3.6 \mathrm{~m}$ (60 planes). The overall dimensions of the detector were designed to guarantee containment of hadronic showers over the full energy range available and also to allow beam muons of up to $2 \mathrm{GeV} / \mathrm{c}$ to stop within the detector volume. The amount of material per plane was $2.5 \mathrm{~cm}: 1 \mathrm{~cm}: 6 \mathrm{~cm}$, for the steel, scintillator and air gap, respectively [91]. Note that the amount of steel is slightly smaller than the values for the ND and FD planes $(2.54 \mathrm{~cm}$ or 1 inch) [65].

Both the ND and FD readout systems were installed at CalDet for the Near/Far hybrid running mode. The FD readout system instrumented the "WEST" side 


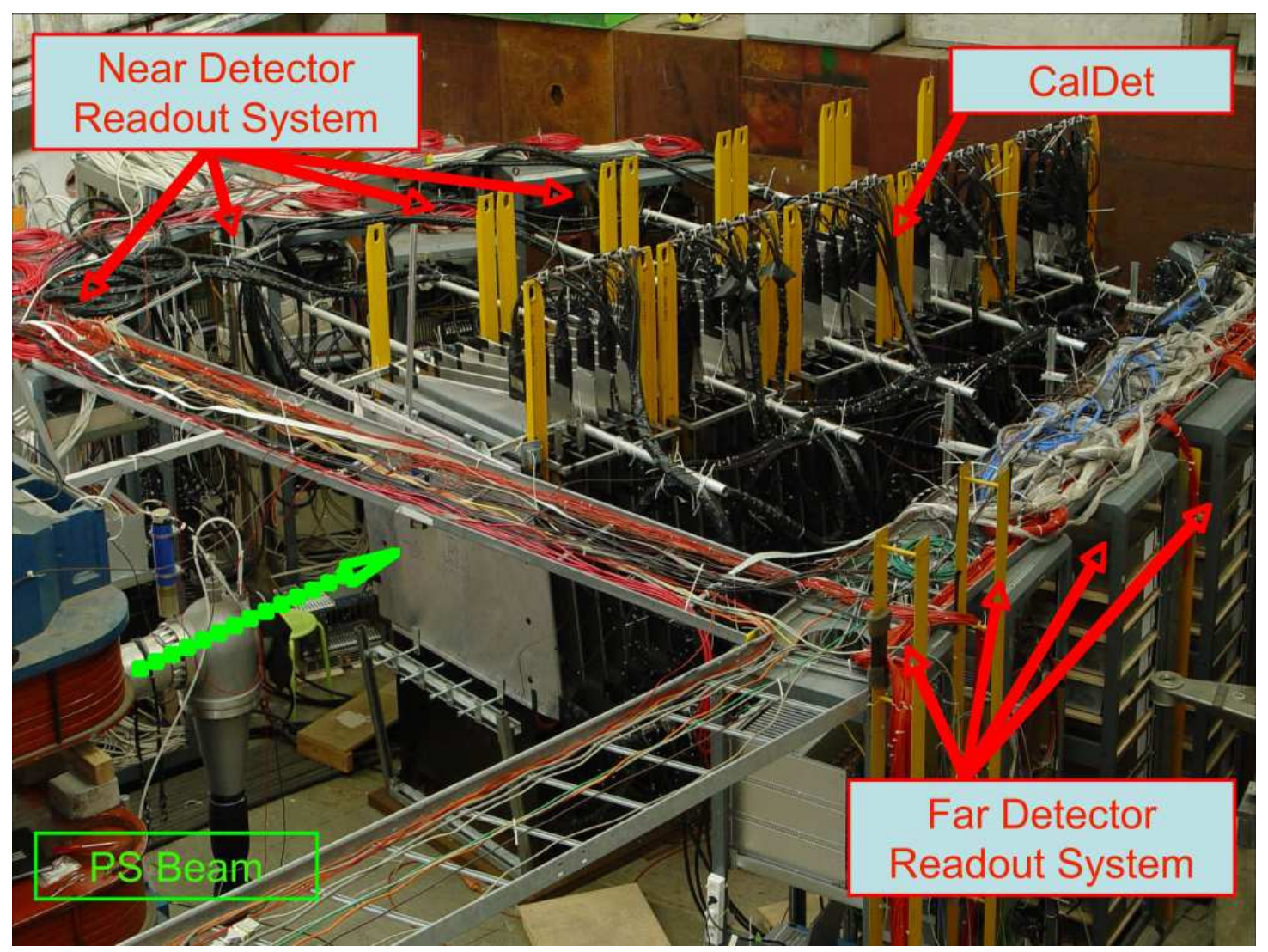

Figure 4.1: The CalDet at Try in Near/Far Comparison Configuration. The CalDet can be seen to have one side instrumented with the ND readout system and the other side by FD readout system. Incoming particles incident onto the CalDet from the PS beam were therefore digitised by both readout systems simultaneously. The aim of this configuration is to enable analysis of potential differences in the events as characterised by either readout systems. (Photo by Phil Adamson).

while the ND readout system instrumented the "EAST" of the CalDet. The lengths and types of readout optical fibres (the ones guiding the light from the strip-ends to the PMTs) were generally different in each "view", as shown in Figure 4.2. Different fibres lengths were used to emulate the lengths found in the other MINOS detectors. Furthermore, using such fibre lengths reduced the overall light levels approaching to the levels expected in the others MINOS detectors. Correcting for the attenuation along the fibre is one of the challenges to be overcome by the calibration chain and was tested with such a setup.

A "view" is the term used to refer to each set of planes sharing a common sideorientation configuration. Therefore the CalDet has four corresponding views: ND-Even/FD-Even (even planes: Horizontal) and ND-Odd/FD-Odd (odd plane: 


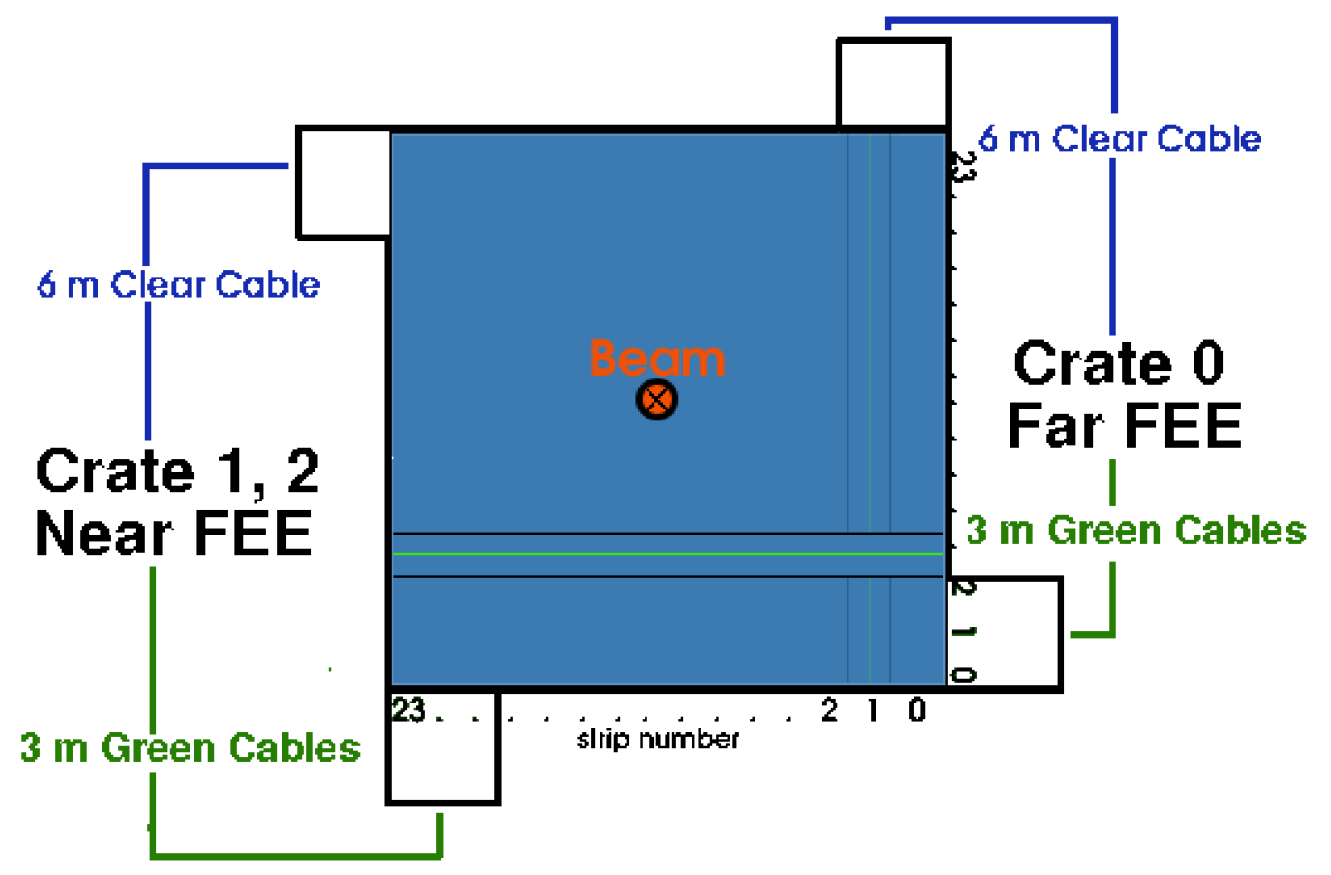

Figure 4.2: The CalDet Planes and the Readout Fibres Configuration. Even planes had $6 \mathrm{~m}$ of clear fibres going to the FD side and $3 \mathrm{~m}$ of green (WLSF) fibres going to ND side. While odd planes, the order was inverted. Plot from [74].

Vertical). Each view has an unique readout-system/readout-fibre combination. Due to the instrumentation differences, each view provides a different perspective of a physical event with some degree of orthogonality. This capability is very useful to understand possible view-wise effects. In fact, much of the forthcoming discussion will extensively exploit the view separation.

Unlike the other two detectors, CalDet was not magnetised. However, studies showed that the magnetic field should have negligible effects on the topology of hadronic and electromagnetic showers [92]. In principle, the performance of the PMTs could deteriorate in a strong magnetic field [93] [94] [95], but measurements showed that if residual fields are kept $<5$ Gauss inside the PMTs isolating boxes, the magnetic field should have negligible effect on the PMTs performance.

The DAQ at CalDet was kept as simple as possible by having relatively little online processing. For example, events were not formed by online algorithms, as in the case of the ND and the FD detectors. Instead, the data files written out by the DAQ were subsequently processed by an offline event-building algorithm, called "OLT" [96]. This algorithm mimics the event formation based on digit clusters (as 
described in Section 3.2.1), which happens online in the other MINOS detectors.

\subsection{The CalDet within the PS Beam Complex at CERN}

CalDet was exposed to test beams at the PS (Proton-Synchrotron) accelerator complex at CERN. The PS test beams are generated from secondary particles arising from the collisions of PS protons onto a selectable target upstream [97]. The contents of the beam are $\mu, e, p$ and $\pi^{ \pm}$. $\mu$ s and es come from the decay of $\pi^{ \pm} \mathrm{S}$ and $\pi^{o} \mathrm{~S}\left(\pi^{o} \rightarrow \gamma \gamma\right)$, respectively created at the target. The relative composition of the beam changes with the selected momenta of the beam and material of target used upstream. Figure 4.3 (right) shows the average relative composition found at CalDet during 2003. The measurements were taken within the most relevant energy range for MINOS (from $0.5 \mathrm{GeV}$ to $8 \mathrm{GeV})^{3}$.
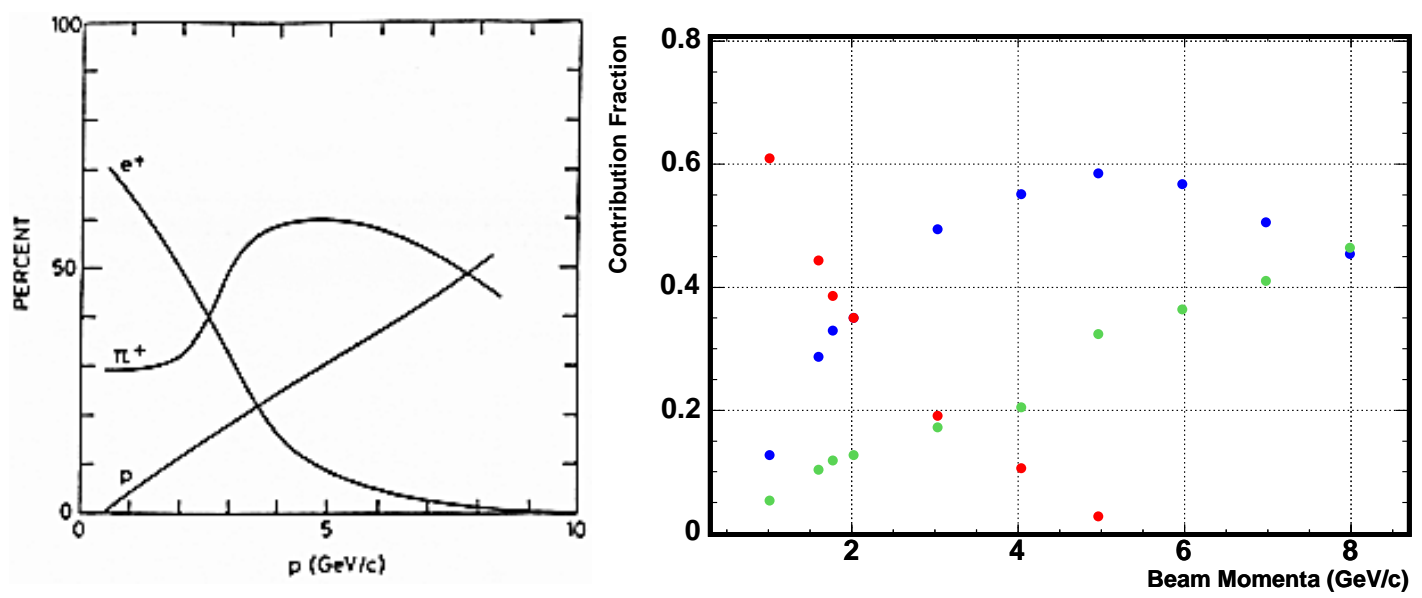

Figure 4.3: Average Relative Particle Composition of the Try Beam. The expected composition of the T7 beam-line is shown on the (left). The measured relative composition of $\mathrm{T} 7$ beam is shown on the (right).

CalDet was exposed to the T7 beam lines, which is shown in Figure 4.4. Long spills $\sim 0.5 \mathrm{~s}$ were available. There were a few super-cycles per PS cycle of $\sim 17 \mathrm{~s}$.

The $\mathrm{T} 7$ beam-line cover reached higher momenta: from $\pm 0.8 \mathrm{GeV} / \mathrm{c}$ up to $\pm 10.0 \mathrm{GeV} / \mathrm{c}$, where the \pm stands for the two possible polarities available in the beam by having the corresponding magnet configuration. The momentum of the

\footnotetext{
${ }^{3}$ Energy range within which the oscillation signature is expected and for which the best calibration would grant the highest possible precision on $\Delta m^{2}$.
} 
beam line was tuned by configuring the current to the magnets, located downstream of the PS target. Configuration tables for the dipole and quadrupoles magnets of the beam-line were produced using the beam simulations [98] by MINOS collaborators.

The T7 beam lines had three collimators. Two of the collimators were used to control the incident rates on the detector, while the third one was used to keep the fractional momentum spread of the beam, i.e., $\Delta p / p$, below $2 \%$.

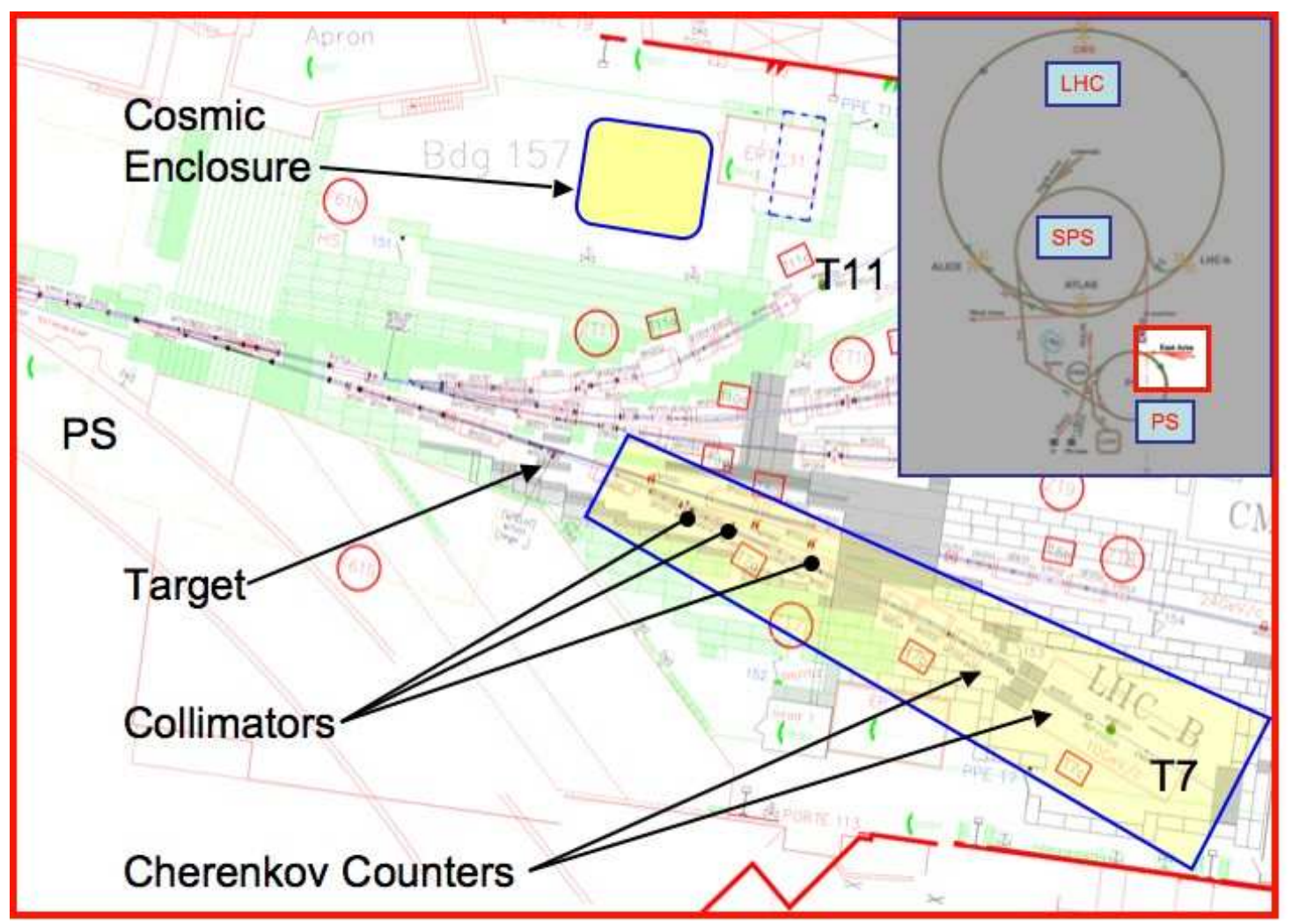

Figure 4.4: Diagram of the Tr Beam Line within the PS Beam Complex. The T7 and T11 beam lines are shown in the diagram of the west-PS-complex building at CERN. The T7 line has been highlighted. The "cosmic enclosure" location is also indicated. The cosmic enclosure was used to store the CalDet when not being exposed to the beam. Plot obtained from.

\subsection{PID and Triggering Instrumentation}

CalDet was endowed with additional instrumentation to yield efficient event-byevent PID (Particle Identification) capability in the beam environment. The PID instrumentation consisted of Cherenkov Detectors (CHD), available as part of the 
beam line: two in T7. A Time of Flight (ToF) system was also developed and installed by the MINOS collaboration. Both sets are illustrated in Figure 4.5.

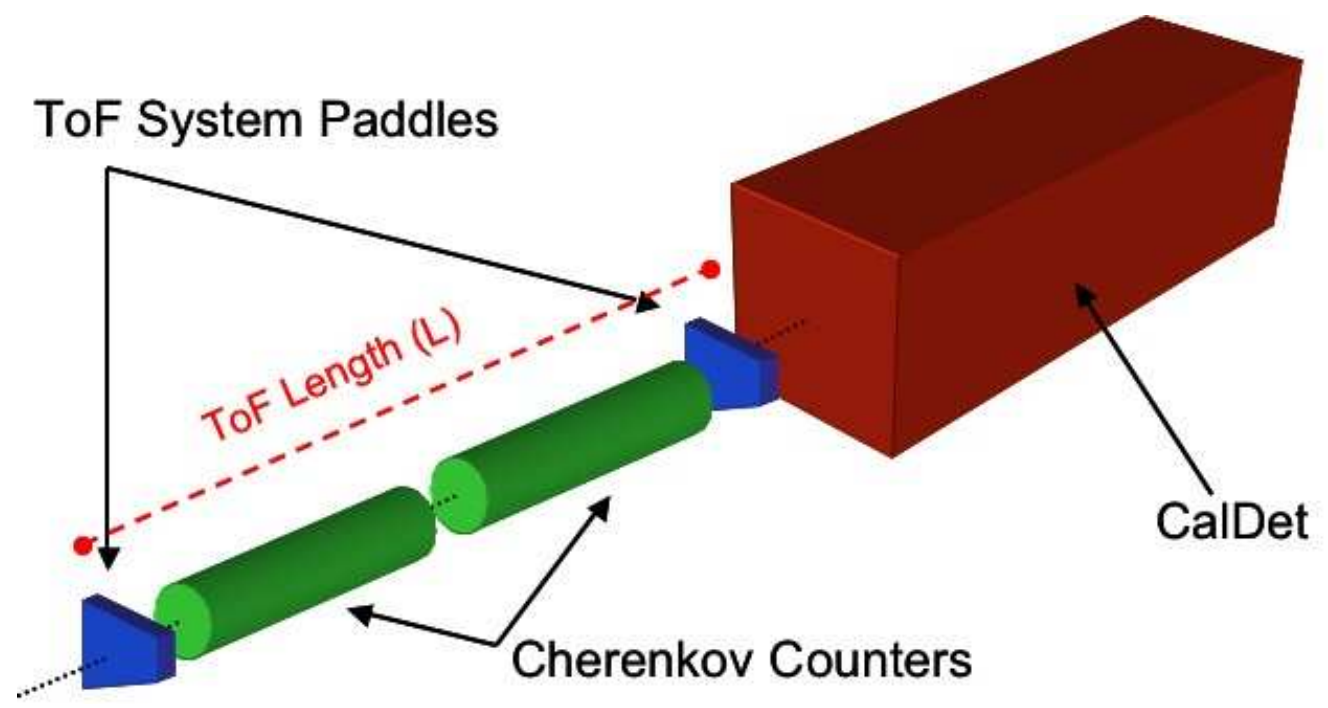

Figure 4.5: Diagram of the CalDet External PID Instrumentation. This diagram illustrates the location of the different PID instruments on the beam-line prior to the CalDet face (not-to-scale). The paddles of the ToF system are coloured blue and the Cherenkov counter detectors are in green.

The output signals from the PMTs of the CHDs and the beam-trigger tag were time-stamped and digitised by VA-FEE, using dedicated modules. This was found to be simplest way to incorporate the PID information into the rest of the CalDet data-stream. The PID information inherits all the features and limitation associated with any VA-FEE digit. Therefore, the DAQ was designed to handle only one set of PID digits per trigger: one digitisation per CHD and one more for the beam-trigger tag. Offline analyses requiring event samples have to be restricted to events with single particle deposition per trigger, for which the PID is unambiguous.

\subsubsection{The Time of Flight System}

The ToF-system was designed to provide proton ID and therefore separation from pions, muons and electrons up to $5.0 \mathrm{GeV} / \mathrm{c}$ momenta. ToF-system discrimination uses the measured travelling time that an ionising particle needs to cover a baseline of know length $(L)$. The travelling time is proportional to the speed of the particle in question. In a momentum selected beam line, faster particles imply lighter particles, therefore one has a handle on the relativistic mass of the particle and, hence, on its ID. At higher energies, particles speeds tend asymptotically to $c$ 
(Ultra-Relativistic limit), making the differences un-resolvable due to the finite time resolution of the experimental system. An example of the time difference distribution of ToF-system is shown in Figure 4.6.

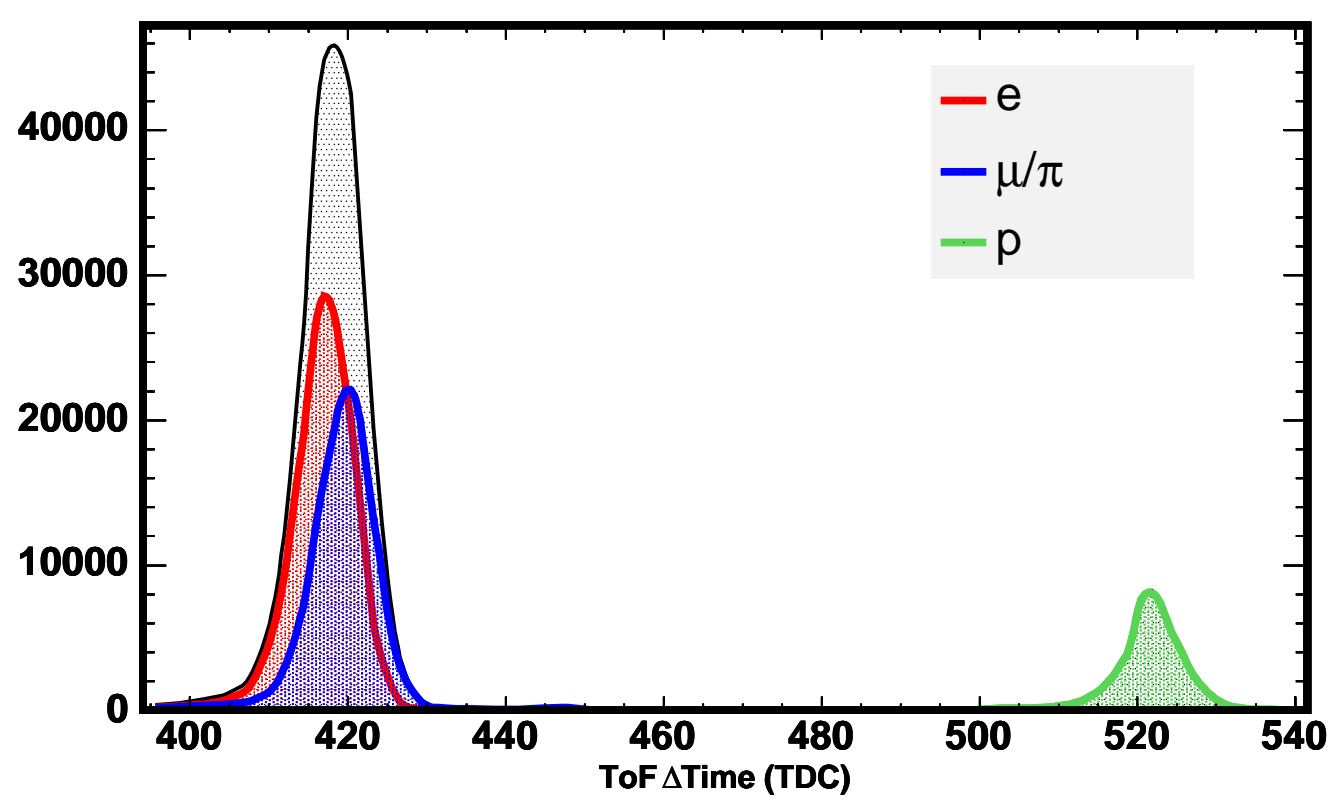

Figure 4.6: ToF System Time Distribution at CalDet. $\Delta$ Time is the time difference between the times measured by each ToF paddles in TDC as the triggering particle goes along the beam line. The distribution shown corresponds to a $1.8 \mathrm{GeV} / \mathrm{c}$ run aided by the CHDs to roughly identify the different particle types contributions to the ToF distribution.

The ToF-system made use of fast scintillator paddles coupled to fast PMTs, which were inserted in the beam line upstream of the CalDet. The distance between paddles ( $L$ : between the up-stream paddle (US) and the down-stream paddle (DS) ) depended on the physical constraints of the beam line. $L$ was $11.8 \mathrm{~m}$ for the $T 7$ beam line.

The output of the PMTs was connected, through low dispersion cables, to constant fraction discriminators. The discriminated pulses were coupled to a TDC module for the time measurement with a resolution of up to 35ps per TDC count. The ToF was required to provide a measurement only when the two paddles were successfully hit within the time window expected from a particle moving at $\sim c$ along $L$. This coincidence requirement eliminated false triggers caused by accidentals or particles with off-axis trajectories hitting a single paddle. The coincidence between paddles was used to issue the ToF coincidence-tag signal that flagged a suc- 
cessful ToF-system PID tag [99]. The TDC module was started by the coincidencetag and stopped by the delayed signals of each paddle, yielding a TDC(Paddle-US) and TDC(Paddle-DS). Thus, $\triangle \mathrm{TDC}=$ TDC(Paddle-DS $)$ - TDC(Paddle-US). The DAQ reads out the buffer of the TDC module with every VARC buffer-swap (20Hz), due to the limited depth of the memory available in the module.

\subsubsection{The Beam Trigger}

The beam-trigger was necessary due to the intrinsic asynchronous dead-time of the VA-FEE. The asynchronous dead-time is a consequence of the fact that each PMT is dynode-triggered. As different channels are hit, they become independently busy (dead) causing different sectors of the detector not be ready when the next beam spill happens. The result of this problem is that most of the collected events have to be rejected since they may not be complete. The pattern behind the dead-time of each VA-chip is deterministic, so unambiguous tracing of dead-chips is possible by algorithms offline.

During the CalDet 2002 and 2003 data-taking campaigns, a beam-trigger was implemented, increasing the efficiency of the data collection enormously. The beamtrigger allowed the coincidence-tag of the ToF system to work as an external trigger for both readout systems. The beam-trigger was endowed with logic gates to allow some degree of dynamic control or even to veto other sub-systems during spills, like the hardware of calibration systems [99]. The beam-trigger was implemented to force a synchronous dead-time across the detector by inhibiting the full readout for $\sim 70 \mu$ s from the last trigger. Hence, the entire readout systems were ready and alive for each new trigger/event.

The FD readout system was not designed for beam data taking and no external trigger gate is available on the FEE. Instead, an enable-input gate is available on each VARC. Such a gate is able to mimic an effective external trigger gate. When the enable-input gate is asserted by the beam-trigger, the VA-FEE is allowed to accept any incoming dynode-triggering to cause the VA-FEE to read out. Otherwise, the incoming dynode-trigger is ignored. The QIE-FEE, instead, was designed with an external trigger capability. Therefore, the beam trigger signal $f$ orced the ND systems to read out. Note that half of the detector (FD side) is dynode triggered while the other half (ND side) is externally triggered. This means that the ND-side readout was independent of dynode trigger thresholds, while the FD-side had the dynode threshold convolved with the external triggering condition. 


\subsubsection{The Cherenkov Counters}

CHD are threshold devices that tag a particle emitting Cherenkov light in a medium with index of refraction $n$. The selection is therefore made in terms of $\beta$, such that $\beta>\beta_{t}=1 / n[16]$. The CHD can be set up such that "light" particles trigger the CHD, while "heavy" particles do not. The threshold of a CHD was set by varying $n$ of the Cherenkov medium. This is generally achieved by either the choice of the Cherenkov medium itself or adjusting the pressure of the medium. Figure 4.7 (top) shows the Cherenkov medium $\left(\mathrm{CO}_{2}\right.$ gas) pressure selected to trigger on different particle according to their momenta. The selection of electrons $(\sim 300 \times$ lighter than a $\mu$ ) in CalDet relied almost entirely in the triggering of at least one CHD. Throughout this document, the two CHDs of T7 will be referred as US up-stream and DS down-stream CHDs.

The efficiency of the CHDs is an important quantity required to compute the probability of mis-tagging events and to measure the absolute beam composition and the degree of contamination of events samples for which the CHD provides most discrimination. The overall efficiency of the CHD (Cavity $\oplus \mathrm{PMT}$ ) is generally dominated by absolute number of Cherenkov photons generated per triggering particle and the efficiency to collect and detect those photons, as shown in Figure 4.7 (bottom). Note that the efficiency of the CHD is strongly dependent upon the $\sin ^{2}\left(\theta_{c}\right)$, where $\theta_{c}$ is the "Cherenkov angle", which is defined as $\cos \left(\theta_{c}\right)=1 / \beta n$. This is proportional to the number of Cherenkov photons detected by the PMT of the CHD. $\sin ^{2}\left(\theta_{c}\right)$ is, to first order, proportional to the pressure of the medium as well as being particle dependent [75]. So, at low pressures, the efficiency of the CHD is lower, which is generally used at high momenta to provide discrimination between electrons and muon/pions.

US-CHD was always used to trigger on electrons, as it was the most efficient CHD. DS-CHD was used for redundancy on electrons at low energies, while the ToF-system provided efficient ID on protons. Beyond $2 \mathrm{GeV} / \mathrm{c}$, the DS-CHD was used to provide additional separation between $p$ from $\pi / \mu / e$. The DS-CHD was the only discrimination between $p$ from $\pi / \mu$ beyond the momenta for which the ToF cannot offer proton ID any more.

The US-CHD signal was added to the beam-trigger logic on to obtain "electronruns" by logic AND-ing the US-CHD signal. Electron-runs were usually necessary at high energies where the relative contribution from electrons is very low with respect to other particle types (see 4.3). 


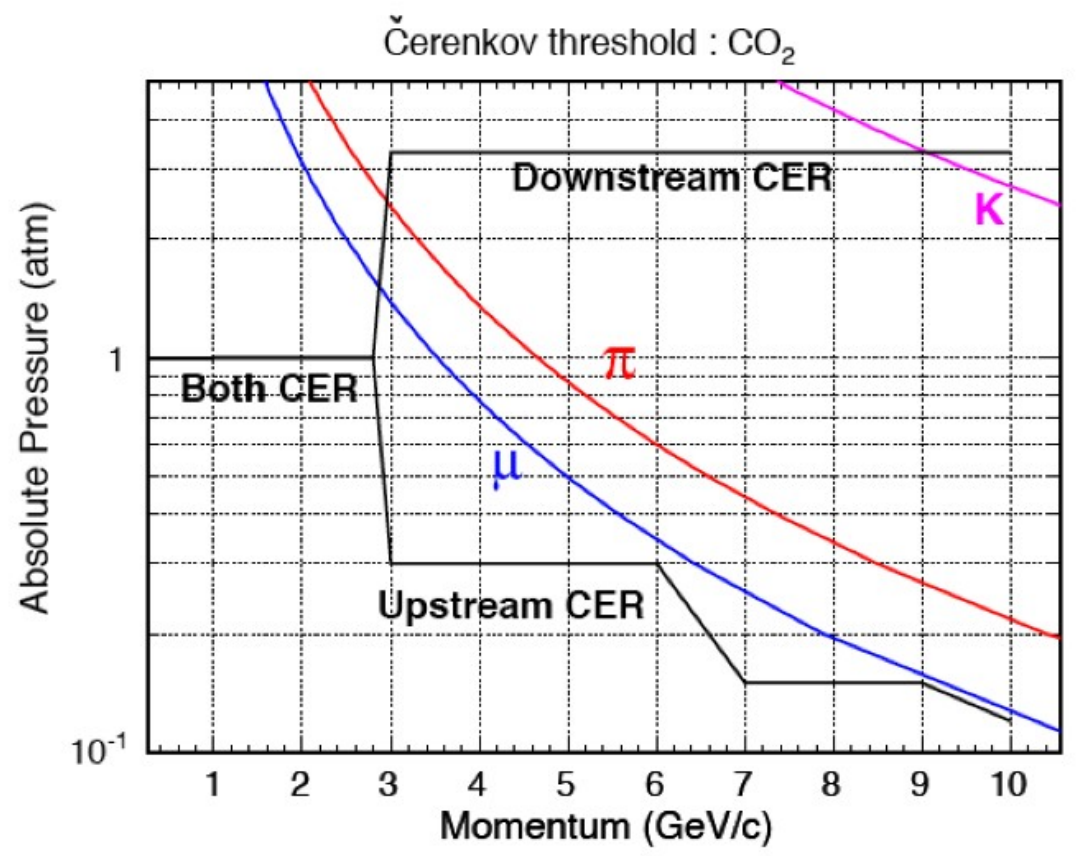

Electron Tagging Efficiency vs. $\sin ^{2} \theta_{c}: T 7: 2003$

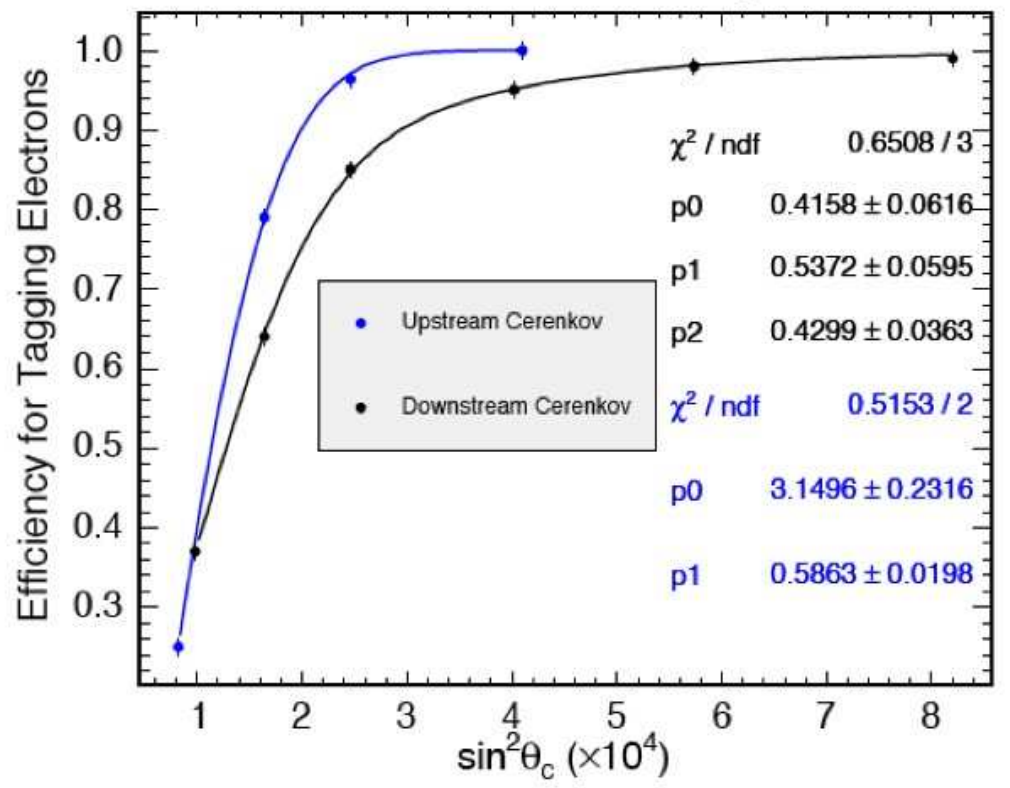

Figure 4.7: Cherenkov Counters: Pressure and Efficiency. Plot from [75].

\subsection{Physics Runs at CalDet}

Different types of data were collected to meet different purposes. The data collected can essentially be divided into two categories calibration and physics data. 
The differences between physics data runs lies in how the readout of CalDet was triggered.

Physics: Null Trigger Run In this mode, the readout systems are dynode-triggered.

This kind of running mode was generally possible and desired when the beam was absent, for the collection of cosmic $\mu$ s for calibration.

Physics: External Trigger Run This mode of running was used for the beam data collection, since only then was the external triggering by the ToFcoincidence signal of both readout systems possible. Since there were a few $0.5 \mathrm{~s}$ spills every $\sim 17 \mathrm{~s}$ at the PS, calibration data was collected in between spills. This was particularly useful since it allowed accurate monitoring of the drift of the readout systems with time, as beam physics data was collected. The trigger-logic allowed the data to be taken at any time. For this purpose the trigger-logic was interfaced to both the PS-beam electronics and calibration system control electronics.

Some calibration runs were taken prior to every physics data run to ensure that the physics data did not suffer from any short time-scale effects. The description of the calibration runs taken during CalDet running is be given in Section 5.2. 


\section{Chapter 5}

\section{The MINOS Calibration Chain}

The MINOS experiment has been designed to detect neutrinos with a high resolution in $\mathrm{L} / \mathrm{E}$, such that the $\mathrm{L} / \mathrm{E}$ dependencies (or features) of the survival probability can be resolved to test the neutrino oscillation hypothesis. Since MINOS is a stationary experiment, $\mathrm{L}$ is fixed and well known. Therefore, the resolution in $\mathrm{L} / \mathrm{E}$ is essentially the precision with which MINOS can measure E, i.e. the energy of the incident neutrino.

MINOS was designed to be in a energy regime (or neutrino interaction regime) over which the fraction of transfered momentum to the hadronic sector in a CC $\nu_{\mu}$ interaction can be up to $30 \%$ of the total energy of the incident neutrino. The precision in the neutrino energy measurement is, to first order, Bjorken-y weighted combination (in quadrature) of the precision with which the muon and hadronic activity energies can be measured. Therefore, the MINOS collaboration chose tracking-calorimeters for the detector technology, so that the fraction of energy going into both the leptonic and hadronic systems can be measured precisely. The precision on the muon energy measurement (based on curvature or range) is typically $<10 \%$, while the hadronic measurement is limited by the relative hadronic energy resolution (quoted in Equation 3.2), which is about $56 \% / \sqrt{E}$. Therefore, a $\Delta m_{23}^{2}$ measurement as good as $4 \%$ precision $(1 \sigma)$ is possible, providing that the relative inter-detector calibration and the absolute calibration are to be understood to $2 \%$ and $5 \%$ precision level, respectively and, of course, if there is enough statistical sensitivity. This represents non trivial challenges to MINOS in the calibration front-end. Moreover, the separation of $\nu_{e} \mathrm{CC}$ events from NC events relies, to first 
order, on the distinction between the energy deposition profiles of each type of events and on the quality of the calibration.

This chapter will be dedicated to describing the different stages of the MINOS calibration to achieve the goals above mentioned. Particular emphasis is given to the stages of the calibration chain exercised at CalDet for the Near/Far running. The validation of the calibration will be described in Chapter 7, upon discussion of the final results. Those stages of the standard MINOS calibration chain omitted at CalDet will also be briefly mentioned for completeness.

Moreover, this chapter will be used to highlight the contributions of the author to the calibration chain.

\subsection{MINOS Calibration Chain Overview}

The full calibration chain of MINOS can be broken down into five stages. Figure 5.1 provides an illustrative summary of the MINOS calibration chain.

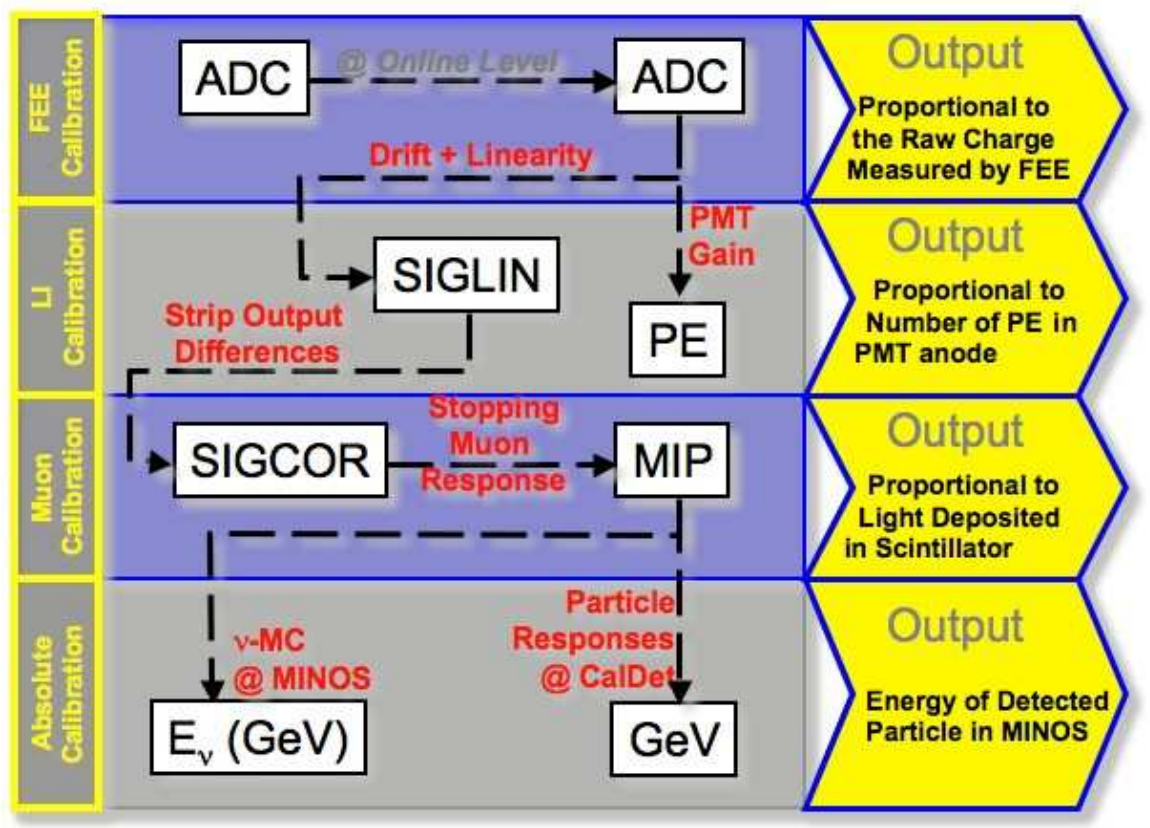

Figure 5.1: The MINOS Calibration in a Nut-shell. All steps of the calibration chain are highlighted, including the origin (yellow boxes on the left), the name of the calibrated scale (boxes), the correction carried out (in red) and the output equivalent (yellow arrows). 
1. First, the FEE was designed such that it performs pedestal subtraction, sparsification (zero suppression) and linearisation of the ADC response online (in the QIE-FEE only). All other calibration stages are implemented offline.

2. Second, the Light Injection (LI) calibration system corrects for both the drift of the responses with time and the non-linearity of the readout systems $(\mathrm{PMT} \oplus \mathrm{FEE})$.

3. Third, muons are used for strip-end calibration, as they are the only copious radiation source available in all MINOS detectors, to determine the scintillator strip and detector differences. Through-going cosmic muons are used to obtain the intra-detector relative calibration for each detector, while stopping muons are used to accomplished the relative inter-detector calibration across all the MINOS detectors. The intra-detector and inter-detector calibrations are colloquially referred to as the "Strip-to-Strip" ("S2S") and "MIP" calibrations, respectively.

4. The attenuation of light along the optical fibres is taken into account by the "MAPPER" calibration. Dedicated data was taken to map the attenuation curves of each single strip-end during construction of the detectors. This stage of the calibration will not be described any further since it was not performed at CalDet.

However, the attenuation of light along the fibres was critical for this analysis and two approaches were developed to this end: the "Integrated Attenuation" and the " $4 \times$ MIP" calibrations.

5. The fourth and final stage of the calibration provides the link between the amount energy deposited in the detector and the energy of the originating particle. Hence, this stage is called the "Absolute Energy" calibration. The absolute energy calibration differs between CalDet and the detectors exposed to neutrinos.

\subsection{Dedicated Calibration Runs at CalDet}

FEE and LI calibration need the collection of specific data for their implementation. A list of the dedicated runs for calibration are briefly summarised together with their objective below. 
VA-Pedestal Run The object of this run is to sample the pedestals of all VAFEE channel. Essentially, pedestal runs can be seen as series of random digitisations of the output voltage of each channel of the VA-chips.

At CalDet, one pedestal run was taken prior to any physics run.

VA-FEE-Linearity Run This run is used to obtain data to study of the nonlinearity of the response of each channel of the VA-chips. Every VFB was endowed with a Charge Injection (CI) circuitry, whose task was to inject charge over the whole dynamic range of the electronics. The comparison between the known amount of charge injected to the measured response provides a measure of the non-linearity of the VA-FEE.

At CalDet, a few VA-FEE Linearity runs were taken per CalDet-configuration.

QIE-FEE-Calibration Run This run is taken every time the QIE-FEE has to be calibrated, either because a new calibration is desired or because there is no calibration at all. Like in the FD side, the QIE-FEE was endowed with CI circuitry for calibration. The CI injects charge throughout the dynamic range of the electronics. The QIE-FEE uses the data to compute calibration curves online which linearise the response of each QIE-FEE channel. Each MASTER stores such an linearisation information into local LUTs, as explained in Section 3.2.3.

QIE-FEE-Calibration-Check Run The data collected for this run in the same principle for QIE-FEE Calibration runs. The CI circuitry inject charge across the dynamic range of the electronics, but this time the data output has already been linearised, as the QIE-FEE had already been calibrated. The output is used to check the linearity of the response of the QIE-FEE.

At CalDet, this run was taken one prior to any physics run.

Light-Injection-Drift Run LI-Drift runs are taken frequently to monitor the drift of the response of the each channel of the readout system in time. The LI-system was set to flash at a single light level. $\sim 30 \mathrm{PE}$ was found, upon dedicated studies, to be optimal, as the PMTs are linear.

Light-Injection-Gain-Curve Run LI-Gain-Curve runs use the LI-system to pulse light across the dynamic range of the readout systems such that the nonlinearity of each channel of the readout systems can be characterised with respect to the response of the LI PIN diodes. 
These runs were taken at CalDet once or twice per CalDet configuration.

\subsection{FEE Calibration}

The FEE calibration stages are often not regarded as part of the MINOS calibration per se, although they should since, strictly speaking, they affect the ability to measure energy in MINOS.

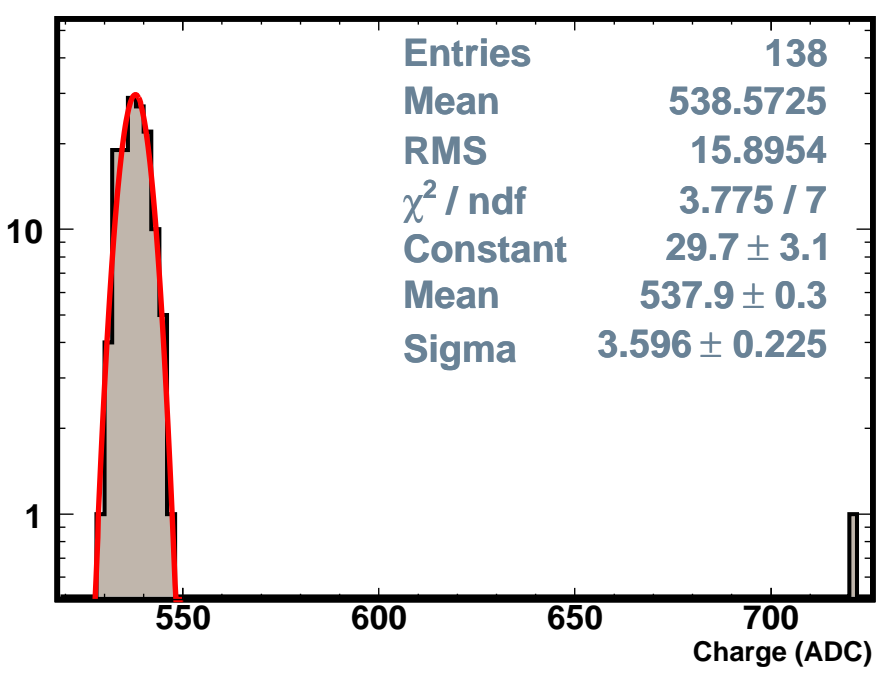

Figure 5.2: Pedestal Distribution. Sample of a pedestal distribution with one outlier (at $\sim 720 \mathrm{ADC}$ ). The effect of the outliers can be appreciated by increase in the RMS with respect to the width of the distribution estimated by a Gaussian fit (red). For this example, the fit width is about $4.5 \times$ less than the RMS. Note that the mean has changed by about $1 \mathrm{ADC}$.

\subsubsection{VA-chips Pedestal}

Pedestal runs are taken in such a way that channels are digitised with no energy deposition (or charge), therefore producing pedestal or zero charge distributions. The mean and width (RMS) of each pedestal are respectively used to infer the zero ADC scale and the noise level of each channel. After every pedestal run, tables of pedestal means are calculated online. The new pedestals tables are then made available to both the offline and the online prior to every subsequent run. The new pedestal tables are uploaded by the FEE such that online pedestal subtraction can 
be performed for each channel. Pedestal information also has important diagnostic power for understanding the stability and noise of each channel.

\section{Pedestal Truncation Analysis:}

When pedestal runs are taken the PMTs must be at their nominal HV ${ }^{1}$. Thus, potential collection of $1 \mathrm{PE}$ accidental events (so-called singles) is possible.

Singles are generally caused by spontaneous light emission of the WLSF, PMT dark noise or, possibly, radioactivity from the cavity rock. Singles generally exhibit a charge spectrum characterised by a distribution peaking at $\sim 1 \mathrm{PE}$. Incident singles or comic muons in the pedestal distribution will appear as outliers, as shown in Figure 5.2.
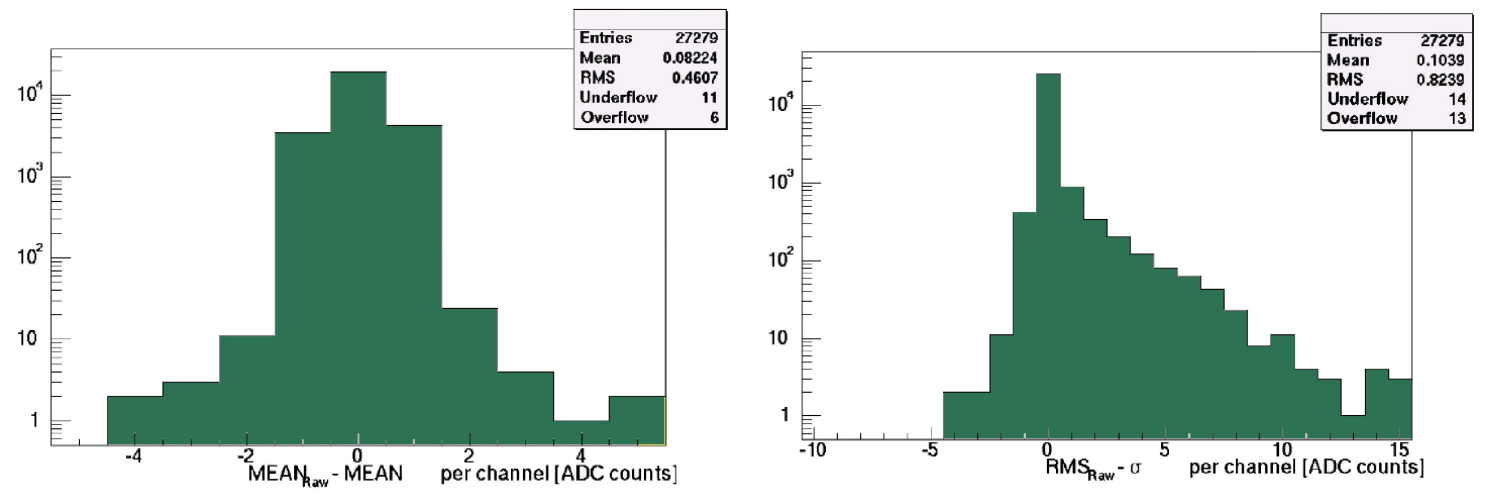

Figure 5.3: Effect of Pedestal Truncation. After truncating every pedestal, the truncated means and RMS were compared with raw means and RMS. The left plot shows the average difference channel-to-channel between the raw and the truncated means. While the right plot shown similar difference for the raw and truncated RMS. Note that although in both cases the average difference is probably negligible, the effect on the RMS channel-to-channel can be sizable.

The outlying entries could potentially distort the calculations of both the mean and the width of the pedestal distributions. Truncation algorithms were devised to understand the consequences of outliers upon the accuracy of pedestal calculation. The mean and the width of the truncated distribution was computed for the study of systematic errors on the calculated non-truncated pedestals. The results [100] showed an average very low single rate per channel: $1.5 \pm 1.2$. Computing the difference between the non-truncated and truncated means of the pedestal showed that the non-truncated average mean was $+0.082 \pm 0.002 \mathrm{ADC}$ higher than the

\footnotetext{
${ }^{1}$ Otherwise the VA-chip channels are set to high impedance by a diode on the base of the PMTs that is expected to be biased by the HV.
} 
average truncated mean. The average width was not sizably enlarged by the outliers by $+0.104 \pm 0.005$ ADC.

Therefore the effect of singles on the pedestal calculations is negligible on average. However, channel-by-channel, the width distortion can be very significant in some cases, with up to a factor $15 \times$ larger widths can be found (see Figure 5.2).

\section{Pedestal Stability Analysis}

The stability of the pedestal was another important question investigated. The object was to understand how stable pedestals are over time and, therefore, estimate how often pedestal runs should be taken to have an accurate sample of the pedestal location. The pedestal stability was studied over three time-scales: 1 hour, 10 hour and 1 week (Table 5.1).

\begin{tabular}{|c|c|c|}
\hline Time Scale & Max. $<$ Drifted Mean $>$ & Max. $<$ Drifted Width $>$ \\
\hline \hline 1 hour & 0.006 & 0.57 \\
10 hour & 0.05 & 0.63 \\
1 week & 0.12 & 1.3 \\
\hline
\end{tabular}

Table 5.1: Studied Time Scale Variations.

The location of the mean pedestal was found to drift with time, characterised by two main components:

- Common Drift: The first component was a "common-drift" for all channels. The VA-FEE has however an internal mechanism to correct for the common-drift of pedestals called common-mode correction [77]. Figure 5.4 (top-left) shows the amplitude of the drift of mean with no common-mode correction applied. Figure 5.4 (top-right) shows the remaining amplitude after common-mode correction was applied. The mean drift is suppressed up to a factor of $15 \times$ when common-mode correction is applied.

- Individual Drift: The second component was caused by channel-to-channel stochastic variations, which can be estimated by measuring the with of the pedestal drift distribution and its evolution over time. The effect of this component is characterised by mapping the divergence of the drifted pedestal mean over time. Figure 5.4 (bottom-left) shows the divergence of the drifted mean over time with no common-mode correction applied, while Figure 5.4 (bottom-right) shows the divergence of the drifted mean over time with 

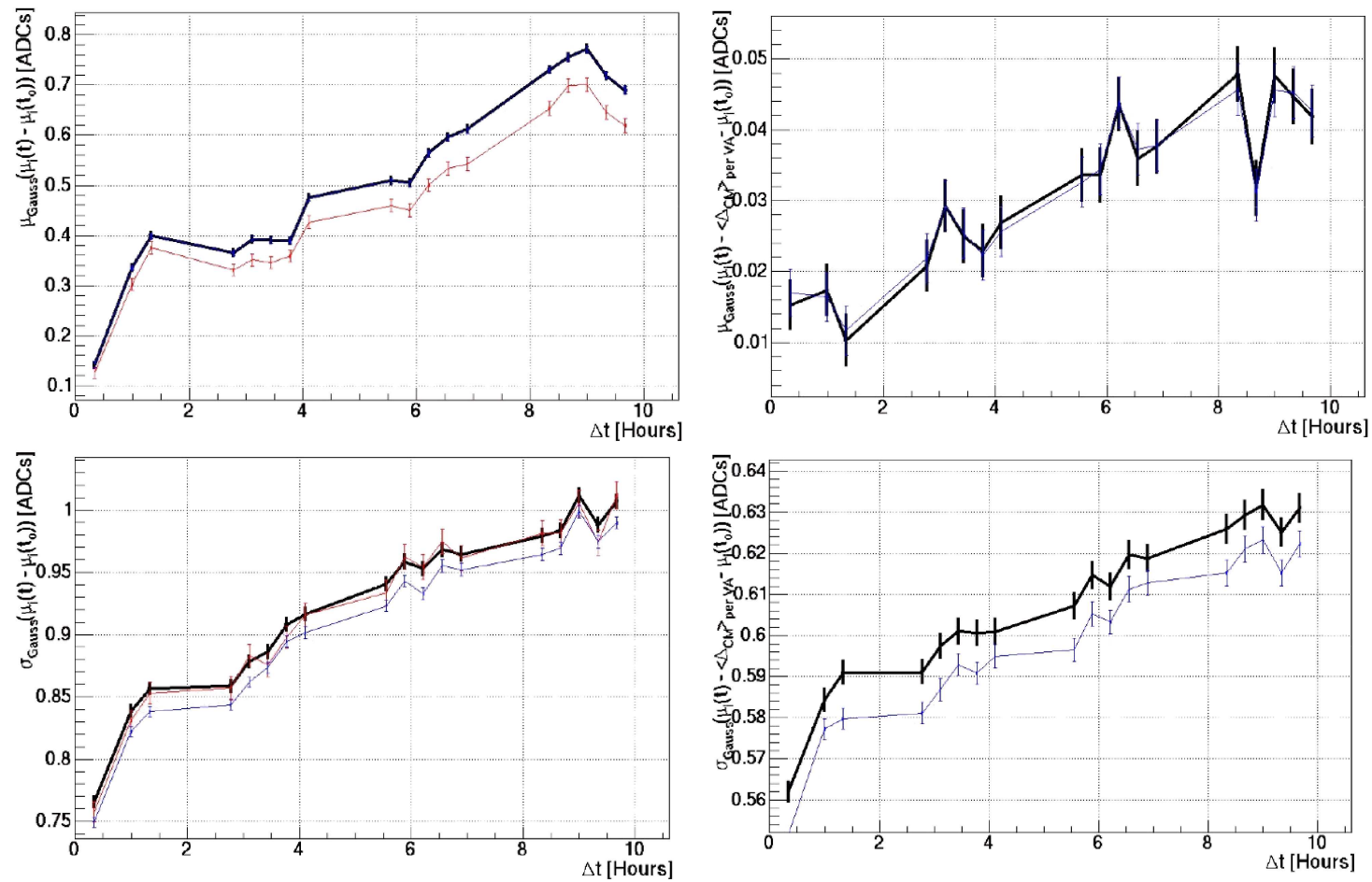

Figure 5.4: Pedestal Stability Studies. The top plots shows the average drift of the mean of the pedestal over time in time scale of $10 \mathrm{~h}$. The bottom plots shows the average divergence of the mean of the pedestal over the time scale. There have been no commonmode correction applied for the left plots, while common-mode correction has been carried out for all plots on the right. The PMT channels, PIN channels and common-mode channels are depicted in black, blue and red respectively.

common-mode correction applied. Note that the common-mode correction does not have such an significant impact on the divergence of the drifted pedestals. The stochastic component was found to exhibit an average negligible divergence $\sqrt{1.30^{2}-0.56^{2}}=1.2 \mathrm{ADC}$ per week (Table 5.1). To limit the divergence of the drifted pedestals, new pedestal runs are taken to force the re-calculation of all pedestals again from scratch.

After these stability studies, 1 pedestal run $(\sim 5 \mathrm{~min}$.) per day is taken. Previously, 1 pedestal run every about 3 hours had been taken in general. Therefore, the overall duty-cycle of the FD has been enhanced by at least $3 \%$.

\subsubsection{VA-FEE Linearity}

The VA-FEE is known to have non-linear response, as shown in Figure 5.5 (left). The mechanism behind the non-linearity of the VA-FEE [77] is out of the scope of 
this discussion. However, the behaviour is such that the non-linearity of the VAchip increases monotonically with the charge of the incoming pulse after 8000ADC, saturating at about 14000ADC, as shown in Figure 5.5 (right).
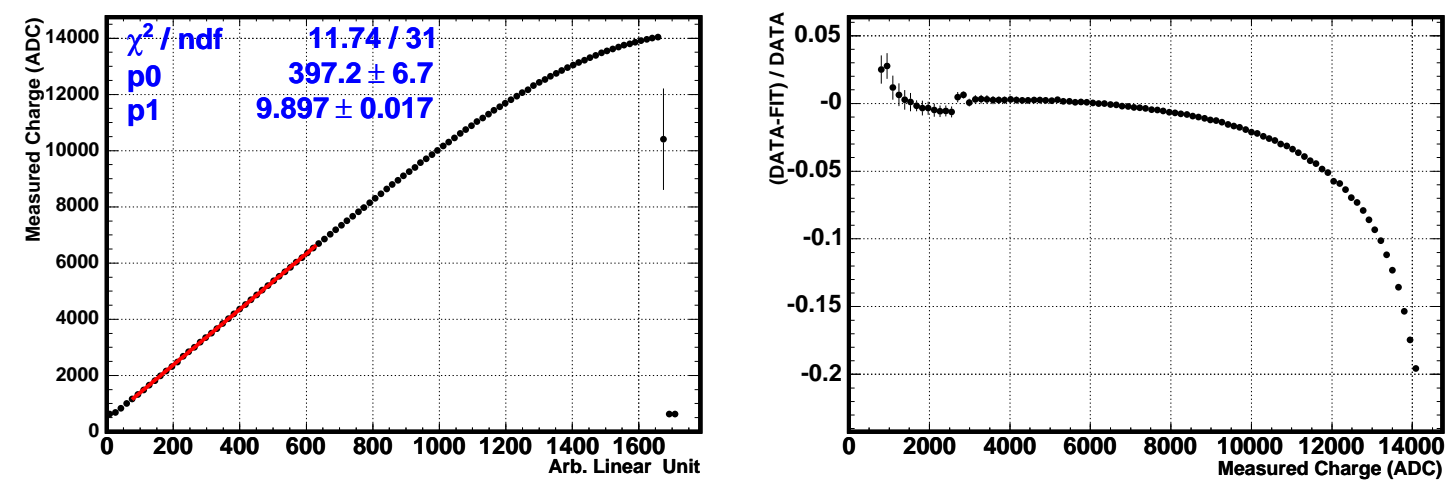

Figure 5.5: VA-FEE Non-linearity Curve. The response of the VA-FEE can be mapped with charge injection runs. The residuals show up to a $20 \%$ non-linearity. A small glitch at low charge can also be identified and the linearity correction deal with both the low and high charge non-linearity. The curves shown correspond to a single channel picked at random.

The data collected during VA-Linearity runs is used to characterise the nonlinearity of the VA-FEE. The response below 4000ADC is used to infer the correction curves that would linearise the response of the VA-FEE at high charges. This process occurs offline and the subsequent calibration is applied, when available, during offline reconstruction. This correction is only necessary for the LI PIN diode response, as will be explained in the Section 5.4.

\subsubsection{QIE-chip Calibration}

The information needed for the electronics to perform the online linearisation is held in LUT. The calibration of the QIE-FEE relies on taking a QIE-FEE-Calibration runs. During this run the computation of the LUTs happens. Once the LUTs are available, the QIE-FEE linearises its own response online. After calibration, the effective response of the QIE-FEE is like a 16-bit-ADC is available.

\subsubsection{QIE-FEE Linearity}

Once calibrated, the online effective response of QIE-FEE is very linear (deviations $<0.5 \%$ ). The data from QIE-FEE-Calibration-Check runs are used to 
evaluate/check the linearity of the response of the QIE-FEE.
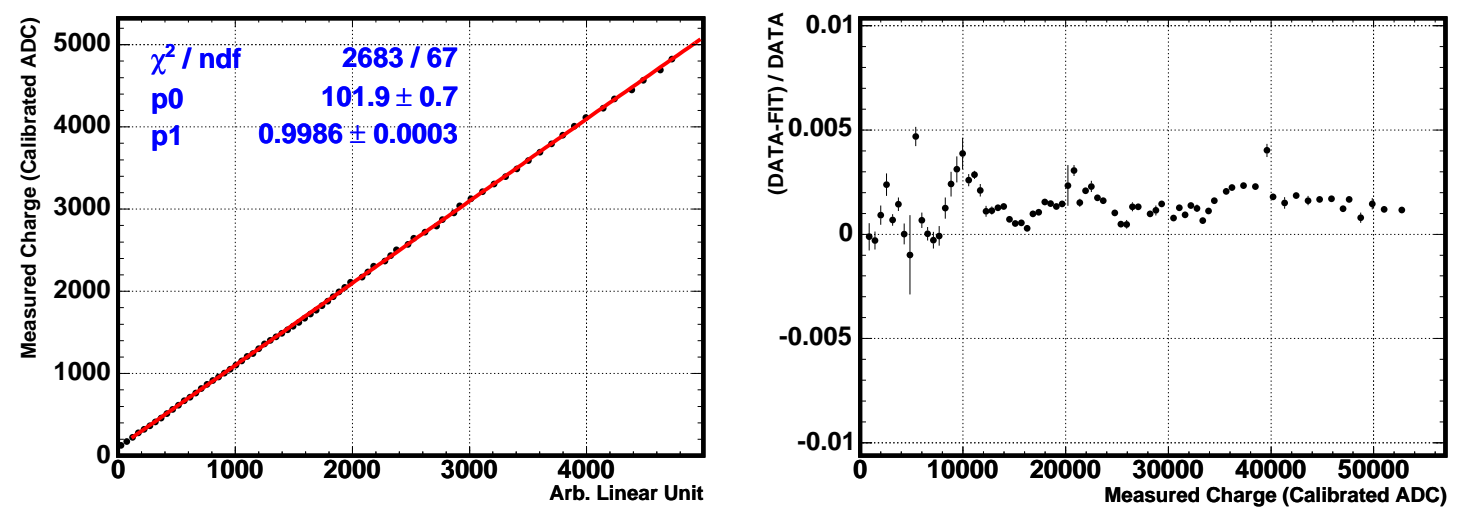

Figure 5.6: QIE-FEE Non-linearity Curve. One channel, picked at random, has been used to show the linearity of the response of the QIE-FEE after calibration has taken place. Note the scale of the residual plot (right): $<0.5 \%$.

Figure 5.6 shows both the response of the QIE-FEE to the CI input (left) and the residuals from the fit (right) that characterise the degree of linearity of the response. The same data can be used to study the stability of the calibration and variations of the gain of the QIE-FEE over time.

\section{QIE Calibration Diagnosis Analysis}

During the CalDet 2003, the QIE-FEE was, for the first time, exposed to beam data taking. A few problems were found, associated to the calibration of the electronics. These problems had negligible impact on the data collected due to the prompt action taken to understand the origin and effects. Data from QIE-FEE-CalibrationCheck runs and the study of the contents of the LUT contents [101] were the major diagnostic tools. The LUT data analysis was particularly useful because the QIEFEE Calibration Check runs are not intended for the detailed study of the contents of the LUT. They merely sample the response of QIE electronics (or the LUT contents) with a very limited number of points across the dynamic range available. Four problems were found:

1. The calibration was found sometimes to fail. An example is shown in Figure 5.7 (left). Whenever the calibration of the QIE-FEE was carried out while there was incident beam this caused bad calibrations. This is a consequence of having no switch that isolates the FEE from the PMTs during FEE calibration runs. Bad calibration also occurred under no beam. 

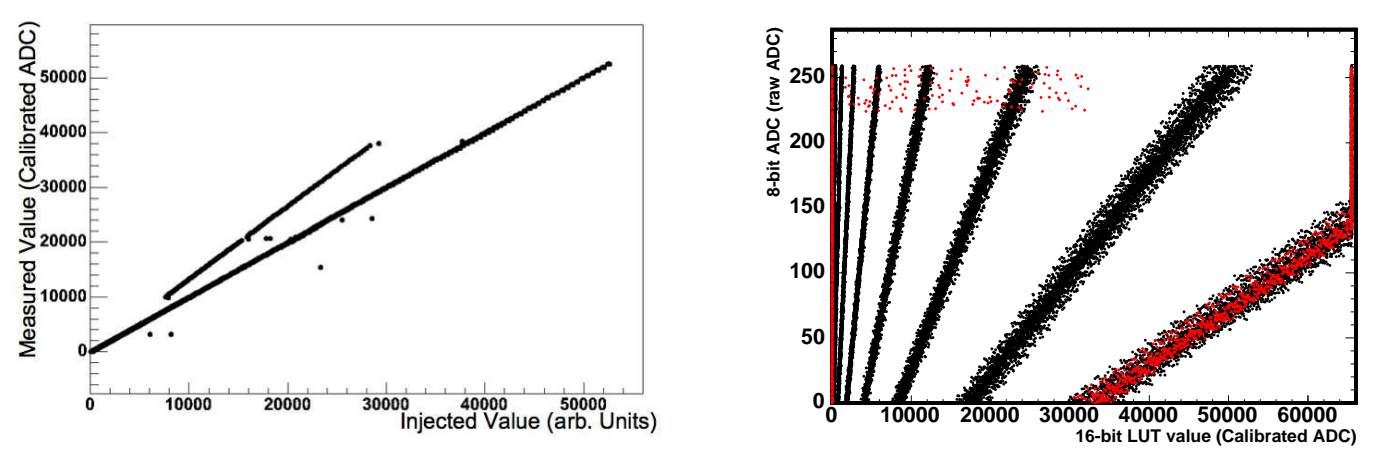

Figure 5.7: QIE-FEE Calibration Quality Studies.

Generally, re-taking the QIE-Calibration run again sufficed to obtain a good calibration.

2. The second problem was found to be intrinsic to the QIE functioning. In the very rare occasions that a digit has the right charge to be within a range transition, the QIE circuit the RANGE-bit can flip. This "RANGE-flipping" causes the QIE-chip to incorrectly select the range, outputting an incorrect calibrated ADC value upon reference to the LUT. This effect was later reproduced in a bench test at FNAL [102] and was estimated to occur very rarely [103].

3. The third problem was found to be some level of corruption in the LUT, as shown in Figure 5.7 (right). This problem, while severe, has no chance to affect the data, as it was restricted to the very top of the dynamic range of QIE-chip, i.e. RANGE-7. This is because the corrupted region of the LUT was only reachable by digits whose charge was $>500 \mathrm{PE}$ occurring over one bucket (19ns). With $9 \mathrm{GeV} / \mathrm{c}$ electrons, at most $200 \mathrm{PE}$ were reached integrating over all buckets hit.

The reported corruption was immediately found to be due to an incomplete DAQ "transaction" during the population of LUT [104] and, once identified, it was successfully corrected. This anticipated some debugging for the later Near Detector installation.

4. Yet another type of corruption was found in the LUTs. One digitisation on about $60 \times 10^{6}$ was off in the LUT. The very rare occurrence of this problem limited the possibility to diagnose it. Its impact on the data is also negligible. 


\subsubsection{Sparsification Thresholds}

The sparsification was designed to reduce the rates of the DAQ by omitting the pedestal peaks of each channel from the data (see section 3.2.2). Sparsification threshold tables are upload by the FEEs prior to any physics and most calibration runs such that the FEE can perform online zero suppression.

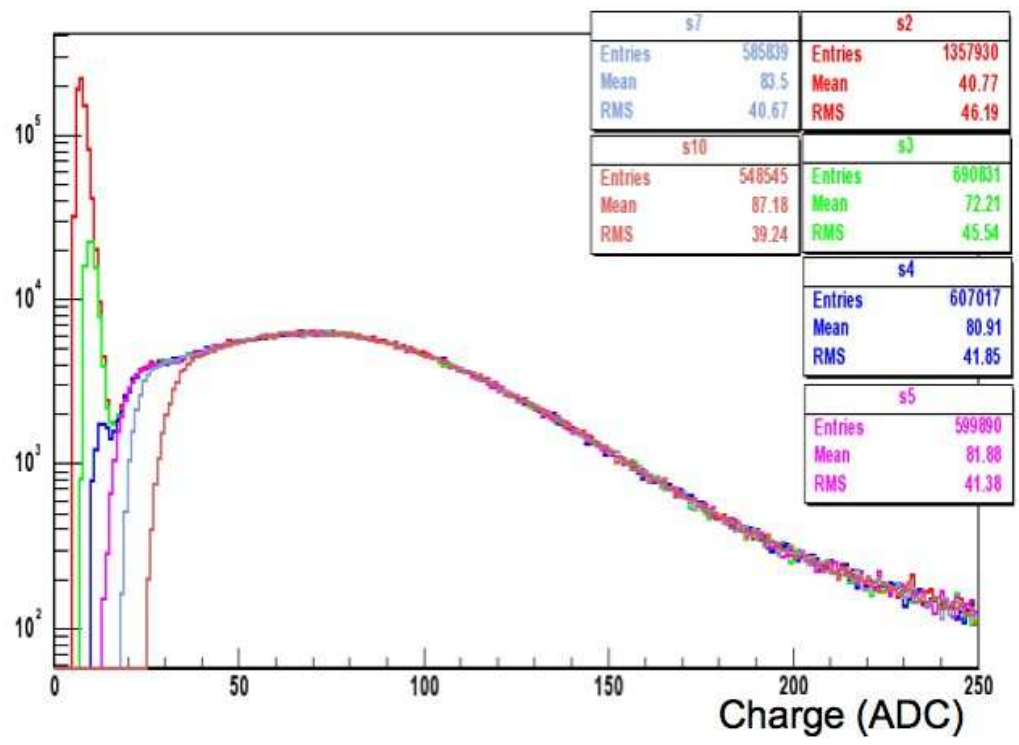

Figure 5.8: Sparsification Threshold Definition Criteria. This plot shows the charge spectra of singles (dominated by 1PE peak) for about 9s exposure with different sparsification thresholds. The sparsification threshold computed as $5 \times$ the width of the truncated pedestal is optimal to efficiently remove the pedestal peak -that can be seen at $<10$ ADC- while keeping the 1PE efficiency $>90 \%$.

\section{Sparsification Threshold Configuration}

A method was developed whereby the sparsification thresholds were set to account for the width of the pedestal for each channel.For this method to work, an accurate estimation of the width of each pedestal is needed, which, as explained in 5.3.1, will need truncation for the calculation. The optimal sparsification threshold was found to be $5 \sigma$ of the truncated pedestal width, as shown in Figure 5.8, such that the pedestal peak is efficiently removed while keeping maximal sensitivity to the 1PE peak.

Figure 5.9 shows, the sparsification threshold as calculated both with and without truncation. Note that this technique accounts for the different readout types, as their intrinsic noise levels and, therefore, the width of their pedestals are slightly 


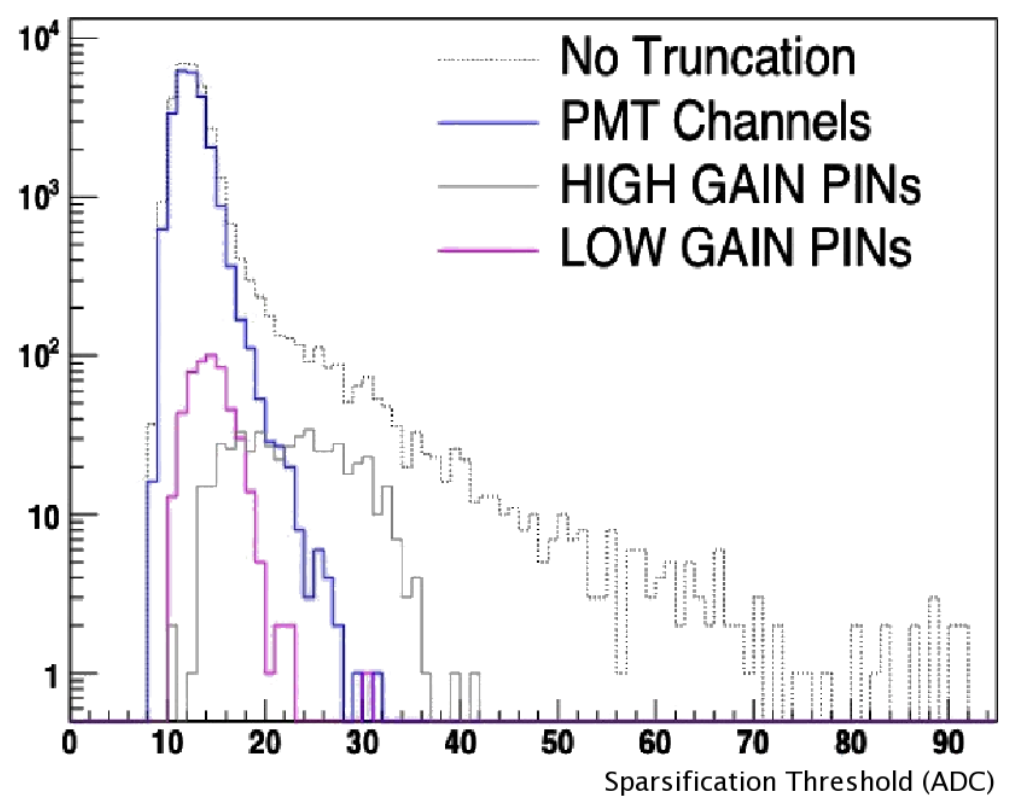

Figure 5.9: Sparsification Thresholds Per Readout Type. This plot shows the result of the analysis: the sparsification thresholds now account for the noise level of each readout type connected to the FEE.

different. The truncated width is about $\sim 3.0 \mathrm{ADC}, \sim 3.2 \mathrm{ADC}$ and $\sim 5.0 \mathrm{ADC}$ PMT, low-gain and high gain PINs.

In the FD, prior to this analysis, the sparsification thresholds were set to up to 20ADC, which is $\sim 7 \sigma$ of the average pedestal width. The DAQ rate, therefore, increased by a $<0.5 \%$-negligible- after the new thresholds have been used, while the sensitivity to the $1 \mathrm{PE}$ peak is now $\sim 95 \%^{2}(\sim 1 / 5 \mathrm{PE})$. The sparsification thresholds are now calculated offline after every pedestal run by the "SexyPedestal" package [105].

In the ND, no tuning of the sparsification thresholds have been envisaged. The threshold is set to 20ADC for all channels, which is about $8 \sigma$ of the average width of the pedestal. Therefore, the average sensitivity to the $1 \mathrm{PE}$ is $\sim 94 \%(\sim 1 / 5 \mathrm{PE})$, which is comparable to the level reached in the FD.

\subsection{Light Injection Calibration}

The Light Injection (LI) system [106] was designed to measure:

- the time drift of each channel gain with time: Drift-Point Calibration.

\footnotetext{
${ }^{2}$ Assuming a Gaussian 1PE distribution with $50 \%$ width
} 
- the gain of each PMT pixel: Gain Calibration.

- the non-linearity of each channel: Linearity Calibration.

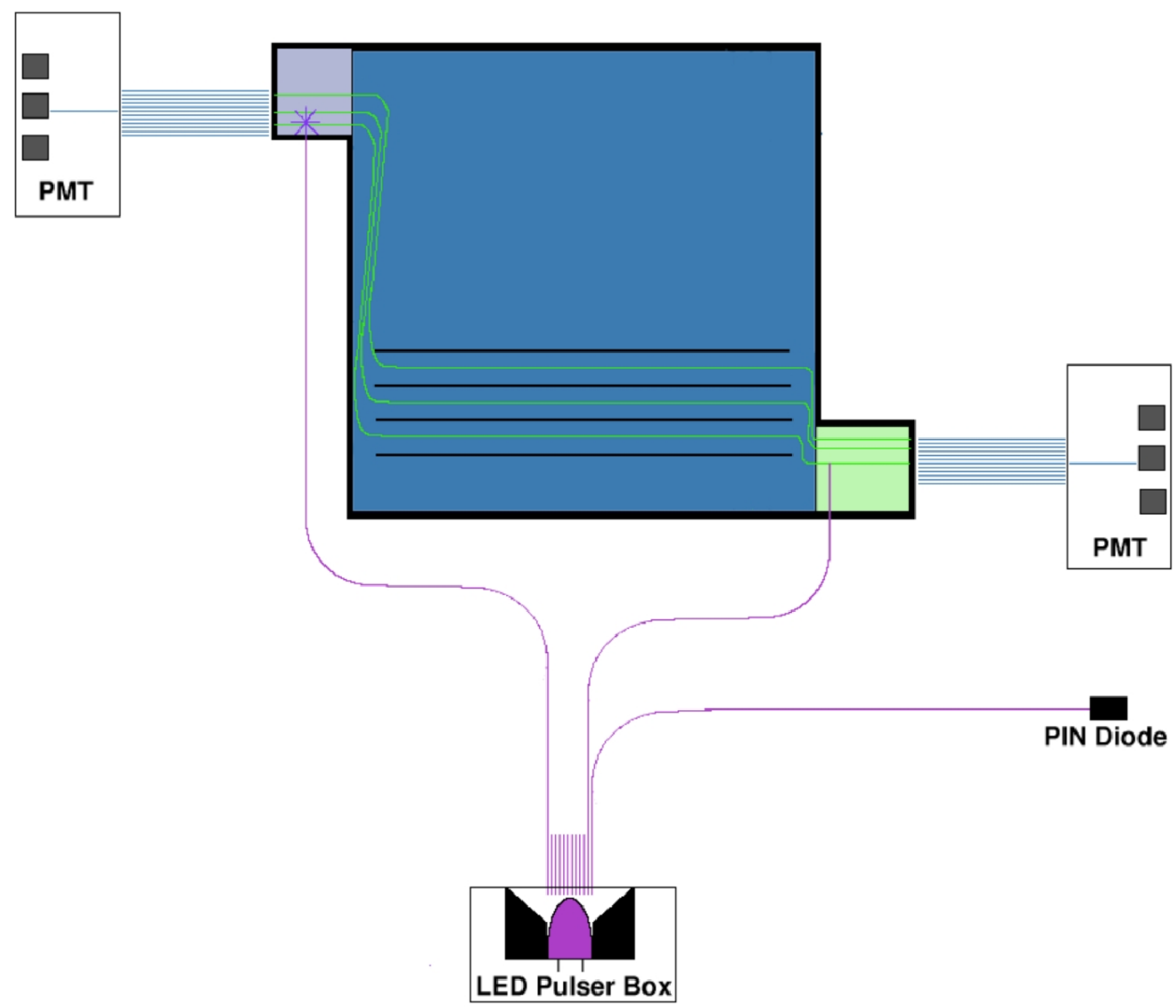

Figure 5.10: Light Injection System Diagram at CalDet. (Drawing from [74].

The LI-system injects light at different light levels into the optical system of the detectors with UV-LEDs . The effective amount of light injected can vary from a fraction of $1 \mathrm{PE}$ up to $>500 \mathrm{PE}$. The injection of light into the optical systems happens at the a specific module, where the light of the LED illuminates the green fibres at either end of each scintillator plane-module. The light of each LED is independently monitored by two PIN diodes (generally referred as "PINs") with different relative gains to allow them to cover the full dynamic range of the LI-system.

The LI system has additional diagnostic abilities. Among the most interesting one are: check the sanity of the light path of each optical channel, validation of the cabling and even measurement the dead-time of each channel. Much of the LI calibration procedures are described in [107] [108] [109]. 
The application of the LI corrections is described in the following equation:

$$
\begin{aligned}
\mu_{i}(\mathrm{SIGLIN}) & =\mu_{i}(\mathrm{ADC}) \times C_{i}^{L I}\left(\mu_{i}\right), \forall i \text {-th strip } \\
\text { where } & \\
C_{i}^{L I}\left(\mu_{i}\right) & =C_{i}^{\text {GainCurve }}\left(\mu_{i}\right) \times C_{i}^{\text {DriftPoint }}
\end{aligned}
$$

where $C_{i}^{\text {GainCurve }}\left(\mu_{i}\right)$ stands for the linearity correction of the $i$-th channel, which depends on the response to be calibrated $\left(\mu_{i}\right)$, while $C_{i}^{\text {DriftPoint }}$ corresponds to the correction for the overall drift of the $i$-th channel. Once the the LI corrections (all or some) have been applied, a pseudo-ADC unit of charge becomes available: SIGLIN, which can be thought of as a magnitude proportional to the number of PEs measured at the photo-cathode ${ }^{3}$ of a linear PMT.

\subsubsection{Gain \& Drift-Point Calibration}

A "drift-point" $\left(D_{i, j}\right)$ is calculated (see Equation 5.3) from the ratio of the response each $i$-th PMT-pixel $\left(\mu_{i}(P M T)\right)$ to the response of its corresponding $j$-th PIN $\left(\mu_{j}(P I N)\right.$, upon a flash of the $\operatorname{LED}\left(L E D_{i, j}\right)$. The $i$ and $j$ stand for the pixel number and PIN number.

$$
\begin{gathered}
D_{i, j}=\mu_{i}(P M T) / \mu_{j}(P I N) \\
\text { where } \\
\mu_{i}(P M T) \propto G_{P M T, i} \times L_{\text {injected }} \times G_{F E E, i} \\
\mu_{j}(P I N) \propto L_{\text {injected }} \times G_{F E E, j}
\end{gathered}
$$

The response of the PIN $\left(\mu_{j}(P I N)\right.$ is proportional to the amount of light injected $\left(L_{\text {injected }}\right)$ by the LED $\left(L E D_{i, j}\right)$, as indicated in Equation 5.5. Whereas the response of PMT $\left(\mu_{j}(P M T)\right)$ is proportional to both gain of the PMT $\left(G_{P M T, i}\right)$ and the amount of light injected, as indicated in Equation 5.4. Note that, since the same FEE reads out both, then $G_{F E E, i} \approx G_{F E E, j}$, causing the ratio to be insensitive to the gain of the FEE. The response of the PIN has a negligible temperature dependence, whereas the PMT is expected to have a temperature-dependent gain

\footnotetext{
${ }^{3}$ Strictly speaking SIGLIN is proportional to the number PEs at the photo-cathode, only if PMT crosstalk did not happen.
} 
due to the stochastic processes. Therefore, the ratio should reflect the changes of the PMT gain with temperature: $G_{P M T, i}=G_{P M T, i}(T)$, to first order. The evolution of the drift-point correction is shown in Figure5.11.

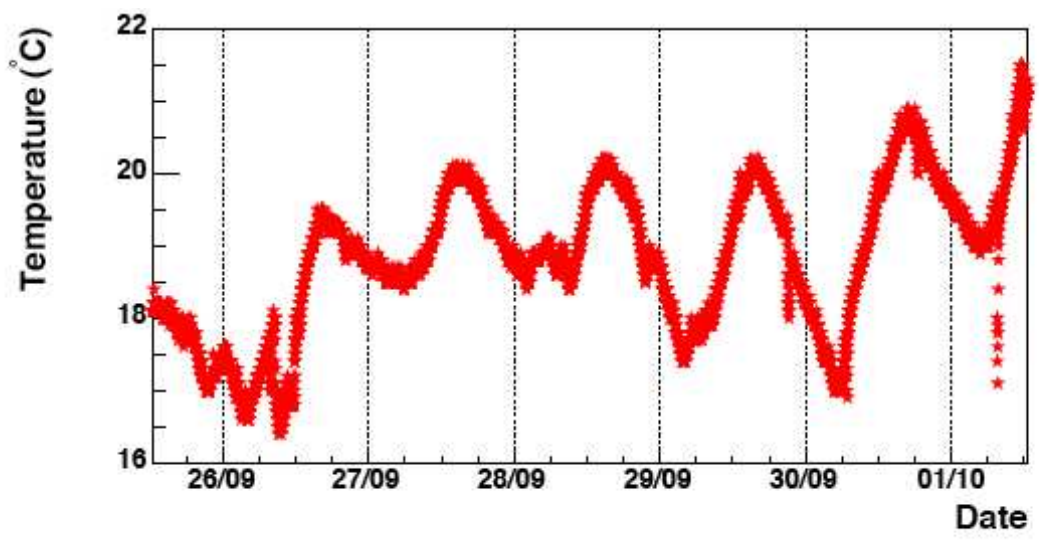

Average Drift Point Correction (Over 6 Days)

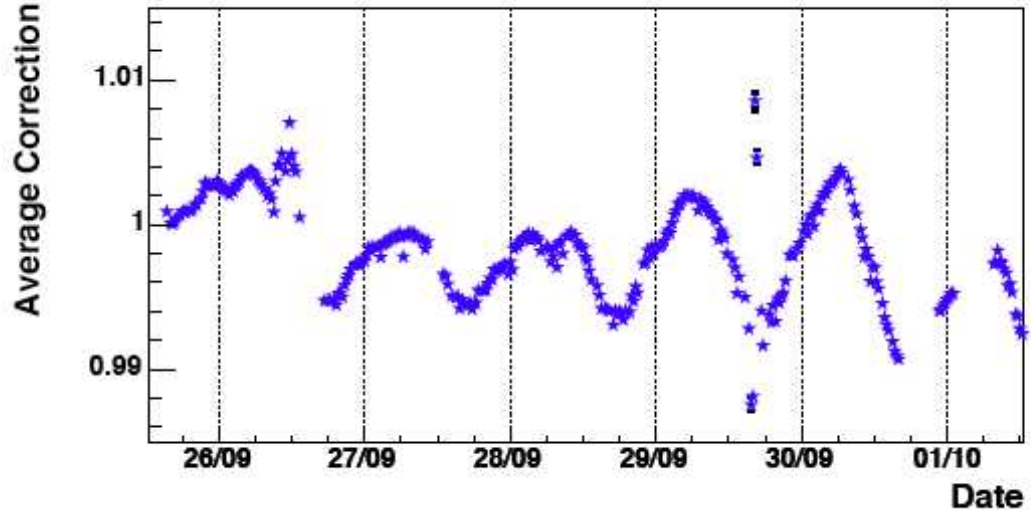

Figure 5.11: Drift-Point Calibration Capability. These plots show the evolution of the temperature (top) and drift point correction (bottom) over 6 days, where a clear anticorrelation between temperature and drift-point correction can be inferred. The gain of the PMTs has been measured to change with temperature by about $-0.5 \% /{ }^{\circ} \mathrm{C}$. (Plot from [107]).

The design of LI does not allow the drift-point correction to account for the variation of the FEE gain $\left(G_{F E E}\right) . G_{F E E}(\mathrm{VA})$ has been measured to vary with temperature as $-0.22 \% /{ }^{\circ} \mathrm{C}$ [110]. This correction is currently incorporated as part of the scintillator temperature correction estimated from the muon calibration for the Far Detector. The QIE-FEE is water-cooled in the Near Detector and expected variations of the temperature are $\pm 0.1^{\circ} \mathrm{C}$. Preliminary studies of $G_{F E E}(\mathrm{QIE})$ measurements obtained an absolute $0.07 \%$ variation over two weeks, although its correlation with temperature has not yet been determined [111]. 
At CalDet 2003, the PIN of the FD readout system was used to for the drift point correction computation. Therefore, the drift of the full ND readout system and the M16 will be characterised with respect to the un-corrected drift of the VA-FEE.

$$
\begin{aligned}
& G_{P M T}=\mu(P M T) / N_{P E} \\
& \text { where } \\
& N_{P E}=(\mu(P M T) / \sigma(P M T))^{2}
\end{aligned}
$$

The LI can also be used to measure the absolute gain of each pixel. This computation can be carried out using photon-statistics, as described in Equation 5.6, where $\mu(P M T)$ and $\sigma(P M T)$ stands for the mean and RMS from the charge distribution measured by a PMT pixel as light at the same light level is injected. The measured the gain is indicated by $G_{P M T}$, which can be known to a few percent level (1000 injections) through this technique -normally limited by statisticsbecause the error in RMS.

\section{Demonstration: PMT Gain Long Term Effect}

Once the LI system was fully functional ${ }^{4}$ in the FD, a trend was identified in the PMT gains. The gain of the readout system was found to depend on the associated crate number. Further studies, not included in this thesis, discarded showed that the pattern did not belong to the FEE, proving therefore to belong to the PMTs, due to a long term variation ("ageing"), as shown in Figure 5.12 (top). The relation between crate numbering and the PMT gain is due to the fact that the crate numbering scheme and time (from the installation of the PMTs) are correlated: the first plane installed was at crate 0 and 1 .

The total amplitude of the effect is $\sim 15 \%$, as shown in Figure 5.12 (bottomleft). Figure 5.12 (bottom-right) shows the rate of gain variation decreases in older PMTs (lower crate number). This is consistent with an asymptotic saturation of the increase of the gain with time. Once this effect was first discovered at the FD [113], it was also identified in the M16 test-stands as summarised in [71]. The mechanism for this "ageing" effect is, however, not well understood.

On the top of being an interesting feature of the M16s, this change of the gains in the PMTs represents no problem for MINOS since the LI-system, used to

\footnotetext{
${ }^{4}$ Particularly after the LED output was tuned for LI-Drift-Point runs.
} 

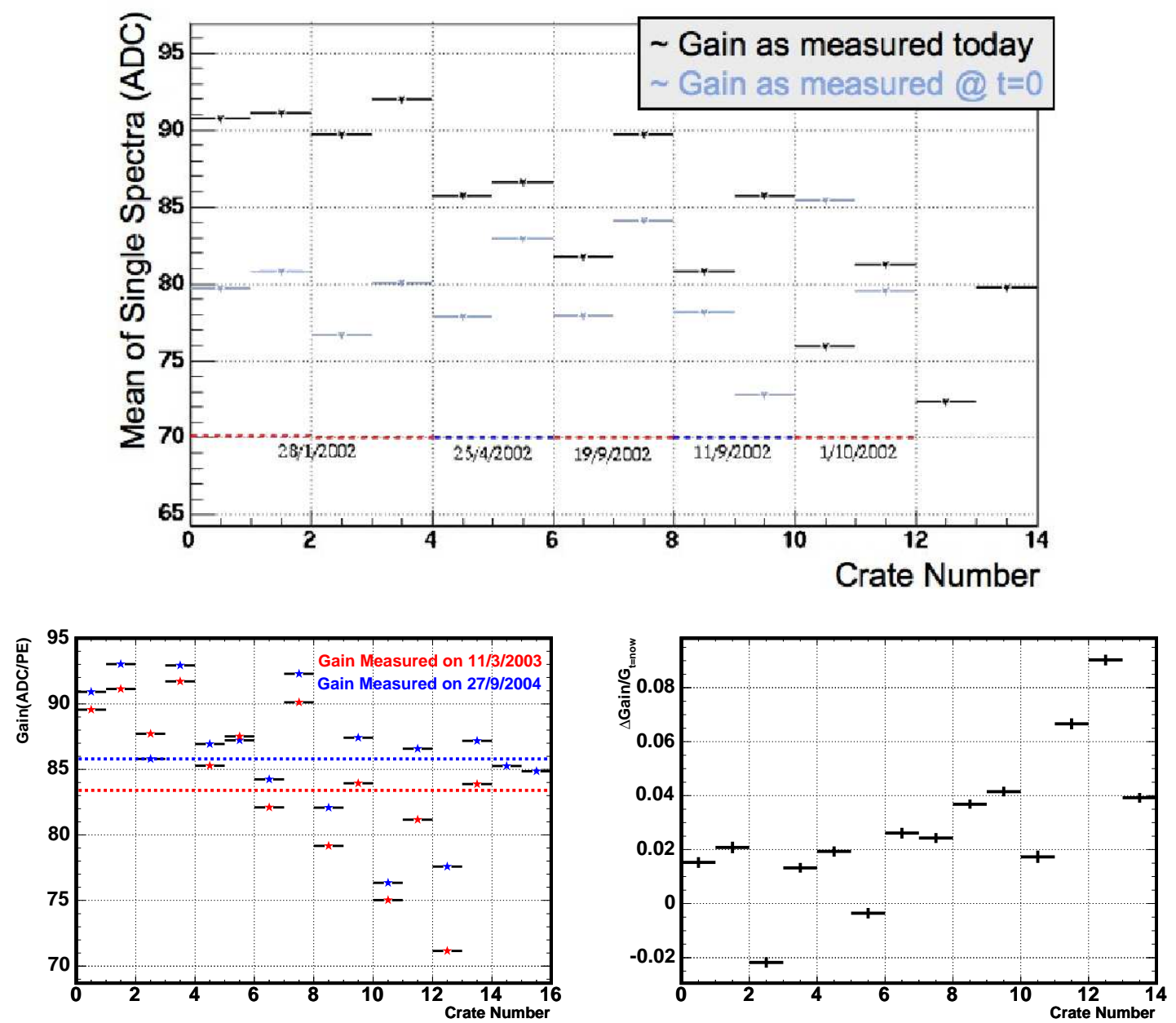

Figure 5.12: Long Term PMT Gain Variation at FD. The top plot shows average gain (as estimated with singles) versus crate number, as measured in March 2003 (labelled "today") in black and as measured when the respective crates have just been commissioned (labelled: "@ $\mathrm{t}=0$ ") - the corresponding dates are explicitly enclosed in the plot. Note the the was no slope at $t=0$ - pale-blue data points, therefore the gain has increased after installation : ageing effect. The plots at the bottom show a few more quantitative features about the effect: i) (bottom-left) the average increase in a year has been about $+2 \%$ (difference between blue and red dashed lines). Note that to the measured $+2 \%$ gain increase, we should add the expected effect due to $\Delta T \sim 2.2^{\circ} C:+1.5 \%$. So, accounting for the gain drift, the effect is sized to be about $+3.5 \%$ altogether. ii) (bottom-left) the rate of change of gain depends on the crate number in a manner which is consistent with an asymptotic saturation of the effect with time.

discover/characterised it, can of course calibrated out. 


\subsubsection{Linearity of Readout Systems}

The linearity of the readout system (PMT $\oplus \mathrm{FEE})$ can be studied/calibrated using the LI-system by taking LI-Gain-Curve runs. The non-linearity of the each channel of the readout system will be characterised/corrected using the linear response of the PIN as a reference. When dealing with the VA-FEE, the non-linearity of the VA-FEE, inherited by the PINs, should be generally corrected for first, as described in Section 5.3.2; so that the response of the PIN becomes linear.
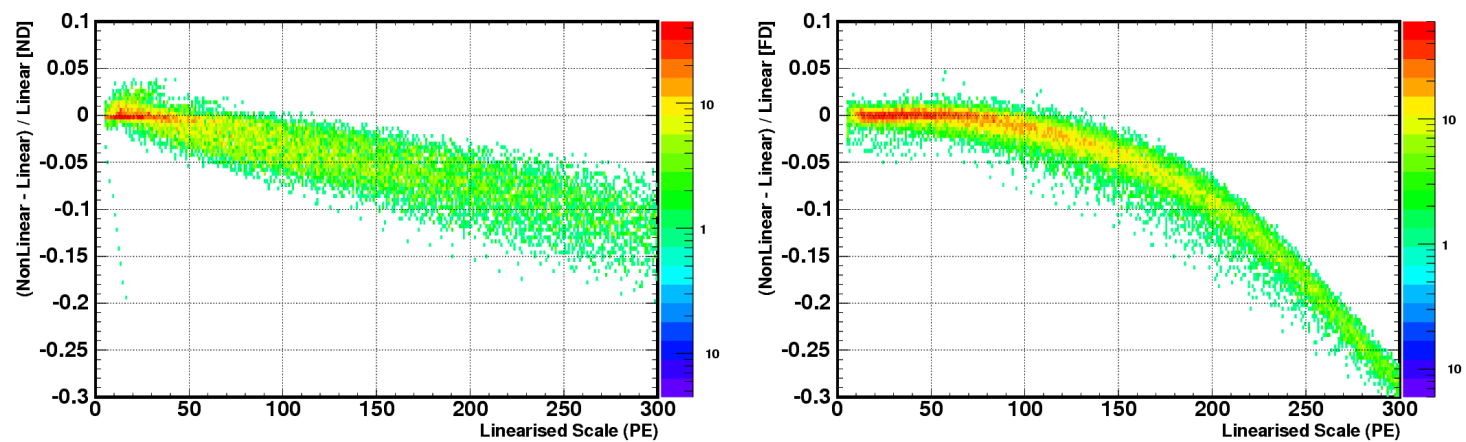

Figure 5.13: Readout System Non-linearity Measurement. The relative non-linearity fraction verses the injected charge is shown for the ND (left) and FD (right) readout systems, as measured by the LI-system. The non-linearity fraction appears to be characterised in PE, rather than ADC. The PE scale used for this plots is actually an ADC scale multiplied by the average PMT gain of each readout system $(65 \mathrm{ADC} / \mathrm{PE}$ for FD and $81 \mathrm{ADC} / \mathrm{PE}$ for ND). This approach was taken such that both patterns are shown in comparable forms.

The LI linearity correction method relies on the readout system to be linear at low charges. A linear fit is carried out over the linear regime of the readout system measured response. The difference between the linear fit and the measured response is then parametrised and used to correct (residuals) for the non-linearity exhibited at high charges (channel-by-channel). The residuals from that fit are shown in Figures 5.13 for the ND (left) and FD (right) side, as measured at CalDet during the Near/Far running.

The non-linearity generated by the PMTs is believed to be a consequence of the field density effect at the anode of each PMT pixel. Therefore, the PMT nonlinearity is determined, to first order, by the rate of charge incident to each pixel's anode. This has two consequences:

- The charge pulses generated at the PMTs by the LI-system and by a particle striking the scintillator must be nearly identical for the LI-system to be able 
to correct for the non-linearity of both readout systems accurately. This is a critical constraint of the LI-system that has been verified during the analyses carried out for this thesis.

- The correct framework to characterise the non-linearity is in ADC (charge).

\subsubsection{ND and FD Non-Linearity Parametrisation}

The need for the parametrisation of the non-linearity of the ND and FD readout systems is two fold.

- The linearity calibration per channel was not made available for the Near/Far running period at CalDet.

- During the main analysis of this thesis, it was realised that none of nonlinearity models then implemented described the data accurately.

Hence, the non-linearity information was extracted from an LI-Gain-Curve run data. The shape of the parametrised non-linearities was then fed into the detector simulation packages. Note that the parametrisation extracted account for the average non-linearity trend, while the channel-to-channel differences are simply ignored.

The overall non-linearity of the ND readout system is shown in Figure 5.14(top), while the non-linearity of the FD readout system is shown in Figure 5.14(bottom). The non-linearity of the M16 is also shown in Figure 5.14(top). The M16 nonlinearity was obtained by subtracting the average effect of the non-linearity of the VA-FEE to the responses used to compute the non-linearity of the FD readout system for each channel. Since the QIE-FEE is virtually linear, Figure 5.14(top) represents the non-linearity of the M64. Note that the non-linearity of both PMTs has resembling shapes. An interesting fact is that the M64 appears to be about $2 \times$ more non-linear than the M16 beyond $\sim 100 \mathrm{PE}$.

The parametrisation of the non-linearity of the M16 and M64 have been implemented in the detector simulation software of MINOS. The parametrisation of both PMTs has been found to be well-described by a simple straight line beyond a threshold. Although evidence will be shown suggesting that the bump shown in the non-linearity curve of the M64 may be real - a $0.4 \%$ effect. 

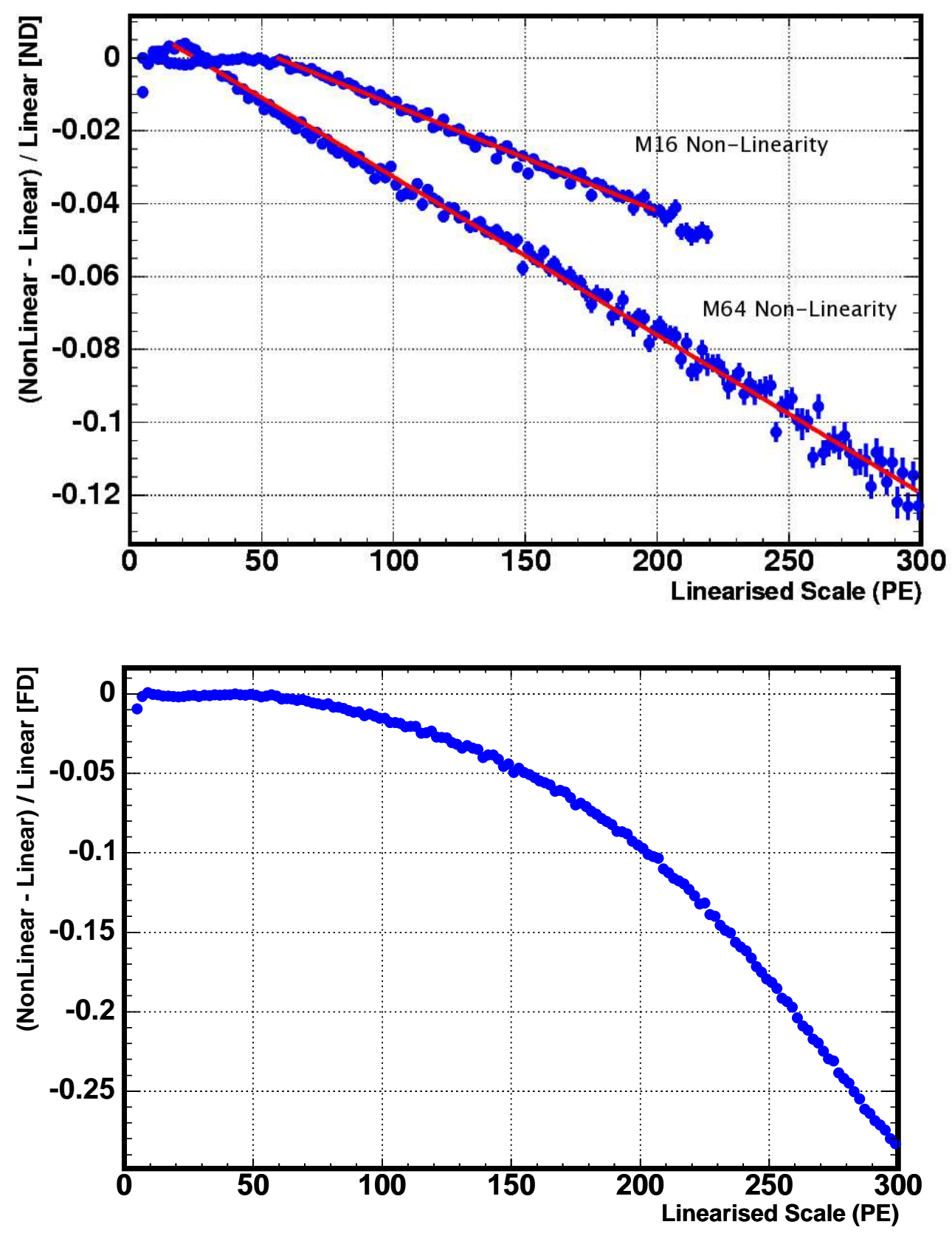

Figure 5.14: ND and FD Readout Systems Non-Linearities. Top figure shows the PMT non-linearity from the M16 and M64. Linear fits are shown in red that appear to characterise the fractional non-linearity of both PMTs accurately. Bottom figure shows the non-linearity of the full FD readout system: M16 $\oplus$ VA-FEE. 


\subsection{Muon Calibration}

Atmospheric muons are used as the common source for calibration across all the MINOS detectors. They are essentially the only source of radiation copiously available in the three detectors and their behaviour is relatively well understood.

\subsubsection{Intra-Detector Calibration: "Strip-to-Strip"}

The intra-detector (or "S2S") calibration uses the response of through-going muons, which have an average energy deposition that is characteristic of minimum-ionising particles. The objective of the S2S calibration is to compute calibration constants that correct for the effective strip-to-strip differences. The inherent strip-to-strip difference is about $\sim 25 \%$. This difference can arise from many sources, such as effective light yield differences, pixel-to-pixel gain variations, optical light-path efficiencies and photo-cathode efficiency ${ }^{5}$.

The S2S calibration was performed whenever certain changes have been made to the detector, such as swaps of fibres, etc. This was particularly critical at CalDet, since every time the detector was dismantled/mounted to allow displacements across the PS-beam complex a new S2S calibration was needed.

$$
\mu_{i}(\mathrm{SIGCOR})=\mu_{i}(\mathrm{SIGLIN}) \times C_{i}^{S 2 S}, \quad \forall i \text {-th strip }
$$

Once the S2S calibration has taken place the strip-to-strip responses will be normalised to the average strip response across each detector. The output of the S2S calibration is a corrected pseudo-ADC unit of charge called SIGCOR, as defined in Equation 5.8, for which the strip-to-strip spread has been reduced. SIGCOR is a unit proportional to the amount of light produced in any strip corrected to behave like the average scintillator strip in the detector. The uncertainty on each calibration constant is generally statistically limited to $\sim 2 \%$, the systematic error will be explored during the forthcoming analysis discussion. Details on the implementation of this calibration are described in [108] [107] [114].

Finally, there are two characteristics intrinsic to the implementation of the S2S calibration, which need to be introduced.

- A tracker has been used to identify the muons used to compute the calibration, and the corresponding hits associated with their energy deposition.

\footnotetext{
${ }^{5} \mathrm{~A}$ convolution of the quantum and collection efficiency.
} 
Therefore, to first order, PMT-crosstalk hits are not included into the computation of the S2S calibration constants. In other words, the S2S calibration is computed from "tracked-responses". This is because the tracker rejects hits which appear not to be topologically linked to the core of the muon track. Most of such isolated hits correspond to PMT-crosstalk. This fact will have an effect on the response normalisation caused by the S2S calibration, depending on whether the responses are "tracked" (without PMT-crosstalk) or "total" (with PMT-crosstalk).

- The tracked-responses used for the S2S calibration computation has been corrected for attenuation along the strip. Therefore, responses are calibrated out by the S2S calibration if they originated from the centre of the strip. In other words, the S2S calibration defines a "calibration-axis" along the geometrical axis of the detector (locus of the centre of all strips) on which the calibration is valid. If the responses originated off the calibration-axis, attenuation has to be taking into account. This constraint is the main motivation for the attenuation calibration implemented for this analysis, described in Section 5.5.3.

\subsubsection{Inter-Detector Calibration: "MIP"}

The relative inter-detector (or "MIP") calibration is intended to determine a common scale for all the MINOS detectors. The principle of this calibration is to define of an arbitrary but specific energy unit (called MIP) based on the response of stopping muons that can be measured precisely in all MINOS detectors. This unit is used as a calibration "candle" to normalise (or inter-calibrate) the scales of all detectors to a common energy scale: the MIP-scale. Stopping muons are obtained at CalDet from the beam, while cosmic muons are used at the other MINOS detectors.

The technique developed [115] defines the MIP based on a window (called MIPwindow) over which a very specific fraction of the energy deposited by the stopping muons is integrated. The MIP-window is located in the most stable part of the energy deposition of a stopping muon, i.e. the logarithmic rise of the muon stopping power $(d E / d x)$, far from the end of the track where the muon decays and large fluctuations occur. The MIP-window is defined with respect to the end of the stopping muon track, therefore the energy deposited over the MIP-window is independent from the initial energy of the stopping muon. Figure 5.15 shows the 


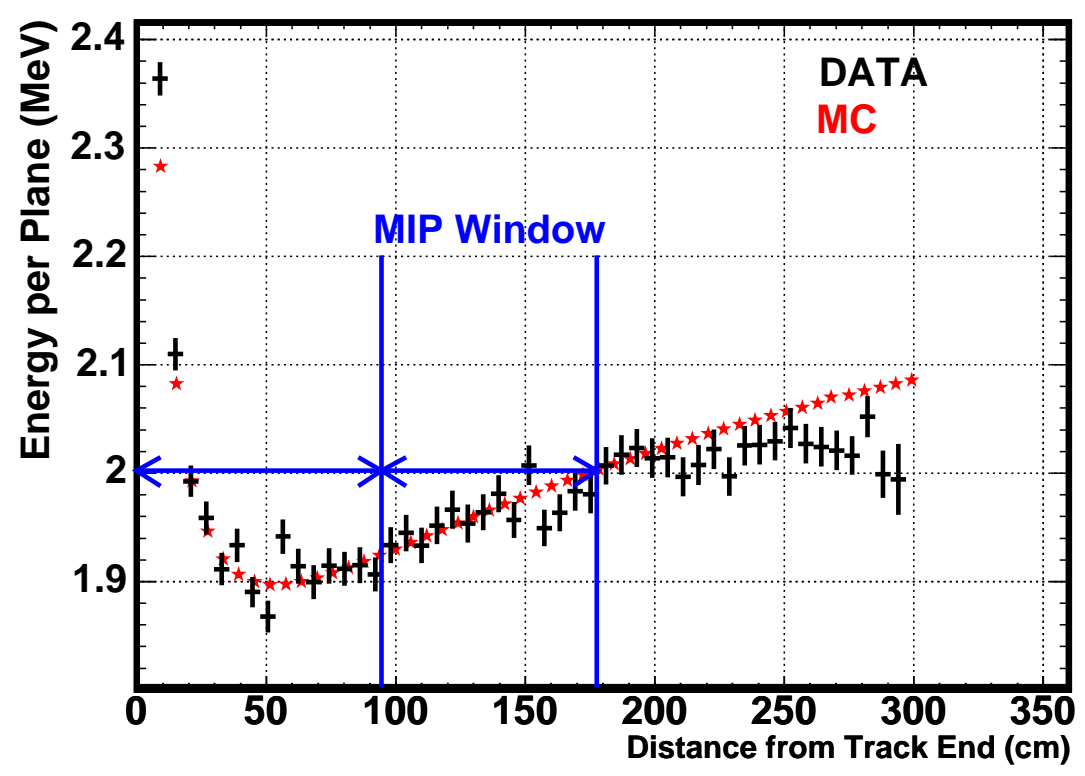

Figure 5.15: MIP Calibration Definition: MIP Window. Plot [115] shows the definition of the MIP-window. The window is situated at an exact location with respected to the end of the track. The energy deposited within the the MIP-Window is used to define the MIP unit. Data (black) and MC (red) are shown. The scatter present on the data (muons at $1.8 \mathrm{GeV} / \mathrm{c}$ ) is due to the combination of low statistics and calibration problems on the S2S calibration constants at few percent level, typically $<2 \%$.

MIP-window definition for the CalDet detector.

$$
\mu_{i}(\mathrm{MIP})=\mu_{i}(\mathrm{SIGCOR}) \times C^{M I P}, \forall i \text {-th strip }
$$

\begin{tabular}{|l|c|}
\hline MIP Constant & Light Level \\
\hline 608.7 (SIGCOR/MIP) & $\sim 9(\mathrm{PE} / \mathrm{MIP})$ \\
\hline
\end{tabular}

Table 5.2: MIP Calibration Constant for CalDet Near/Far 2003.

The MIP calibration relies strongly the intra-detector calibration. Therefore, the MIP calibration is performed whenever the S2S calibration is computed. The output of the MIP calibration is a constant, as explicitly indicated in Equation 5.9. Each detector has its own MIP constant. The MIP constant normalises the calibrated responses measured in each detector. Once the inter-detector calibration has been carried out, results obtained with one detector can be translated across all MINOS detectors. 


\subsubsection{Attenuation Calibration at CalDet}

The need for attenuation measurements, as explained before, is due to the fact that the S2S calibration has effectively defined a calibration-axis along which the responses on both sides of the detector have been calibrated. As the Near/Far Comparison analysis relies on the comparison of the responses as measured at either end of the strips, attenuation has to be taken into account. Otherwise, if the energy deposition (a hit) happened off the calibration axis, the readout system at the closer end of the strip should measure a higher response, inducing an apparent systematic difference between both readout systems.
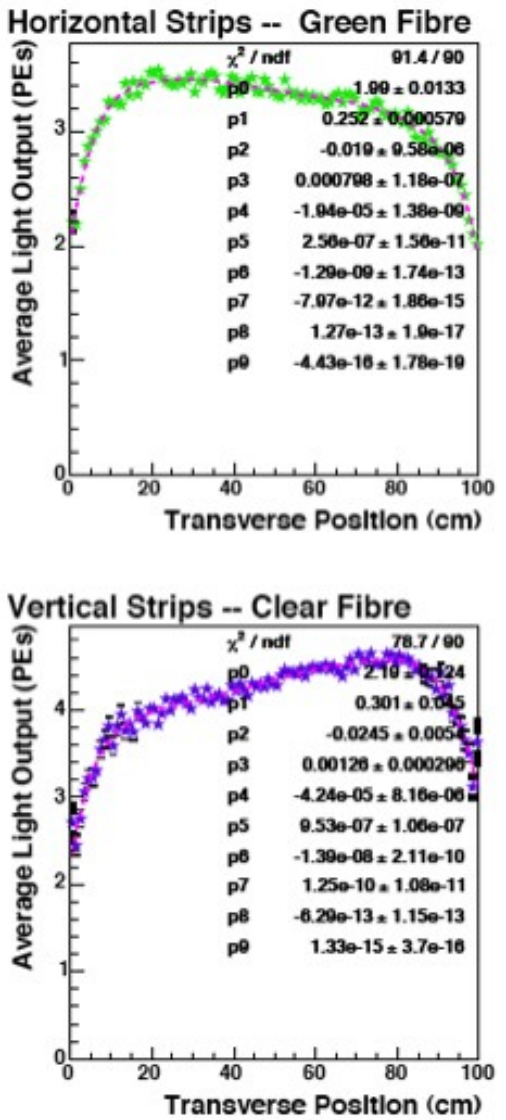
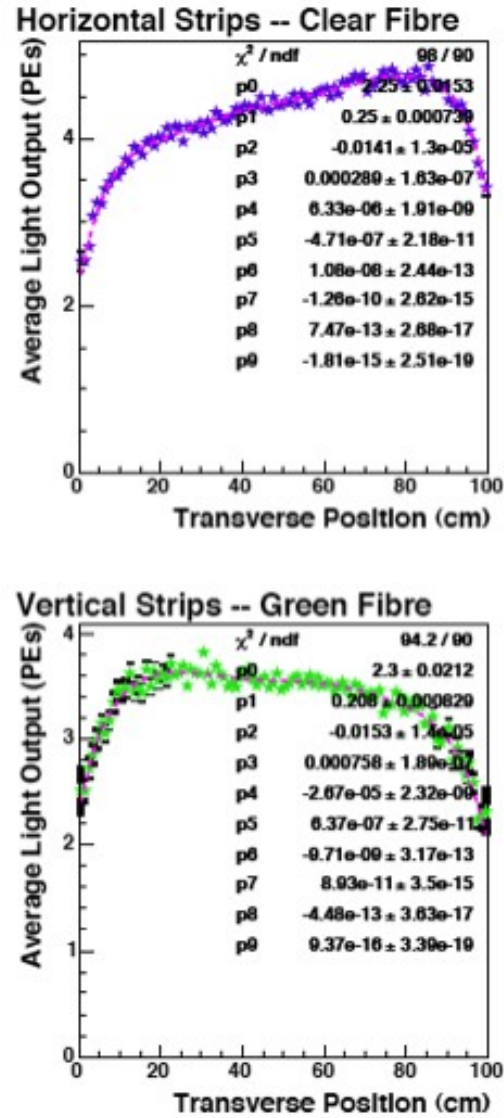

Figure 5.16: Attenuation Measured with Cosmic Muons Plot from [107].

At CalDet, the standard attenuation correction (MAPPER calibration) was not available [116]. This was generally not a problem at CalDet as the beamspot was set to be almost coincidental to the geometrical centre of the detector, and therefore the calibration-axis. The difference between the beam-spot and the 
detector centre is $\sim 2 \mathrm{~cm}$ at most per view. Therefore, the effect of attenuation is essentially negligible for all analyses which add the responses over both sides. The effect of attenuation is illustrated in Figure 5.16, as measured with cosmic muons, where the average response of cosmic muons is mapped across the length of the CalDet strips, as seen by the four views.

This section, therefore, describes the two attenuation calibrations developed for the specific needs of the Near/Far comparison.

\section{Integrated Attenuation Correction}

This approach relies on MC events to quantify the expected attenuation as a function of the beam-spot location relative to the calibration-axis. MC electrons were "injected" at different locations with respect to the calibration-axis ranging over a couple of strips. The parameter $\alpha$ was computed, as shown in Equation 5.11, to characterise the measured relative response difference between both readout systems for each electron injection.

$$
\alpha=\frac{\mathcal{N}-\mathcal{F}}{\frac{1}{2}(\mathcal{N}+\mathcal{F})}
$$

Hence, a linear map of the integrated attenuation can be computed as electrons sweep the face of the detector. The integrated attenuation correction $\left(f_{a}\right)$ corresponds to the slope of the of the curve shown in Figure 5.17, where $\alpha$ is shown as a function of the location of the electron injection, when varied vertically (Ydirection). The exhibited variation is only distinguishable in the EVEN-view, as strips are oriented horizontally. Every injection corresponds to 5000 electrons.

Note that this correction has been computed using the calorimetric responses of the $\mathrm{ND}(\mathcal{N})$ and $\mathrm{FD}(\mathcal{F})$, i.e. the summed effect of attenuation over all strips hit by the MC electrons. The beam-spot was varied independently in X (not shown) and $\mathrm{Y}$ directions, such that the respective ODD and EVEN integrated attenuation corrections could be parametrised. The parametrisation of both curves is summarised in Table 5.3.

This approach allows the measurement of the overall attenuation correction for a whole event merely by knowing the location of the event relative to the S2S calibration-axis (at $\mathrm{X}=12$ strip and $\mathrm{Y}=12$ strip). Once the attenuation has been parametrised, it can be applied to either readout system, as indicated in Equation 5.11. 


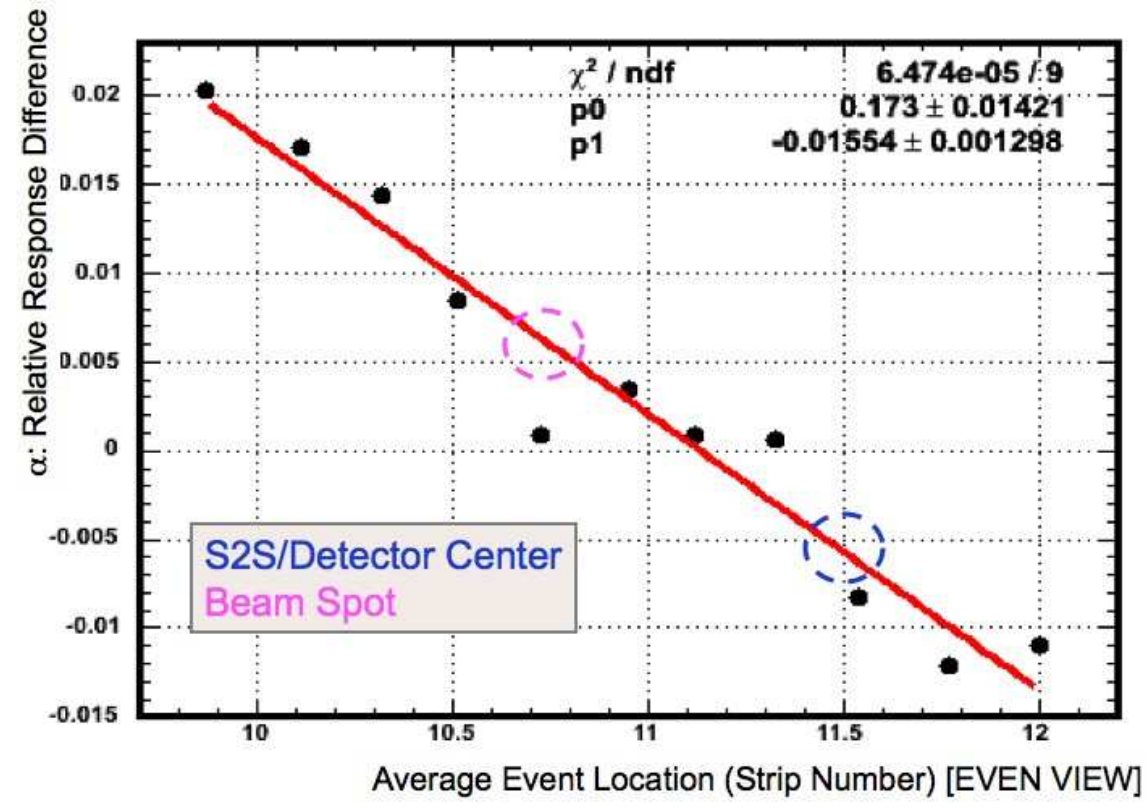

Figure 5.17: Integrated Attenuation Curve at CalDet.

$$
\text { hence }\left\{\begin{array}{l}
\mathcal{N}(x)=\mathcal{N}-\frac{f_{a}}{2}\left(x-X_{\text {BeamSpot }}\right) \times \mathcal{N} \\
\mathcal{F}(x)=\mathcal{F}+\frac{f_{a}}{2}\left(x-X_{\text {BeamSpot }}\right) \times \mathcal{F}
\end{array}\right.
$$

\begin{tabular}{|l|c|}
\hline Zero Attenuation @ & Strip 12 in X and Y \\
\hline Slope EVEN $(\mathrm{Y})$ & $(-1.55 \pm 0.13) \% /$ Strip \\
Slope ODD $(\mathrm{X})$ & $(+1.70 \pm 0.14) \% /$ Strip \\
\hline Beam-spot EVEN $(\mathrm{Y})$ & Strip $\in[11.1,11.5]$ \\
Beam-spot ODD $(\mathrm{X})$ & Strip $\sim 11.4$ \\
\hline
\end{tabular}

Table 5.3: Integrated Attenuation Computation Measurements.

An interesting property of this technique is that the attenuation correction has the ability to account precisely for the location of the beam-spot (event-by-event and/or run-by-run). This ability matches is necessary since the beam-spot changes with the beam momenta. The amplitude of the beam-spot variation can be up to $\sim 0.4$ strip $(\sim 1.6 \mathrm{~cm})$ in the vertical plane for electrons, as shown in Figure 5.18. The variations in the horizontal view are consistent with being fixed for all beam momenta. This is agreement with the fact that the last dipole magnet prior to the detector stirs the beam vertically. 
Implementation Details \& Uncertainties The integrated attenuation calibration essentially depends on two contributions: the measurement of the average event location and the computation of $f_{a}$.

The Beam-Spot Location Algorithm: The event location algorithm is based on charge weighted centre of mass calculation using hits within the first 24 planes. The PMT-crosstalk hits were rejected by limiting the calculations to digits falling within a central fiducial volume: only most central 12 strips. Additionally, digits whose charge was $<1.5 \mathrm{PE}$ were also rejected from the calculations. This low charge digits would contribute little to the accuracy of centre-of-mass location while they may degrade the precision.

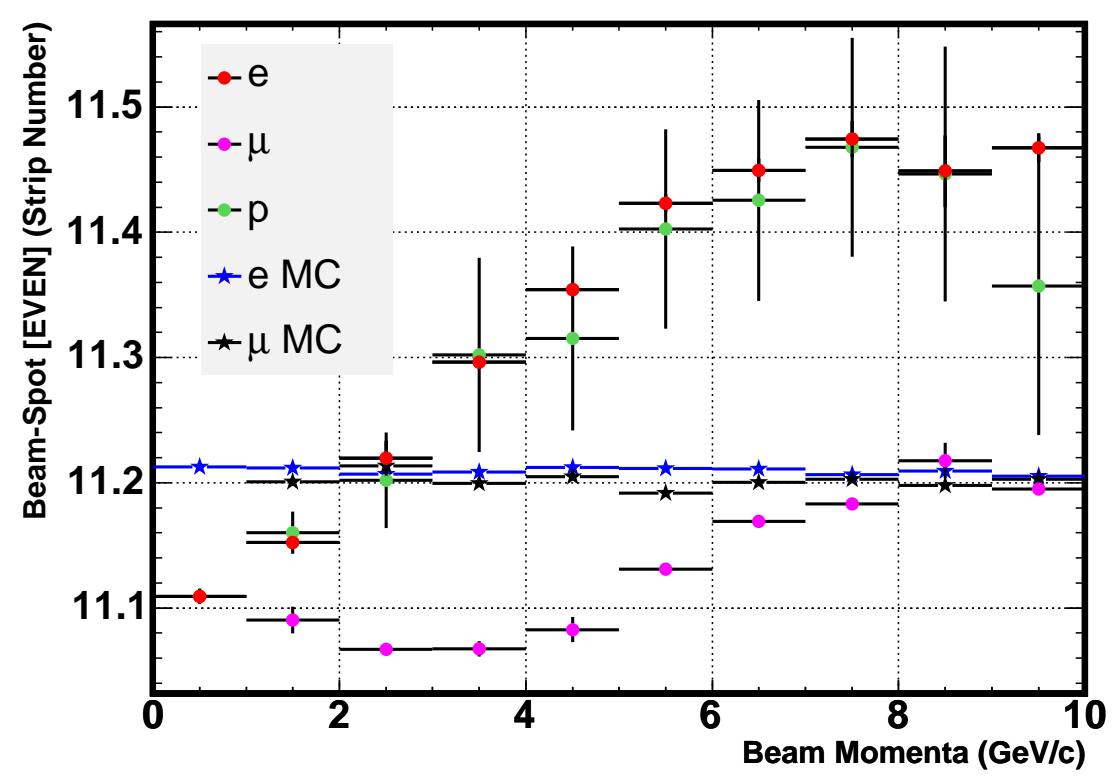

Figure 5.18: Beam Spot Variation at CalDet.

The precision of the algorithm was excellent even for electrons at the lowest energies. This was demonstrated with MC electrons (and muons) generated at the same beam-spot for all energies, as shown in Figure 5.18. The average location of the events exhibits very little scatter across all energies. The amplitude of the scattering is $<0.01$ strips, which would induce a negligible $<0.02 \%$ relative uncertainty on the attenuation correction.

The accuracy of the event location determination is, in principle, not very important, as the same algorithm is used for the implementation -with MC 
events- and application -on data and MC events- of the attenuation calibration. Therefore, any possible systematic bias in the event location determination cancels on the application of the attenuation correction. However, the accuracy of the algorithm was somewhat proved by the fact that its output is independent particle. MC muons and electrons injected at the same beam spot, but having very different hit patterns, are found to be located at the same beam-spot within uncertainties, as shown also in Figure 5.18.

The variation of the beam-spot of electrons was in good agreement with that for protons and pions. The muon beam-spot seem to have its own trend speculated to be due to the fact that muons arise from the decay from pions which could change the average beam-spot location.

Computation of $f_{a}$ : The computation of $f_{a}$ relies intrinsically on the average knowledge of the attenuation parametrisation (available in MC), as no specific attenuation parametrisation of the CalDet fibres exist. No large deviations are expected between the two, but the attenuation correction must be very accurate indeed, as the magnitude of the correction is about $\sim 1 \%$ per view, at most. The level of agreement between the average attenuation parametrisation with respect to CalDet specific values is not known. Therefore, this attenuation correction needs to be cross-checked (see Section 7.5.4) with the results obtained from the $4 \times$ MIP calibration, which will be described next.

The computation of $f_{a}$ is believed to be particle-independent as its calculation used:

- MC events having no dependence on the status of calibration constants, that may affect different hit-patterns.

- same attenuation parameters over all strips, therefore, again, there is no hit-pattern dependence.

- events well within the linear regime, therefore, no event light level dependence.

So, the uncertainty of the attenuation correction is dominated by the uncertainty on the slope computed for every calibration curved (summarised in Table 5.3), which can be as high as $\sim 8.5 \%$, leading to a $\sim 0.2 \%$ as the relative error on the attenuation correction. 


\section{$4 \times$ MIP Calibration}

The second approach to correct for attenuation is to use the response of stopping muons to calibrate out the effect of light attenuation. Actually, this calibration does more than merely correcting for the attenuation correction. This calibration uses the framework already set up for the MIP calibration to effectively "intercalibrate" both readout systems, just like the MIP calibration would do for the actual Near and Far detectors, but within the same physical detector: CalDet. The implementation of the $4 \times$ MIP calibration is indicated in Equation 5.12.

$$
\mu_{i}(\mathrm{MIP})=\mu_{i, V}(\mathrm{SIGCOR}) \times C_{V}^{M I P}, \forall i \text {-th strip and } \mathrm{V} \text {-th view }
$$

Beam stopping muons are incident to the CalDet at the beam-spot, as well as all other beam particles. Therefore, the response of muons should reflect the corresponding attenuation effect in all different views. So, four MIP constants (one per view) are provided such that the effect of attenuation, as measured by stopping muons, is calibrated. The principle of this calibration is to equalise the response of each view to that of beam stopping muons so that all possible effects common to all beam particles, like attenuation, become calibrated. An illustration of the principle is shown Figure 5.19.

\begin{tabular}{|l|c|}
\hline Views & MIP Constant \\
\hline \hline ND-Even & 296.7 \\
ND-Odd & 305.4 \\
FD-Even & 307.4 \\
FD-Odd & 307.9 \\
\hline
\end{tabular}

Table 5.4: $4 \times$ MIP Calibration for Near/Far Running.

An important advantage of the $4 \times$ MIP calibration is that it could also correct for a calibration systematic uncertainty evenly affecting any view, as induced by the S2S calibration. This is the reason why we compute a " $4 \times$ MIP" rather than a " $2 \times$ MIP" calibrations, that would suffice to account for the attenuation between ND and FD sides. The motivation is a known EVEN/ODD asymmetry that has been introduced in the past by the S2S calibration.

The one disadvantage of the $4 \times$ MIP calibration is the intrinsic assumption that stopping muons would account for all possible calibration uncertainties the response of any other beam particle. This assumption is not true as we already 

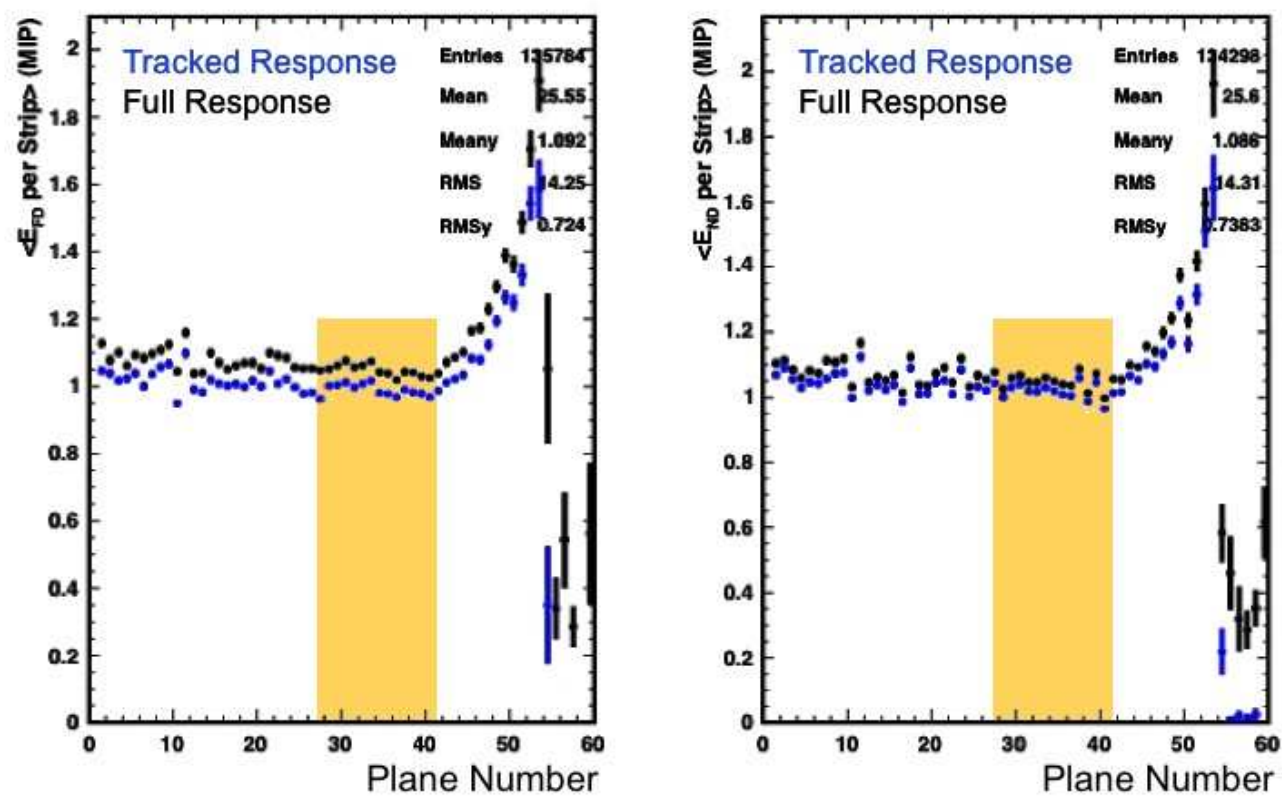

Figure 5.19: $4 \times M I P$ Calibration Definition. This figure shows the average muon energy per plane total and tracked blue for the FD (left)and ND (right). The run used in an $1.8 \mathrm{GeV} / \mathrm{c}$, for which beam muons stop in the detector, as illustrated by the sharp increase in the energy deposited per plane peaking at plane $\sim 55$. The MIP constants are computed by integrated the energy within the MIP-window (orange) for each different view. The tracked to total response different provides a quantitative account of the PMT-crosstalk - relative difference between black and blue curves. Note that there is some up-down periodicity in the plane responses, which is thought to be caused by the mentioned EVEN/ODD asymmetry induced by the S2S calibration (using tracked responses). Computing a MIP constant per view $(4 \times$ MIP calibration $)$ the attenuation, the PMT-crosstalk differences and some of the calibration systematic observed can be corrected in principle.

know (see Figure 5.18). Muons have a generally different beam-spot, therefore the attenuation correction delivered by the $4 \times \mathrm{MIP}$ calibration is estimated to differ by up to $\sim 0.5 \%$ per view. Further discussion about the performance of the $4 \times \mathrm{MIP}$ calibration will be covered in Section 7.5.4.

\subsection{Absolute Energy Calibration}

This is the stage of the calibration chain where the light measured by the detector, which is proportional to the energy deposited by the different particles, is associated to the actual energy of the incident particle. At CalDet, a conversion factor for each different particle is obtained: "Single Particle Absolute Calibration Factor". 
Whereas, for the MINOS detectors exposed to neutrinos, the absolute calibration consists of estimating the energy of the originating neutrino from the visible energy measured in the detector. In this case, not only the particles observed by the type of neutrino interaction may play a role on the neutrino energy determination.

\subsubsection{Energy Calibration at CalDet}

The absolute energy calibration for beam particles at CalDet is the stage of the calibration that sets the MIP/GeV ratio. This ratio quantifies the amount of light produced in the scintillator (related to the sampling ratio) per $\mathrm{GeV}$ of the incident particle traversing the detector. The MIP/GeV ratio is different for different particle types, as different particles have different energy deposition mechanisms given by the physics dominating their interactions with matter over the energy range in question.

$$
E_{\lambda}(\mathrm{GeV})=C_{\lambda}^{A B S} \times \sum_{\text {Over }_{i}} \mu_{i}(\mathrm{MIP}), \quad \text { where } \lambda \equiv \mathrm{e}, \mu, \text { hadrons. }
$$

Once the MIP $/ \mathrm{GeV}$ ratio is known for the particle $\lambda$ (i.e. $e / \mu / \pi / p$ ), the response of such a particle can be expressed in $\mathrm{GeV}$, as indicated in 5.13. The amount of light produced in the scintillator is proportional to the amount of energy deposited in the scintillator, which in principle is independent from the particle type. However, scintillator light emission effects such as saturation (characterised by "Birk's Law") and/or light thresholds, could affect the apparent energy deposition of particles in particle-type dependant way.

\begin{tabular}{|l|l|}
\hline Absolute Calibration (electrons) & $(50.54 \pm 0.10) \mathrm{MIP} /(\mathrm{GeV} / \mathrm{c})$ \\
Absolute Calibration per Side (electrons) & $(25.27 \pm 0.05) \mathrm{MIP} /(\mathrm{GeV} / \mathrm{c})$ \\
\hline
\end{tabular}

Table 5.5: Absolute Calibration of Electrons.

An electron absolute energy calibration has been performed for this thesis, relying on the knowledge of the beam momenta as a reference. The beam momenta is believed to be known to $\sim 1 \%$. Figure 5.20 shows the fit from which the MIP $/ \mathrm{GeV}$ calibration ratio of electrons is obtained. The $\mathrm{MIP} / \mathrm{GeV}$ ratio is the slope of the curve, while the non-zero but negative intercept (about -4MIP) is due to the average energy loss of the electrons prior to the instrumented region of CalDet used, such as plane-0 (ignored) and material in the beam-line (like PID instrumentation). 


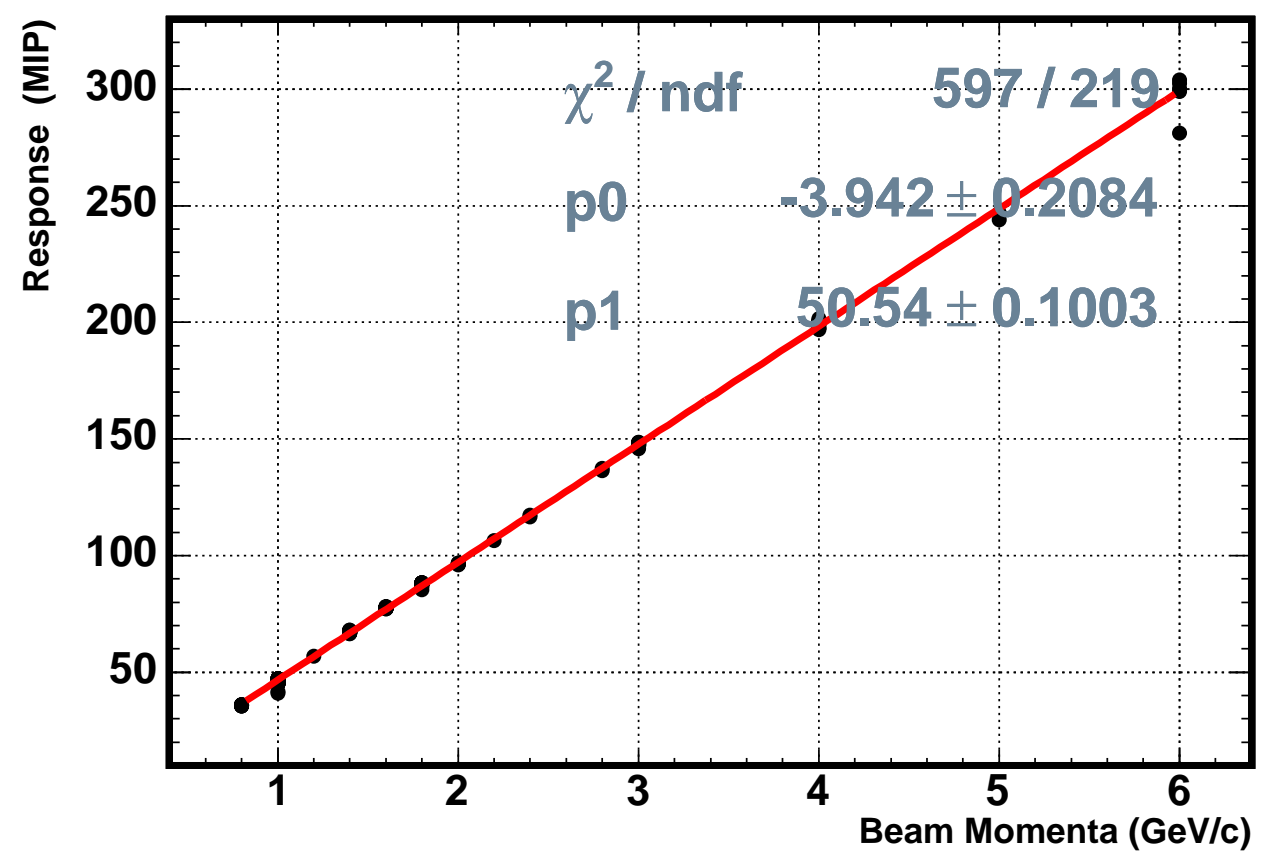

Figure 5.20: Absolute Energy Calibration of Electrons.

Note that, for electrons, energy and momenta are essentially interchangeable (the difference is $\left.\mathcal{O}\left(10^{-3}\right)\right)$.

The absolute energy calibration at all particles available at CalDet have been studied thoroughly in [75] [74] [115].

\subsubsection{Neutrino Energy Calibration}

The energy of the neutrino is inferred from the energy deposited in the detector, the so-called $E_{\text {visible. }}$. The amount of energy visible is dependent on the type of neutrino interaction and the nature of the detector. Energy thresholds, sampling ratios and target material of the detector-dependent factors, which enter into the determination of the neutrino energy. 


$$
\begin{aligned}
& E_{\text {visible }}(\mathrm{GeV})=E_{\mu}(\mathrm{GeV})+E_{\text {hadron }}(\mathrm{GeV}) \\
& E_{\text {hadron }}(\mathrm{GeV})=\sum_{\text {Over } \lambda}\left(C_{\lambda}^{A B S} \times \sum_{\text {Over }} \mu_{i}(\mathrm{MIP})\right)
\end{aligned}
$$

where $\lambda \equiv$ each particle created in the interaction.

Equation 5.15 refers to the estimation of $E_{\text {visible }}$ for the main MINOS neutrino oscillation channel: $\nu_{m u}$ CC. The energy of the muon $\left(E_{\mu}\right)$ is estimated from spectrometry (i.e. from "curvature") at high momenta and from range at low energies, if the muon is fully contained. The energy on the hadronic system ( $E_{\text {hadron }}$ ) is obtained from the energy sum (calorimetry) of all single daughter particles (indicated by the index $\lambda$ ) identified in each neutrino interaction.

$$
E_{\nu}(\mathrm{GeV})=f^{M C}\left(E_{\mu}(\mathrm{GeV}), E_{\text {hadron }}(\mathrm{GeV}), \nu \text { Interaction }\right)
$$

The fraction of the neutrino energy still invisible is to be estimated by the MC (through " $f^{M C}$ "), as suggested in Equation 5.16. 
Chapter 6

\section{Selection of Events}

In this chapter, the criteria and techniques developed for event selection will be described. The principle is very simple: the analysis will use the energy deposition of known particles incident onto the CalDet as a probe. The major concern behind the event selection is the possibility of biasing the analysis in any way. Therefore, a series of measures and cuts have been developed to ensure that both readout systems are exposed to the same physical events. As will be explained later on, this is not necessarily trivial due to the different triggering/functioning of both FEEs. The number of events accumulated during the data acquisition was generally far beyond that necessary for the level of precision needed. Therefore, the overall approach taken was to set rather stringent conditions to ensure purity in the event selection at the expense of statistics.

In summary, the goal of the event selection was, first, to minimise the likelihood of inducing systematic bias in the analysis and, second, to keep the purity of the event selection as high as possible.

\section{Meaning and Colour Coding of PDFs}

Much of the event selection relies on the application of 1D-cuts to distributions. Each distribution used for event selection will be referred as a PDF, standing for "Probability Distribution Function", even though the normalisation of the distribution will be kept to the number of events.

For clarity, a specific colour coding scheme for the PDFs has been adopted throughout this chapter. When showing the effect of a cut on the PDFs used, three PDFs will be shown simultaneously per cut. First, the "Raw PDF", to which 
no cut has been applied, will be coloured in red. Second, the "N-1 PDF", to which all cuts but the one under consideration have been applied, will be coloured in blue. And finally, the "Selected PDF", to which all cuts have been applied, will be coloured in yellow.

The N-1 distribution is particularly useful to illustrate the relevance of the cut in question in attaining a certain event sample relative to the other cuts applied. So, for example, if the N-1 distribution and the selected distribution were very similar, then the cut in question would not be crucial for the event selection intended. Conversely, if the same distributions were very different, then the cut would be critical for the event selection.

\subsection{Event Selection Cuts}

The cuts applied to each event can be broken down into four categories: quality, comparison, PID and topology cuts. These categories has been created to reflect both the nature of the implementation and of the objective of the cut, which is generally different.

\subsubsection{Quality Cuts}

The first series of cuts are called Quality Cuts. Quality cuts are designed to discard events suffering from any kind of known hardware anomaly which occurred during data taking. The impact of quality cuts was the rejection of, at most, $5 \%$ of all the accumulated events per run. The many reasons why an event was rejected by the quality cuts are listed below.

- VA Dead-Chips. Dead-chips (chips which are dead due to FEE intrinsic dead-time caused by a previous trigger) can be found on the VA-FEE by the OLT (see Section 4.4.2). The effect of dead-chips would bias the analysis since the FD response would only reflect some fraction of the total event energy. While the ND would record the full response. Events tagged as possibly affected by dead-chips are, therefore, rejected.

The only way that dead-chips can occur is if a beam-trigger becomes split into two events by the OLT. Therefore, an event tagged with dead-chips could be accompanied by an earlier event within the same beam-trigger whose information has also been truncated. Hence, the event prior to any event 
tagged as having dead-chips is also rejected. This was the major source for rejection of events.

- FEE Errors. Both readout systems were endowed with the capability of flagging different type of malfunctions at the FEE level. Any event having any kind of FEE error reported is rejected since this could, once again, bias the analysis.

- Light Injection Flashes during Beam Spill. During beam spill, the LIsystem was inhibited by the beam-trigger. However, very rarely, some LI events (i.e. LED flashes) occurred during the beam data taking mode. An LI event would contaminate the purity of the particle sample to be used for the analysis. The "triggering-PMT" ${ }^{1}$ is used to assert whether the event in question is itself or is contaminated by an LI flash and, if so, the event would be rejected.

- Check for Beam-Trigger Tag Quality. The beam-trigger tag (or ToFcoincidence signal) was a pulse whose output was set by the beam-trigger electronics to be within the dynamic range of the VA-chip and well above sparsification level. If the charge from the beam-trigger tag was anomalous (i.e. too low and too high) relative to its nominal value, the event was rejected. The rejection is issued under the suspicion that the beam-trigger could have been caused by a potential accidental or a malfunction of the trigger logic electronics.

- Check for the Quality of the ND Clock Jitter. During the Near/Far running, the time systems of the FD-side and the ND-side had to be synchronised together. The FD timing system was configured to send a signal to the ND timing system to restart every time-frame (time-frame $=1 \mathrm{~s}$ ). Since the internal clocks of both FEEs work at different frequencies $(40 \mathrm{MHz}$ for the VA-FEE and 53MHz for the QIE-FEE), the restart of the time-frame in the ND jitters with respect of the time-frame signal of the FD clock. The amplitude of the relative jitter should be about twice of the period of the ND clock ( $\sim 38 \mathrm{~ns})$. The system was set such that the time-frame start of the ND was recorded by a dedicated channel on the FD side. Therefore, the FD

\footnotetext{
${ }^{1}$ The Triggering-PMT is a dedicated PMT used to identify the occurrence of LI flashes. Dedicated fibres from the LI pulser box illuminate the triggering-PMT whenever a LI flash occurs.
} 
clock measures the jitter which is stored for offline analyses as a correction to attain accurate timing correlation between both readout systems.

The ND time-frame start signal was verified to have the expected charge (arbitrarily set by electronics, but known) and the correct timing features: amplitude of the jitter was about two buckets wide plus an known offset. If either (or both) conditions failed, the event would be rejected.

- Events within the Forced Dead-Time. A forced dead-time of about $70 \mu \mathrm{s}$ was implemented as part of the beam-trigger logic (see Section 4.4.2). If any two events were found to be separated by a smaller time interval than the forced dead-time, the later event was rejected. The occurrence of such events is either the indication that the trigger-logic did not work well or that the OLT has split a beam-trigger into more than one event. In either case, the event was not used for further analysis.

\subsubsection{Comparison Cuts}

The second kind of cuts are called Comparison Cuts. Comparison cuts were designed to prevent any bias due to the different intrinsic functioning of the VAFEE and the QIE-FEE. All events used on the analysis must fulfil the comparisoncuts.

\section{Time Fiducial Cut}

The time fiducial cut was designed to ensure that both readout systems have an equivalent acceptance to energy deposition. Upon trigger readout, the VA-FEE and the QIE-FEE are alive for different lengths of time, and, therefore, have different acceptances. The VA-FEE allows PMT dynode signals to trigger the readout for about $620 \mathrm{~ns}$ around the beam-trigger signal $(\sim 320$ before and $\sim 300$ after the beam-trigger $)$. The QIE-FEE reads out for about $\sim 380$ ns $(\sim 20$ buckets $\times 19$ ns per bucket), starting at about 50ns prior to the arrival of the beam-trigger. Therefore, the FD-side would be more likely to measure more events (and their depositions) than the ND-side per trigger. This poses a potential bias to the analysis, for which the comparison of the responses of both readout systems to the same event is essential.

The time fiducial cut aims to restrict the acceptance of both readout systems such their effective acceptances become equivalent. Achieving comparable accep- 


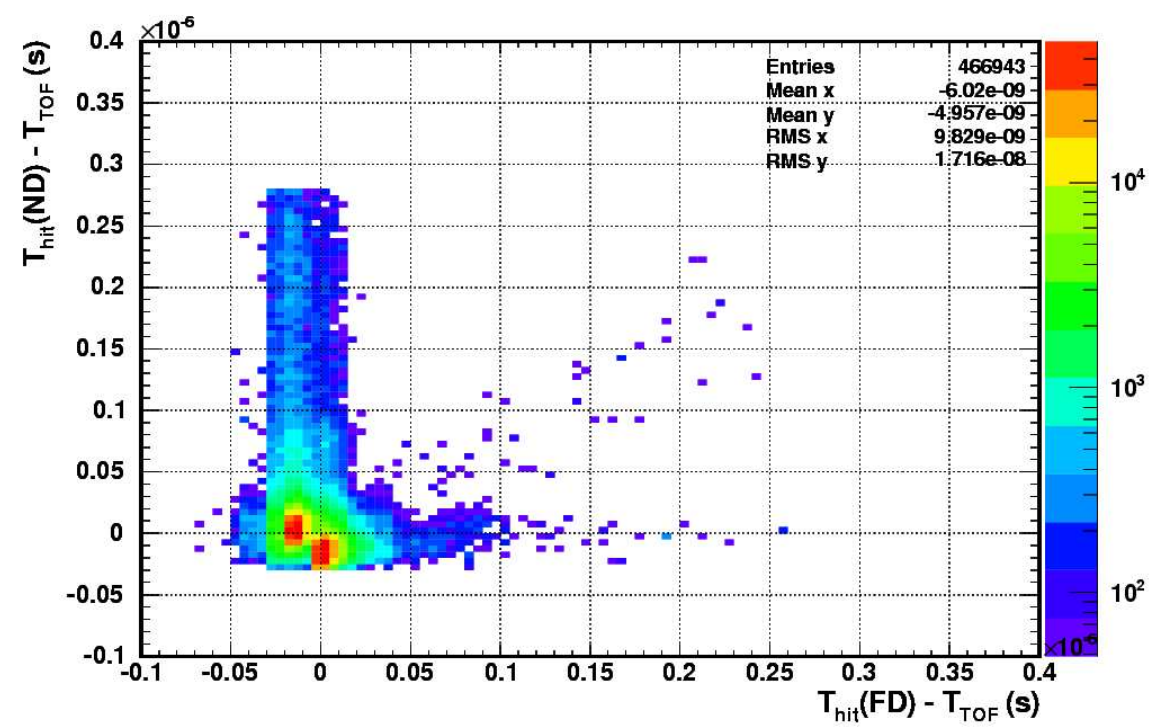

Figure 6.1: Readout Systems Time Correlation. This plot is meant to serve as an illustration on how the time-stamping works on both readout systems. The time of the digits with respected to beam-trigger $(a t t=0)$ is shown for the ND versus FD. The $\mathrm{z}$-axis has been weighted by the energy as measured by ND side. There are four identifiable regions: i) $T_{N D}=0 \& T_{F D}=0$ : both detectors measure the arrival of the beam events - large energy deposition. ii) $T_{N D}>0 \& T_{F D}>0$ : a very few low energy and time correlated later hits are incident in the detector. iii) $T_{N D}=0 \& T_{F D}>0$ : very few digits low energy hits are detected by the FD only: $1 \mathrm{PE}$ noise. iv) $T_{N D}>0 \& T_{F D}=0$ : the ND time-stamps every bucket accounting for the time structure of the energy deposition in time: exponential de-excitation of optical system (WLSF and scintillator). Note that, for the FD (unlike the ND), once it time-stamps the first hit, there appears to be no more incident hits, as expected.

tances is the first step towards attaining a comparable sample between both readout systems. The time fiducial cut is based on the definition of a time-window (called Time-Fiducial-Window) from both readout systems within which all hits whose energy deposition is not consistent with noise $(>1.5 P E)$ are to fall. If this condition is met, the event would be accepted for comparison purposes. Otherwise, the event is rejected, as the comparability is not guaranteed. This cut intrinsically demands the time correlation on the digits between both readout systems, as shown in Figure 6.1. Therefore, the correction for jitter between clocks is critical for the accurate implementation of this cut.

The time distributions of the digits as measured with respect to the beamtrigger shows the definition of the time fiducial cut, as illustrated in Figure 6.2. Note that in order to implement the time fiducial cut a time calibration has to be carried out on both sides in order to define the time-window accurately with 
respect to the beam-trigger tag time. The fiducial window is defined to be from $-30 \mathrm{~ns}$ to $280 \mathrm{~ns}$ in the time calibrated scale, for which zero refers to the arrival of the beam-trigger tag.
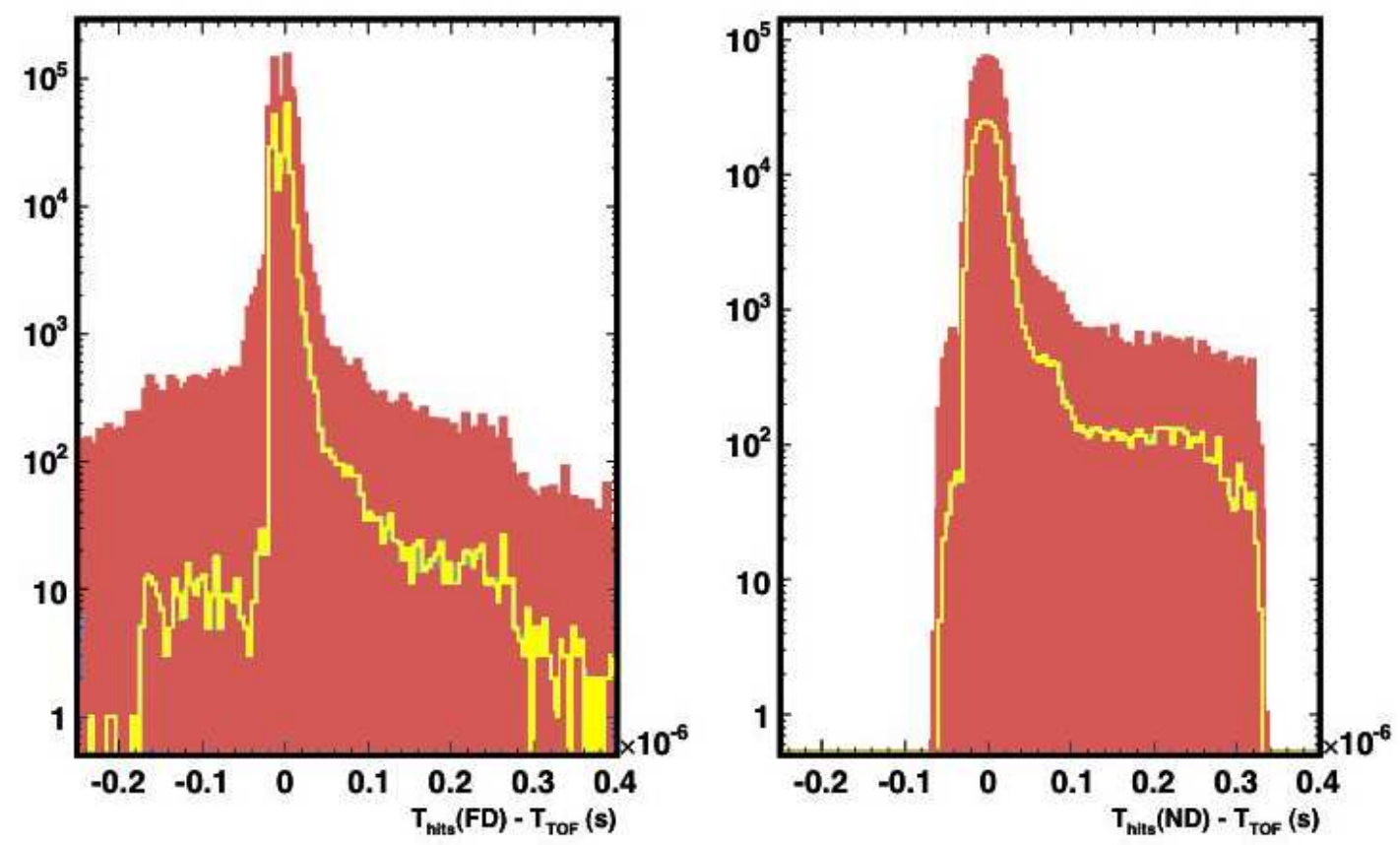

Figure 6.2: Time Fiducial Cut Definition. Plots show the digits time relative to the beam-trigger distributions for the ND (left) \& FD (right). The peaks determine when the beam-trigger, the detector and events strike the detector: $T \rightarrow 0$. The raw distribution is shown in red and the distribution after the time-fiducial cut has been applied in shown in yellow. Note that after the cut the acceptance in time of both readout systems has become equivalent.

There is yet another complication that has to be considered to make this cut work based on a simple time-window cut. The time-stamping works differently in both readout systems, as discussed in Sections 3.2.2 and 3.2.3. On the FD side, the time of the digit corresponds to the time of the first hit per triggered VA-chip. If a VA-chip was hit multiple times within a beam-trigger, there would not be explicit tag of the arrival of any later hit. On the other hand, the charge measured would be, to first order, the integrated sum of all hits incident on the VA chip per beamtrigger. Therefore, the VA-FEE is not capable of unambiguously telling whether a VA-chip has been hit more than once, as illustrated in Figure 6.1. On the ND side, all digits (one per buckets) are time-stamped and, therefore, there is no ambiguity as to which hits fall within the fiducial window. 
The discussed degree of ambiguity exhibited due to the VA-FEE time-stamping is a problem if we wish to allow the time-fiducial-cut to force both readout systems to have identical acceptances to energy depositions. To overcome the problem, we will seek to ensure that, in hardware, the QIE-FEE is active during the entire period that the VA-FEE is active after the beam-trigger. That is why CalDet was configured to have 20-buckets readout window for the ND electronics [117]. This allowed us to define the time-fiducial-window to be equivalent for both readout systems, making the FD time-stamping ambiguities irrelevant. Failure to do this would make it impossible to know whether the QIE-FEE was missing hits relative to the VA-FEE.

\section{Multi-Particle Rejection Cut}

This cut has been designed to use the timing capabilities of the detector to flag the deposition of more than one particle per beam-trigger. Such multi-particle triggers are not to be used by any CalDet analysis. The main reason for this is that the DAQ was designed to handle beam PID information only when one particle per beamtrigger occurs, as explained in Section 4.4. Furthermore, multi-particle triggers could give rise to subtle response differences between both readout systems, as the QIE-FEE was aimed to be robust at high intensities while the VA-FEE was not designed to work at such a regime.

A "Slicer" 2 was developed to flag beam-triggers that had more than one particle energy deposition in the detector. The Slicer algorithm relies only on the time of the energy depositions to identify event multiplicity within a beam-trigger. The beam flux was kept as low as possible (see Section 4.3) to avoid multi-particle triggers. However, some fraction of multi-particle triggers, generally $<10 \%$, were found.

The multi-particle trigger cut is implemented by estimating the multiplicity of energy depositions in time by the Slicer algorithm. If the beam-trigger in question was found to have more than one particle energy deposition, the event would be rejected from the analysis sample. The Slicer algorithm was based on an algorithm to identify timing peaks in the distribution of energy deposition with respect to the time for each beam-trigger (see Appendix C). Some illustrative examples on the performance of the Slicer are shown in Figure 6.3.

\footnotetext{
${ }^{2}$ The name Slicer is inspired on the algorithm to be used for actual MINOS ND data where a full spill $(10 \mu \mathrm{s})$ worth of data is stored as one single event (or "snarl") and later "sliced" into all its particle deposition contents as part of the offline reconstruction.
} 


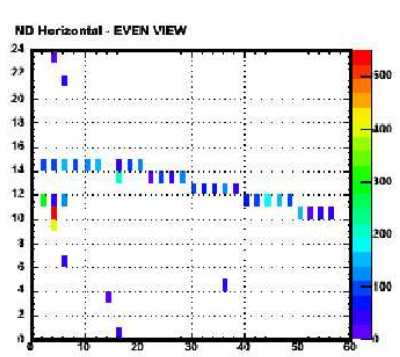

Easy Event:
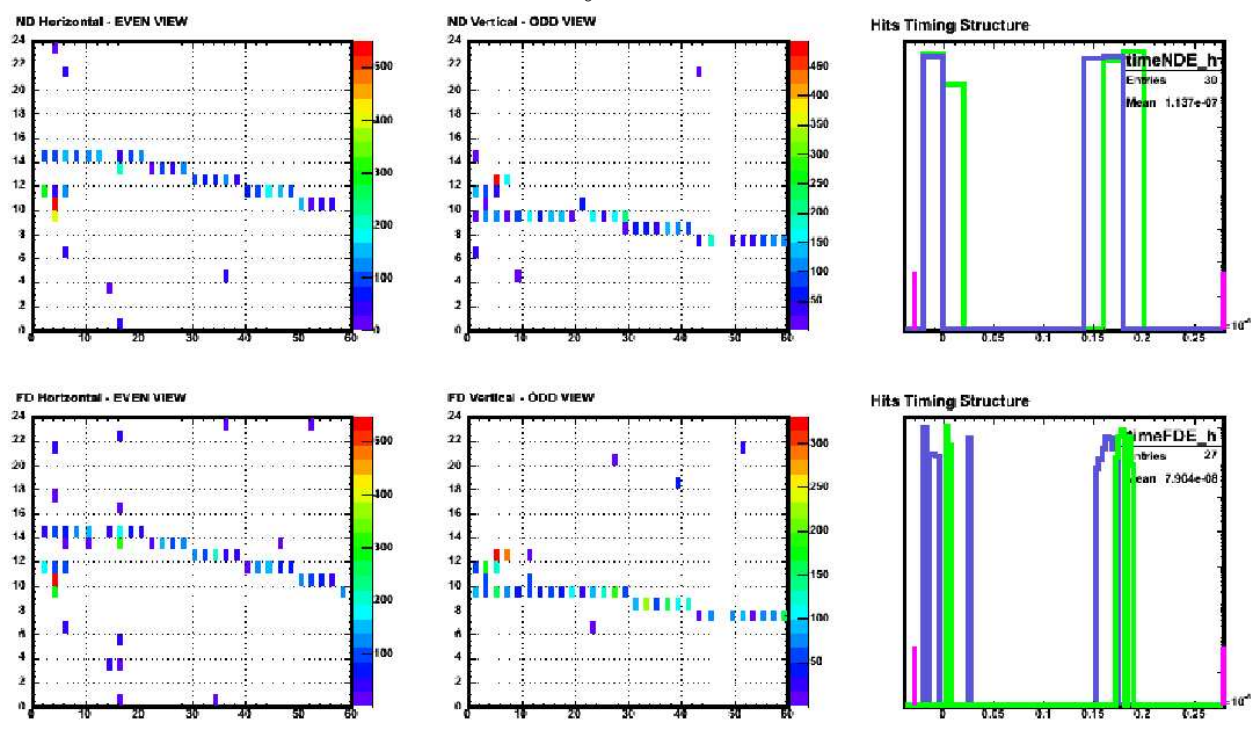

Difficult Event:
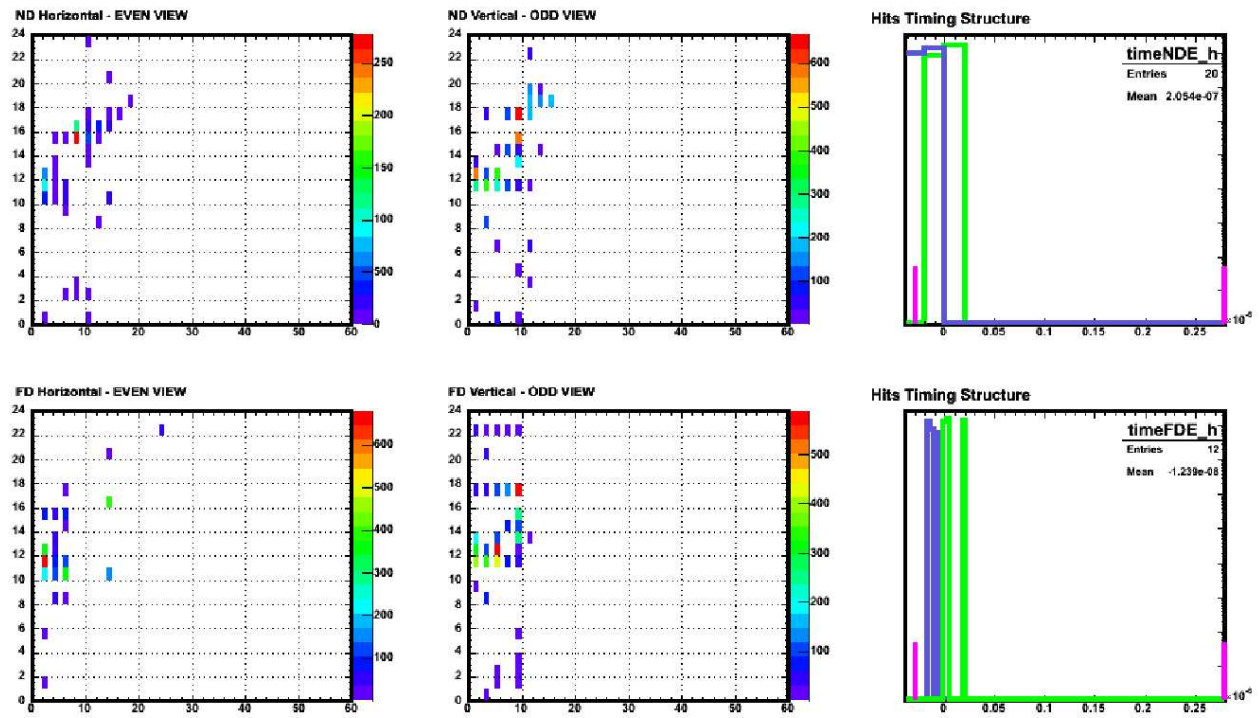

Figure 6.3: Example of a Multiple Particle Trigger Tagged by the Slicer. Two event displays are shown corresponding to two multi-particle triggers flagged by the Slicer. Each event display show six boxes: (top-first) ND-EVEN, (top-second) ND-ODD, (topthird) ND time distribution, (bottom-first) FD-EVEN, (bottom-second) FD-ODD and (bottom-third) FD time distribution. In the time distribution the corresponding hit to EVEN (green) and ODD (blue) views are shown. The lines (magenta), suggest the limits of the time-fiducial window. The first event display shows an electron and an offaxis-muon from the PS complex. The second event display shows another beam-trigger with more than one particle. This event is difficult to spot as the particles interact over about the same detector region and time. The excellent timing capabilities of FD help significantly to flag this type of events. 


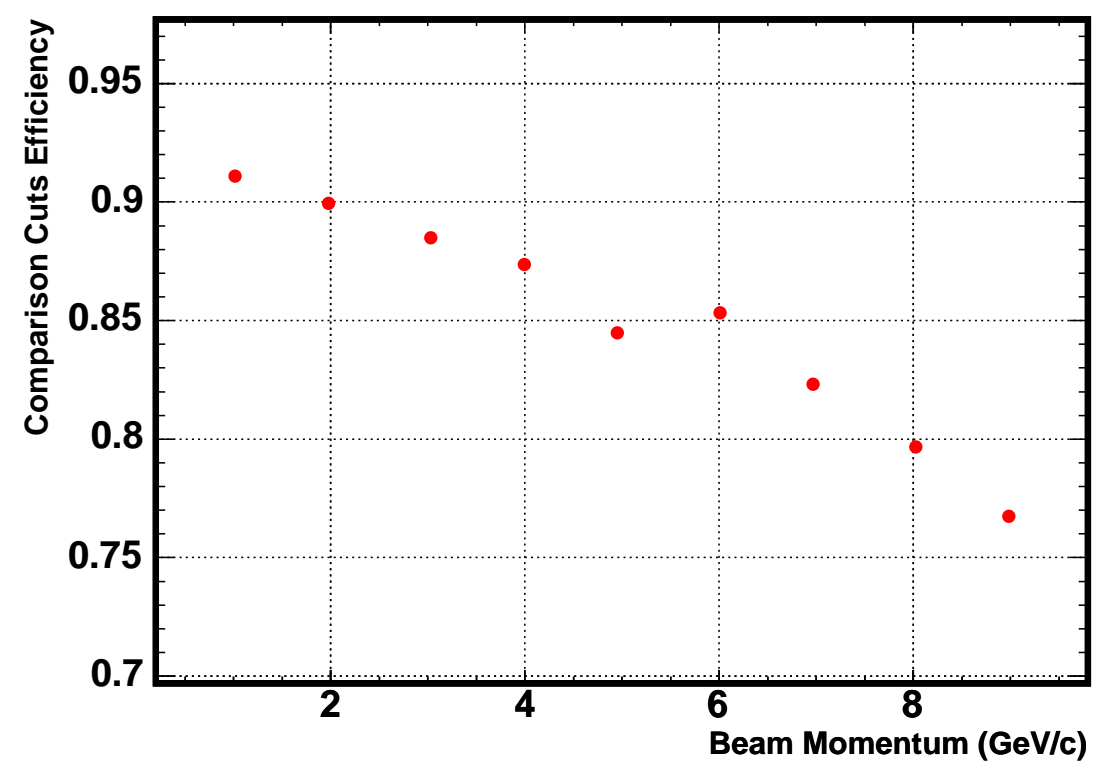

Figure 6.4: Efficiency of Comparison Cuts. This plots shows the estimated efficiency of the comparison cuts as a function of the beam momenta. The efficiency was calculated as the ratio between all event that passed the comparison cuts over the total number events collected. The MC was not subjected to the comparison cuts, as the object of the cuts is to remove beam effects, which are only present in the data.

\section{Performance of the Comparison Cuts}

The main shortcoming of the time fiducial cut is that the efficiency of the cut falls with beam momenta, as shown in Figure 6.4, where the efficiency of both comparison cuts is plotted versus beam run momenta. This is believed to be caused by the correlation between the spread in time of energy deposited and the average light level of the event due to the exponential decay of the de-excitation of the WLSF.

This trend in the efficiency with respect to energy would not be desired in an absolute response study, as events at a certain energy would be more accepted if fluctuating towards lower energy values with respect to higher values. However, the Near/Far comparison analysis results are not affected by this trend as any bias on the shower fluctuations will equally measured by both readout systems.

As intended, the comparison-cuts reduce the acceptance to multi-particle triggers. This is illustrated in Figure 6.5, where the fraction of single particle events is plotted against the average number of triggers per spill. A larger average number trigger per spill means a larger flux onto the detector. Therefore, the probability 


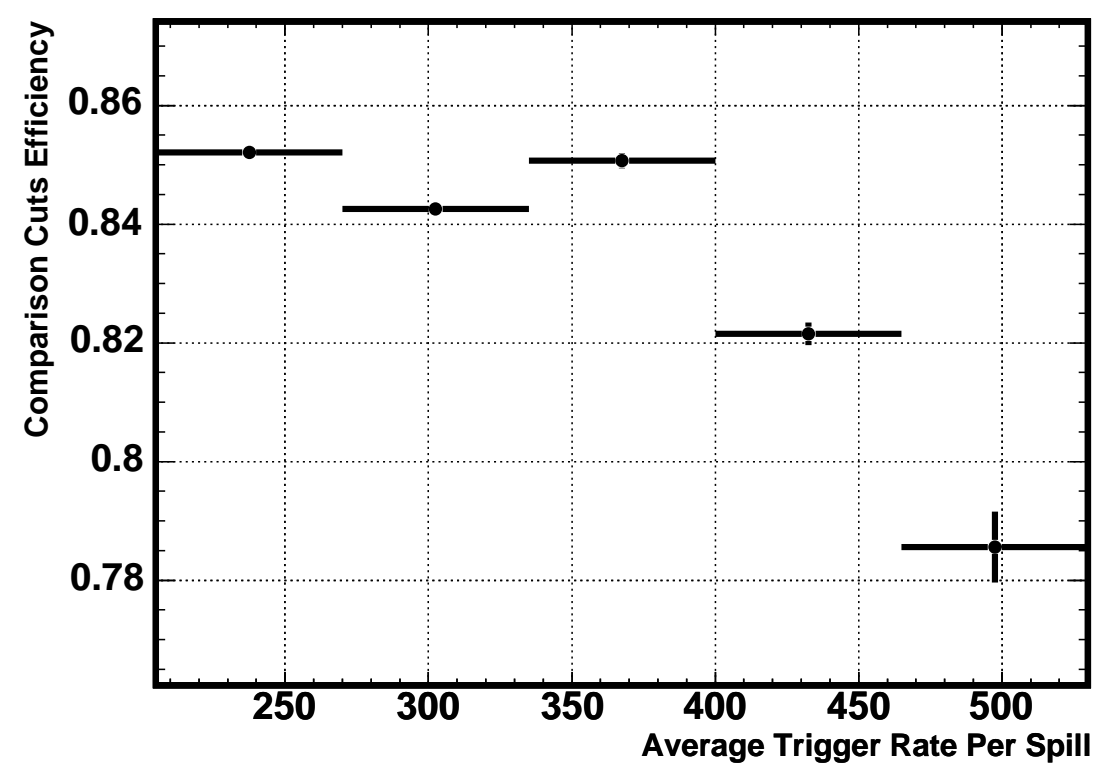

Figure 6.5: Overall Multiple Events Rejection Performance. The figure shows the fraction of events passing the comparison-cuts plotted versus the average rate of beamtriggers per spill. If the comparison-cuts were correctly designed, the efficiency of single particle events collection anti-correlates with the rate of events beam-triggered per spill, as observed.

of multi-particle triggers should increase, thus causing a larger rejection of events (i.e. less efficient data taking).

\subsubsection{PID Cuts}

The third type of cuts are called the PID Cuts. These cuts rely on the PID beam instrumentation added to the CalDet (see Section 4.4) for particle type ID. Once the PID cuts have been applied, an event will be labelled as an $e, \mu / \pi$ or $p$ particle candidates. The PID cuts tend to exhibit the tightness on the cuts alluded to earlier.

\section{ToF Timing Cut}

The $p$ identification is essentially granted by the ToF-system information up to $4-5 \mathrm{GeV} / \mathrm{c}$. A simple cut on the time distribution of the ToF-system allows the separation the $p$ peak from the ultra-relativistic $e / \mu / \pi$ peaks, as shown in Figure 6.6. The ToF system cut was used to isolate each peak, providing further consistency constraint on the expected behaviour of the particles searched. Isolating 
specific peaks with the ToF information, allows rejection of background and undesired particles also present in the beam (like K,deuterium, etc), which would, otherwise, contaminate the particle candidate samples.

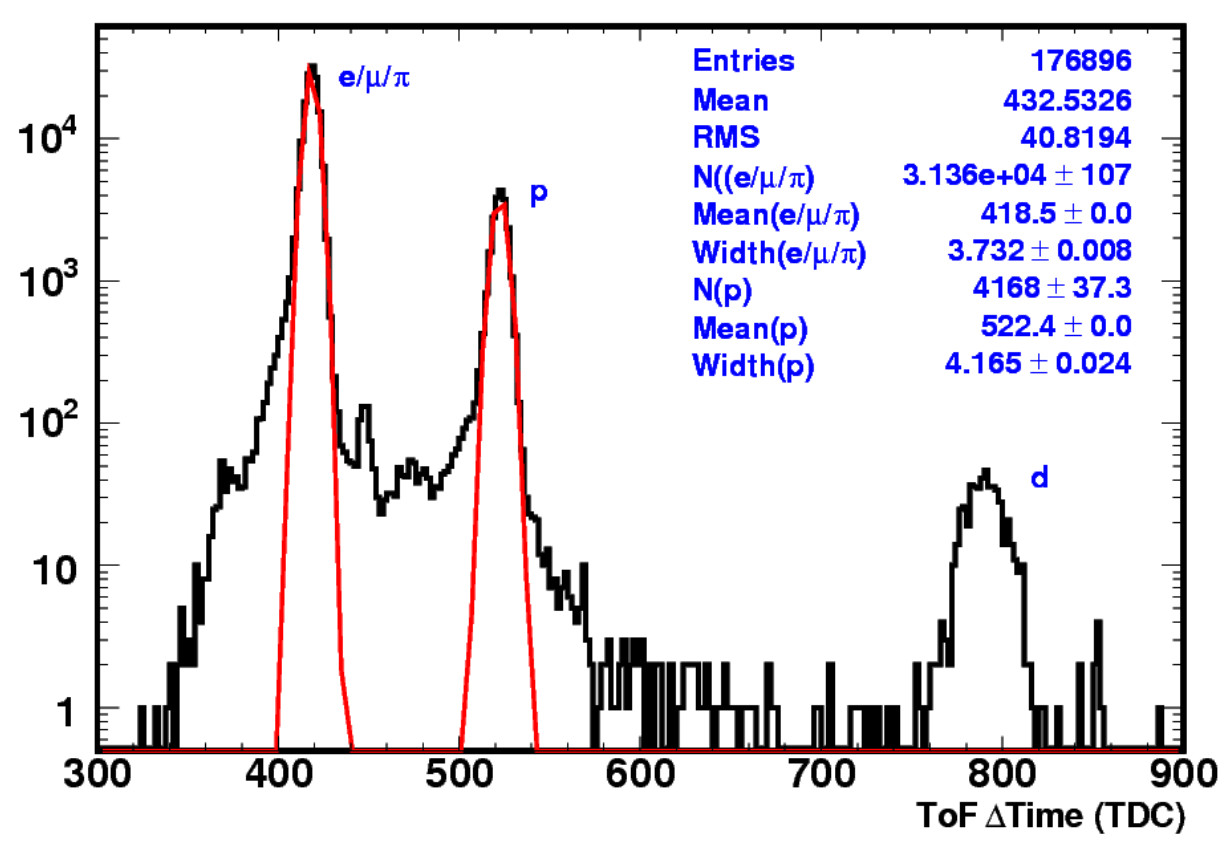

Figure 6.6: ToF Time Difference Distribution Sample. This is the ToF-system $\Delta$ TDC distribution for a $+1.8 \mathrm{GeV} / \mathrm{c}$ run where $\Delta \mathrm{TDC}$ refers to the difference between the ToF-paddles times, which is proportional to the speed of the particle. The $e / \mu / \pi$ ultrarelativistic peak (at $\sim 400 \mathrm{TDC})$, the proton peak (at $\sim 550 \mathrm{TDC}$ ) and even the deuteron peak (at $\sim 800 \mathrm{TDC}$ ) can be easily observed and fitted (red curve) to determine their locations.

Therefore, the implementation of the PDF cut was based on the information from Gaussian fits to each peak, as illustrated. At energies above $5 \mathrm{GeV}$, only the ultra-relativistic peak was distinguishable and the ToF cut was used to isolate such a peak from any other particle whose $\triangle T D C$ ToF was still distinct. Details on the ToF-system are described in Section 4.4.

\section{Cherenkov Counter Cut}

Two Cherenkov Detectors (CHD) were used. They were used for different PID capabilities according to the momentum of the beam. Electrons at all energies and muons/pions at high energies (beyond $2 \mathrm{GeV} / \mathrm{c}$ ) relied on the CHD for their identification. Details on the functioning of the CHD were given in Section 4.4.3. 


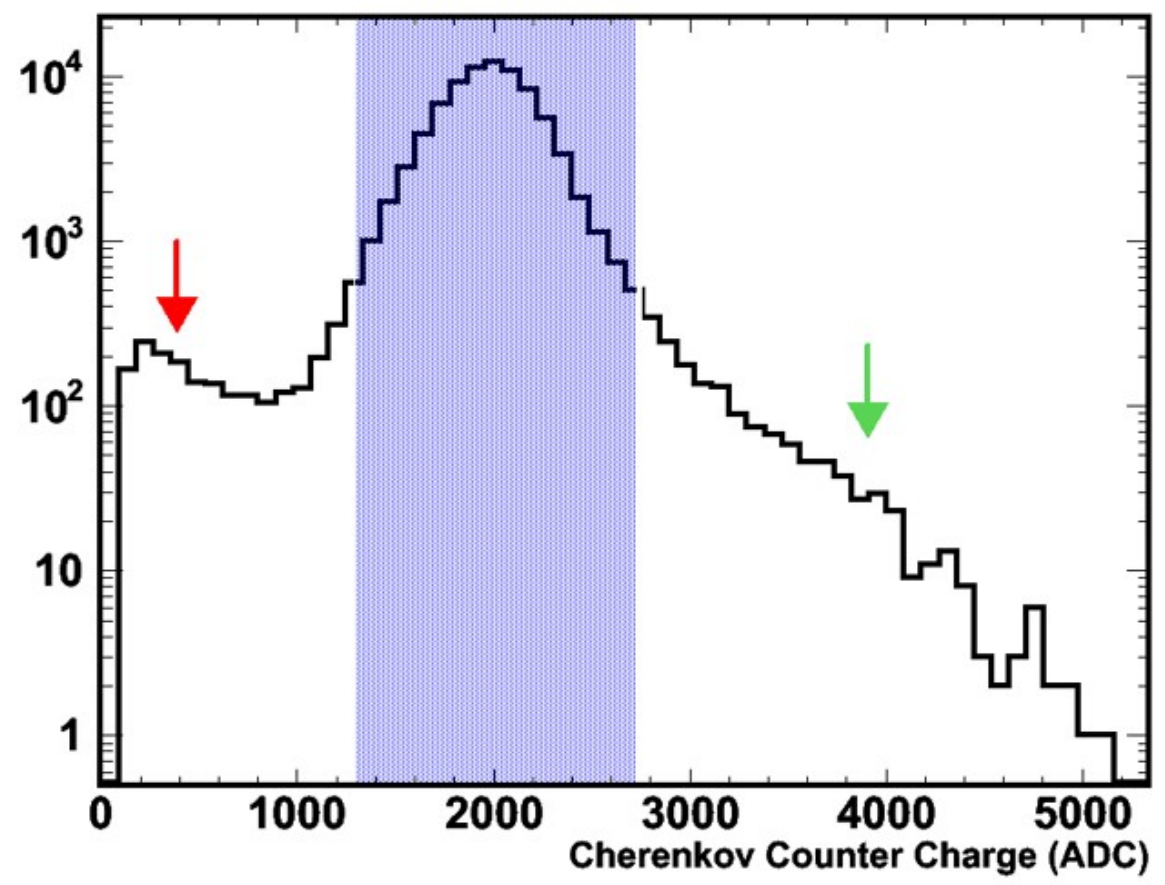

Figure 6.7: Cherenkov Counters Charge Distribution Sample. The plot shows a typical charge distribtution of a CHD. The pronounced peak (note logarithmic scale) correspond to the single particles set to trigger the CHD (shadded blue region). The shoulder towards high ADC counts (green arrow) corresponds to events with more than one particle detected by the CHD per trigger. The tail towards low ADC counts (red arrow) corresponds to a combination of noise and particles which deposited less light than expected in the CHD. The reason for the lower deposition can happen in the CHD is believed to be due to the beam-off-axis events, which do not go through the whole body of the CHD.

The most basic requirement that can be obtained from a CHD is whether any Cherenkov light was detected. However, the charge recored by the PMT in the CHD was also digitised, providing extra information on the selected events. An illustration of a typical charge distribution is shown in Figure 6.7. The structure of the CHD charge distribution is the convolution of the photon-statistics response of the CHD PMT and a Poisson distribution representing, to first order, the average number of particles triggering the CHD (occupancy) per trigger. The main peak (shaded in blue) corresponds to the CHD single particle per beam-trigger peak. The shoulder of the distribution, indicated by a green-arrow, is due to the contribution of two particles incident in the CHD per beam-trigger. While the plateau, indicated by the red-arrow, corresponds to particles that could trigger the CHD but that did 
not go through the whole length of the CHD (beam off-axis particle) and possibly also includes some noise at the lowest ADC. An off-axis particle would generate only a fraction of Cherenkov photons in the volume of the CHD from what the same kind of particle would do if passing through the whole detector. Hence, cutting on the main peak of the CHD charge distribution, one demands a sample of single particles triggering the CHD, which are on-axis.

The cut was implemented by fitting a Gaussian to the CHD charge distribution in order to infer the approximate location and width of the main peak. The cut was applied by accepting event being within $2 \sigma$ about the peak of the CHD charge distribution, as suggested by the blue shaded region.

This cut, in addition, increases the rejection capabilities of multi-particle triggers (already addressed by other cuts) by enhancing the rejection of similar particles that are able to triggered the CHD. This is good news, because multiple identical particles per beam-trigger are expected to be the weakness of the Slicer algorithm. The Slicer limitation reflects the fact that identical particle would have very similar hit patterns in detector-space. Therefore, approximately the same set of chips are hit on the FD side. If exactly the same chips were hit, the FD readout system would not time-stamp any later energy deposition, therefore leaving the toy-slicer algorithm "blind" to such an occurrence. The ND does not suffer from this problem, but it is very limited by its coarse time resolution hit-to-hit. The number of identical multi-particle triggers is generally very low: less than a few per thousand.

\section{Cherenkov-to-Beam-Trigger Coincidence Cut}

Whenever the CHD are used to select a particle type, one could additionally require the coincidence in time between the beam-trigger and the hit in the CHD. The coincidence between the two ensures that the particle selected with the CHD is also the triggering particle.

An example of the time difference distributions between the the beam-trigger and the one of the CHDs is shown in Figure 6.8. Events whose beam-trigger tag and CHD timing coincide correspond to the narrow spike. Therefore, the cut consist in rejecting any events not belonging to the peak, as suggested by the red arrow.

\subsubsection{Topology Cuts}

The fourth type of cuts are called Topology Cuts. These cuts can be regarded as PID cuts too. Their aim is to improve upon the PID selection criteria, depending on 


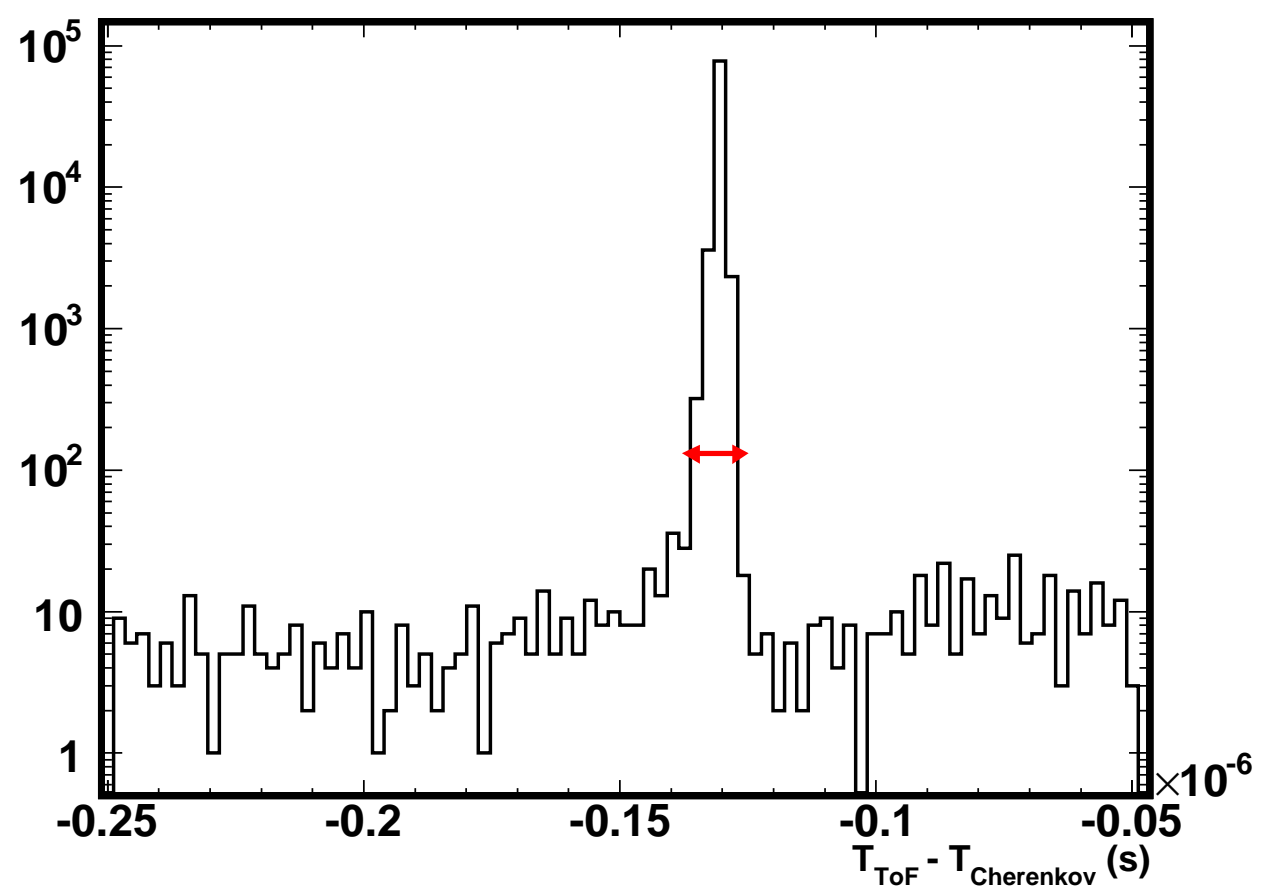

Figure 6.8: Beam-Trigger Tag to Cherenkov Counters Difference Distribution Sample. Note that the actual value of the difference is arbitrary (determined by cable length differences). All that matters is that the events to be used are in coincidence: the peak of the distribution. Note that there is a low flat background of accidentals in the distribution. Therefore, the narrower the peak the better the rejection will be to such accidentals. The width of peak ( $\sim 10 \mathrm{~ns}$, as indicated by the red arrow) was very narrow, thanks to the excellent time resolution of the VA-FEE used to time-stamped both signals.

the particle type in question. The distinction between the PID and topology cuts is made to help the reader understand the different nature of their implementations.

As its name suggests, the basis of topology cuts arises from the reconstructed pattern of the event energy deposition in the CalDet and not from the usage of dedicated PID instrumentation. They are important for the $\pi / \mu$ separation, which was not handled by the PID cuts. Topology cuts also help to improve the purity of the those particle candidate samples whose ID was essentially granted by the PID cuts. Much tuning of these cuts was generally aided by MC generated events in order to better understand the observations and optimise the purity to statistics ratio of the selection. 


\section{Range Cut}

Different particle have different penetration capabilities as their energy deposition mechanism (showering or only ionisation: $d E / d x$ ) is different. The Range is a measure of the penetration capability of a particle through the detector. Range is measured as the difference between the last plane hit and the first plane hit providing the hits have at least 1.5PE worth of charge. The charge cut in PE tackles the undesired possibility that PMT-crosstalk could bias the range estimation. A gap of up to 4 planes was allowed for computation of the range. The range cut was developed based on the strong correlation between particle types and their corresponding range at CalDet. The range was calculated for both the ND and FD sides independently. Likewise, the implementation of the range cut happens in both sides independently, so there is no coupling between both the readout systems results at the selection level.

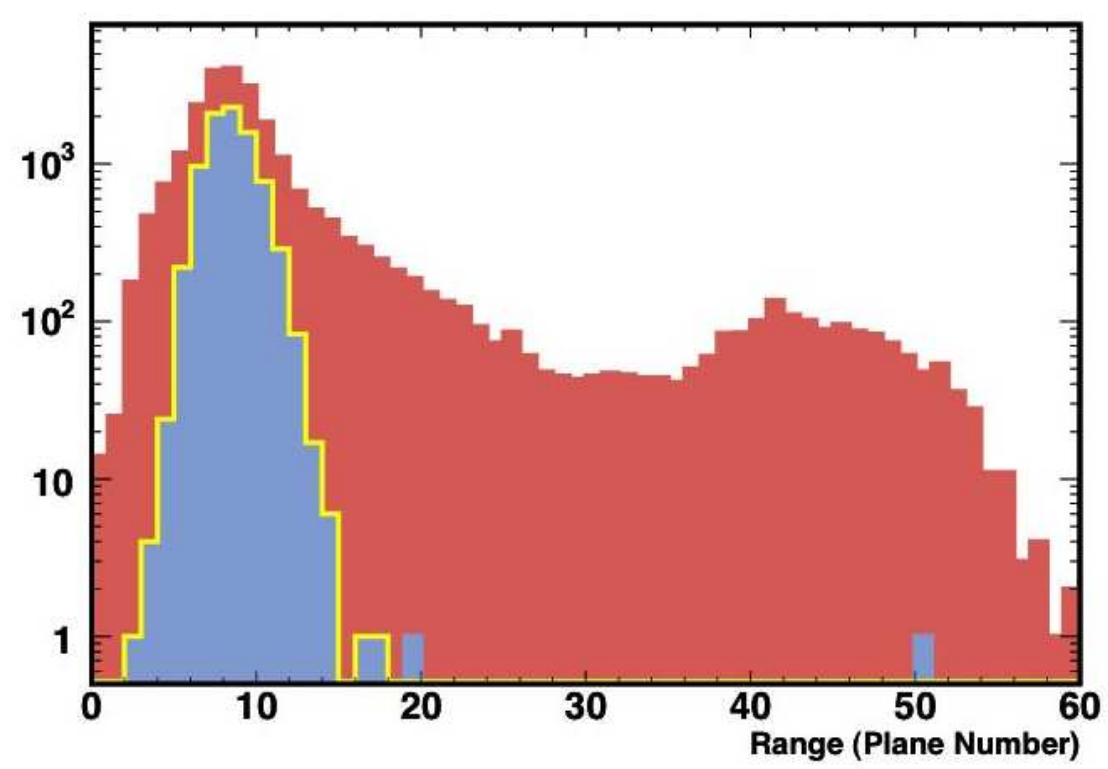

Figure 6.9: Range Distribution Sample for Electrons Candidates. In this plot -as anticipated- three PDFs per cut are shown: "Raw" (red), "N-1" (blue) and "Selected" (yellow). For electrons, the range cut removes on average 1 in 3000 events. The kind of events removed present an electron contaminated by a pion or a muon as the range distribution indicates.

An example of the range distribution for an simple case, namely electron candidates. The range of electrons is never beyond 25 planes for all energies. Figure 6.9 shows the range distribution for electrons, where a few events can be seen to exhibit a larger range. Therefore, a simple cut on range at $20-25$ planes for the electron 
range helps to remove some contamination from the electron sample while the cut has not impact on the electron events collected, which are nearly $100 \%$ efficient, as estimated by MC. Those very few electron-candidates with suspiciously larger range were generally found to contain an electron (that triggered the CHD) but also had an associated pion or a muon. Therefore, there were a few multi-particle events that had escaped the dedicated cuts for their rejection.

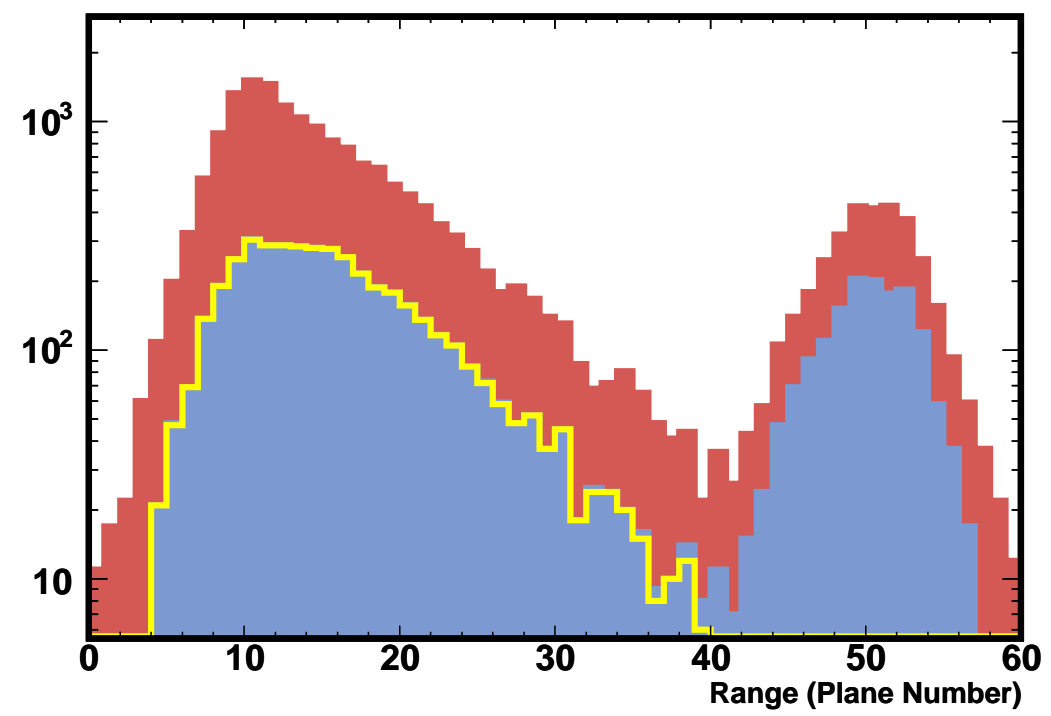

Figure 6.10: Range Distribution for Pions Candidates. The range cut is the most important element on the separation of pions and muons, as suggested by the $\mathrm{N}-1$ distribution.

An extreme case of the application of the range cut is the pion/muon separation. In such a case, the range cut becomes the major factor in their PID criteria. If all muons and pions had the exact momentum of the beam setting (i.e. a monochromatic beam), the range cut should separate the event sample accurately. The MC suggests that there is still a small number of events that may be consistent with being both muons and pions, about $<1 \%$. Therefore a perfect muon/pion separation is not possible, but there are two effects that degrade the separation even more:

1. A small fraction of muons would come from the decay of the pions as the pions are about to strike the face of the CalDet. Those muons would exhibit less momentum than that of the beam. These off-beam-momentum muons would range less than expected from a on-beam-momentum muon. The low 
momentum muons contaminate the pion sample, if the criterion of selection was based only on the range cut. Estimating the fraction of these events would require a full beam-line MC which is was not needed for this analysis.

2. A small fraction of pions hardly interacts strongly at all with the CalDet material. Instead, they "punch-through" the planes of the detector as a minimum ionising particle would, losing energy through ionisation only. Therefore, the pattern observed is identical to that of a muon, since their stopping powers are very similar due to their similar masses.

A sample of minimum ionising particle going through the detector was desired for the analysis, for which absolute responses are not particularly relevant. Whether the particles were muons or a few pions behaving just like muons has negligible impact on to the conclusions of the analysis. Due to the described difficulties, muon candidates, and not pion candidates, were used for the analysis.

The cut for the muon candidate selection has a more involved criteria to ensure the high purity of the sample. The selection of muon candidates was performed in three stages:

Pre-selection of Muon. Muon and pion candidates were separated based on a range cut. Note that the range criterion is, at low energies, defined by a window in range. The reason why such a definition is needed is two fold: i) the lower limits rejects most pions, while $i i$ ) the higher limit eliminates high energy muons that were found to originate from the PS beam facilities rather than the $\mathrm{T} 7$ beam-line pipe, as they were off-axis. Note that this range cut implicitly demands some degree of "track-ness" and straightness on the selected muons. A summary of the range cut criteria for all energies is summarised in Table 6.1.

The pion sample obtained after the range cut was simply not used any further since it could be contaminated by off-beam-momentum muons and electrons arising from the increasingly inefficient CHDs at higher energies (Section 4.4.3) used for the $\pi$ /e separation. However, the electron contamination should fall with energy, as the number of electrons available in the beam falls exponentially (see Figure 4.3).

Track-like Events. Track events should hit one strip per plane on average. Therefore, a loose cut was defined by which the number of strips hit per planes was demanded to be $<1.3$ for events to become muons candidates. 


\begin{tabular}{|c|c|c|}
\hline Beam Momentum (P) & Range-Min & Range-Max \\
\hline \hline $1.0 \mathrm{GeV} / \mathrm{c}$ & @ plane 17 & @ plane 29 \\
$1.4 \mathrm{GeV} / \mathrm{c}$ & @ plane 26 & @ plane 42 \\
$1.6 \mathrm{GeV} / \mathrm{c}$ & @ plane 31 & @ plane 47 \\
$1.8 \mathrm{GeV} / \mathrm{c}$ & @ plane 34 & @ plane 53 \\
$2.0 \mathrm{GeV} / \mathrm{c}$ & @ plane 40 & @ plane 57 \\
$>2.0 \mathrm{GeV} / \mathrm{c}$ & @ plane $42+n(p)$ & none \\
\hline
\end{tabular}

Table 6.1: Range Cut for $\mu / \pi$ Separation. Where $n(p)$ indicates an increasing function with the beam momenta $(p)$, to account for increasing rage range of pions with momenta.

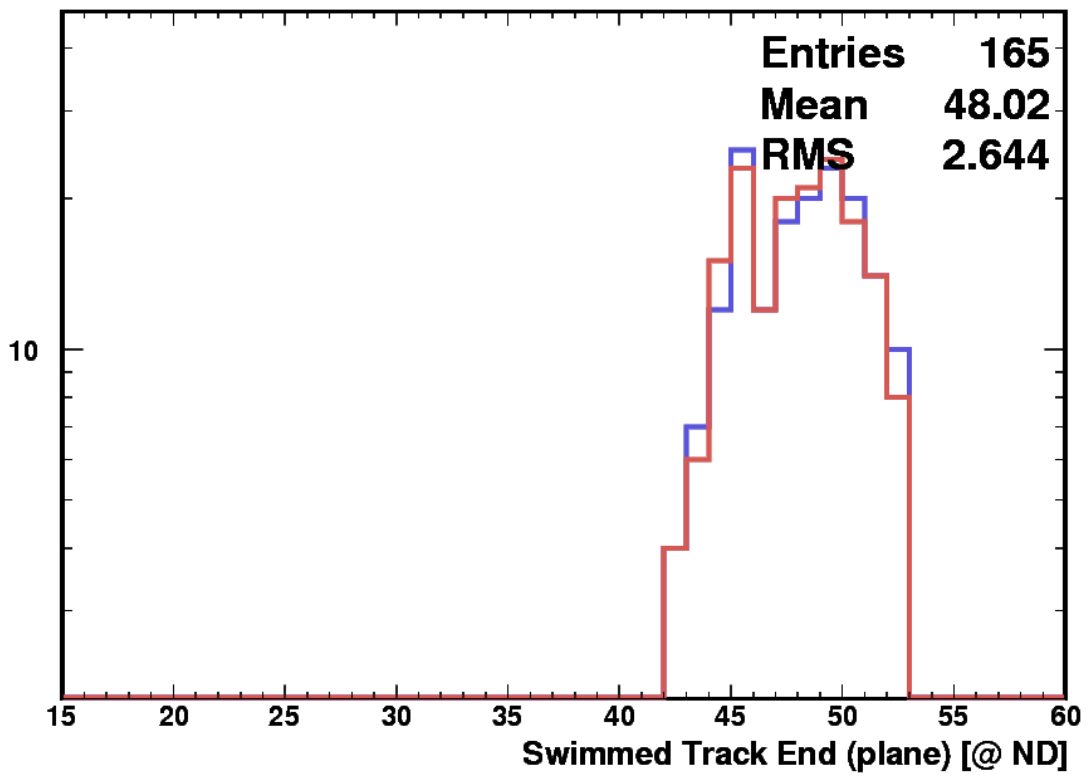

Figure 6.11: Range Distribution of Muons after Tracking. Blue shows the range of muons as tracked on the ND side, whereas red corresponds to the tracking output carried out on the FD side. The spread shown by the distribution is dominated by the scatter of the muons (not corrected), not by the spread in momenta of the beam, which is expected to be $<2 \%$. Of course, the effect of the cut has biased the measured range spread.

Track-end Finder: The Swimmer The Swimmer was devised to essentially trace (or "swim") the path of the muon until the muon stopped or escaped the detector. The description of the implementation of the Swimmer is covered in Appendix C.

The Swimmer was used to accurately find the end of the tracks, to measure the muon range. Thus, the rejection of possible pions was enhanced by cutting 
tighter on the accurate location of the track end. Figure 6.11 shows the range distribution after the application of the Swimmer, as measured by both readout systems. If the momentum was $>2 \mathrm{GeV}$, the muons were forced to escape within the last 8 planes of the detector.
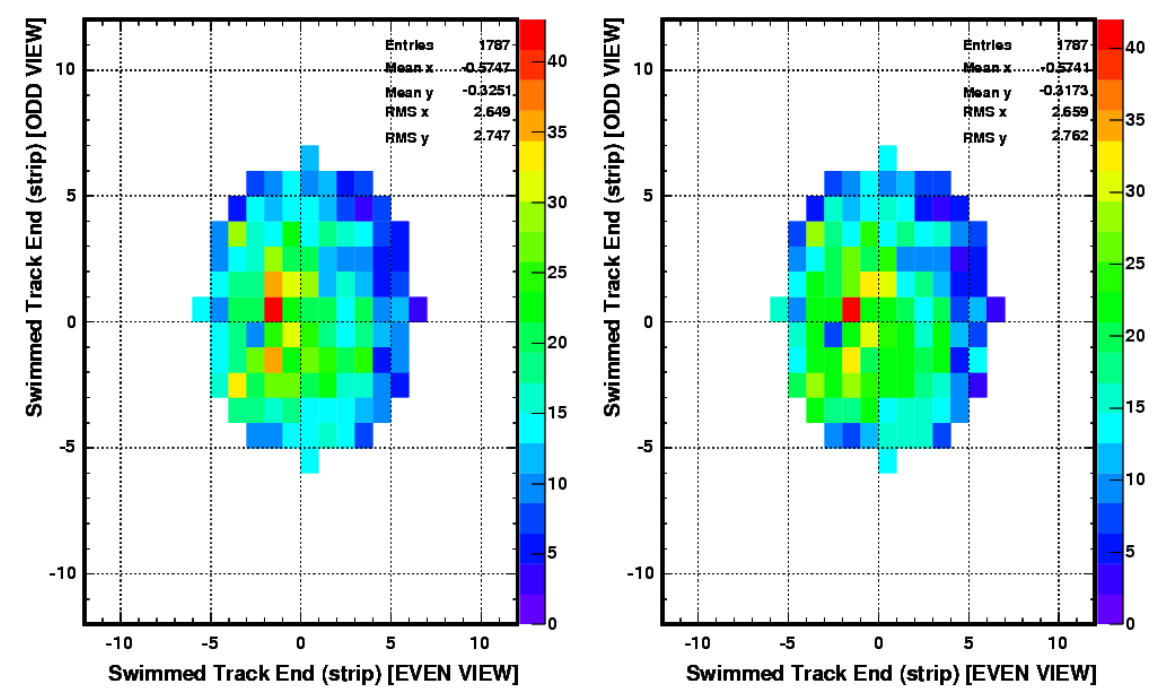

Figure 6.12: Track-End Location for Calibration Quality Muons. Straightness was a requirement for muons used to monitor the quality of the calibration. This was done by demanding that the last strip hit of the track was close to the central axis of the detector. The distribution of the location of the last strip hit of the track for both the ND (left) and FD (right) sides is shown in this plot. The plots also illustrate the fact that the beam-spot does not match the detector symmetry axis.

\section{Calibration Muons Selection}

Some muons were used for monitoring the quality of the calibration run-by-run: calibration checker. For those muons an extra condition was required: straightness. The straightness was imposed by requiring that the last strip hit by the track was within the most central 12 strips, i.e. a 6 strips radius, as shown in Figure 6.12. Straightness was demanded because, in this way, the muon responses depended less on attenuation corrections when the muons scatter by the back of the detector.

\section{High Energy Muons Selection}

In order to collect muons at energies beyond $2.0 \mathrm{GeV} / \mathrm{c}$, a special requirement was added to the already described muon selection criteria. The extra requirement is 
due to fact that muons escape, therefore the range cut only ensures that the last plane hit was by the end of the detector. The condition required was based on the response of muons within the last third of the detector. The selected high energy muons has to have $<1.6 \mathrm{MIP}$ per plane on average over the last third of the detector.

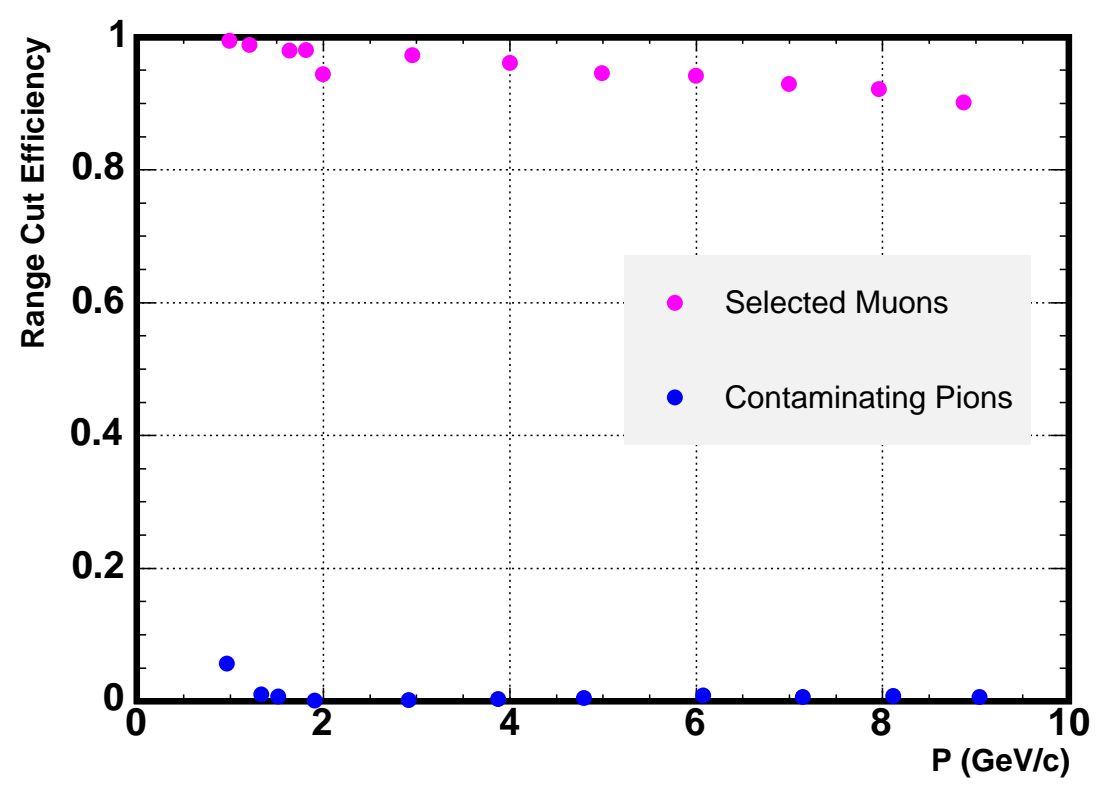

Figure 6.13: Efficiency of Range Cut for Muons.

The muon selection criteria efficiency and pion contamination in the muon sample is shown in Figure 6.11, as estimated using MC events. Note that, generally, the pion contamination was $<3 \%$, while the efficiency was generally $>90 \%$. The falling efficiency with energies of the muon selection is believed to be due the increasing rate of delta-rays generated by the muons over the region that the response cut was applied at high energies.

\subsection{Particle Candidates Samples}

The order in which the cuts has been presented does not reflect the order in which the cuts are applied. First, the quality cuts are applied rejecting events having hardware problems. Once a sample of good events is obtained, each event is pro- 
cessed $^{3}$ in order to compute all information needed to infer its ID through the remaining cuts. Finally, to obtain samples of any particular particle type, very specific combinations of cuts were applied (as described below) after which events were labelled as electron, muon or proton candidates correspondingly.

The series of cuts has been split into two regimes: Low Energy $\left(p_{\text {beam }} \leq 2 \mathrm{GeV} / \mathrm{c}\right)$ and High Energy $\left(p_{\text {beam }}>2 \mathrm{GeV} / \mathrm{c}\right)$. The inflection between both regimes is based on the use of the DS-CHD, which is, itself, based on the fact that muons are not contained beyond $2 \mathrm{GeV} / \mathrm{c}$. The DS-CHD was used at low energies for electrons (for redundancy) while, at high energies, it was used to trigger on $\mu / \pi / e$ which help the $\mu / \pi$ identification.

\subsubsection{Electron Candidates Sample}

\begin{tabular}{|r|c|c|}
\hline & Low Energy & High Energy \\
\hline \hline Quality Checks & $\sqrt{ }$ & $\sqrt{ }$ \\
Time Fiducial & $\sqrt{ }$ & $\sqrt{ }$ \\
Reject Multi-Particle & $\sqrt{ }$ & $\sqrt{ }$ \\
ToF $\Delta$ TDC & $e / \mu / \pi$ & $e / \mu / \pi / p$ \\
US-Cherenkov Counter & $\sqrt{ }$ & $\sqrt{ }$ \\
DS-Cherenkov Counter & $\sqrt{ }$ & veto \\
US-Ckov./Beam Coincidence & $\sqrt{ }$ & $\sqrt{ }$ \\
DS-Ckov./Beam Coincidence & $\sqrt{ }$ & $\mathrm{X}$ \\
Range & $\leq 20$ planes & $\leq 25$ planes \\
\hline
\end{tabular}

Table 6.2: Sequence of Cuts for Electron Selection.

Table 6.2 summarises the combinations of cuts needed to obtained an electron candidate sample over the energy range available at CalDet. Figure 6.14 shows a typical map for electrons in CalDet. Figures 6.15 and 6.16 illustrates the cuts on the PDFs for the electrons selection:

- Figure 6.15 (top) shows the most important cut for the electrons selection: the cut on the spectra of both CHD (up-stream and down-stream) for a run at $1.8 \mathrm{GeV} / \mathrm{c}$. This cut essentially obtains a electrons selection with a contamination to the $1 \%$ level. The remaining cuts help to enhance the

\footnotetext{
${ }^{3}$ Alluding to some basic tailor-made reconstruction for the analysis. No standard MINOS reconstruction was used because this analysis was intended to be reconstruction independent. Furthermore, the standard MINOS reconstruction software was still under heavy development at the time of this analysis.
} 


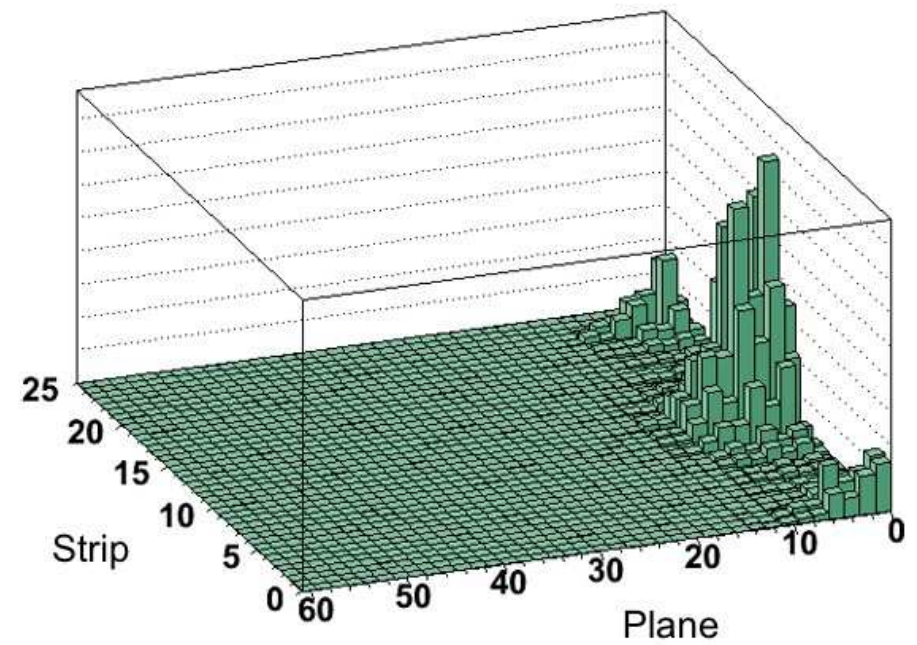

Figure 6.14: Electrons 3D Hit Map. Plot shows the occupancy (z-axis) of each stripend according to their location in terms of plane and strip numbers. Note that beam is incident at plane-0 and strip-12. The hits on the edges of the detector correspond to PMT-crosstalk.

purity of the sample by about 2 orders of magnitude, i.e.less than $1: 10000$ contaminating events.

Note that, in Figure 6.15 (top), the "N-1" and the "Selected" PDFs are pretty similar, despite the fact that this is the most important cut for the selection. This is because most of the events rejected by this cut will pile up at zero charge for both CHDs. For the sake of illustration purposes, that peak has been removed in order not to alter the abscissa scale. In addition, there is an artifact arising from the fact that the cut on each CHD has been treated as independent. Therefore, the "N-1" PDF of the up-stream CHD has been affected by cutting on the down-stream CHD and vice versa.

CHDs are applied for the selection of electrons (up-stream CHD) and muon/pions (down-stream CHD) for momenta beyond $2.0 \mathrm{GeV} / \mathrm{c}$. No PDF is shown for those cases as the distributions look essentially identical to ones shown in Figure 6.15 (top).

- Figure 6.15 (middle) shows the corresponding PDFs to demand the coincidence in time between the CHDs and the beam-trigger to ensure that the triggering particle hits both the CHD and the ToF-system.

- Figure 6.15 (bottom) shows the PDFs for the time-fiducial cut. This cut 

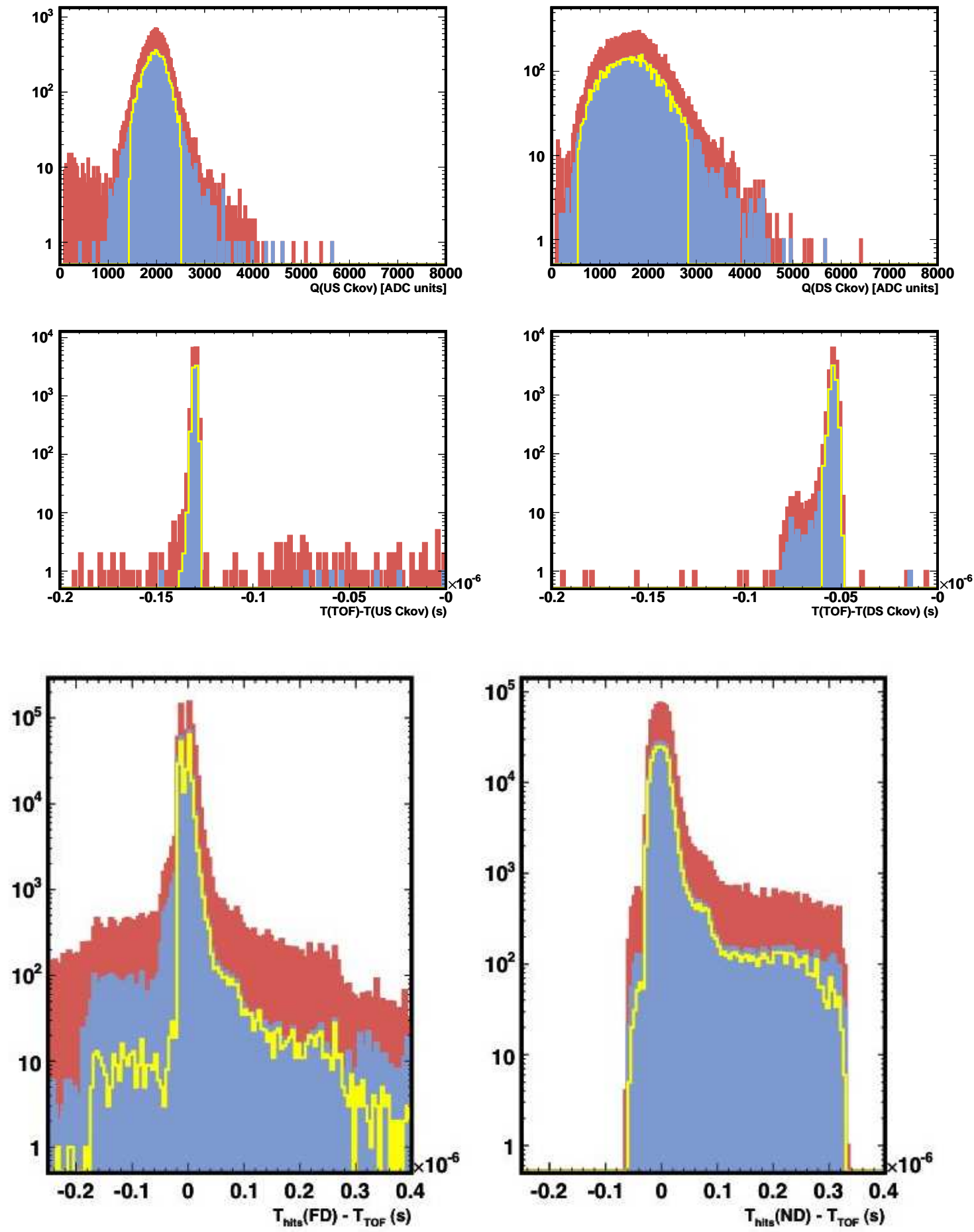

Figure 6.15: Electron Candidate Sample Selection Cuts (1). 

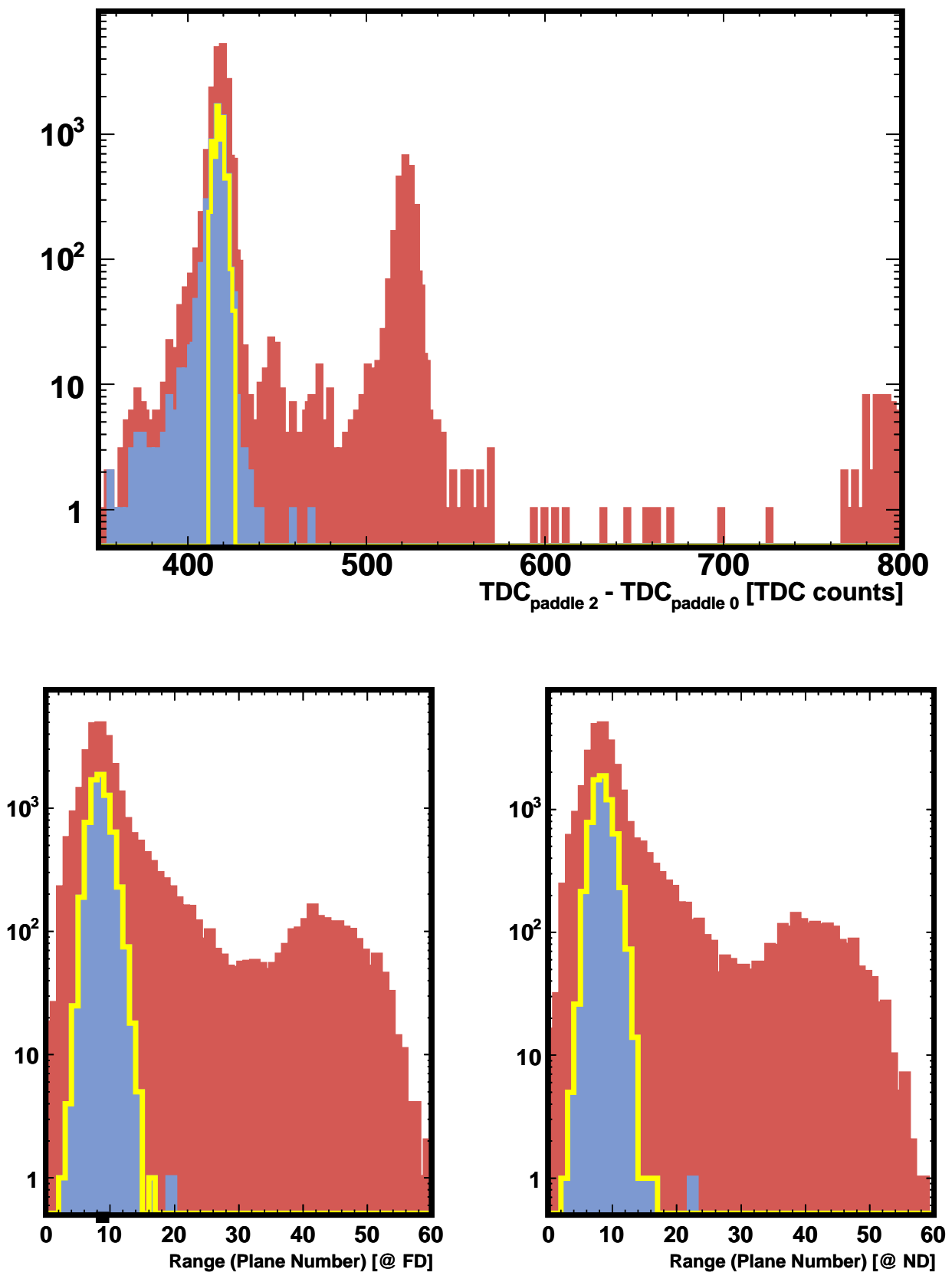

Figure 6.16: Electron Candidate Sample Selection Cuts (2). 
is common to all particle samples. Despite the fact the "N-1" and "Selected" PDF will be affected by all other cuts specific to each event sample, Figure 6.15 (bottom) represents a good sample of the application of the time fiducial cut for all particle samples, so it will not be shown for muons and protons.

- Figure 6.16 (top) shows the PDF for the TOF system correspondingly to $1.8 \mathrm{GeV} / \mathrm{c}$. The peaks for proton and deuteron can also be clearly seen. This distribution is identical for muons.

- Figure 6.16 (bottom) shows the PDF for the range cut for electrons.

\subsubsection{Muon Candidates Sample}

\begin{tabular}{|r|c|c|}
\hline & Low Energy & High Energy \\
\hline \hline Quality Checks & $\sqrt{ }$ & $\sqrt{ }$ \\
Time Fiducial & $\sqrt{ }$ & $\sqrt{ }$ \\
Reject Multi-Particle & $\sqrt{ }$ & $\sqrt{ }$ \\
ToF $\Delta$ TDC & $e / \mu / \pi$ & $e / \mu / \pi / p$ \\
US-Cherenkov Counter & veto & veto \\
DS-Cherenkov Counter & veto & $\sqrt{ }$ \\
US-Ckov./Beam Coincidence & $\mathrm{X}$ & $\mathrm{X}$ \\
DS-Ckov./Beam Coincidence & $\mathrm{X}$ & $\sqrt{ }$ \\
Range & muon range & muon range \\
\hline
\end{tabular}

Table 6.3: Sequence of Cuts for Muon Selection.

Table 6.3 summarises the combinations of cuts needed to obtained a muon candidate sample over the energy range available at CalDet. Figure 6.17 shows a typical hit map for muons in CalDet. Figure 6.18 shows an example of good muon event as tracked by the Swimmer that has successfully met all conditions. 


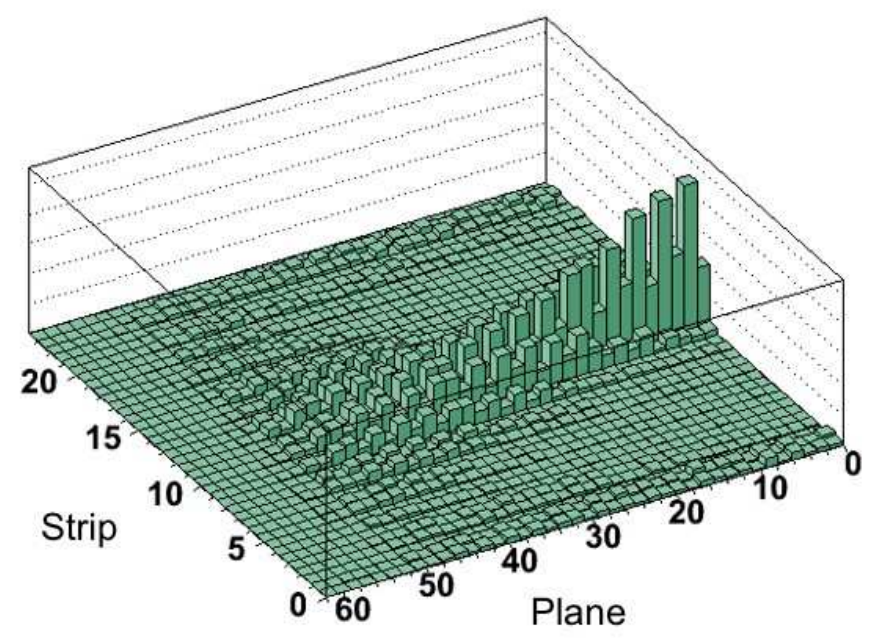

Figure 6.17: Muons 3D Hit Map. Plot shows the occupancy (z-axis) of each stripend according to their location in terms of plane and strip numbers. Note that beam is incident at plane- 0 and strip- 12 . The hits on the edges of the detector correspond to PMT-crosstalk.
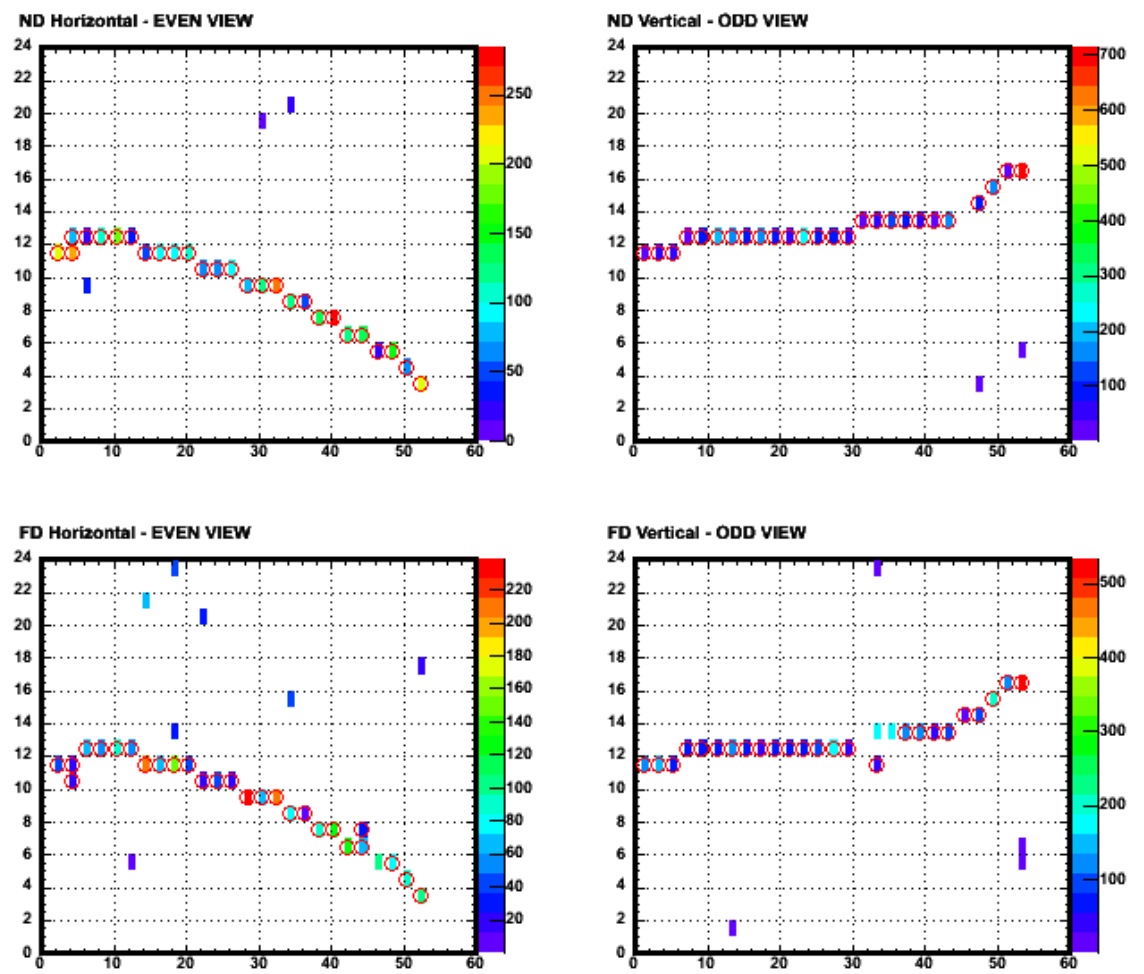

Figure 6.18: A Well-defined Stopping Muon. 
Figure 6.19 (top) corresponds to the range cut for muons. This is the most important cut for the muon/pion separation, as can be seen from the discrepancy between the "N-1" and "Selected" PDFs. Note that the pions are the events indicated by $\mathrm{N}-1$ distribution which have not been selected. The contamination on the muon sample is dominated by an average $<2-3 \%$ of pions.
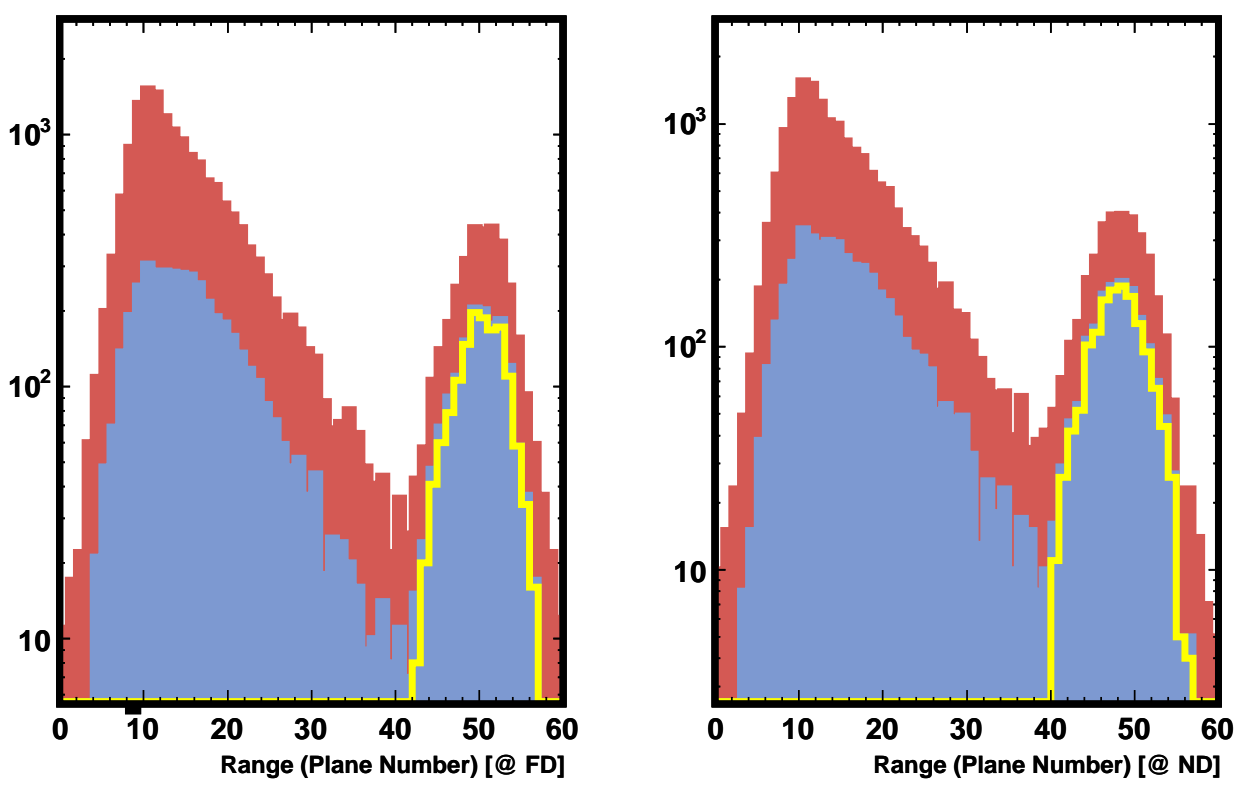

Figure 6.19: Muon Candidate Sample Selection Cuts. 


\subsubsection{Proton Candidates Sample}

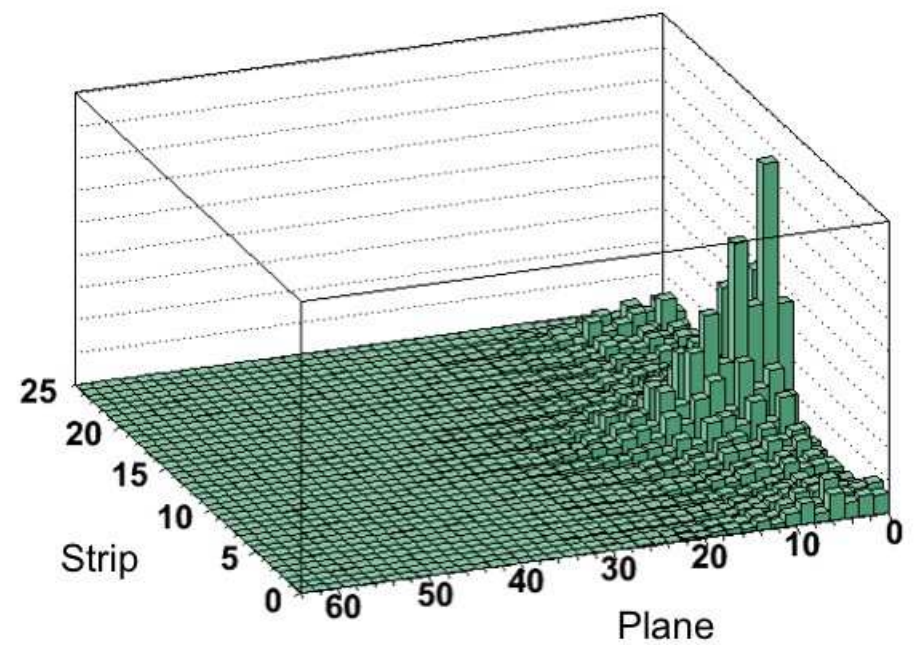

Figure 6.20: Protons 3D Hit Map. Plot shows the occupancy (z-axis) of each stripend according to their location in terms of plane and strip numbers. Note that beam is incident at plane-0 and strip-12. The hits on the edges of the detector correspond to PMT-crosstalk.

\begin{tabular}{|r|c|c|}
\hline & Low Energy & High Energy \\
\hline \hline Quality Checks & $\sqrt{ }$ & $\sqrt{ }$ \\
Time Fiducial & $\sqrt{ }$ & $\sqrt{ }$ \\
Reject Multi-Particle & $\sqrt{ }$ & $\sqrt{ }$ \\
ToF $\Delta$ TDC & $\mathrm{p}$ & $e / \mu / \pi / p$ \\
US-Cherenkov Counter & veto & veto \\
DS-Cherenkov Counter & veto & veto \\
US-Ckov./Beam Coincidence & $\mathrm{X}$ & $\mathrm{X}$ \\
DS-Ckov./Beam Coincidence & $\mathrm{X}$ & $\mathrm{X}$ \\
Range & hadron range & hadron range \\
\hline
\end{tabular}

Table 6.4: Sequence of Cuts for Proton Selection.

Table 6.4 summarises the combination of cuts needed to obtained a proton candidate sample over the energy range available at CalDet. Figure 6.20 shows a typical hit map for protons in CalDet.

Figure 6.21 illustrates the cuts on the PDFs for the protons selection.

- Figure 6.21 (top) corresponds to the TOF PDF for protons at $4 \mathrm{GeV} / \mathrm{c}$. This is the most important cut for the proton identification up to $2.0 \mathrm{GeV} / \mathrm{c}$. Beyond 

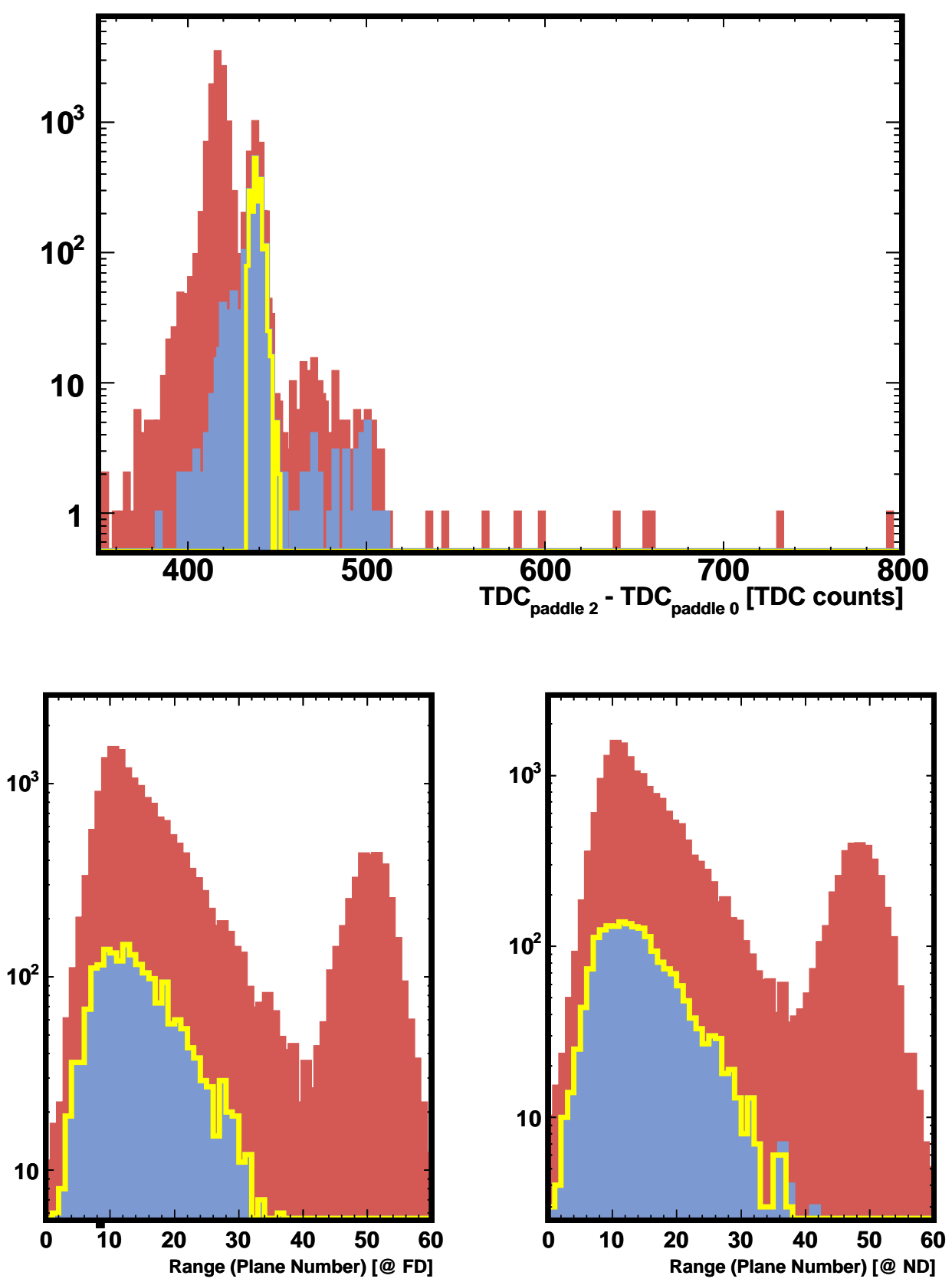

Figure 6.21: Proton Candidate Sample Selection Cuts.

$2 \mathrm{GeV} / \mathrm{c}$ the proton identification relies mainly in vetoing the signal of both CHDs, as the down-stream CHD triggers on electrons and the down-stream CHD triggers on $e / \pi / \mu$. Note that beyond $4 \mathrm{GeV} / \mathrm{c}$ the TOF system does no longer provide resolvable discrimination of protons with respect to the $e / \pi / \mu$ peak. 
The level of contamination at high energies is expected to be dominated by the inefficiency of the down-stream CHD, which depends on the pressure at which the CHD was pumped, as described in Section 4.4.3. Therefore the proton sample will be contaminated by pions to the few percent level. The rejection of muons is handled by the range cut while electrons are virtually rejected by vetoing in the two CHDs simultaneously.

- Figure 6.21 (bottom) shows the range PDFs for protons. The definition of the range for hadrons can be found implicitly in the Table 6.1. The definition of the range of hadrons is given by events whose range is lower than the "Range-Min". This cut is used to enhance the purity of single proton events from possible muons or long-range pions. 


\section{Chapter 7}

\section{The Near/Far Readout Systems Comparison}

This chapter describes the main topic of this thesis: "the Near/Far Detector Readout Systems Comparison" (NFC). This analysis assesses a long-standing assumption within MINOS: the relative energy reconstruction and topology of events will not be systematically affected by having chosen two different readout systems (particularly the electronics) for the MINOS Near and Far detectors. Therefore, the main goal of this analysis is to understand the extent to which this assumption is correct and to provide quantification on the performance of both readout systems in order for MINOS to achieve its physics goals.

After reviewing the objectives and strategy of the analysis, this chapter will be broken down into four main sections. The first part will be dedicated to the studies of the different aspects of the energy reconstruction (and calibration) comparison. The second part will investigate the comparison of the event topology as measured by both readout systems. The time structure of the ND will be explored too, as the subject of the third part. Finally, the fourth part will be devoted to summarising a few interesting instrumental effects discovered and/or studied in detail during the course of this analysis.

\subsection{Analysis Objectives}

The objectives of the NFC can be essentially be summarised into three correlated aspects: 
- Milestone on the MINOS Calibration Validation.

Two intrinsically different readout systems are installed in the MINOS Near and Far detectors. Agreement of the measured responses by the two readout systems can only be achieved by having a robust calibration that has to work for both systems simultaneously. Therefore, the NFC is one of the most important demonstrations of the MINOS calibration chain.

\section{- Milestone on the MINOS Detector Simulation.}

The NFC not only focuses on assessing how equal/different the Near/Far readout systems perform relative to each other, but also focuses on exploring how accurately the MINOS "Detector Simulation" chain describes the response and performance of each readout system. So, $\mathbf{N D}_{D A T A}$ versus $\mathbf{N D}_{M C}$ and $\mathbf{F D}_{D A T A}$ versus $\mathbf{F D}_{M C}$ comparisons will be carried out to validate and tune MC generated events with CalDet data. In fact, current MINOS thoughts seem to suggest that DATA $=\mathbf{M C}$ agreement may be more important than $\mathbf{N D}=\mathbf{F D}$ since straight comparison between MINOS Near and Far detectors may not be the most optimal approach for the oscillation analyses. On this front, the agreement of responses as well as event topology are explored.

\section{- MINOS Detector Commissioning.}

The process of configuring the two readout systems to work side-by-side at CalDet was a fruitful experience for MINOS. In addition to the expertise gained, much was learnt about how to run both readout systems in order to ensure their differences were minimised such that a similar effective overall response was achieved. This was particularly true for the ND electronics, since the system was first tested at CalDet.

\subsection{Analysis Strategy}

Although this chapter describes the results of the NFC per se, much critical preparation work has already been described in previous chapters. Two of the most important "ingredients" needed for an un-bias NFC are:

Firstly, obtaining a pure sample of single particles events per trigger incident in the CalDet, such that both readout systems have effective equivalent acceptances. This step was described in Chapter 6 . 
Secondly, performing the full calibration chain to correct for all known readout system-dependent effects. The calibration essentially brings the measured responses for both readout systems to a common framework where comparison becomes possible. Chapter 5 was fully devoted to the calibration for the NFC. Sections 3.2.2 and 3.2.3 cover the most important notions about the logic structure and functioning of the FD and the ND readout systems respectively.

\subsubsection{Analysis Probes}

Electrons are used as the main probe for the comparison of the responses from both readout systems. There are many reasons for this decision. First, the physics of electromagnetic showers is well understood and described by MC packages, like GEANT3, on which the GMINOS MC generator is based. The studies of event topology particularly benefit from having a good MC physics description. Having a reliable physics description allows for identification of possible discrepancies between MC and data in terms of effects related to the performance of one readout system with respect to the other. Second, the isolation of very pure single electron events per trigger was simpler than for other particle types with the PID instrumentation in CalDet during beam running. Third, the response of the MINOS calorimeter to electrons is the largest. The MINOS detectors are non-compensated calorimeters meaning that the calorimeters are more efficient to the electromagnetic energy depositions than those depositions of hadronic origin. Therefore, electrons are ideal to explore most of the useful energy range relevant to MINOS for oscillation physics and almost all whole dynamic range of the FD electronics. For example, electrons at $5 \mathrm{GeV} / \mathrm{c}$ generate hits which reach the end of the dynamic range of the FD electronics, beyond which the digitised responses are saturated. The energy deposition of electrons spreads only over a few strips due to electromagnetic showers. Generally there is one strip (called "Shower-Max-Strip" in this document) that obtains most of the energy deposition: an injection of light subjected to shower fluctuations. This is ideal for the NFC, since the response of the shower-max-strip serves to explore the relative non-linearity of the readout systems.

Muons are also used for the analysis. The interested in muons is two fold. First, the muon energy deposition pattern and scale is very different from that of electrons so that they explore the performance of the readout system response in a somewhat different regime, where thresholds and PMT crosstalk may become more important. Second, muons are worth studying because the major measurements 
envisaged and most critical calibration procedures in MINOS rely on the efficient description of the muon responses across all the MINOS detectors. However, the capability of using CalDet for muon studies is somewhat limited since muons escape from the CalDet instrumented volume when their momenta is beyond $2 \mathrm{GeV} / \mathrm{c}$.

Hadrons, in principle, do not offer more information to this analysis. Hadrons will also shower, but yielding a lower response than electrons. Therefore, with hadrons, one cannot explore the dynamic range of the readout systems as vastly as with electrons. However, one interesting use of hadrons is the fact that the hit pattern of hadrons is broader (broader showers), allowing a larger fraction of strips to be sampled. This in not, intrinsically, the goal of the comparison, but does allow a calibration verification. Moreover, the physics simulation of hadrons at energies of about $\mathcal{O}(1 \mathrm{GeV})$ is known to be more susceptible to deviations between $\mathrm{MC}$ and data, as have been reported in [75], due to the inaccurate modelling of the hadron response around the $\mathcal{O}(1 \mathrm{GeV})$ regime. Therefore, hadrons will be generally ignored for this analysis and only proton responses will be briefly discussed at some point during the analysis. 


\subsubsection{Generated Events}

A great deal of the scope of this analysis lies in the data and $\mathrm{MC}$ comparison. Therefore, it is useful to summarise the default configuration used for the generation of the MC events.

\begin{tabular}{|l|c|}
\hline Validity Time & $10^{\text {th }} / 9 / 2003$ \\
\hline Gain M64 & $81 \mathrm{ADC} / \mathrm{PE}$ \\
M64 non-linearity & $\sqrt{ }$ \\
\hline Gain M16 & $65 \mathrm{ADC} / \mathrm{PE}$ \\
M16 non-linearity & $\sqrt{ }$ \\
\hline Sparsification Threshold & $20 \mathrm{ADC}$ \\
Pedestal Width & $3 \mathrm{ADC}$ \\
Range0 Gain & 1.92 \\
\hline Sparsification Threshold & $12 \mathrm{ADC}$ \\
Pedestal Width & $3 \mathrm{ADC}$ \\
VA-chip non-linearity & $\sqrt{ }$ \\
VARC Trigger & $\mathrm{X}$ \\
\hline DAQ Trigger & $\mathrm{X}$ \\
\hline Dark Noise & $100 \mathrm{~Hz}$ \\
Green Fibre Noise & $300 \mathrm{~Hz}$ \\
Noise Window & $350 \mathrm{~ns}$ \\
\hline
\end{tabular}

Table 7.1: MC Generation Configuration: Detector Simulation

\begin{tabular}{|l|c|}
\hline PMT Gain Drift & X \\
Use Data PMT Gain Table & $\sqrt{ }$ \\
Use Data Linearity Calibration & $\sqrt{ }(*)$ \\
Use Data S2S Constants & $\sqrt{ }$ \\
Use Data MIP Constants & $\sqrt{ }$ \\
Use Data $4 \times$ MIP Constants & $\sqrt{ }$ \\
\hline MIP/GeV Ratio & $1.760 \times 10^{-3}$ \\
\hline
\end{tabular}

Table 7.2: MC Generation Configuration: Calibration $(*)$ : The MC has been generated assuming that both readout systems have linear responses. Then the MC responses have been "de-calibrated" (i.e. invert the effect of the calibration), incorporating the ND and FD non-linearities as measured by the LI-system on data. This non-standard approach was taken because there was no LI-linearity calibration available for the data and the non-linearities MC models were not mature enough to reproduce the non-linearity of the readout systems to the accuracy level required by this analysis.

In order to simply the forthcoming descriptions, "MC" refers to "MC events generated with the default settings", unless otherwise stated. 


\begin{tabular}{|l|c|}
\hline Beam Spot X & $0 \mathrm{~cm}$ \\
Beam Spot Y & $-1.2 \mathrm{~cm}$ \\
Beam Spot Z & 0cm (CalDet face) \\
Beam Spot Spread: $\Delta \mathrm{X}$ & $1.5 \mathrm{~cm}$ \\
Beam Spot Spread: $\Delta \mathrm{Y}$ & $1.6 \mathrm{~cm}$ \\
Angle X & $0^{\circ}$ \\
Angle Y & $0^{\circ}$ \\
Momentum Spread: $\Delta \mathrm{p} / \mathrm{p}$ & $2 \%$ \\
\hline Birk's Constant & $0.133 \mathrm{~m} / \mathrm{GeV}$ \\
GEANT Threshold & $10 \mathrm{keV}$ \\
\hline
\end{tabular}

Table 7.3: MC Generation Configuration: Event Location

\subsubsection{Data Status}

This section summarises the status of the data used. Data runs used are from the Near/Far running CalDet 2003. Only run taking with positive charge particles (as selected with the beam-line dipole magnets) were used to obtained protons, as the production of anti-proton is very inefficient. Therefore, references to electrons, pions, muons; implicitly means $e^{+}, \pi^{+} \& \mu^{+}$respectively.

The calibration corrections applied to the data are shown in Table 7.4.

\begin{tabular}{|l|c|}
\hline LI Drift Point & $\sqrt{ }$ \\
LI Gain & $\sqrt{ }$ \\
LI Linearity & X \\
S2S & $\sqrt{ }$ \\
MIP & $\sqrt{ }$ \\
$4 \times$ MIP & $\sqrt{ }$ \\
Absolute & $\sqrt{ }$ (e only $)$ \\
\hline
\end{tabular}

Table 7.4: Data Calibration

The details of the data acquisition were explained in Chapter 4 while the calibration steps were summarised in Chapter 5 - those steps performed online are always applied and, therefore, are not specifically mentioned.

\section{Known Calibration Problems}

A few strip-end associated calibration problems were identified due to their spurious behaviour throughout the running period. The contribution of such strips, meaning both ends (ND and FD readout) of the strip in question, are therefore ignored in 
the analysis. No event was rejected for this reason since ignoring a few strips on the outskirts of the beam-spot should not induce any sizable bias on average. To first order, double-ended hits within the core of the beam-spot dominate the overall response budget. The same procedure was applied to simulated events, so that MC data and beam data could be compared on the same basis. Small systematic differences could develop between the measured responses of the FD and the ND sides and between data and MC if this rejection had not been not accomplished. Table 7.5 summarises the location of the strip-end associated problems in detectorspace.

\begin{tabular}{|c|c|l|}
\hline Plane & Strip & Problem \\
\hline \hline 0 & all & Bad calibration constant \\
6 & 18 & Bad calibration constant \\
21 & 19 & Wrong pedestal computation \\
41 & 19 & Wrong pedestal computation \\
43 & 1 & Bad calibration constant \\
47 & 7 & Bad calibration constant \\
\hline
\end{tabular}

Table 7.5: This table shows a list of strips dismissed along with the problems causing their rejection. Note that none of the strips are in the central region occupied by events during the beam running (strip $\in[8,16]$ and planes $\in[1,20]$ ). Thus, about $2 \%$ (29 strips out of 1440) of the detector were not used for analysis.

\subsection{Energy Comparison Framework}

The energy comparison will be broken down into three sections. This first section will introduce the reader to the framework developed to enable a response comparison across the two readout systems. The second section will concentrate on analysis results obtained at the individual hit level: i.e. double-ended hit and single-ended hit comparisons. While the third section will concentrate on the event energy comparison, which is inherently related to the previous section.

\subsubsection{The Energy Asymmetry Definition: $A_{N / F}$}

Responses will be generally compared by calculating the relative difference between the responses of both readouts: the "Near/Far Energy Asymmetry" $\left(A_{N / F}\right)$. The general form of $A_{N / F}$ is given in Equation 7.2, where $\mathcal{N}$ and $\mathcal{F}$ stand for the responses measured in the ND and FD respectively. The same notation will be 
used throughout this document. When $A_{N / F}=0$, this means that the calibrated responses of both readout systems are identical, i.e. maximal correlation (Figure 7.1). $A_{N / F}>0$ means that the ND side dominates relative to the FD side and vice versa for $A_{N / F}<0$.

$$
\begin{aligned}
& \qquad A_{N / F}=\frac{\mathcal{N}-\mathcal{F}}{\frac{1}{2}(\mathcal{N}+\mathcal{F})} \\
& \text { such that } A_{N / F}\left\{\begin{array}{r}
>0 \longrightarrow \mathcal{N}>\mathcal{F} \\
=0 \longrightarrow \mathcal{N}=\mathcal{F} \\
<0 \longrightarrow \mathcal{N}<\mathcal{F}
\end{array}\right.
\end{aligned}
$$

$A_{N / F}$ has been normalised by the average deposition $\frac{1}{2}(\mathcal{N}+\mathcal{F})$, which has the advantage of making $A_{N / F}$ proportional to the relative fraction of the effect by a factor $\times 100$, therefore, $A_{N / F} \in[-2,2]$.

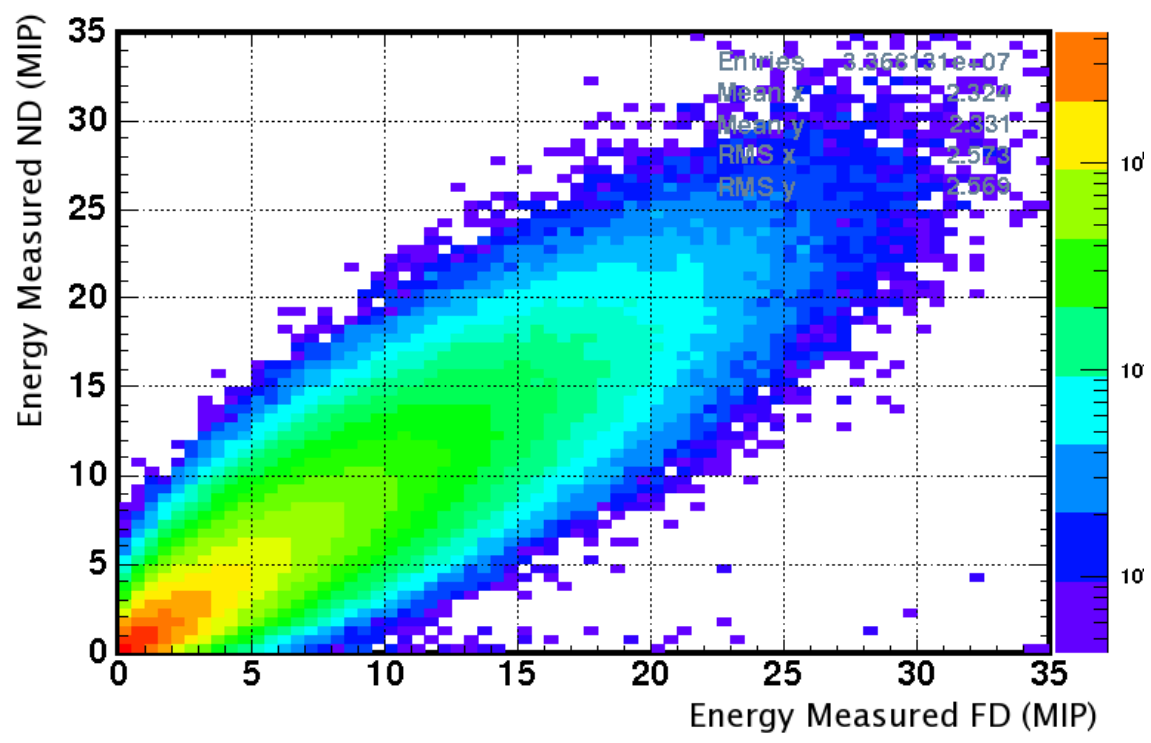

Figure 7.1: Correlation of Responses Strip-by-Strip.

The reason why the comparison is based on a difference rather than on, for example, a ratio is that ratios are likely to diverge as the denominator tends to zero. So, for example, when studying low energy deposition strip-wise, as seen in Figure 7.1, it may well happen that one side measures "A", a small amount of energy, whereas the other side measures "B", that happens to be zero because, for 
example, an instrumental effect like sparsification or merely because of statistical fluctuations.

Note that $A_{N / F}$ can be computed either on an event-by-event basis or a stripby-strip basis. The former refers to the comparison between the calorimetric energy measurement made by either readout systems, while the latter, refers to the comparison of the signal as measured on either side of the same strip (double-ended hits).

Single-ended hits also contain information regarding readout asymmetry, but they would trivially cause $A_{N / F}$ to be maximal $( \pm 2)$ (by definition) and, so, are not useful in this comparison framework. Instead, their effect is studied in terms of their contribution to the event total energy. By looking at the event-by-event and strip-by-strip energy comparison, one addresses different, but correlated, aspects of the performance of the readout systems and its relative calibration.

\subsection{Strip-wise Energy Comparison}

\subsubsection{Double-Ended Strip Relative Comparison}

The aim of this section is to explore and understand the effective relative response of both readout systems to energy deposition at the strip level. Only double-ended hits are used such that the energy asymmetry can be computed. The $A_{N / F}^{\text {Strip }}$ is calculated according to Equation 7.2.

$$
A_{\text {NtF }}^{\text {Strip }}=\frac{\mathcal{N}^{\text {Strip }}-\mathcal{F}^{\text {Strip }}}{\frac{1}{2}\left(\mathcal{N}^{\text {Strip }}+\mathcal{F}^{\text {Strip }}\right)}
$$

It should be noted that there is a distinction between "strips" and "digits" because the energy associated with a strip is the sum of all digits in a strip per trigger. In the FD, the charge integration is carried out at the level of the electronics, therefore outputting only one digit per trigger. However, the ND electronics integrate the current pulses in separate buckets $(\sim 19 \mathrm{~ns})$, reflecting, coarsely, the time structure of the light pulses incident at the PMTs. Every bucket is then digitised separately. Therefore a "strip" corresponds to the total energy (offline sum over buckets in the ND) deposited in the detector per beam-trigger.

Because of the way $A_{N / F}^{\text {Strip }}$ has been computed and as a result of the calibration applied (summarised in Table 7.4), two main observations are expected:

1. Since the readout system LI-linearity calibration was not performed, the 
$A_{N / F}^{\text {Strip }}$ is expected to be very sensitive to the non-linearity of both readout systems - that is, the relative difference in their non-linearities. Therefore, the $\mathrm{MC}$ is our best reference for understanding the structure of $A_{N / F}^{\text {Strip }}$ with energy in the data as it gathers all our knowledge for both readout systems non-linearities and threshold effects.

2. The S2S calibration plays a critical role in the structure of $A_{N / F}^{\text {Strip }} \cdot A_{N / F}^{\text {Strip }}$ uses only double-ended hits. Hence, it has been constructed very similarly to the way the S2S calibration has been constructed - i.e. using response whose crosstalk has been rejected. This resemblance allows us to predict that $A_{N / F}^{\text {Strip }}$ must be zero at about $\sim 1.2-1.5 \mathrm{MIP}$. This is because the S2S calibration equalises the average energy deposited by cosmic muons (about $1.5 \mathrm{MIP})$ across all strips in the detector. Deviations of $A_{N / F}^{\text {Strip }}$ from zero across the energy range explored are expected to be dominated by the non-linearity contribution of both readout systems, as mentioned above.

The $A_{N / F}^{\text {Strip }}$ evolution with average strip energy $\left(\frac{1}{2}\left(\mathcal{N}^{\text {Strip }}+\mathcal{F}^{\text {Strip }}\right)\right)$, as computed for electrons, is shown in Figure 7.2 for MC (red) and data (black). The top plot shows the contour of the evolution of $A_{N / F}^{\text {Strip }}$ while the bottom plot shows the profile version of the same distribution. These plots cover the entire strip-wise dynamic range at CalDet: from 0 to $\sim 40 \mathrm{MIP}$, which corresponds to about $\sim 180 \mathrm{PE}$ or $\sim 12000 \mathrm{ADC}(\mathrm{FD})$ and $\sim 15000 \mathrm{ADC}(\mathrm{ND})$.

The structure shown in the contour plot is intrinsic to the energy deposition of electrons convolved with Poisson statistics, which dictate the probability of a photon going to either side of a strip. The Poisson contribution is responsible for the broadening of the distribution at very low energies, where the fluctuations can even be maximal $\left(A_{N / F}^{\text {Strip }}= \pm 2\right)$, reflecting the physical scenario of a few photons going to either one side or the other. The same plot indicates that most $(>90 \%)$ of the hits associated with electrons are at energies below 5MIP. Note that these plots have been generated with using $\sim 0.5 \times 10^{6}$ electrons across all energies up to $6 \mathrm{GeV} / \mathrm{c}$ giving rise to more than $6 \times 10^{6}$ double-ended hits.

As explained in Table 7.4, the MC events have been generated by explicitly adding the average non-linearity of the readout systems as measured in data by the LI system, as summarised in Section 5.4.3. Note that this approach is almost equivalent to linearising the data (by calibrating out the non-linearity of each channel as measured by the LI-system) and then performing comparisons with a MC generated without non-linearity. Therefore, Figure 7.2 also shows that the 

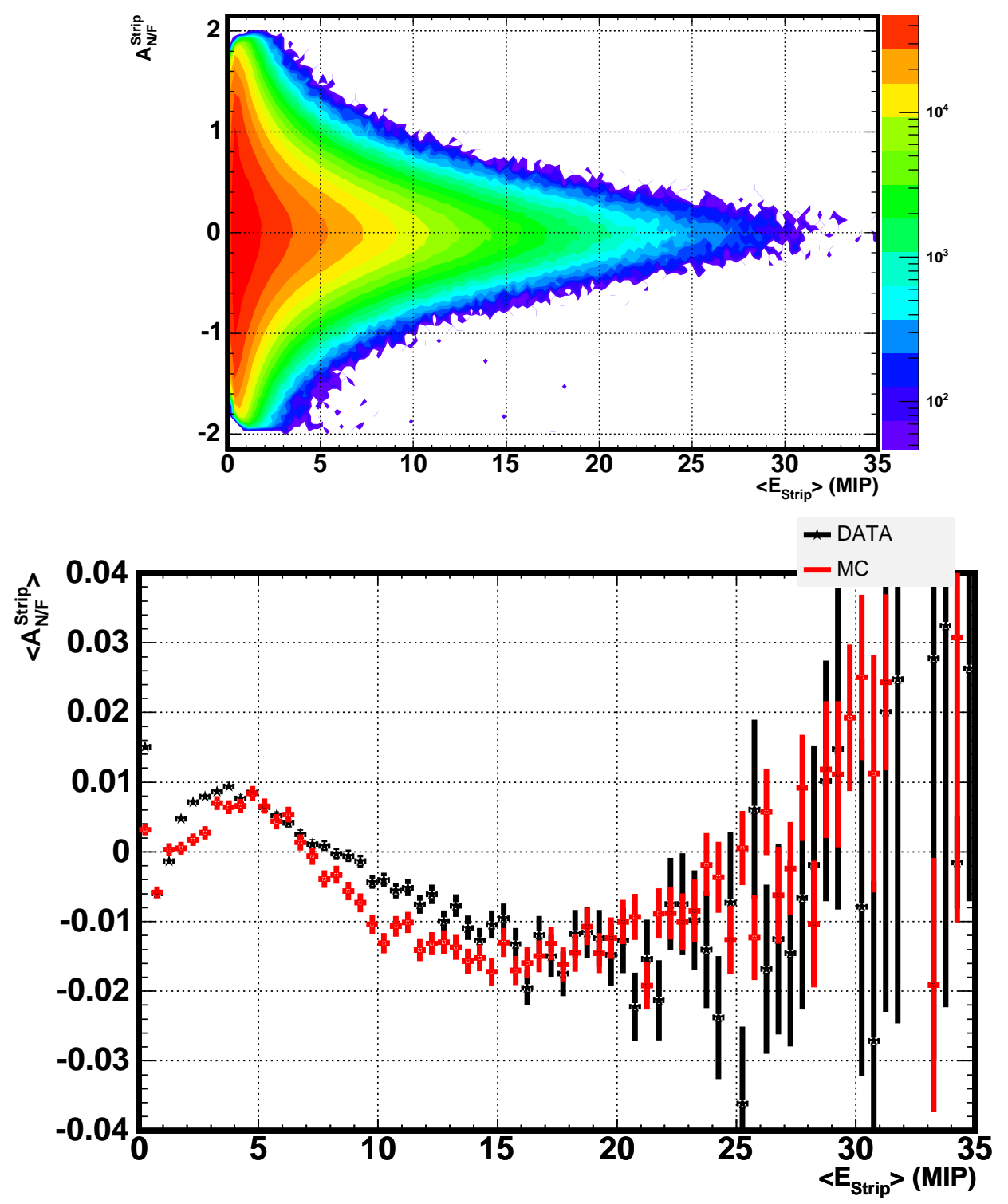

Figure 7.2: Strip-by-Strip $A_{N / F}$ Evolution. This plot shows $A_{N / F}^{\text {Strip }}$ versus the average energy on the strip for double-ended hits. The structure exhibited is a convolution of the effect of the calibration, the relative non-linearity and the sparsification on both readout systems. Electrons from $0.8 \mathrm{GeV}$ to $6 \mathrm{GeV}$ have been used to produce this plot.

LI-linearity correction could be used to calibrate the non-linearity of both readout system accurately. This result is important because it was not obvious that the non-linearity measured by the LI-system would be the same as the non-linearity exhibited by the detector response to electrons. This uncertainty is due to the assumption that PMT non-linearity is driven by the rate of charge incident at the 


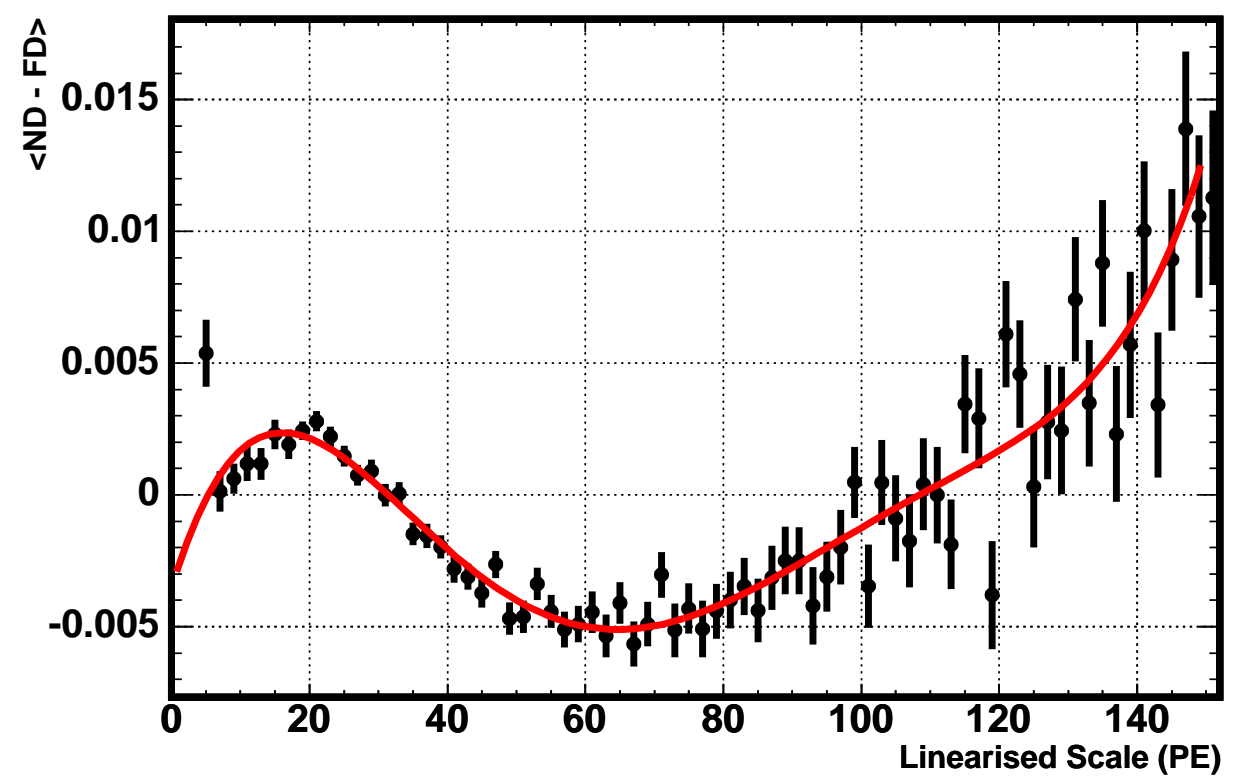

Figure 7.3: Relative Non-Linearities as Measured by LI. This plot shows the relative difference of the non-linearity fractions of each readout systems (ND-FD) as measured by the LI-system. In other words, this plot represents the "prediction" of the structure of $A_{N / F}$ caused by the intrinsic non-linearities of the systems as characterised by the LI-system.

anode (for each pixel). Therefore, if the time structure of the charge pulses in the PMTs was different between the LI system and electrons, the LI system would not have been able to correct for the non-linearity.

The LI-system was designed to deliver pulses that resemble those generated by energy depositions in the MINOS scintillator [106]. Therefore, Figure 7.2 constitutes a success for the MINOS calibration chain.

Figure 7.3 shows the expected relative non-linearity as measured by the LIsystem. Note that the two curves shown in Figure 7.3 and Figure 7.2 are not meant to be identical, but strongly correlated. Figure 7.2 is a convolution including the effect of the S2S calibration (not present in the LI estimation - Figure 7.3) as well as the relative non-linearity.

The evolution of $A_{N / F}^{\text {Strip }}$, shown in Figure 7.2 (bottom) is rich in features to which we shall dedicate further attention by breaking down the curve into different ranges:

From 0 MIP to 2.5MIP: The "Dip". This range is characterised by exhibiting a "dip" at about $0.5 \mathrm{MIP}(\mathcal{N}<\mathcal{F})$, then followed by a "hill" from 2.5-5MIP, 
over which $\mathcal{N}>\mathcal{F}$. The main characteristics of this regime are:

- As anticipated, $A_{N / F}^{\text {Strip }} \rightarrow 0$ between approximately $1.25 \mathrm{MIP}$ to $1.5 \mathrm{MIP}$, which is a consequence of the S2S calibration normalisation.

- The observed dip is caused by bucket sparsification on the ND side, with respect to the effect of sparsification on the FD, which is applied to the total integrated charge per strip. Figure 7.4 shows a finer detail view of the dip area as measured by electrons. Three curves are shown: black corresponds to data, red corresponds to $\mathrm{MC}$ and blue corresponds to $\mathrm{MC}$ generated with the sparsification switched off for the ND side only. The difference between the blue and the red curves (both MC) represents the effect of bucket sparsification, since both curves were generated with the same FD settings, allowing the FD response to serves as a reference. The absolute size of effect of bucket sparsification is of the order of $\sim 4.5 \%$ at $0.5 \mathrm{MIP}$, decreasing the effective response of the ND readout system.

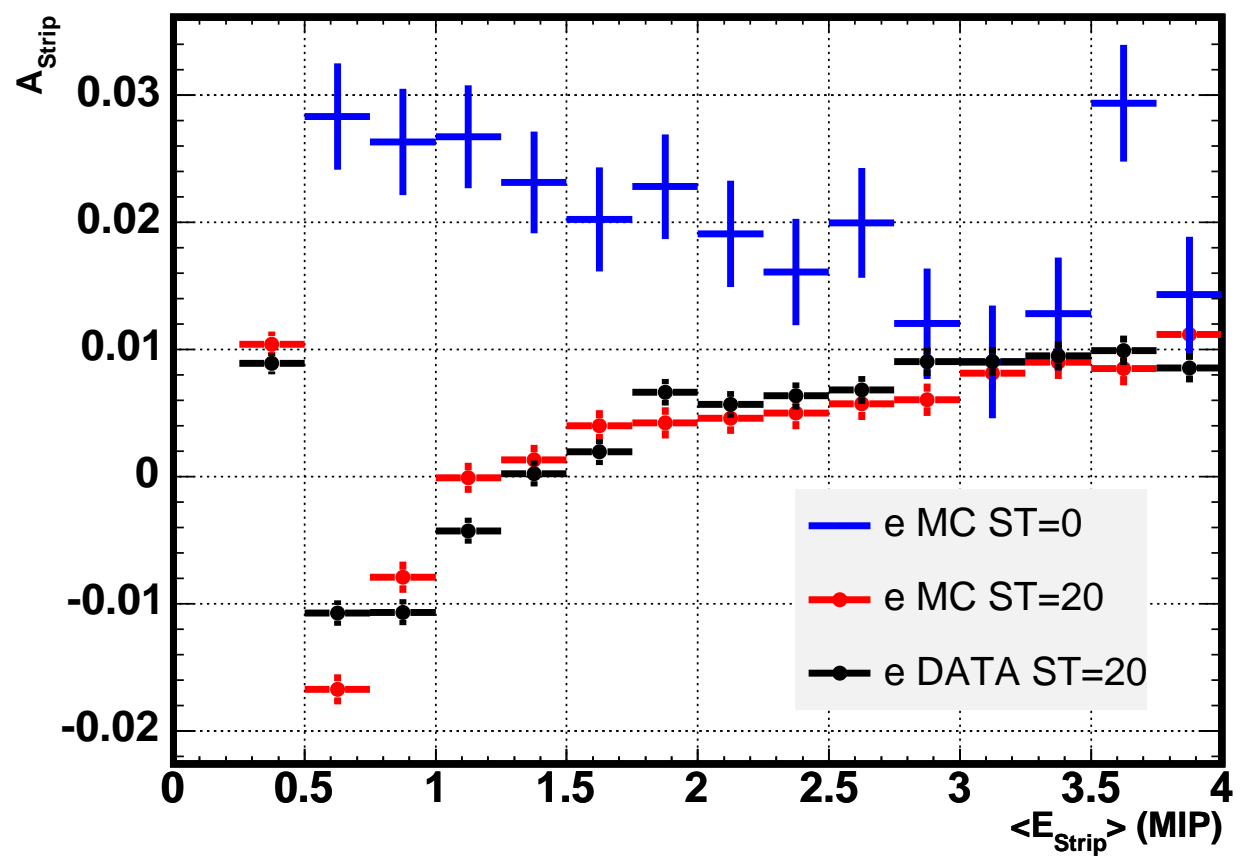

Figure 7.4: Bucket Sparsification Effect. This plots shows $A_{N / F}^{\text {Strip }}$, in detail, over the region in which the ND response has been suppressed by bucket sparsification. The black curve corresponds to data while the red and blue curves correspond to MC generated with and without sparsification, respectively. Therefore, the difference between the MC curves represent the MC prediction for the magnitude of the effect. 
The structure observed in Figure 7.4 can be qualitatively justified by simple arguments. As charge increases beyond 0.5MIP (first bin seen), the average probability that the pulses can span more than one bucket by $\sim 20 \%$ (where $0.2=4 \mathrm{~ns} / 19 \mathrm{~ns}^{1}$ ) at $0.75 \mathrm{MIP}$. Charge spreading over more than one bucket will produce a more dramatic sparsification effect. The loss of charge due to sparsification can be as high as $\sim 25 \%$ (where $0.25=0.5 \mathrm{PE} / 2 \mathrm{PE})$ at $0.5 \mathrm{MIP}$. To first order, the combination of the charge loss with probability that that occurs explains the magnitude of the decrease in response exhibited by the data: $\sim 5 \%=20 \% \times 25 \%$. The first bin $(<0.5 \mathrm{MIP})$ is expected to be less affected by bucket sparsification as the charge is so low that it hardly spreads beyond one bucket; therefore the average suppression is expected to be less, as observed. On the other hand, as charge increases, the fraction of charge loss due to sparsification becomes more negligible causing the dip to effectively disappear.

Bucket sparsification was preliminarily studied using simple simulations in [119]. The findings from such studies are in good agreement with the results reported here, for the first time using MINOS data.

- Note that the whole mechanism behind bucket sparsification is strongly dependent on the time structure of the charge pulses digitised by the QIE-FEE. The agreement between MC and data suggests that the MC accurately simulates the real time structure of the pulses.

- The first bin shown $(<0.5 \mathrm{MIP}$ in Figure 7.4$)$ is affected not only by ND bucket sparsification, but also by the FD sparsification thresholds and possibly electrical PMT crosstalk (see Appendix A) that may leak into the double-ended hit sample. A cut was applied at $>0.14 \mathrm{MIP}$ (about 0.5PE) to emulate a common sparsification threshold across both readout systems. This cut significantly improves the shown agreement between $\mathrm{MC}$ and data for the lowest bin. Before the cut there was a discrepancy between MC and data of up to $3 \%$.

The bucket sparsification can have a dramatic effect on the MINOS calibration chain, as will be discussed in Section 7.8.1.

\footnotetext{
${ }^{1}$ This is in agreement with measurements carried out with the Oxford PMT test-stand, where the average pulse width of a $1 \mathrm{PE}$ pulse is about $3-4 \mathrm{~ns}$ [118].
} 
From 2.5 MIP to 5MIP: The "Hill". The hill describes the region, which follows the dip, over which there is an apparent $\mathcal{N}>\mathcal{F}$ excess. This apparent hill is a consequence of the combination of the $\mathrm{S} 2 \mathrm{~S}$ calibration normalisation (which sets the relative scale between both readout systems responses ) and the bucket sparsification effect. Because the S2S calibration was carried out within the regime affected by the dip caused by the bucket sparsification, consequently zeroing $A_{N / F}^{\text {Strip }}$ at $\sim 1.5 \mathrm{MIP}$, the region where the bucket sparsification becomes negligible appears like "hill".

However, during the LI-linearity studies summarised in Section 5.4.3, it was found the M64 had a small positive contribution to the non-linearity: $+0.4 \%$ at about $20 \mathrm{PE}$, which is shown as a bump in Figure 7.3. The $A_{N / F}^{\text {Strip }}$, as computed with data, seems to support the hypothesis that such a feature may be real. Its origin and the verification of the effect has not been settled by the time of the writing of this thesis.

In summary, the hill in $A_{N / F}^{\text {Strip }}$ is believed to be dominated by the combination of the calibration and the bucket sparsification effect causing the dip. In addition, there may be an instrumental effect arising from the M64s that could also be contributing to the measured magnitude and shape of the hill.

From 5MIP to 20MIP This region is dominated by the relative difference between the non-linearity rates of the M64 and M16. The fact that $A_{N / F}^{\text {Strip }}$ effectively falls with the average energy per strip, shown in Figure 7.2(bottom), is because over most of this range the M64 non-linearity dominates with respect to the M16 non-linearity. The non-linearity of the M16 effectively does not "switch-on" until $\sim 60 \mathrm{PE}(\sim 15 \mathrm{MIP})$, which causes the flattening of the response difference near the end of this range. The VA-chip non-linearity does not become appreciable until about 20 - 25MIP, which determines the end of this regime. This range is characterised by an excellent agreement between $\mathrm{MC}$ and data.

The relative contribution of the each readout systems non-linearity was measured with the LI-system, as reported in Section 5.4.3. The slope of $A_{N / F}^{\text {Strip }}$ between $5 \mathrm{MIP}$ and $15 \mathrm{MIP}$ is about $-0.22 \%$ /MIP, which roughly corresponds to the slope expected from the contribution of the M64 non-linearity, measured to be $\sim-0.20 \% / \mathrm{MIP}$. The expected contribution due to the M16 nonlinearity is $\sim+0.14 \% / \mathrm{MIP}^{2}$. Beyond $15 \mathrm{MIP}$, the relative difference becomes

\footnotetext{
${ }^{2}$ The positive sign serves to indicate the positive trend of the M16 non-linearity within the
} 
hard to define accurately due to the low statistics available.

Beyond 20MIP The structure of $A_{N / F}^{\text {Strip }}$ from 20MIP is dominated by the VA-chip non-linearity whose dramatic effect can be observed despite the increasingly low statistics. MC and data agree once again, but given the poor statistics, little can be said regarding the underlying structure and the degree of agreement. The VA non-linearity should overwhelm the fate of the curve beyond $\sim 8000 \mathrm{ADC}$, i.e. $\sim 28 \mathrm{MIP}$ as shown by the $\mathrm{MC}$ in Figure 5.5 .

The reason why the statistics are so poor over this region is because the analysis has to be constrained to electron runs up to $6 \mathrm{GeV} / \mathrm{c}$. Higher energy runs taking with dedicated "electron-trigger", have the HV lowered by $30 \%$ for the PMTs of the FD side. This was done in order to prevent the VA-chip electronics from saturating at about 14000ADC. By lowering the $\mathrm{HV}$, the effective non-linearity regimen of the PMTs has changed, as the $\mathrm{MIP} / \mathrm{ADC}$ ratio has been increased. With the HV having been lowered, 4000ADC becomes 25MIP and therefore any comparison across data with different HV would simply be misleading.

In conclusion, all features of the structure of $A_{N / F}^{\text {Strip }}$ are not only well understood but also accurately described by the MC, which represents a success for both the calibration and detector simulation efforts in MINOS. It is particularly remarkable that the $\mathrm{MC}$, once given the average non-linearity information as measured by the LI-system (shown in Figure 7.2), describes the data with deviations within the 1\% level.

\subsubsection{Single-End Strip Relative Comparison}

Single-ended hits are interesting because they account for the contribution generated through mainly PMT crosstalk and, to a lesser extent, single-PE noise. The single-ended responses are not expected to be comparable across readout systems, as their crosstalk fractions are not equal. Therefore, our interest in the single-ended responses is to use them as a test-bed for the data/MC comparison. Moreover, the single-ended energy contribution will have an impact on the event-by-event energy comparison. The energy fraction of single-ended hits can account for up to $\sim 5 \%$ of the measured calorimetric energy, while the remaning $\sim 95 \%$ of the energy occurs as double-ended hits.

scope of $A_{N / F}^{\text {Strip }}$ sign convention. 
The energy contribution of single-ended hits and double-ended hits to the total energy per event is shown in Figure 7.5 (bottom and top, respectively) versus the total energy of the event. The plots are also divided into FD and ND, which are left and right, respectively. The plots correspond to electrons from 0.8 to $6 \mathrm{GeV} / \mathrm{c}$.

Double-Ended(FD)

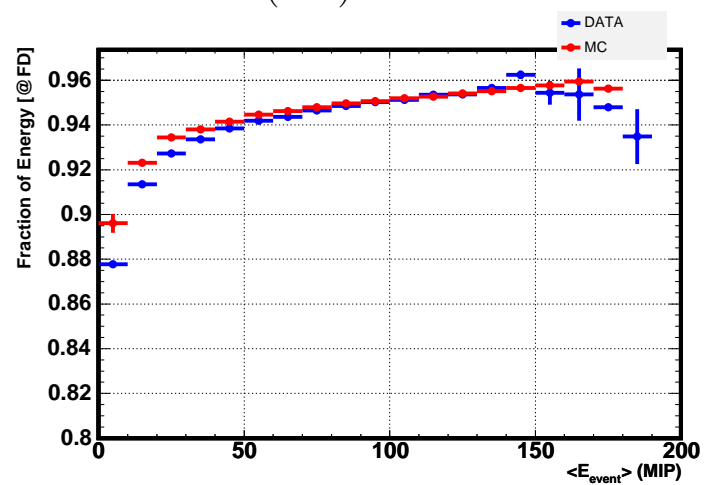

Single-Ended(FD)

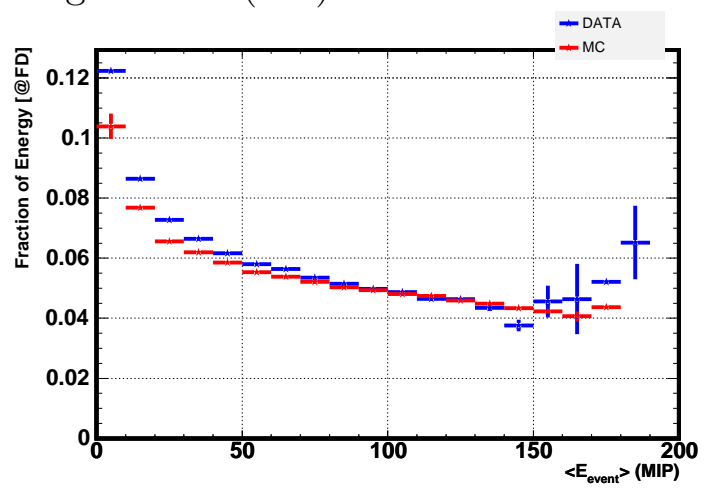

Double-Ended(ND)

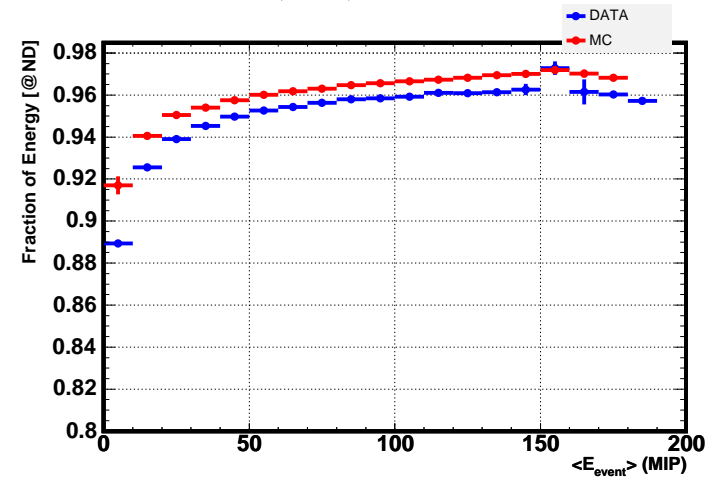

Single-Ended(ND)

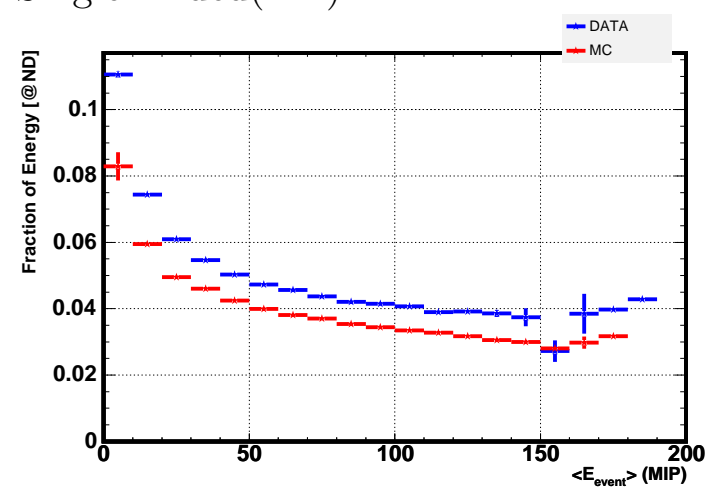

Figure 7.5: Double/Single Ended Hits Contributions. These four plots correspond to the relative contribution of double-ended and single-ended hits to the total energy of the event. They provide a test-bed for the MC/data comparison for both readout systems.

The slope exhibited in all curves is believed to be caused by the fact that single-ended hits can sometimes match up, faking double-ended hits, particularly in shower events. This "random matching" occurs more frequently as the energy of the events increases. This is because the region where hits are confined increases logarithmically (in correlation with "shower-max") while the light level of the events increases linearly with energy, as does the amount of charge due to crosstalk. Therefore, as the event energy increases, the energy density increases, almost linearly, thus increasing the probability for single-ended hits to match up.

Note that the the MC and data estimations of the single-ended and doubleended hits energy fractions are in reasonable good agreement, particularly for the 
FD side. For the ND, however, the MC seems to overestimate the fraction of energy going into double-ended hits (by a constant $1 \%$ with event energy), and, consequently, underestimates the fraction of energy going into single-ended hits. This effect could, in principle, have many sources: such as a calibration problem on the data or a MC problem, by which there is something not well taken into account. In fact, both avenues are possible, as will be shown. The calibration has problems at the $\sim 1.0 \%$ level, while the MC seems to underestimate the ND crosstalk slightly.

However, there is yet another effect on the data not simulated by the MC that could partially explain this observation, namely that the ND side was found to exhibit a larger rate of single-PE noise than expected (see Appendix B). On the hand, the MC has been generated with the same rate of noise as measured on the FD side $\sim 2 \mathrm{kHz}$ per plane. Hence, this mechanism would explain why agreement between MC and data appears better on the FD side. Independent measurements with the ND showed up to $20 \mathrm{kHz}$ per plane or $53 \mathrm{kHz}$ per M64. The expected energy contribution due to noise for the ND could add up to $\sim 0.4 \%^{3}$ per event for electrons at $1 \mathrm{GeV} / \mathrm{c}$. However, their contribution of this effect to the total energy of the event is expected to fade with increasing event energy.

\subsection{Event-wise Energy Comparison}

This section will be broken down into five sub-sections. The first section covers analytical calculation of the expected value on $A_{N / F}$. The second part will be dedicated to the presentation of the results obtained. The third part will be devoted to further investigate the quality of the calibration, which is critical for the subsequent discussion and interpretation of the results. The fourth part studies the nature of the width of the $A_{N / F}$ distribution. The fifth part will be focused on interpreting and discussing about the all results obtained.

\footnotetext{
${ }^{3}$ The average rate of noise per event is about $0.45=30 \times 53 \mathrm{kHz} \times \sim 300 \mathrm{~ns} \sim 8 \%(30 \mathrm{PMTs}$ on the ND side). The measured spectra of the noise peaks at $0.4 \mathrm{MIP}(1.5 \mathrm{PE})$, therefore the average contribution of noise per event is about $0.18 \mathrm{MIP}$ per event $=0.45$ noise hits per event $\times 0.4 \mathrm{MIP}$ per noise hit. Therefore, the expected increase in energy per event depends upon the response of the event -for example, at $1 \mathrm{GeV} / \mathrm{c}$ electrons deposit $\sim 50 \mathrm{MIP}-$, hence the noise amounts to up to an additional $0.4 \%$ to the total event energy.
} 


\subsubsection{Analytical Calculations of $A_{N / F}$}

This section is dedicated to explicitly carrying out calculations of the expected values for the different $A_{N / F}$ measured. In this calculation, the effect of the S2S calibration will be taking into account.

The S2S calibration is expected to equalise (or calibrate) the responses of both readout systems, providing the energy deposition has happened along the calibration-axis and the responses employed are "tracked responses" (no crosstalk), as previously explained. This equality between "tracked responses" and "crosstalkrejected responses" is possible because of the ability of the tracker to reject (used for the computation of the S2S calibration) any hit which is topologically not being associated with the track (typically true for all crosstalk hits). The crosstalk of the $\mathrm{ND}$ and FD will be indicated respectively by $\mathcal{X}_{N D}$ and $\mathcal{X}_{F D}$. Therefore, the effect of the S2S calibration can be summarised in the following equation:

$$
\mathcal{N}\left(1-\mathcal{X}_{N D}\right)=\mathcal{F}\left(1-\mathcal{X}_{F D}\right)
$$

The calculations in this section will assume the following conditions:

i) the measured responses are generated by particles interacting along the calibrationaxis, so that there no need for attenuation corrections

ii) the responses are perfectly linear for both readout systems

These two conditions are used at this stage to simplify the calculations, but also because the data, once fully calibrated, should be compatible with them.

- Calculation of $A_{N / F}^{S 2 S}$

First, we shall try to construct an $A_{N / F}$, which is calibrated by the S2S calibration: $A_{N / F}^{S 2 S}$. Such an $A_{N / F}$ must, therefore, use tracked-responses or responses whose crosstalk have been fully subtracted. The former can be achieved only with muons, while the latter becomes possible for any particle, if we know both the ND and FD crosstalks. As will be shown in Section 7.8.2, we do indeed know the crosstalk of both readout systems, therefore,

$$
A_{N / F}^{S 2 S}=\frac{\mathcal{N}\left(1-\mathcal{X}_{N D}\right)-\mathcal{F}\left(1-\mathcal{X}_{F D}\right)}{\frac{1}{2}(\mathcal{N}+\mathcal{F})}
$$


Note that the numerator of $A_{N / F}^{S 2 S}$ cancels upon imposing the effect of the S2S calibration (Equation 7.3). Therefore, $A_{N / F}^{S 2 S}$ is zero, as expected, leading to the expected fact that

$$
A_{N / F}^{\text {Tracked }} \equiv A_{N / F}^{\text {NoCrosstalk }} \rightarrow 0
$$

- Calculation of $A_{N / F}^{O n A x i s}$

It is instructive to explore the expected value of the bare $A_{N / F}^{O n A x i s}$.

$$
\begin{aligned}
A_{N / F}^{\text {OnAxis }} & =\frac{\mathcal{N}-\mathcal{F}}{\frac{1}{2}(\mathcal{N}+\mathcal{F})} \\
& =\frac{\mathcal{N}\left(1-\mathcal{X}_{N D}+\mathcal{X}_{N D}\right)-\mathcal{F}\left(1-\mathcal{X}_{F D}+\mathcal{X}_{F D}\right)}{\frac{1}{2}(\mathcal{N}+\mathcal{F})} \\
& \stackrel{\mathcal{N}}{\approx} \mathcal{F} \\
& \approx A_{N / F}^{S 2 S}+\mathcal{X}_{N D}-\mathcal{X}_{F D} \\
& A_{N / F}^{S 2 S}+\Delta \mathcal{X}
\end{aligned}
$$

Substituting Equation 7.3 into Equation 7.9 imposes normalisation the S2S calibration on the measured responses, which is the same as $A_{N / F}^{S 2 S} \rightarrow 0$. This way, one obtains a prediction for the measured value of $A_{N / F}^{O n A x i s}$, as a function of difference between $\mathcal{X}_{N D}$ and $\mathcal{X}_{F D}$, which have been measured to be $\sim$ $3.2 \%$ and $\sim 5.2 \%$ respectively. Hence, taking into account the effect of the calibration and the measured crosstalks, we obtain:

$$
\begin{aligned}
& A_{N / F}^{\text {OnAxis }}=\Delta \mathcal{X} \\
& A_{N / F}^{\text {OnAxis }} \rightarrow-2.0 \%
\end{aligned}
$$

Note that $A_{N / F}^{O n A x i s}$ is not expected to be zero, as a consequence of the S2S calibration.

Since the data has not been linearised (Table 7.4), the calculated predictions are not fully comparable with the measured $A_{N / F}$. Therefore, as we cannot easily introduce an analytical correction for the non-linearity, we shall associate a conservative uncertainty with the provided predictions. The uncertainty was calculated 
to be a $0.2 \%$, which corresponds to the variation of $A_{N / F}$ when the non-linearity is switched on and off: $\left(\left|A_{N / F}^{N o n L I N}-A_{N / F}^{L I N}\right|\right)_{M C}$ using MC. Where $A_{N / F}^{L I N}$ and $A_{N / F}^{N o n L I N}$ correspond to the value of $A_{N / F}$ with and without linearity correction. Different attenuation corrections/calibrations have been computed, so a better comparison between analytical calculations and data can be performed.

\subsubsection{Measurements of $A_{N / F}$}

The event-by-event energy comparison is studied based on $A_{N / F}$, which, for this analysis, is computed as the relative difference in calorimetric responses measured by both readout systems. In order to compare the effective integrated responses across both sides of the detector, the effect of light attenuation along the strips has to be taken into account. This is because the responses of both readout systems are calibrated to be the same only at the calibration-axis (centre of the detector) by construction of the $\mathrm{S} 2 \mathrm{~S}$ calibration. If the beam-spot is off from the detector centre, the $A_{N / F}$ would measure an apparent bias between the responses of the ND and FD - because the amount of light measured becomes larger for the readout system located the closest to the beam-spot.

In order to isolated $A_{N / F}$ from the specific configuration of CalDet, two different approaches have been followed:

- Implementation of a calibration that is able to account for both the attenuation of light along the fibres and PMT-crosstalk differences. The calibration in question is the $4 \times$ MIP calibration that was described in Section 5.5.3.

- Measurement and correction for both the attenuation (integral attenuation calibration, described in Section 5.5.3) and the PMT-crosstalk on an eventby-event basis. This approach allows the $A_{N / F}$ to be calculated as if it had been computed from particles interacting at the centre of the detector, i.e. along the calibration-axis.

The two methods are complementary as they have different advantages and disadvantages. It is particularly fruitful to use both, as they provide a different insight into the results obtained. An extreme case of complementarity is the fact that there is no way to validate the magnitude of the integral attenuation correction other than by the comparison of the results to those obtained with the $4 \times \mathrm{MIP}$ calibration. 
The forthcoming presentation of results will be broken down into three parts. The first part concentrates on those results obtained using the $4 \times \mathrm{MIP}$ calibration including a discussion of the specific problems associated with the implementation of this method at CalDet. In the second part, the measurements of $A_{N / F}$ using the integrated attenuation correction will be presented. While the third part will be dedicated to the study of the evolution of all measured $A_{N / F}$ with event energy.

Measurements of $A_{N / F}^{4 \times \mathrm{MIP}}$

This approach is particularly interesting for MINOS, as it relies on the framework set up for the MINOS inter-detector or MIP calibration. The principle was to use beam stopping muons between $1.4-2.0 \mathrm{GeV} / \mathrm{c}$ to compute one MIP constant per view: ND-ODD, ND-EVEN, FD-ODD and FD-EVEN. This approach can be viewed as a relative "inter-detector" calibration across the ND and FD readout system responses while both readout systems are physically located in the same detector (CalDet). The MINOS inter-detector calibration will aim to do the same goal when the two readout systems are located in their corresponding detectors kilometres apart.

A more pragmatic view of the principle is that, by applying the $4 \times \mathrm{MIP}$ calibration, we are normalising the responses of any incident particle to that of beam stopping muons per view. Therefore, the whole procedure relies on a critical assumption: all underlying effects, manifested on the response of muons over the MIP-window must have a similar effect on the other beam particles and over the entire detector. The $4 \times$ MIP calibration will, therefore, take into account the following effects:

1. Attenuation Beam stopping muons strike the detector at the beam-spot and not at the calibration-axis.

2. Crosstalk The MIP calibration is computed based on the calorimetric response of muons within the MIP window. Therefore, the $4 \times$ MIP calibration accounts for crosstalk contributions present in the calorimetric responses, and rejected during the S2S calibration definition.

3. Calibration Systematic Bias If there was a calibration systematic bias that was evenly propagated across a whole view(s) of the detector, the $4 \times \mathrm{MIP}$ calibration could also correct for this. An example of such a case is the socalled "EVEN/ODD" asymmetry that has been induced in the past by the S2S calibration [120]. 
However, if there was a calibration problem that varied heterogeneously across the length $\mathrm{z}$-direction of the detector, then the $4 \times \mathrm{MIP}$ calibration would not be able to correct for that or may even introduce possible artifacts.
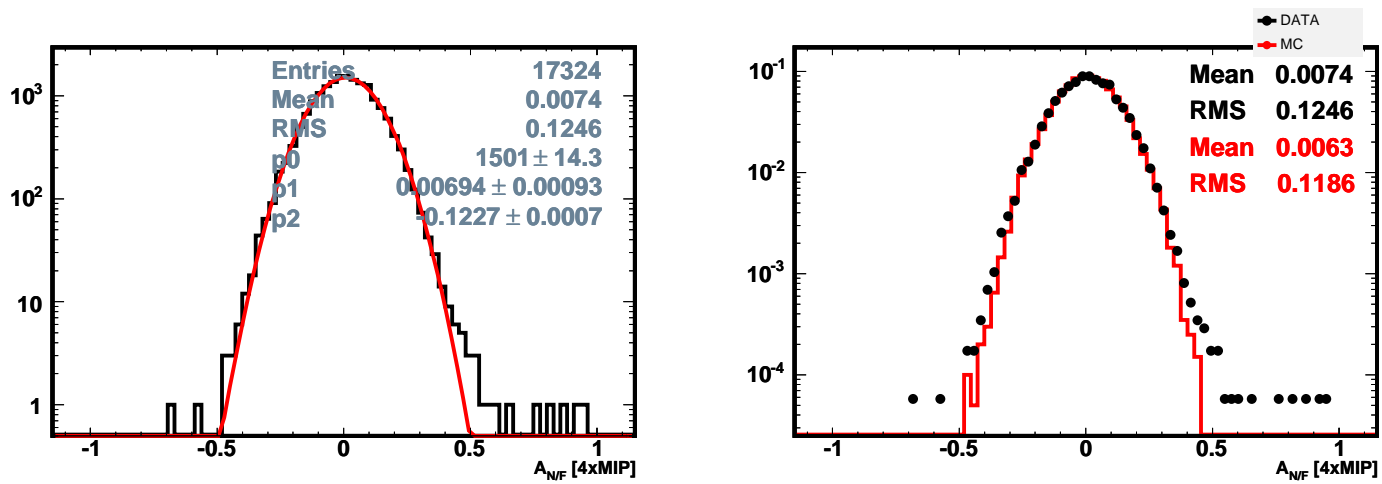

Figure 7.6: $A_{N / F}^{4 \times M I P}$ for Electrons: Data versus $M C$ predictions. These plots show the distribution of $A_{N / F}^{4 \times \text { MIP }}$. Left shows data to which a Gaussian has been fitted to accurately measure the mean and width of the distribution. Right shows a comparison between data and MC predictions.

$$
A_{N / F}^{4 \times \mathrm{MIP}}=\frac{\mathcal{N}^{4 \times \mathrm{MIP}}-\mathcal{F}^{4 \times \mathrm{MIP}}}{\frac{1}{2}\left(\mathcal{N}^{4 \times \mathrm{MIP}}+\mathcal{F}^{4 \times \mathrm{MIP}}\right)}
$$

Once the $4 \times$ MIP calibration has been applied to each hit according to the view it belongs to, the $A_{N / F}^{4 \times M I P}$ can be computed, as indicated by Equation 7.12. from the calorimetric responses. The corresponding $A_{N / F}^{4 \times \mathrm{MIP}}$ distribution is shown in Figure 7.6. Note that $A_{N / F}^{4 \times \text { MIP }}$ should peak at zero, as the $4 \times$ MIP calibration corrects for all known (listed above) and unknown effects, which may cause any energy asymmetry across the readout systems responses. A vanishing $A_{N / F}$ means that the readout systems have been calibrated correctly, leading to $\mathcal{N}=\mathcal{F}$ as a result. However, $A_{N / F}^{4 \times \text { MIP }}$ is about $0.7 \%$ for both data and MC for electrons at $1.8 \mathrm{GeV} / \mathrm{c}$.

There are two problems introduced in the value of $A_{N / F}^{4 \times \text { MIP }}$ arising from the $4 \times$ MIP calibration implementation at CalDet.

1. Muon Beam-Spot Muons have been found to have slightly different beamspot with respect to that of electrons, pions and protons across beam momenta settings. This fact was reported during the attenuation calibration 
implementation discussion, as shown in Figure 5.18. The effective attenuation correction provided by the $4 \times$ MIP calibration is not better than $30 \%$ accuracy, depending on the run. The usual attenuation correction is about $1 \%-1.5 \%$ per view, therefore, $A_{N / F}^{4 \times \text { MIP }}$ is expected to exhibit a systematic bias of the order of $+0.5 \%$ due to the attenuation correction.

2. Crosstalk Differences The $4 \times$ MIP calibration also provides an empirical correction for the crosstalk differences, as measured by muons. However, the crosstalk difference varies for different particles, since their hit-pattern does too. This fact was measured and will be reported in Section 7.8.2. Therefore, the $4 \times$ MIP calibration constants are expected to over-correct by about $+0.3 \%$, which contributes linearly into a possible offset for the $A_{N / F}^{4 \times \text { MIP }}$ as measured by electrons.

Therefore, it has been shown that the measured $A_{N / F}^{4 \times \mathrm{MIP}}$ for electrons can be affected by systematic effects as high as $+0.8 \%$, which are intrinsic to the $4 \times$ MIP calibration implementation at CalDet. Note that $A_{N / F}^{4 \times \text { MIP }}$ can also be affected by calibration systematic uncertainties. This is indeed the case, but this problem will be discussed later as it is common to all measured $A_{N / F}$. Further discussion on the significance of the non-zero value of $A_{N / F}^{4 \times M I P}$ will take place in Section 7.5.6, once all uncertainties have been considered.

\section{Measurements of $A_{N / F}$ with the Integral Attenuation Correction}

$A_{N / F}$ has been calculated in two ways. The difference between the calculations vary in terms of the corrections used for determining each $A_{N / F}$. The interest in doing this lies in the insight obtained about the quality and uncertainties inherited from the applied corrections.

Measurements of $A_{N / F}^{O n A x i s}$

$$
A_{N / F}^{\text {OnAxis }}=A_{N / F}^{\text {Beam-Spot }}-f_{a} \times \bar{x}_{\text {event }}
$$

$A_{N / F}^{O n A x i s}$ is computed (see Equation 7.13) by correcting for the attenuation using the integral attenuation calibration $\left(f_{a} \times \bar{x}_{\text {event }}\right): A_{N / F}^{\text {Beam-Spot }} \rightarrow A_{N / F}^{\text {OnAxis }}$. $\bar{x}_{\text {event }}$ stands for the relative distance between the beam-spot location and the S2S calibration-axis. 

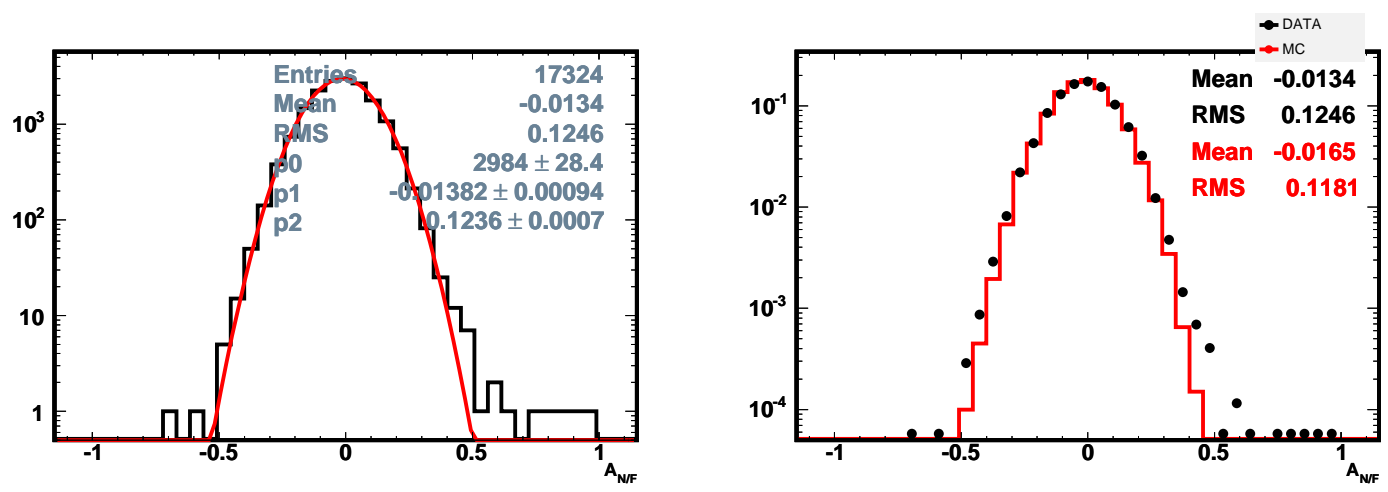

Figure 7.7: $A_{N / F}^{\text {Beam-Spot }}$ for Electrons: Data versus $M C$ predictions. These plots show the distribution of $A_{N / F}^{B e a m-S p o t}$. Left shows data to which a Gaussian has been fitted to accurately measure the mean and width of the distribution. Right shows a comparison between data and MC.

Figure 7.7 shows that $A_{N / F}^{\text {Beam-Spot }} \sim-1.3 \%$, indicating the expected apparent $\mathcal{N}<\mathcal{F}$ for an electron run at $1.8 \mathrm{GeV} /$ c. This is in rough agreement with MC: $A_{N / F}^{\text {Beam-Spot }} \sim-1.6 \%$. Once the attenuation correction is taken into account $\left(+0.4 \%=-f_{a} \times \bar{x}_{\text {event }}\right.$, averaged over both views $)$, we obtain $A_{N / F}^{O n A x i s} \sim-0.9 \%$, for this run. The reason why $A_{N / F}^{O n A x i s}$ is not expected to be zero, is because of the $\mathrm{S} 2 \mathrm{~S}$ calibration, as explained in the previous section. $A_{N / F}^{O n A x i s}$ was calculated to be about $\sim-2.0 \%$, which is about $1 \%$ lower that the measurements.

Measurements of $A_{N / F}^{S 2 S}$

$$
\begin{aligned}
A_{N / F}^{S 2 S} & =\frac{\mathcal{N}-\mathcal{F}}{\frac{1}{2}(\mathcal{N}+\mathcal{F})}-f_{a} \times \bar{x}_{\text {event }}-\Delta \mathcal{X} \\
A_{N / F}^{S 2 S} & =A_{N / F}^{\text {OnAxis }}-\Delta \mathcal{X}
\end{aligned}
$$

where

$$
\Delta \mathcal{X}=\mathcal{X}_{N D}-\mathcal{X}_{F D}
$$

The reason why $A_{N / F}^{\text {OnAxis }}$ is not zero is that the $\mathrm{S} 2 \mathrm{~S}$ calibration has been constructed in such a way that only "tracked responses" (unlike "total responses") would lead to an $A_{N / F}$, which is meant to be zero. This is the principle behind the definition of $A_{N / F}^{S 2 S}$, which is obtained by subtracting the total crosstalk contribution in order to mimic a tracked response. Therefore, subtracting the FD crosstalk $\left(\mathcal{X}_{F D}\right)$ and the ND crosstalk $\left(\mathcal{X}_{N D}\right)$ from 
$A_{N / F}^{O n A x i s}$ (Equation 7.15) gives $A_{N / F}^{S 2 S}$, which must be zero, as the responses used have been calibrated by the S2S calibration.
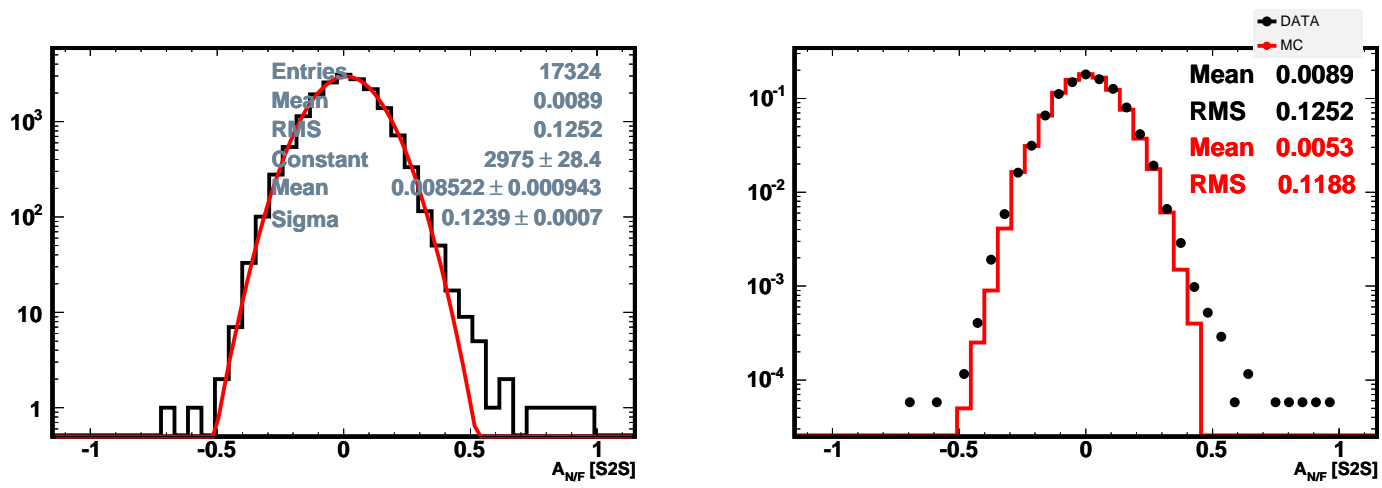

Figure 7.8: $A_{N / F}^{S 2 S}$ for Electrons: Data versus $M C$ predictions. These plots show the distribution of $A_{N / F}^{S 2 S}$. Left shows data to which a Gaussian has been fitted to accurately measure the mean and width of the distribution. Right shows a comparison between data and MC predictions.

Figure 7.8 shows that $A_{N / F}^{S 2 S}$ is about $\sim+0.9 \%$ for the data and about $+0.5 \%$ for MC, both for electrons at $1.8 \mathrm{GeV} / \mathrm{c} . A_{N / F}^{S 2 S}$ approaches zero, although the meaning of the unexpected non-zero value will be discussed after the quality of the calibration and the corresponding uncertainties have been assessed. For now, however, this result is consistent with an apparent $\mathcal{N}>\mathcal{F}$.

Note that the discrepancy between MC and data is about the same as measured with $A_{N / F}^{O x A x i s}$ (shown previously), suggesting that neither the crosstalks (measured for each data and MC) nor the attenuation correction has caused any sizable deviation between MC and data. This observation provides confidence (while not proof) in the accuracy of the corrections. 


\subsection{3 $A_{N / F}$ Evolution with Energy}

In this section, we shall study the shape of $A_{N / F}$ as measured with different particles over all available energies. The error bars shown in this section only account for the statistical uncertainty. The inclusion of all relevant uncertainties, including systematic uncertainties, will be discussed in next section.
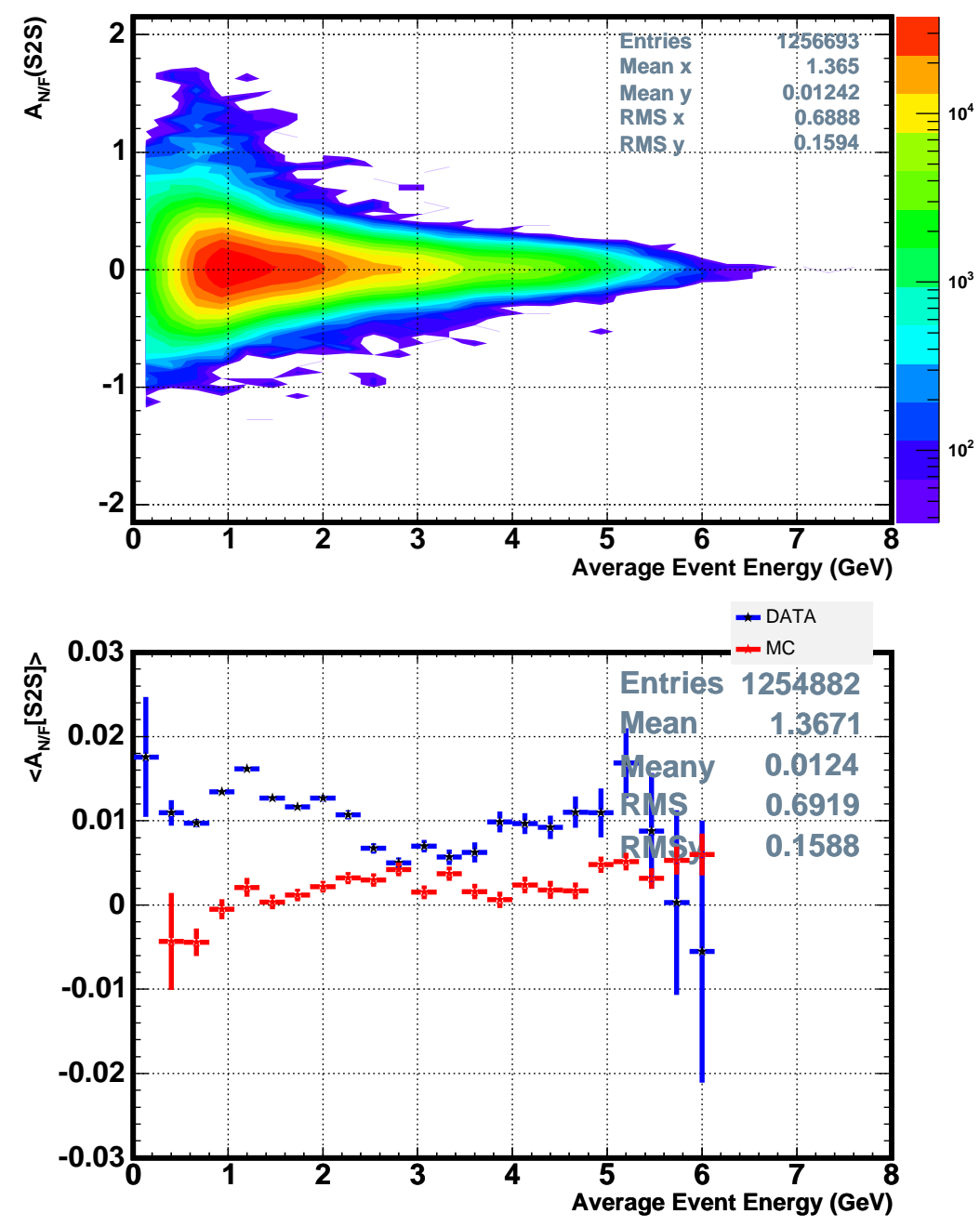

Figure 7.9: Event-by-Event $A_{N / F}^{S 2 S}$ Evolution for Electrons. This figure shows the contour and profile plots of the evolution of $A_{N / F}$ with energy for electrons.

The dependence of $A_{N / F}^{S 2 S}$ with the energy of the electron is shown in Figure 7.9 , using $\sim 1.3 \times 10^{6}$ electrons ranging $0.8 \mathrm{GeV} / \mathrm{c}$ to $6.0 \mathrm{GeV} / \mathrm{c}$. The contour plot illustrates that most of the available electrons are at low energies. This is a feature of the $\mathrm{T} 7$ beam-line, for which the contribution of electrons falls approximately exponentially with energy, as shown in Figure 4.3. 

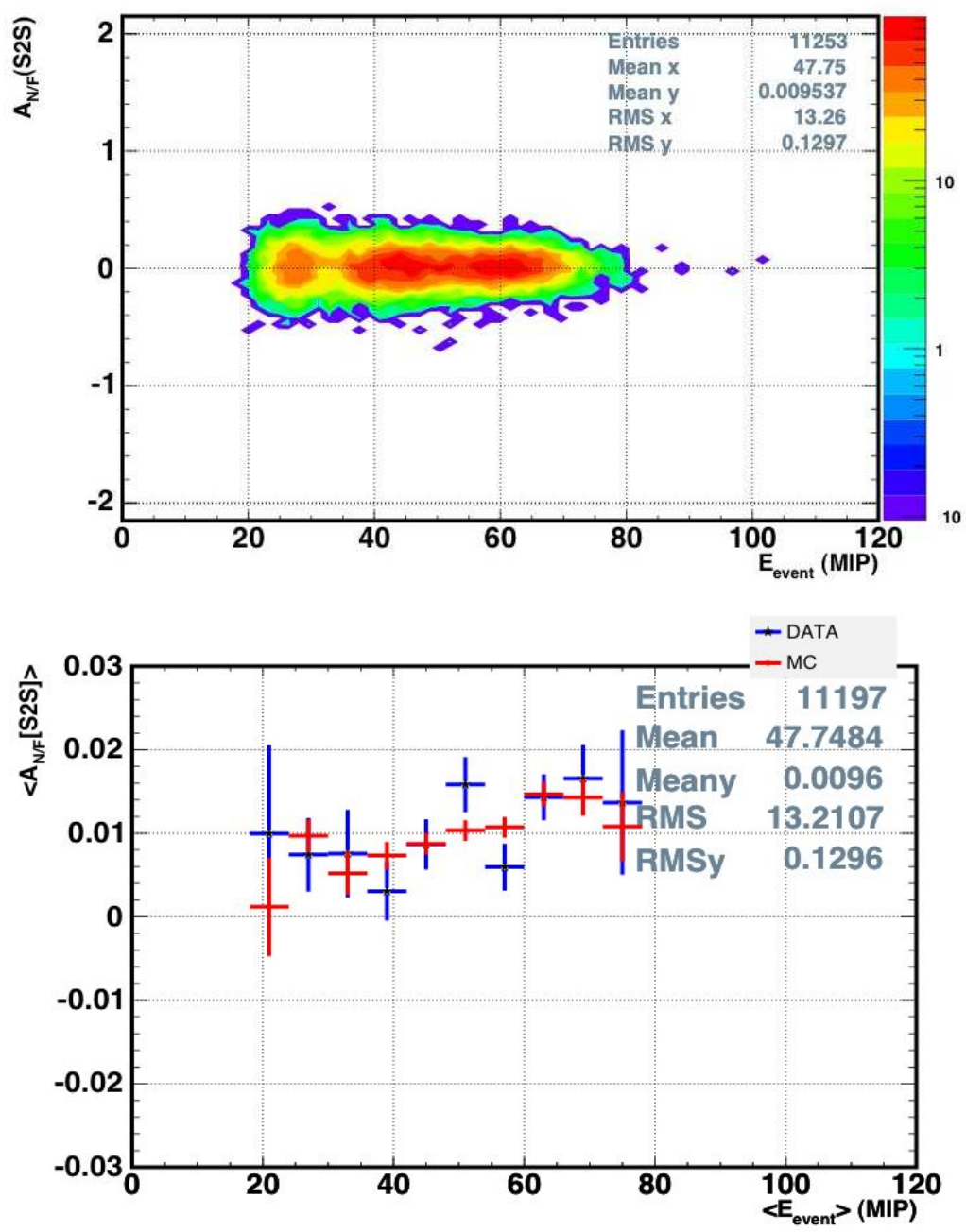

Figure 7.10: Event-by-Event $A_{N / F}^{S 2 S}$ Evolution for Muons. This figure shows the contour and profile plots of the evolution of $A_{N / F}$ with energy for muons.

Figure 7.10 shows the evolution of $A_{N / F}^{S 2 S}$ with event energy computed using 11,000 muons from $1.0 \mathrm{GeV} / \mathrm{c}$ to $3.0 \mathrm{GeV} / \mathrm{c}$. Beyond $2.0 \mathrm{GeV} / \mathrm{c}$ muons escape the detector and their response is expected to increase with energy according to the logarithmic rise of the $\mathrm{dE} / \mathrm{dx}$ curve, as dictated by the Bethe-Bloch formula. Muons at higher energies are ignored as it is impossible to verify that they have the beam selected momentum, which is generally checked by the cut on range.

Similarly, Figure 7.11 shows an equivalent plot of the evolution of $A_{N / F}$ with event energy for protons between $1.0 \mathrm{GeV} / \mathrm{c}$ to $8.0 \mathrm{GeV} / \mathrm{c}$. Only about 250,000 protons were collected.

Note that the behaviour of $A_{N / F}^{S 2 S}$ is not expected to be flat necessarily with 

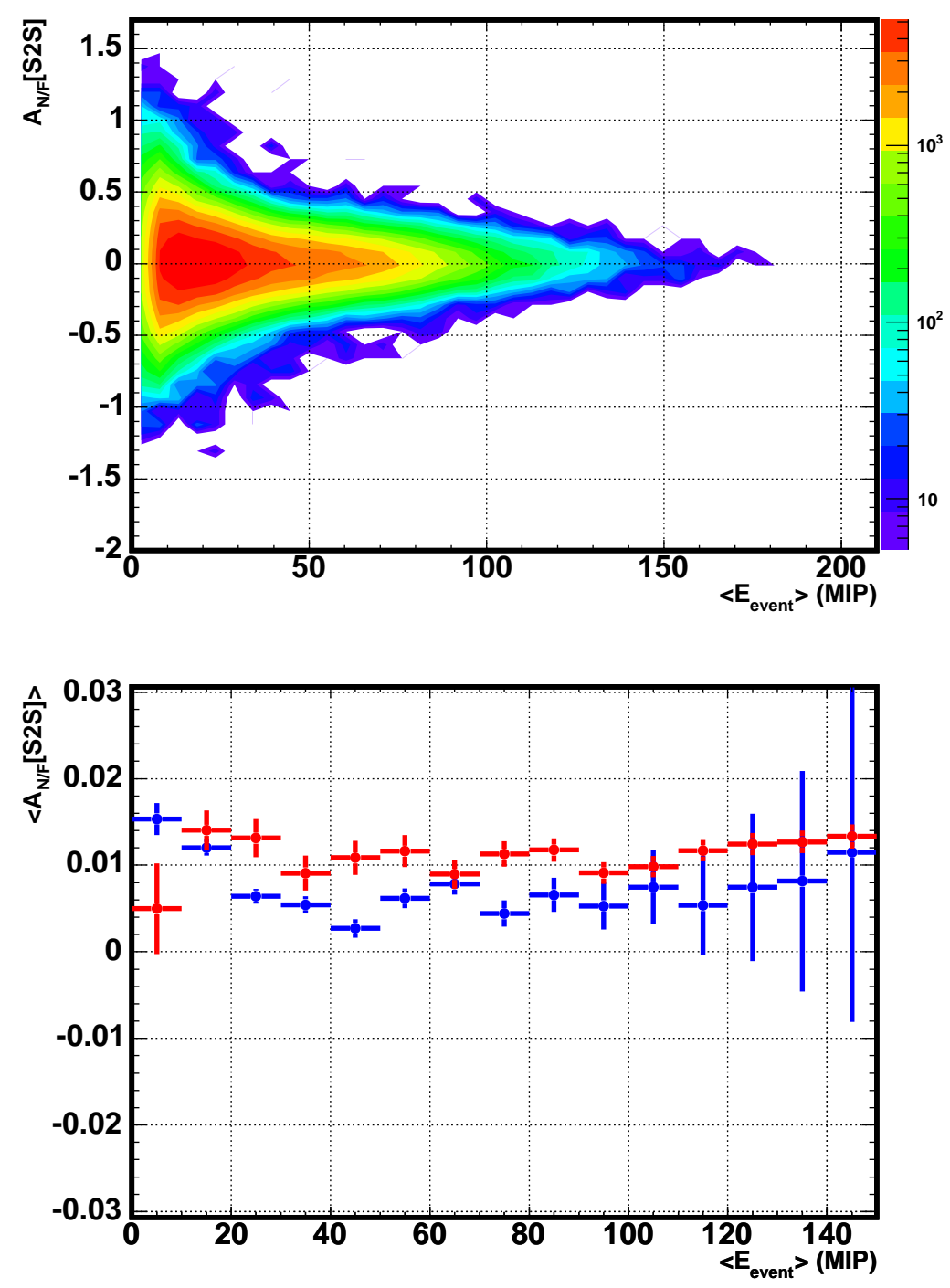

Figure 7.11: Event-by-Event $A_{N / F}^{S 2 S}$ Evolution for Protons. This figure shows the contour and profile plots of the evolution of $A_{N / F}$ with energy for protons.

energy as no linearity calibration was available. For the same reason, $A_{N / F}^{S 2 S}$ is unlikely to be exactly zero on average. The rich structure of $A_{N / F}^{\text {Strip }}$ (Figure 7.2) is convolved into this curve, contributing up to $95 \%$ of the energy budget of each event. The origin of the "wiggles" in the electron curve for the behaviour of $A_{N / F}^{S 2 S}$ is not well understood as the MC, which also inherits its structure from $A_{N / F}^{\text {Strip }}$ (relative non-linearity) does not seem to agree with the data. The behaviour of $A_{N / F}^{4 \times \text { MIP }}$ with energy is shown in Figure 7.12 for electrons and protons.

Both $A_{N / F}^{4 \times M I P}$ and $A_{N / F}^{S 2 S}$ were expected to vanish, one by construction and the 

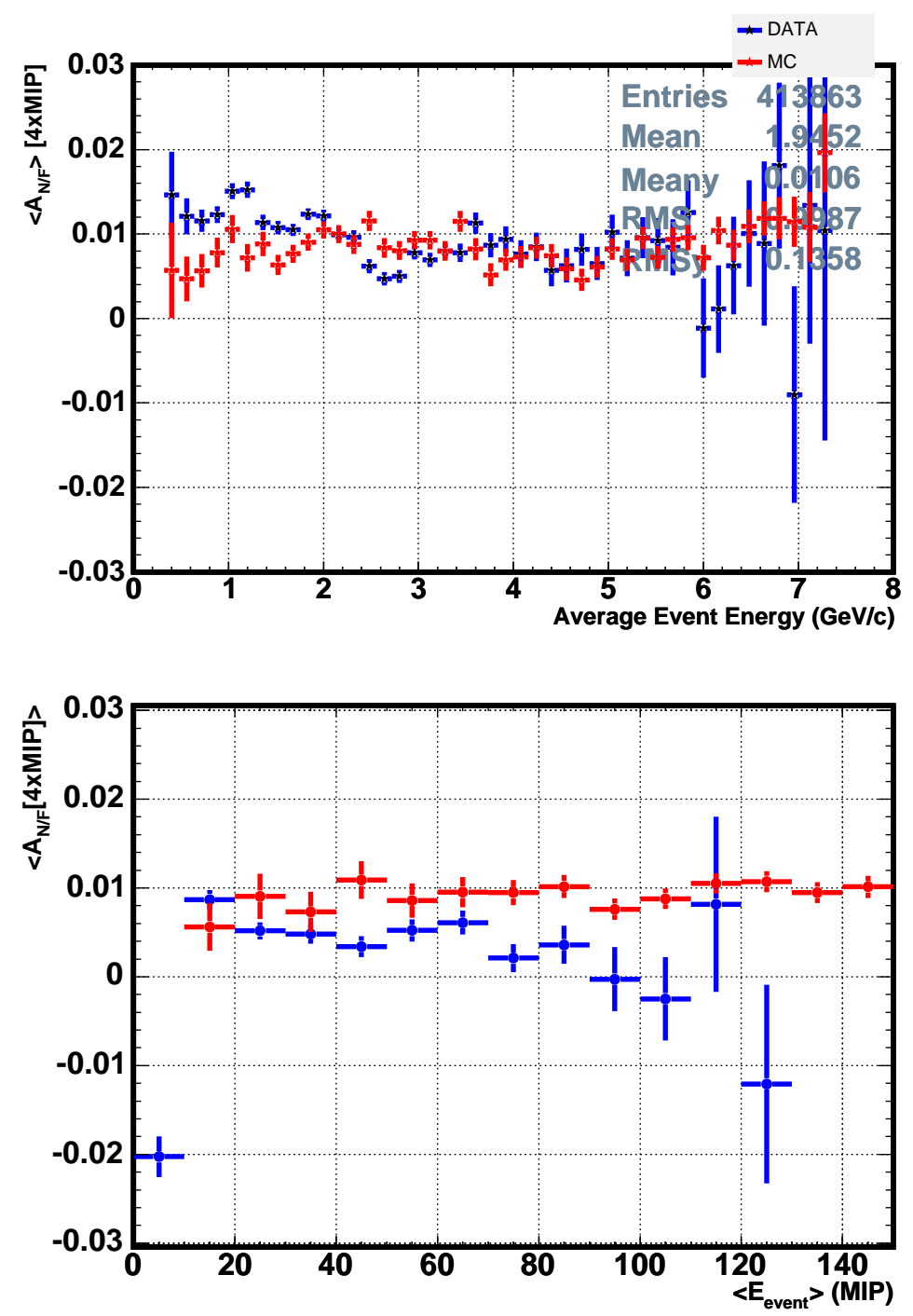

Figure 7.12: Event-by-Event $A_{N / F}^{4 \times M I P}$ Evolution. This figure shows the profile plots of the evolution of $A_{N / F}^{4 \times M I P}$ with energy for electrons (top) and protons (bottom).

other by the definition of the S2S calibration. Both $A_{N / F}^{4 \times \text { MIP }}$ and $A_{N / F}^{S 2 S}$ show a consistent, systematic, positive offset of about $1.2 \%$ with variations of up to $1 \%$ in amplitude for different particles and energies. The $\mathrm{MC}$ is generally predicting a $\sim 0.5 \%$ (energy dependent) lower value for $A_{N / F}^{4 \times \text { MIP }}$ and $A_{N / F}^{S 2 S}$, almost vanishing in some cases.

In order to understand the origin and significance of the possible systematic effects which could cause $A_{N / F}^{4 \times \text { MIP }}$ and $A_{N / F}^{S 2 S}$ to be not only non-zero but positive, we shall investigate the quality of the calibration, which is the subject of the next section. 


\subsubsection{Calibration Repercussions on $A_{N / F}$}

In this section, we shall look deeper into all the stages of the calibration which could give rise to the observed offsets in the measurements of $A_{N / F}$.

\section{Attenuation Corrections Validation}

The effect of the attenuation, and even calibration, can be appreciated by separating $A_{N / F}$ into its EVEN and ODD component views. Each view will exhibit a different attenuation effect corresponding to the orthogonal mapping of the location of beam-spot in $\mathrm{X} / \mathrm{Y}$.
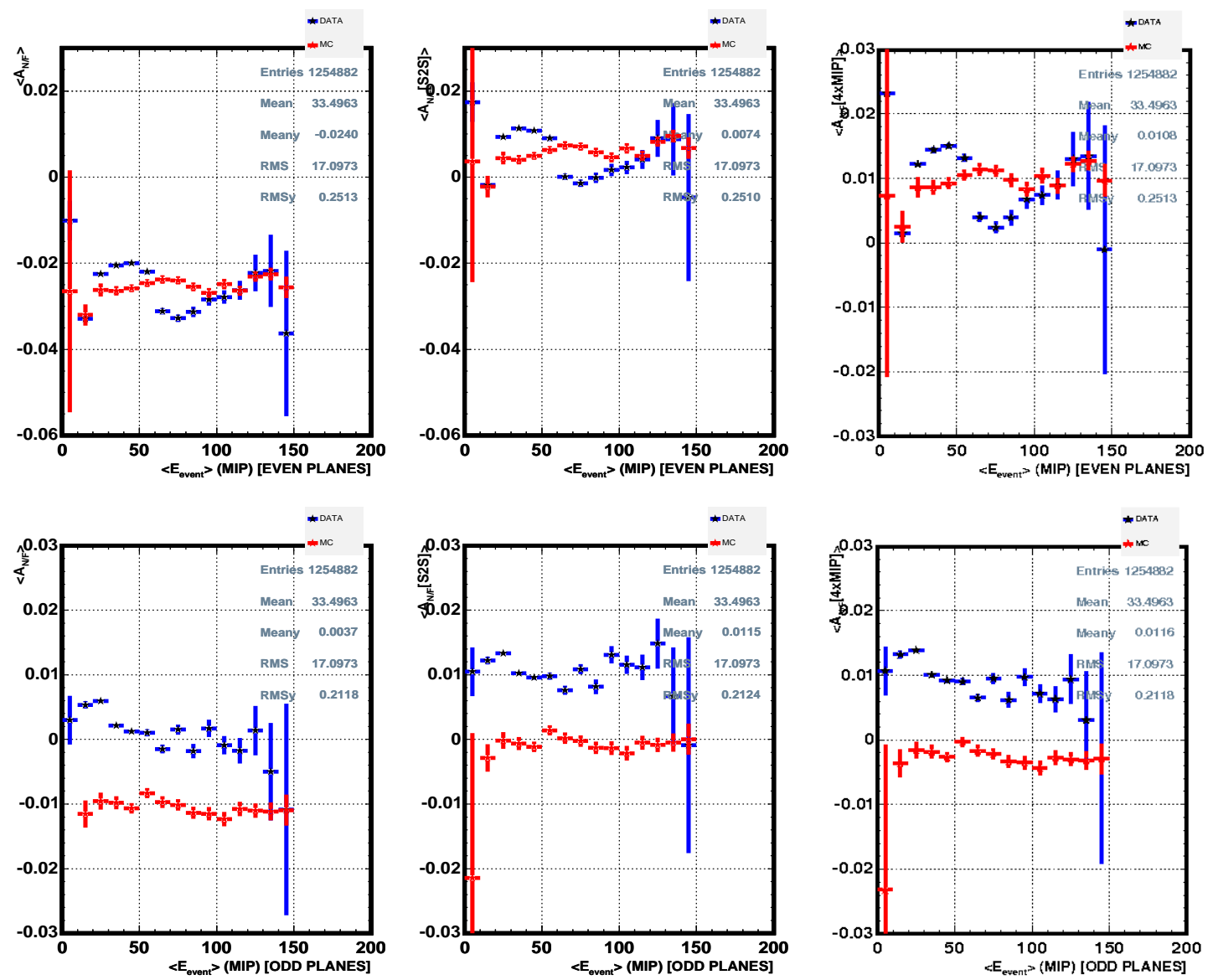

Figure 7.13: Demonstration of the Attenuation Correction. These plots show the evolution of $A_{N / F}$ with energy for EVEN (top) and ODD (bottom) views. The effect of the attenuation correction is exemplified and assessed by comparing $A_{N / F}$ (left), $A_{N / F}^{S 2 S}$ (middle) and $A_{N / F}^{4 \times M I P}$ (right).

Figure 7.13 shows the effect of attenuation on the EVEN (top) and ODD (bot- 
tom) views. The first column corresponds to $A_{N / F}$ prior to any attenuation correction. The effect of the integral attenuation correction to compute $A_{N / F}^{S 2 S}$ can be seen in the middle column, while the $A_{N / F}^{4 \times \text { MIP }}$ is shown in the last column. Note that the agreement between $A_{N / F}^{S 2 S}$ and $A_{N / F}^{4 \times \text { MIP }}$ for the different views serves as the only validation available of the integral attenuation correction accuracy. This was because the attenuation correction factor $\left(f_{a}\right)$ was obtained from MC, but the accuracy of those calculations were based on assumptions which, while reasonable, had not been verified.

There is substantial information in these plots, indicating the possible mechanism causing $A_{N / F}^{S 2 S}$ to be non-zero:

- The discrepancy between MC and data observed per view is independent of the application of the attenuation correction. The same relative differences are observed for all measured $A_{N / F}$.

- $A_{N / F}(\mathrm{EVEN})$ shows reasonable agreement between $\mathrm{MC}$ and data. There is an about constant $1 \%$ offset between $\mathrm{MC}$ and data on the measurements of $A_{N / F}(\mathrm{ODD})$. This observation is consistent with a calibration problem affecting the data on the ODD view only.

- $A_{N / F}$ (data EVEN) is the source of the "curvy" structure, as previously discussed, while $A_{N / F}$ (data ODD) is flat. The amplitude of the variations in the EVEN view can be as high as about $\sim 1 \%$. This observation supports the calibration problem hypothesis.

- Both MC and data $A_{N / F}^{S 2 S}$ and $A_{N / F}^{4 \times \text { MIP }}($ EVEN) are $\sim 1 \%$, while the MCODD is $\sim 0 \%$ and data-ODD is $\sim 1 \%$. Therefore, the MC have a slight EVEN/ODD asymmetry. This observation suggests that in addition to calibration problems, affecting the data, there may be some MC issues $<1 \%$ level.

Therefore, both attenuation corrections seem to perform accurately while not influencing the non-zero values on the measured $A_{N / F}$. However, there is increasing evidence pointing to dubious quality of the calibration.

\section{Response Temperature Dependence}

No remnant temperature dependence is expected on $A_{N / F}$ after the LI-drift calibration has been carried out, as any drift in gain of either readout system has been 
corrected. The major agent causing the readout systems to drift with time is the temperature dependence of the PMT and FEE gains.
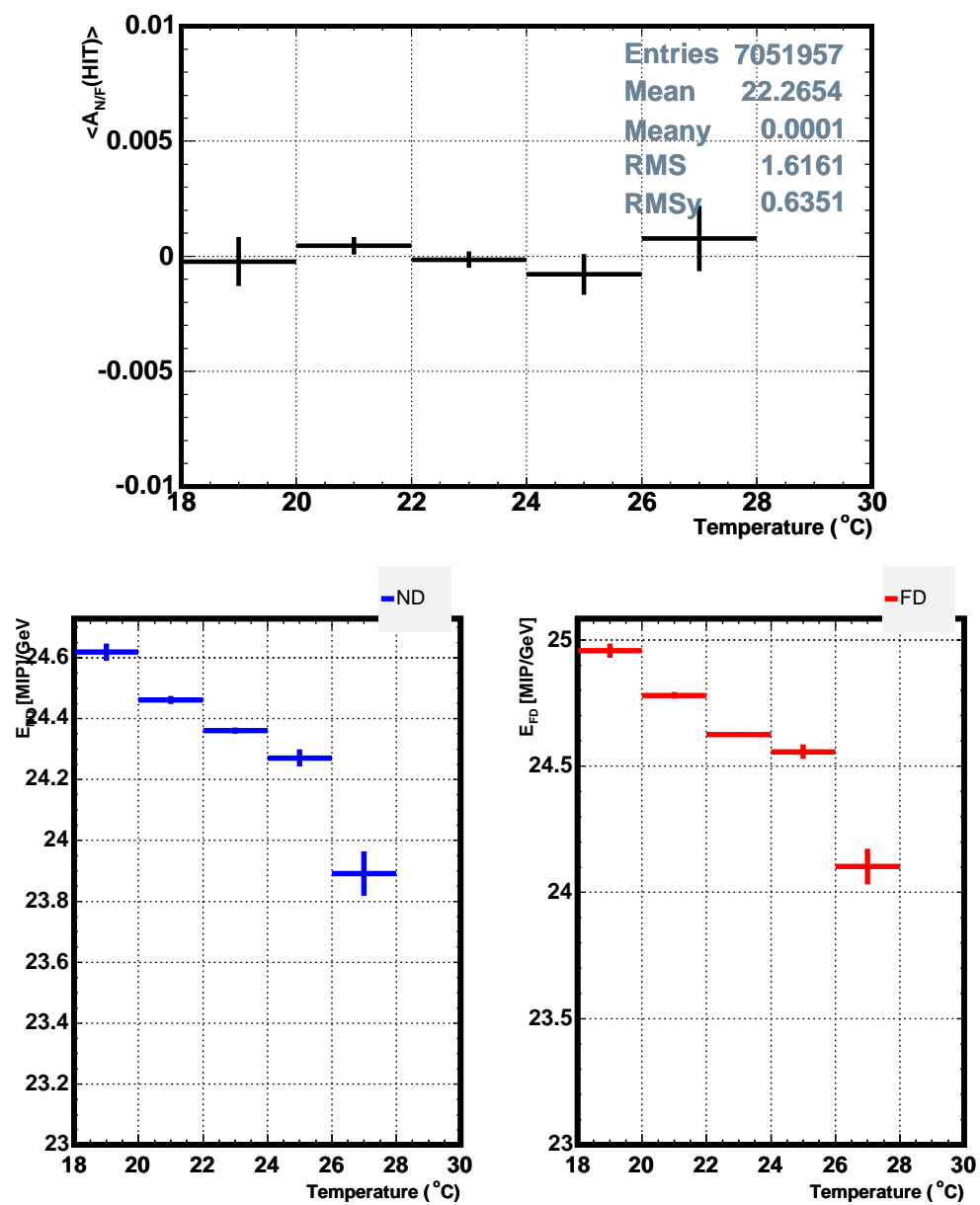

Figure 7.14: Remnant Temperature Dependence of $A_{N / F}$.

The top plot shows the temperature dependence of $A_{N / F}$, which is negligible. The bottom plots show the remnant temperature dependence of the absolute responses of both readout systems, which arise from the VA-FEE variations with temperature not corrected by the LI-Drift calibration by construction.

The drift of both readout systems (actually only the M16 and the M64 $\oplus$ QIEFEE) has been corrected with respect to the response of the LI-PINs, which were read out by the VA-FEE. Note that VA-FEE temperature dependence has not been corrected for by the LI-drift calibration, as the drift has been measured with respect to the VA-FEE PINs. Therefore, we shall expect a temperature dependence of the absolute responses for both readout systems in agreement with that expected for the VA-FEE. 
- As expected, relative variations of $A_{N / F}$ have been corrected, as shown in Figure 7.14 (top). This observation tests the level of success of the LI-drift calibration at MINOS.

- However, the absolute response of both readout systems exhibit a variation with temperature consistent with that of the gain of the VA-FEE $\left(-0.22 \% /{ }^{\circ} \mathrm{C}\right)$ [121] plus scintillator variations $\left(-0.08 \% /{ }^{\circ} \mathrm{C}\right)[122]$, as shown in Figure 7.14 (bottom). The expected temperature dependence is $-0.30 \% /{ }^{\circ} \mathrm{C}$ while the temperature variations measured are $-0.27 \% /{ }^{\circ} \mathrm{C}$ for both the QIE-FEE and the VA-FEE readout systems. The measurements are in rough consistency with expectations.

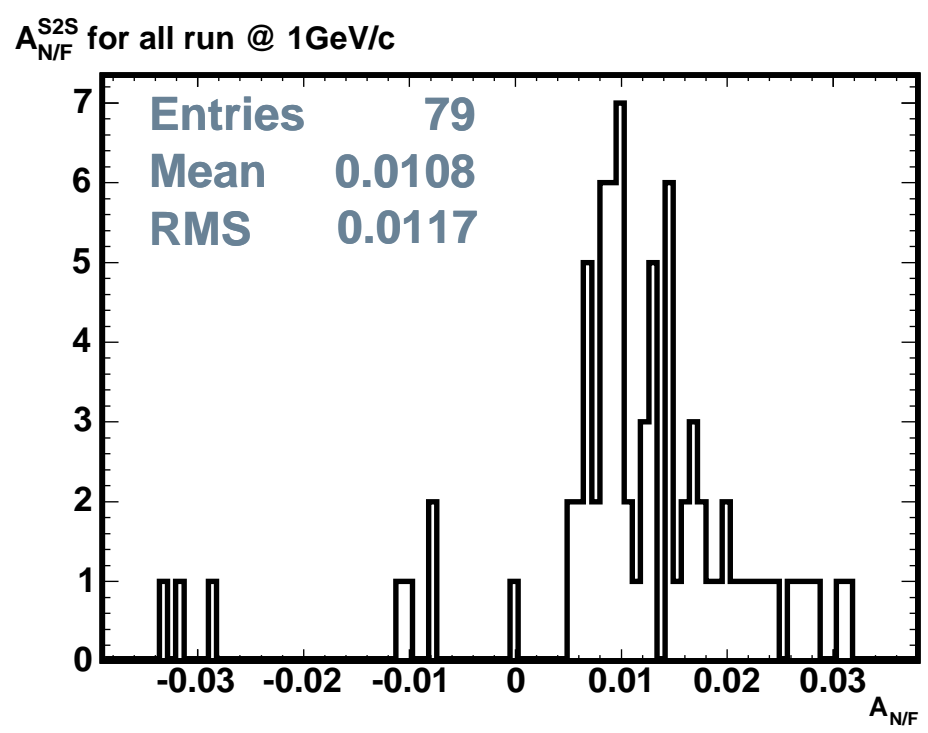

Figure 7.15: Systematic Uncertainty on $A_{N / F}$ due to the LI-drift Calibration. This plot shows the distribution of all $A_{N / F}$ measured in $1 \mathrm{GeV} / \mathrm{c}$ runs. An upper limit on the systematic uncertainty due to the drift calibration can be inferred from the spread of the distribution to be $0.13 \%$, which is the error on the mean $(\mathrm{RMS} / \sqrt{N})$.

- The systematic uncertainty associated with the LI-drift calibration can be estimated, in turn, by estimating the width of the distribution of all $A_{N / F}$ measured in $1 \mathrm{GeV} / \mathrm{c}$ runs, as shown in Figure 7.15. The most dominant effect that could give rise to variations in $A_{N / F}$ is the drift of the readout systems with temperature, as other major components such the attenuation and the non-linearity are fixed. The former because the beam-spot is the fixed same and the latter because event light level is about the same for all runs. Therefore, we can set upper limit on the systematic uncertainty arising 
from the drift calibration from the width of the shown distribution to be $0.13 \%$.

\section{Trigger Rate Dependence}

As mentioned before, the QIE-FEE, as compared to the VA-FEE, is a rate-robust system. So, it is conceivable that, if high rates were allowed during data taking then the value of $A_{N / F}$ could be biased in a very subtle way. This was an identified possibility for which dedicated cuts were aimed at the level of the event selection, as described in Section 6.1.2.

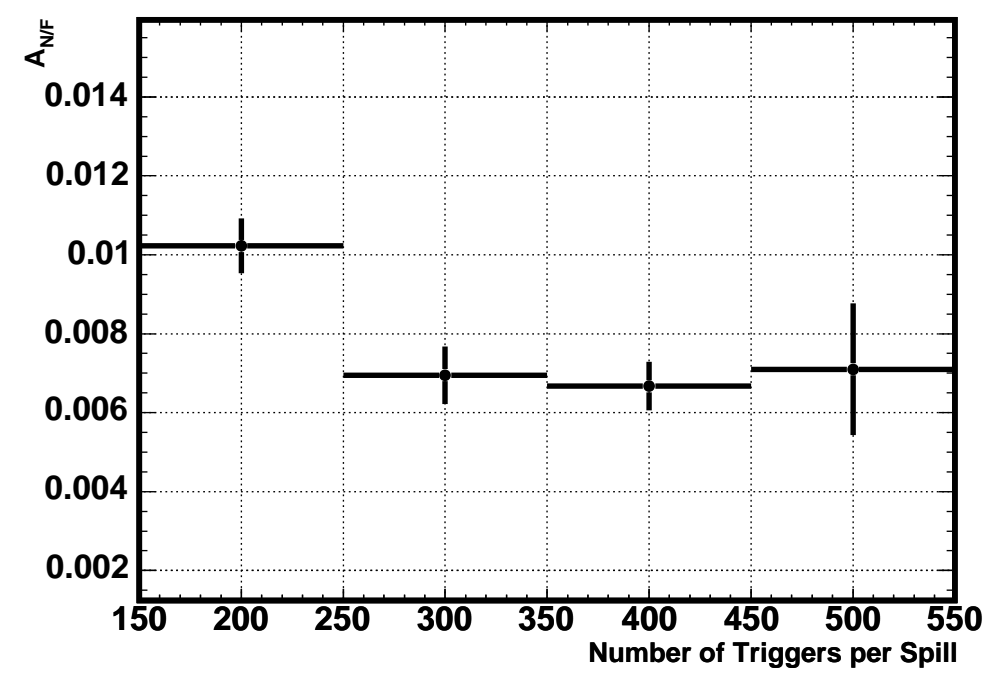

Figure 7.16: Dependence of $A_{N / F}$ on the Trigger Rate. This plot shows that $A_{N / F}$ has no dependence on the incident event rate at CalDet.

Figure 7.16 shows negligible dependence of $A_{N / F}$ on the trigger rate per spill. This is because of the success of the event selection performed for this analysis, whereby the time-fiducial cut and Slicer rejected most multi-particle triggers, causing the selection efficiency to consequently fall with the trigger rate, as shown in Figure 6.5. 


\section{S2S Calibration Systematics Bias}

The quality of the S2S calibration was evaluated using "calibration muons" ${ }^{4}$. Figure 7.17 shows a hypothetical event display of a calibration muon in a view of the detector.

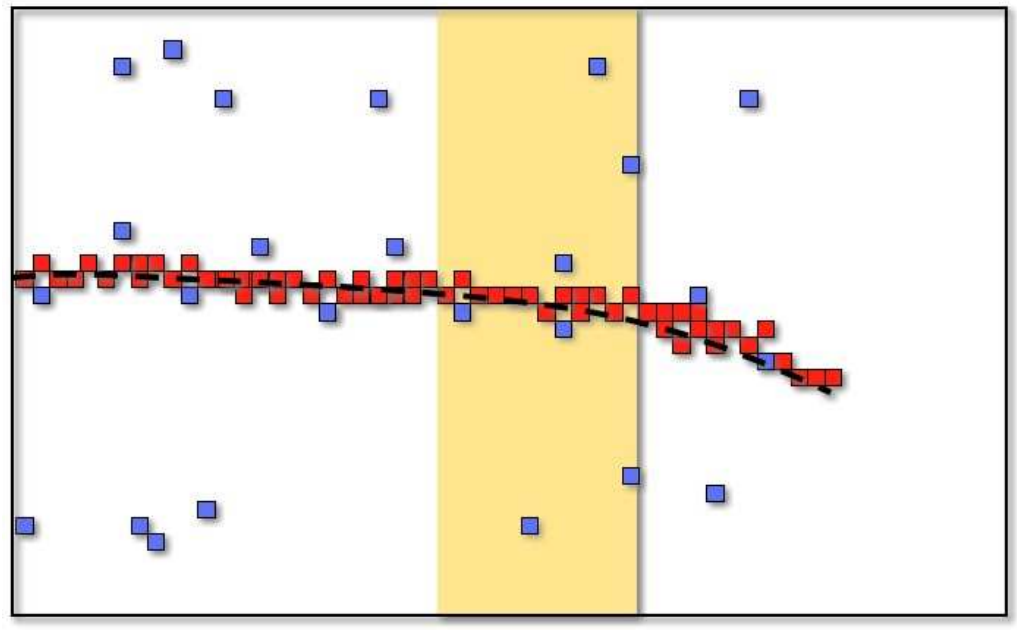

Figure 7.17: Calibration Muon Diagram. Swimmer is used to trace the muon tracks distinguishing between track-hits (red) and crosstalk-hits (blue) based on their topological association with the muon track.

For calibration purposes the Swimmer algorithm becomes very useful at distinguishing the hits associated with the track (red) from those hits due to crosstalk (blue), as shown in the diagram. The rationale is that tracked-responses can be use to better mimic the responses employed for the S2S calibration construction and to check the validity/quality of the calibration.

Figure 7.18 (top) shows the absolute tracked response per plane versus plane number corresponding to $\sim 4500$ muons at $4.0 \mathrm{GeV} / \mathrm{c}$ that go through the detector without stopping. By definition, the response of perpendicular muon going through the detector centre is $1 \mathrm{MIP}$ on average.

The most clear conclusions that can be drawn regarding the sanity of the S2S calibration are the following:

- Figure 7.18 (top) shows clear evidence of so-called "EVEN/ODD effect" over the first-third of the detector: visible irregular pattern (up and down) in

\footnotetext{
${ }^{4}$ Calibration muons are those muons chosen to have very central paths (little multiplescattering), so their responses suffer less from attenuation effects by the end of the detector, where muons scatter the most.
} 

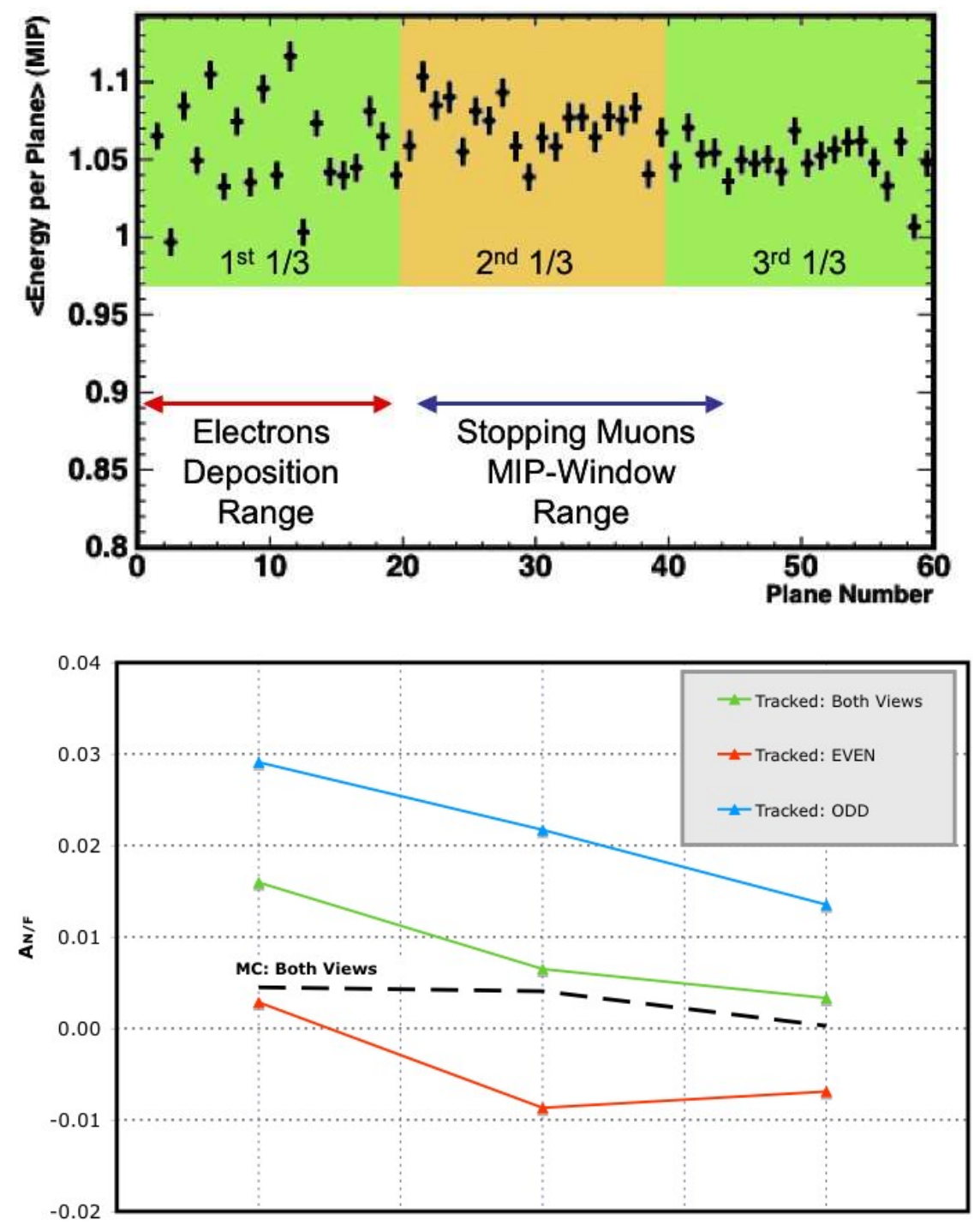

Figure 7.18: Calibration Muon Results. The top plot shows the response per plane of muons going through the CalDet. The irregular structure in the first-third of the detector illustrates poor quality of the S2S calibration constants. The bottom plots quantifies a systematic shift in the value of $A_{N / F}$ over the first-third of the detector (relative to the other two thirds), which affects the response of electrons, as this is the region hit by electrons.

the muon response over difference planes. The response of ODD planes is generally higher (up to 10\%, plane-to-plane) than that on EVEN planes.

- The same first-third appears to have a lower average absolute response with respect to the rest of the detector. An average fall of the average response with plane number is expected, which corresponds to the logarithmic "fall" 
of the muons $d E / d x$ as they lose energy towards the back of the detector. This falling trend is more evident and homogeneous in the last two thirds of the detector.

- Figure 7.18 (bottom) shows $A_{N / F}$ as computed across the three thirds of the detector. $A_{N / F}$ is about $1.1 \%$ higher in the first-third of the detector. This, in combination with the apparent fall of the of the absolute response, suggests that the S2S calibration has caused an underestimation of the responses in the FD side.

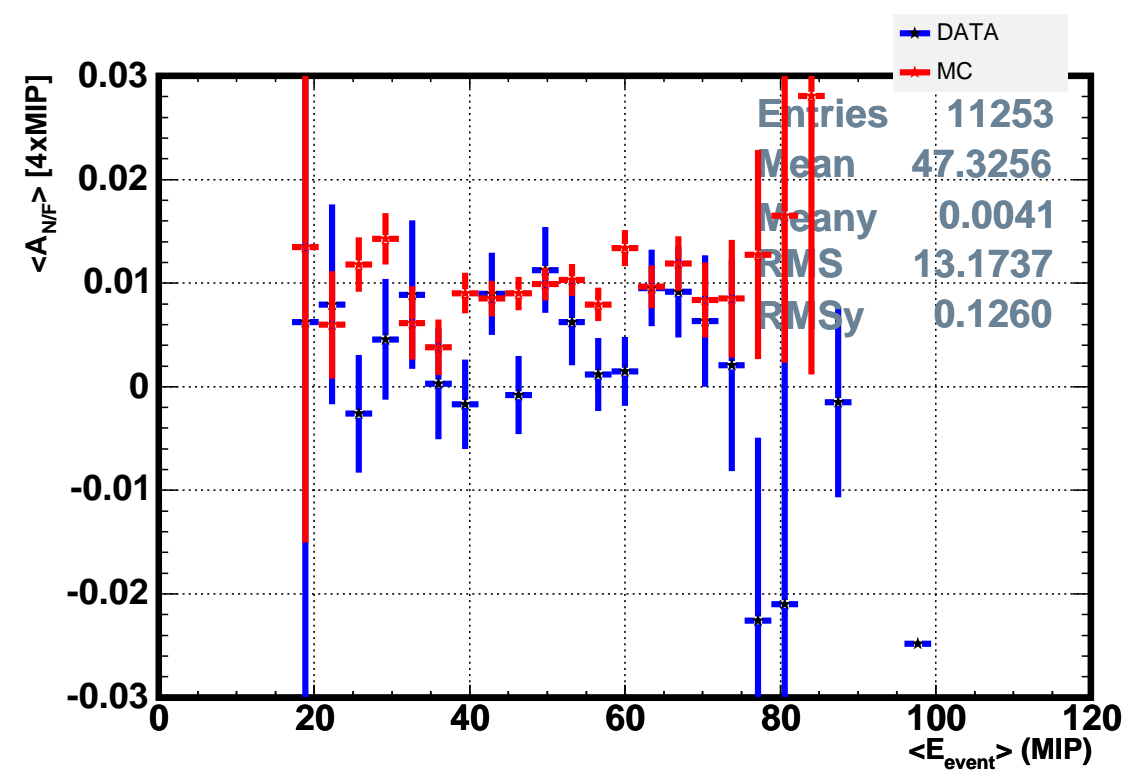

Figure 7.19: Event-by-Event $4 \times M I P A_{N / F}$ Evolution for Muons. This plot shows the evolution of $A_{N / F}^{4 \times \mathrm{MIP}}$ with event energy for muons. $A_{N / F}^{4 \times \mathrm{MIP}}$ ought to be zero, by construction, for muons. The non-zero value of the muons $A_{N / F}^{4 \times \mathrm{MIP}}$ is in good agreement with the existence of calibration systematics found in the first-third of the detector.

Note that this observation is in good agreement with the "self-consistency" check that can be performed with the $4 \times$ MIP calibration by computing the $A_{N / F}^{4 \times \text { MIP }}$ for muons, Figure 7.19, which ought to be zero (by construction). $A_{N / F}^{4 \times \text { MIP }}$ has been measured to be $0.4 \%$. This offset is consistent with the fact that $A_{N / F}^{4 \times M I P}$ is measured using the response of the muon along its entire track, while the $4 \times$ MIP calibration relies on the response computed over the second-third of the detector. That second-third of the detector does not exhibit sizable "EVEN/ODD" asymmetry or slope in $A_{N / F}$ - which was the initial motivation of such calibration. 
The observed increase in $A_{N / F}$ could also be caused by a higher rate of incident radiation in the first planes for which the ND was designed to be more efficient than the FD. This hypothesis was tested by measuring $A_{N / F}$ only over the first-third: $[0,20] \rightarrow[5,20]$ planes as no effect due to incident beam radiation should show up beyond the $5^{\text {th }}$ plane. The corresponding change in $A_{N / F}$ was $-0.2 \%$, proving that up to $\left(+0.9 \pm 0.4^{\text {stat }}\right) \%$ of $A_{N / F}$ is consistent with having its origin in a calibration systematic bias.

The magnitude of the effect shown in Figure 7.18 (bottom) depends on the particle, as the calibration constants sampled are hit pattern dependent. So, we cannot predict the expected bias on $A_{N / F}$ as measured with electrons or protons, however an overall systematic bias of the calibration has been measured to be $\left(+1.1 \pm 0.4^{\text {stat }}\right) \%$ over the region where electrons and protons deposit their energy. Note that, in addition, the magnitude of the measured systematic bias is in excellent agreement with the systematic bias measured with electrons and protons. This measurement can be further exploited in our understanding of $A_{N / F}$ for electrons once all uncertainties have been taken into account (forthcoming section). However, such a discussion will be postponed until the end of next section where the intrinsic width of $A_{N / F}$ distribution is studied.

\subsection{5 $A_{N / F}$ Width Studies}

The width of the $A_{N / F}$ distribution should be dominated by photon-statistics (PS) as $A_{N / F}$ is not sensitive to the intrinsic width associated with the shower fluctuation. This is because the same event (i.e. with the same shower fluctuations) is readout by both readout system and compared. However, as the event energy increases the overall light level of the events increases as they produce more scintillation light per event. PMTs measure light more precisely at higher light level as the relative width $(\sigma / \mu)^{5}$ decreases with the average light level. This is because the Poissonian fluctuations of the electron multiplication driving the gain of PMTs increase as $\sigma=\sqrt{\mu}$ as a function of the mean light level injected. Hence the relative width decreases with the mean as $1 / \sqrt{\mu}$.

An example of the PS contribution with respect to the total width is shown in Figure 7.20. The blue curve is a Gaussian that illustrates the width computed from PS relative to the fitted width of the $A_{N / F}$ depicted in red. Note that, although PS accounts for most of the width of the curve, they do not account for the entire

\footnotetext{
${ }^{5}$ Where $\mu$ and $\sigma$ correspond to the mean and the width of the charge distribution measured.
} 


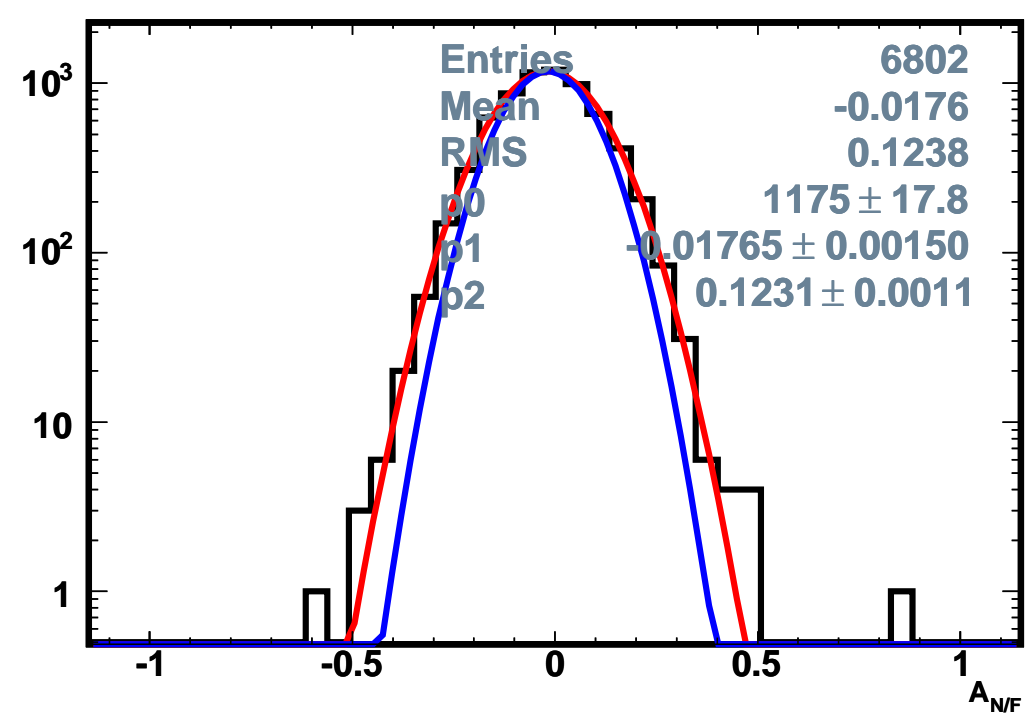

Figure 7.20: Near/Far Energy Asymmetry Width. The distribution shows the $A_{N / F}$ distribution for electrons at $1.8 \mathrm{GeV} / \mathrm{c}$. A Gaussian fit (red) allows to measured the width of the distribution accurately, which is used to be compared with the expected width from Photo-Statistics (blue Gaussian curve).

width, suggesting that there is yet another component causing fluctuations eventby-event that is different for both readout systems.

Figure 7.21 (left) shows the evolution of the width of $A_{N / F}$ (black) as compared with the expected width from PS (blue). The width from PS dominates the width of $A_{N / F}$ across all energies. Figure 7.21 (right) shows that the MC (red) can account for the observed width (black) of $A_{N / F}$ accurately, even if we did not understand their origin. Note that the $\mathrm{MC}$ is able to describe the width of the data accurately. The source for the extra width beyond the PS contribution was investigated but no one single contribution was unambiguously identified in isolation as the responsible. Therefore, we are more willing to believe that extra contribution is caused by a combination of a few small effects.

\subsubsection{Systematic Uncertainties}

So far, $A_{N / F}$ has been measured to be $\sim 1 \%$ on average. However, after evaluating the quality of the calibration, we have gathered evidence that the S2S calibration suffers from systematic effects. Therefore a thorough analysis of systematic uncertainties from all contributions used for the calculation of $A_{N / F}$ is needed to 

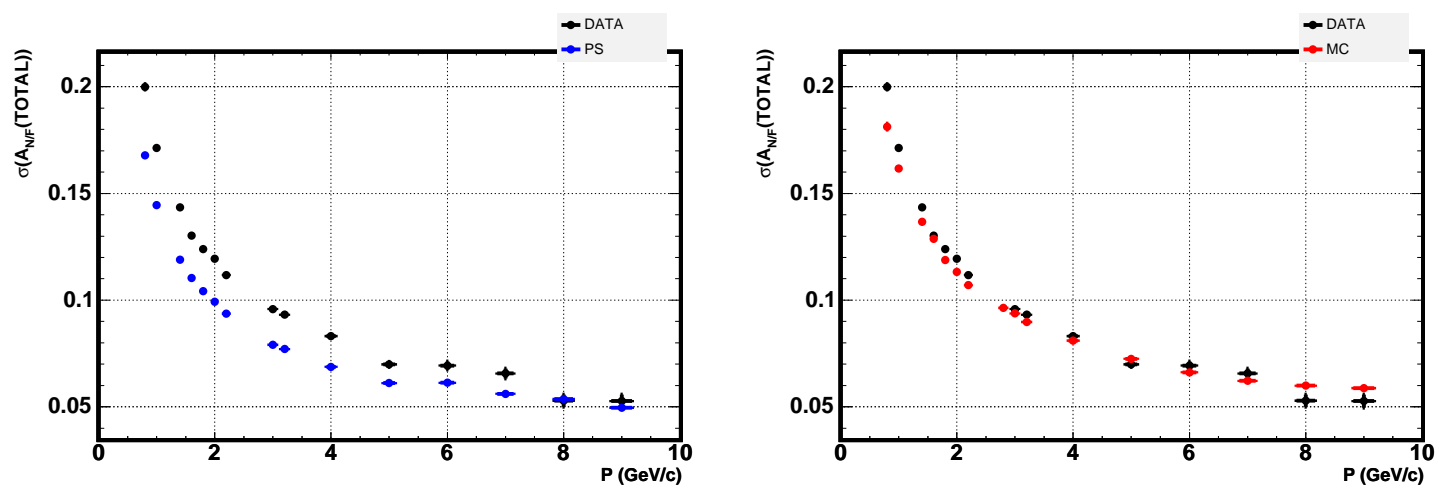

Figure 7.21: Near/Far Energy Asymmetry Width Evolution. These plots show the evolution of the width of $A_{N / F}$ with beam momenta, as compared with PS (left) and MC (right). The data points corresponding to the width of $A_{N / F}$ at $8 \mathrm{GeV} / \mathrm{c}$ and $9 \mathrm{GeV} / \mathrm{c}$ are affected by the poor measurements of the width due to the poor statistics available.

understand the degree of significance of the offset measured.

\begin{tabular}{|c|c|c|c|}
\hline & Typical Value & $\sigma_{D A T A}\left(A_{N / F}\right)$ & $\sigma_{M C}\left(A_{N / F}\right)$ \\
\hline \hline Statistical & & $\sim 0.1 \%$ & $\sim 0.2 \%$ \\
\hline Attenuation & & & \\
$f_{a}$ & $<2.0 \%$ (per view) & $\pm 0.2 \%$ & $\pm 0.2 \%$ \\
$\bar{x}_{\text {event }}$ & \pm 0.5 strip & $\sim 0 \%$ & $\sim 0 \%$ \\
\hline Crosstalk & & & \\
$\mathcal{X}_{N D}$ & $4.8 \%$ & $\pm 0.1 \%$ (syst) & $\pm 0.1 \%$ (syst) \\
$\mathcal{X}_{F D}$ & $5.1 \%$ & $\pm 0.1 \%$ (syst) & $\pm 0.1 \%$ (syst) \\
Calibration & & & \\
$4 \times$ MIP & $\sim 600 S I G C O R$ & $\pm 0.8 \%$ (syst) & $\pm 0.8 \%$ (syst) \\
S2S & $25 \%$ & $\pm 1.1 \%$ (syst) & $\mathrm{X}$ \\
LI-Drift & $<5 \%$ & $\pm 0.13 \%$ (syst) & $\mathrm{X}$ \\
LI-Linearity & $<15 \%$ & $\mathrm{X}$ & $\pm 0.2 \%$ (syst) \\
\hline
\end{tabular}

Table 7.6: $A_{N / F}$ Uncertainty Budget

Table 7.6 summarises the uncertainty budget associated with all contributing sources to the measurement of $A_{N / F}$. The name, a typical value and the respective uncertainties for data and $\mathrm{MC}$ are shown respectively in first through fourth columns.

Here is a summary of the origin behind each uncertainty as well as the rationale of how it propagates through to an uncertainty in $A_{N / F}$ :

- Uncertainty Associated to the Attenuation Correction: the contribution to the uncertainty in $A_{N / F}$ is dominated by the uncertainty on the slope 
of the attenuation calibration curve $\left(f_{a}\right)$, which can be up to $9 \%$. This leads to a consequent uncertainty on $A_{N / F}$ to be $A_{N / F} \times \frac{\Delta f_{a}}{f_{a}}=2 \% \times 9 \%=0.2 \%$.

- Uncertainty Associated to the Crosstalk Correction: the crosstalk contributes to $A_{N / F}$ through the absolute error estimated for each crosstalk measurement propagated in quadrature.

- Uncertainty Associated to the Calibration: calibration constants contribute to the $A_{N / F}$ prior to any correction. Their effect is propagated through their fractional errors. The uncertainty on the LI-drift and S2S calibration was estimated in Section 7.5.4.

The uncertainty associated with the LI-linearity calibration applied to linearise the MC was calculated by estimating the full of effect on the value of $A_{N / F}:\left(\left|A_{N / F}^{N o n L I N}-A_{N / F}^{L I N}\right|\right)_{M C} \sim 0.2 \%$. Where $A_{N / F}^{L I N}$ and $A_{N / F}^{N o n L I N}$ correspond to the value of $A_{N / F}$ with and without linearity correction with MC. The effect of linearity correction changes $A_{N / F}$ by $0.2 \%$. This provides a rough and generous account for all possible effects, for which there is no easy way to check the true value and accounting for the ignored channel-to-channel variations.

The uncertainty in the $\mathrm{S} 2 \mathrm{~S}$ calibration is dominated systematic bias measured to be $\left(+1.1 \pm 0.4^{\text {stat }}\right) \%$ with muons. This allows to set an upper limit on the uncertainty of the S2S calibration to be $1.1 \%$ for electrons. However a possible scenario is to use the bias measured with muons to corrected the measured $A_{N / F}^{S 2 S}$. If so, a conservative relative systematic uncertainty of $50 \%$ should be associated to the measurement of the bias due to the extrapolation between muon and electrons. Therefore, the correcting terms to be subtracted to $A_{N / F}^{S 2 S}$ is $\left(+1.1 \pm 0.4^{\text {stat }} \pm 0.6^{\text {syst }}\right) \%$.

The uncertainties of the $4 \times$ MIP calibration affect both MC and data. In Section 7.5.2, the intrinsic systematic uncertainties associated with the technique, as implemented at CalDet, were assessed to be up to $0.8 \%$.

Table 7.7 shows the $A_{N / F}$ measured by data and MC compared with all uncertainties taken into account. The last quoted $A_{N / F}(\mathrm{c})$ has been corrected by the systematic bias measured with muons, as discussed previously in this section. Note that the analytical expectations for the values of the different $A_{N / F}$ are also enclosed for comparison. 


\begin{tabular}{c|c|c|c} 
& DATA & MC & Prediction \\
\hline$A_{N / F}^{S 2 S}$ & $\left(1.24 \pm 0.01 \%^{\text {stat }} \pm 1.26^{\text {syst }}\right) \%$ & $\left(0.27 \pm 0.02^{\text {stat }} \pm 0.32^{\text {syst }}\right) \%$ & $\left(0.0 \pm 0.2^{\text {syst }}\right) \%$ \\
$A_{N / F}^{4 \times \mathrm{MIP}}$ & $\left(1.22 \pm 0.01^{\text {stat }} \pm 1.39^{\text {syst }}\right) \%$ & $\left(0.71 \pm 0.02^{\text {stat }} \pm 0.86^{\text {syst }}\right) \%$ & $\left(0.0 \pm 0.2^{\text {syst }}\right) \%$ \\
\hline$A_{N / F}^{S 2 S}(\mathrm{c})$ & $\left(0.14 \pm 0.01 \%^{\text {stat }} \pm 0.61^{\text {syst }}\right) \%$ & $\left(0.27 \pm 0.02^{\text {stat }} \pm 0.32^{\text {syst }}\right) \%$ & $\left(0.0 \pm 0.2^{\text {syst }}\right) \%$ \\
\hline
\end{tabular}

Table 7.7: Final $A_{N / F}$ Measurements.

\section{Discussion}

- Mean Value of $A_{N / F}$. Once all uncertainties have been taken into account, both the $\mathrm{MC}$ or the data do not exhibit significant deviation from zero. All deviation are well within $1 \sigma$ level. In other words, with the precision available the measured $A_{N / F}$ are consistent with $\mathcal{N} \sim \mathcal{F}$.

The uncertainty level and systematic bias measured on the first two measurements of $A_{N / F}$ quoted in Table 7.7, are dominated by the uncertainties from the S2S calibration over the first third of the detector. Therefore, further sensitivity on the value $A_{N / F}$ could be achieved if the uncertainties in the S2S calibration were significantly reduced up to $\sim 0.3 \%$ ( $5 \times$ lower $)$, the level at which the uncertainties of other contributions become significant.

The $A_{N / F}^{S 2 S}(\mathrm{c})$ is the most powerful limit that we can set on the relative responses between the ND and FD readout systems. Both the mean value and the associated uncertainty are constraint the most, whereby up to $\sim 4 \sigma$ fluctuations on the measurements obtained are still below the inter-calibration uncertainty goal of $2 \%$, suggesting that the readout system performance is equivalent. Even in this case the systematic uncertainties are still dominated by the S2S calibration systematics. As expected, once the data have been corrected from calibration bias, the agreement between data and MC (and even the theoretical expectation) is excellent.

The uncertainty in the $\mathrm{MC}$ is dominated by the attenuation correction or by the $4 \times$ MIP calibration uncertainties which can be as high as $0.8 \%$, as discussed in Section 7.5.2. The margin for improvement with this approach is small, as the intrinsic uncertainties to the method would generally dominate when sensitivity improves beyond the $1.0 \%$ level.

- Width of $A_{N / F}$. The spread of $A_{N / F}$ is was studied and found to be 
dominated by photon-statistics fluctuations, as expected. MC was able to accurately describe the behaviour exhibited by the data (Figure 7.21 right).

- Behaviour of $A_{N / F}$ with the Energy.

The dependence of $A_{N / F}$ with event energy is consistent with possible fluctuations well within the estimated uncertainty level. $A_{N / F}$ was however, not expected to be flat as the LI-linearity calibration was not made available for the data accurately.

However, it is suggestive that most of the "wavy" structure measured with electrons is inherited from the $A_{N / F}$ measured with EVEN view responses. This kind of behaviour strongly suggest for calibration problems, which are known to dominate the uncertainties on $A_{N / F}$.

In summary, within the uncertainty level, there is no sizable energy dependent artifact in the energy spectra explored arising from the readout systems. Structure arising from the non-linearity has also been shown in this analysis that can be calibrated out by the LI-linearity calibration. Therefore, the visible energy spectra measured by the Near and Far detectors readout systems are identical to about $<1 \%$ providing that both detector have been inter-calibrated, even if no linearity calibration has been carried out.

- Extrapolating to the Near and Far Detectors: $A_{N / F}^{T o t a l}$. As will be explained in full detail in section 7.8.2, a fraction of the crosstalk of the ND PMTs has not been taken into account during the calorimetric measurements because of an instrumental setup of CalDet. The results are not affected by this, as the calibration mechanism does not depend on crosstalk. However, it is interesting and possible to measure this fraction of crosstalk event-by-event (to be called "unread-crosstalk"). The unread-crosstalk has been measured to be about $1.8 \%$ but, most importantly, this figure is CalDet-specific (cabling), which is not to be extrapolated to the configuration of any other detector. Therefore, this measured fraction of crosstalk could be regarded as "missing energy" from a configuration in which all the PMT-crosstalk was readout, like, for example, the configuration of the MINOS Near Detector at FNAL. So an interesting measurement is to compute $A_{N / F}^{\text {Total }}$ for which we have added the unread-crosstalk contribution, as indicated in Equation 7.18 and quoted in Table 7.8 . 


$$
\begin{aligned}
A_{N / F}^{\text {Total }} & =\frac{\mathcal{N}\left(1+\mathcal{X}^{\text {Unread }}\right)-\mathcal{F}}{\frac{1}{2}(\mathcal{N}+\mathcal{F})}-f_{a} \times \bar{x}_{\text {event }} \\
A_{N / F}^{\text {Total }} & =A_{N / F}^{\text {OnAxis }}+\mathcal{X}^{\text {Unread }}
\end{aligned}
$$

\begin{tabular}{l|c} 
& $A_{N / F}^{\text {Total }}$ \\
\hline DATA & $\left(-0.16 \pm 0.01^{\text {stat }} \pm 0.60 \%{ }^{\text {syst }}\right) \%$ \\
MC & $\left(0.05 \pm 0.02^{\text {stat }} \pm 0.32^{\text {syst }}\right) \%$ \\
Prediction & $(-0.3 \pm 0.2) \%$ \\
\hline
\end{tabular}

Table 7.8: Final $A_{N / F}^{\text {Total }}$ Measurements.

Note that $A_{N / F}^{\text {Total }}$ has been corrected such that the S2S calibration systematic bias $\left(+\left(1.1 \pm 0.6^{\text {syst }}\right) \%\right)$ has been subtracted. The interest in this $A_{N / F}$ is because the responses measured in the MINOS Near and Far detector are closer to the ones employed to compute $A_{N / F}^{T o t a l}$. Therefore, $A_{N / F}^{T o t a l}$ represents the most accurate extrapolation from the relative comparison made at CalDet to a relative comparison made at the level the Near and Far detectors, limited to the scope of the readout system. Table 7.8 shows agreement among the average measured value of $A_{N / F}^{\text {Total }}, \mathrm{MC}$ and the analytical calculations expectation. $A_{N / F}^{\text {Total }}$ is expected to be $-0.3 \%$, since $A_{N / F}^{S 2 S}-A_{N / F}^{\text {Total }} \approx \Delta \mathcal{X}$ which has been measured (see Section 7.8 .2 ) to be $+0.3 \%$, in agreement with observations.

From another perspective, -0.3 is the expected average bias between the responses of both readout systems arising from having been calibrated by a method which rejects the PMT-crosstalk, while the calorimetric measurements (showers) integrate over the full crosstalk contributions. This is only true at CalDet, because the measured $A_{N / F}$ are based on the normalisation of the S2S calibration (when no $4 \times$ MIP calibration was used). The MIP calibration is meant to account for the crosstalk difference, making $A_{N / F}^{T o t a l} \rightarrow 0$, across Near and Far MINOS detectors. 


\subsection{Topology Comparison}

MINOS relies heavily on the event-by-event neutrino interaction identification (NC/CC) to achieve the physics goals. This is because the main scope of MINOS is the study of neutrino oscillations among active neutrinos, which are only sensitive through $\mathrm{CC}$ events. NC events within the $\mathrm{CC}$ sample can reduce the sensitivity of MINOS since NC contamination can bias the measured survival probability, particularly in the lowest visible energy bins. The separation between NC/CC events in MINOS relies fully on the event topological distinction.

In this section, we will concentrate on the study of both readout system to characterise the topology of events. The readout systems are not expected to significantly affect the event topology apart from the action of PMT crosstalk. In addition much of the differences between ND and FD measured in this analysis have the intrinsic dependence of the CalDet idiosyncrasies (cabling and size mainly), which are not to be extrapolated to the actual Near and Far detectors. In fact, none of the conclusions drawn during this section can be extrapolated to the Near and Far detector apart from discrepancies between MC and data led by a possible instrumental effect not described by the detector simulation software. Therefore, the main focus of this section is to study the accuracy with which the MINOS detector simulation is able to replicate the features measured by each readout system, i.e. a MC/data comparison rather than a ND/FD comparison.

The topology comparison will be performed with muons and electrons. Electrons are representative of shower-like events while muons are representative of minimum ionising track-like events in a MINOS detector. Pions and protons can fall within either category, but mainly within the former. Note that during the event topology characterisation one becomes very sensitive to inaccuracies of the MC physics generators used.

\subsubsection{Basic Topology Comparison}

In order to compare the hit-pattern of events in the detector, a few characterising variables are computed such that an event-by-event comparison can be established across both readout systems and/or MC and data. Some of the variables in question are similar to those used in the Near and Far detectors for neutrino physics analysis; for example the number of planes hit.

The variables in question are the following: 


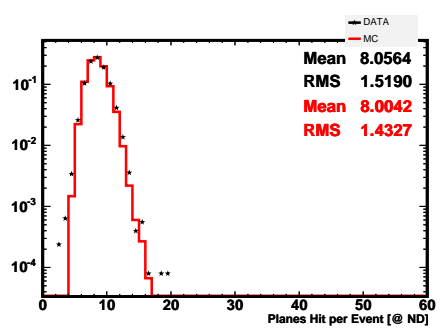

Number of Planes Hit
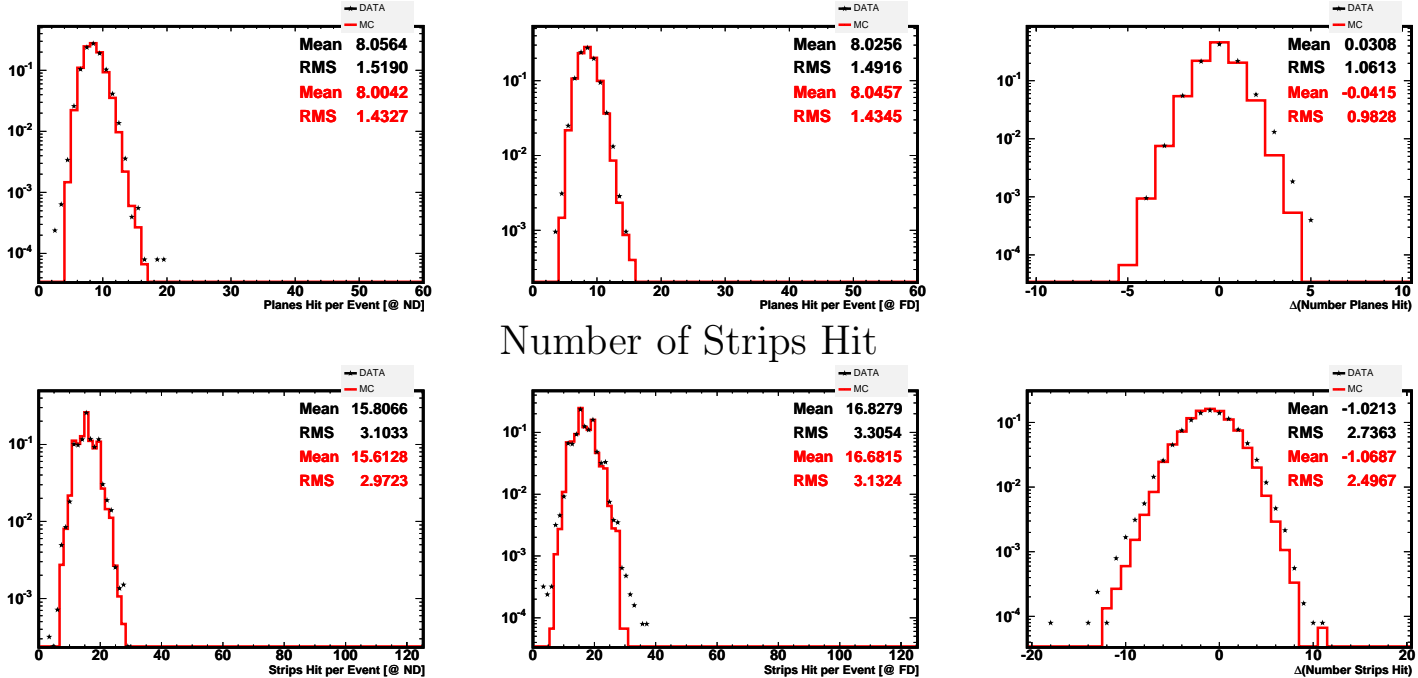

Number of Strips Hit
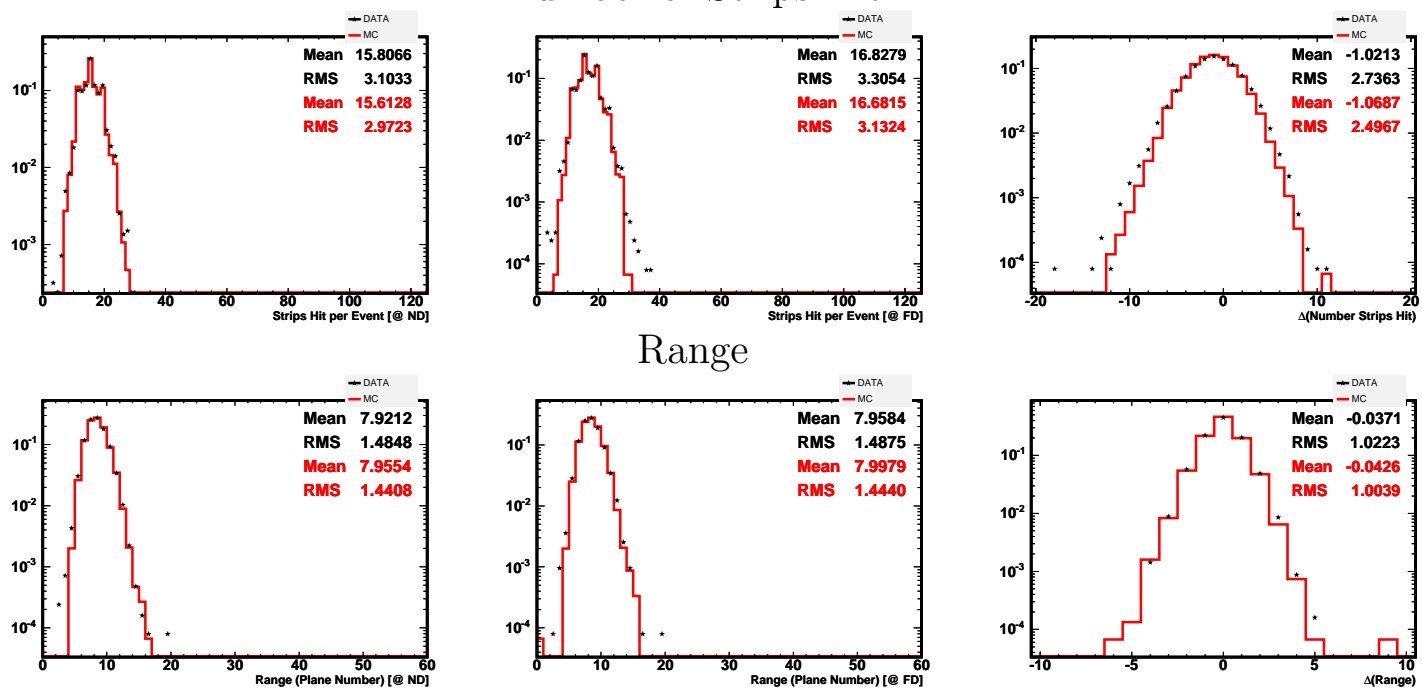

Figure 7.22: Basic Topology Features. The distributions of some basic event topology variables are shown as computed by the ND (left), FD (centre). The difference between the ND and FD measurements are shown in the distributions on the right. Both MC (red) and data (black) are shown. Note that generally negligible discrepancies can be seen, which are overemphasised by the log scale on the abscissa axis.

Number of Planes Hit (Figure 7.22(top)) Number of planes hit is computed by counting the number of planes hit with charge $>1.5 \mathrm{PE}$. This cut on the charge was designed to reject PMT crosstalk hits from the calculations. This cut imposes agreement between the ND and FD calculations as their crosstalk patterns are different due to the intrinsically different cabling for each readout system.

Number of Strips Hit (Figure 7.22(middle)) Likewise, the number of strips hit was also computed by imposing a similar $>1.5 \mathrm{PE}$ cut before including a strip. The object of the cut is the same as above.

Event Length or Range (Figure 7.22(bottom)) The "length" or "range" of an event is computed by computing the number planes between the first and last plane hit. Note that this calculation assumes that events are horizontally in- 
cident into the detector, which is the case as they are beam events. Assigning the last hit plane was subjected to a tighter criterion: the last plane hit has to have more than 1.5PE worth of charge in a single hit. Gaps of up to 4 planes were allowed.

"Shower-Max" Spot The "Shower-Max"spot (SM) is the name given to the strip with the largest amount of energy deposition in an event. Clearly, calculating the location of SM is only sensible for electrons, because the typical energy deposition of electrons is characterised by a large deposition of charge on one strip (i.e. SM) along with a few strips in the middle range energy and many strips with very low charges. The location of SM is, to first order, the location of the centre of mass of an electron event.

Charge Weighted Centre of Mass of Event The calculation of the calibrated charge weighted centre of mass of each event was used in the computation of a few variables used in the event topology comparison. For instance, the event location and direction were based on this type of calculation. Also the longitudinal and transverse energy profiles rely on a centre of mass calculation over each plane and over each strip respectively. So, even though the actual centre of mass was calculated per every event, the most interesting results will be indirectly illustrated through the energy profiles analysis and the event location results. Note that the location of the centre of mass and the location of the shower-max-strip are very correlated for electrons, unlike for muons.

Event Location The event location is computed by calculating the centre of mass in each plane. This calculation associates the average strip position with the charge in that plane. The calculations were restricted to use hits within a fiducial volume defined to be the 12 most central strips of each plane up to plane 24. The fiducial volume was used to remove contributions from crosstalk hits - generally located in the outer strips due to the cabling at CalDet. The cut below 24 planes was chosen to prevent the calculations from being affected by the random scattering of muons in the remaining part of the detector. Therefore, the event location algorithm actually computes the average incident location of the event in the detector. The average event location in each view was obtained simply from the mean of the distribution in each view.

The average incident location of the events was used to tune the beam-spot 
in the MC with respect to the data. The beam-spot involved electrons at $1.8 \mathrm{GeV}$ because most of the electrons are at low energies, setting the MC beam-spot to where most of the statistical power is. Note that the beamspot tuning is very important for the topological comparison, since the main distorting effect is PMT crosstalk, which is very sensitive to the hit pattern of events and their location in detector space. Small discrepancies can be seen in the shown figures, however, their effect is generally negligible or at most of the order $1 \%$.

Figure 7.22 shows the distributions of some of the topology variables explored during this studies. The distributions are ordered such that the ND on is the left, the FD in is the middle and the "ND-FD" (the difference) distribution is on the right. $\mathrm{MC}$ is shown in red, while data is in black. Note the overall excellent agreement across ND and FD and between data and MC.

\section{Topology Evolution with Beam Momentum}

The dependence of the topology variable with the beam momenta provides a clearer framework to understand the mechanisms behind the features observed. The following plots will show that there are two main ingredients responsible for the observations: the PMT crosstalk and PMT gain (due to HV variations).

As mentioned before, the PMT HV was lowered to effectively lower the gain of the readout systems for electrons runs beyond $6 \mathrm{GeV} / \mathrm{c}$, to prevent the saturation of the dynamic range in the FD. So the range, the number of strips hit and the number of planes hit will exhibit a decrease in the FD side beyond $6 \mathrm{GeV} / \mathrm{c}$. This is illustrated in all plots in Figure 7.23 (middle). Figure 7.23 (right) shows the difference between the ND and the FD, called "Near/Far-difference" $(\Delta)$. Note that $\mathrm{MC}$ and data are in excellent agreement up to $6 \mathrm{GeV} / \mathrm{c}$ where the data is affected by the lowered HV. This is because the MC was generated with the PMT gains at nominal values for both readout systems.

The overall trend of the Near/Far-difference plot is dictated by PMT crosstalk. The topology variables in question were computed with an $1.5 \mathrm{PE}$ cut to reduce the effect of crosstalk. This is why the distribution is approximately zero at nominal HV. The evolution of the Near/Far-difference distribution is towards negative values (at nominal HV), suggesting that the FD measures more (range, strips and planes hit) at higher energies. This is because, as discussed before, all the FD crosstalk $(\sim 5.2 \%)$ contributes to these plots while only the ND read-crosstalk 

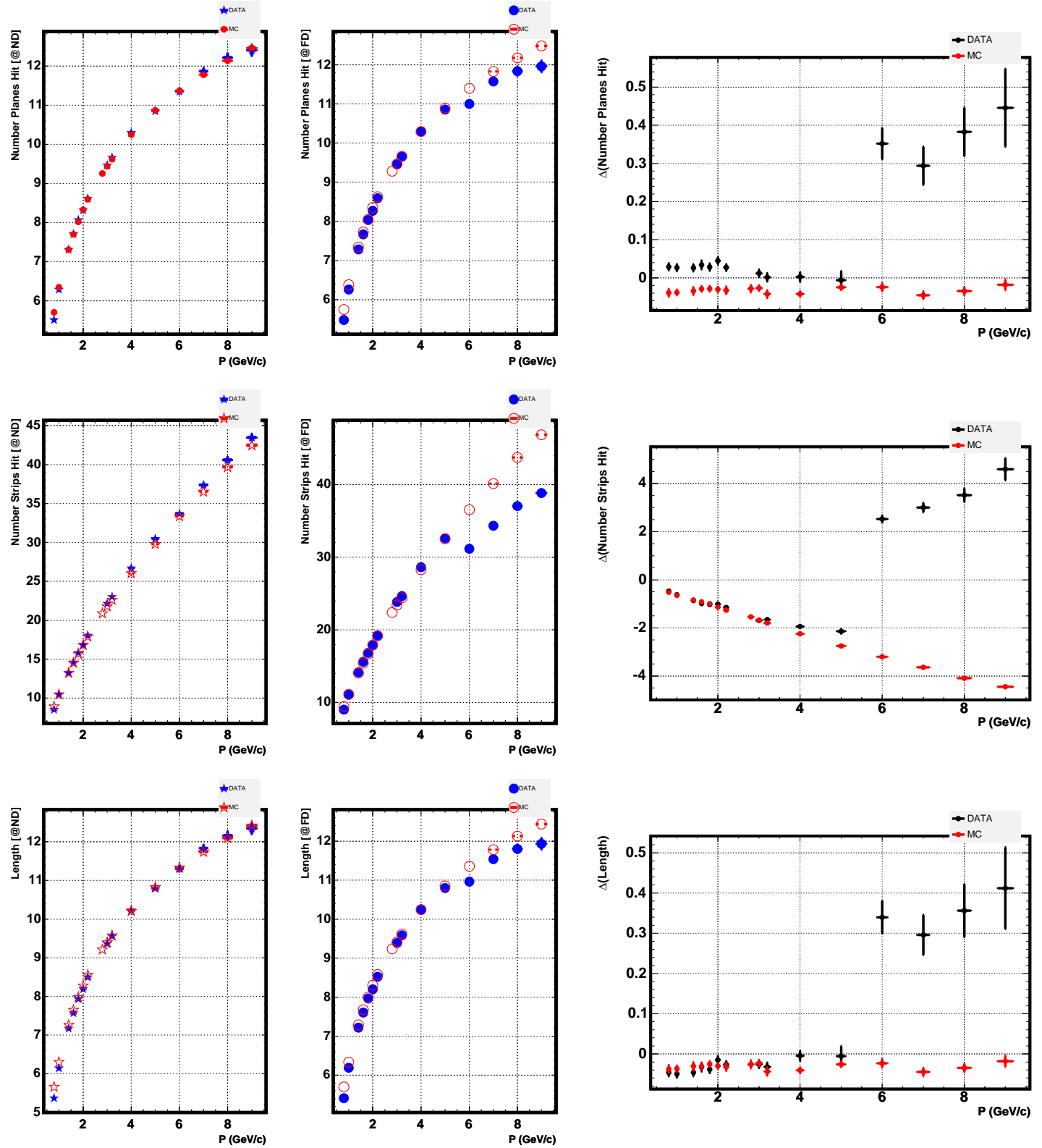

Figure 7.23: Topology Characterisation Evolution for Electrons. The evolution with beam momenta of the event topology variables studied are shown as computed for electrons by the ND (left), FD (centre). Likewise, the difference between the ND and FD measurements are shown in the distributions on the right. Both $\mathrm{MC}$ (red) and data (black) are shown.

$(\sim 2.6 \%)$ produces hits within the detector volume (see Section 7.8.2). As the energy of the events increases, the number of hits generated per event is larger. This gives rise to a larger absolute difference due to a larger amount of crosstalk hits available on the FD side relative to the ND side. Therefore, the relative difference 

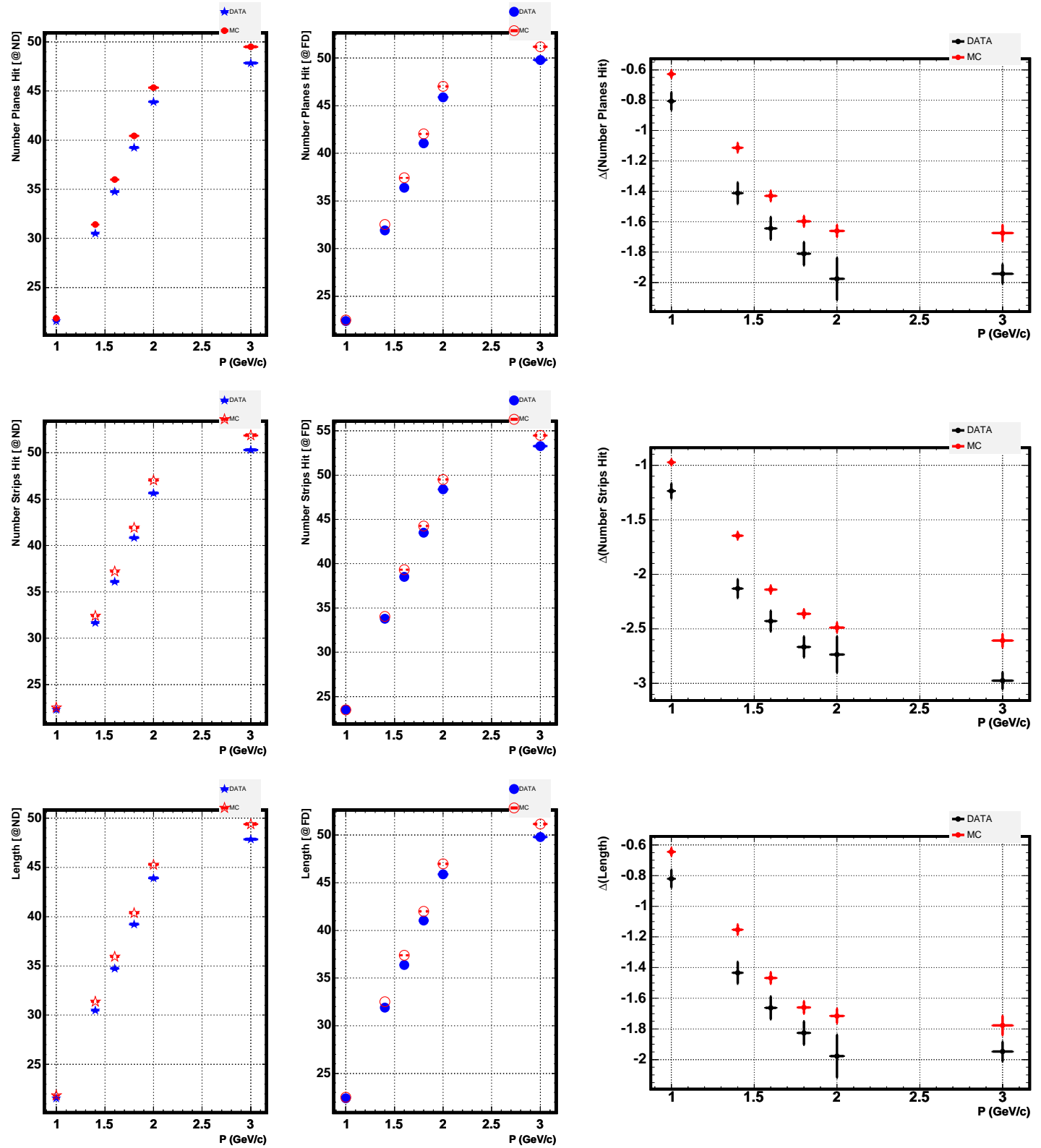

Figure 7.24: Topology Characterisation Evolution for Muons. The evolution with beam momenta of the event topology variables studied are shown as computed for muons by the ND (left), FD (centre). Likewise, the difference between the ND and FD measurements are shown in the distributions on the right. Both MC (red) and data (black) are shown.

should be constant, leading to a constant slope in the observed absolute difference.

Crosstalk can induce a larger number of planes to appear to be hit than really were. This is due to the cabling of CalDet: one M64 and three M16 PMTs cover the same two planes per view (even/odd). Therefore, if energy was deposited in the 
first plane , crosstalk could well induce hits on the second plane, which is located 2 planes forward in detector-space, as both share a common view.

Figure 7.24 shows similar trends for muons as were shown with electrons above. Note that the muon light level per hit changes logarithmically with energy. Therefore, the trend of the Near/Far-difference distributions towards FD is likely due to an larger average number of hits due to crosstalk on the FD with respect to the ND.
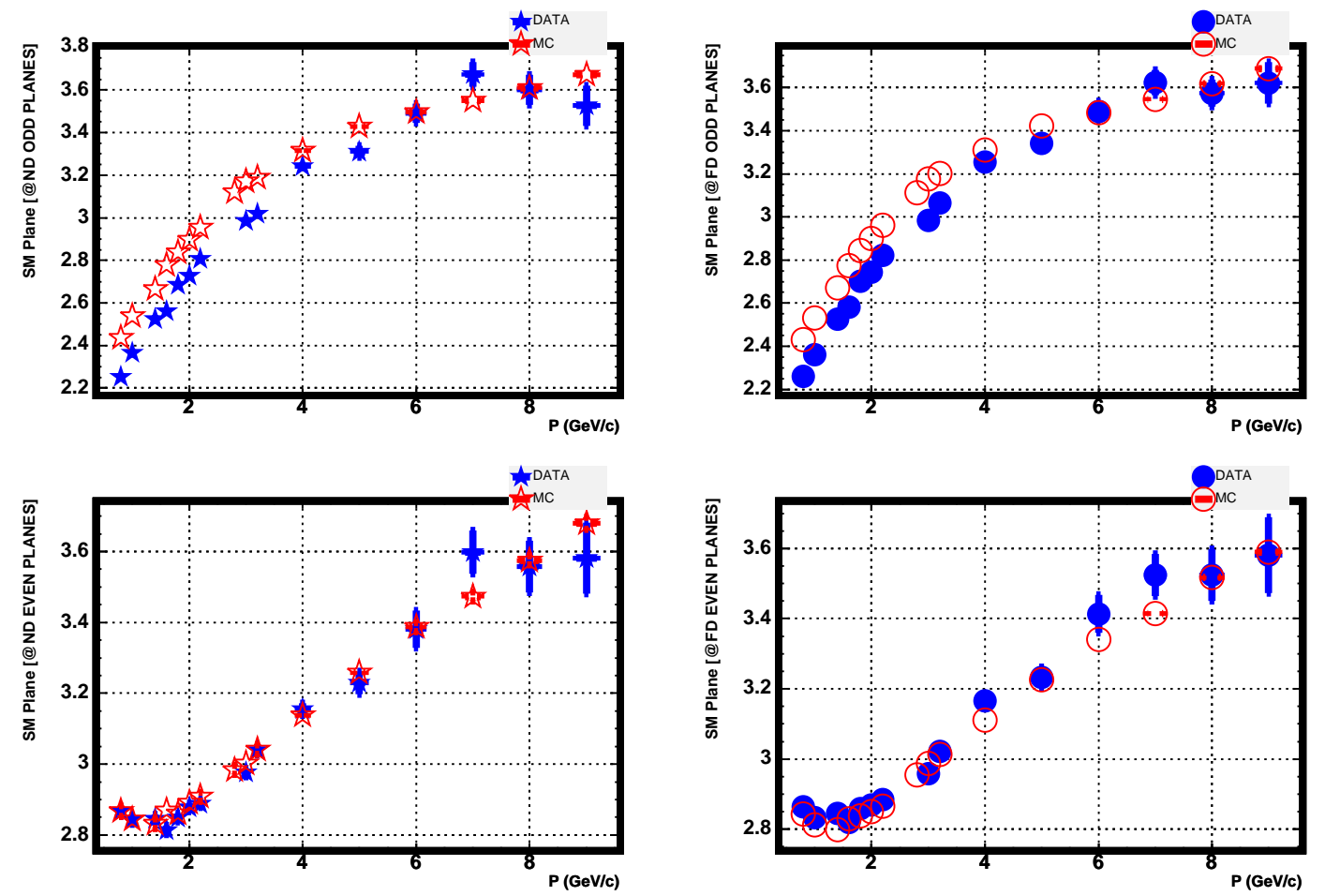

Figure 7.25: "Shower-Max" Plane Location. The location of "Shower-Max" is shown as it varies with beam momenta for ND and FD.

The difference between ND and FD is also more dramatic in the case of muons, as compared with the Near/Far-difference distributions for electrons, while the fractional difference is about constant. To first order, this is caused by a larger number of strips being hit by muons with respect to electrons. In addition, the minimum ionising response of muons is likely to be more sensitive to crosstalk hits and the crosstalk cut than electrons. This is because crosstalk hits are more likely to appear in the same strip of a genuine hit for electrons whose strip hit multiplicity is larger than for muons. The average light level of muon per strip-end is about $4 \mathrm{PE}$, which is reasonably comparable with that of a $1 \mathrm{PE}$ crosstalk hit allowing 
$4-5 \%$ chances for a zero and $20 \%$ chances for $1 \mathrm{PE}$.

Figure 7.25 and 7.26 show the location of the SM in the detector: plane and strip respectively. The location of SM dominates the location of the centre of mass of the electrons and, therefore, also the average event location. The measured plane of SM has a different pattern for EVEN and ODD views (well modelled by the MC). This is because plane- 0 has been removed completely from the analysis. As the average beam momenta increases, the absence of plane- 0 becomes more negligible and, therefore, the structure of the SM plane in the EVEN view agrees better with the behaviour exhibited in the ODD view.
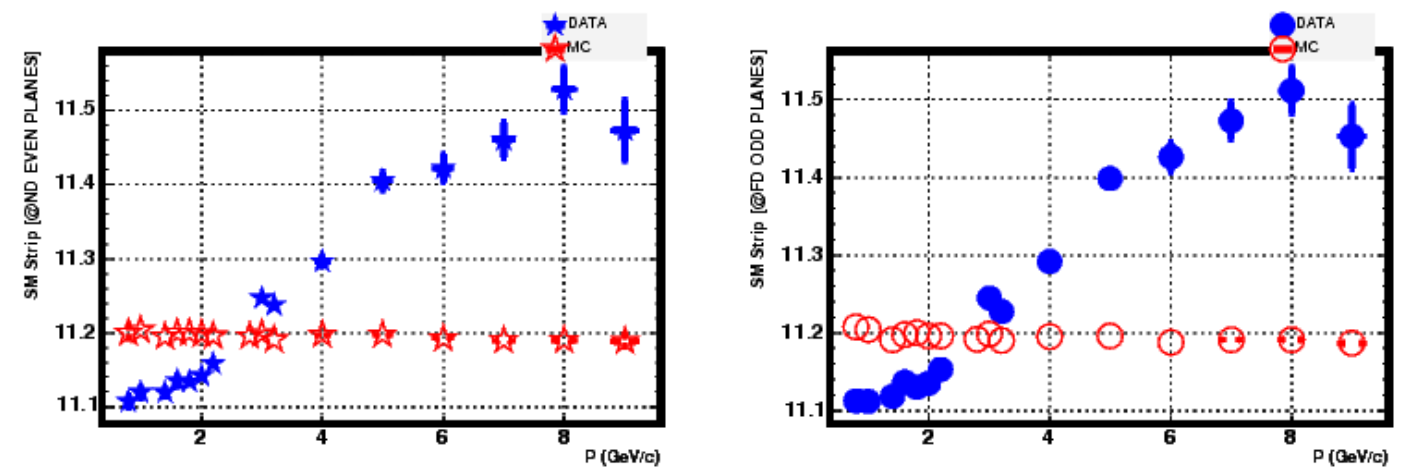

Figure 7.26: "Shower-Max" Strip Location. The evolution of the location of "ShowerMax" in terms of the strip number provides a good illustration of the beam-spot evolution for electrons in the beam.

\section{Summary}

The main discrepancies between MC and data appear in

- Number of Planes Hit by Electrons: there is an overall 0.05 strips difference between MC and data. The actual mechanism for the difference is not well understood but the effect far beyond the sensitivity needed to perform cuts in MINOS; for example for the separation of long/short events, which is the first step towards any $\mathrm{CC} / \mathrm{NC}$ separation.

- Topology of Muons: there is systematic 0.2 strips or plane difference between MC and data in all Near/Far-difference distributions for muons. The muon events have been generated with standard GEANT3, which is known to underestimate the muons $\mathrm{dE} / \mathrm{dx}$ slightly, as shown in Figure 7.27. 

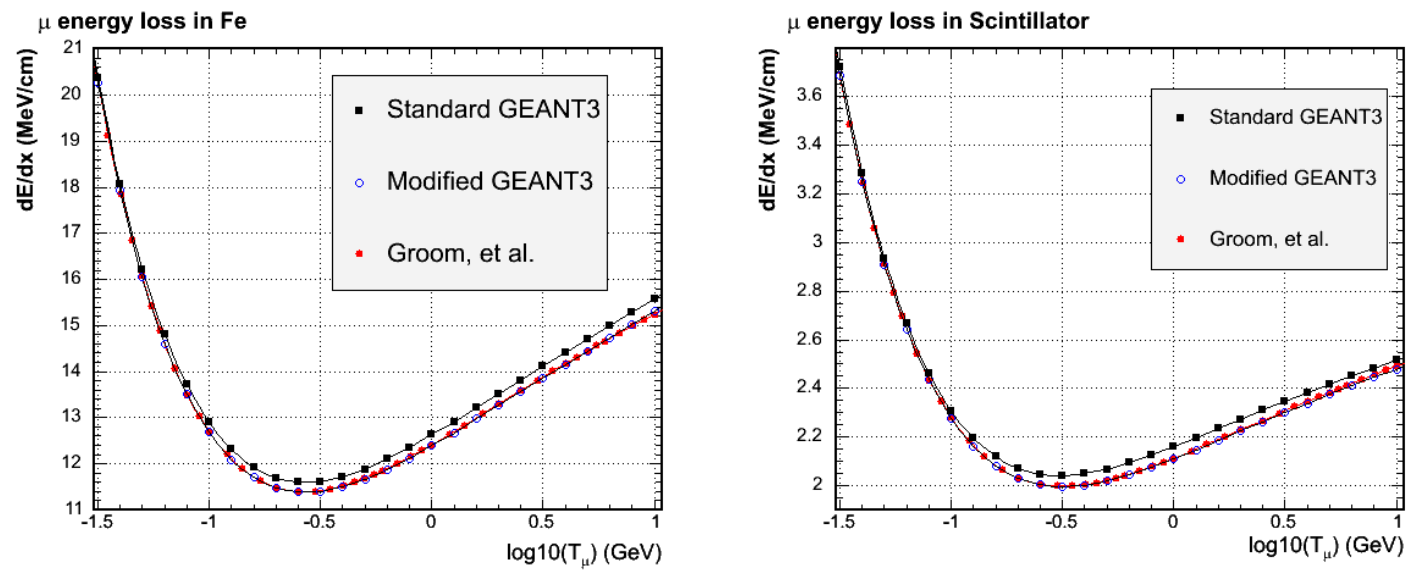

Figure 7.27: Muon Simulation Accuracy in GEANT3. The GMINOS (standard GEANT3) muon $\mathrm{dE} / \mathrm{dx}$ is known to accurate to $1-2 \%$ with respect to theory (Groom et $a l$.), leading to some of the small discrepancies between data and MC. Further effort is being carried out in MINOS to improve the description of the muon $\mathrm{dE} / \mathrm{dx}$ (modified GEANT3). This figures illustrates the current status of some dedicated studies [123], changes not implemented in the present studies.

- Beam-Spot Tuning: The MC and data beam-spot differ at most by 0.3 strips. The MC beam-spot was tuned to match the low energy electrons where higher statistics are available reduces this effect of the discrepancy when the correction for attenuation cannot be carried out.

\subsubsection{Longitudinal Energy Profile}

Another very important aspect of the topology of events are the energy deposition profiles. These profiles are likely to be used in MINOS NC $/ \nu_{e}-\mathrm{CC}$ analyses for the separation of hadronic induced showers from electromagnetic showers. This may be particularly important for $\nu_{e}$-CC appearance channels. So, the aim of this section is to use the longitudinal and transverse energy deposition profiles for electrons and muons to test the accuracy of the detector simulation in this regard. Note that the aim of this analysis in generally limited to relative comparison between both readout systems rather than the absolute modelling of electrons. Studies on the absolute responses of electrons was studied in [74].

Figure 7.28 shows the longitudinal energy profile of electrons as measured by both readout systems. Note that plane-0 has been removed.

Figure 7.28 (bottom) shows the longitudinal energy deposition profile of electrons for data and MC. The agreement between the MC and data is reasonable 

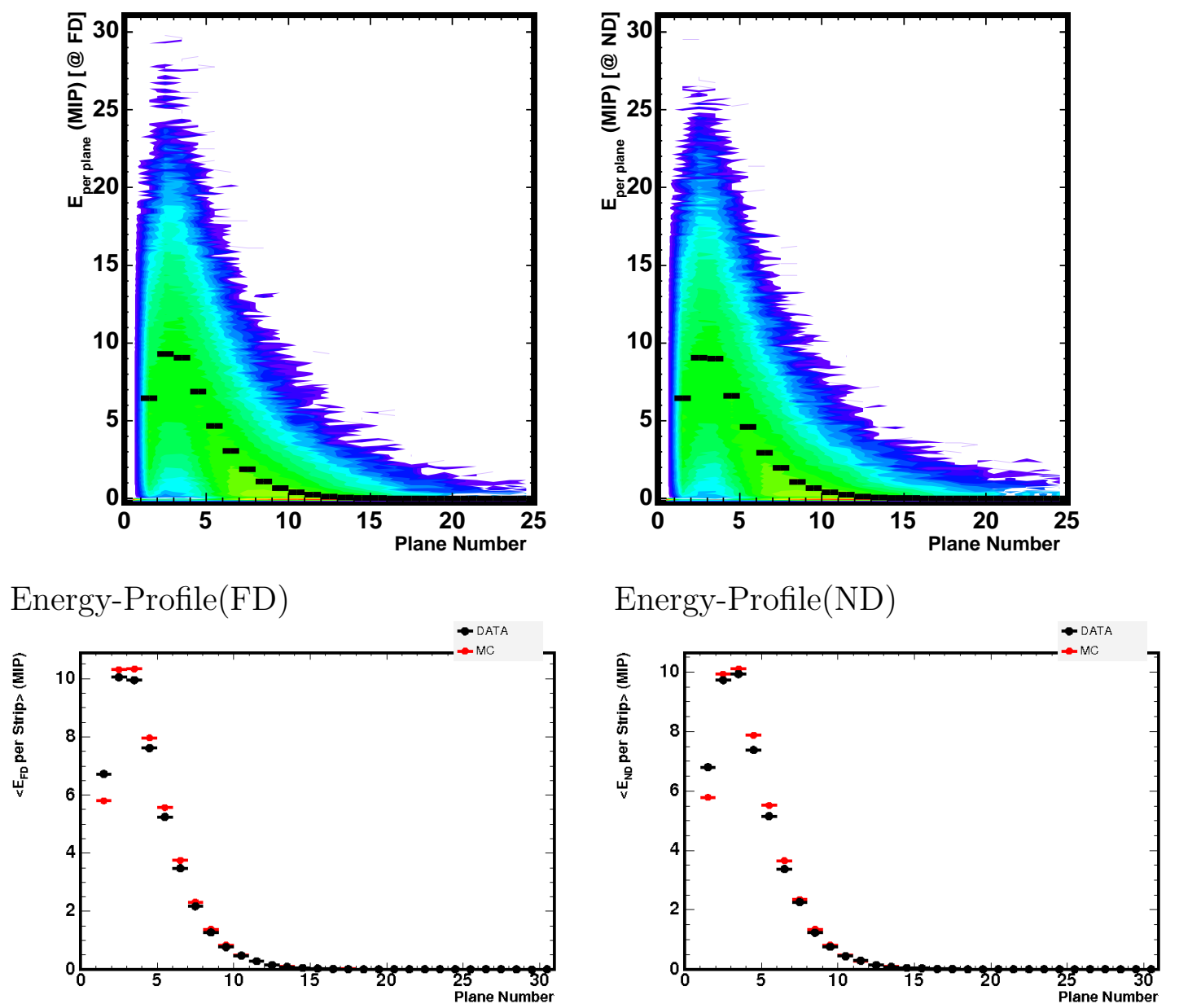

Figure 7.28: Longitudinal Energy Profiles of Electrons. The discrepancy between MC and data is due to the fact that MC was generated without taking into account energy losses upstream the detector. This causes a strong discrepancy in the overall shape of the longitudinal profile of the shower, appearing to be particularly severe during the rise of the shower profile.

despite the fact that MC was generated not taking into account upstream detector energy losses as MC did not have a full beam-line simulation. This effect looks particularly bad in the energy deposition on plane-1: $~ 15 \%$ discrepancy between MC and data. This effect was found to less pronounced for more massive particles such as muons, protons and pions, as one would expect.

A Kolmogorov and $\chi^{2}$ tests were carried out over the longitudinal energy deposition profiles in order to measure the agreement between the observations of the ND and the FD (for MC and data). The compatibility probability of the profiles was generally measured to be $>99 \%$ no matter the energy of the electrons 
used. We conclude from this that the shower profiles are essentially identical across ND/FD readout systems. Such tests also suggest that the agreement between MC and data is very good, despite the discrepancies mentioned.

\subsubsection{Transverse Energy Profile}

The transverse energy profile are particularly important in CalDet because they can be used to check the beam-spot location and measure the effect of PMT crosstalk.

Because of the small dimensions of CalDet the transverse profiles are affected by CalDet cabling effects. As mentioned before, the neighbour pixels of those strip-ends located centrally in the detector (at the beam-spot) are associated with outlying strip-ends in the same plane. Therefore the crosstalk induced by large depositions of energy in the central strips will appear at the "wings" of the detector plane.

Figure 7.29 shows the MC/data comparison for the transverse profiles for electrons. The peak in the centre is due to the main energy deposition at the beam-spot. While the two peaks at both ends ("wings") are due to the PMT crosstalk induced routed to those strips by the CalDet cabling.

Transverse distributions are very sensitive to the position of the beam-spot at CalDet. During the tuning of the MC beam-spot, it was found that small changes in the beam-spot lead to large pattern differences between MC and data in the transverse profiles measured. Figure 7.29 were generated with electrons at $1.8 \mathrm{GeV} / \mathrm{c}$, which are well tuned by construction.

\subsection{Time Comparison of the ND Readout Sys- tem}

A full spill $(10 \mu$ s worth of data) is stored and digitised by the ND FEE in synchronisation with the MI clock. All events interacting within the detector within the spill will be read out in the same snarl. Offline reconstruction, namely the Slicer algorithm, is applied to separate all events that happen within the same snarl. Therefore, for the NFC, some emphasis was put on investigating the time structure of the ND - namely the accuracy with which the MINOS detector simulation packages are able to describe the timing characteristics of the readout system. The most important goal is to validate the simulated time structure of the ND, which 
Occupancy (FD)

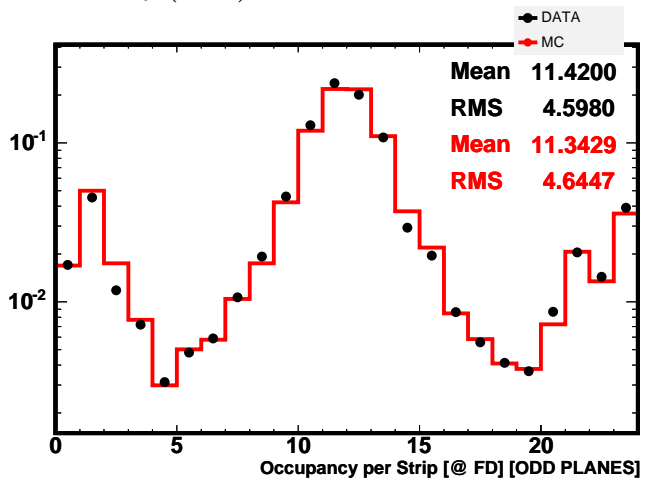

Energy-Profile(FD)

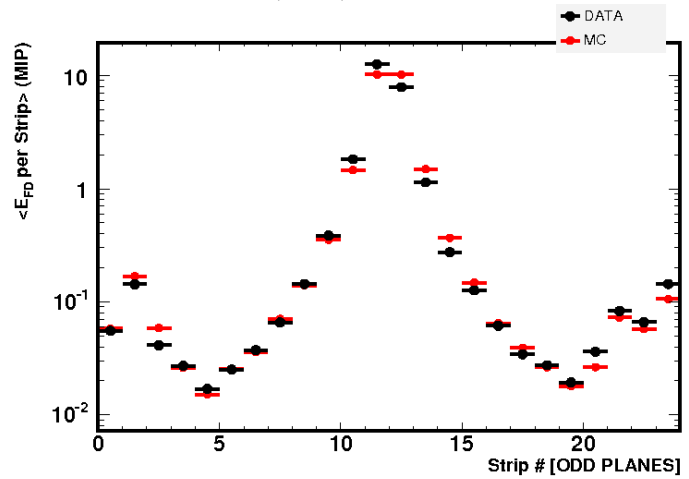

Occupancy(ND)

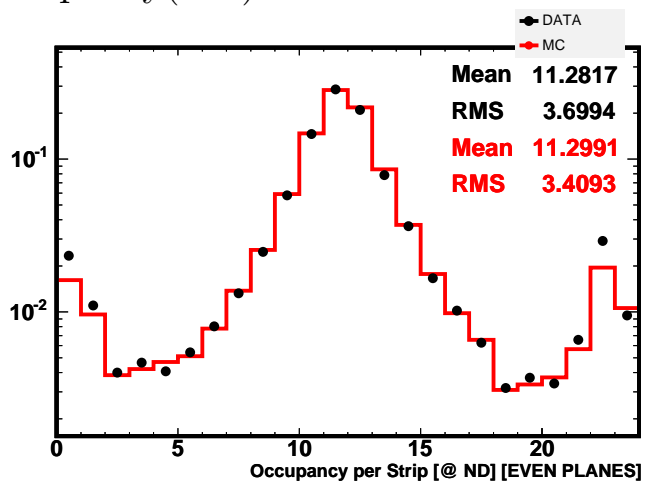

Energy-Profile(ND)

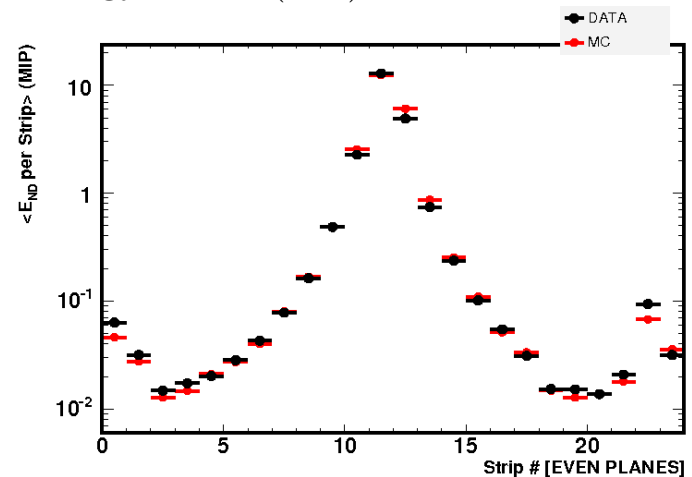

Figure 7.29: Transverse Energy Profiles of Electrons. These plots show the strip occupancy (top) and the transverse energy profile (bottom) for electrons, where the average event location can be seen to be about the same for data and MC. Note that the occupancy plots show good agreement in the beam-spot location between data and MC. The peaks in the "wings" of the distributions correspond to PMT crosstalk that becomes "routed" by the cabling setup at CalDet. Describing accurately the crosstalk peaks on the wings of the detector can be used as test-bed for MC PMT crosstalk modelling.

is used for tuning the Slicer algorithm, since this stage of reconstruction relies, to first order, on timing for the event separation.

\subsubsection{Time Structure of Pulses into the Readout System}

The time structure measured is a convolution of the intrinsic time structure of the PMTs, which are very fast $(\sim 3$ ns rise-time, since they are very small, and that of the scintillation light dispersion through the optical system of the detector up to the face of the PMTs. Therefore the time structure is dominated by the slowest components: the scintillation de-excitation exponential intrinsic to the green WLSF, whose time constant is $\sim 7 \mathrm{~ns}$. Therefore the time structure is light level dependent, due to the exponential decay contributions. Since the amount of WLSF 


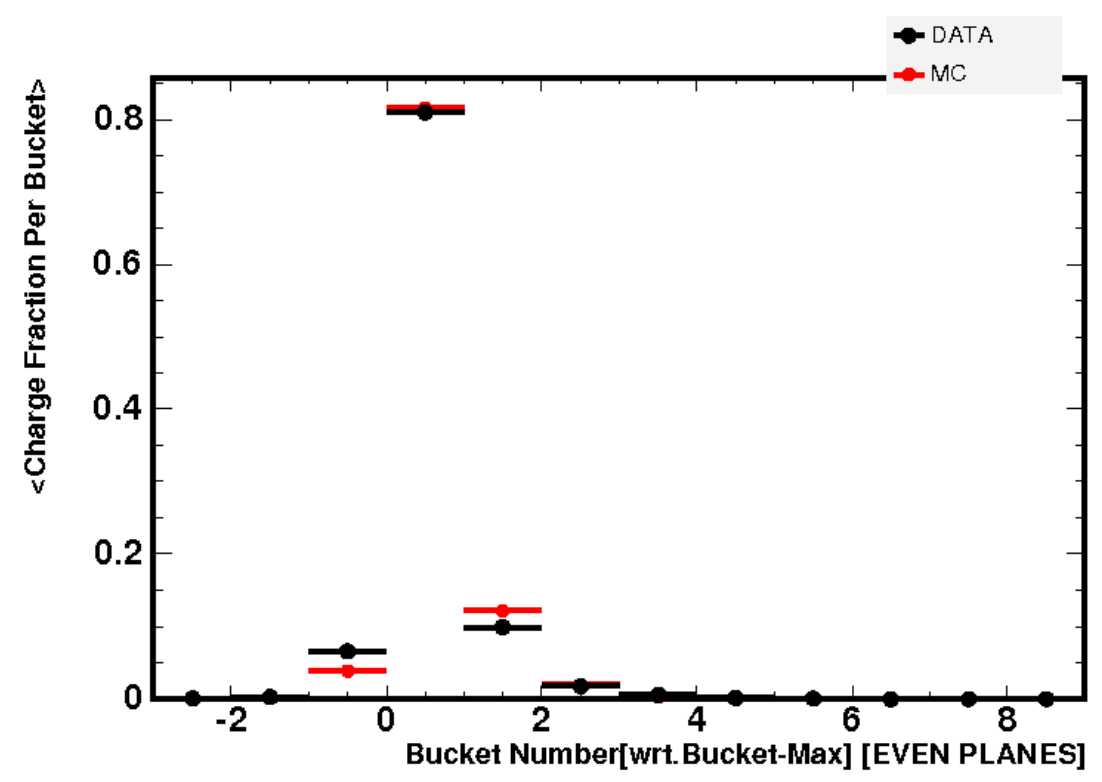

Figure 7.30: Time Pulse Shape. This distribution shows the pulse shape as measured by the ND for data and MC. The time structure is coarsely sampled by the buckets. The $\mathrm{MC}$ appears to deliver charge slightly more slowly in this plot.

is different for the EVEN and ODD views, most plots will be separated per view.

Figure 7.30 shows the time structure of pulses for MC and data. They show the fraction of charge in each bucket with respect to the total charge measured in each strip. The bucket alignment has been obtained by using the buckets with the largest among of charge per strip ("Bucket-Max") as the reference (zero in the abscissa axis). MC and data are in very good agreement and more than adequate for the precision needed by MINOS.

A more quantitative measurement of the spread of the pulses is shown in Figure 7.31, where the RMS of the arrival hits with respect to the event "triggering" 6 time has been computed. Once again, MC and data are in good agreement (note the logarithmic scale of the plots). The tail towards high RMS in the data not present in the $\mathrm{MC}$ is believed to be due to the fact that the 1PE noise was found to be up to $4 \times$ larger in some data runs than expected. This effect of $1 \mathrm{PE}$ noise will be studied in detail in Appendix B.

\footnotetext{
${ }^{6}$ In data, this time corresponds to the arrival time of the beam-trigger. While in MC this corresponds to zero time, when the event happened. The time references should differ by no more than $5 \mathrm{~ns}$ absolute time, as a rough time calibration of the data was carried out to zero the arrival time of the beam-trigger.
} 


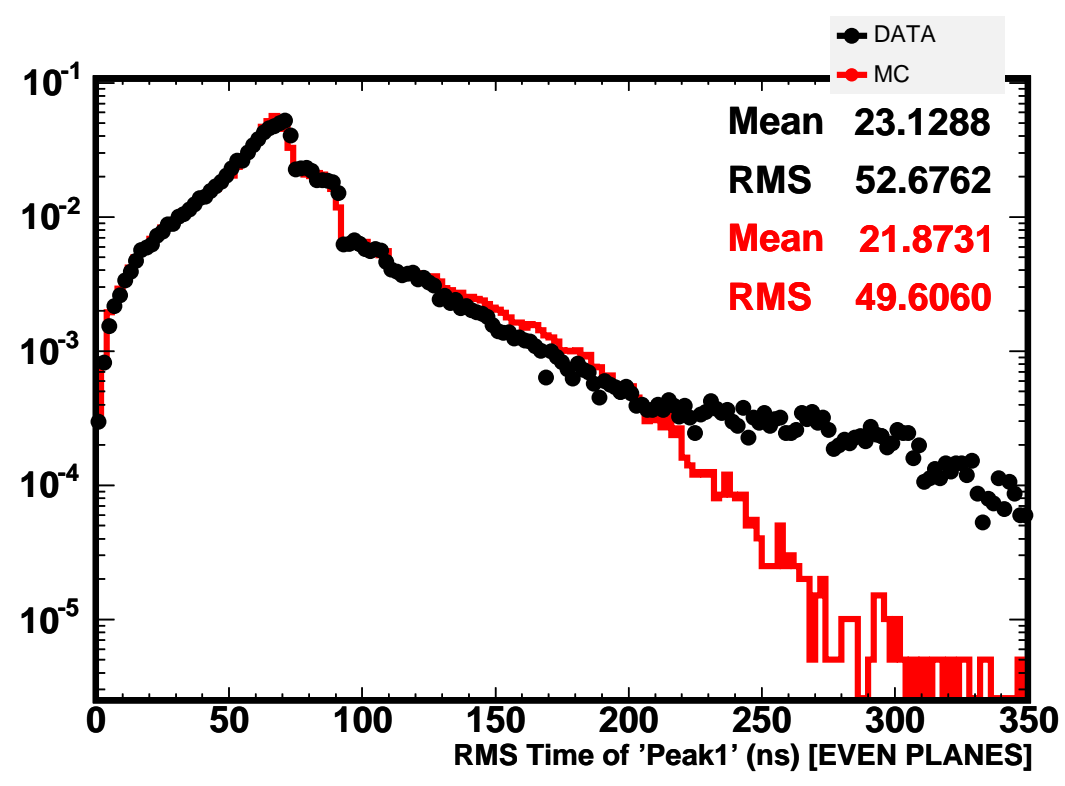

Figure 7.31: Time Spread. This plot shows the spread in time of the hits with respect to the average mean time for the energy deposition per each strip. The difference shown between MC and data is believed to be due to the higher rate of 1PE noise on the data described in Appendix B.

\subsubsection{Time Spread over Readout Buckets}

Another issue, linked to the previous discussion, is the charge spread over the readout buckets.

Figure 7.32 shows a linear correlation between the number of buckets hit and the amount of charge per strip-end. Both MC and data show a similar trend, however the steeper gradient in the MC suggests that the MC pulses are slightly narrower: less buckets hit for the same amount of charge per strip. The difference is believed to be negligible for the purposes of MINOS, therefore no further tuning was carried out.

\subsubsection{Definition of the QIE-FEE Readout Window}

The ND readout system was run to read out 20-buckets upon a dynode or external trigger during Near/Far running. This offered an opportunity to investigate the optimum number of buckets to be read out upon trigger in the comic mode for the MINOS Near detector.

Figure 7.33(left) shows $\left(1-E_{\text {bucket-i }} / E_{\text {Strip }}\right)$ versus $i-t h$ bucket number. Therefore, $\left(1-E_{\text {bucket-i }} / E_{\text {Strip }}\right)$ represents the amount of energy missing if we integrated 

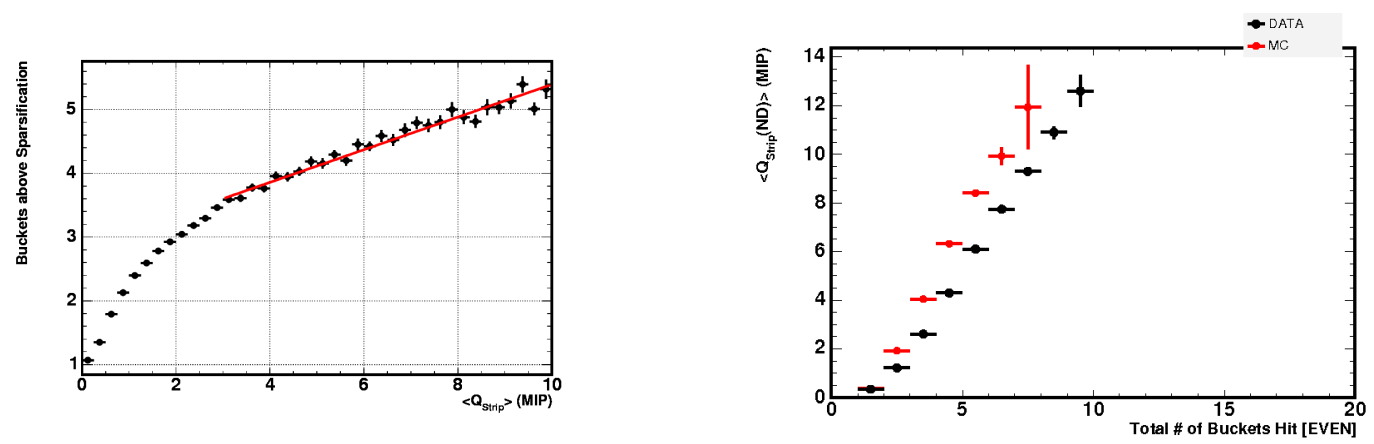

Figure 7.32: Number of Buckets Hit. These plots show the intrinsic relation between the number of buckets hit and the amount of energy deposited per strip. The left plot shows the peculiar low energy structure of the distribution which, to some extent, is shaped by bucket sparsification. The right plot shows the average behaviour at high energies (for MC and data): 5 buckets hits per every 4MIP worth of energy.
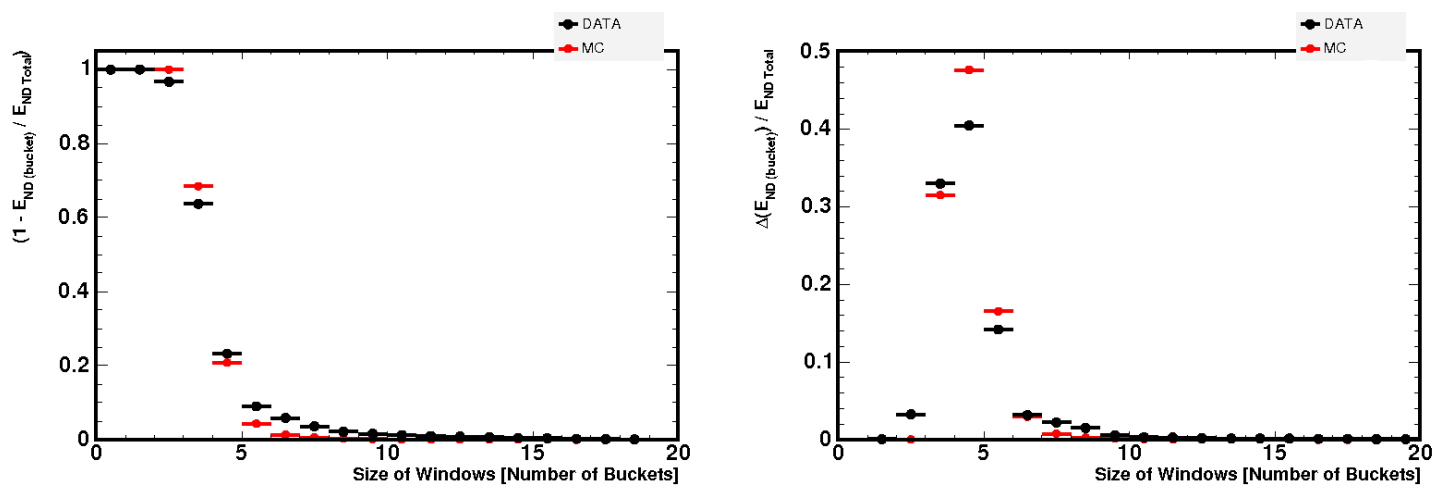

Figure 7.33: ND Bucket Integration: MC/Data Comparison. The contents of these plots are explained in the text in full details.

from bucket-0 (first bucket above sparsification per strip) to the $i$-th bucket. For example, at bucket-5, $\left(1-E_{\text {bucket-i }} / E_{\text {Strip }}\right) \sim 0.2$, which means that, if we were to have only five buckets per trigger, the ND would miss $20 \%$ of the energy depositited per strip on average. Therefore, we are interested in determining how many buckets we should integrated over to contained most of the energy deposited, while minimising the integration over noise. To answer that question, we should study the evolution of the slope of $\left(1-E_{\text {bucket-i }} / E_{\text {Strip }}\right)$ versus bucket number, which is shown in Figure 7.33(right). Note that the derivative increases very rapidly as the integration window spans over the peak of the energy deposition, after which the derivative dramatically falls asymptotically tending to zero when most of the energy has already been contained within the integration window.

In this analysis, it was found that the optimum width of the readout window 
is about 6 buckets in order to contain most of the energy deposited, providing good synchronisation with respect to the triggering is achieved. Charge integrated beyond the sixth bucket appeared consistent with noise. With the contribution of the findings shown, it was verified as correct the usage of 8 bucket wide ( 6 buckets needed plus 2 extra buckets) readout window for the Near detector cosmic mode running.

\subsection{Specific Instrumental Effects}

A few interesting effects, characteristic of and/or discovered in either or both readout systems will be the subject of this this last section. Some of these effects have been mentioned already but were not the central theme of the discussion. Therefore, the investigations carried out will be documented here for completeness.

\subsubsection{Bucket Sparsification on Muons}

The most important repercussion of the bucket sparsification effect is that it is likely to affect the response of muons (within $0-5 \mathrm{MIP}$ ) in a manner which is light level dependent, as shown in Figure 7.34. Muons are important because they are used to generate calibration constants in all MINOS detectors, namely the intra and inter detector calibrations. Note that the $A_{N / F}^{\text {Strip }}$ for muons exhibits a somewhat more dramatic effect compared with electrons in the region where the bucket sparsification is known to be important. The actual nature of the difference was not investigated, as the $\mathrm{MC}$ was able to describe the effect accurately. However, this effect was speculated to be a subtle artifact caused by crosstalk.

Bucket sparsification should not sizably affect the S2S calibration in the MINOS Near detector. This is because the response of the cosmic muons per strip, as measured with the same readout system across the detector (unlike CalDet N/F running), are compared and normalised to compute the S2S constants. The bucket sparsification would cause a systematic bias between the Near and Far detectors normalisation, if no correction was applied. In this thesis, it has been shown that a correction (possibly as large as $4-5 \%$ ) based on the MC prediction should be able to account for the effect to about $0.5 \%$ accuracy over the dip region.

The QIE-FEE calibration data cannot account for the effect, mainly because the calibration data is collected with no sparsification and by means of DC injector. The LI-linearity data seems to be marginally sensitive to sparsification of buckets, 


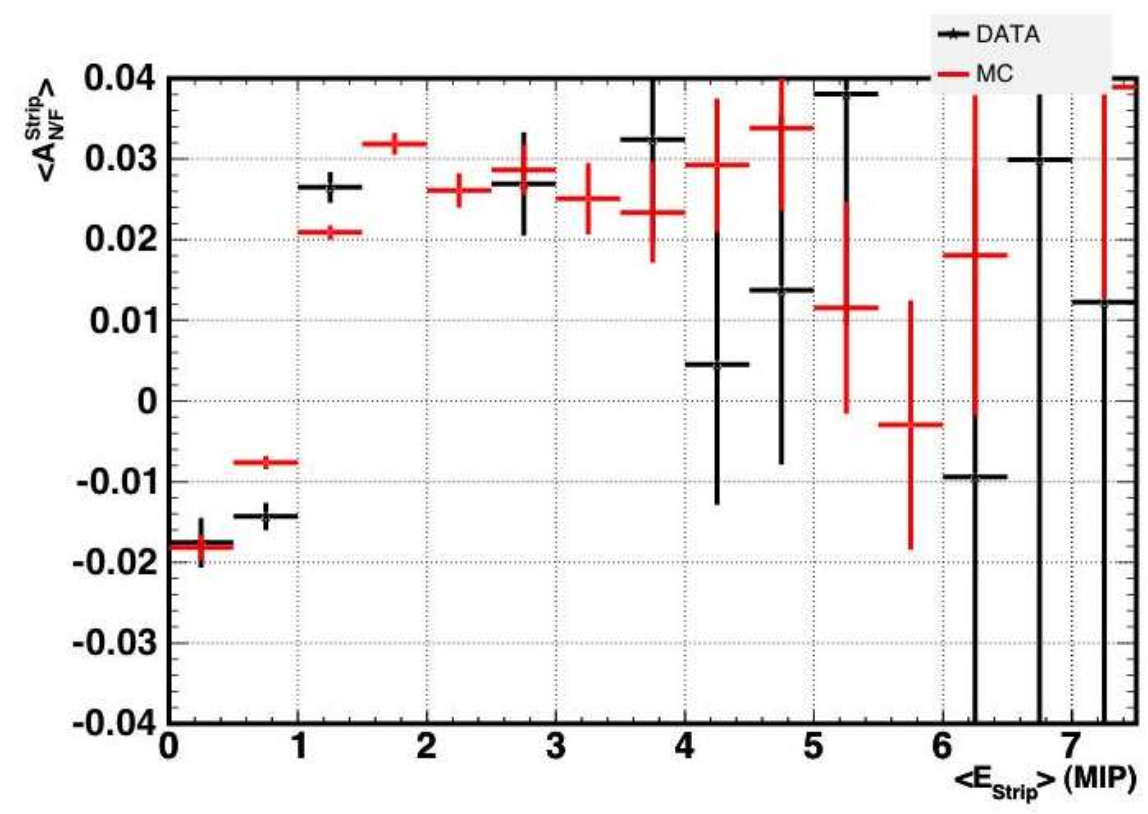

Figure 7.34: Strip-by-Strip $A_{N / F}$ Evolution for Muons. This plot shows the evolution of $A_{N / F}^{\text {Strip }}$ with strip energy for double-ended hits for muons. The bucket sparsification effect dominates the structure.

as shown in Figure 7.3 (small deficit at $x \rightarrow 0$ ). However, the region over which the bucket sparsification is more prominent $(<10 \mathrm{PE})$ is the region over which the LI-Linearity analysis tends to be less sensitive. This is because that same region is used for a linear fit to extrapolate over the full dynamic range. However, using LI-Linearity data to characterise the effect of bucket sparsification is a possibility not explored thoroughly in this thesis.

\subsubsection{Crosstalk Measurements}

Crosstalk has proved to a play major role in this analysis because the precision of the results were intended to be better than 1\%. Moreover, study of crosstalk is complicated by the peculiar way CalDet was setup for the Near/Far comparison. This analysis has also been particularly useful for validating the the ND crosstalk simulation. In the end, no tuning was performed to match the M64 crosstalk at CalDet with the measurements taken at the Oxford M64 test-stand. The algorithm developed for the measurement of the M64 crosstalk (including the separation of crosstalk components) is the subject of of Appendix A. The FD crosstalk has also been measured and investigated, finding very good agreement with the MC. 


$$
\mathcal{X}=\frac{\mu(\text { non-injected })}{\mu(\text { injected })}
$$

Crosstalk has been measured in both readout systems using muons. By tracking (Swimmer), one can separate the crosstalk hits from genuine track hits based on event topology. A diagram of the tracking principle is shown in Figure 7.17. Then, the crosstalk is defined as the ratio of the average untracked charge to the average charge tracked, as indicated in Equation 7.19. Note that provided we are dealing with pulses $<\mathcal{O}(100) \mathrm{PE}$ (see Appendix A), the only measurable crosstalk is optical crosstalk and, therefore, crosstalk should be measured in terms of photo-electrons: charge in $\mathrm{PE}$.

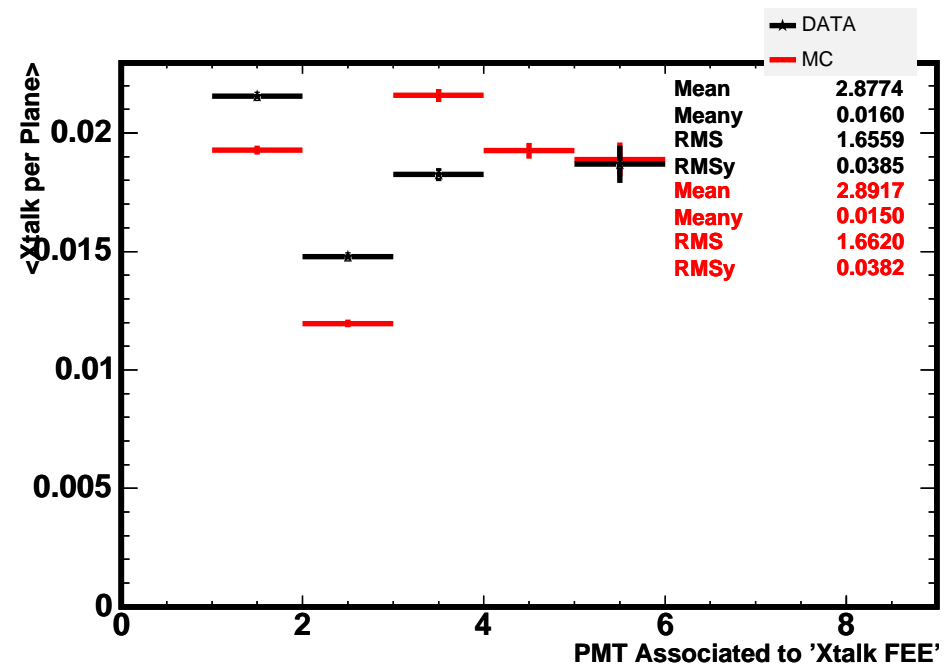

Figure 7.35: Unread-Crosstalk Measurements. The unread-crosstalk is plotted versus the correspond PMT used to measured for MC and data. The PMT-to-PMT variations are dominated by the different amount of hits found each PMT, as they cover different regions of the detector.

The total crosstalk of the M64 is split into two component (due to the detector configuration): "read" and "unread-crosstalk". The read-crosstalk (normal) is the crosstalk that appears in detector-space as isolated hits, since the crosstalked pixels are associated with a strip-end of the detector. While the unread-crosstalk is that associated with pixels that are not coupled to any strip-end. This was the case for $1 / 4$ of the pixels of each M64 at CalDet, of which most were not even provided with FEE to be read out. Only nine PMTs were fully read out and those PMTs were used to measure the unread-crosstalk. The unread-crosstalk turned out to be an excellent test-bed to validate the crosstalk MC simulation, as this represented 
an unique environment to isolate crosstalk without ambiguity in order to confront data and MC results.

Figure 7.35 shows the measurements of the unread-crosstalk per PMT used for one electron run. Note that despite the PMT-to-PMT differences, the average unread-crosstalk is very similar between MC and data.

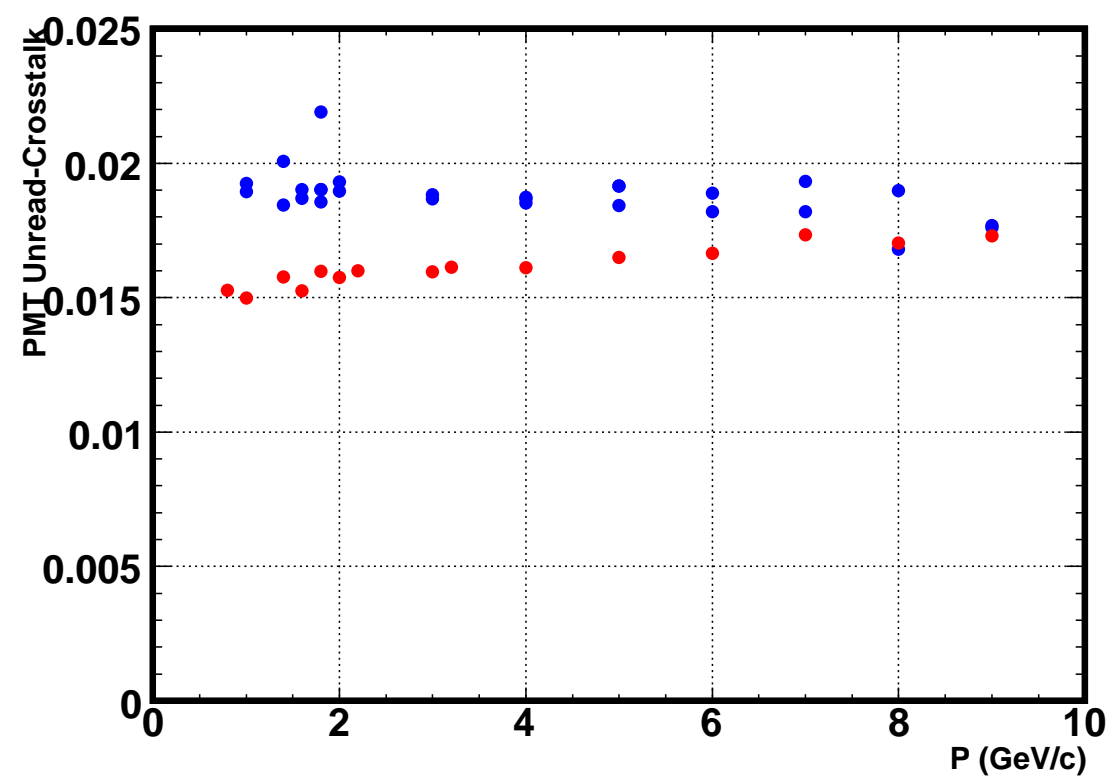

Figure 7.36: Unread-Crosstalk for Muons and Electrons. The unread-crosstalk has been measured to be different for different particles. This is expected as the crosstalk is hit pattern dependent.

The PMT-to-PMT variations are believed to be dominated by the difference in hit patterns between data and MC. This is because the beam-spot for MC and data are not identical, even though they were tuned to be as close as possible. All crosstalk patterns were found to vary very dramatically with the beam-spot location. In addition, there may be some differences in pixel-to-pixel crosstalk normalisation between MC and data that convolute into the differences observed. Note that, for electrons, all the statistics in the first two bins of Figure 7.35. The measured unread-crosstalk measured for electrons and muons is different, as shown in Figure 7.36.

Figure 7.37 shows the total crosstalk as measured with muons. The total ND crosstalk is the sum of the unread and read crosstalk. The read crosstalk was measured as the ratio of charges measured in PE, while the unread-crosstalk was measured as the ratio of ADC charges, since there was no pixel-gain information 


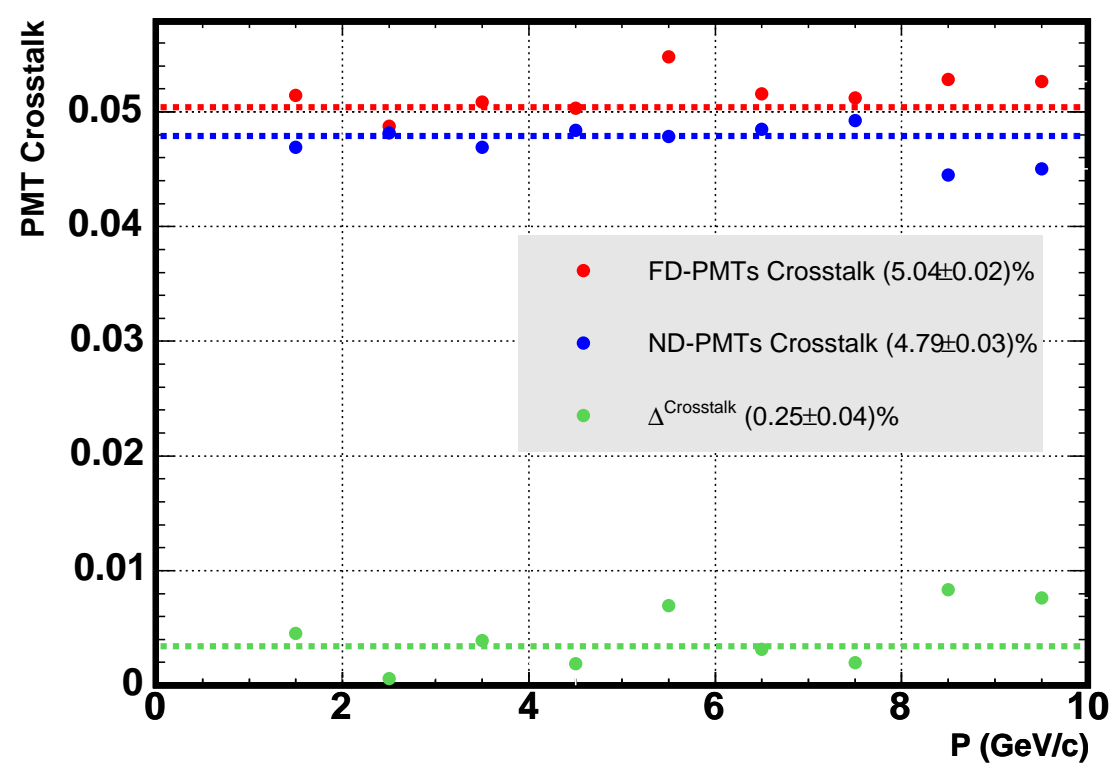

Figure 7.37: PMT Crosstalk from Muons. Muons have been used to measure the PMT crosstalk of both readout systems using the Swimmer. Note that the total ND crosstalk is the sum of the read and "unread" crosstalk measurements.

for the pixel not associated with a strip-end. The bias induced by calculating the unread-crosstalk from the ratio of ADC was measured to be $<0.5 \%$.

The total crosstalk of the ND was measured to be $\left.\left(4.79 \pm 0.03^{\text {stat }}\right)\right) \%$ (DATA), which are to be compared with the optical crosstalk measured in the test-stand at Oxford: $(4.0 \pm 0.8) \%$ [70]. The FD total crosstalk was measured to be $(5.04 \pm$ $\left.\left.0.02^{\text {stat }}\right)\right) \%($ DATA). 


\section{CHAPTER 8}

\section{Conclusions}

This thesis has been devoted to study the performance of the readout systems of the Near and Far detectors as compared one to each other. The data used comes from the measurements taken at the MINOS Calibration detector, while the most interesting issue is to understand how such measurements translate to the MINOS Near and Far detectors and eventually to the physics capabilities of MINOS to measured $\Delta m_{a t m}^{2}$. Hence, the conclusions will be broken down into these three logical domains: CalDet, MINOS, and physics capabilities in MINOS.

\subsection{Readout Systems Comparison at CalDet}

The $A_{N / F}^{S 2 S}$, the relative response asymmetry between the Near/Far readout systems, has been measured at CalDet with electrons between $0.8 \mathrm{GeV} / \mathrm{c}$ and $6 \mathrm{GeV} / \mathrm{c}$ to be on average $\left(0.14 \pm 0.01 \%\right.$ stat $\left.\pm 0.61^{\text {syst }}\right) \%$ (data) upong the subtraction of an systematic offset due to the S2S calibration measured with muons to be $(+1.1 \pm$ $\left.0.4^{\text {stat }} \pm 0.6^{\text {syst }}\right) \%$. The MC exhibits a measurement consistent with the data: $\left(0.27 \pm 0.01^{\text {stat }} \pm 0.32^{\text {sys }}\right) \%(\mathrm{MC})$. Equivalent measurements with muons and protons have yielded consistent results.

- The $A_{N / F}$ measured is consistent with the ND and FD readout systems having equivalent performance when exposed to the same energy deposition to within $0.5 \%$, once the MINOS muon calibration stages have been carried out.

The sensitivity of all measured $A_{N / F}$ is limited by the systematic uncertainties measured in the $\mathrm{S} 2 \mathrm{~S}$ calibration, which particularly affect the responses measured over the first 20-planes of CalDet. 
No linearity calibration was available for the CalDet period in question. However, it was estimated, with the MC, that the expected variation on $A_{N / F}$ due to the non-linearity of both readout systems should be about $0.2 \%$.

- The measured $A_{N / F}^{4 \times \text { MIP }}$ obtained with the $4 \times$ MIP calibration is also dominated by the S2S calibration systematics uncertainties. However, the precision obtained with such a calibration is also degraded by the systematic uncertainties specific to implementation of the $4 \times$ MIP calibration at CalDet. In principle Those uncertainties limiting the technique at CalDet are not to be extrapolated to the same technique, if developed in any other MINOS detectors.

- The event topology has been demonstrated to cause negligible distortion on the results measured with either readout systems. The topological features found are generally CalDet-specific and, in any case, are generally accurately described by the MINOS detector simulation.

- The MINOS detector simulation packages have been found to accurately describe both the ND and FD readout systems. Almost all possible aspect of the detector simulation have been explored during the course the NFC analysis and discrepancies have been found to be generally well $<1 \%$ level.

The timing of the ND has been investigated in especial detail, as this feature has primary importance of the neutrino reconstruction in the ND. Once again, remarkable agreement between MC and data has been found.

- Both the ND and FD intrinsic crosstalk have been measured at CalDet to be $(4.79 \pm 0.03) \%$ and $(5.04 \pm 0.02) \%$, respectively. The measurements are in good agreement with the results from dedicated studies carried out at the MINOS PMT test-stands.

- An attenuation calibration has been developed for the specific purposes of the analysis presented, whose success validates the accuracy (with respect to CalDet $<0.2 \%$ ) of the average parametrisation of the light attenuation along the fibres used in MINOS. This attenuation correction however cannot be extrapolated to MINOS Near and Far detector, where light attenuation is far more delicate due to the dimensions of the detectors. Specific light attenuation calibration has been devised for the MINOS Near and Far detectors, namely the MAPPER calibration [65], that is expected to allow the characterisation of the light attenuation for each single detector strip. 


\subsection{Readout Systems Comparison in MINOS}

The conclusion that can be drawn from the CalDet results into the MINOS FD and ND are restricted to those aspect which are common among the three detectors. Therefore, little can be anticipated about differences arising from the respective S2S calibration, as the implementation of such calibration is dominated by detectorspecific effects, such as attenuation corrections, cabling features, etc. This thesis provides, however, useful studies to the implementation of the inter-detector calibration of the ND and FD MINOS, when a common energy scale or normalisation is defined. Namely, it measures to what extent a systematic differences is expected whose origin lies on the readout system performance differences. Detector-specific systematics effects beyond the readout systems affecting the inter-detector energy normalisation are, therefore, beyond the scope of the studies hereby presented.

- From the NFC at CalDet the best estimate to be transported to the MINOS Near and Far detectors is the measured $A_{N / F}^{T o t a l}$ (relative asymmetry taking into account all crosstalk contributions), which at CalDet is expected to be about $\left(-0.3 \pm 0.2^{\text {syst }}\right) \%$. The measured value is in good agreement with predictions: $\left(-0.16 \pm 0.01^{\text {stat }} \pm 0.60 \%{ }^{\text {syst }}\right) \%$ for data and $\left(0.05 \pm 0.02^{\text {stat }} \pm 0.32^{\text {syst }}\right) \%$ for $\mathrm{MC}$, once all calibration systematic have been taken into account.

The negative prediction arises from the PMT crosstalk difference between readout systems due to the fact that that no separate MIP calibrations were performed for the CalDet data. At the ND and FD the expectation is to be null asymmetry as the MIP calibration should calibrate the crosstalk differences.

- The capabilities of the LI-systems has been validated and quantitative measurements of its capabilities have been achieved:

1. The LI-Drift calibration has been proved to be able to correct for the drift of both readout systems successfully to about $<0.2 \%$ over a month.

2. The LI-system capability to measure and correct for the non-linearity of both readout systems has been proved for the first time to a level of accuracy $\sim 0.5 \%$.

- The intrinsic uncertainties associated to the $4 \times \mathrm{MIP}$ calibration discussed in this thesis do not translate to the MINOS Near and Far detectors MIP calibrations. 


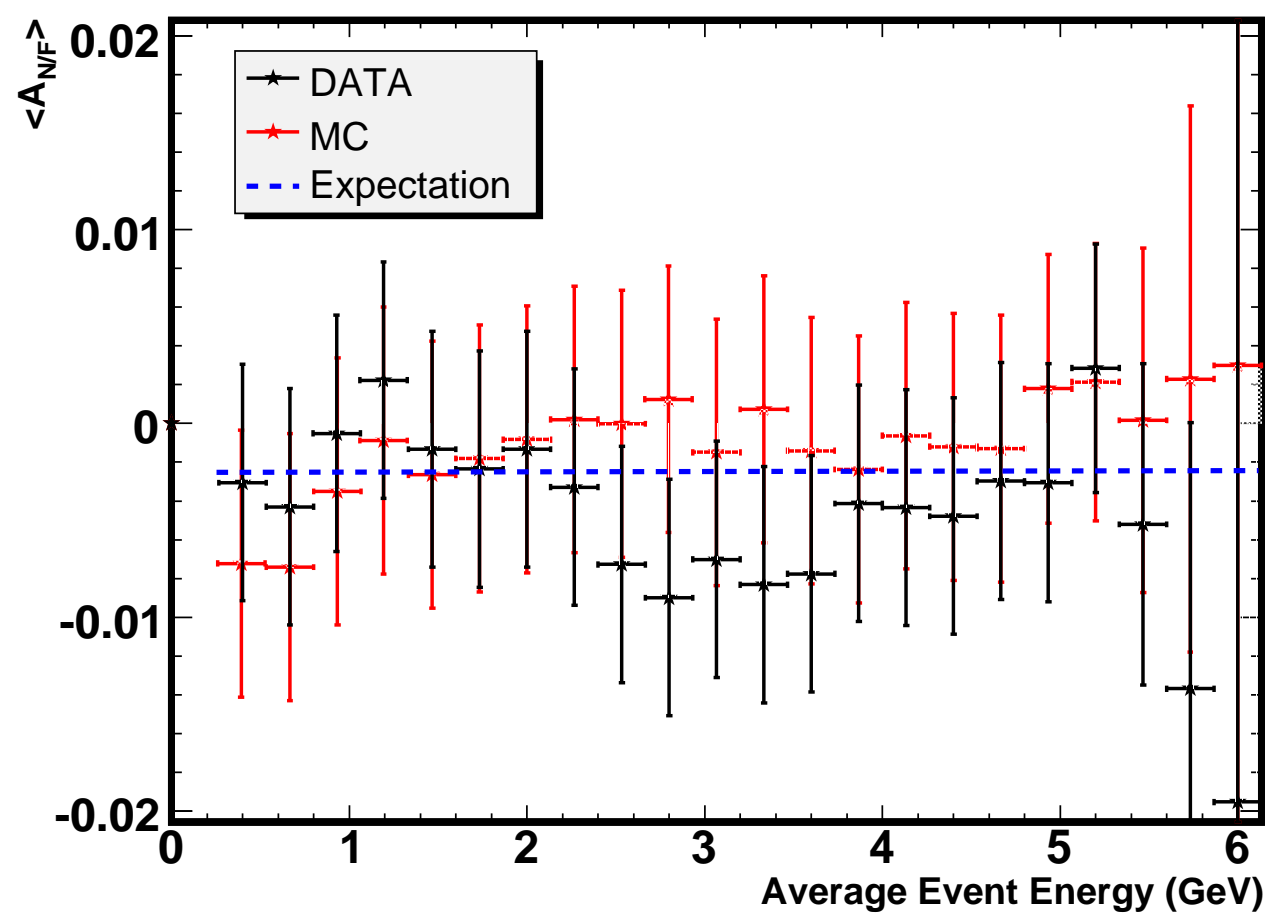

Figure 8.1: $A_{N / F}^{\text {Total }}$ versus Event Energy

- The topology comparison has shown that $\mathrm{ND}_{D A T A} \sim \mathrm{ND}_{M C}$ and $\mathrm{FD}_{D A T A} \sim \mathrm{FD}_{M C}$, with discrepancies being of the order of $1 \%$ level. The ND/FD differences discussed during this thesis are CalDet-specific: cabling, crosstalk pattern, beam-spot, occupancy, etc.

- The effect of bucket sparsification has been measured and characterised with data for the first time. It has been identified that such an effect can lead to a significant discrepancy (light level dependent) on the relative normalisation level between the MINOS detectors unless a correction is provided. The effect has been measured to lead to an effective decrease on the response of the ND readout system of up to $4-5 \%$. The structure and magnitude of the effect have been found to be well described by the MC, with discrepancies of the order of $0.5 \%$.

The most important consequence of the this effect is that it affects the range of the pulse-heights generated by muons, while muons responses are used critically for the MINOS calibration scheme, namely the inter-detector calibration. Therefore, this thesis anticipates the need for a correction for the 
implementation during the inter-detector calibration of MINOS, which has been shown to be feasibly obtained from the MC.

Finally, running both ND and FD readout systems at CalDet has proved to be a very fruitful experience from which much has been learnt about both systems in terms of their performance, calibration and simulation generally from relative point of view.

\subsection{Repercussions on the Physics Capabilities of MINOS}

The measured $A_{N / F}$ is consistent with zero, however, due to the limited uncertainty associated to the measurements possible few $\%$ offsets could still be compatible with the observation reported. Independent to whether an offset originates from a subtle difference between the responses of the readout systems or a limitation of the MINOS calibration chain, we should contemplate the possible impact into the MINOS physics capabilities.

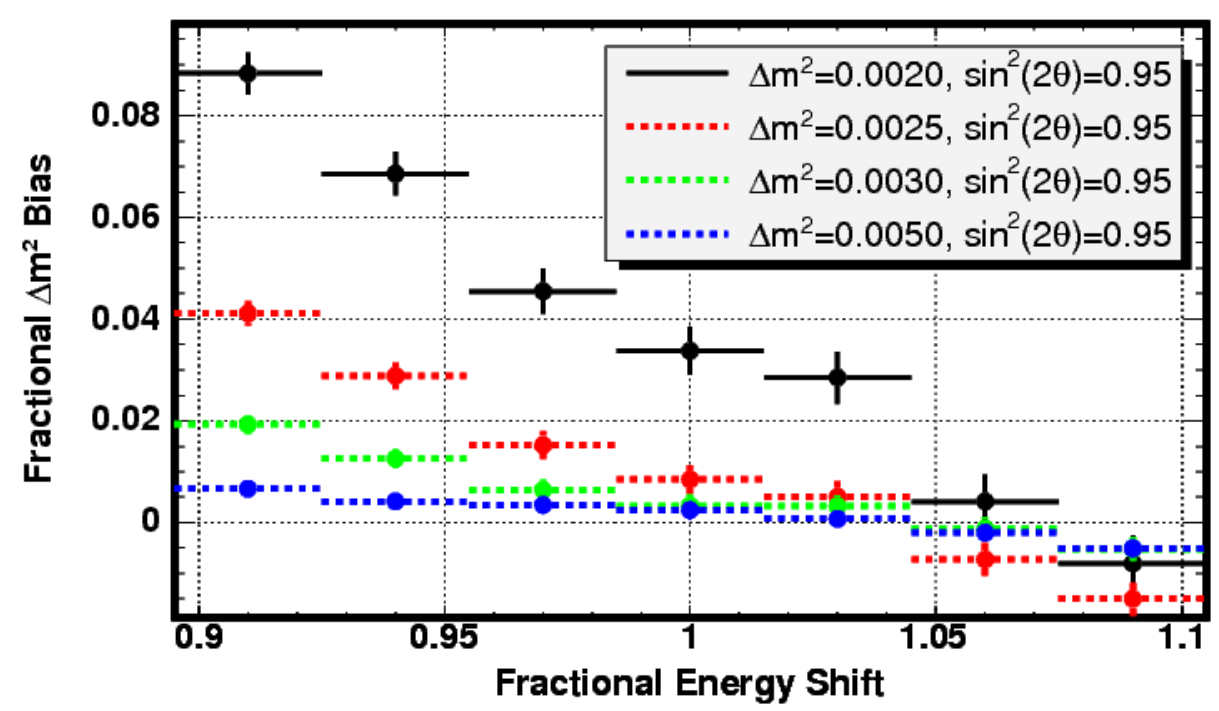

Figure 8.2: Fractional Error Induced in $\Delta m^{2}$ Plot from [115].

Figure 8.2 shows the relative bias in the MINOS measurement of $\Delta m_{a t m}^{2}$ versus the magnitude of a possible systematic shift in energy between the Near and Far 
Detector: $\mathcal{F} / \mathcal{N}$. The shift is $>1$ if $\mathcal{F}>\mathcal{N}$ and $<1$ otherwise. The different curves correspond to the different assumed values of $\Delta m_{\text {atm }}^{2}$ used for their generation. Note that all curves should go through null bias on $\Delta m_{\text {atm }}^{2}$ for null fractional energy shift. The fact that the curves do not go through zero, indicates a bias in the fitting algorithm used for the generation of this plot, which by the time of the writing of this thesis has not yet been resolved. Therefore, this plot should be interpreted for each curve in a relative basis, i.e. the offset between different curves is an artifact.

The asymmetrical structure of the curves reflects the fact that the MINOS L/E ratio aims for somewhat large $\Delta m_{\text {atm }}^{2}$. At the lowest $\Delta m_{\text {atm }}^{2}$ MINOS would suffer from seeing the neutrino oscillation "dip" over the region where the NuMI beam spectrum dies off (towards the low energy neutrinos). This reduces the overall sensitivity of the experiment and causes the stronger dependence between the bias and the shift in energy towards shifts $\mathcal{F}<\mathcal{N}$.

The NFC have proved that a possible shift in energy as measured by the ND and FD readout systems is restricted to be within 0.985 and 1.015 to about $3 \sigma$ level. Therefore, the bias in $\Delta m_{a t m}^{2}$ is expected to be below $1.0 \%$, for any $\Delta m_{a t m}^{2}$. The small effect bias on the measurement of $\Delta m_{a t m}^{2}$ reflects the fact that shift in the calorimetric energy measurement in MINOS would only affect the energy measurement of the hadronic activity in a $\mathrm{CC} \nu_{\mu}$ interaction, as the muon energy is measured by range or curvature - independent from the pulse-height. 


\section{Cross-Talk Studies of the Hamamatsu M64}

This section describes the first studies accomplished at the Oxford PMT test-stand to understand crosstalk in the R5900-00-M64 [125] [126] (M64) PMT. These studies made evident that the PMT crosstalk has two independent components. The experimental setup is described in Section A.2. The algorithm for the separation of the two crosstalk components is described in Section A.3. Once, separated both components will be characterised, Sections A.4 and A.5.

\section{A.1 PMT Crosstalk}

M64s are multi-anode PMTs and therefore, they have 64 independent pixels sharing the same enclosure where electron multiplication happens. Hamamatsu has deployed focusing wires along the paths of each pixels in order to reduce to the minimum the interplay between signals across pixels. However, previous measurements have shown that as a pixel is illuminated, some charge is drawn in other non-illuminated pixels. PMT Crosstalk is therefore quantified as the percentage amount of charge found in the an non-illuminated pixels $\left(Q_{j}^{\text {non-injected }}\right)$ relative to the injected pixel $\left(Q_{i}^{\text {injected }}\right)$, as shown indicated in Equation A.1.

$$
X_{j, i}=\frac{Q_{n o n-\operatorname{injected}(j)}}{Q_{\text {injected }(i)}}
$$

Preliminary quantifications suggest that the average total crosstalk in M64s can be as high as $10 \%[127]$. MINOS is tracking calorimeter for which the event 
topology provides the capability to recognise $\mathrm{NC} / \mathrm{CC}$ events, particularly at the lowest visible energies. The effect of crosstalk on calorimetry information could be calibrated out, if necessary. However, the possibility that crosstalk would blur the topology of neutrino interactions in the MINOS detectors is somewhat more worrisome. Therefore, the main objective of the output of this PMT crosstalk studies is the implementation into the MINOS detector simulation packages.

\section{A.2 Experimental Setup: the Oxford PMT Test- Stand}

The Oxford MINOS group built a test-stand to characterise every single MINOS M64 PMT. Dedicated crosstalk runs were taken as part of the standard run sequence for each PMT. During crosstalk runs, light was injected 10,000 times in each pixel, while all the pixels were read out with every injection. Therefore, the charge of every single pixel was measured with every injection. Before every crosstalk run, a pedestal run was taken to allow accurate pedestal subtraction.

A sample of 9 photo-multipliers was used to identify and measure crosstalk in the M64s for this analysis. The PMT chosen met all the conditions agreed between Hamamatsu for MINOS to accept such PMTs. For this studies, data taken at only one light level was used. The light level was such that the PMTs were well within the linear regime, i.e. 40PE (Photo-Electron). Further details about Oxford test-stand can be found in [124].

\section{A.3 PMT Crosstalk Separation}

Looking a the charge spectra of a non-illuminated pixel illustrates the effect of crosstalk in M64s. A non-illuminated pixel should exhibit a clean pedestal distribution centred at zero charge. However, a clear distortion can be seen in Figure A.1.

This kind of distortion is signature of the M64 crosstalk. The distortion is characterised by two major features. First, the pedestal peak (and the whole distribution) has been shifted slightly toward positive charge values. Second, a small peak is observed at about 190ADC. This corresponds to the $1 \mathrm{PE}$ observed on the pixel.

A different view of the effect is given by Figure A.2, in which the charge of the 


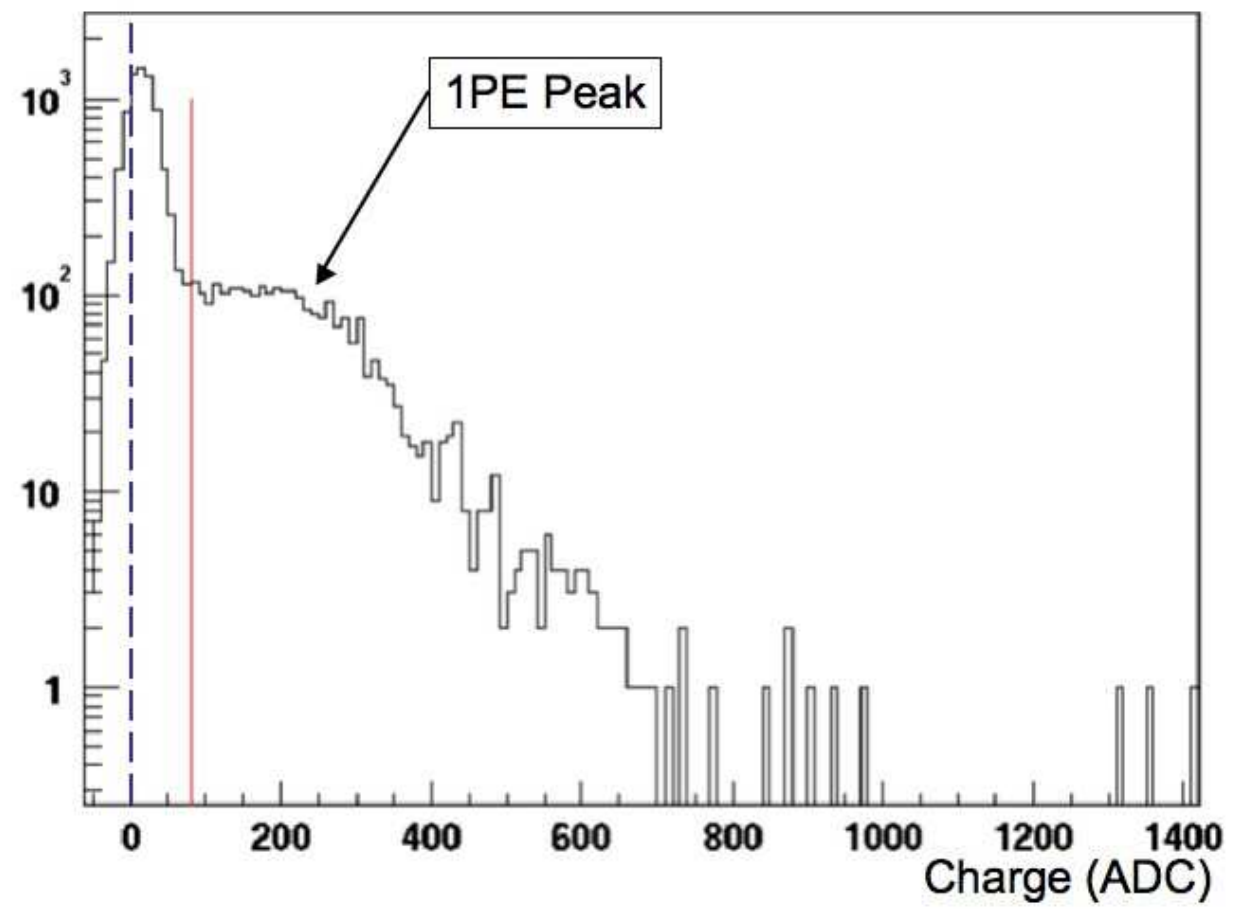

Figure A.1: Charge Spectrum of a Cross-Talked Pixel The black line emphasises the position of the zero charge, after pedestal subtraction. The green line is the $1 \mathrm{D}$ projection of the 2D-cut used to separate the two crosstalk components found in M64s. EXT is the shifted pedestal distribution toward positive charge values (shown to the left of green line). Whereas OXT causes the 1PE dominated peak (shown to the right of the green line).

non-injected pixel is plotted versus the charge of the injected pixel. From this new perspective, one further feature is revealed: correlation. The small drift of pedestal peak arises from shift in zero charge position of the whole distribution, which is proportional to the charge injected in the illuminated pixel. This also implies that shift of the pedestal is accompanied by an effective increase in its width due to the shown correlation.

These two very different components in the charge spectra pointed to two different sources of crosstalk. The first one is related to the effective drift of the pedestal peak. The low charge drawn $(<1 \mathrm{PE})$ appears to point to some kind of mechanism "post-first-dynode" somewhere along the dynode chains as the electrons cascade towards the pixels anodes. This component will be therefore called "Electrical Crosstalk" (EXT). It should not be mistakenly inferred that it is caused by the electronics. The second component causing the 1PE peak appears to have its origin consistent with a "pre-first-dynode" mechanism. Therefore, this second component 


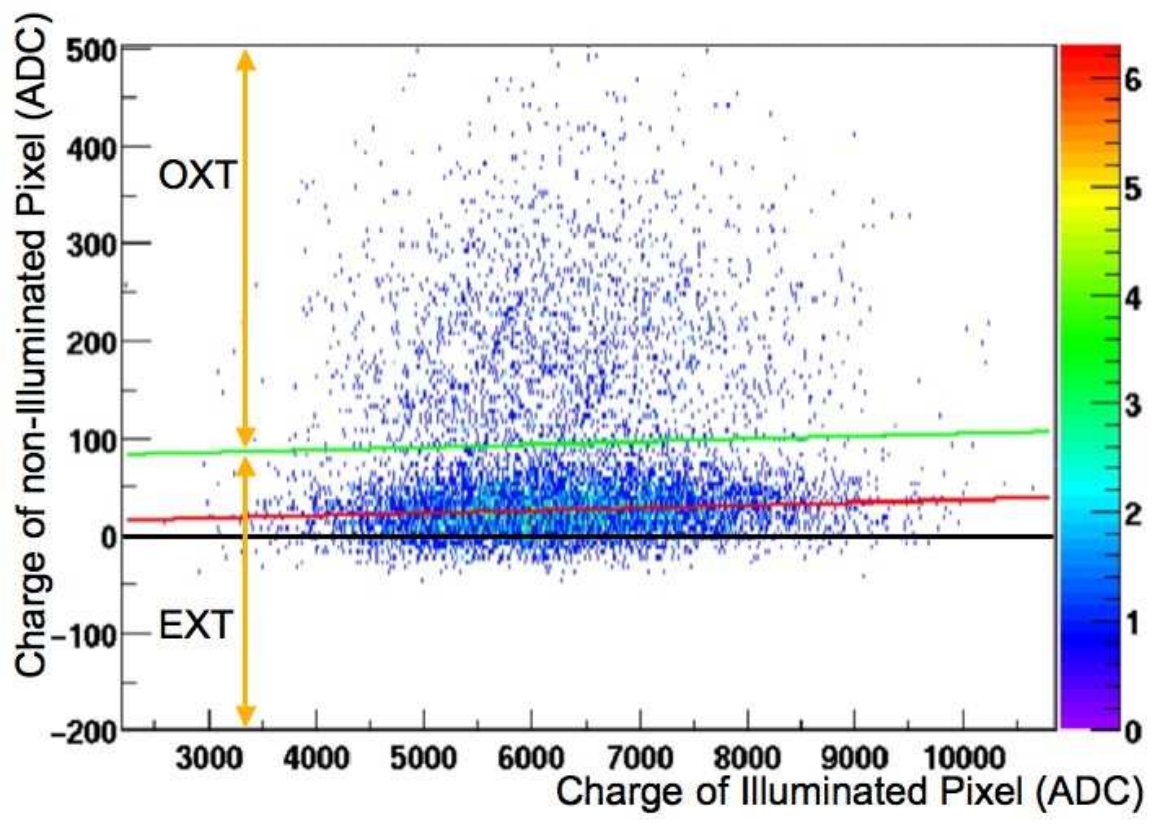

Figure A.2: Correlation Between Injected Pixel versus Cross-Talked Pixel. Correlation between the two spectra can be clearly seen. The red line is a fitted strait line to the EXT distribution used to measure the slope of the correlation. The information from the fit is fed into definition of a 2D-cut to separate both OXT (above the green line)and EXT (below the green line). The 2D-cut is illustrated by the green line.

will be called "Optical Crosstalk" (OXT). As it will be shown, the crosstalk in the M64 appears to be fully characterised by OXT and EXT. A diagram of the crosstalk in the M64 is illustrated in Figure A.3.

In order to characterise each single crosstalk component one should separate them from each other. Care must be taken at this stage not to bias the characterisation of the different crosstalk components by a clumsy cut. Note that the degree of correlation with the charge on the illuminated pixel and the location of the $1 \mathrm{PE}$ peak would vary from pixel-to-pixel as the gain is different in every pixel. The fact that the EXT arises from a correlation made us opt to develop a 2D-cut, as it is also shown in Figure A.2. Whether the 1PE component is correlated at all, was not possible to prove during these studies, not even after the separation of the components.

\section{A.3.1 Implementation of the 2D Cut}

The implementation of the $2 \mathrm{D}$ cut took the following steps to preserve the correlation information of every pixel: 


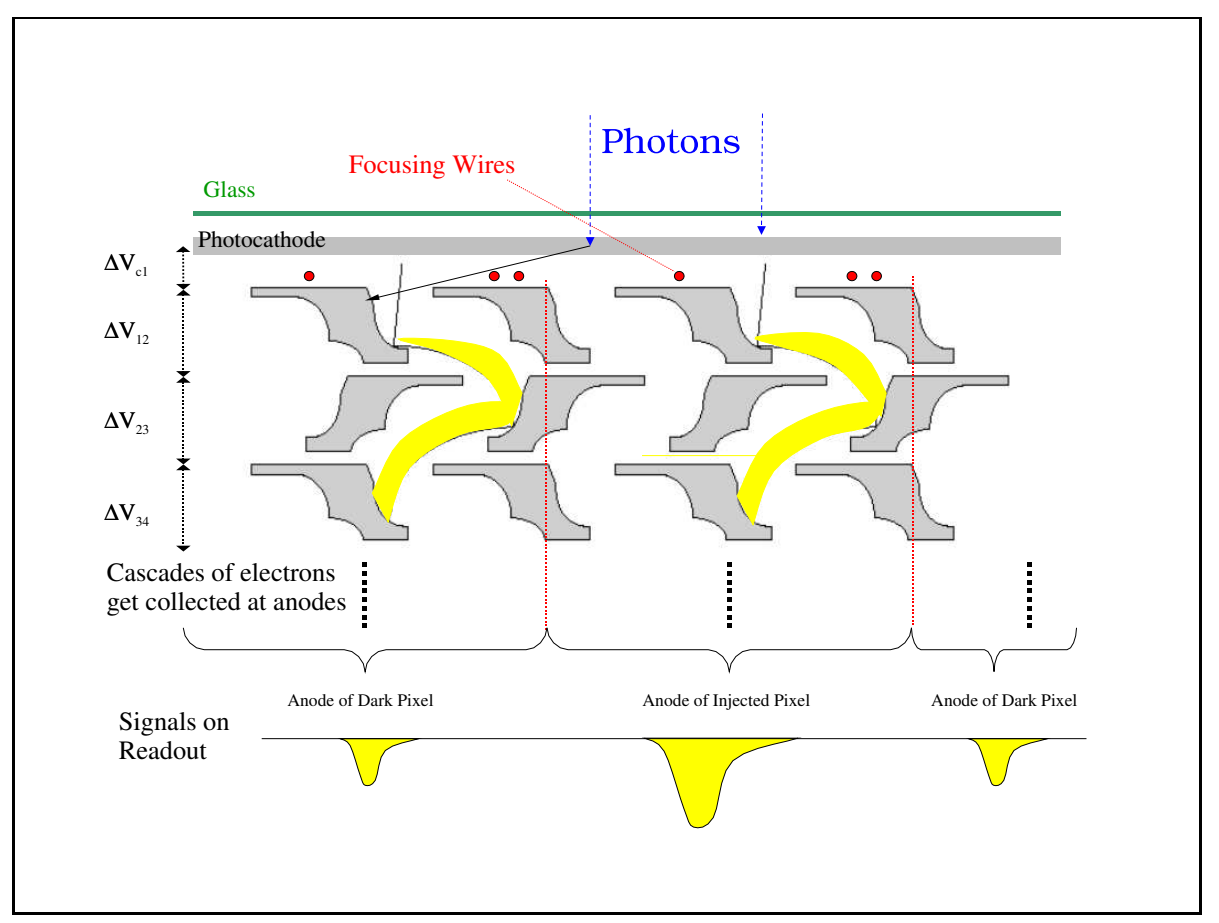

Figure A.3: Diagram of Crosstalk in the M64 This drawing intends to guide intuition on how crosstalk of the M64 works. The OXT occurs by a PE creating a cascade of secondary electrons in a non-illuminated pixel, while EXT happens somewhat downstream in the dynode chain due to the leaking of secondary electrons into neighbouring pixels.

1. Perform a first coarse separation of the between EXT and OXT distribution: red line in Figure A.1.

2. Then, fit a straight line to the profile histogram from the EXT, once the OXT removed has been removed. The fit is performed to measure the slope of the correlation, as indicated by the red line in Figure A.2.

3. The 2D-cut was then defined by a parallel straight line to the fit curve to the correlated distribution. The cut was carried out at $2.5 \sigma$ of the pedestal width toward positive charge values as indicated by the green line illustrated in Figure A.2.

Hence, the green line in Figure A.2 defines the boundary between EXT (below) and OXT (above).

\section{A.4 Characterisation of PMT Electrical Crosstalk}

As mentioned before this component is responsible for shifting the whole nonilluminated charge distribution by a certain amount which is proportional to the 
injected charge in the illuminated pixel. Figure A.4 shows that amount of EXT have an unambiguous dependence upon the distance between the cross-talked pixel and the illuminated pixel This structure strongly supports the hypothesis that the EXT is caused by the PMT, as Figure A.6 already show no sizable effect related to the readout electronics.

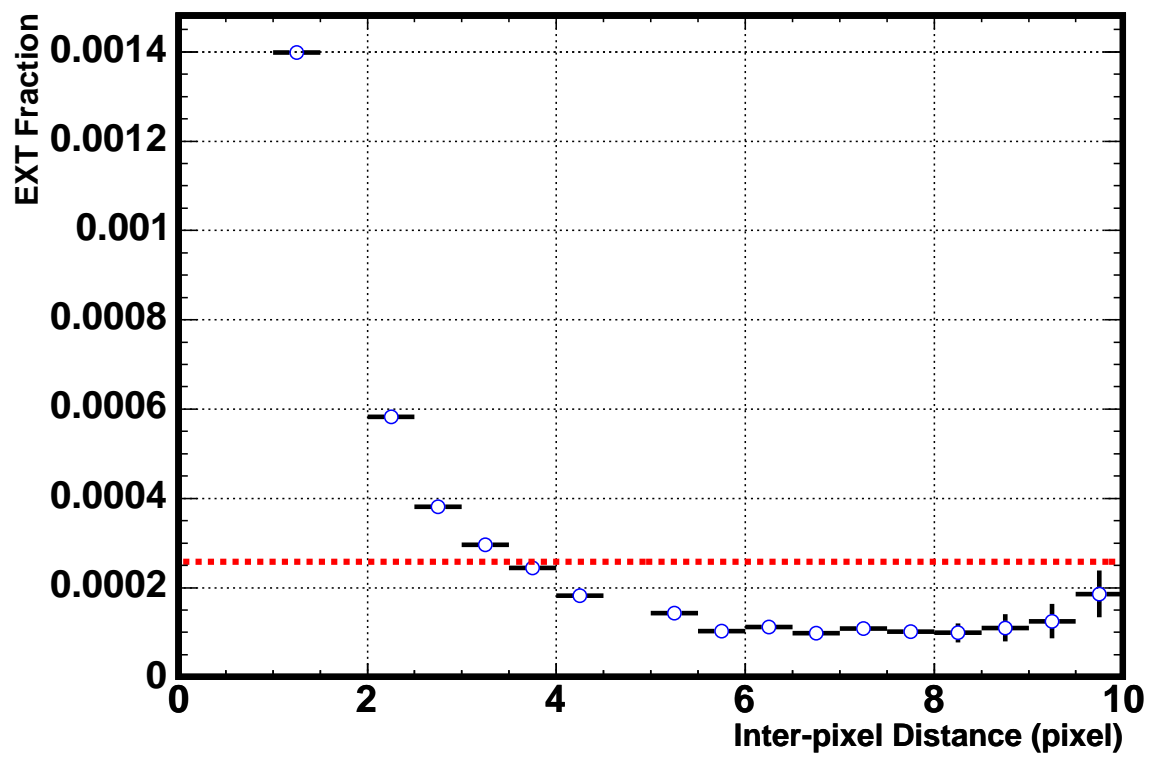

Figure A.4: EXT versus Inter-Pixel Distance. Up to approximately 0.1PEs can be drawn by EXT.

EXT is also characterised by happening very often while drawing little charge, i.e. always less than $1 \mathrm{PE}$ supporting the hypothesis that its mechanism is somewhere along the dynode chain. A hypothetical mechanism suggested is that this crosstalk is caused by a constant small fraction of the leakage of cascading electrons from one across pixels. The correlation observed in Figure A.2 is consistent with such a possibility. Further studies on the nature of EXT for different light levels can be found in [124].

An increase of the RMS of the EXT distribution was also appreciated in the closest pixels to the injected pixel as is shown in Figure A.5. The cause for this broadening can be generated by the correlation shown in Figure A.2. However, part of this broadening can also be affected by the leakage of the 1PE into the EXT distribution. In other words, by the intrinsic inaccuracy of the technique based on the 2D-cut applied for the crosstalk component separation. The 2D-cut was performed at $2.5 \sigma$ (98.8\% probability) of each pedestal width, hence $\sim 45$ ADC. In 


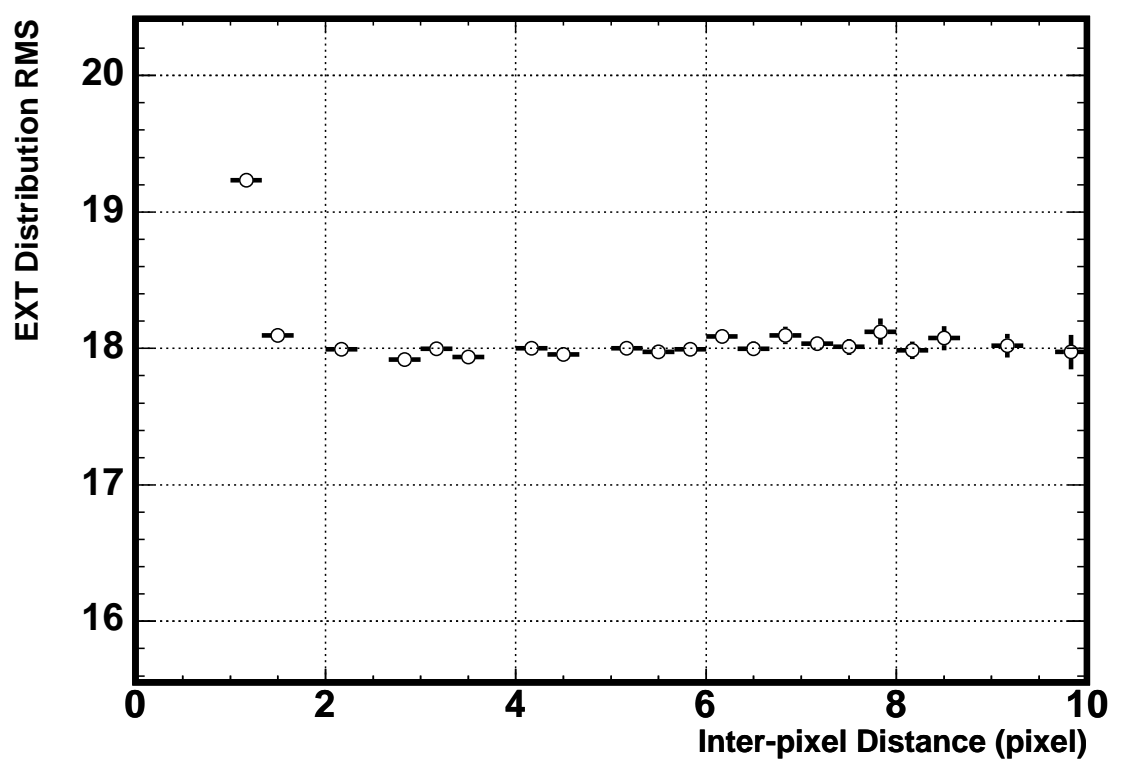

Figure A.5: Broadening of the EXT Distribution. The EXT distribution tends to get wider in the closest pixels to the injected pixel.

terms of the width of the $1 \mathrm{PE}$ peak, $\sim 45 \mathrm{ADC}$ corresponds to $\sim 1.5 \sigma$ on average. Therefore there is on average a $\sim 11 \%$ probability that $1 \mathrm{PE}$ entries leak below the cut, which would increase the width too.

\section{A.4.1 Readout Electronics Crosstalk Studies}

In order to study the PMT crosstalk, parallel studies have to be performed about the possible contribution of the readout electronics crosstalk, if any. The results of those investigations are summarised in Figure A.6.

The small correlation between the EXT charge and the channel distance in electronics space is believed to be dominated by the coincidence between closest channels in the electronics and neighbouring pixels in the PMT. This correlation is far less significant than that found in PMT-space. Therefore, although we have not proved the absence of electronics crosstalk from the measurements, we are demonstrating that the observation are dominated by the PMT crosstalk contribution.

\section{A.5 Characterisation of PMT Optical Crosstalk}

This component causes the 1PE peak in the charge distribution of cross-talked pixels. Hence its mechanism has to be pre-first-dynode. In order to characterise 


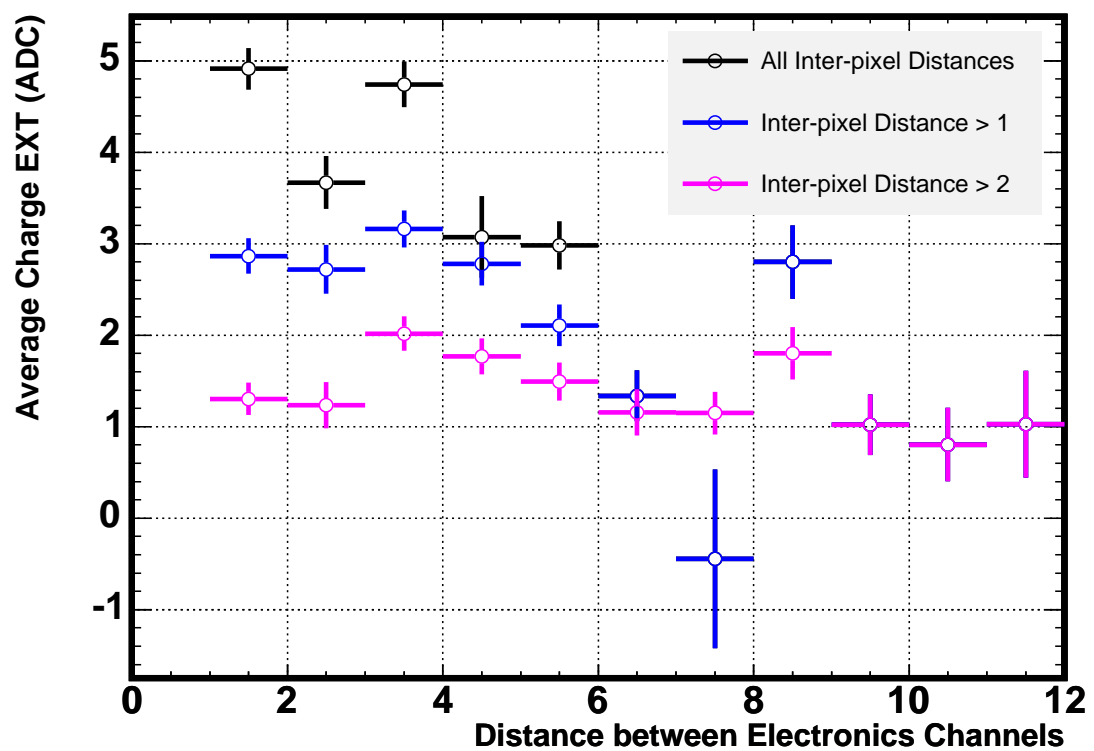

Figure A.6: Readout Electronics Crosstalk.

OXT, the contribution of EXT should subtracted.

We tested whether a Poissonian distribution (see Equation A.2) would accurately describe the average number of PEs drawn by OXT, indicated by $\lambda$.

$$
P(\lambda, n)=\frac{\lambda^{n} \exp -\lambda}{n !}
$$

The average number of PEs injected (or $\lambda$ ) due to OXT can be estimated in two different ways.

- Use the mean charge in OXT (in PE units) as shown in Equation A.3 for which the gain of the cross-talked pixel (Gain) needs to be known.

$$
\lambda=\frac{<Q_{O X T}>-<Q_{E X T}>}{\text { Gain }} \times \frac{N_{O X T}}{N_{\text {total }}}
$$

Where $N_{O X T}$ and $\left\langle Q_{O X T}>\right.$ are the number of entries and average charge of the OXT peak, respectively. $N_{\text {total }}$ is the total number of injections, i.e. 10,000 flashes.

- An alternative approach assumes that the probability of OXT is described by a Poissonian distribution. Hence $\lambda$ is obtained from Equation A.4. 


$$
\lambda=-\ln P(n=0)=-\ln \frac{N_{E X T}}{N_{\text {total }}}
$$

Where $N_{E X T}$ is the number of injections there was no $1 \mathrm{PE}$ occurrences, i.e. number of entries in EXT.

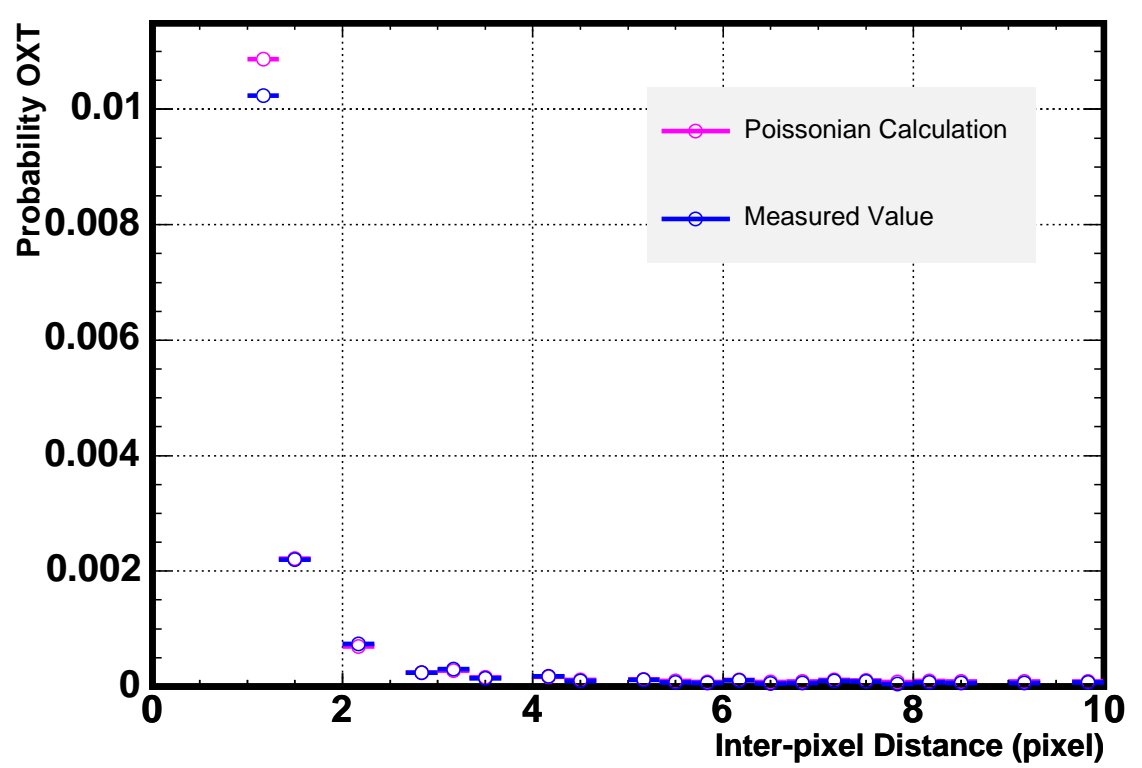

Figure A.7: Characterisation Model for OXT. Two models are used to measure the probability of OXT as a function of the distance between the cross-talked and the injected pixel. The blue curve shows the Method 1 in which OXT probability is calculated from the mean charge in the OXT distribution. Whereas in red curve shows the Method 2 uses a Poissonian distribution to estimate the average number of PEs caused by OXT. There is general agreement in the pattern between the two methods, however $10 \%$ disagreement remains at the closest non-diagonal pixels from the estimation of the average number of PEs due to OXT.

The OXT probability is the ratio of $\lambda$ to the total number of PE measured at the anode of the illuminated pixel. Figure A.7 shows the strong dependence between the OXT probability and the distance between the illuminated and crosstalked pixels. The OXT probability has been computed in two ways suggested above and compared in the figure. The methods describe the dependence upon the distance between the illuminated and cross-talked pixels similarly. General good agreement is also found between the two methods, which suggests that the Poissonian description of the phenomena would be good enough approximation. There is a non-statistical $\sim 10 \%$ discrepancy for the closest neighboring pixels 
(distance equals to 1). The cause for such a difference was not well understood. It may well be related to inaccuracies of the 2D cut. Having had Monte-Carlo generated crosstalk distribution would have provided a handle on the actual nature of the disagreement.

If the OXT was generated by genuine $1 \mathrm{PE}$ peak occurrence, the average mean of the OXT distribution should reflect the gain of the cross-talked pixel as indicated in Equation A.5. This fact provides an interesting test to the nature of OXT.

$$
\text { Gain }_{\text {Crosstalk }}=\frac{<Q_{\text {total }}>-<Q_{E X T}>}{\lambda}
$$

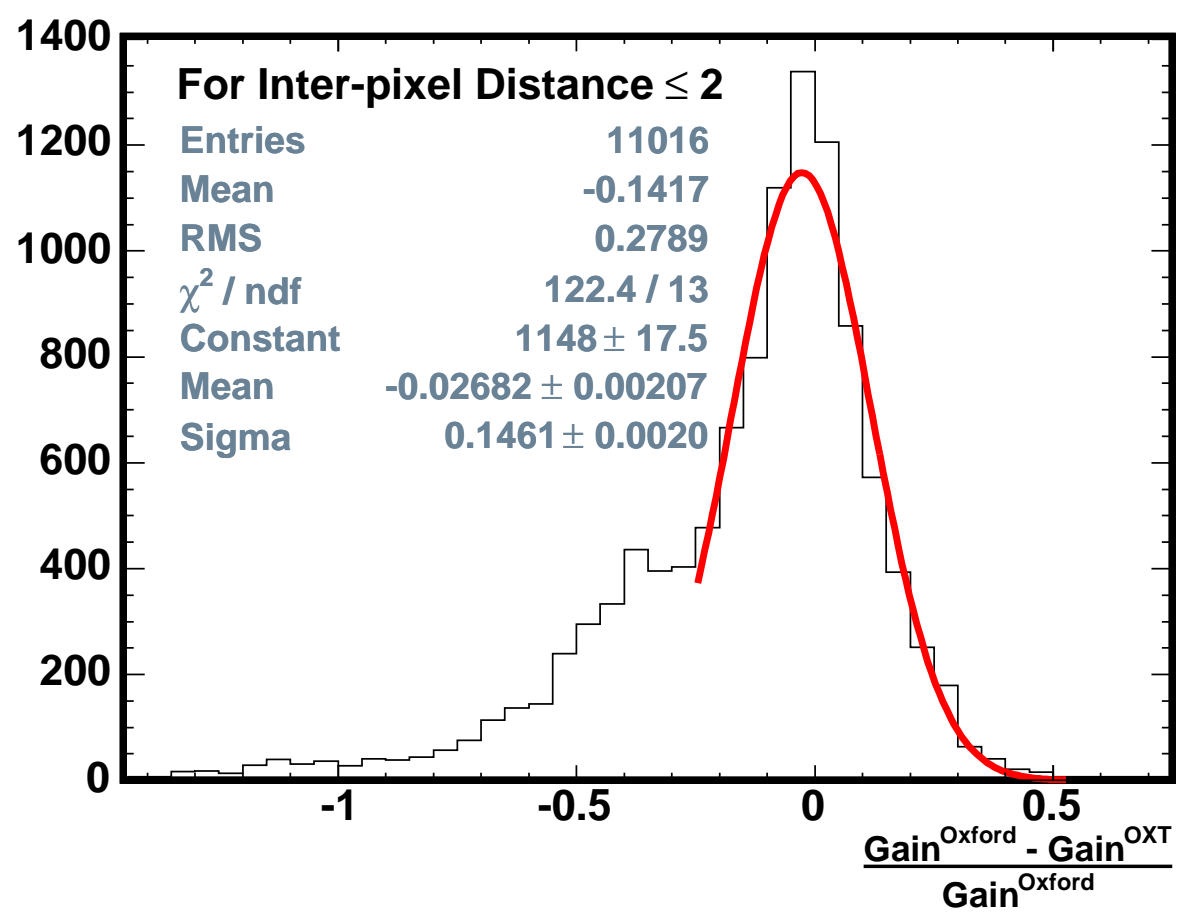

Figure A.8: Evidence for Pre-First-Dynode Nature of OXT. The fact that the OXT distribution is dominated by the occurrence of $\mathrm{PE}$ peaks with a very low mean allows sensitivity for a gain estimate for the cross-talked pixels. The comparing the crosstalk gain estimate with dedicated measurements taken at Oxford test stand reveal good agreement between the two methods.

Figure A.8 shows the difference between the gain estimated using the crosstalk distributions and the gain measured at the Oxford test stand in dedicated runs. General good agreement between both measurements is shown, proving that OXT is dominated by the 1PE peak, proving its pre-first-dynode nature. There is a $2 \%$ offset in the difference (as shown by the Gaussian fit), whose origin is not well understood and was not investigated. 
Two mechanisms are consistent with a pre-first-dynode caused crosstalk. However, the results do not offer discrimination between there alternatives.

- Mechanism 1: the 1PE get collected by a neighbouring pixel after photoconversion.

- Mechanism 2: the photon scatters in the glass prior to the photo-cathode causing eventually the PE somewhere else in another pixels collection region.
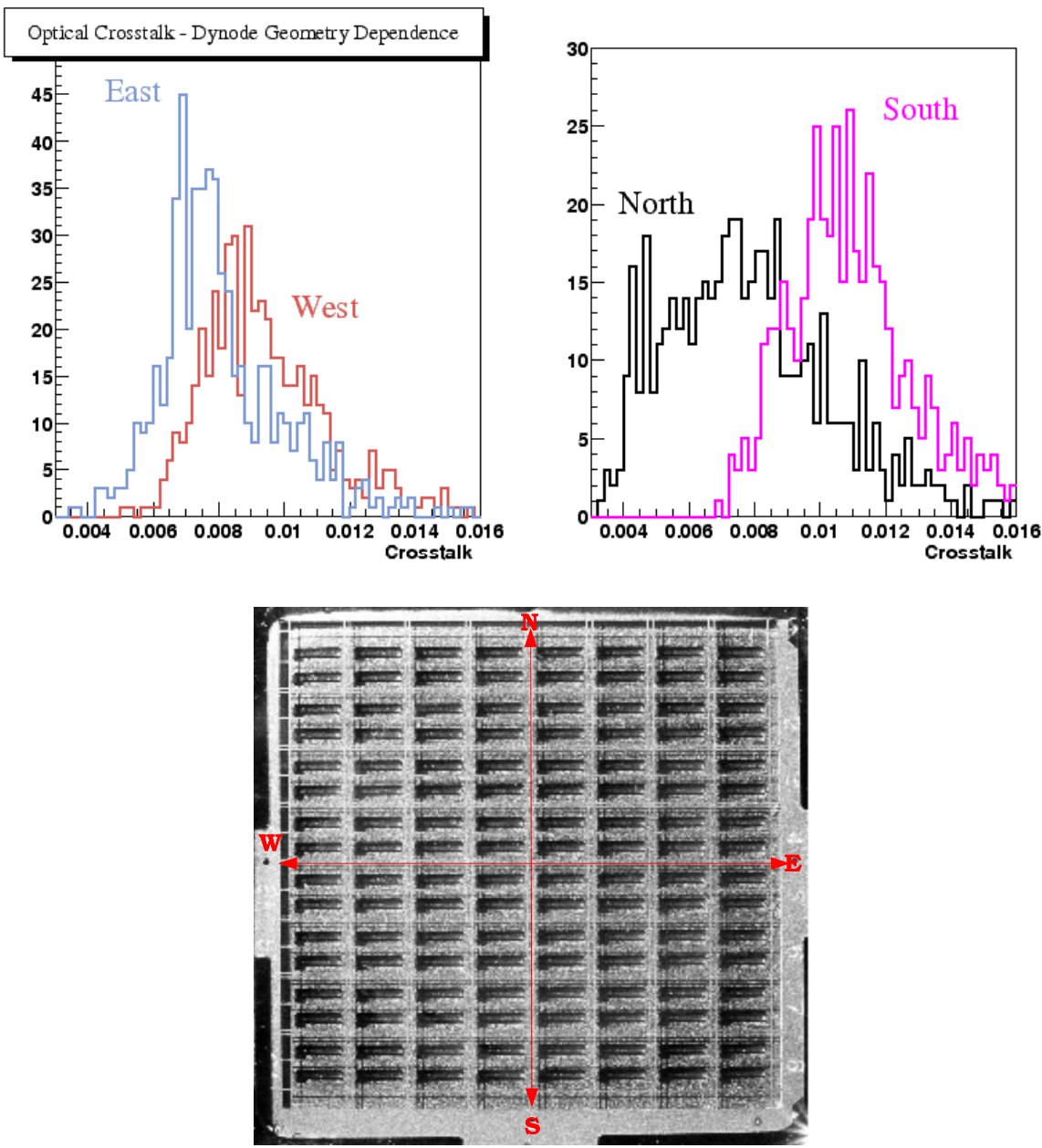

Figure A.9: Evidence for OXT Origin Pattern. The cause of the asymmetry is not well understood. A systematic misalignment as well as the reflection by OXT of the structure of the first dynode in the M64 could be possible explanations. This plot shows the geometrical structure of the first dynodes of the M64. Collection efficiency may reflect the asymmetries of such an structure. We considered using this handle to discriminate between different mechanisms of OXT. However other potential effects like misalignment could fake the pattern expected. (Courtesy of the Oxford University Photographic Unit) 
OXT shows also some asymmetries in the amount of cross-talked exhibited in the closest non-diagonal cross-talked pixels, as shown by Figure A.9. This may be consistent with systematic misalignment of the "cookie" with respect to the pixel of the $\mathrm{PMT}^{1}$ or that OXT reflects the geometrical structure of the first dynodes of the PMT, which favours mechanism 2. No other way for the discrimination of the two mechanisms was found.

\section{A.6 Summary of Analysis}

Crosstalk in the M64 PMTs has been identified and measured. We found that the crosstalk of the M64s is dominated by two components. A 2D-cut was developed to separate both contributions since their origins were thought to be different.

The first component called Electrical Crosstalk causes the the whole distribution to shift toward positive charge values. It can be measured from the position of the shifted pedestal. Its origin must be post-first-dynode. Our hypothesis for the mechanism behind is the leakage of the secondary electrons from the injected to the cross-talked pixels as electrons travel toward the anodes. This component of crosstalk will generally not be observed in the MINOS detectors due to the sparsification of the pedestal peak.

The second component was called Optical Crosstalk. It is characterised by the occurrence of PE peaks in the charge spectrum of the cross-talked pixels. The amount of cross-talked charge is high enough to be above sparsification and therefore it will be seen in MINOS. Its probability seems to be well characterised by a Poissonian distributions to a $10 \%$ accuracy.

\footnotetext{
${ }^{1}$ Note that this effect would be systematic for all the PMTs tested.
} 


\section{Near/Far Single Noise Measurements}

"Singles" are 1PE random noise found in the detector due to PMT dark-noise and WLSF spontaneous emission of light. The latter has been found to dominate the rate measured at both CalDet and the FD. The dark noise expected rate per pixel is about $\sim 100 \mathrm{~Hz}$. The dark noise rate of each PMT was measured in dedicated MINOS PMT test-stands before being accepted and installed in the MINOS detectors [124]. The rate of WLSF singles correlates with the amount of WLSF associated with each channel.

The reason for dedicating this section to singles-noise is because the ND was found to exhibit up to a factor of $4 \times$ larger rate of single-noise than the FD during beam running. The origin of this effect is still a mystery. This section, however, summarises the investigations carried out to understand the origin of the noise and the possible scenarios that have so far been ruled out.

\section{Optical-Noise during Beam Running}

In order to measure the singles-noise during beam running, a method was devised to sample the last 35 planes of the detector whenever a single electrons event was triggered. Electrons events are virtually confined to the front 20-25 planes. Beyond plane-25, only single-noise can be observed, as shown in Figure B.1. This figure, already illustrates the main finding of this analysis, the ND has a larger number of singles-noise hits than the FD. The plot shown was obtained with 15, 000 electrons at $1.8 \mathrm{GeV} / \mathrm{c}$. In Figure B.1 the entries are made charge weighted in PE. Charge 
weighting helps with finding large depositions of charge, such as a muon going through the detector in one of the events, which would leave a visible of trace of $4 P E$ per strip hit on average. Light leaks are in principle discarded, as they would cause a plane-wise pattern, that also should generally propagate to the other side of the plane, if the light leak was at the level of the planes.
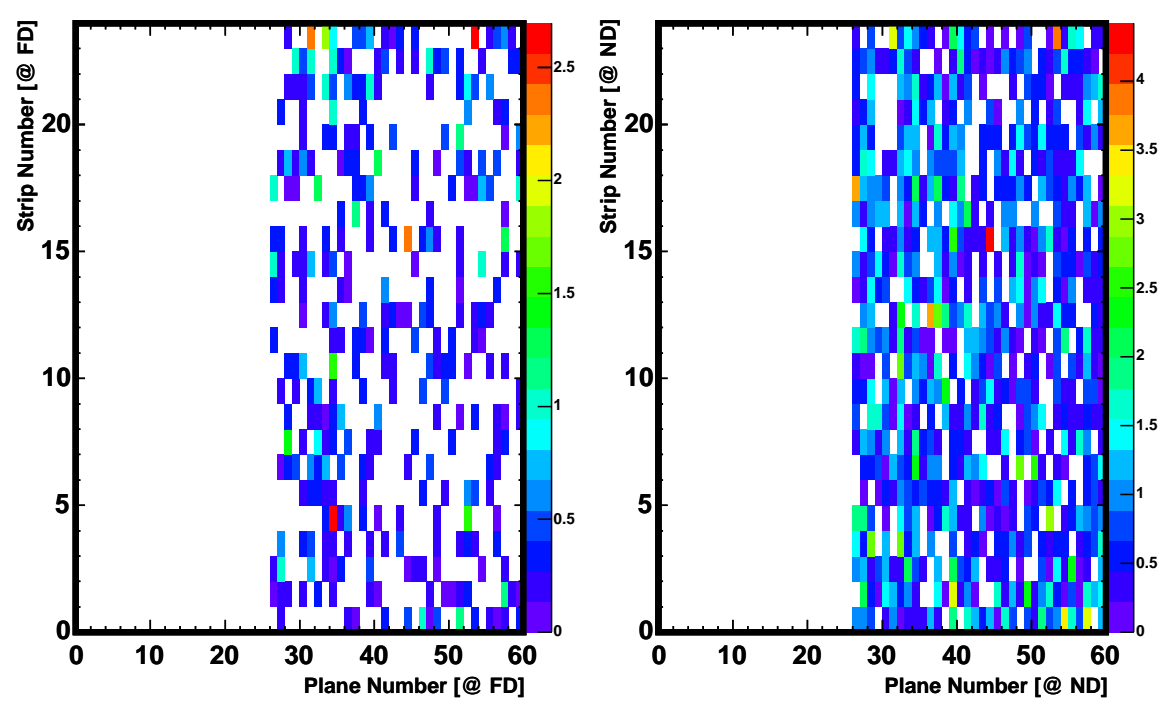

Figure B.1: Single-Noise Occupancy during Beam Running

This method provides an ideal setup for singles-noise measurements as the exposure to both readout systems is also well known by the definition of the time fiducial time window: 300ns per event. The time distribution for both readout systems is shown in Figure B.2 (top). The black distributions shows the very flat distribution of single-end hits in either readout system, while the red distributions correspond to the very few double-ended hits found within the sampled region/time. None of the distributions show any remnant structure of the beam spill/trigger, that would cause a peak at about zero time, when the beam trigger-tag was calibrated to be.

Hence, once we know the the time window ( $\Delta t=300 \mathrm{~ns})$, the number of electron events $\left(\mathcal{N}_{e}=15000\right)$ used and count the total number of singles-noise hits found in either detector $\left(N_{F D}=266\right.$ and $\left.N_{N D}=1264\right)$, then the rate per channel can be calculated from Equation B.2. 

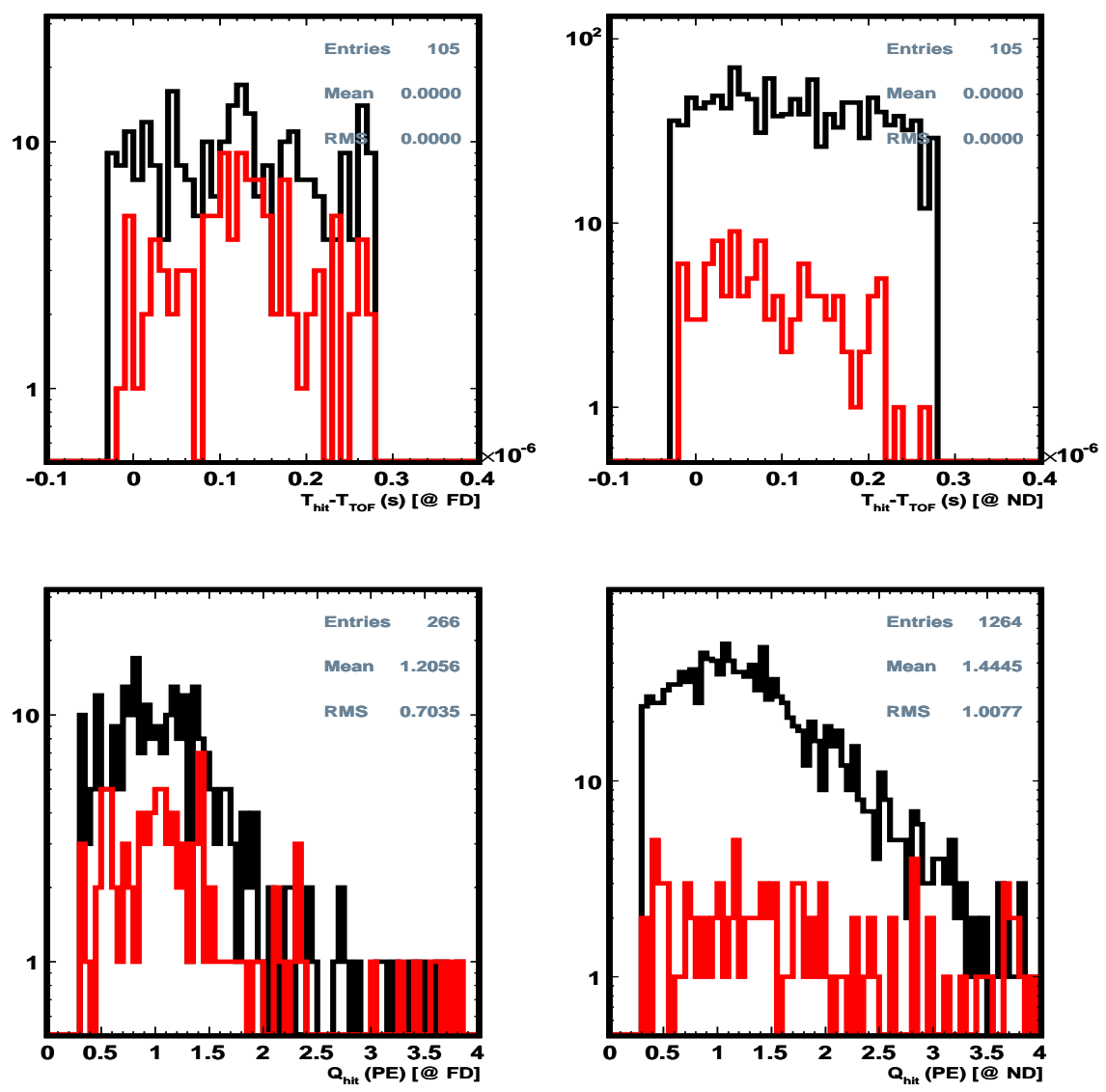

Figure B.2: Charge \& Time Distribution of Single

$$
\begin{aligned}
\mathcal{R}_{N D} & =\frac{N_{N D}}{35 \times \Delta t \times \mathcal{N}_{e}} \times 10^{-3} \mathrm{kHz} \\
\mathcal{R}_{F D} & =\frac{N_{F D}}{35 \times \Delta t \times \mathcal{N}_{e}} \times 10^{-3} \mathrm{kHz}
\end{aligned}
$$

Where the 35 corresponds to the number of planes sampled. The rates $(\mathcal{R})$ measured were $\sim 2.0 \mathrm{kHz}$ and $\sim 10 \mathrm{kHz}$ for the FD and ND respectively.

Figure B.2 (bottom) shows the charge distribution in PE for both single-ended and double-ended hits for both readout systems. All charge distributions are peaking roughly at about $1 \mathrm{PE}$ as expected since this is characteristic of the singles-noise. Note that there is no pedestal leakage, or any kind of low charge contribution/peak in neither of the distributions shown. Both charge distributions peak at about 1.2PE, although the means are different. 

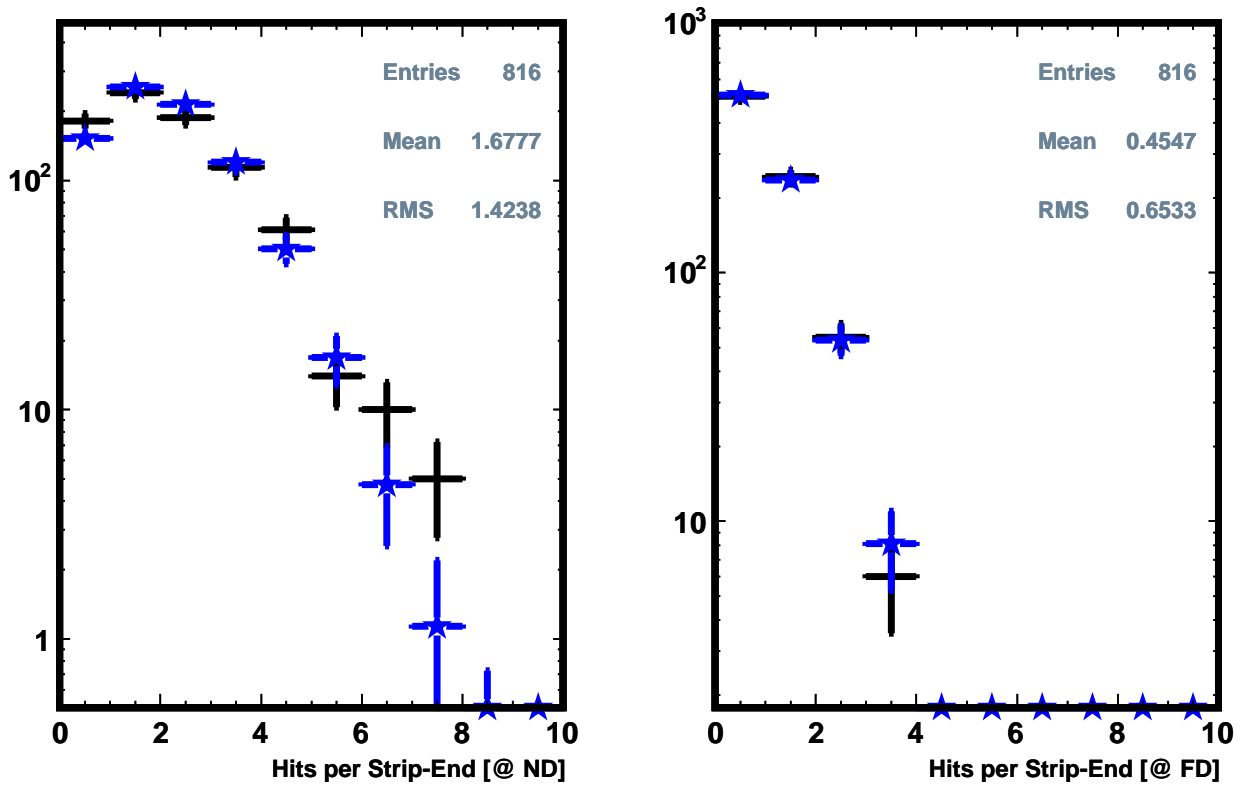

Figure B.3: Number of Hits per Strip-end

Figure B.3 shows the average number of hits per strip. If there was a "hot spot" pulling up the number of singles measured in the ND it should be shown, as well as in the hit-map shown in Figure B.1. No such a "hot spot" was found. However, this distribution can also be used to gain insight on the nature of the noise measured in both sides, i.e. whether the noise is random and homogeneously distributed across the sampled region. This can be done by testing the shape of the black histogram against a Poissonian probability distribution. The blue histograms correspond to a Poissonian distribution whose $\lambda_{\text {Poisson }}$ was the mean number of hits per strip-end measured. The agreement between the two curves suggests that the source of noise driving the ND and FD distribution is random and homogeneously distributed.

Another interesting test, not shown, is whether the mean number of hits per strip-end measured in the ND EVEN view and ODD view is different. If singles were caused by WLSF, we would expect that the EVEN view measures about a factor of $4 x$ less singles than the ODD view. In fact, the EVEN view measured $10 \%$ higher rate. Therefore, we can conclude that the additional rate of singles measured by the ND side is not generated by the WLSF; and the FD singles measurements are in full agreement with singles rate dominated by the WLSF, previously measured at CalDet during 2002.

Two further pieces of information were found. They are more intriguing than 

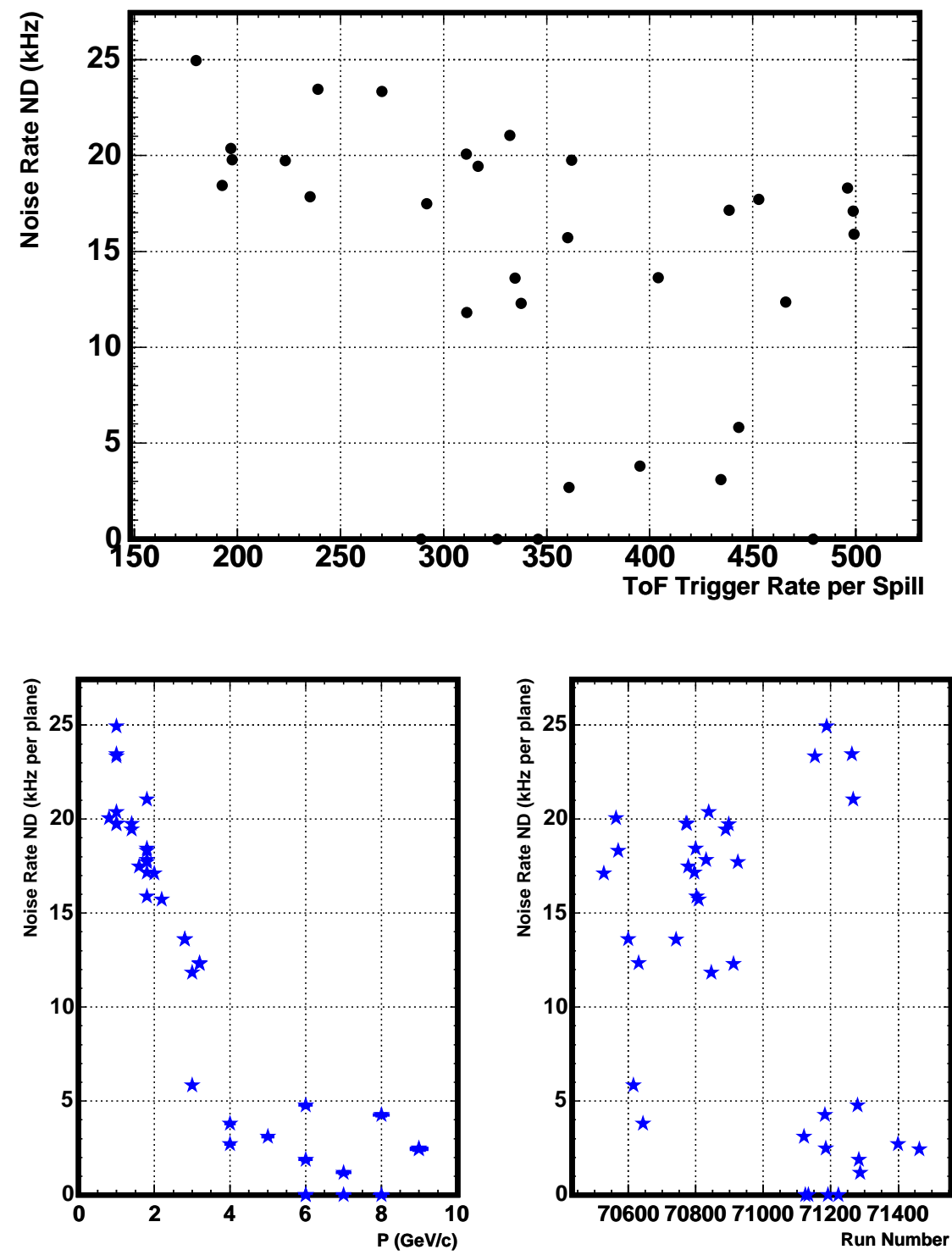

Figure B.4: Beam Single versus Beam Momentum 
elucidating though. Both are shown in Figure B.4. The top plot shows that the noise measured in the ND seems to correlate with the rate of triggers per spill. Whether this correlation is meaningful or not could not be investigated.

An even more unexpected correlation suggest (bottom plots) that the noise measured in the ND seems strongly anti-correlated with the momenta of the run. This leads to two possibilities. First, that some of the electron hits are leaking into the sample region as the electron range is logarithmically increasing with energy. However, this hypothesis would give the opposite trend: more noise hits at higher energies and the excess hit should be seen by both readout systems. Second, at higher energies, the amount of charge digitised by the FEE is larger. Therefore, maybe there was some degree of electronics crosstalk/ringing caused by an overwhelming amount of charge into the system. This however, should also correlate, not anti-correlate, with beam momenta. It is also interesting to investigate whether the low/high energy runs are clustered in time, between which something may have been changed in the electronics. This is shown not to be the case, as low and high energy runs interleave each other in time, as suggested by the run number monotonically increasing with time (bottom-left in Figure B.4).

So far, no mechanism consistent with the evidence has been found to be responsible for the higher noise exhibited by the ND with respect the FD. The absolute rate of this effect has nonetheless negligible consequences on the results shown so far.

\section{Optical-Noise during Cosmic Running}

With beam data, we have measured the noise rate of the ND readout systems to be up to $\sim 10 \mathrm{kHz}$ per plane. The FD rate is however, $\sim 2.0 \mathrm{kHz}$ per plane. The associate dead-time with such rates, if the detector was allowed to be randomly trigger, rather than triggered by the beam-trigger, would be have been disastrous. The detector is indeed allowed to be randomly triggered for cosmic muons data collection: Null Trigger runs. Therefore, one of the Null-Trigger runs used for computing the S2S muon calibration constants was used to investigate the rate of noise in the ND under such circumstances.

Figure B.5 (top) shows the selection criterion used to identified singles: use events whose number of strips hit is $<5$ strips. The $2.5 \mathrm{~s}$ worth of singles were used for the noise calculations. Figure B.7 shows the charge distribution (PE) and time distribution of the selected singles. Note that there the amount of FD singles, for the same exposures, suggest a larger singles rate in the FD than in ND during 


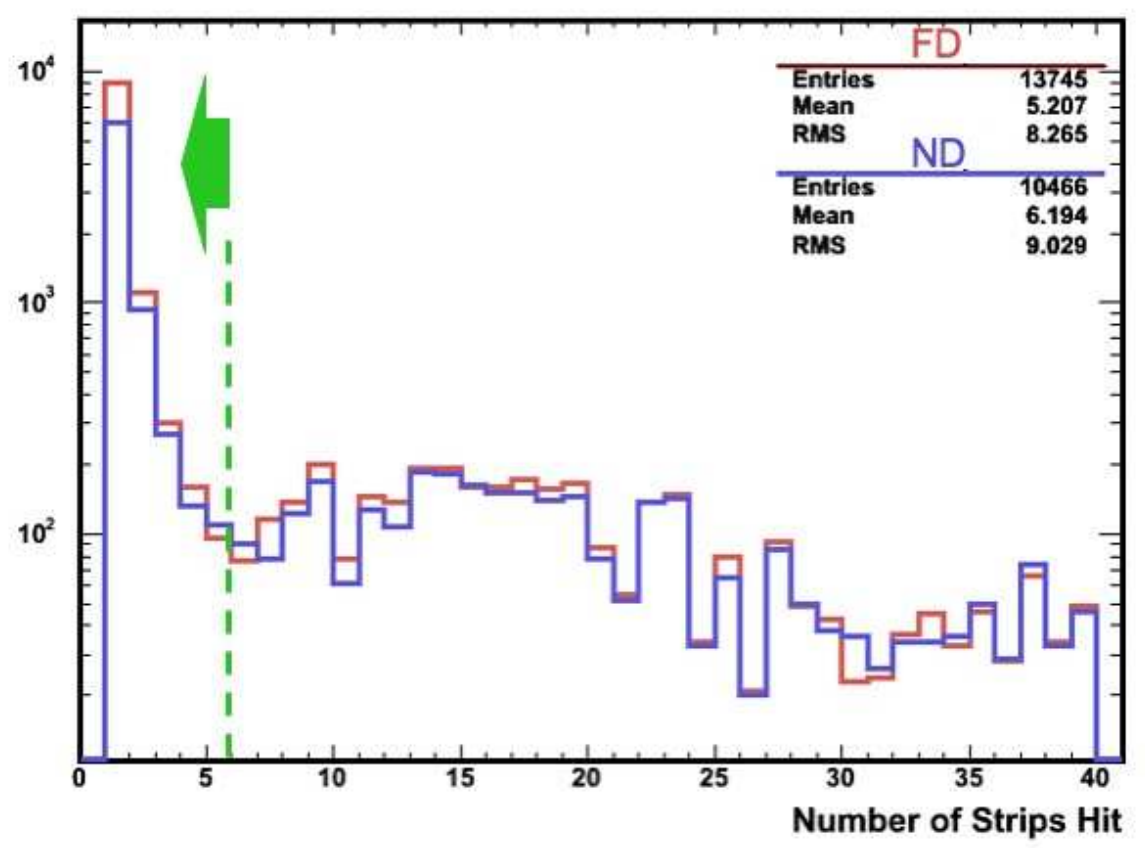

Figure B.5: Selection of Singles During Cosmic Runs

Null-Trigger runs. This is contrary to what was observed during beam data taking. The charge distributions show, in good agreement with observations during beam data taking. The rates measured were about $1.5 \mathrm{kHz}$ per plane for both readout systems for the cosmic run used.
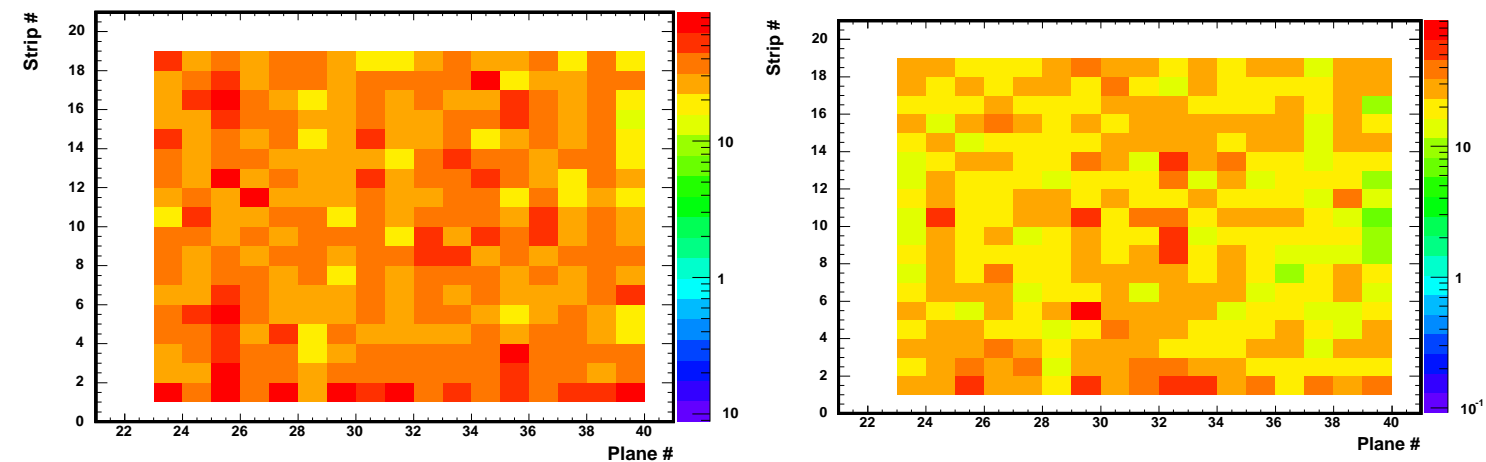

Figure B.6: Pattern of Single During Cosmic Runs

Figure B.6 shows the hit map for the ND and FD. Note the whole detector has not been shown. This is because a few hardware problems encountered would bias the results. Those problems were handled by the "sanity cuts" (previously described) during the beam data analysis previously shown. 

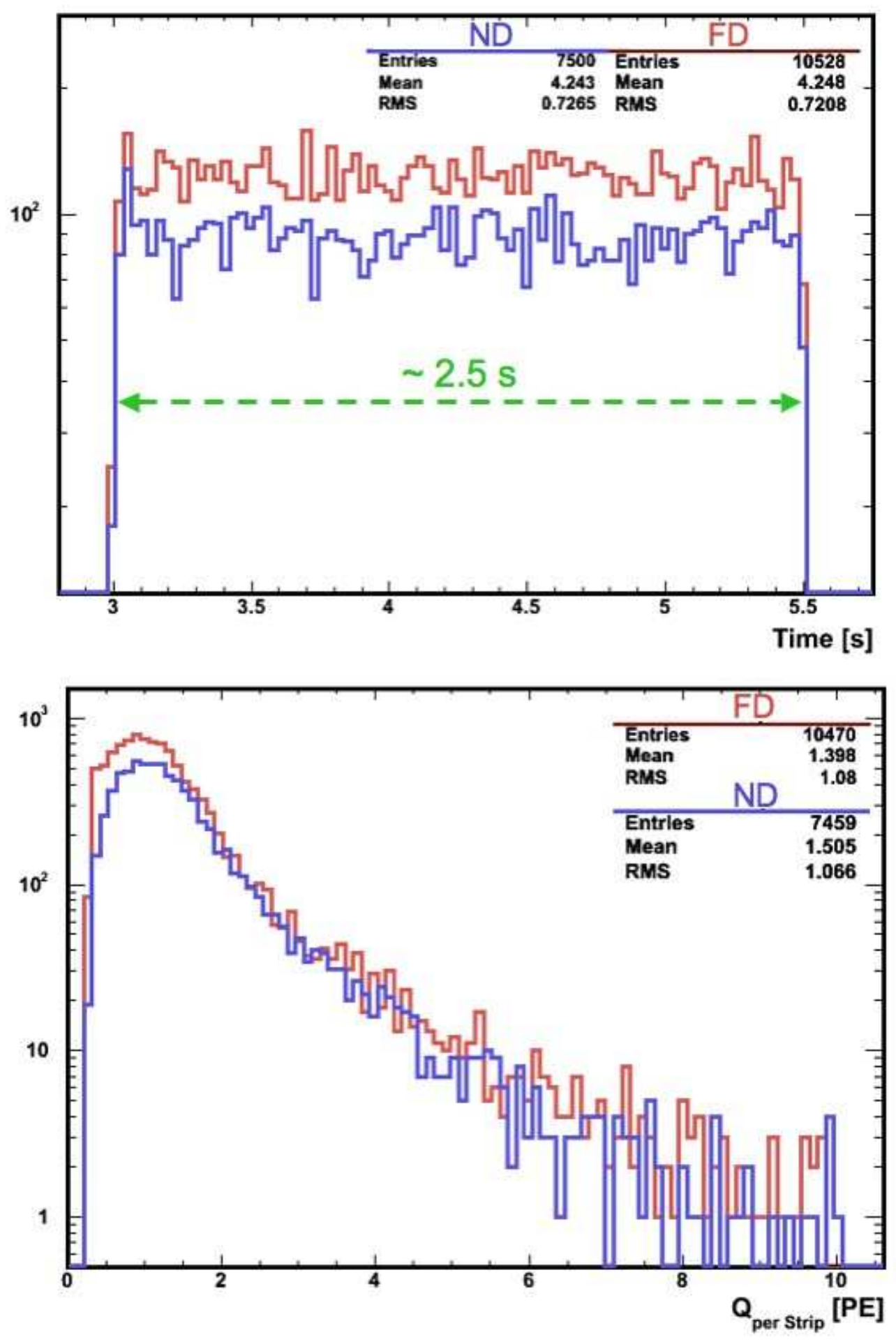

Figure B.7: Charge and Time Distribution of Singles 
Therefore, the results observed with Null-Trigger runs suggest that the noise measured during beam data run in the ND may be to do with the way the ND FEE is triggered. Beam data runs were taking by externally triggering the ND FEE, while Null-Trigger runs were taken by dynode triggering the detector. Therefore, there seems to be a hardware behaviour associated with the observation, although the signature is fully consistent with that of $1 \mathrm{PE}$ optical noise. The origin of this observation is still unknown at the time of writing of this thesis. 
ApPendix C

\section{Slicer Implementation}

This appendix is devoted to describe the algorithm behind the Slicer, which was developed to identify triggers with contribution of more than one particle.

The basic principle of the Slicer is to identify in time the number of energy depositions occurring for every beam-trigger and from that information infer whether the observations are consistent or not with the incidence of more than one particle. Triggers with more than one particle are not desired for analysis purposes, therefore, the Slicer assesses the rejection of those triggers.

The Slicer is confined to run within the fiducial-window with (plus extra 50ns on each end), as this is the effective acceptance time window for both readout systems. The Slicer, therefore, effectively relies on the time-fiducial cut to be an intrinsic part of the selection chain, which is indeed the case. The time distribution of energy deposition, shown in Figure C.1, is the seed (actual input) for the algorithm. The steps taken to assess whether each event has the contribution of more than one particle are:

1. The binning of the distributions have been chosen to represent the time resolution hit-to-hit of each readout system: 20ns for the ND side and 4ns for the FD side, as respectively shown in Figure C.1 (top) and (bottom).

2. The hits are energy weighted providing that they are at least $0.5 \mathrm{MIP}(\sim$ 2PE). The threshold was imposed to prevent the inclusion of 1PE-noise hits into the distributions.

3. The peak-finding algorithm (from the class TSpectrum in ROOT [72]) is used to find and count peaks in the time distribution - one time distribution per 

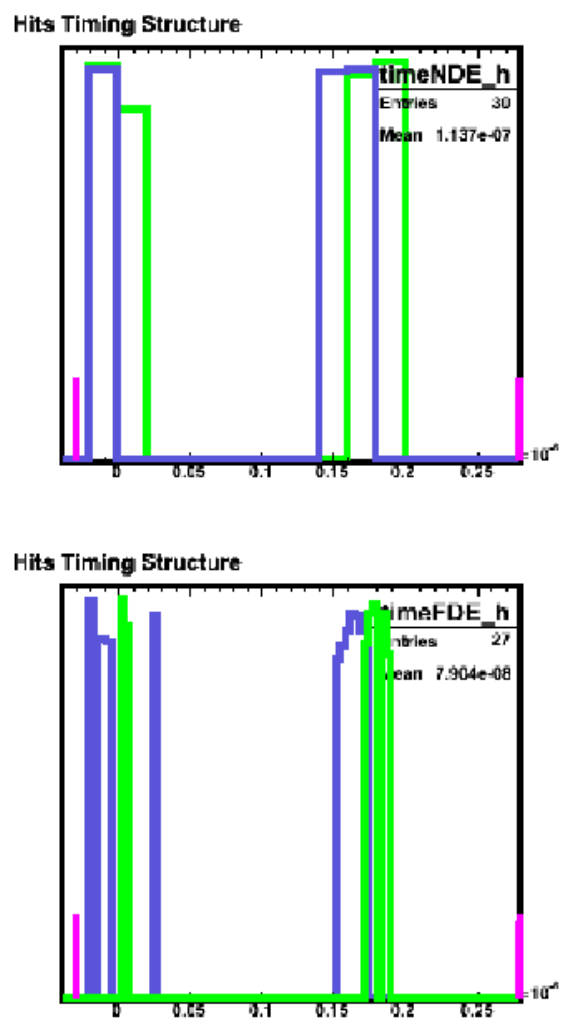

Figure C.1: Time Distribution of Energy Deposition.

view. The peak-finding algorithm also returned the location (in time) of each found peak.

4. The peaking-finding algorithm has an inbuilt relative threshold set to $10 \%$.

5. A final requirement was applied: only "resolvable" peaks will count. If any two peaks were found, they have to be resolvable. By resolvable is meant that the difference between the average time of the peaks found should be at least 15ns and 20ns (one bin) for them to be considered as distinct contributions of different particles. The aim of the resolution thresholds is to prevent the peakfinding algorithm from splitting energy deposition corresponding to the same particle. For example, it would take about $9 \mathrm{~ns}\left(\sim 3 \mathrm{~m} / \mathrm{c}=3 \times(1 \mathrm{~m} / 3 \mathrm{~ns})^{-1}\right.$ ) for a muon to go through the entire CalDet. The resolution criterion was applied to each view: ND-ODD, ND-EVEN, FD-ODD and FD-EVEN.

6. If any two "resolvable peaks" were found by either readout systems, providing any two views agree, the event becomes flagged as a multi-particle event. 


\section{The Swimmer Algorithm}

The Swimmer is a simple algorithm developed to trace (or follow) the path of tracklike events. Note that the Swimmer was not a tracker. The Swimmer did not assess whether the event "swum" was a track or not, but instead this was an assumption made, so generally pre-selected muon candidates were fed to the Swimmer. Once an event was given to the Swimmer, this was was used for two main purposes: event selection and calibration.

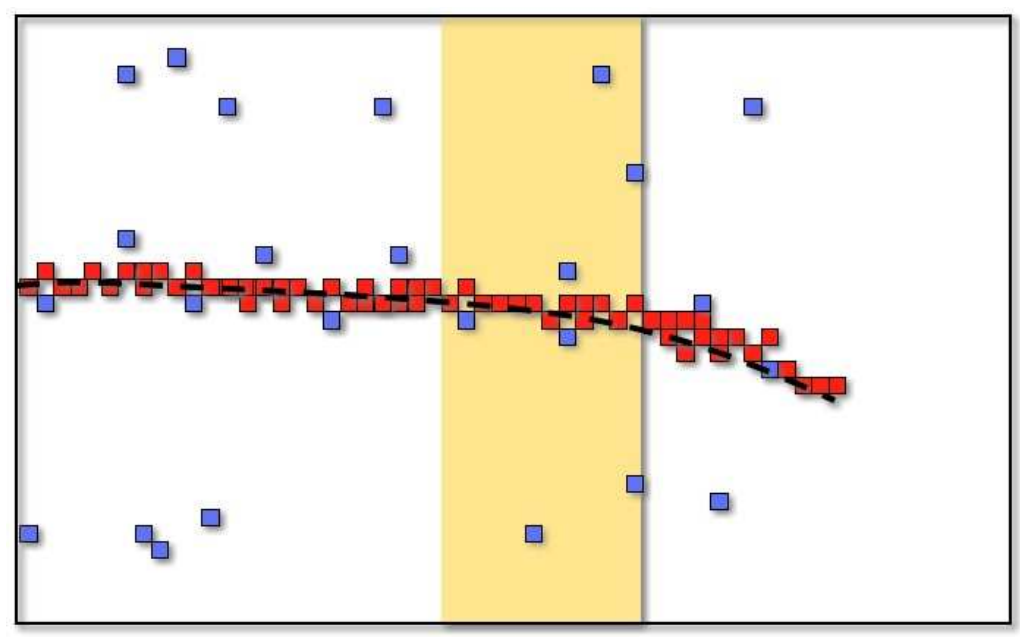

Figure D.1: Swimmer Diagram

The contribution of the Swimmer to the event selection was based on its excellent capability to find the end of the track accurately. Measuring the end of track accurately is identical to measuring the range of a track-like event. As explained 
in Section 6.1.4, this was the basis of the discrimination between muons and pions. Therefore the Swimmer was critical to yield a pure muon selection.

The Swimmer was also critical to distinguish tracked/untracked hits, respectively depicted in red/blue in Figure D.1. This capability was particularly useful to evaluate the quality of the $\mathrm{S} 2 \mathrm{~S}$ calibration, as this calibration is computed with the responses of tracked hits of cosmic muons. In addition, the charge ratio of untracked to tracked hits provided a measurement of crosstalk of both PMTs as explained in Section 7.8.2.

\section{D.1 Swimmer Algorithm Criteria}

The Swimmer algorithm was based on the simplified case that the events at CalDet were known to come from the beam, i.e. they would hit the centre (about strip 12) and they hit strips as the plane number increases. As muons go through the detector, they lose energy through ionisation, as characterised by the $d E / d x$ curves from Bethe-Bloch formula [16]. Muons exhibit random scattering as they go through the detector which becomes more dramatic as the energy of the muon decreases. This effect can be very dramatic for stopping muons.

The known the expected behaviour of muons was built into the Swimmer to allow simplicity of the algorithm while granting high accuracy. The hit of a muons are, therefore, "swum" by the Swimmer in all four view simultaneously and orthogonally within one iteration by following the sequences of steps:

1. First, pre-select muons by a preliminary somewhat loose range cut. At this point the range was coarsely calculated by computing the difference between the first and last planes hit, for which the energy deposition was $>1.5 \mathrm{PE}$ while allowing a gap of up to 2 planes per view.

2. In order to account for the fact that events are deposit energy along the centre of the detector from the front to the back of the detector, the hits of every pre-selected muon candidate are sorted as follows:

- First, hits whose plane $\in[0,20]$ are sorted by their relative position with respect to the centre of the detector weighted by $1 / \mathrm{E}$. Therefore, hits whose $\mid$ Strip-Number -12|/E per plane is smallest will be presented during the hit iteration, therefore, obliging the Swimmer to stir towards central hits whose energy deposition is largest. 


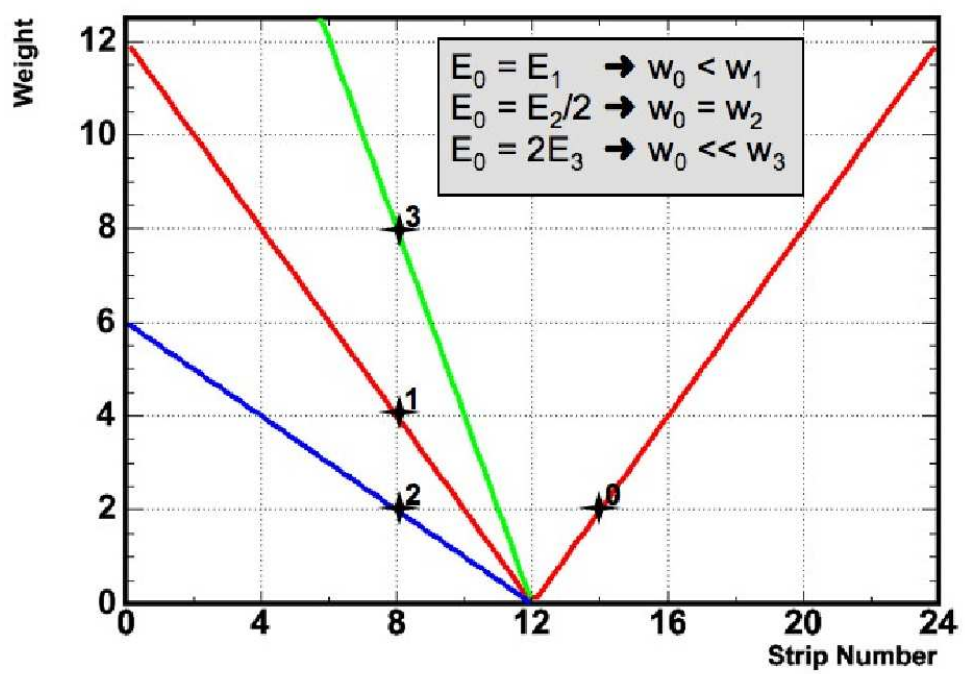

Figure D.2: Swimmer Weight Diagram

A diagram summarising the criteria is illustrated in Figure D.2. Note that the sorting is confined to the first 20 planes, since random scattering causes the track to diverge from the centre of the detector.

- Second, hits are sorted in terms of the plane number, so that the Swimmer is given first all the hits in plane-1 (as plane-0 was ignored) and then in plane- 2 and so on.

Once the hits have been sorted, the Swimmer iterates over the hits so that hits belonging to the track are recognised by "remembering" the information of the previous plane.

3. The Swimmer is seeded to start with the hit whose charge is largest and closest to the centre of the plane for each view. The Swimmer moves to the next plane $(i-t h$ plane) and uses the following criteria to find out the corresponding tracked hit(s):

- The Swimmer first tries to to continue in a straight line (Figure D.3) by checking whether there is a hit in the $i-t h$ plane with the same strip number as the previous plane $(i-t h-1$ plane). Note that the tracked hit in the previous plane is colour-coded in red, while the hypothetical new tracked hit is colour coded in yellow. This colour coding will remain throughout the rest of this section. 


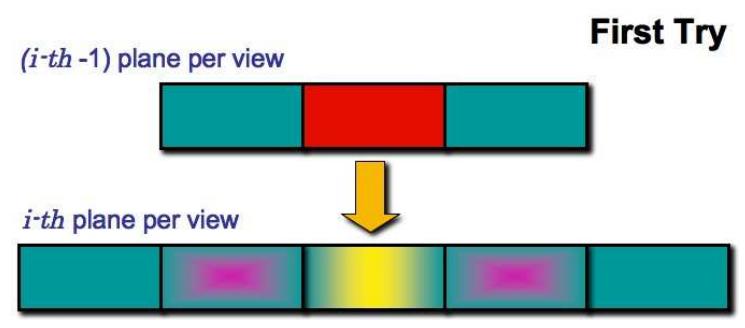

Figure D.3: Swimmer Step-1

Whenever the Swimmer finds the tracked hit, it allows at most one adjacent hit to be associated with the track, coloured in magenta.

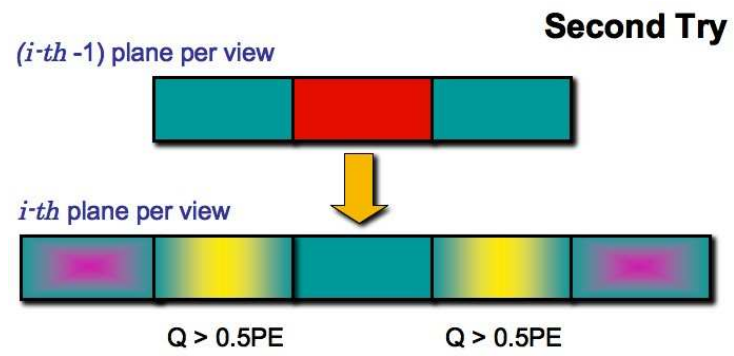

Figure D.4: Swimmer Step-2

- If the Swimmer failed to find the tracked hit, then it tries to deviate by one strip (Figure D.4) which at least must have 0.5PE worth of charge.

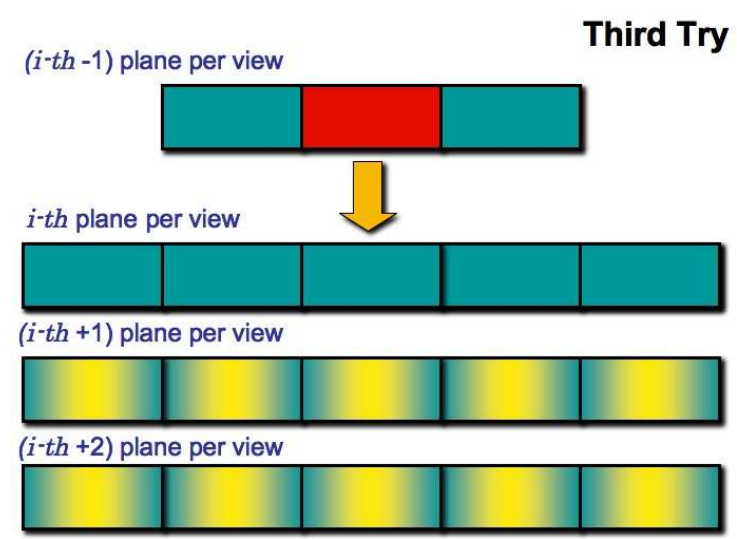

Figure D.5: Swimmer Step-3

- If the Swimmer still did not find consistent tracked hit, the it assumes that the particle did not interact with the that $i-t h$ plane so it allows a gap of up to 2 planes to find the next tracked hit. In this occasion the 
next tracked hit is allowed to be have deviated by up to three strips but providing that the charge measured is at least $1.5 \mathrm{PE}$.

4. As soon as the Swimmer succeeds to find the new tracked hit for the plane in questions in moves to the nest plane until it cannot find any more hits consistent with being part of the tracked, following the above criteria. If no more tracked hits are found, the tracked is assume to finish and the track-end location is recorded. The end of track can happen either because the muon stopped in the detector $($ momenta $<2.0 \mathrm{GeV} / \mathrm{c}$ ) or because the muon escaped either because it was a high energy muon or because it random-scattered so much that it escaped between planes.

A few examples of muons selected and "tracked" by the Swimmer are shown in Figure D.6. The first muons has been shown to be tracked by the Swimmer as well as one can desire. However, the Swimmer is not infallible, but sometimes it can go wrong, like in the second muon shown, whereby the Swimmer got fooled and although it recovers the track, it misses a few hit belonging to the track. Note, however, that despite the small mistake, the Swimmer is able to tell the end of the track very accurately.

200 events were eye-inspected finding that the Swimmer was "fooled" dramatically in one view, causing that one view be in disagreement with the others. Generally, such an occurrence ( $<5 \%$ of the times) was easy to spot. Those muons badly "swum" were not used for any high precision calculations, such as the assessment of the quality of the $\mathrm{S} 2 \mathrm{~S}$ calibration. In about $<1 \%$ of the times the Swimmer measured the end of the track wrongly by, at most one, plane. 
Muon with a perfect tracking:
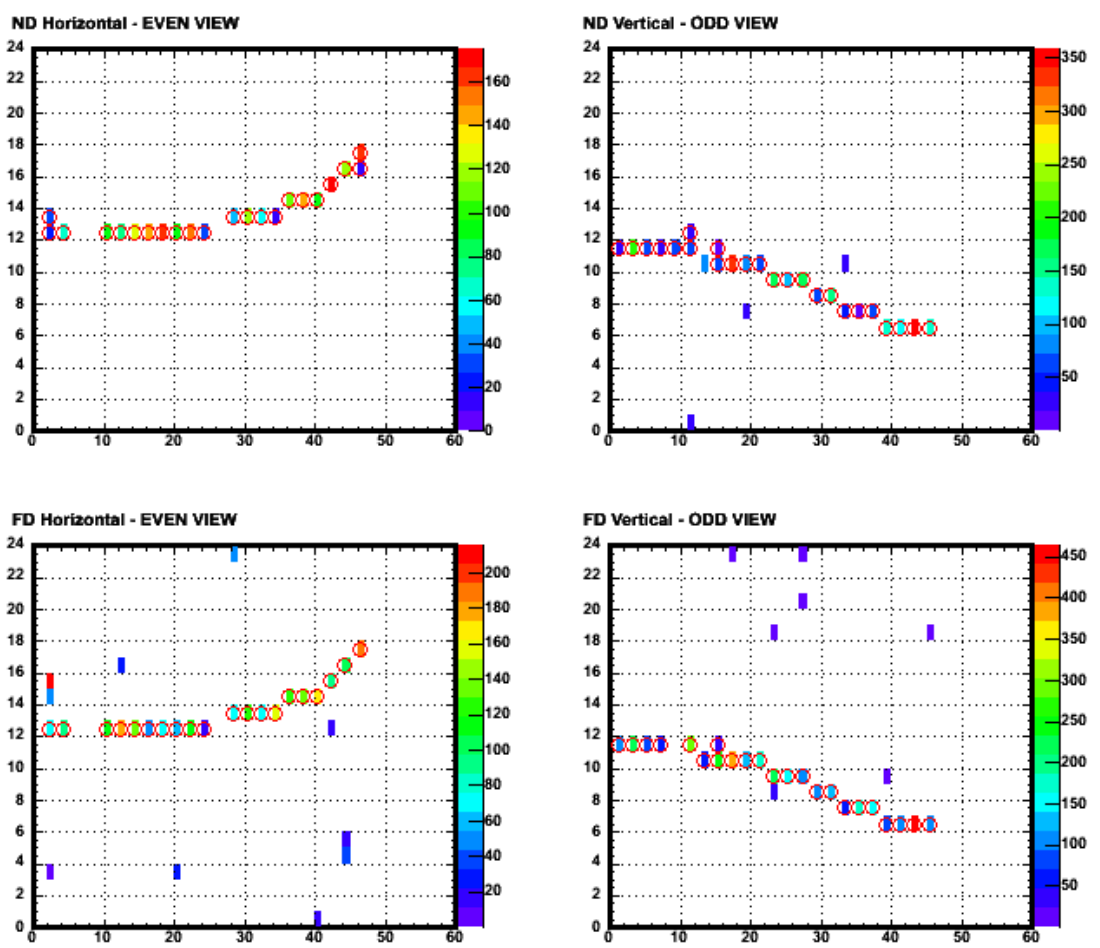

Muon with a small tracking problem:
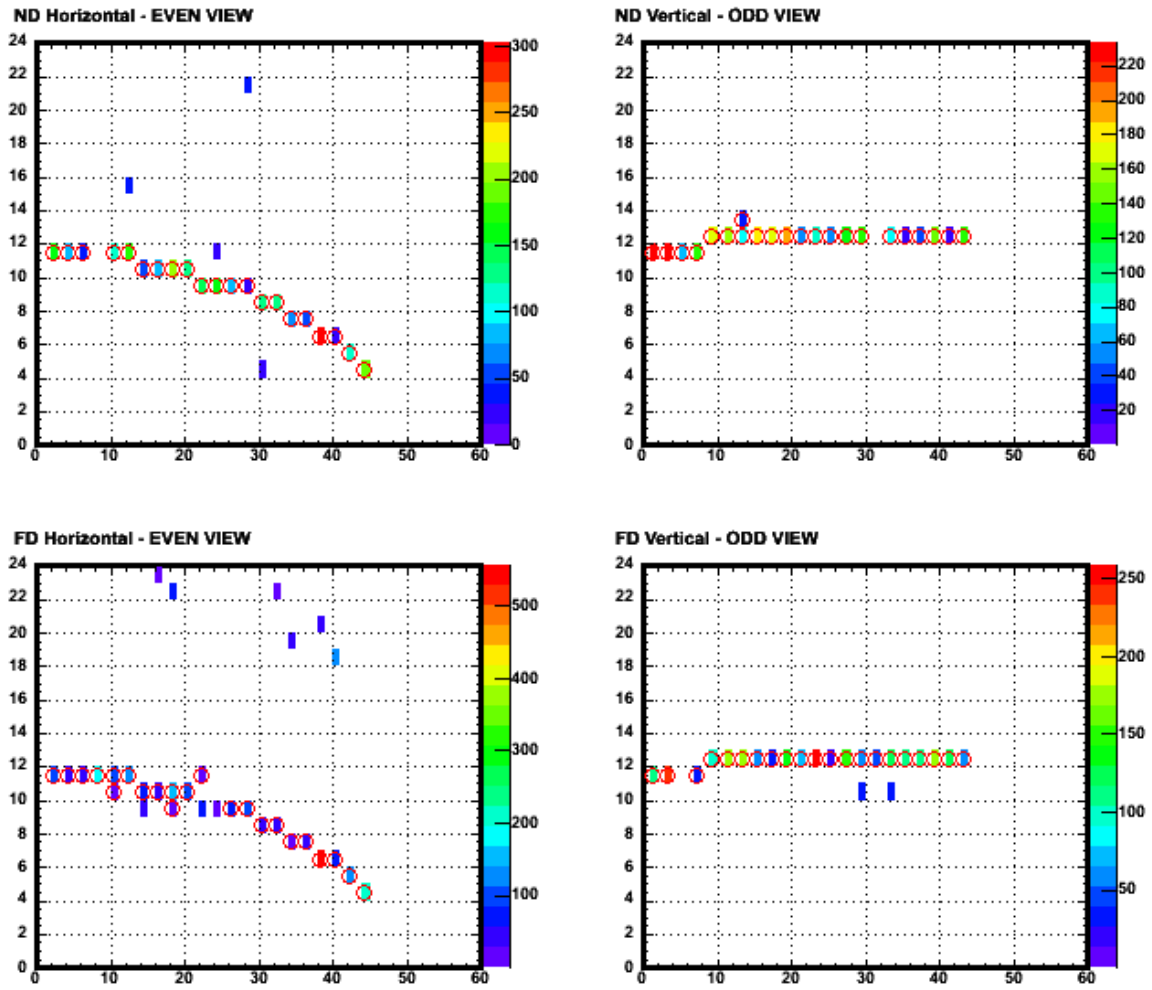

Figure D.6: Swimmer Example Each example shows four event displays corresponding to each view tracked by the Swimmer. Each view is labelled correspondingly. Tracked hits are circled, while untracked hits are not. Hits are colour coded in terms their energy as measured in SIGCOR. 


\section{Analysis Software Developed for MINOS}

\section{E.1 "SexyPedestal"}

SexyPedestal is a package made up two main pieces of software: "SexyPedReco" and "SexyPedAna".

SexyPedReco is in charge of the "Reco" stage, meaning the acquisition of the data from the offline MINOS data streams. The main function of SexyPedReco is to output a ROOT TTree with all the information from the pedestals (from a FD pedestal run) that is needed to perform analysis: charge, channel info, plane number, etc.

SexyPedAna is the pedestal analysis software, which uses an an input the TTree output by SexyPedReco. SexyPedAna is actually a pedestal analysis framework, while the actual calculations are performed by "SexyPedestal", one per channel. So, essentially SexyPedAna directs the operations which each SexyPedestal can do, such as:

- Compute its mean and RMS.

- Calculate its mean and RMS using two methods of truncation[105].

- Compute the sparsification threshold based on the truncated width of the pedestal. 
- Compares the new pedestal calculations (mean and width) with previous calculations.

- Compares the new sparsification thresholds with respect to current sparsification tables used online.

This package was documented in [105], where far more information is provided.

\section{E.2 "PedStability"}

"PedStability" is a package created for pedestal analysis that preceded SexyPedestal.

Function: Performs the pedestal stability analysis as well as similar calculations later re-implemented in SexyPedestal. SexyPedestal should be used by default, unless pedestal stability is desired. This package was coded to perform the analysis summarised in Section 5.3.1.

Input: Uses the TTrees provided by the "RawDigitNt" package available at Soudan. Output: Returns a postscript file with the all the plots selected for the analysis.

\section{E.3 "LookAtLUT"}

"LookAtLUT" is a package that used at CalDet to analysis the QIE-FEE calibration LUT dumped from the FEE by the VME computer. The package consist of two elements: the "TreeMyLUT" macro \& the "LookAtLUT" analysis framework.

\section{E.3.1 "TreeMyLUT"}

Function: Decodes the contents from the each ASCII file and stores them into ROOT TTrees, allowing subsequent analysis.

Input: ASCII files with the LUT dump from the VME computer.

Output: Returns a TTree with all the contents of each LUT, one per MASTER. 


\section{E.3.2 "LookAtLUT"}

LookAtLUT is an OO analysis framework.

Function: LookAtLUT uses "QieRangeResponse" objects to analyse the quality of the contents of each LUT. The calculations are performed by QieRangeResponse, while LookAtLUT essentially stirs the fate of the analysis. QieRangeResponse measures the gain of each single range-response ${ }^{1}$ and find whether there are outliers and some types of corruption.

Input: Uses the output TTree by the TreeMyLUT.

Output: Returns postscript files with the summaries of the analysis as well as ASCII files with some numerical summaries of the findings.

\section{E.4 "LIGainCurve"}

"LIGainCurve" is an independent piece of software added to LISummary [130] for Gain-Curve analysis.

Function: Study the non-linearity of the readout systems. It was developed for CalDet N/F running, there it is able to deal with both types of electronics, however, it needs some adaptation if to be used for the actual ND or FD data analysis, since some of the CalDet specific issues are hard-wire into the code. LIGainCurve uses the "LILinResp" objects to perform the analysis of the linearity of each single channel in terms of their residuals from the linear response of the PINs. Essentially there is one LILinResp per channel. The actual analysis happens at the level of each LILinResp.

This package can be used to measure the non-linearity of the M16 and M64. Results obtained from this software were reported in Section 5.4.3.

Input: Uses the TTree output by LIReco with all the LI-summaries information.

Output: Returns postscript file with summary plots of analysis performed.

\section{E.5 "NFComp" \& "PIDSurveyor"}

This the software developed for the analysis presented in this thesis.

\footnotetext{
${ }^{1}$ Corresponding to each "8-bit ADC vs calibrated 16-bit-like ADC" curve for each channels, RANGE and CAPID.
} 


\section{E.5.1 "NFCompReco"}

Function: The functioning of NFCompReco is summarised in Figure E.1. NFCompReco takes the data from the MINOS offline software and stores them into TTrees according to the kind of data per event.

Input: NFCompReco extracts the data from "TDAQ" files that have been obtained from CalDet "MDAQ" files, once they have been processed by the OLT offline.

Output: NFCompReco return a ROOT file with four TTrees in each. The "GenTTree" stores general information about the run. "CompTTree" and "XtalkTTree" stores the information about hits genuine and related to "crosstalk channels" respectively. Then "PIDTree" stores the information relative to the beam PID instrumentation used for identifying events.

\section{E.5.2 "PIDSurveyor"}

Function: "PIDSurveyor" is a dedicate module that extracts the PID beam instrumentation data of each run and calculates the cuts for event selection, which will become stored in a local database.

The main goal of separating the PID cut calculation from the analysis routine is that the overhead of calculating PID cuts for each run is done only once, speeding up the analysis process. The other advantage of this approach is that one can tune the cuts, preliminary calculated automatically, at a later time and will. Most of the time the automatic values for the cuts are adequate, but sometimes, when lacking statistics, for example, one may desire to sacrifice some purity against statistics by re-tuning the cuts by hand.

Input: "PIDSurveyor" uses the output from "NFCompReco", in particular it uses the data from "PIDTree".

Output: "PIDSurveyor" stores the calculations as the cuts into the PID-Run database file - an ASCII file. Those cuts are available to be used later for event selection during the analysis chain later at any time. 


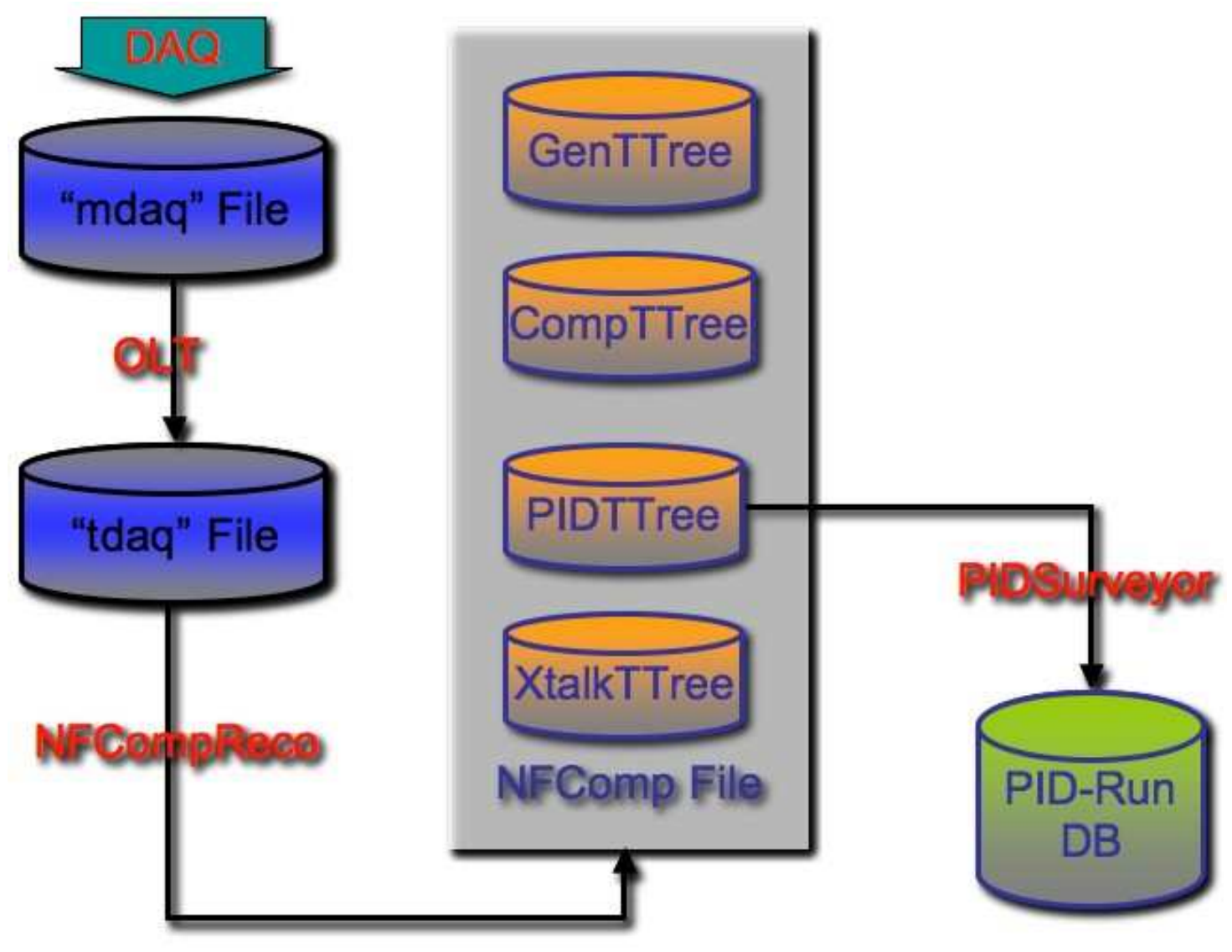

Figure E.1: NFComp: Data Flow Diagram

\section{E.5.3 "NFCompAna"}

Function: "NFCompAna" is the core of the Near/Far comparison data analysis software. The logic structure and dependencies are illustrated in Figure E.2. "NFCompAna" is an OO data analysis based on ROOT and marginally on the MINOS offline software, which uses objects like NFCRuns, NFCSnarls and NFCHit to perform analysis at the run, snarl and hit levels, respectively. Further documentation is in the code itself, which will be made available through the MINOS CVS repository [131].

Input: "NFCompAna" uses the TTrees output by "NFCompReco", explained above.

Output: "NFCompAna" returns, upon configuration, four files, two of which contain relevant data for further analysis at the snarl and run levels: "NFCSnarlTree" and "NFCRunTree"; and two files storing all analysis plots. Of 
the files storing the analysis plots, one stores them in postscript format, as a '.ps' file and the other stores them in a ROOT format, into a '.root' file.

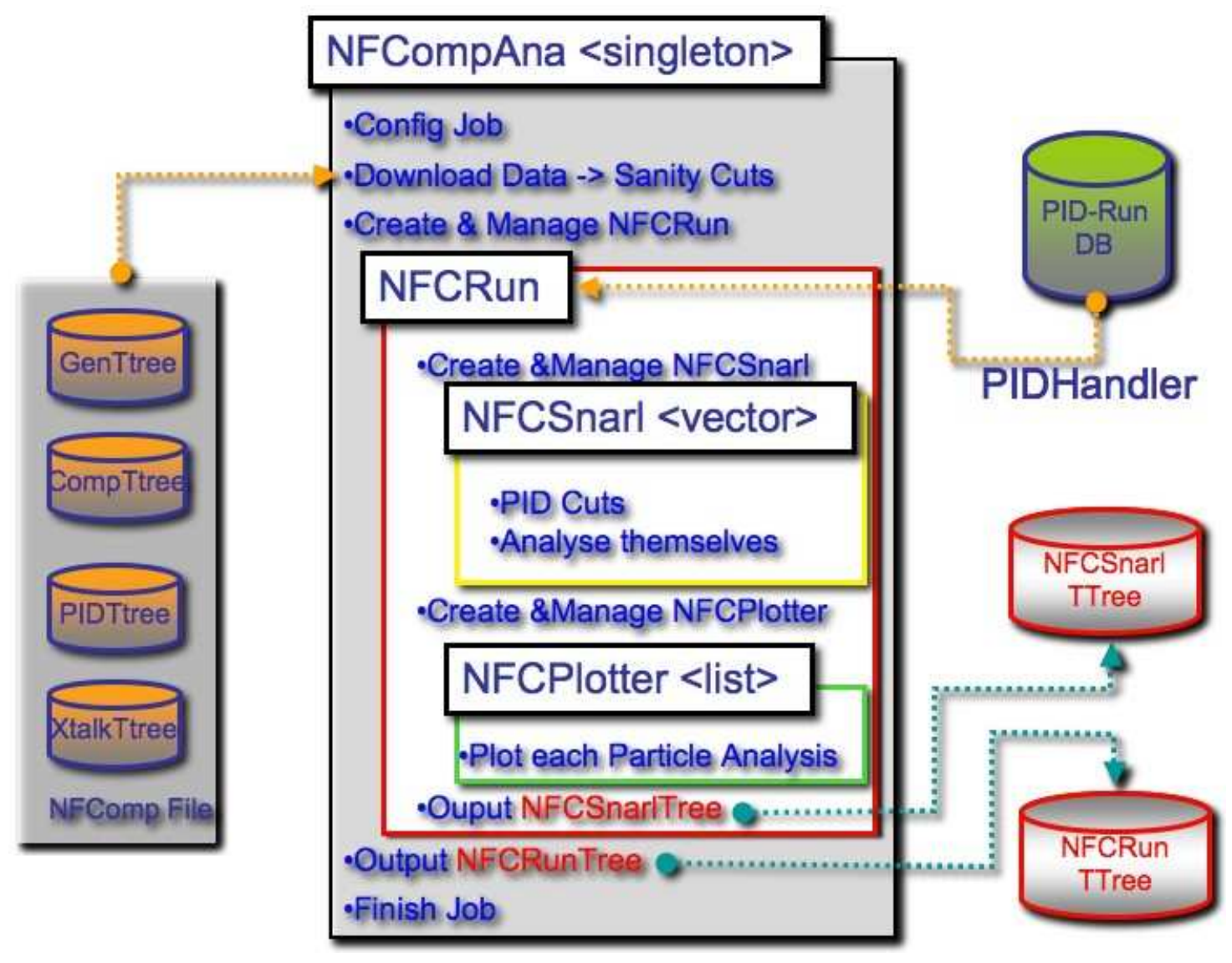

Figure E.2: Execution Structure

\section{E.5.4 "NFCompMvsD" and "NFCompVsE"}

Function: "NFCompMvsD" and "NFCompVsE" are respectively used for data/MC systematic and energy evolution comparisons.

Input: Both programs use the output files from "NFCompAna".

Output: Both programs return files with the respective analysis plots in postscript format. 


\section{BIBLIOGRAPHY}

[1] Glashow S. Nucl. Phys. 22. 579. 1961.

Salam A. Elementary Particle Theory ed N Swarthohn, Almquist and Wiksell (Stochholm) p 367. 1968.

Weinberg S. Phys. Rev. Lett. 19. 1264. 1967.

[2] The Particle Adventure. URL: http://particleadventure.org/particleadventure/

[3] Chadwick J. Verh. der Duetchen Physikalischen Ges. 16. 383. 1914.

[4] Fermi E. Z.Phys. 88. 161. 1934.

[5] Reines F. and Cowan C. Phys. Rev. 92. 330. 1953.

[6] Reines F. and Cowan C. Nature. 178. 446 and 523. 1956.

[7] Reines F. and Cowan C. Science. 124. 103. 1956.

[8] Lee A. and Yang C. Phys. Rev. 104. 254. 1956.

[9] Wu C. Phys. Rev. 105. 1413. 1957.

[10] Goldhaber M., Grodzin L. and Sunyar A. Phys. Rev. 109. 1015. 1958.

[11] Christenson J. et al. Phys. Rev.Lett. 13. 138. 1964.

[12] Danby et al. Phys. Rev. 9. 36. 1962.

[13] Hasert F. et al. Phys. Lett. B. 46. 121. 1973.

[14] Hasert F. et al. Nucl. Phys. B. 73. 1. 1974. 
[15] Arnison R. et al. (UA1). Phys. Lett. B. 122. 103. 1983.

Arnison R. et al. Phys. Lett. B. 126. 398. 1983.

Bagnaia P. et al. (UA2). Phys. Lett. B. 129. 130. 1983.

Banner M. et al. (UA2). Phys. Lett. B. 122. 476. 1983.

[16] Hagiwara K. et al. [PDG-2002] Phys. Rev. D. 66. 010001. 2002.

[17] Kodama K. et al. (DONUT). Phys. Lett. B. 592. 1. 2004.

[18] Davis R. et al. Phys. Rev. Lett. 20. 1205. 1968.

[19] Bahcall J. Pinsonneault M and Basu S. Astrophys. J. 555. 990. 2001.

[20] Basu S. et al. Mon. Not. R. Astron. Soc. 292. 243. 1997.

[21] Kai Zuber. "Neutrino Physics". IoP. 2004.

[22] Cleveland et al. Astrophys. J. 495. 505. 1998.

[23] Abdurashitov J.et al. Phys. Rev. C. 60 055801. 1999.

[24] Ansermann P. et al. Phys. Lett. B. 285. 376. 1992.

[25] Altmann M. et al. Phys. Lett. B. 490. 16. 2000.

Bellotti E. et al. Nucl. Phys. Proc. Suppl. 91. 44. 2001.

[26] Cattadori C. Nu2004 Conference. 2004.

[27] Hirata K. et al. Phys. Rev. D. 44. 2241. 1991.

[28] Borger J. et al. Nucl. Meth. Instrum. A. 449. 172. 2000.

[29] Fukuda Y. et al. (SK) Nucl. Instrum. Meth. A. 501. 418. 2003

[30] Ahmad Q. et al. Phys. Rev. Lett. 89. 011301. 2002.

Ahmed S. nucl-ex/0309004. 2004.

[31] Fukuda Y. et al. (SK) Phys Rev. Lett. 77. 1683. 1996

Suzuki Y. et al. Nucl. Phys Proc. Suppl. 91. 29. 2001

[32] Bachall J., Gonzalez-Garcia M. and C. Peña-Garay. JHEP. 07. 054. 2002.

[33] Becker-Szendy R. et al.. Phys. Rev. Lett. 69. 1010. 1992.

Becker-Szendy R. et al.. Nucl. Phys.Proc.Suppl. 38. 331. 1995. 
[34] Gaisser T. and Honda M. Ann. Rev. Nucl. Part. Sci. 52. 153. 2002.

[35] Fukuda Y. et al. (SK) Phys Rev. Lett. 81. 1562. 2001

[36] Apollonio M. et al. (Chooz) Eur. Phys. J. C. 27. 331. 2003.

Apollonio M. et al. (Chooz) Phys. Rev. Lett. B. 466. 415. 1999.

[37] Mohapatra R. and Senjanovic G. Phys. Rev. Lett. 49. 652. 1980.

[38] de Gouvêa A. Nu2004 Conference. 2004.

[39] Barger V. et al. Phys. Rev. Lett. 82. 2640. 1999.

[40] Lisi E. et al. Phys. Rev. Lett. 85 1166. 2000.

[41] Piepke A. et al. (KamLAND) Nucl. Phys. Proc. Suppl. 91. 99. 2001. Araki T. et al. (KamLAND) hep-ex/04060035. 2004.

[42] Nakamura K. et al. (K2K) Nucl. Phys. Proc. Suppl. 91. 203. 2001.

[43] Aliu E.et al. (K2K) hep-ex/0411038. 2004.

[44] Kearns E. Nu2004 Conference. 2004.

[45] Gratta G. Nu2004 Conference. 2004.

[46] Aguilar A. et al. Phys. Rev. D. 64. 112007. 2001.

[47] Tayloe R. et al. Nucl. Phys. Proc. Suppl. 118. 157. 2003.

[48] Autiero D. Nu2004 Conference. 2004.

[49] Bueno A. Nu2004 Conference. 2004.

[50] Anderson K. hep-ex/0402041. 2004.

[51] Double-Chooz. Letter of Intent: hep-ex/0405032. 2004

[52] URL: http://mwtheta13.uchicago.edu/

[53] Hayato Y. Nu2004 Conference. 2004.

[54] Messier M. Nu2004 Conference. 2004.

[55] Wolfenstein L. Phys. Rev. D. 17. 2369. 1978.

Mikheev S. and Smirnov A. Sov. J. Nucl. Phys. 42. 913. 1985. 
[56] Kraus C. et al. Nucl. Phys. A. 721. 533. 2003.

[57] Assamagan K. et al. Phys. Rev. D. 53. 6065. 1996.

[58] Roney J. Nucl. Phys. Proc. Suppl. 91. 287. 2001.

[59] Elliot S. and Vogel P. Annu. Rev. Nucl. Part. Sci. 52. 115. 2002.

[60] Spergel D. et al. Astrophys. J. Suppl. 148. 1752003.

Elgaroy O. and Lahev O. JCAP. 0304. 004. 2003. (astro-ph/030389)

Hannestead S. JCAP. 0305. 004. 2003. (astro-ph/030376)

[61] Klapdor-Kleingrothaus H. Nu2004 Conference. 2004.

[62] Barger V. et al. hep-ph/0205290. 2002.

[63] The MINOS Collaboration. "The NuMI Technical Design Handbook". 1999.

[64] http://ppd.fnal.gov/experiments/e907/e907.htm

[65] The MINOS Collaboration. NuMI Note 337. 1999.

[66] Holder M. et al. Nucl. Instrum. Meth. 148. 235. 1978.

Holder M. et al. Nucl. Instrum. Meth. 151. 69. 1978.

[67] Sakamoto W. et al. Nucl. Instrum. Meth. A. 294. 179. 1990.

[68] Bolton T. et al. Fermilab Proposal P-815. 1990.

[69] Weber A. et al. NuMI Note 720. 2001.

[70] N. Tagg et al. Nucl. Instrum. Meth. A. 539. 668. 2005.

[71] K. Lang et al. Accepted for publication on Nucl. Instrum. Meth.

[72] Brun R. and Rademakers F. Nucl. Instrum. Meth. A. 521. 81. 1997.

[73] Belias A. et al. IEEE Trans Nucl. Sci. 51. 451. 2004.

[74] Vahle T. PhD Thesis. University of Texas. 2004.

[75] Kordosky M. PhD Thesis. University of Texas. 2004.

[76] Felt N. et al. IEEE Transactions on Nuclear Science. 51. 5. 2193. 2003.

[77] Felt N. et al. NuMI Note 901. 2003. 
[78] Drake G. et al. NuMI Note 628. 1999.

[79] Gallagher H. and Goodman M. NuMI Note 112. 1995.

[80] Zeller G. 2003. hep-ex/0312061.

[81] Diwan M. et al. NuMI Note 714. 2001.

[82] Lindner M. Talk at ECT*, Trento. 2004.

[83] Petyt D. NuMI Note. 691. 2000.

[84] Third International Workshop on Neutrino-Nucleus Interactions In the FewGeV Region (NuInt04), GranSasso, Italy. Nucl. Phys. B Proc.Suppl. 139. 2004.

[85] Letter of Intent: http://www.pas.rochester.edu/ ksmcf/minerva/

[86] http://castore.mi.infn.it/ monolith/

[87] http://www.imsc.res.in/ ino/

[88] Allison W. et al. Phys. Lett. B391. 491. 1997.

Allison W. et al. Phys. Lett. B449. 137. 1999.

Sanchez M. et al. Phys. Rev. D68. 113004. 2003.

[89] Ambrosio M. et al. Phys. Lett. B566. 35. 2003.

[90] http://www-numi.fnal.gov/offline_software/srt_public_context/

WebDocs/detsim/DetSim_Documentation.html

[91] Durkin T. NuMI Note 1073. 2005.

[92] Abramov V. et al. IFVE-96-91.

[93] Eilerts S. et al. NuMI Note 568. 1999.

[94] Barker M. et al. NuMI Note 602. 2000.

[95] Barker M. et al. NuMI Note 650. 2000.

[96] Adamson P. and Vahle T. NuMI Note 978. 2003.

[97] http://psschedule.web.cern.ch/PSschedule/pindex.html.

[98] Saakyan R. Provided to the collaboration. 
[99] M. Kodorsky et al. NuMI Note 946. 2003.

[100] Cabrera A. MINOS Internal Talk @ FNAL. 2002.

Cabrera A. MINOS Internal Talk@ Cambridge. 2003.

[101] Cabrera A. MINOS Internal Talk @ FNAL. 2003.

[102] Nelson C. (FNAL). Private Communication.

[103] Shanahan P. MINOS Internal Talk @ FNAL.. 2003.

[104] Reyna D. (ANL). Private communication.

[105] Cabrera A. NuMI Note 998. 2003.

[106] Adamson P. et al. Nucl. Instum. Meth. A. 492. 325. 2002.

[107] Nichol R. PhD Thesis. University College. 2003.

[108] Smith C. PhD Thesis. University College London. 2002.

[109] Adamson P. PhD Thesis. University Sussex. 2001.

[110] Raufer T. MINOS Internal Talk @ FNAL. 2004.

[111] Shanahan P. (FNAL) Private Communication.

[112] Hartnell J. MINOS Internal Talk @ FNAL. 2003.

[113] Cabrera A. MINOS Internal Talk@ FNAL. 2003.

[114] Lee R. and Seun S. NuMI Note 694. 2000.

[115] Hartnell J. PhD Thesis. University of Oxford. 2005.

[116] Mualem L. (Minnesota) Private Communication.

[117] Cabrera A. MINOS Internal Talk @ FNAL. 2003.

[118] Litchfield P. Private Communication.

[119] Reyna D. NuMI Note 757. 2001.

[120] Smith C. http://www.hep.caltech.edu/ smith/CalDet/Calib_Apr04/

[121] Reufer T. and Litchfield P. MINOS Internal Talk @ FNAL. 2004. 
[122] http://www.hep.caltech.edu/ smith/CalDet/Calib_Dec04/

Temperature_Calibration_Dec04.html

[123] Kordosky M. MINOS Internal Talk. 2005.

[124] Cabrera A. et al. NuMI Note 934. 2003.

[125] Hamamatsu Photonics K.K. "Photomultipliers Tubes - Basics and Applications". 1999.

[126] Hamamatsu Photonics K.K. "R5900-00-M64 PMT Data Sheet". 2000.

[127] Barker M., De Santo A., Weber A. NuMI-L-682. 2000.

[128] Leo W. "Techniques for Nuclear and Particle Physics Experiments". Springer. 1994.

[129] Knoll G. "Radiation Detection and Measurements". John Wiley \& Sons. 1999.

[130] Hartnell J. http://www-numi.fnal.gov/offline_software/srt_public_context/ WebDocs/Package_Overviews/LISummary.html

[131] http://www-numi.fnal.gov/offline_software/srt_public_context/ WebDocs/WebDocs.html 